Enrico Benetto

Kilian Gericke

Mélanie Guiton Editors

Designing Sustainable

Technologies,

Products and Policies

From Science to Innovation
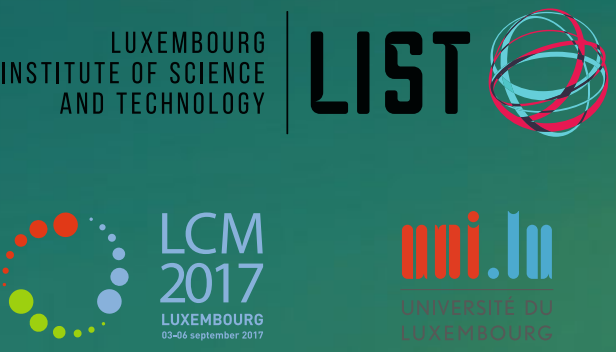

Springer Open 
Designing Sustainable Technologies, Products and Policies 
Enrico Benetto - Kilian Gericke

Mélanie Guiton

Editors

\section{Designing Sustainable Technologies, Products and Policies}

From Science to Innovation

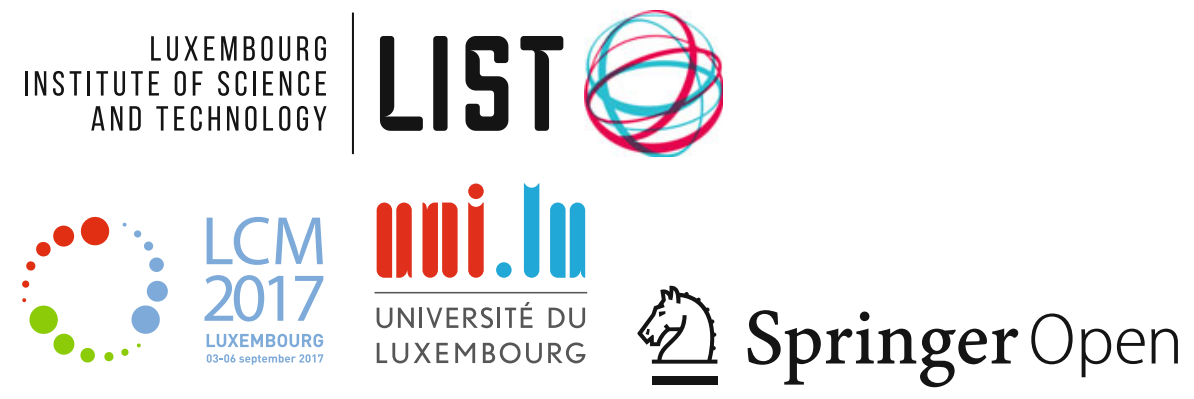




\section{Editors}

\author{
Enrico Benetto \\ Department ERIN-Environmental \\ Research and Innovation \\ Luxembourg Institute of Science \\ and Technology (LIST) \\ Esch-sur-Alzette \\ Luxembourg
}

Kilian Gericke

Department of Engineering

University of Luxembourg, Campus

Kirchberg

Kirchberg, Luxembourg

Luxembourg

\author{
Mélanie Guiton \\ Department ERIN-Environmental \\ Research and Innovation \\ Luxembourg Institute of Science \\ and Technology (LIST) \\ Esch-sur-Alzette \\ Luxembourg
}

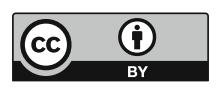

ISBN 978-3-319-66980-9 ISBN 978-3-319-66981-6 (eBook)
https://doi.org/10.1007/978-3-319-66981-6

Library of Congress Control Number: 2018938354

(C) The Editor(s) (if applicable) and The Author(s) 2018. This book is an open access publication.

Open Access This book is licensed under the terms of the Creative Commons Attribution 4.0 International License (http://creativecommons.org/licenses/by/4.0/), which permits use, sharing, adaptation, distribution and reproduction in any medium or format, as long as you give appropriate credit to the original author(s) and the source, provide a link to the Creative Commons license and indicate if changes were made.

The images or other third party material in this book are included in the book's Creative Commons license, unless indicated otherwise in a credit line to the material. If material is not included in the book's Creative Commons license and your intended use is not permitted by statutory regulation or exceeds the permitted use, you will need to obtain permission directly from the copyright holder.

The use of general descriptive names, registered names, trademarks, service marks, etc. in this publication does not imply, even in the absence of a specific statement, that such names are exempt from the relevant protective laws and regulations and therefore free for general use.

The publisher, the authors and the editors are safe to assume that the advice and information in this book are believed to be true and accurate at the date of publication. Neither the publisher nor the authors or the editors give a warranty, express or implied, with respect to the material contained herein or for any errors or omissions that may have been made. The publisher remains neutral with regard to jurisdictional claims in published maps and institutional affiliations.

Printed on acid-free paper

This Springer imprint is published by the registered company Springer International Publishing AG part of Springer Nature

The registered company address is: Gewerbestrasse 11, 6330 Cham, Switzerland 


\section{Preface}

Life Cycle Management (LCM) can be considered an integrated concept and toolbox of methods and operational approaches aimed at improving the environmental, social and economic sustainability of technologies, products, services, policies and organizations from a life cycle perspective. Businesses and policy-making organizations use LCM frameworks to explicitly identify, document, inform and communicate their strategy and to chart a course from this strategy towards a more sustainable society.

The LCM conference series is the leading Life Cycle Assessment forum worldwide, bringing together 700+ LCM scholars and practitioners from 40+ countries working in industry, academia and public institutions. LCM 2017 has been the 8th time this conference has taken place, following the inaugural conference in 2001 in Copenhagen, and highly successful events in Barcelona (2005), Zurich (2007), Cape Town (2009), Berlin (2011), Gothenburg (2013) and Bordeaux (2015).

LCM 2017 was held from the 3 to 6 September 2017 at the European Convention Centre Luxembourg (ECCL) and was organized by the Luxembourg Institute of Science and Technology (LIST), in collaboration with the University of Luxembourg and ArcelorMittal. LCM 2017 attracted 730 international scientists and practitioners of Life Cycle Management from 46 countries. Top-tier international companies, along with senior representatives of European institutions and national governments, took part in the event, which featured a series of star speakers and visionary leaders of the field, such as Bertrand Piccard and Mathis Wackernagel. Delegates also had the opportunity to meet with His Royal Highness Grand Duke Henri of Luxembourg who, accompanied by his son, His Royal Highness Prince Louis, spontaneously took part in the closing session of the conference.

LCM 2017 was thus an opportunity for the whole community of researchers who believe in the effectiveness of LCM to gather together, but it was more than that. With the rise of the Circular Economy as a new paradigm for market growth, several methods and certification schemes to quantify and communicate the impacts and benefits of circular systems have emerged. The LCM toolbox can indeed 
provide a legitimate and scientifically founded, quantitative basis to steer and monitor the transition towards a more sustainable and healthier society. The LCM community must, however, confront important challenges to reach this objective, in particular, in terms of positioning LCM on the political and business agendas, simplifying and standardizing LCM methods without oversimplifying and neglecting complexity, ensuring efficient communication and promoting innovation based on LCM concepts and tools.

LCM 2017 was designed to tackle these challenges and offer to the LCM community the opportunity to take LCM to the next level, to effectively contribute to the journey towards a safer and more sustainable society.

First, the programme of LCM 2017 was designed around new (smart) technologies (and related implementation sectors) which, on the one hand, are likely to disrupt the LCM practice in the coming years (e.g. smart sensors making data collection much easier) and, on the other hand, for which the LCM toolbox has great development potential. In over more than 35 sessions, there were sessions on smart agricultural, smart manufacturing and smart mobility systems, including urban infrastructures and energy for the built environment, sessions specifically targeting the role of LCM for Circular Economies and vice versa, sessions on sectors which are historically under-represented in the LCM series (like pharmaceutical industry or textiles). New technologies such as Blockchain, Building Information Management (BIM), Nature-based Solutions for Cities, and Artificial Intelligence were discussed. The focus on bridging the gap between science and innovation was also pursued in the exhibition area, where companies have showcase technologies developed using the LCM toolbox.

Second, communication (BtoB and BtoC) is historically an improvement lever for our community. The LCM toolbox is often considered by policy-makers and business leaders too difficult, complex and expensive to understand, implement and communicate. Indeed, this is certainly one of the reasons why other approaches, simpler albeit sometimes less rigorous scientifically speaking, could have a much stronger impact on business than LCM.

LCM 2017 included a number of sessions focusing on current standardization approaches, LCM guidelines and best practices as well as on the visualization and interpretation of LCM results.

Finally, from our perspective, it is very important for the LCM community to bridge the gap with other scientific and business communities, pursuing similar objectives, often in a complementary way. This combination can bring higher visibility to policy to the LCM toolbox and to other influential institutions and thus avoid the risk of being considered the result of a niche community of users and academics. With this objective in mind, a number of transversal sessions were run, such as a discussion panel devoted to the funding of LCM and Circular Economies, thanks to the involvement of the European Investment Bank.

This book is a selection of the most relevant contributions to the LCM 2017 together with a resume of the discussion and outcomes from each session. 
I would like to thank my co-chairs, Kilian Gericke (University of Luxembourg) and Jan Bollen (ArcelorMittal), for their support in shaping the programme of the conference and the members of the organizing committee, whose tireless work has made LCM 2017 happen: Mélanie Guiton, Marylène Martin, Céline Goncalves, Tomás Navarrete Gutierrez and Lugdivine Unfer. The Event and Communication departments of LIST and Uni.lu are also gratefully acknowledged for their support in the communication and dissemination actions.

A large conference such as LCM 2017 would never have been possible without the financial help of external institutions. I am very grateful to the Luxembourg National Research Fund (FNR), the Luxembourg Ministry of the Economy and the European Investment Bank for their invaluable support. I would also like to acknowledge the extraordinary level of financial funding from private companies: Evonik Nutrition \& Care GmbH, PRé Consultants B.V., Thinkstep AG and Kronospan Luxembourg S.A. (Platinum Sponsors); BASF SE, Ipoint Systems $\mathrm{GmbH}$ and Quantis International (Gold Sponsors); Delphi Automotive Systems Luxembourg, Plastics Europe, Nestlé S.A., Steelcase Werndl AG and Tarkett GDL S.A. (Silver Sponsors). The sponsors contributed to shape an attractive programme of keynote lectures and discussion panels in a fair and constructive atmosphere, to add valuable content to the conference programme.

We acknowledge the partnership with the Luxembourg Chamber of Commerce for the b2fair matchmaking event, a new feature introduced to the LCM conference series to facilitate networking among participants.

Finally, I would like to thank all the abstract and session contributors, chairs and reviewers, exhibitors and delegates. It is your commitment and interest that made it all happen.

Esch-sur-Alzette, Luxembourg

Enrico Benetto

Chair LCM 2017 


\section{Contents}

Part I LCM, Circular Economy and Product Value Chain

Life Cycle Management Approaches to Support Circular

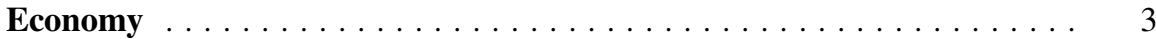

Sébastien Zinck, Anne-Christine Ayed, Monia Niero, Megann Head,

Friedrich-W. Wellmer, Roland W. Scholz and Stéphane Morel

Sustainability Performance Evaluation for Selecting the Best

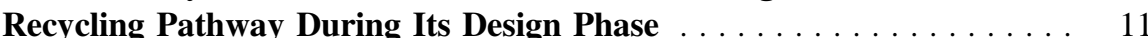

Guilhem Grimaud, Nicolas Perry and Bertrand Laratte

A Synthesis of Optimization Approaches for LCA-Integrated

Industrial Process Modeling: Application to Potable Water

Production Plants . . . . . . . . . . . . . . . . . . . . . . . 21

Florin Capitanescu, Antonino Marvuglia and Enrico Benetto

A Bi-dimensional Assessment to Measure the Performance of Circular

Economy: A Case Study of Tires End-of-Life Management . . . . . . . . . 33

Geoffrey Lonca, Romain Muggéo, Hugue Tétreault-Imbeault,

Sophie Bernard and Manuele Margni

Bio-based Materials Within the Circular Economy: Opportunities

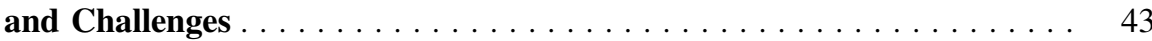

Birgit Brunklaus and Ellen Riise

Bio-Economy Contribution to Circular Economy . . . . . . . . . . . . . . . . 49

Sara Corrado and Serenella Sala

Life Cycle Management and Circular Economy Challenges for the

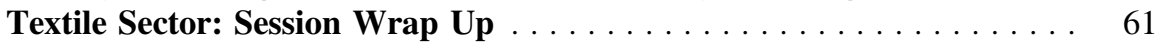

Keith James and Julian Lings

Life Cycle Assessment of Organic, BCI and Conventional Cotton:

A Comparative Study of Cotton Cultivation Practices in India . . . . . . 67

Pragnesh Shah, Abhishek Bansal and Rajesh Kumar Singh 
Life Cycle Management in the Pharmaceutical Industry Using an Applicable and Robust LCA-Based Environmental Sustainability Assessment Approach .

Yasmine Emara, Marc-William Siegert, Annekatrin Lehmann

and Matthias Finkbeiner

Establishing LCA in the Healthcare Sector

Nanja Hedal Kløverpris

Improving the Life Cycle Performance of Chemical Products and Materials Through Data Exchange Along the Value Chain-Synthesis of LCM2017 Session Presentations . . . . . . . . . . . . . . . . . . .

Guido Sonnemann and Carmen Alvarado

Biomass Balance: An Innovative and Complementary Method

for Using Biomass as Feedstock in the Chemical Industry . . . . . . . . . . 101

Christian Krüger, Andreas Kicherer, Claudius Kormann

and Nikolaus Raupp

Greening Agri-food Value Chains in Emerging Economies

Matthias Stucki and Anél Blignaut

Assessment of Cleantech Options to Mitigate the Environmental

Impact of South African Dairy Farming

Regula Keller, Lea Eymann, Sarah Wettstein, Deborah Scharfy

and Matthias Stucki

Food Waste Management (Sector) in a Circular Economy .

Nicole Unger and Francesco Razza

The Role of Compost in Bio-waste Management and Circular

Economy

Francesco Razza, Lorenzo D’Avino, Giovanni L'Abate and Luca Lazzeri

Agri-Food Waste Streams Utilization for Development of More

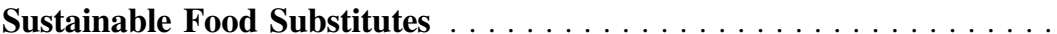

Sergiy Smetana, Kemal Aganovic, Stefan Irmscher and Volker Heinz

Implementation and Management of Life Cycle Approaches in

Business-Challenges, Opportunities, Business Learnings and

Best Practice.

Lena Landström and Sara Palander

Neuro-marketing Tools for Assessing the Communication

Effectiveness of Life Cycle Based Environmental

Labelling-Procedure and Methodology

Anna Lewandowska, Barbara Borusiak, Christian Dierks,

Pasquale Giungato, Ewa Jerzyk, Przemyslaw Kurczewski,

Jagna Sobierajewicz, Sangwon Suh and Joanna Witczak 
Part II Innovation for Sustainable Production and Urban Systems

LCM for Transport and Mobility

Stephan Krinke and Mara Neef

Conceptual Development of Hybrid Structures Towards Eco-Efficient

Vehicle Lightweighting .

Alexander Kaluza, Tim Fröhlich, Sebastian Kleemann, Wolfgang Walk,

Christoph Herrmann, Stephan Krinke and Thomas Vietor

LCA in Strategic Decision Making for Long Term Urban

Transportation System Transformation

Florian Ansgar Jaeger, Katrin Müller, Cornelia Petermann and Eric Lesage

Management of Construction Waste: LCA and Complex System

Modeling

Anne Ventura and Maxime Trocmé

Guidelines for Effective and Sustainable Recycling of Construction

and Demolition Waste

Serena Giorgi, Monica Lavagna and Andrea Campioli

Environmental Assessment of Energy Related Products

and Energy Systems Across Their Life Cycle.

N. Espinosa and Y. J. Suh

Is It Useful to Improve Modelling of Usage Scenarios to Improve

the Environmental Footprint of Energy-Using Product?

Charlotte Heslouin, Véronique Perrot-Bernardet, Lionel Pourcheresse and Nicolas Perry

Life Cycle Management of Energy and Energy TransitionsManaging the Complexity of Todays and Future Energy Systems with a Life Cycle Focus: Challenges and Methodological

Solutions

Karin Treyer, Roberto Turconi and Alicia Boyano

Integrating Energy System Models in Life Cycle Management

Miguel F. Astudillo, Kathleen Vaillancourt, Pierre-Olivier Pineau and Ben Amor

LCM at the Urban Scale: BIM and Nature Based Solutions

Antonino Marvuglia, Benedetto Rugani and Germain Adell

Design-Integrated LCA Using Early BIM

Alexander Hollberg, Julia Tschetwertak, Sven Schneider and Guillaume Habert 
A Proposition to Extend CityGML and ADE Energy Standards for Exchanging Information for LCA Simulation at Urban Scale 281

Adélaïde Mailhac, Emmanuelle Cor, Marine Vesson, Elisa Rolland,

Pascal Schetelat, Nicoleta Schiopu and Alexandra Lebert

Dynamic Assessment of Nature Based Solutions Through Urban

Level LCA

Duygu Başoğlu, Emre Yöntem, Seda Yöntem, Beril Șenyurt

and Özge Yılmaz

Role of Carbon Capture and Storage (CCS) or Use (CCU)

on Climate Mitigation

Jan Bollen

\section{Part III Development of LCM Methods and Tools}

Potentials and Limitations of Combined Life Cycle Approaches and Multi-dimensional Assessment

Johanna Berlin and Diego Iribarren

Propagating Uncertainty in Life Cycle Sustainability Assessment into Decision-Making Problems: A Multiple Criteria Decision Aid Approach

Breno Barros Telles do Carmo, Manuele Margni and Pierre Baptiste

The Value of Transdisciplinary Perspectives During Transition to a Bio-based Economy: The Prospect for Converting Mixed Food Waste into Bio-based Chemicals . . . . . . . . . . . . . . . . . . . . . . . . . . 327

Birgit Brunklaus, Emma Rex, Johanna Berlin, Frida Røyne,

Johanna Ulmanen and Graham Aid

Improving Interpretation, Presentation and Visualisation of LCA Studies for Decision Making Support

Serenella Sala and Jessica Andreasson

Visualizing the Effects of Parameter Variability on Comparative

LCA Results

Céline Alexandre, Elisabeth van Overbeke, Maxime Dupriez,

Johan Lhotellier and Bernard De Caevel

Life Cycle Management in Industry-Supporting Business with Life

Cycle Based Assessments

Andreas Bednarz, Julia Beier, Thomas Grünenwald, Birgit Himmelreich,

Bärbel Hundt, Florian A. Jaeger, Martin Kirchner, Stephan Krinke, Ulla Létinois, Christina Merz, Lisa Mohr, David Morris, Nikolaj Otte, Gerald Rebitzer, Peter Saling, Urs W. Schenker, Diederik Schowanek, Gerd Vollmer, Niklas von der Assen and Alain Wathelet 
Sustainable Design of Complex Systems, Products and Services

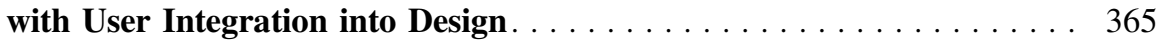

Nicolas Perry and Julien Garcia

Implementation of Environmental Considerations in the Innovation Process of Complex Systems: Groupe PSA Case Study . . . . . . . . . . . . 371 Julien Garcia, Pierre Tonnelier and Sophie Richet

Development of an Environmental Evaluation Tool in the Transport Sector and Its Impact on Decision-Making in the Early Stages of Design 381

Sergio A. Brambila-Macias, Lisbeth Dahllöf, Karin Eriksson and Tomohiko Sakao

Discussion Panel-Assessment of Externalities: Monetisation and Social LCA

Stéphane Morel, Marzia Traverso and Philipp Preiss

Applying LCA to Estimate Development Energy Needs: The Cases of India and Brazil .

Narasimha D. Rao, Alessio Mastrucci and Jihoon Min

Integrating the Concept of Planetary Boundaries into Decision

Making Processes .

Marcial Vargas-Gonzalez

Exploring the Linkages Between the Environmental Sustainable

Development Goals and Planetary Boundaries Using the DPSIR

Impact Pathway Framework

Chanjief Chandrakumar and Sarah J. McLaren

Part IV How to Develop and Sustain LCM-Based Innovations

Financing Innovation and Circular Economy .

L. Goovaerts, C. Schempp, L. Busato, A. Smits, L. Žutelija and R. Piechocki

Life Cycle Approaches to Sustainable Regional Development

Fritz Balkau and Timothy Grant

Turning the Lens Around: LCA Success Stories Outside-In

Eric Mieras and Alain Wathelet

Integrated Product Development at Nestlé

Namy Espinoza-Orias, Karen Cooper and Sofiane Lariani

The City Performance Tool-How Cities Use LCM Based

Decision Support .

Jens-Christian Holst, Katrin Müller, Florian-Ansgar Jäger

and Klaus Heidinger 
Sustainability of Bio-based Products: Linking Life Cycle Thinking with Standards, Certification and Labelling Schemes . . . . . . . . . . . . 469 Mauro Cordella and Renata Kaps

Sustainability Assessment of Blue Biotechnology Processes:

Addressing Environmental, Social and Economic Dimensions

Paula Pérez-López, Gumersindo Feijoo and María Teresa Moreira

Integrating Life Cycle Assessment and Eco-design Strategies for a Sustainable Production of Bio-based Plastics .

Venkateshwaran Venkatachalam, Sebastian Spierling, Hans-Josef Endres and Andrea Siebert-Raths

Using LCA and EPD in Public Procurement Within the Construction

Sector .

Kristian Jelse and Kristof Peerens

Green Public Procurement and Construction Sector: EPD and LCA

Based Benchmarks of the Whole-Building

Sara Ganassali, Monica Lavagna, Andrea Campioli

and Sergio Saporetti

Special Session on Product Environmental Footprint

Mélanie Guiton and Enrico Benetto 


\section{Part I \\ LCM, Circular Economy and Product Value Chain}




\title{
Life Cycle Management Approaches to Support Circular Economy
}

\author{
Sébastien Zinck, Anne-Christine Ayed, Monia Niero, Megann Head, \\ Friedrich-W. Wellmer, Roland W. Scholz and Stéphane Morel
}

\begin{abstract}
This article summarizes the panel session "Life Cycle Management approaches to support Circular Economy" of the 8th International Conference on Life Cycle Management (LCM2017 conference, Luxembourg). Four panellists were invited to share their point of view on this topic. Each of them brought a different perspective, addressing the topic from both the academic and industrial point of view; focusing on a raw materials aspect or considering a life cycle (or eco-design) related scope; in the context of a certification process (for products or activities) or of an eco-innovation process (including new business models for circular economy). After short presentation by each of the panellists, the discussion especially addressed the complementarity between several LCM concepts to be considered jointly when developing circular concepts and models.
\end{abstract}

\footnotetext{
S. Zinck (ه)

Environmental Research and Innovation, Life Cycle Sustainability

and Risk Assessment, Luxembourg Institute of Science and Technology,

4422 Belvaux, Luxembourg

e-mail: sebastien.zinck@list.lu
}

A.-C. Ayed

Tarkett, R\&I Centre, Z.A. Salzbaach, 9559 Wiltz, Luxembourg

M. Niero

Division for Quantitative Sustainability Assessment, Technical University

of Denmark, DTU Management Engineering, 2800 Kongens Lyngby, Denmark

M. Head

Steelcase Inc., Global Sustainability, 901 44th St. SE, Grand Rapids MI, USA

F.-W. Wellmer

Federal German Institute of Geosciences and Natural Resources (BGR),

Neue Sachlichkeit 32, 30655 Hannover, Germany

R. W. Scholz

Natural and Social Science Interface, Swiss Federal Institute of Technology,

Zurich, Switzerland

S. Morel

Renault, Technocentre, 1, Avenue du Golf, 78280 Guyancourt, France

(C) The Author(s) 2018

E. Benetto et al. (eds.), Designing Sustainable Technologies,

Products and Policies, https://doi.org/10.1007/978-3-319-66981-6_1 


\section{Introduction}

Circular Economy (CE) is a concept that has gained some significant traction for some years, both on the policy and the industrial levels. It helps further structure Sustainability strategies and initiatives. And it can be described as an organizational principle which aims at evolving from the current linear economic model — where resources are extracted, manufactured, consumed and wasted - to an economic model which values resource efficiency, not only from a today's perspective, at every stage of the value chain and enables the biodiversity protection, as well as a development suitable for the well-being of individuals.

From the definition of a CE strategy to the implementation of action plans, as well as for the development of new business models in this field, processes, indicators and tools are necessary to support decision-making [1]. Life Cycle Management (LCM) approaches and expertise are thus suitable to ensure the Sustainability performance of decision-making. In this discussion panel session, 4 speakers from companies and academia presented some examples of LCM approaches (e.g. environmental impact assessment methods, eco-design, recycling, etc.), but also collaborative tools, in support of CE strategy definition and implementation.

\section{A Decision Support Framework for Circular Economy Implementation in the Packaging Sector}

Monia Niero (Technical University of Denmark) presented a decision support framework for the development of continuous loop packaging systems, which builds on the combined use of Life Cycle Assessment (LCA) and the Cradle to Cradle $^{\circledR}$ (C2C) certification program [2]. The C2C design framework [3] inspired the creation in January 2014 of the Carlsberg Circular Community, i.e. a cooperation platform involving Carlsberg and a selection of global partners with the ambition to develop packaging products that are optimized for recycling and reuse, while retaining their quality and value [4]. As a first step of the framework, the environmentally optimal beverage packaging life cycle scenario is identified, both in terms of defined use and reuse. Second, the limiting factors for the continuous use of materials in multiple loops are identified considering the two requirements in the $\mathrm{C} 2 \mathrm{C}$ certification process that address the material level (i.e. "material health" and "material reutilization" criteria) and the "renewable energy" criterion [5]. Then, alternative scenarios are built to meet $\mathrm{C} 2 \mathrm{C}$ certification criteria, and LCA is used to quantify the environmental impacts of the resulting improvement strategies, for example, change in material composition, in order to guide the identification of the optimal scenario from an eco-efficiency point of view. Finally, the business perspective is addressed by assessing the potential for a green value network business model for a closed-loop supply [6]. The outcome is a list of prioritized actions 
needed to implement the most eco-efficient and eco-effective strategy for the beverage packaging, both from an environmental and an economic point of view. The decision support framework was tested in the case of the aluminium cans, with main recommendation from both the LCA [7] and C2C perspective [8] to ensure a system that enables can-to-can recycling. Designing packaging for "zero contamination" and improving transparency in materials composition to assure high quality recycling were the main lessons learnt from the Carlsberg Circular Community [2]. The suggested framework for optimization of continuous loop system can be applied and adapted by any other company familiar with LCA and C2C certification tools, based on eco-efficiency and eco-effectiveness approaches, respectively.

\section{Leveraging an Ecodesign Foundation to Enable Circular Value Creation}

Megann Head (Steelcase) presented Steelcase's strong foundation of eco-design practices, rooted in LCM principles. In the company, the three pillars that guide efforts to innovate, improve, and deliver on product promises include materials chemistry, life cycle thinking, and reuse/recycle. Each of these pillars are necessary aspects of product performance as the company transitions to circular business models. However, they may need to adapt, and new capabilities will need to emerge. New design sensibilities need to be utilized, expanding upon those already existing, such as design for disassembly and recycling. The new design sensibilities could be designing for refurbishment and remanufacturing and harvesting parts that feed new products. The products and services (business models) need to work together in a circular economy, so they both need to be developed with a systems mind-set. The existing tools used to evaluate these product-service systems, like Life Cycle Assessment, can be useful in initial evaluations, but do need some updates, such as for allocation, in a circular economy.

\section{Raw Materials Are Products of Our Brain-What Does This Mean for LCM?}

For Friedrich-W. Wellmer (Federal German Institute of Geosciences and Natural Resources) and Roland W. Scholz (former chair of Natural and Social Science Interface, Swiss Federal Institute of Technology), raw materials are products of the brain [9]. They are not a fixed parameter or quantity. They vary according to creativity, demand and supply, and technology. This means that URR (ultimate recoverable resources) cannot be a fixed quantity, as long as economic activity and innovations continue. Individuals do not need raw materials as such. They need an intrinsic property to fulfil a function. For finding solutions for functions, individuals have three 


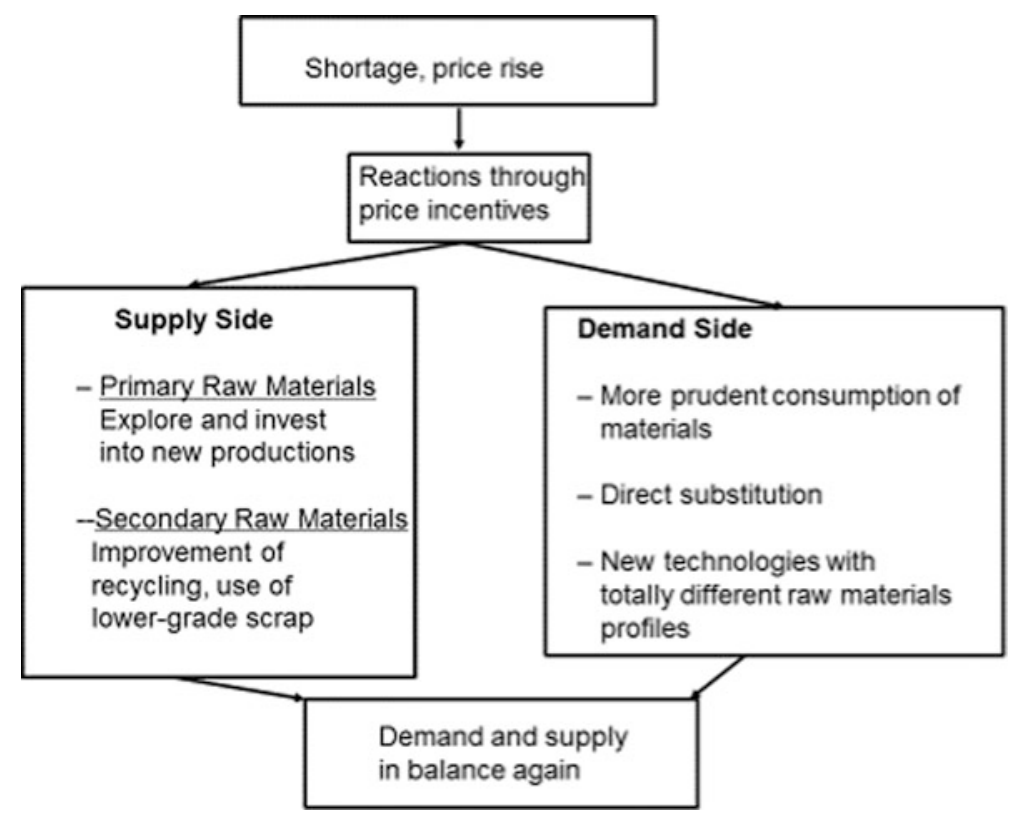

Fig. 1 The feedback control cycle of mineral supply [9]

spheres at their disposal: resources of the geosphere (natural resources), resources of the technosphere (atoms do not get lost) and the human ingenuity. Therefore, the driver proposed for finding solutions in a market economy is the price. Technology and human demand decide what of mineral resources are needed with what properties and to what amount. We also have to acknowledge that an increase of prices induces an increase of reserves. Thus the concept of scarcity is relative and may have to be adjusted also from a mid- and long-term perspective. Via the feedback control cycle of mineral supply in times of shortages and price peaks, there are incentives on the supply side to produce more functions from primary and secondary materials, and on the demand side to use less or to substitute materials, Fig. 1 [10]. More production, less consumption will re-establish a market equilibrium. This feedback control cycle also regulates the finding of the optimum in LCM. LCA helps to find better solutions while the market optimum will be determined by price incentives.

\section{Collective Action to Settled New Circular Economy Business Models}

A recent report from AFEP (2017) pinpointed that Multi Actor Action is a lever to set up new circular economy activities. Stéphane Morel (Renault) proposal is to discuss this statement and the potential for the life cycle community to contribute. 
The creation of a new business model needs two pillars [11]. In one hand it is an explorative activity, on the other hand, it is a collective action. In the case of Circular Economy Business Models, we can particularly point out the necessity to set a dialogue between stakeholders from various horizons and motivations. In shorter recycling loops, you may introduce the notion of second hand parts and remanufacturing to customers and industrial plants. In longer loops you may design the product in a way that improve dismantling and sorting efficiency at its end of life. Collective action will involve all stakeholders committed in the new business model construction. But they are not spontaneous and need to be managed.

One proposal to manage this dialogue is to use the Collaborative Life Cycle Activities (Co-LCA) way [12] developed during the environmental footprint assessment of Renault first electric vehicle [13]. This scheme embeds three levels (Purpose, People and Action) and follow five steps: E1: Explore the topic; E2: Engage with appropriate stakeholders; E3: Elucidate the questions; E4: Evaluate the benefits; and finally E5: Extend to other activities.

To anchor the new business model and transform it into a dominant model, Stéphane Morel underlined the need for economic actors to measure the creation of shared value [14]. In order to proceed, four fields of benefits are proposed: 1/ financial income; 2/brand improvement; 3/knowledge sharing and 4/decision and anticipation accuracy.

As a conclusion, the LCA community is well grounded in collective action to carry comprehensive and complex studies [15]. Therefore, they are effective support to facilitate the creation of circular economy businesses. As a challenge for the next decade, though, this community shall continue to dig into data, but shall also open more largely to social and management science to build the bridge from utopia to real life businesses.

\section{Key Issues and Learnings from the Discussion}

One of the conclusion is that LCA and C2C can be complementary approaches to address circular economy issues. Indeed, C2C provides a vision for continuous use of materials through the avoidance of chemicals of concern, therefore facilitating the valorisation of materials over biological or technical cycles, while LCA allows to identify the intermediary milestones to be reached and provides a quantitative assessment of environmental impacts, both required to reach the vision set through $\mathrm{C} 2 \mathrm{C}$ concept in a sustainable way.

It was also pointed out that one of the main challenge to overcome in the LCA field, within the circular economy context is the quantification of the benefits from recycling, both in terms of substituted materials and quality of the secondary material [16-20]. It was also noticed that an important contribution to solve the related issue of multi-functionality and substitution of primary material has been provided by the Product Environmental Footprint (PEF) pilots initiative through the definition of the Circular Footprint Formula [21]. 
As a more general finding, the assessment of circularity through the consistent measurement of positive aspects/benefits of circular economy strategies on the environment and society is one of the major evolutions to be addressed by LCM methodologies.

Another technical challenge for LCM tools is to bridge the gap between the measurement of CE indicators at a micro-level (e.g. product) and at a macro-level (e.g. national policy). This would support a better translation and management of top-down strategic initiatives into action plans (e.g. European Commission CE objectives implementation at a company level).

Finally, it was demonstrated that considering a market incentive (i.e. economic indicator, like a price signal) is often necessary to find the optimum level of resources consumption, when applying LCM approaches. A methodological challenge would be to integrate this indicator into LCA practices.

\section{References}

1. Bertrand A, Guiton M, Zinck S, Scientific approaches and tools supporting Circular Economy concepts, Revue Technique Luxembourgeoise, 2-2017.

2. Niero M, Hauschild MZ, Hoffmeyer SB, Olsen SI, Combining eco-efficiency and eco-effectiveness for continuous loop beverage packaging systems: learnings from the Carlsberg Circular Community. Journal of Industrial Ecology Vol. 21, No 3, 2017, pp. 742753.

3. McDonough W, Braungart M, Cradle to cradle, New York: North Point Press, 2002.

4. Carlsberg Group, Corporate Sustainability Report 2014, Growing Responsibly Together, 2015.

5. Cradle to Cradle Products Innovation Institute, Cradle to Cradle Certified Product Standard Version 3.1., 2016.

6. Stewart R, Niero M, Murdock K, Olsen SI, Exploring the implementation of a circular economy strategy: the case of a closed-loop supply of aluminum beverage cans, To be submitted to Procedia CIRP (September 2017).

7. Niero M, Olsen SI, Circular economy: to be or not to be in a closed product loop? A Life Cycle Assessment of aluminium cans with inclusion of alloying elements, Resources Conservation and Recycling, Vol. 114, 2016, pp. 18-31.

8. Niero M, Negrelli AJ, Hoffmeyer SB, Olsen SI, Birkved M, Closing the loop for aluminium cans: Life cycle assessment of progression in Cradle-to-Cradle certification levels, Journal of Cleaner Production, Vol.126, 2016, pp. 352-362.

9. Wellmer FW, Scholz RW, Peak minerals: what we can learn from the history of mineral economics and the cases of gold and phosphorus?, Mineral Economics, 30,2, 2017, 73-93.

10. Wellmer FW, Hagelüken C, The feedback control cycle of mineral supply, increase of raw material efficiency, and sustainable development, Minerals, 5, 2015, 815-836.

11. Beulque R, Franck A, "Circular Business Model Innovation: Key Patterns and Challenges to unleash recycling value creation potential", EGOS, 2016.

12. Morel S, Unger L, Buet G, "Behind-the-scenes of eco-innovation at Renault: from collective action to breakthrough concepts", International Journal on Interactive Design and Manufacturing (IJIDeM), 10.3 (2016): 251-255.

13. Morel, S. L'empreinte environnementale à l'ère de la société collaborative: de l'Analyse du Cycle de Vie comme outil expert à une instrumentation collaborative pour conduire une transition organisationnelle Diss. Ecole Nationale Supérieure des Mines de Paris, 2014. 
14. Kramer MR, Porter M, "Creating shared value", Harvard business review, 89.1/2 (2011): 62-77.

15. Morel, S., Beulque R, "Dynamiques collaboratives de co-construction et pilotage renforcé de l'action collective: quels enseignements de l'ACV pour l'économie circulaire?", 7th International Conference on Life Cycle Management-Life Cycle Management for product sustainability value creation, 2015.

16. Rigamonti L, Grosso M, Sunseri MC, Influence of assumptions about selection and recycling efficiencies on the LCA of integrated waste management systems, International Journal of Life Cycle Assessment, Vol.14 No.5, 2009, pp. 411-419.

17. Gala $\mathrm{AB}$, Raugei $\mathrm{M}$, Fullana-i-Palmer $\mathrm{P}$, Introducing a new method for calculating the environmental credits of end-of-life material recovery in attributional LCA, International Journal of Life Cycle Assessment, Vol.20, 2015, pp. 645-654.

18. Schrijvers DL, Loubet P, Sonnemann G, Developing a systematic framework for consistent allocation in LCA, International Journal of Life Cycle Assessment, Vol.21, No.7, 2016, pp. 976-993.

19. Vadenbo C, Astrup T F, Hellweg S, Let's be clear(er) about substitution-a reporting framework to account for product displacement in LCA, Journal of Industrial Ecology, 2016 https://doi.org/10.1111/jiec.12519.

20. Zink T, Geyer R, Startz R, A Market-Based Framework for Quantifying Displaced Production from Recycling or Reuse, Journal of Industrial Ecology, Vol.20 No.4, 2015, pp. 719-729.

21. Zampori L, Pant R, Schau EM, De Schryver A, Galatola M, Circular Footprint Formula, Circular Footprint Formula, Ispra, Italy: European Commission, 2016. display/EUENVFP/ Technical + Advisory + Board + workspace. (Accessed 04.01.2017).

Open Access This chapter is licensed under the terms of the Creative Commons Attribution 4.0 International License (http://creativecommons.org/licenses/by/4.0/), which permits use, sharing, adaptation, distribution and reproduction in any medium or format, as long as you give appropriate credit to the original author(s) and the source, provide a link to the Creative Commons license and indicate if changes were made.

The images or other third party material in this chapter are included in the chapter's Creative Commons license, unless indicated otherwise in a credit line to the material. If material is not included in the chapter's Creative Commons license and your intended use is not permitted by statutory regulation or exceeds the permitted use, you will need to obtain permission directly from the copyright holder. 


\title{
Sustainability Performance Evaluation for Selecting the Best Recycling Pathway During Its Design Phase
}

\author{
Guilhem Grimaud, Nicolas Perry and Bertrand Laratte
}

\begin{abstract}
As the end of life products are becoming more and more complex, the recycling systems encountered many difficulties in valuing all the materials contained in each product. This involves not only recovering a large number of materials but also doing so with the minimal environmental impact. Although the benefits of recycling are well established, the industrial processes need to be designed in regard with their environmental impacts. Therefore recyclers need robust assessment tools to make the right choices during the design of recycling processes. This approach should enable them to choose the right recycling solutions for a wide range of end of life products. In this article, we present a methodology developped for evaluating the performance of recycling processes during their design phase. This methodology is our answer to help the optimisation of the recycling of multi materials products based on the evaluation of the sustainability performance of the processes chosen.
\end{abstract}

\section{Introduction}

The growth of world's population and its life conditions go hand in hand with the growth of energy and raw material consumption as well as the steady growth of $\mathrm{CO}_{2}$ concentration in the atmosphere $[1,2]$. As the economy is mostly linear, the growth of consumption comes with an increase in the amount of waste produced annually [3]. In this context, the demand for primary resources is not tenable in a long-term $[4,5]$. It is therefore required to find industrial solutions to maintain or improve standards of living while also decoupling resource use and demand [6].

\section{G. Grimaud ( $\square)$}

MTB Recycling, Trept, France

e-mail: guilhem.grimaud@ensam.eu

G. Grimaud · N. Perry · B. Laratte

Arts et Métiers ParisTech, CNRS, I2M Bordeaux, F-33400 Talence, France

B. Laratte

APESA-Innovation, Talence, France

(C) The Author(s) 2018

E. Benetto et al. (eds.), Designing Sustainable Technologies,

Products and Policies, https://doi.org/10.1007/978-3-319-66981-6_2 


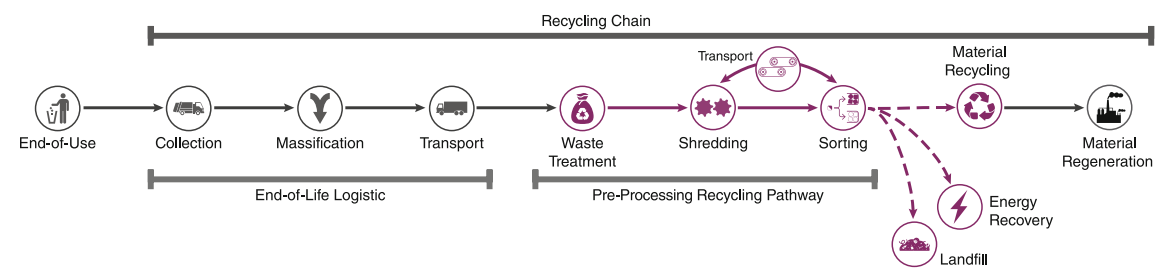

Fig. 1 Main steps of the end-of-life chain including recycling pathway

The circular economy offers a partial answer to resource depletion [7]. Recycling is inherent in the circular economy strategies making industrial companies looking for stepping recycling rates up. To do so one of the most efficient way is to implement product centric End-of-Life (EoL) strategies using closed loop recycling [8]. It shows good environmental performances but they rely on specific EoL processes. Furthermore, those EoL strategies require a suitable and efficient supply chain to reach the recycling plant. The different steps of an EoL scenario are shown on the Fig. 1. Unfortunately, the generalization of closed loop recycling is slowed down, if the associated economic balance is not favourable [9-11].

MTB company, an international manufacturer of recycling technologies and a recycling operator in France, has launched a sustainability strategy. The aim of the strategy is to reduce the environmental impact of its industrial activities. To do so, MTB started to evaluate its environmental performance with evaluation tools such as Life Cycle Assessment (LCA) and Materials Flow Analysis (MFA). The first evaluation has been realised on an aluminium recycling process using only mechanical separation process instead of smelting. Results show the advantages of mechanical processes [12]. Based on these results from environmental evaluations, MTB implemented corrective measures to increase its environmental performance level [13]. Beyond optimizing recycling pathways in operation, these results also helped us to guide the research for new recycling processes which have been designed to be more sustainable [14]. All these steps help to enrich the company's own knowledge, but the evaluation process is long and requires strong stakeholder involvement at each assessment step.

In order to make this new practice more systematic and provide relevant data to decision makers, a methodology was needed to integrate the Life Cycle Management (LCM) approach during the design phase. The technologies used for pre-recycling processes are multiple and it is important to determine the best combination according to different categories of indicators and not only financial performance. The purpose of our work is to provide the engineering team with the results of the environmental evaluation during the design phase. Based on this information, the engineering team will be able to select the best recycling pathway. This method is intended more specifically to assess waste that are not recycled so far.

The construction of our approach has been broken up into several key stages. First, the evaluation tools (LCA, MFA) were used to characterize technologies and to identify the key impact category indicators. Next, the Environmental Technology 
Verification (ETV) was used to draw the evaluation framework for recycling pathway. Then, the final stage was the implementation of a decision-making tool for the engineering team. In this article, we present the interlinking of tools to conduct an evaluation during the design phase of recycling processes.

\section{Methodological Framework}

\subsection{Segmentation of Recycling Processes}

The recycling pathways are mostly based on common elementary technologies. The elementary technology selection and order have a strong influence on the overall performance of the recycling chain [15]. This assembly achieves the targets of purity and quality specific to processed waste. The performances largely depend on the pathway rather than technological innovations [16], hence, the assembly choices of common sub-processes are one of the key points to design efficient recycling pathways. The Fig. 2 shows EoL's pathway alternatives for the same waste. The technologies used and the streams vary with recycling process choices. We have determined that recycling processes can be classified in three types [17]: shredding, separation and transport. In addition to these three families of process unit, there is the flow unit family.

\subsection{Unit Process Database}

To support the evaluation, we launched the construction of a database for recycling processes. This database includes technical, environmental and economic datasets. On the one hand, for each data a part of the values is fixed. They are invariant data regardless the type of transformation performed by the unit process. This is mainly the impact of manufacturing, its price without the options or the weight of the

(a)

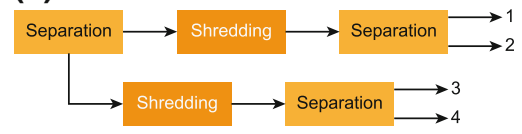

(b)

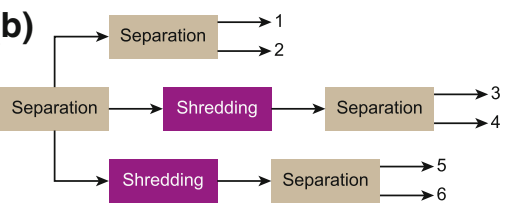

(c)

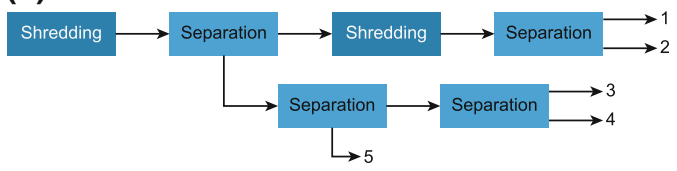

Fig. 2 Presentation of different pathways for the same waste 


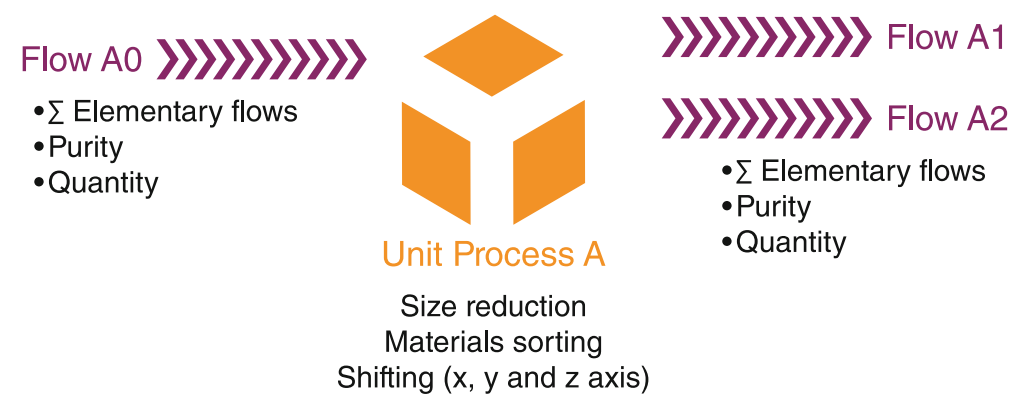

Fig. 3 Modeling of a recycling pathway step with a separation unit process

equipment. On the other hand, in addition to these fixed values, the engineering team define values to adjust the unit process to the specificities of the customer needs. These are the operating variables. These actions will have a direct effect on the performance of the recycling pathway. Each unit process and its associate in/output flows can be modelled as shown on the Fig. 3.

In order to define the technical characterization of each unit process, we have chosen to implement the Environmental Technology Verification (ETV) protocol $[18,19]$. The main steps of the ETV program are given on Fig. 4. The whole ETV verification steps combine together take eight to eighteen months to be performed [20]. In comparison, the average designing time for a recycling pathway is between three and six months. Although ETV's verification time is too long for designers, the program provided general requirements, allowing to develop a self-assessment framework [21].

For the three families of unit process, the Table 1 gives the associate operational details and the technical characterization defined using the ETV program. For each specific unit process, technical characterization will help to define the most suitable process for each purpose of the recycling pathway step.

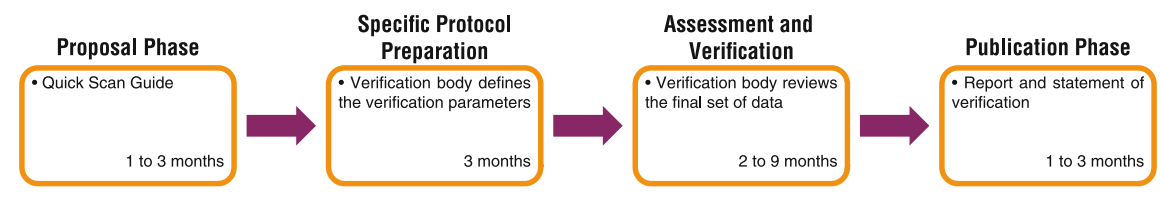

Fig. 4 Main steps of the European environmental technology verification process 
Table 1 Variables and characterization for recycling each unit process family

\begin{tabular}{l|l|l}
\hline Type & Operational details & Characterization \\
\hline Shredding & $\begin{array}{l}\text { Type of technology (constraint) } \\
\text { Cost of purchase } \\
\text { Material losses } \\
\text { Capacity }\end{array}$ & Reduction rate/fineness \\
\hline Separation & $\begin{array}{l}\text { Type of technology (constraint) } \\
\text { Cost of purchase } \\
\text { Material losses } \\
\text { Capacity }\end{array}$ & Effectiveness/separation quality \\
\hline Transport & $\begin{array}{l}\text { Type of technology (constraint) } \\
\text { Environmental characterization } \\
\text { Cost of purchase } \\
\text { Material losses } \\
\text { Capacity }\end{array}$ & Rate flow \\
\hline Elementary flow & $\begin{array}{l}\text { Composition flow } \\
\text { Physical properties } \\
\text { Input or output } \\
\text { Market price }\end{array}$ & Purity \\
\hline
\end{tabular}

\section{Results}

\subsection{Step by Step Evaluation Methodology}

Our assessment methodology has been developed to provide a coarse result in early design phases and to promote sustainable solutions. The methodology can be divided into several key steps. First, the general framework is built based on the customer needs and with the waste type specifications. This step allows to determine the specific constraints, delays and costs of the project in order to determine the initial specifications for the recycling pathway. In the continuity, the customer provides its main orientations for the recycling process purpose. The customer defines the purpose and objectives for the recycling pathway. Next, the engineering team validate or not the main orientation of the recycling chain. From this orientation, the engineering team starts working on the recycling pathway proposal. The aim is to provide treatment synoptic definition, selection of the main steps and the choice of technological bricks.

According to the recycling chain synoptic, for each step of the recycling pathway, MTB's Sales Team needs to select the appropriate technology and thanks to the expertise from MTB's Engineering Team the operating variables are selected. It is from this point that the database makes it possible to calculate the unit performances. This calculation is made according to the general settings, the specific information flow and the variables. At the end, a synthetic evaluation of the global process and unit steps is provided to allow discussion. 


\subsection{Unit Process Performance Calculation}

\subsubsection{Technical Performances}

The technical performance indicators are oriented towards the capacity of the pathway to recycle the waste, so each unit process is described by three indicators.

- Recycling rate

- Recovery rate

- Landfill rate

The calculation of these rates is made according to the standard [22].

\subsubsection{Economic Performances}

For the economic dataset, data is easily accessible through the information provided by manufacturers and recyclers feedback. The Life Cycle Cost (LCC) analysis is used to determine the economic performance of each unit process. The LCC methodology used to consider both the costs of each system in addition to the profit from the sales of the sorted materials. However we do not include the costs of the environmental impact [23]. The economic performance is described by using three results:

- Initial investment costs

- Operating costs (cost per ton)

- Profit from recycled materials sales

\subsubsection{Environmental Performances}

Inventory data characterising recycling processes are rare and usually not available in the most currently used generic Life Cycle Inventory database (ELCD, Gabi, Ecoinvent). Specific inventory data remain to be collected and assessed to build reliable and representative datasets. Our team has started to build an environmental database for recycling processes. The results of environmental performance assessment are given through one inventory indicator and two environmental impacts indicators (using ILCD methodology [24]):

- Total energy consumption

- Climate change

- Non-renewable resource depletion 


\section{Discussion}

The decision tool aims to help the design team to implement more sustainable recycling pathway. It is not a matter of providing a comprehensive assessment for each recycling pathway during the design phase, but it is to communicate to industrial customers the performance indicators in addition to the economic indicators. These additional performance indicators should allow designers to propose optimization on recycling pathways and give a quantified result of the improvements. With an iterative approach, designers could optimize the flows and processes to contain impacts.

Although recycling process lines are not new, industrial optimization has not been fully conducted yet [25]. The unconstructive approach, the complexity of waste and the lack of control over incoming flows limit the drafting of theoretical principles. The increasing interest in waste recycling and the evolving regulations in force steer the waste sector to adopt an increasingly industrial approach. In order to support this transition, it is a question of advancing the design methods with specific tools.

\section{Conclusion}

Even though plenty of technical options exist for developing recycling products, the recycling solutions selecting motivations are too often led by the pursuit of profit growth which leads to a greater inefficiency [26]. By communicating additional performance indicators, we are convinced that this approach can evolve. And that new issues will be introduced in trade negotiations for recycling pathway.

As a next step, we need to build a sufficiently complete and robust database to support the evaluation of recycling pathway. This approach must be enriched in the future. It is also required to facilitate the improvement of the quality of results during the refining process variables and input parameters.

Acknowledgements The authors want to thank MTB Recycling and the French National Association for Technical Research (ANRT) for the funding of the Ph.D. study (CIFRE Convention $\left.\mathrm{N}^{\circ} 2015 / 0226\right)$ of the first author.

\section{References}

1. Stocker T.F, Qin D, Plattner G.K, Tignor M.M.B, Allen S.K, Boschung J, et al., Climate change 2013: the physical science basis, Intergovernmental Panel on Climate Change, Cambridge, 2013.

2. Yao C, Feng $\mathrm{K}$, Hubacek $\mathrm{K}$, Driving forces of $\mathrm{CO}_{2}$ emissions in the $\mathrm{G} 20$ countries: An index decomposition analysis from 1971 to 2010, Ecol. Inform. 26 (2015) 93-100. https://doi.org/ 10.1016/j.ecoinf.2014.02.003. 
3. EUROSTAT, Statistics on Waste in Europe, Stat. Explain. (2015). <http://ec.europa.eu/ eurostat/statistics-explained/index.php/Waste_statistics/fr> (accessed April 3, 2016).

4. Miehe R, Schneider R, Baaij F, Bauernhansl T, Criticality of Material Resources in Industrial Enterprises - Structural Basics of an Operational Model, 23rd CIRP Conf. Life Cycle Eng. 48 (2016) 1-9. https://doi.org/10.1016/j.procir.2016.03.035.

5. Hametner M, Dimitrova A, Schindler P, Sustainable development in the European Union2016 Edition, EUROSTAT, Brussels, 2016, doi:10.1002/ (SICI)1099-0976 (199711) 7:6 <181::AID-EET134> 3.0.CO; 2-S.

6. Schandl H, Decoupling global environmental pressure and economic growth: scenarios for energy use, materials use and carbon emissions, J. Clean. Prod. (2015) 1-12. https://doi.org/ 10.1016/j.jclepro.2015.06.100.

7. McDonough W, Braungart M, Cradle to cradle: Remaking the Way We Make Things, Edition al, Manifesto, Paris, 2012.

8. Rebitzer G, Fullana P, Weidema B.P, Jolliet O, Recycling, Close-Loop Economy, Secondary Resources, in: 10th LCA Case Study Symp., 2003: pp. 106-108.

9. Butterworth J, Morlet A, Nguyen H.P, Oppenheim J, Stuchtey M, Towards the Circular Economy: Economic and Business Rationale for an Accelerated Transition, Ellen MacArthur Foundation, Vol. 1, 2013 98. https://doi.org/10.1162/108819806775545321.

10. Gahleitner A, Closing the loop: Next steps critical for Europe's Circular Economy, Eur. Alum. Assoc. 2, 2015, 2.

11. Lavery G, Pennell N, Le Nouveau Modèle Industriel : Plus de bénéfices, plus d'emplois et moins d'impact sur l'environnement, Interface, 2014.

12. Grimaud G, Perry N, Laratte B, Life Cycle Assessment of Aluminium Recycling Process: Case of Shredder Cables, in: Procedia CIRP, Berlin, 2016. https://doi.org/10.1016/j.procir. 2016.03.097.

13. Grimaud G, Perry N, Laratte B, Reducing Environmental Impacts of Aluminium Recycling Process Using Life Cycle Assessment, 12th Bienn. Int. Conf. EcoBalance. October, 2016, 7. https://doi.org/10.1016/j.procir.2016.03.097.

14. Grimaud G, Laratte B, Perry N, To Transport Waste or Transport Recycling Plant: Insights from Life-Cycle Analysis, in: Soc. Mater. Int. Conf. (SAM 11), SOVAMAT, Trondheim, 2017: pp. 1-18.

15. UNEP, Recycling Rates of Metals, Paris, 2011.

16. Fisher A, Functional versus Innovative Products, 2012, 8.

17. Heiskanen K, Theory and Tools of Physical Separation/Recycling, in: Handb. Recycl., Elsevier Inc., Amsterdam, 2014: pp. 39-61. https://doi.org/10.1016/b978-0-12-396459-5. 00005-2.

18. European Commission, General Verification Protocol for EU Environmental Technology Verification programme-Version 1.1, 2014, 74.

19. European Commission, EU Environmental Technology Verification, Environ. Technol. Verif. Progr., 2016, 15. <http://ec.europa.eu/environment/etv/> (accessed July 13, 2016).

20. European Commission, A Comprehensive Guide for Proposers to the EU Environmental Technologies Verification Pilot Programme, Project Ad, European Commission, Luxembourg, 2012.

21. Grimaud G, Perry N, Laratte B, Decision Support Methodology for Designing Sustainable Recycling Process Based on ETV Standards, Int. Conf. Sustain. Mater. Process. Manuf. SMPM 2017. 7, 2017, 72-78. doi:http://dx.doi.org/10.1016/j.promfg.2016.12.020.

22. International Standard Organization, ISO 22628:2002 - Road vehicles-Recyclability and recoverability - Calculation method, 2002. <https://www.iso.org/standard/35061.html > (accessed May 31, 2017).

23. Office of Acquisition and Project Management, LIFE CYCLE COST HANDBOOK Guidance for Life Cycle Cost Estimation and Analysis, 2014, 89. http://energy.gov/sites/prod/files/ 2014/10/f18/LCC Handbook Final Version 9-30-14. pdf. 
24. JRC, European Commission - Institute for Environment and Sustainability, Characterization factors of the ILCD Recommended Life Cycle Impact Assessment methods-EUR 25167, Brussels, 2012. https://doi.org/10.2788/60825.

25. Martínez Leal J, Charbuillet C, Pompidou S, Perry N, Recycling Chains: A proposal for an Exhaustive Definition, 10th Int. Conf. Soc. Mater., Roma, 2016: p. 21.

26. Allwood J.M, Ashby M.F, Gutowski T.G, Worrell E, Material efficiency: A white paper, Resour. Conserv. Recycl., 55, 2011, 362-381. https://doi.org/10.1016/j.resconrec.2010.11.002.

Open Access This chapter is licensed under the terms of the Creative Commons Attribution 4.0 International License (http://creativecommons.org/licenses/by/4.0/), which permits use, sharing, adaptation, distribution and reproduction in any medium or format, as long as you give appropriate credit to the original author(s) and the source, provide a link to the Creative Commons license and indicate if changes were made.

The images or other third party material in this chapter are included in the chapter's Creative Commons license, unless indicated otherwise in a credit line to the material. If material is not included in the chapter's Creative Commons license and your intended use is not permitted by statutory regulation or exceeds the permitted use, you will need to obtain permission directly from the copyright holder. 


\title{
A Synthesis of Optimization Approaches for LCA-Integrated Industrial Process Modeling: Application to Potable Water Production Plants
}

\author{
Florin Capitanescu, Antonino Marvuglia and Enrico Benetto
}

\begin{abstract}
This paper synthesizes the authors' experience in the area of integrated approaches coupling multi-objective optimization (MOO), industrial process modeling and simulation, and life cycle assessment (LCA), with particular application to the sector of drinking water production. An industrial process is intended as any process using a certain technology to produce a product or deliver a service. The paper discusses comparatively the suitability for the optimization of a real-world drinking water production plant (DWPP) of four optimization approaches, namely: (1) off-the-shelf global search metaheuristic algorithms, (2) hybrid optimizers combining global search and local search, (3) surrogate model based optimizers, and (4) local search.
\end{abstract}

\section{Introduction}

The combination between various optimization methods and life cycle assessment (LCA) has been initiated two decades ago [1], with the aim to empower decision makers with Pareto trade-off cost-effective solutions to decrease environmental impacts of processes. Many approaches have been proposed since then (e.g. [2-4]) in this research area; the reader is referred to [5] for a relatively comprehensive survey. Rooted in the same research field, but focusing on the computationally expensive optimization problem of eco-design of drinking water production plants (DWPPs), the 3-year project "Optimization based integrated process modellingLCA: application to potable water production" (OASIS) has further explored the best paths for the threefold coupling (process modeling, LCA and optimization) along four major optimization research streams namely: (1) off-the-shelf global search metaheuristic optimization algorithms [6], (2) hybrid optimizers combining global search and local search [7], (3) surrogate model based optimizers [8, 9], and (4) local search [10].

\footnotetext{
F. Capitanescu $(\square) \cdot$ A. Marvuglia $\cdot$ E. Benetto

Luxembourg Institute of Science and Technology (LIST), Belvaux, Luxembourg

e-mail: florin.capitanescu@ list.lu

(C) The Author(s) 2018

E. Benetto et al. (eds.), Designing Sustainable Technologies,

Products and Policies, https://doi.org/10.1007/978-3-319-66981-6_3
} 
The outline of the remaining of the paper is as follows. Section 2 presents the statement of the problem and the tool components. Section 3 briefly describes the main optimization algorithms employed in the four research streams. Section 4 provides optimization results with these algorithms for a realistic model of a real-world DWPP. Section 5 concludes and provides directions for future work.

\section{Statement of the Optimization-Process Modelling-LCA Problem}

\subsection{Problem Formulation}

The multi-objective optimization (MOO) problem corresponding to a DWPP can be compactly expressed, assuming a single relevant aggregated operating scenario, as follows:

$$
\begin{array}{cc}
\min _{\mathbf{x}} & \left\{f_{1}(\mathbf{x}), f_{2}(\mathbf{x})\right\} \\
\text { subject to: } & \mathbf{g}(\mathbf{x})=\mathbf{0} \\
& \mathbf{h}(\mathbf{x}) \geq \underline{\mathbf{h}} \\
& \underline{\mathbf{x}} \leq \mathbf{x} \leq \overline{\mathbf{x}}
\end{array}
$$

where: $\mathbf{x}$ denotes the vector of decision variables (e.g. design and operation parameters of the DWPP unit processes), $f_{1}$ models the operation cost of the DWPP (comprising especially raw materials, chemicals, and electricity), $f_{2}$ models the environmental impacts of the DWPP (calculated using ReCiPe method applied at midpoint level [11]). The equality constraints $\mathbf{g}(\mathbf{x})=\mathbf{0}$ model the input-output mass flow for each unit process in the entire chain. The inequality constraints $\mathbf{h}(\mathbf{x}) \geq \underline{\mathbf{h}}$ enforce the outlet water quality [6]. The latter is represented only by seven relevant aggregated parameters (e.g. total coliforms, total trihalomethanes, total organic carbon, Escherichia coli, faecal streptococci, turbidity, and conductivity). Finally, the inequality constraints $\underline{\mathbf{x}} \leq \mathbf{x} \leq \overline{\mathbf{x}}$ model the physical bounds of the decision variables.

Note that, because there is no qualitative or quantitative benefit to express analytically the hundreds of complex chemical reactions involved in the optimization problem (1), these are assessed (by the specialized software PHREEQC ${ }^{\circledR}[12]$ ) by running the DWPP simulator, called EVALEAU [13], for specific values of the decision variables. 


\subsection{EVALEAU: The DWPP Simulator}

EVALEAU is a simulator of DWPPs (see Fig. 1) which integrates detailed modelling of DWPPs unit operations with state of the art LCA tools [13]. It consists in a library of unit process (UPs) models for water treatment, allowing life cycle inventory calculation (LCI) as a function of process parameters. The tool is embedded in the LCA software Umberto ${ }^{\circledR}$ and relies on the Ecoinvent database. It also includes a sensitivity analysis toolbox based on the Morris method for the identification of the process parameters mainly affecting the life cycle impact assessment (LCIA) results. More details about it can be found in the literature [13-15].

\subsection{The Proposed Integrated Simulation-Optimization Approach}

The approach proposed in the OASIS project for solving the simulationoptimization problem (1) consists in decomposing the problem into two modules (the EVALEAU simulator and a multi-objective optimizer) which interact in a loop, as shown in Fig. 2.

Because the simulator is intrinsically computationally expensive (it takes in average around two minutes per simulation) and can be seen by the optimizer as a black-box, suitable optimizers have been sought as described in the next section.

\section{Optimization Approaches}

While the benefits of using optimization techniques for DWPP eco-design (e.g. reduction in both operating cost and environmental impacts) have been quantified in our previous works (e.g. [6-10, 16]), this section summarizes chronologically the main four lines of research pursued in the OASIS project, whose performances will be compared in the next section.

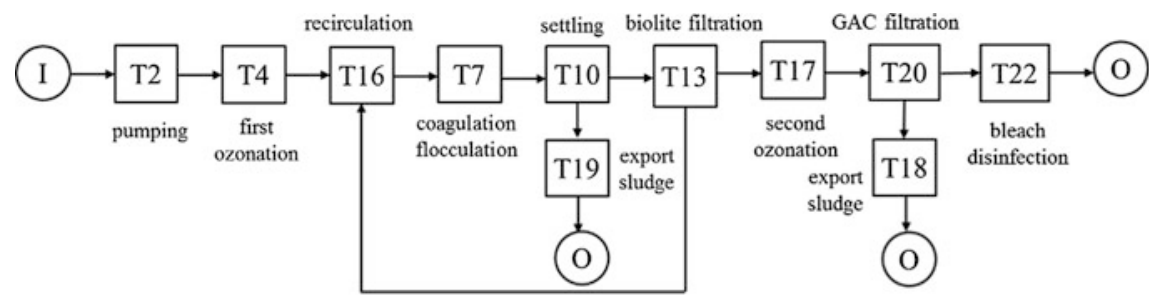

Fig. 1 Modular model of a real-world DWPP (I, O and T stand for input, output, and unit process, respectively) 


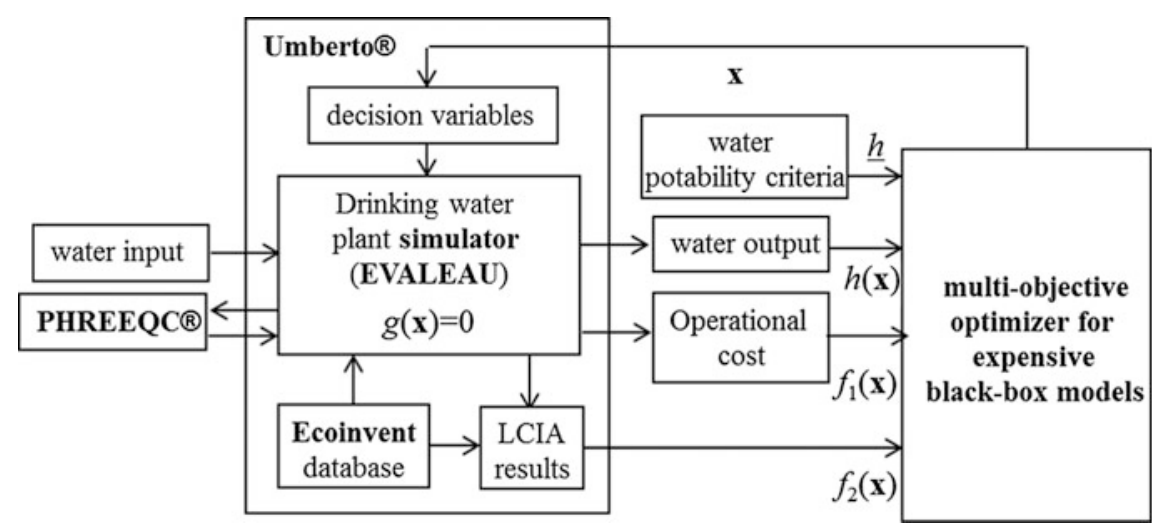

Fig. 2 Architecture of the integrated tool coupling the DWPP EVALEAU simulator with an optimizer

\subsection{Off-the-Shelf Global Search Metaheuristic Algorithms}

Global search generic metaheuristic algorithms have been naturally the first optimizers tested. The detailed results obtained with six algorithms of this class have been reported in [6]. Two algorithms, namely the Strength Pareto Evolutionary Algorithm (SPEA2) [17] and the Non-dominated Sorting Genetic Algorithm (NSGA-II) [18], have proven consistently best performances in terms of convergence speed to the Pareto front. However, these algorithms involve heavy computations, due to their inherent slow convergence near to the optimum and genericity (i.e. they make no attempt to take advantage of the problem structure). This fact motivates further research among the three following lines.

\subsection{Hybrid Algorithms Combining Global and Local Search}

Hybrid algorithms combine global search (or exploration) and local search (or exploitation) so as to take advantage of their assets while offsetting their drawbacks.

Two coupling schemes between global search and local search have been used:

(1) sequential approach (explored to some extent in [10]): global search identifies first the most promising regions of the design space and then its final solutions are transferred to the local search method (described in Sect. 3.4) for further local refinement;

(2) integrated approach (explored in [7]): the local search module is embedded in the global search algorithm; the local search is called at a certain pace to improve locally the best current candidate solutions. Specifically, [7] has 
proposed such a hybrid approach in which a memory-based adaptive partitioning algorithm was embedded into an archive-based multi-objective evolutionary algorithm, developed relying on the structure of NSGA II [18].

\subsection{Surrogate Model-Based Optimizers}

This research line consists in building tailored computationally cheap surrogate models of the optimization problem, aiming to accelerate the convergence and reduce the computational burden. Two approaches have been developed $[8,9]$ in which the surrogate model of the optimization problem is based on:

(1) mixed-integer linear programming (MILP) $[8,16]$. This surrogate model relies on piecewise linear approximations, via brute-force sensitivity computation, of the objective functions and inequality constraints. Additionally, the use of constraint programming [19] for solving the MILP problem at the core of the surrogate model has been explored in [9].

(2) nonlinear programming (NLP). This surrogate model relies on curve fitting of objectives and inequality constraints via either quadratic polynomial functions or higher order polynomial functions (e.g. cubic) [9].

In both proposed methodologies which include such surrogate models, the approximation of the Pareto front is generated upon applying the well-known $\varepsilon$-constraint method [20] to the MOO surrogate problems.

\subsection{Local Search}

The last optimization research strand investigated in this project concerns the local search [10]. The latter is useful not only in the context of hybrid algorithms (see Sect. 3.2) but also in many real-world computationally expensive simulation-based applications, where the aim is to improve a given system state locally with limited computational budget. To this end, a new neighborhood-based iterative local search method has been proposed [10]. This method aims at steering the search along any desired direction in the objectives space and resorting to first derivatives approximation and linear programming optimization. 


\section{Numerical Results}

\subsection{Short Description of the DWPP}

The optimization approaches developed in the frame of the OASIS project have been applied to a realistic model of an existing DWPP from France [13]. The DWPP treatment chain of the inlet river water contains the main unit process (see Fig. 1): pumping, a first ozonation, coagulation/flocculation, settling, biolite filtration, a second ozonation, granular activated carbon filtration, and bleach disinfection. A set of six relevant decision variables is considered, namely (see Fig. 1): the ozone transfer efficiency and the pure oxygen fraction in feed gas in units T4 and $\mathrm{T} 17$, the coagulant dose in unit $\mathrm{T} 7$, and the granular activated carbon regeneration frequency in unit $\mathrm{T} 20$.

\subsection{Results Using off-the-Shelf Global Search Algorithms}

Figures 3 shows three snapshots retrieved during the convergence to the Pareto front obtained with SPEA2 and NSGA-II algorithms. For all the algorithms the Pareto front corresponds to roughly 50 generations of simulation.

By comparing the two sides of Fig. 3 one can note that, while after 10 generations NSGA-II front approximation is substantially better (in terms of non-dominance and spread of solutions) than the one of SPEA2, as generations evolve, SPEA2 slightly outperforms NSGA-II.
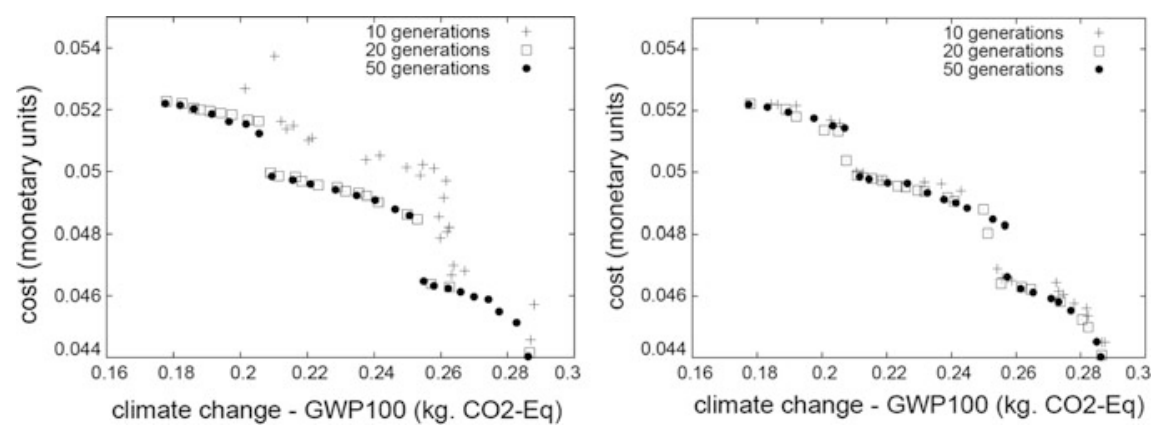

Fig. 3 Progress toward the Pareto front obtained with SPEA2 (left) and NSGA II (right) optimizers [6] 


\subsection{Results Using Hybrid Algorithms}

Figures 4 and 5 present results obtained with two hybrid algorithms: a sequential two step algorithm (Fig. 4) and an integrated algorithm (Fig. 5).

Figure 4 shows that, although the local search is launched (starting from best solutions provided by the global search algorithm SPEA2 after 120 evaluations) relatively far from the Pareto front, despite the limited budget, it behaves well (i.e. a few solutions converge already on the front and their spread is good) [10].

Figure 5 shows the results obtained with an integrated hybrid algorithm called Archive-based Multi-Objective Evolutionary Algorithm with Memory-based Adaptive Partitioning of search space (AMOEA-MAP) [7]. Despite the limited computational budget allowed (200 evaluations) this algorithm exhibits excellent performances compared to NSGA-II. It also outperforms the sequential algorithm.

Fig. 4 Solution path of the local search method applied after 120 evaluations of global search algorithm SPEA2 [8]
Fig. 5 Approximation of the Pareto front as a function of the number of simulator evaluations: hybrid algorithm (AMOEA-MAP) versus NSGAII optimizer [7]
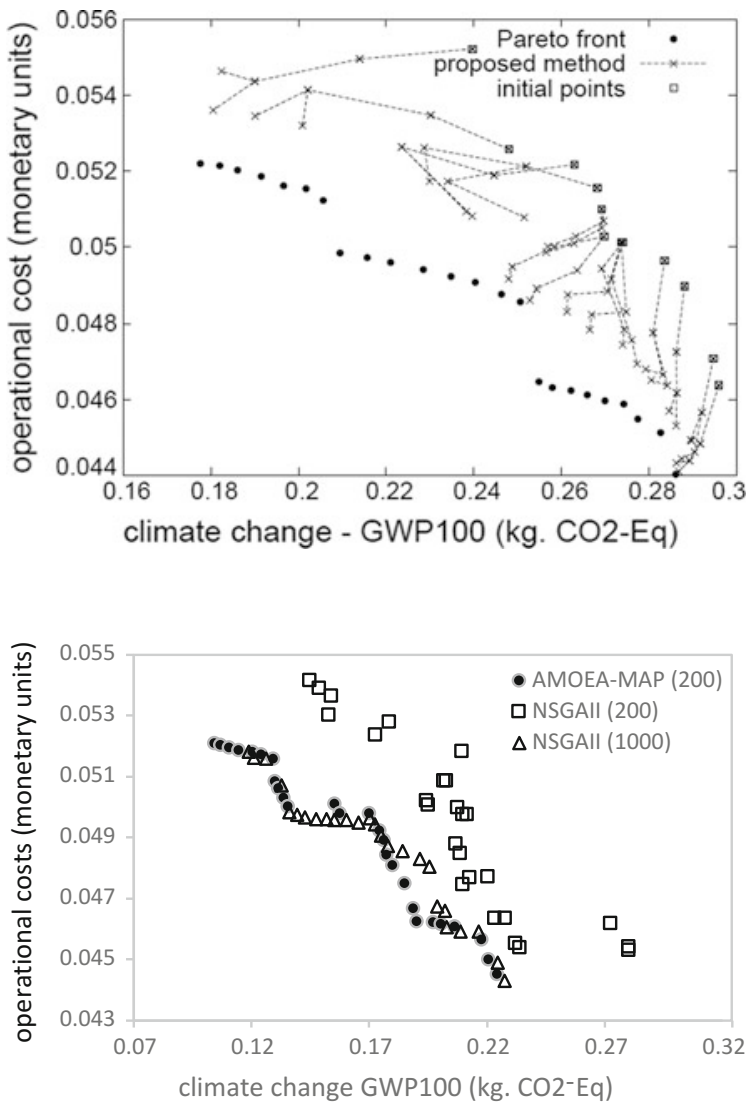


\subsection{Results Using Surrogate Model-Based Optimizers}

Figure 6 plots the front approximations obtained with the MILP-based surrogate model using 10 function linearization intervals [8]. One can observe that, for the same number of evaluations, the MILP-based algorithm outperforms SPEA2.

Due to space limitation, the results obtained with NLP-based surrogate models [8, 9] and constraint programming [16], both performing less well than MILP model, are not shown. For detailed results the reader is referred to $[8,9,16]$.

\subsection{Results Using Local Search}

Figure 7 displays the solution path obtained with the LP-based local search method [10], starting from an initial operating point where each decision variable is set to the half value of its physical range. One can first observe that the method has a good ability to steer the search along the desired direction in the objectives space. Furthermore, the method produces generally a high quality approximation of the Pareto front (in terms of accuracy and distribution of solutions), especially in the upper concave part of the front, except of one search trajectory in the middle which gets stuck.

Fig. 6 Approximation of the Pareto front for MILP-based surrogate algorithm [8]

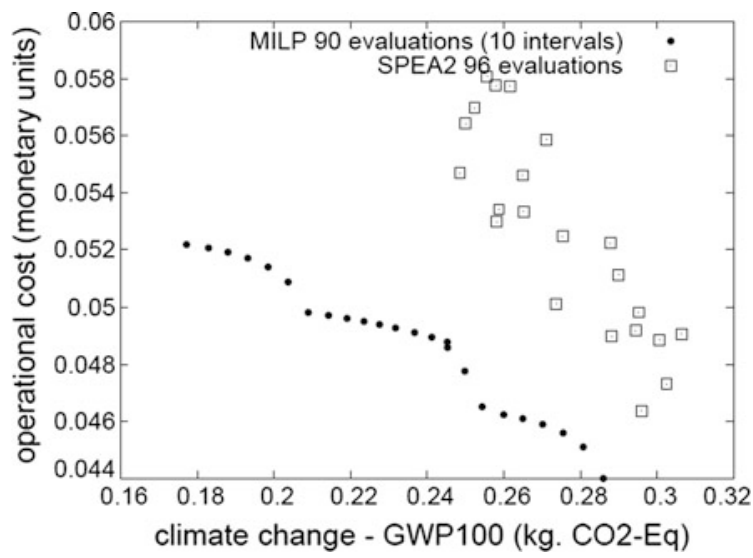


Fig. 7 Solution path and approximated Pareto front via the LP-based local search method [10]

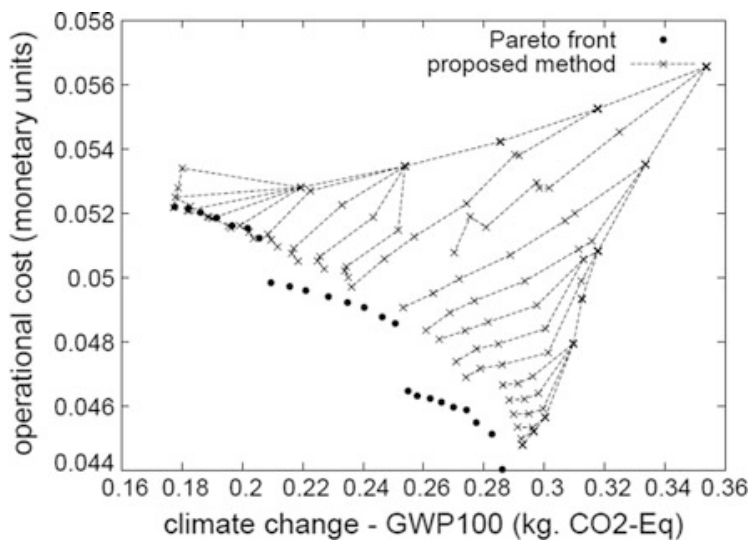

\section{Conclusions and Outlook}

This paper has synthesized the main findings of our experiments with four classes of optimization approaches for the MOO of the DWPP in the frame of the project OASIS. The main conclusions drawn can be summarized as follows.

(1) Hybrid algorithms, and particularly the integrated hybrid algorithm AMOEAMAP, and MILP-based surrogate model have shown the best performances, compared to other competing alternatives.

(2) The off-the-shelf generic global search metaheuristic algorithms are substantially less efficient than the best classes of algorithms. However, given especially their application straightforwardness, these alternatives cannot be discarded. Among the tested algorithms, NSGA-II has shown the best performances overall, being closely followed by SPEA2. The experiments with four other popular metaheuristic algorithms have indicated that, in the myriad of existing meta-heuristic algorithms with various pros and cons, the best algorithm for a given problem should be chosen carefully.

(3) Constraint (integer) programming is less suitable than MILP classical algorithms in our context of loosely constrained small size surrogate optimization problem, where feasibility is not a major concern.

(4) The proposed LP-based local search method has shown good performances and keeps intact its promises for other mildly nonlinear computationally expensive optimization problems.

Although the explored optimization algorithms have been applied to the bi-objective (e.g. cost versus LCA-based environmental impact) optimization of DWPP at planning stage, they remain generic to other application fields dealing with (computationally expensive) MOO problems. Furthermore, our results with these algorithms could serve to evaluate their suitability for different problems. In our experiments we have noticed that the water quality constraints are not severely 
constraining the feasible region of the problem; this is however case-dependent and the impact of these constraints may be more pronounced for other DWPPs.

As a side complementary experiment of the project, a detailed discussion regarding the pros and cons of expressing environmental impacts in MOO utilizing the midpoint categories versus endpoint score has been conducted in [21].

Finally, the algorithms developed and the experience gained in this project, could be applied (with due adaptations) to other case studies. In particular, a very appealing and timely research area would be the optimization of supply chains, under resiliency constraints and risk-based decision making.

Acknowledgements The authors acknowledge the funding from Luxembourg National Research Fund (FNR) in the framework of the OASIS project (CR13/SR/5871061).

\section{References}

1. A. Azapagic, R. Clift, Life cycle assessment and multiobjective optimisation, Journal of Cleaner Production, Vol. 7, 1999, pp. 135-143.

2. I.E. Grossman, G Guillén-Gosálbez, Scope for the Application of Mathematical Programming Techniques in the Synthesis and Planning of Sustainable Processes, Computers \& Chemical Engineering, Vol. 34, 2010, pp. 1365-1376.

3. G. Guillén-Gosálbez, J.A. Caballero, L. Jiménez, Application of Life Cycle Assessment to the Structural Optimization of Process Flowsheets, Industrial \& Engineering Chemistry Research, Vol. 47, 2008, pp. 777-789.

4. F. You, L. Tao, D.J. Graziano, S.W. Snyder, Optimal design of sustainable cellulosic biofuel supply chains: Multiobjective optimization coupled with life cycle assessment and inputoutput analysis, AIChE Journal, Vol. 58, 2012, pp. 1157-1180.

5. C. Pieragostini, M.C. Mussati, $P$ Aguirre, On process optimization considering LCA methodology, Journal of Environmental Management, Vol. 96, 2012, pp. 43-54.

6. F. Capitanescu, S. Rege, A. Marvuglia, E. Benetto, A. Ahmadi, T. Navarrete-Gutierrez, L. Barna, Cost versus life cycle assessment-based optimization of drinking water production plants, Journal of Environmental Management, Vol. 177, 2016, pp. 278-287.

7. A. Ahmadi, L. Barna, F. Capitanescu, A. Marvuglia, E. Benetto, An archive-based multi-objective evolutionary algorithm with adaptive search space partitioning to deal with expensive optimisation problems: application to process eco-design, Computers \& Chemical Engineering, Vol. 87, 2016, pp. 95-110.

8. F. Capitanescu, A. Ahmadi, E. Benetto, A. Marvuglia, L. Barna, Some efficient approaches for multi-objective constrained optimization of computationally expensive black-box model problems, Computers \& Chemical Engineering, Vol. 82, No. 2, 2015, pp. 228-239.

9. F. Capitanescu, A. Marvuglia, E. Benetto, A. Ahmadi, L. Barna, Assessing the uses of NLP-based surrogate models for solving expensive multi-objective optimization problems: application to potable water chains, Enviroinfo Conference, Copenhagen (Denmark), 2015.

10. F. Capitanescu, A. Marvuglia, E. Benetto, A. Ahmadi, L. Barna, Linear programming-based directed local search for expensive multi-objective optimization problems: application to drinking water production plants, European Journal of Operational Research, Vol. 262, 2017, pp. 322-334.

11. M.J. Goedkoop, R. Heijungs, M. Huijbregts, A. De Schryver, J. Struijs, R. Van Zelm, ReCiPe 2008-A life cycle impact assessment method which comprises harmonised category indicators at the midpoint and the endpoint level, First edition Report I: Characterisation, 6 January 2009. 
12. D.L. Parkhurst, C.A.J. Appelo, User's guide to PHREEQC (version 2)-A computer program for speciation, batch-reaction, one-dimensional transport, and inverse geochemical calculations. U.S.G.S., 1999.

13. Y. Méry, L. Tiruta-Barna, E. Benetto, I. Baudin, An integrated "process modelling-life cycle assessment" tool for the assessment and design of water treatment processes, International Journal of Life Cycle Assessment, Vol. 18, 2013, pp. 1062-1070.

14. Y. Méry, L. Tiruta-Barna, I. Baudin, E. Benetto, E. Igos, Formalization of a technical procedure for process ecodesign dedicated to drinking water treatment plants, Journal of Cleaner Production, Vol. 68, 2014, pp. 16-24.

15. Y. Méry, Development of an integrated tool for Process Modelling and Life Cycle Assessment-Ecodesign of process plants and application to drinking water treatment. Chemical and Process Engineering. INSA de Toulouse, 2012. English.

16. F. Capitanescu, A. Marvuglia, E. Benetto, A. Ahmadi, L. Barna, Constraint Programming versus MIP for LCA-based Multi-Objective Optimization of Sustainable Potable Water Production Plant, iEMSs Conference, Toulouse (France), 2016.

17. E. Zitzler, M. Laumanns, L. Thiele, SPEA2: Improving the Strength Pareto Evolutionary Algorithm for Multiobjective Optimization, In: Evolutionary Methods for Design, Optimisation and Control with Application to Industrial Problems (Eds. Giannakoglou K.C. et al.), Proceedings of the EUROGEN2001 Conference, Athens, Greece, 2002, pp. 95-100.

18. K. Deb, S. Agrawal, A. Pratap, T. Meyarivan, A Fast Elitist Non-dominated Sorting Genetic Algorithm for Multi-objective Optimization: NSGA-II, PPSN VI, LNCS, 1917, 2000, pp. 849-858.

19. T. Achterberg, SCIP: solving constraint integer programs, Mathematical Programming Computation, 2009, pp. 1-41.

20. G. Mavrotas, Effective implementation of the $\varepsilon$-constraint method in Multi-Objective Mathematical Programming problems. Applied Mathematics and Computation, 2009; Vol. 2013, pp. 455-65.

21. F. Capitanescu, E. Igos, A. Marvuglia, E. Benetto, Midpoint vs single score in multi-criteria optimization under life cycle assessment constraints: the case of potable water treatment chains, Enviroinfo Conference, Copenhagen (Denmark), 2015.

Open Access This chapter is licensed under the terms of the Creative Commons Attribution 4.0 International License (http://creativecommons.org/licenses/by/4.0/), which permits use, sharing, adaptation, distribution and reproduction in any medium or format, as long as you give appropriate credit to the original author(s) and the source, provide a link to the Creative Commons license and indicate if changes were made.

The images or other third party material in this chapter are included in the chapter's Creative Commons license, unless indicated otherwise in a credit line to the material. If material is not included in the chapter's Creative Commons license and your intended use is not permitted by statutory regulation or exceeds the permitted use, you will need to obtain permission directly from the copyright holder.

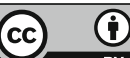




\title{
A Bi-dimensional Assessment to Measure the Performance of Circular Economy: A Case Study of Tires End-of-Life Management
}

\author{
Geoffrey Lonca, Romain Muggéo, Hugue Tétreault-Imbeault, \\ Sophie Bernard and Manuele Margni
}

\begin{abstract}
Although circular initiatives emerge around the world, the process of decoupling the economic activity from resource consumption and environmental impacts is far of being achieved. The concept of circular economy embodies the opportunity to reconcile an improved resource use while reducing the environmental footprint. Appropriate assessment metrics and methodologies are needed to identify potential trade-off between these 2 sides of a single coin. In this paper, we apply the Material Circularity Indicator (MCI) and Life Cycle Assessment (LCA) to analyse tires end-of-life strategies aiming at improving the circular flow of all tire materials. Results reveal re-treading is interesting to produce trade-offs on environmental impacts while re-grooving offers a fully decoupled strategy that improves material circularity avoiding environmental burdens. Further improvements should integrate environmental assessment as well as economic factors to link micro scale to macro scale contributions to sustainable development.
\end{abstract}

\section{Introduction}

Decoupling the economic activity from any form of social and ecological degradation is gaining recognition as a universal condition to increase - or at least maintain-social welfare throughout generations (e.g. [1]). Since the idea of a sustainable development appeared, it gave birth to some concepts related to more

\footnotetext{
G. Lonca $(\bowtie) \cdot H$. Tétreault-Imbeault · M. Margni Department of Chemical Engineering, CIRAIG, École Polytechnique de Montréal, PO Box 6079 Stn. Centre-Ville, Montréal, QC H3C 3A7, Canada e-mail: geoffrey.lonca@polytml.ca

R. Muggéo

Centre de Technologies de Ladoux, M.F.P Michelin, 23 place des Carmes Déchaux, Clermont-Ferrand 63040, France
}

\section{S. Bernard}

Department of Mathematical and Industrial Engineering, École Polytechnique de Montréal, PO Box 6079 Stn. Centre-Ville, Montréal, QC H3C 3A7, Canada

E. Benetto et al. (eds.), Designing Sustainable Technologies,

Products and Policies, https://doi.org/10.1007/978-3-319-66981-6_4 
specific goals. Among them, the circular economy (CE) focuses on decoupling the economic activity from resource depletion, providing an appealing concept for companies to support the development of strategies benefitting from both saving money and resources.

CE is still a not well-defined concept that heavily builds on past ones, as suggested in the CIRAIG's white paper on circular economy released in 2015. Nonetheless, a narrow scope definition of CE jeopardizes its contribution to the bigger picture of a sustainable development. Without a clear identification of the dimensions that define a $\mathrm{CE}$, practitioners are willing to choose inadequate assessment tools that account for only a small part of the complete $\mathrm{CE}$ model. According to several definitions and metrics of CE, a company that consumes more materials and less energy is less circular than the one that consumes fewer materials and more energy [2]. This is due to an unclear definition of the boundaries between of material circularity and burden shifting, and all the more, environmental benefits are not quantitatively linked to circular strategies (i.e. circular economy loops) [3]. As the world faces a growing amount of complex products coming to their end-of-life to manage as well as recycling efficiency challenges, recycling strategies tend to consume more energy as the amount of recovered material rises $[4,5]$. Besides, technological progress make sometimes the reusing a worse environmental strategy [6], e.g. innovation on energetically efficient products leads reusing old products less efficient to increase its overall environmental impacts. Hence, the need for adequate quantifying tools for circular strategies - to enlighten decision makers towards sustainable practices - is gaining increasing attention [7].

This paper attempts to provide an approach that allows identifying trade-offs between increasing material circularity and decreasing environmental burdens to assess CE strategies through a case study on tire end-of-life management. We first identify suitable tools to assess both circularities of material flows and environmental burdens and we provide a novel approach to evaluate CE strategies (See Chapter "Sustainability performance evaluation for selecting the Best Recycling Pathway During its Design Phase"). We apply it to a case study on tire end-of-life management to identify trade-offs of CE strategies (See Chapter "A synthesis of optimization approaches for LCA-integrated industrial process modeling: application to potable water production plants"), we then discuss some limitations of our approach (See in this Chapter). Finally, concluding remarks provide insights for future works on $\mathrm{CE}$ assessment (See Chapter "Bio-based materials within the circular economy: opportunities and challenges"). 


\section{Approaches for Circular Economy Assessment}

\subsection{A Short Review on Circular Economy Assessment}

Elia et al. provide a critical review of environmental assessment tools and CE index methods [8]. They evaluate the level of alignment of the identified tools regarding five CE requirements inspired by the European Environmental Agency [9] - the report provides an outlook on resource-efficient, low-carbon economic and social development goals to achieve in 2050:

- Reducing inputs and use of natural resources;

- Reducing emission levels;

- Reducing valuable materials losses;

- Increasing the share of renewable and recyclable resources;

- Increasing the value durability of products.

Amongst the 14 environmental assessment methodologies analysed, the Life Cycle Assessment (LCA) turns out to have the best level of alignment with the $5 \mathrm{CE}$ requirements aforementioned. This is the same level of alignment as the Substance Flow Analysis (SFA), and better than the Material Flow Analysis (MFA) that does not complete requirement 3 and the Water Footprint (WF) deprived of the second requirement.

Only three out of the sixteen CE index methods published in the scientific literature in the last ten years, suits for micro-scale material circularity assessment, i.e. product or company level. Amongst the Reuse Potential Indicator (RPI) developed by Park and Chertow [10], the Circular Economy Index (CEI) by Di Maio and Rem [11] and the Material Circularity Indicator (MCI) by Ellen Mc Arthur Foundation and Granta Design [12], only the latter one accounts for the loss of material as well as product durability [8]. Even if it does not account for emission reduction (requirement 2) - but none of the micro scale CE indexes does-it appears to be the best attempt to comply with the mentioned $\mathrm{CE}$ requirements (Table 1 ).

The MCI is an index ranging between 0 and 1 . It derives from the multiplication of a Linear Flow Index (LFI) with a Utility Factor $(\mathrm{F}(\mathrm{X}))$ :

$$
\begin{aligned}
\mathrm{MCI} & =\max \left(0, \mathrm{MCI}^{*}\right) \\
\mathrm{MCI}^{*} & =1-\mathrm{LFI} \times \mathrm{F}(\mathrm{X})
\end{aligned}
$$

The LFI is a mass-based indicator that can be considered as an adapted MFA layout [13]. The LFI integrates 4 elements [see Eq. (3)]:

- The virgin feedstock fraction (v) deducted from the mass fraction of a product's feedstock from recycled sources $\left(\mathrm{F}_{\mathrm{R}}\right)$ and from reused sources $\left(\mathrm{F}_{\mathrm{U}}\right)$;

- The total waste $(\mathrm{w})$ including the waste fraction from upstream $\left(\mathrm{w}_{\mathrm{F}}\right)$ and downstream $\left(\mathrm{w}_{\mathrm{c}}\right)$ recycling processes and waste fraction directly to landfill $\left(\mathrm{w}_{0}\right)$ 
Table 1 State of the art analysis about CE measurement at micro scale retrieved from Elia et al. [8]

\begin{tabular}{l|l|l|l|l|l|l}
\hline Indicator & Author & $\begin{array}{l}\text { Reducing } \\
\text { input and } \\
\text { use of } \\
\text { natural } \\
\text { resources }\end{array}$ & $\begin{array}{l}\text { Increasing } \\
\text { share of } \\
\text { renewable and } \\
\text { recyclable } \\
\text { resources }\end{array}$ & $\begin{array}{l}\text { Reducing } \\
\text { emissions }\end{array}$ & $\begin{array}{l}\text { Reducing } \\
\text { valuable } \\
\text { material } \\
\text { losses }\end{array}$ & $\begin{array}{l}\text { Increasing } \\
\text { the value } \\
\text { durability } \\
\text { of goods }\end{array}$ \\
\hline MCI & $\begin{array}{l}\text { Ellen } \\
\text { McArthur } \\
\text { Foundation } \\
{[12]}\end{array}$ & $\mathrm{x}$ & $\mathrm{x}$ & & $\mathrm{x}$ & $\mathrm{x}$ \\
\hline CEI & $\begin{array}{l}\text { Di Maio } \\
\text { and Rem } \\
{[11]}\end{array}$ & & $\mathrm{x}$ & & & \\
\hline RPI & $\begin{array}{l}\text { Park and } \\
\text { Chertow } \\
{[10]}\end{array}$ & & $\mathrm{x}$ & & & \\
\hline
\end{tabular}

deduced from the fraction of mass of a product being collected to go to a recycling process $\left(\mathrm{C}_{\mathrm{R}}\right)$ and to reuse $\left(\mathrm{C}_{\mathrm{U}}\right)$.

- The waste fraction from upstream process $\left(\mathrm{w}_{\mathrm{F}}\right)$ derived from the efficiency of the recycling process used to produce recycled feedstock for a product $\left(\mathrm{E}_{\mathrm{F}}\right)$;

- The waste fraction from downstream process $\left(\mathrm{w}_{\mathrm{C}}\right)$ derived from the fraction of mass of a product being collected to go to a recycling process $\left(\mathrm{C}_{\mathrm{R}}\right)$ and the efficiency of the recycling process used for the portion of a product collected for recycling $\left(\mathrm{E}_{\mathrm{C}}\right)$;

$$
\begin{aligned}
& \mathrm{LFI}=\frac{\mathrm{v}+\mathrm{w}}{2+\frac{\mathrm{w}_{\mathrm{F}}-\mathrm{w}_{\mathrm{C}}}{2}} \\
& \mathrm{v}=1-\mathrm{F}_{\mathrm{R}}-\mathrm{F}_{\mathrm{U}} \\
& \mathrm{w}_{\mathrm{F}}=\mathrm{F}_{\mathrm{R}} \frac{\left(1-\mathrm{E}_{\mathrm{F}}\right)}{\mathrm{E}_{\mathrm{F}}} \\
& \mathrm{w}_{\mathrm{C}}=\mathrm{C}_{\mathrm{R}}\left(1-\mathrm{E}_{\mathrm{C}}\right) \\
& \mathrm{w}=\mathrm{w}_{0}+\frac{\mathrm{w}_{\mathrm{C}}+\mathrm{w}_{\mathrm{F}}}{2} \\
& \mathrm{w}_{0}=1-\mathrm{C}_{\mathrm{R}}-\mathrm{C}_{\mathrm{U}}
\end{aligned}
$$

$\mathrm{X}$ yields the multiplication of 2 ratios where the user is free to choose the one that better represents the product duration reality. In one case the lifetime $(\mathrm{L})$ of the assessed product is compared to the average product lifetime in the industry $\left(\mathrm{L}_{\mathrm{av}}\right)$ and in another case, the number of functional units $(\mathrm{U})$ - as per in LCA-is 
compared to the industry average $\left(\mathrm{U}_{\mathrm{av}}\right)$. It is up to the user either to choose the ratio that best fits and then to set the other at 1, or in some particular cases to use both as long as it does not produce any double counting.

$$
\begin{gathered}
\mathrm{F}(\mathrm{X})=\frac{0.9}{\mathrm{X}} \\
\mathrm{X}=\left(\frac{\mathrm{L}}{\mathrm{L}_{\mathrm{av}}}\right) \times\left(\frac{\mathrm{U}}{\mathrm{U}_{\mathrm{av}}}\right)
\end{gathered}
$$

As the MCI builds on a simplified methodology, some calculation features can be hard to identify in reality. For instance, the distinction between lifetime extension and reuse is not always clear. Furthermore, calculating the MCI of a product along one life cycle is ineffective to embrace the service it provides. E.g. in our case, one sole tire life cycle cannot be sufficient to provide a transportation service for a whole truck. It requires a repairing or a replacement to provide the same service along the whole truck's lifetime. Then, some components add up to the original product to make it last longer, resulting in a weight surplus in the product mass balance sheet. To avoid any confusion, we applied a mass-based weighting calculation that consists in dividing the product into components with any special circular feature. Thus, the $\mathrm{MCI}_{\text {total }}$ of the product accounts for the number $\left(\mathrm{n}_{\mathrm{i}}\right)$, the mass $\left(\mathrm{m}_{\mathrm{i}}\right)$ and the $\mathrm{MCI}_{\mathrm{i}}$ of every component (i) of the product [see Eq. (9)]. In our case, this equation allows incorporating the MCI value of the tire casing, the tread and the retreaded rubber band.

$$
\mathrm{MCI}_{\text {total }}=\frac{\sum_{\mathrm{i}}\left(\mathrm{n}_{\mathrm{i}} \times \mathrm{m}_{\mathrm{i}} \times \mathrm{MCI}_{\mathrm{i}}\right)}{\sum_{\mathrm{i}}\left(\mathrm{n}_{\mathrm{i}} \times \mathrm{m}_{\mathrm{i}}\right)}
$$

\subsection{An Approach Based on Trade-off Issues in Circular Economy}

Capital theory, in the field of natural resources economics, translates the objective of decoupling the economic activity from environmental degradation as an increasing substitutability between manufactured capital (capital goods) and non-renewable resources (natural capital) [14], traducing the essentialness of a resource and thus, providing a good indication of progress toward a CE. Besides, the ecological economics divides the use of the environment in 2 subsets: (1) a "use of mineral and biotic resources" that we call here "natural feedstock" and (2) a depository for waste products or residuals that we call here "environmental reservoirs" [15]. The CE should embody both environmental issues to align the capital theory of resources economics with the definition of environmental preservation from ecological economics. 


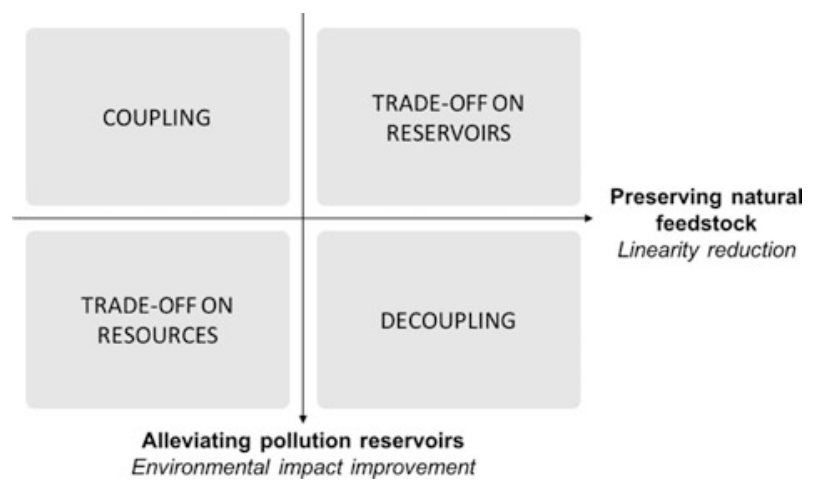

Fig. 1 A bi-dimensional approach to identify trade-off issues based on improvements on resource circularity and on environmental impacts

Let's assume a static state of the economy and disregard any consideration about the dynamic of economic activity over time. If we consider CE assessment to be bidimensional-related to the 2 subsets of use of the environment-then we identify 4 possible areas as per Fig. 1, each of them identifying a pathway departing from a baseline situation (at the origin of both axes):

- Coupling reinforcement, it reveals a pathway toward a stronger dependence on the inputs from the environment (lower circularity) ensuing more output in natural sinks (greater environmental impacts);

- Decoupling, it defines a circular strategy that is eco-efficient;

- Trade-off on resources, it means that the progress made in terms of environmental impacts require drawing on more natural resources;

- Trade-off on reservoirs, it corresponds to the particular case of burden shifting we discussed in the introduction, i.e. when saving natural resources costs more environmental externalities.

The question whether it is a relative or an absolute decoupling as Tim Jackson defined it in Prosperity without growth [16] does not interfere in our demonstration as it depends on quantitative economic features to showcase the overall level of consumption.

\section{Case Study: Tire Closed Loop Recycling}

\subsection{Data, Assumptions and Choices Modeling}

LCA has been performed in a Brazilian context in 2012 initially to guide business models of Michelin Fleet Solutions. The product analyzed is an average tire from a 
haulage truck with 32 tons' average load which usual lifetime is $600,000 \mathrm{~km}$. Data come from the Research Center of Michelin in Clermont-Ferrand (France) and the EcoInvent 2.2 database.

Hereafter is some relevant features to interpret the results. $63.5 \%$ of end-of-life tires go to energy recovery in cement clink processes and $36.5 \%$ go to material recovery (data initially retrieved from the organization responsible for collecting end-of-life tires in Brazil, Reciclanip, in 2012). For every multifunctional process of different recovery route but cast iron and extended boundaries have been considered and the co-products issued from soy esterification were considered through economic allocation. The method ReCiPe (Hierarchical perspective) Endpoint has been used to perform LCA results. For the MCI, the weighting approach for the re-treading case has been used, as per Eq. 11.

It has been assumed that tires are re-treaded 1.5 times on average after their first use. A $13.90 \mathrm{~kg}$ re-treaded rubber band (on average) replaces a tread one weighting $20.78 \mathrm{~kg}$ on average. Tire lifetime after being re-treaded is assumed to be equal to its original lifetime. However, fuel consumption increases $6.8 \%$, i.e. from $12.04 \mathrm{l} / 100$ to $12.87 \mathrm{l} / 100 \mathrm{~km}$ attributable to the re-treaded tire due to an increasing rolling resistance. In Brazil, haulage trucks consume Biofuel B5 from soy esterification. Re-grooving increases tire lifetime $30.46 \%$ on average and decreases the fuel consumption to $11.391 / 100 \mathrm{~km}(-5.4 \%)$.

\subsection{Results}

On Fig. 2, the re-grooving and re-treading scenarios are plotted relative to the baseline scenario $(0,0)$. The $\mathrm{x}$-axe represents linearity reduction which traduces

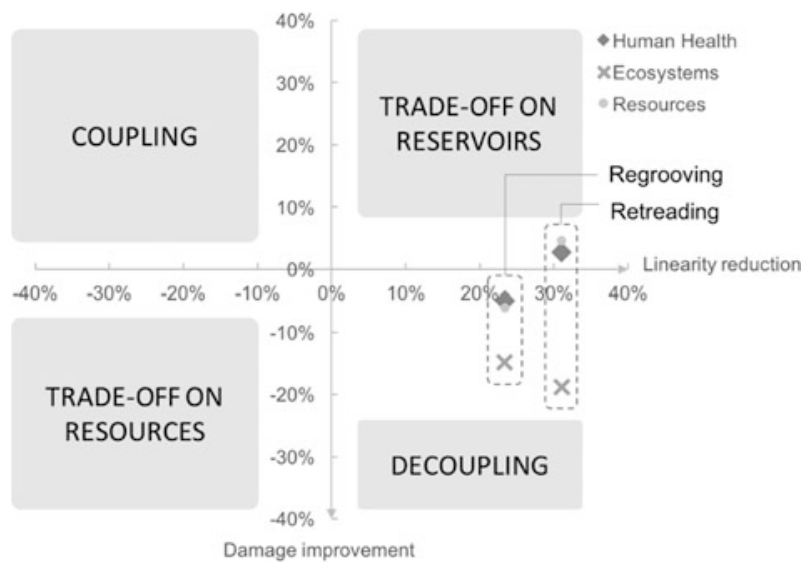

Fig. 2 Interpreting the results of 2 end-of-life tire management scenarios according to the bi-dimensional approach for circular economy assessment 
improvements on the MCI whilst y-axe represents improvements on human health, ecosystems and resources damage scores.

Results reveal that the two types of tire end-of-life management analysed tend to improve the level of circularity from the baseline scenario, as per the MCI, but it is not fully conclusive on environmental damages. Re-grooving follows on a decoupling pathway on all impact categories, whereas re-treading potentially shifts burdens on human health and resources. This is due to the increased fuel consumption required to offset the rolling resistance.

It could be tempting to conclude that re-treading improvements on circularity and ecosystem quality indicators are substantial enough to balance the small increase of damages on human health and resources, but this typical conclusion jeopardizes the preservation of natural capital itself-and consequently the contribution of circular strategies to a sustainable development. A more conservative approach then would be to conclude that only re-grooving improve natural capital preservation.

\section{Discussion}

Preceding results display areas of protection - as per $\mathrm{ReCiPe}$ - as the ultimate expression of environmental reservoirs subsets. Two important discussions stem from this observation. First, one could say that other trade-off on environmental reservoirs can occur when downscaling to mid-point categories. We considered reasonable to assume that mid-point categories can offset one another when contributing to the same damage category. Note that this works also for circularity when resource preservation refers to different subsets of stock of resources yet, this does not concern the MCI as it has no subcategories. The second logical issue is about comparing resources at damage category level and the MCI. The preceding illustration makes them appearing complementary as they derive from distinct assessment methodology. However, as both refer to natural feedstock preservation, they should follow the same trends, yet they do not.

The MCI methodology contains many limitations. For instance, system boundaries definition is narrower than in LCA, which makes the MCI ineffective to prevent from consequences of shifting resources consumption at macro scale as it refers only to one specific resource at the foreground level. Moreover, it excludes energy flows which entail neither consideration for the fuel consumption due to the increased rolling resistance nor for end-of-life energy recovery routes. Whereas, the LCA resource indicator refers to the marginal increase in costs for future extraction due to the extraction of a resource over the life cycle of the assessed product, i.e. the additional costs society has to pay as a result of an extraction [17], embodying resource depletion issues, unlike the MCI.

Finally, as the CE is meant to contribute to a sustainable development, the other sustainability dimensions are also relevant concerns. Not only social aspects are here neglected, but excluding economic factors fails to account for the dreaded 
rebound effects as per the Jevons' paradox. Economic features require special attention for future research in the field for the preceding reason, and also to assess absolute improvements on both natural resource and pollution reservoir preservation according to the approach presented in this paper.

\section{Conclusion}

Through this work, we developed a novel bi-dimensional approach to measure the performance of circular economy beyond the sole objective of resource preservation. It allows a straight forward interpretation of MCI and LCA results revealing trade-offs between improved material circularity and the risk of burden shifting over given LCA damages categories. A case study comparing 2 end-of-life tire management CE strategies revealed that re-grooving offers a fully decoupled pathway, compared to re-treading where trade-offs are identified with the increase of potential human health and resources damages. In fact, re-treading worse off impacts on human health by only $2.72 \%$, while improving circularity by $31.1 \%$ from the baseline scenario, we could question the significance of such results. Performing an uncertainty analysis would help to get fair conclusions on this issue.

To identify resource impact category as one particular environmental reservoir subset is controversial in the approach we propose. This point definitely requires further clarifications for which we recommend future research on $\mathrm{CE}$ tools to focus on the connection between micro scale and macro scale assessment. Substantial improvements should be made on the MCI, as it fails to prevent from any kind of burden shifting by excluding energy and background flows of its boundaries definition. Although the bi-dimensional analysis provides a good representation of trade-off issues, it is relative to a baseline scenario whereas absolute values are preferable, requiring economic features to be included as well.

\section{References}

1. Hotelling H, The economics of exhaustible resources, Journal of political Economy, 1931. 39 (2): p. 137-175.

2. Kampelmann S, Mesurer l'économie circulaire à l'échelle territoriale, Revue de l'OFCE, 2016 (1): p. 161-184.

3. Geyer R, et al., Common misconceptions about recycling, Journal of Industrial Ecology, 2015.

4. Bihouix P, L'Âge des low tech. Vers une civilisation techniquement soutenable, Éditions du Seuil, collection Anthropocène, 2014.

5. Bihouix P, De Guillebon B, Quel futur pour les métaux? raréfaction des métaux: un nouveau défi pour la société, 2010: EDP sciences.

6. Cooper D.R, Gutowski T.G, The Environmental Impacts of Reuse: A Review, Journal of Industrial Ecology, 2015. 
7. Su B, et al., A review of the circular economy in China: moving from rhetoric to implementation, Journal of Cleaner Production, 2013, 42: p. 215-227.

8. Elia V, Gnoni M.G, Tornese F, Measuring circular economy strategies through index methods: A critical analysis, Journal of Cleaner Production, 2017, 142: p. 2741-2751.

9. European Environmental Agency, Circular Economy in Europe-Developing the Knowledge Base (No. 2), 2016.

10. Park J.Y, Chertow M.R, Establishing and testing the "reuse potential" indicator for managing wastes as resources, Journal of environmental management, 2014, 137: p. 45-53.

11. Di Maio F, Rem P.C, A robust indicator for promoting circular economy through recycling, Journal of Environmental Protection, 2015, 6(10): p. 1095.

12. Ellen Mc Arthur Foundation and Granta Design, Circularity indicators: an approach to measuring circular economy, in Project Overview, 2015.

13. Linder M, Sarasini S, Loon P, A Metric for Quantifying Product-Level Circularity, Journal of Industrial Ecology, 2017.

14. Perman R, Natural resource and environmental economics, Pearson Education, 2003.

15. Victor P.A, Indicators of sustainable development: some lessons from capital theory, Ecological economics, 1991, 4(3): p. 191-213.

16. Jackson T, Senker P, Prosperity without growth: Economics for a finite planet, Energy \& Environment, 2011. 22(7): p. 1013-1016.

17. Goedkoop M, et al., ReCiPe 2008, A life cycle impact assessment method which comprises harmonised category indicators at the midpoint and the endpoint level, 2009. 1.

Open Access This chapter is licensed under the terms of the Creative Commons Attribution 4.0 International License (http://creativecommons.org/licenses/by/4.0/), which permits use, sharing, adaptation, distribution and reproduction in any medium or format, as long as you give appropriate credit to the original author(s) and the source, provide a link to the Creative Commons license and indicate if changes were made.

The images or other third party material in this chapter are included in the chapter's Creative Commons license, unless indicated otherwise in a credit line to the material. If material is not included in the chapter's Creative Commons license and your intended use is not permitted by statutory regulation or exceeds the permitted use, you will need to obtain permission directly from the copyright holder.

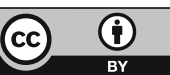




\title{
Bio-based Materials Within the Circular Economy: Opportunities and Challenges
}

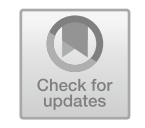

\author{
Birgit Brunklaus and Ellen Riise
}

\begin{abstract}
In a circular society, material consumption should be a circular process where renewable resources and waste streams are used for new bio-based materials. In such a society, bio-based materials are also reused, repaired, recycled, and remanufactured. Not only choices on resources, but also other life cycle choices pertaining to circularity must be done based on technological, environmental and economic basis. For this session, presentations and discussions regarding life cycle management of bio-based materials were suggested. The session had five oral presentations and six poster presentations that gave a general picture of a broader environmental and a positive economic result on a life cycle basis when renewable raw materials are used, while further exploration of the technical aspects within circularity and end-of-life challenges are needed in the future.
\end{abstract}

\section{Introduction}

The linear economy is based on the notion "Take, make and dispose", which is not in line with resource restriction posed on society today. Instead, there is a need for an economy that aims for circularity based on the notion "Restore, rebuilt and regenerate". In a circular society, material consumption should be a circular process where both renewable resources as well as finite resources are reused, recovered or recycled, inducing not only choices on resources, but also other life cycle choices pertaining to circularity that must be done based on technological, environmental and economic basis. This turns the linear life cycle "upside-down" to some extent

\footnotetext{
B. Brunklaus $(\square)$

RISE Research Institutes of Sweden, Energy and Circular Economy,

Sustainable Society, Energy and Environmental Systems Analysis,

41296 Göteborg, Sweden

e-mail: birgit.brunklaus@ri.se

E. Riise

Essity, SCA Hygiene Products AB Group Function Sustainability, 40503 Göteborg, Sweden

(C) The Author(s) 2018

E. Benetto et al. (eds.), Designing Sustainable Technologies,

Products and Policies, https://doi.org/10.1007/978-3-319-66981-6_5
} 
since it also puts another value of resources that earlier was only regarded as waste and as a burden to be minimised. This is one of the differences to be considered in the life cycles of the two material streams, linear and circular. In circular material streams, there are also some differences to be done when considering renewable resources or finite resources cycles. Therefore, the session was focusing on circularity opportunities and challenges for bio-based materials.

\section{Session Contributions}

For this session, both theoretical and practical examples, relating to a variety of industrial sectors and bio-based materials were welcomed, and the submission of abstracts resulted in five oral presentations and six posters. The session ended with a brief panel discussion where the presenters were invited to share their view on the variety of challenges and opportunities for their product or sector.

\subsection{Oral Presentations}

The first presentation on "Wood products circularity and the biodiversity challenge" was given by Christian Bauer, SIG [1]. Wood is a major input for many circular products, including half of all packaging material. Within the assessment methods to quantify the circularity potential, such as Life Cycle Assessment, there are limitations regarding the evaluation of impacts on biodiversity, for instance due to the complexity of the characterization of land use and land use change, and to the lack of consideration of forest management on landscape level and labelling, as done through certified forestry management. Therefore, practitioners and decision-makers prefer to look at other impact categories, such as climate change and water use. Starting from the existing work of UNEP SETAC and other significant existing methods, a new methodology relying on landscape management system was proposed in order to establish characterization factors aiming at integrating forest management practices and biodiversity.

The second presentation by Serenella Sala, European Commissions had the title "Bioeconomy contribution to Circular Economy" [2]. Focusing on food waste, the presentation demonstrated the potential for circularity from the bio-economy and highlighted the added-value and shortcomings of Life Cycle Thinking based approaches applied as supporting tools. Based on the quantification of food losses and waste at the EU scale, and the valorisation options, LCT and LCA approaches were applied in order to support the identification of the best options. The main challenges in order to improve LCT methods are related to the characterization of biotic resources in LCIA, and to the integration of eco-system, water, food, land and energy into LCA, considering all these issues as correlated. 
"Assessing the availability of bio-based materials in product design" was presented by Vanessa Bach from TU Berlin [3]. The use of bio-based materials can be considered as a key strategy in eco-design approach, also contributing to circular economy at some points. However, several socio-economic factors induced by market conditions or disrupting political structures within society can constrain their availability. The presented work proposed a method for assessing bio-based materials availability constraints, aiming at supporting eco-design strategies. The method considers five main sources of constrains (environmental, socio-economic and physical constraints to plants growth), over the materials supply chain, and results in a set of availability indicators. For this study, the indicators were applied to energy-related products, such as production of biodiesel from rapeseed and soy beans, and showed that the indicators seem appropriate and that interpretation leads to meaningful conclusions while methodological robustness of some indicators could be refined.

The fourth presentation dealt with "Integrated market orientation in technical R\&D processes - opportunities and challenges for environmentally friendly bio-based resins" by Miriam Lettner from K Plus, Austria [4]. The use of lignin, an under-utilized by-product from the pulp and paper industry, has the potential to be used as a valuable source for sustainable bio-based products. The market assessment of emerging lignin products aims at support R\&D process by providing information on challenges and opportunities for such products at early design stage. Based on the Smartli H2020 project outcomes and a case study performed with Delphi, it was shown that the life cycle thinking integrated technical R\&D processes based on the involvement of stakeholders and on a multi-perspective assessment (economic, environmental and technological) of newly developed products are highly valuable to face the barriers and incentives for the product market diffusion.

Sebastian Sperling, Institute for Bioplastics and Bio-composites, University of Hannover, gave the presentation "The sustainability of bio-based plastics-quantifying environmental and socio-economic aspects of a computer mouse for a circular economy" [5]. Through the presentation of the environmental and socio-economic impacts of a bio-based plastic housing of a computer mouse, the importance of sourcing bio-based plastics and the associated social aspects in different countries was revealed, despite the fact that these socio-economic aspects are often neglected and the availability of such information is limited. An important conclusion is that a joint guideline for bio-based plastic and fossil-based plastics is needed to reduce the methodological gaps existing for the assessment for such materials.

\section{Messages and Outcomes of the Session}

The messages and outcomes of the session are manifold. Among the bio-based processes there are both the well-proven processes (e.g. corn-based) and new processes (e.g. waste-based), which span a large technical variety of technological opportunities for the future to match with the objectives of circular economy. 
However, the session demonstrated that to ensure the sustainability of these processes and materials, it is required to develop further the characterization of the related environmental impacts, and to integrate the systematic assessment of socio-economic aspects along the bio-based products value chain. Also the waste-based processes will turn the waste into economic value and so would reverse the value chain from end-of-life to new resource, leading to adapt consideration in Life Cycle modelling approaches. Indeed, taking the example of wood-based lignin or coconut fibre as waste-based (by-product) bio-materials, they become more valuable than the main product (timber, coconut), which leads to rethink the allocation system from mass to economic allocation.

At the end of the session, every presenter got the chance to give their view on the variety of challenges: the technical, the environmental or the economic challenge, as well as future implications. The discussion addressed many new aspects, such as the variety of related environmental and socio-economic challenges (impacts on biodiversity [1], resource availability and market of bio-based materials [3-5]), while the technical challenges were addressed in a lower extent.

\section{Future Perspectives Based on the Session}

The opportunity of bio-based materials to contribute to circular economy goes hand in hand with challenges mentioned for the business society, such as the challenge of reverse logistics and infrastructure, the new resource management and value creation from no cost waste to high profit products, the business opportunity to create new services, placing the consumer at the centre of the value chain.

Bio-based materials within the circular economy are one of the emerging areas where life cycle management can connect bio-based resources supply chain management with waste management systems. One of the next practical challenge for bio-based materials will be the management of their End of Life (EoL). Indeed, these resources and products they are part of will be sooner or later subject to European waste legislation or directives, as it is already the case for many sectors in Europe. For instance EoL directives exist for the automotive and electronic products, setting high targets for products and materials recycling and/or reuse rates. At the time these objectives are not achievable for bio-based materials and products, due to the lack of technological solutions. However, the biodegradability of some of these resources could present an advantage.

To our knowledge there are several other technical challenges based on ongoing and recent research projects the Research Institute of Sweden RISE have been involved in with the area of: food waste and bio-refinery, eco-cycle in building construction, forest-based plastics, recycling of green plastics and industrial waste. Within the construction sector, companies recycle and apply their products, but rarely sell further or even buy recycled products! Within bio-based plastics the durability and quality requirements for industrial products are important, while the 
health aspects are important for consumer products. Within bio-based waste and bio-refineries the upgrading process is essential and the downcycling of poorer quality products occurs.

\section{References}

1. Vionnet S, Bauer C, Cockburn D, Brunberg B, Stevenson M, Wood products circularity and the biodiversity. challenge, LCM 2017 Conference, Luxembourg, 2017.

2. Sala S, Bioeconomy contribution to Circular Economy, International Conference on Life Cycle Management, 3-6 September, Luxemburg, 2017.

3. Bach V, Berger M, Finogenova N, Finkbeiner M, Assessing the availability of bio-based materials in product design, LCM 2017 Conference, Luxembourg, 2017.

4. Lettner M, Greiner D, Stern T, Hesser F, Integrated Market Orientation in Technical R\&D Processes-Opportunities and Challenges for environmentally Friendly Bio-Based Resins, LCM 2017 Conference, Luxembourg, 2017.

5. Spierling S, Knüpffer E, Venkatachalam V, Mudersbach M, Behnsen H, Krieg H, Albrecht S, Endres H-J, The sustainability of bio-based plastics-quantifying environmental and socio-economic aspects of a computer mouse for a circular economy, LCM 2017 Conference, Luxembourg, 2017.

Open Access This chapter is licensed under the terms of the Creative Commons Attribution 4.0 International License (http://creativecommons.org/licenses/by/4.0/), which permits use, sharing, adaptation, distribution and reproduction in any medium or format, as long as you give appropriate credit to the original author(s) and the source, provide a link to the Creative Commons license and indicate if changes were made.

The images or other third party material in this chapter are included in the chapter's Creative Commons license, unless indicated otherwise in a credit line to the material. If material is not included in the chapter's Creative Commons license and your intended use is not permitted by statutory regulation or exceeds the permitted use, you will need to obtain permission directly from the copyright holder. 


\title{
Bio-Economy Contribution to Circular Economy
}

\author{
Sara Corrado and Serenella Sala
}

\begin{abstract}
European policies are advocating a transition toward "bio-economy", an economy aiming at reducing the dependence from fossil-based resources, limiting greenhouse gas emissions and environmental impacts, safeguarding food security and ensuring a sustainable economic growth. Besides, circular economy policies are aiming at closing the loop of resources as much as possible. The application of circular economy principles to bio-economy could represent a valuable contribution to bio-economy performance optimisation. The present paper investigates the contribution of bio-economy to circular economy. It proposes a conceptual framework to assess the potential for circularity for bio-waste and related by-products and it puts forward some considerations on the application of this framework to food waste. However, both bio-economy and circular economy may imply environmental burdens if an integrated assessment encompassing all life cycle stages of production and consumption is missing. Hence, adopting life cycle assessment is crucial to unveil trade-offs and ensuring identifying the best options for bio-economy and circular economy implementation.
\end{abstract}

\section{Introduction}

Natural resources provided by the Earth, both biotic and abiotic (i.e. raw materials, energy, water, air, land and soil as well as biodiversity and ecosystems) represent crucial economy and life-support elements for human societies worldwide. Indeed, natural resources are a building block in the supply chain, thus pushing the economic growth, and providing global functions, as in climate regulation. However, in a globalised world where population is in continuous expansion and the demand for finite resources continues to increase, the current production and consumption patterns in developed and developing countries are generating concerns about their

\footnotetext{
S. Corrado $\cdot$ S. Sala $(\square)$

European Commission, Joint Research Centre, Directorate D-Sustainable Resources,

Bio-Economy Unit, via Enrico Fermi 2749, 21027 I-Ispra, VA, Italy

e-mail: serenella.sala@ec.europa.eu

(C) The Author(s) 2018

E. Benetto et al. (eds.), Designing Sustainable Technologies,

Products and Policies, https://doi.org/10.1007/978-3-319-66981-6_6
} 
sustainability, with particular regard to the potential repercussion on the environment and climate. On such a background, a transition toward bio-based economy, called "bio-economy", represents an opportunity to comprehensively address inter-connected societal challenges such as food security, natural resource scarcity, fossil resource dependence and climate change, while achieving sustainable economic growth [1, 2]. However, not only fossil-based products carry an environmental burden, but also bio-based ones. Furthermore, the use of bio-based resources may raise issues on land competition for food production. Hence, to be effective, bio-economy strategies should be founded on resource efficiency and eco-innovation principles [3].

The concept of circular economy - a system in which the final disposal of waste and by-products is minimised by promoting their reuse and valorisation - can be successfully applied to bio-based production chains. Recent research developments, indeed, have highlighted several options for the conversion of bio-waste and bio-based by-products into either bioenergy, biofuels or valuable secondary raw materials [4]. Bio-based waste, indeed, represents a considerable amount of material at the global level, in the order of magnitude of gigatonnes per year [5]. The bio-economy contribution to circular economy may arise from very different typologies of bio-based products, such as wood, food, fibres, leathers and rubbers. Moreover, each step in the supply chain of bio-based products may provide waste or by-products with different characteristics, which can be valorised in various ways [2].

The present paper proposes a framework which may unveil the potential for circularity of bio-waste and bio-based by-products, coherently with the European legislative framework for waste management [6]. Furthermore, some considerations on the application of such framework to food waste and by-products, taken as a case-study are reported. Firstly, strengths and weaknesses of existing data sources and estimations approaches for food waste and by-products accounting are highlighted. Then, an overview of the options for valorisation for food waste and by-products is presented. Finally, the challenges of optimising the use of Life Cycle Assessment (LCA) when assessing the environmental performance of bio-economy value chains are discussed.

\section{Valorisation of Bio-Waste and Bio-Based By-Products}

In the European context, bio-economy is considered an important area of research, whose potential has still to be further disclosed. At present, the production of bio-materials and bioenergy is using about $36 \%$ of the biomass in Europe. However, further investigations are required to evaluate the potential availability of additional biomass, currently unused, without compromising the positive effects that biomass not removed from the field may have on soil fertility [7]. In this context, the European bio-economy sector is growing, showing a $7 \%$ increase in the turnover between 2008 and 2014. Particularly, some bio-economy branches 
increased faster than others and the highest turnover rise occurred in the manufacturing of liquid biofuels $(+25 \%)$, the production of bio-based chemicals, pharmaceuticals, plastics and rubber $(+22 \%)$, and in the forestry sector $(+21 \%)$ [2]. Furthermore, biofuels use is estimated to increase and cover between $27 \%$ and $48 \%$ of road transport fuel need in 2030 [8], whereas the demand for bioplastics, currently representing $1 \%$ of the total plastic used in Europe, is expected to increase by $50 \%$ in the period 2016-2021 [9].

Nowadays, the use of bio-waste and bio-based residues in bio-refineries is still a niche. Indeed, despite bio-waste and bio-based residues are potential sources of several high-values compounds [15], technical and non-technical barriers, such as uncertainty on the quality of materials and limited experiences at the industrial scale, are currently affecting the diffusion of their cascading use [10]. Therefore, further investigations on the practicability of valorisation strategies based on a holistic perspective are needed to foster the contribution of bio-economy to circular economy.

\section{Assessing the Potential for Circularity: The Overall Conceptual Framework and Application to Food Waste}

In order to assess the potential for circularity of bio-waste and bio-based by-products, a conceptual framework has been defined. It aims to guide in the identification of most preferable valorisation options for bio-based waste and by-products streams, as defined within the European legislation on waste [6] (Fig. 1). Therefore, it can be applied for either the identification of valorisation routes for material streams commonly disposed or the investigation of more valuable options for material flows, which are already somehow valorised.

The proposed framework, for which an example of application to food waste is reported in Fig. 2, combines the principles of the European waste hierarchy [6] and the European circular economy action plan for biomass and bio-based products [11], which promotes cascading use of renewable resource, with several reuse and recycling cycles. The underlying and key principle for defining the hierarchy of preferred actions is the minimisation of the dissipative use of high-value resources and the maximisation of the cascading use of bio-materials, meaning that reused bio-materials or the residues from the extraction of high values compounds may be reused for other purposes, e.g. animal feeding. Coherently with the European Waste Framework Directive [6], the proposed logical framework considers that a departure from the "standard" hierarchy may be justified according to the environmental preferability criterion, evaluated through a life cycle thinking approach. Other identified elements, which may limit the applicability of a certain valorisation route, are technical feasibility, economical profitability and legislation compliance.

Waste prevention should, in principle, be the preferred option. However, this might not be applicable, e.g. in case of certain by-products unavoidably delivered by a transformation process, such as bones and peels separated from the edible 
Definitions according to the European Waste Framework Directive [6]:

- waste: any substance or object which the holder discards or intends or is required to discard;

- bio-waste: biodegradable garden and park waste, food and kitchen waste from households, restaurants, caterers and retail premises and comparable waste from food processing plants;

- by product: substance or object, resulting from a production process, the primary aim of which is not the production of that item, may be regarded as not being waste referred to in point (1) of Article 3 but as being a by-product only if the following conditions are met:

(a) further use of the substance or object is certain;

(b) the substance or object can be used directly without any further processing other than normal industrial practice;

(c) the substance or object is produced as an integral part of a production process; and

(d) further use is lawful, i.e. the substance or object fulfils all relevant product, environmental and health protection requirements for the specific use and will not lead to overall adverse environmental or human health impacts.

Fig. 1 Definitions reported in the European Waste Framework Directive [6]

fractions of food or sawdust generated during wood transformation. The second option, the re-use, implies that the material can be utilised for the same purpose for which it was initially conceived. It applies, for example, to the harder discarded fractions of vegetables, which may be not suitable to be eaten directly, but can be used in the other preparations, such as soups. Bio-waste and by-products allow a wide range of recycling options, and their preferability strongly depends on the material characteristics, e.g. homogeneity and composition. Lignocellulosic biomass, for example, is composed mainly by the polymers cellulose, hemicellulose and lignin and the specific content of each of them may influence the preferable option for valorisation [12]. Furthermore, the extraction of high-value compounds is practicable when a homogeneous material stream is available, whereas it is hardly feasible in case of heterogeneous streams. Among the less preferable recycling options, anaerobic digestion was considered better than composting, because it allows a less dissipative use of the bio-based resources, recycling nutrients and producing methane at the same time. It has to be noted that the hierarchy could be subjected to modifications, according to specific sustainability priorities. For example, the maximisation of the uses for sustaining the food system may imply giving preference to the use of food waste as animal feed, rather than to be exploited as basis for bio-based materials.

Considering Fig. 2, "practicable" implies that the option is environmentally preferable, technically feasible, economically profitable and compliant to 


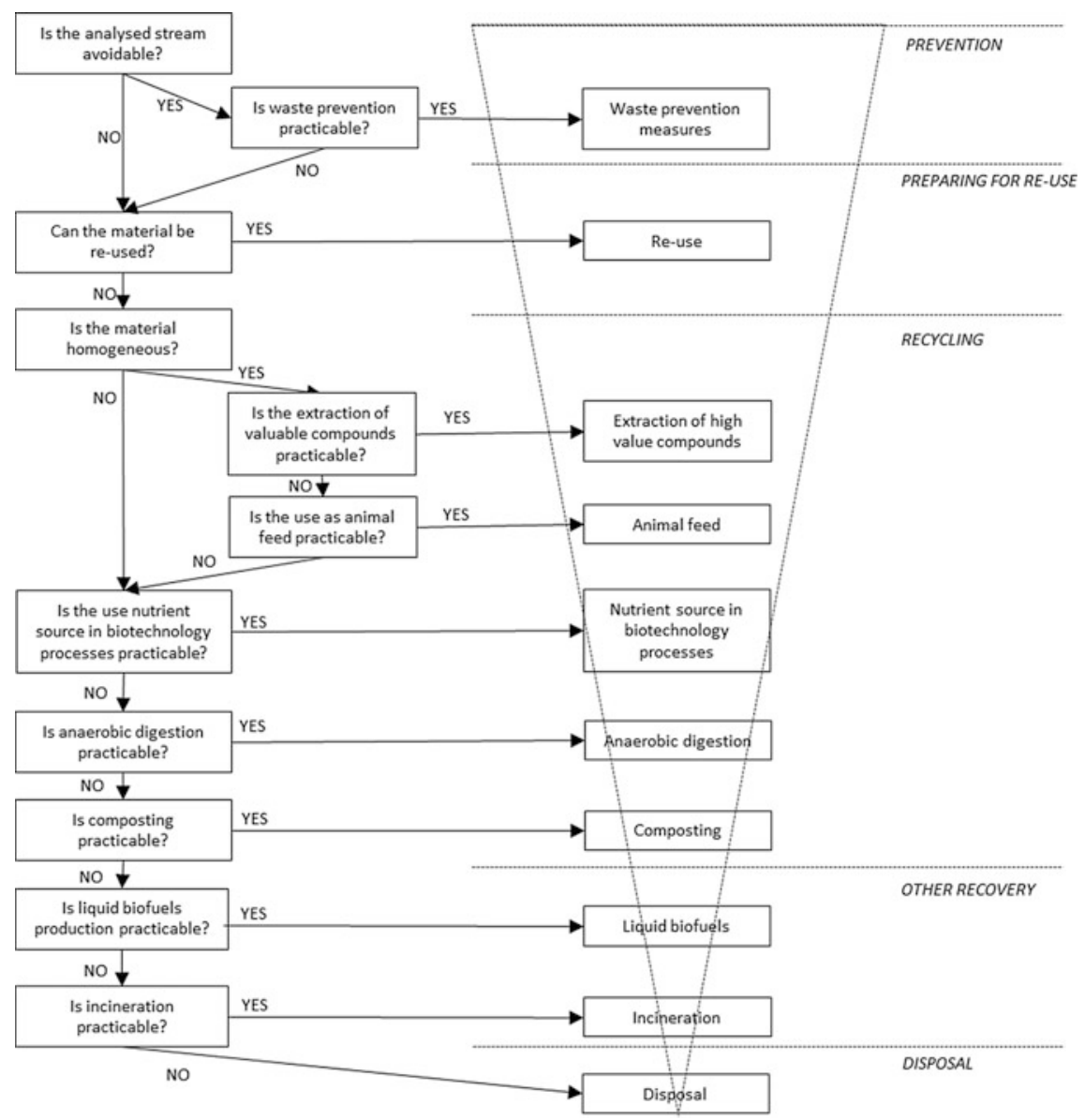

Fig. 2 Conceptual framework to identify the most valuable options for bio-waste and bio-based by-products valorisation, applied to food waste

legislation. The pyramid on the right side of the figure reports the coherence with the waste management hierarchy proposed by the European Waste Framework Directive [6].

\section{Considerations on Food Waste Valorisation from a Circular Economy Perspective}

The United Nations Food and Agriculture Organization (FAO) estimated that globally about one third of the food produced for human consumption is wasted along the supply chain [13]. In Europe, biowaste generated in households is often 
mixed with other waste [14]. However, food waste has generally a moisture content such that makes it unsuitable for incineration or thermal treatment and its presence in landfills may raise environmental concerns due to the production of leachate and methane emissions [15]. Therefore, this huge amount of bio-based materials represents a considerable potential contribution of bio-economy to circular-economy, which not only may optimise resources use, but also help facing the problem of food waste management.

\subsection{Food Waste Generation Accounting}

Being aware of the extent and the type of the waste and by-products streams is the first step towards the identification of valuable valorisation pathways for bio-based materials in general, including food waste [11]. Food waste and by-products generation encompasses all the stages of the food supply chain, from primary production to consumption. Therefore the adoption of a life cycle thinking (LCT) approach for supporting the accounting is advisable to fully capture the potentialities of food waste and by-products valorisation.

Currently, waste generation has been investigated on different geographical scales and breakdowns of the food supply chain (Fig. 3). Besides its relevance for addressing the type of food waste accounting, the matrix in Fig. 3 is also useful for the definition of specific interventions. For example, data on food waste for a specific commodity group at national/regional level may allow depicting tailored scenarios of food waste valorisation.

Results of the studies may differ due to various elements, such data sources, quantification methods, food waste definitions, system boundaries. The selection of the waste flows accounted may be a critical element, influenced by food waste definition and data sources considered. In the study by FAO on global food waste generation [13], for example, only the edible fractions of food are accounted, whereas inedible parts such as bones and fruit peels are excluded. Another criticality may be the inhomogeneity and the uncertainty of statistical data, which may limit the comparability of statistical data provided by different countires $[13,16]$.

These criticalities highlight the need of an accurate understanding of the underlying assumptions on the quantification approaches, in the interpretation of existing studies [17].

\subsection{Valorisation of Food Waste, Possible Options}

The rapidly growing attention for the circular use of bio-based resources has led to the realisation of several studies exploring potential for circularity for food waste and by-products. Not all the analysed solutions are currently developed at the industrial scale application [18]. 


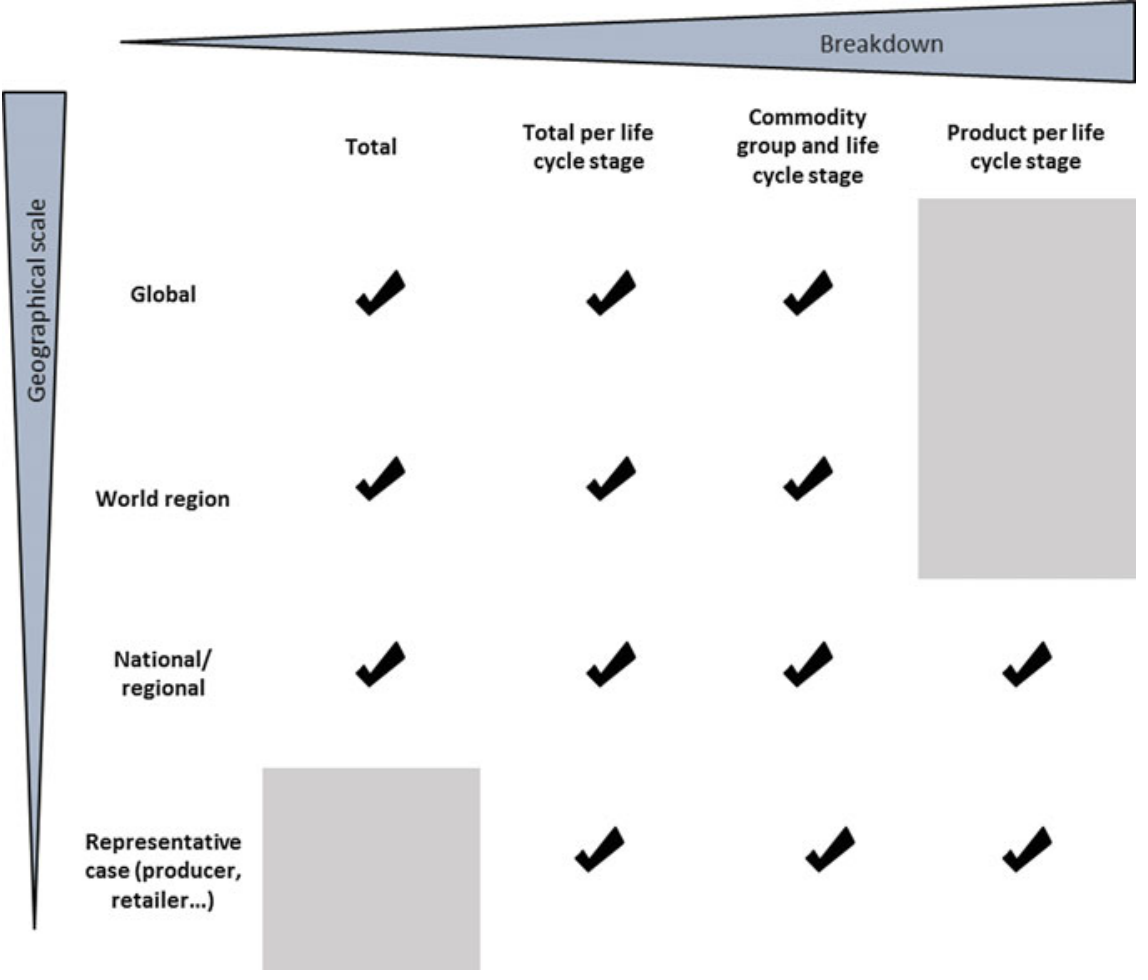

Fig. 3 Combination of geographical scale and breakdown of life cycle stages in the existing studies on food waste accounting. The checks indicate existing combination, whereas grey cells indicate fields not yet investigated

Several studies have highlighted that, being easily accessible and collected under controlled conditions, food waste and by-products from processing has homogeneous features, which give it a noteworthy potential for the extraction of high-value chemicals and for the direct valorisation as feed. Each stream of materials from processing may have in principle various routes for valorisation. However, currently the main research focus is on material flows from fruit and vegetables as source of antioxidants, fibers, phenols, polyphenols and carotenoids and waste from meat and dairy industries, vinasse, distiller's grains with solubles, press cake, fish silage, containing a large amount of recoverable proteins [15, 19].

On the contrary, the variable composition of food waste streams generated at the consumption stage, limits the possibility of extracting systematically specific molecules [18]. However, both processing waste and food waste generated at the consumption stage can be used either as nutrient sources in biotechnological processes [5] or for biofuels production [20,21]. In the last years, indeed, the interest for the production of biological metabolites to be used as biodegradable and renewable substitutes for fossil-based products, such as lactic acid and succinic 
acid, is growing fast [5, 18]. Furthermore, the valorisation of food waste for the production of bioethanol, biogas, hydrogen and biodiesel has attracted increasing attention [20].

\subsection{LCA Applied to Bio-Economy Value Chains: Future Challenges}

The assessment of the environmental preferability of the different pathways is one of the pillars of the proposed conceptual framework. On such purpose, the use of LCA, based on LCT approach, has had large diffusion both in the research and in the legislative fields. LCA, indeed, has been widely used to compare different waste management and treatment options [22, 23] and to assess the environmental performance of various bio-economy value chains [24, 25]. Furthermore, LCT is at the core of the European policies on waste management [6] to support the identification of the most environmentally sound options. One of the main advantages of this approach is the accounting of the so-called burden shifting, namely the transfer of environmental impacts between environmental compartments or supply chain stages, which may happen when pushing for resource efficiency. However, despite LCA broad acceptance and diffusion, past experiences have highlighted some shortcomings, which may limit a clear quantitative understanding of the environmental aspects of bio-economy value chains [25]. These shortcomings encompass both the product system modelling and the impact assessment side.

As mentioned previously, the interest for the valorisation of food waste and by-products as a resource is quite recent, therefore inventory data on innovative recovery processes are currently lacking. Besides, when considering circular system, setting the system boundaries may not be straightforward, influencing considerably the results of the study [26, 27]. Allocation of impacts can be another issue when dealing with food waste valorisation. Indeed, the production of food waste or by-product is, per definition, not the first target of the food system. Therefore, all the steps of the food supply chain, from primary production to consumption, can be considered multi-functional processes delivering food and food waste or by-products. The approach used to partition the impacts between co-products, namely, e.g., system expansion, bio-physical allocation or economic allocation, can importantly influence the LCA results and consequent considerations on food waste [28], limiting, therefore, their comparability [25]. Furthermore, if allocation of the impacts is performed, the allocation criteria can influence importantly the environmental performance of a bio-based product. For example, a by-product, which is commonly disposed has no economic value, whereas if it starts to be valorised as resource it would probably acquire economic value. If economic allocation is performed, the environmental burden of the by-product will increase together with its commercial value, with the potential risk of reaching or overcoming the environmental impacts of the virgin material, which is supposed to 
substitute. On the contrary, bio-physical allocation may attribute to the by-product a considerable burden of the production process, above all when a huge amount of by-product is delivered.

Concerning the impact assessment, Cristobal and colleagues [25] noted that it is a common practice to consider only a narrow number of impact categories, affecting the comprehensiveness of the results. Besides, some impact categories are based on weak calculations models, which limit their applicability. Furthermore, impact categories traditionally considered in LCA are often not enough to capture the environmental pressures exerted by food waste generation [26]. The impacts on biotic resources, for example, are generally not investigated in LCA [29]. Other relevant impact categories are still under refinement for being comprehensive in addressing issues such soil quality, biodiversity, water scarcity. All these aspects are crucial when accounting for the impacts of food waste or when comparing the environmental performance of bio-based and fossil-based products.

\section{Conclusions}

Applying circular economy principles to bio-economy represents a valuable opportunity for our society, which is called to cope with complex and important challenges, such as food security, competition for natural resources, fossil resources dependence and climate change.

A broad range of options exists for valorising bio-based waste and by-products, including already accomplished and innovative solutions, and the present paper introduced a conceptual framework to support the identification of the most valuable ones. The application of such framework is not straightforward, reflecting the complexity of multi-criteria decision-making processes and of the biological systems to which it is applied.

The main elements of the framework have been briefly introduced and discussed in relation to a specific type of bio-based material: food waste and related by-products. The accounting of food waste should be based on LCT, encompassing all the potential sources of food waste within the supply chain. Existing estimations showed some shortcomings mainly due to lack of data, uncertainties and data inhomogeneity, therefore their interpretation requires a deep knowledge of the underlying assumptions. The exploration of valorisation routes for food waste and bio-based by-products have raised considerable interests in the last years in the research field, however, part of the innovative solutions need to be further investigated, assessing their applicability at the industrial scale. Finally, LCA has shown great potentialities in supporting the assessment of the most preferable environmental options for food waste management, however still some efforts are needed to improve the comprehensiveness and robustness of LCA applied to bio-based and circular systems. 
Future industrial developments focused on valorisation of food waste should be based on robust accounting of available quantities, analysis of techno-economic potential coupled with life cycle based environmental assessment of benefits and burdens.

\section{References}

1. European Commission (EC), Innovating for Sustainable Growth: A Bioeconomy for Europe Communication from the Commission, COM (2012) 60.

2. Ronzon T, Lusser M, Klinkenberg M (ed.), Landa L, Sanchez Lopez J (ed.), M'Barek R, Hadjamu G (ed.), Belward A (ed.), Camia A (ed.), Giuntoli J, Cristobal J, Parisi C, Ferrari E, Marelli L, Torres de Matos C, Gomez Barbero M, Rodriguez Cerezo E, Bioeconomy Report 2016. JRC Scientific and Policy Report, 2017.

3. Mahro B, Timm M, Potential of biowaste from the food industry as a biomass resource, Engineering in Life Science, Vol. 7, No. 5, 2007, pp. 457-468.

4. Mohan S.V, Nikhil G.N, Chiranjeevi P, Reddy C.N, Rohit M.V, Kumar A.N, Sarkar O, Waste biorefinery models towards sustainable circular bioeconomy: critical review and future perspectives, Bioresource technology, Vol. 215, 2016, pp. 2-12.

5. Pleissner D, Qi Q, Gao C, Rivero C.P, Webb C, Lin C.S.K, Venus J, Valorization of organic residues for the production of added value chemicals: a contribution to the bio-based economy, Biochemical Engineering Journal, Vol. 116, 2016, pp. 3-16.

6. European Parliament, Directive 2008/98/EC of the European Parliament and of the Council of 19 November 2008 on waste and repealing certain Directives, DIRECTIVE 2008/98/EC.

7. Ronzon T, Santini F, M'Barek R, The Bioeconomy in the European Union in numbers - Facts and figures on biomass, turnover and employment, European Commission, Joint Research Centre, Institute for Prospective Technological Studies, Spain, 2015.

8. European Commission (EC), Biofuels in the European Union - A vision for 2030 and beyond, 2006.

9. European Bioplastics, Nova Institute, Bioplastics market data, 2016.

10. Ladu L, Quitzow R, Bio-Based Economy: Policy Framework and Foresight Thinking, Springer International Publishing, 2017.

11. European Commission (EC), Closing the loop - An EU action plan for the Circular Economy, COM (2015) 0614.

12. Isikgor F.H, Becer C.R, Lignocellulosic biomass: a sustainable platform for the production of bio-based chemicals and polymers. Polymer Chemistry, Vol 6 (25), 20015, pp. 4497-4559.

13. Food and Agriculture Organization of the United Nations (FAO), Global food losses and food waste - Extent, causes and prevention, 2011.

14. European Commission (EC), Measuring progress towards circular economy in the European Union - Key indicators for a monitoring framework, SWD (2018) 17 final.

15. Mirabella N, Castellani V, Sala S, Current options for the valorization of food manufacturing waste: a review. Journal of Cleaner Production, Vol. 65, 2014, pp. 28-41.

16. Monier V, Mudgal S, Escalon V, O'Connor C, Gibon T, Anderson G, Morton G, Preparatory study on food waste across EU 27, Report for the European Commission [DG ENVDirectorate C], 2010.

17. Tostivint C, Östergren K, Quested T, Soethoudt J.M, Stenmarck A, Svanes E, O'Connor C, Food waste quantification manual to monitor food waste amounts and progression, 2016.

18. Girotto F, Alibardi L, Cossu R, Food waste generation and industrial uses: a review, Waste Management, Vol. 45, 2015, pp. 32-41.

19. Tuck C, Pérez E, Horváth I.T, Sheldon R.A, Poliakoff M, Valorization of Biomass: Deriving More Value from Waste, Science, Vol. 337, 2012, pp. 695-699. 
20. Kiran E.U, Trzcinski A. P, Ng W.J, Liu Y., Bioconversion of food waste to energy: a review, Fuel, 2014, Vol. 134, 2014, pp. 389-399.

21. Pham T. P. T, Kaushik R, Parshetti G. K, Mahmood R, Balasubramanian, R, Food waste-to-energy conversion technologies: Current status and future directions, Waste Management, Vol. 38, 2015, pp. 399-408.

22. Manfredi S, Cristobal J, de Matos C.T, Giavini M, Vasta A, Sala S, Tuomisto H, Improving Sustainability and Circularity of European Food Waste Management with a Life Cycle Approach, JRC technical report, 2015.

23. Laurent A, Bakas I, Clavreul J, Bernstad A, Niero M, Gentil E, Christensen T.H, Review of LCA studies of solid waste management systems-Part I: Lessons learned and perspecties, Waste Management, Vol. 34, No. 3, 2014, pp. 573-588.

24. Secchi M, Castellani V, Collina E, Mirabella N, Sala S, Assessing eco-innovations in green chemistry: Life Cycle Assessment (LCA) of a cosmetic product with a bio-based ingredient, Journal of Cleaner Production, Vol. 129, 2016, pp. 269-281.

25. Cristóbal J, Torre de Matos C, Aurambout J.P, Manfredi S, Kavalov B, Environmental sustainability assessment of bioeconomy value chains, Biomass and Bioenergy, 89, 2016, 159-171.

26. Corrado S, Ardente F, Sala S, Saouter E, Modelling of food loss within life cycle assessment: from current practice towards a systematisation, Journal of Cleaner Production, Vol. 140, 2017, pp. 847-859.

27. Saraiva A.B, System boundary setting in life cycle assessment of biorefineries: a review, International Journal of Environmental Science and Technology, Vol. 14, No. 2, 2017, pp. 435-452.

28. Bernstad A.K, Cánovas A, Valle R, Consideration of food wastage along the supply chain in lifecycle assessments: A mini-review based on the case of tomatoes, Waste Management \& Research, Vol. 35, No. 1, 2017, pp. 29-39.

29. Crenna E, Sozzo S, Sala S, Natural biotic resources: towards an impact assessment framework for sustainable supply chain management, Journal of Cleaner Production, Vol. 172, 2017, pp. 3669-3684.

Open Access This chapter is licensed under the terms of the Creative Commons Attribution 4.0 International License (http://creativecommons.org/licenses/by/4.0/), which permits use, sharing, adaptation, distribution and reproduction in any medium or format, as long as you give appropriate credit to the original author(s) and the source, provide a link to the Creative Commons license and indicate if changes were made.

The images or other third party material in this chapter are included in the chapter's Creative Commons license, unless indicated otherwise in a credit line to the material. If material is not included in the chapter's Creative Commons license and your intended use is not permitted by statutory regulation or exceeds the permitted use, you will need to obtain permission directly from the copyright holder.

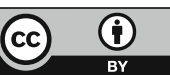




\title{
Life Cycle Management and Circular Economy Challenges for the Textile Sector: Session Wrap Up
}

\author{
Keith James and Julian Lings
}

\begin{abstract}
This session demonstrated the added-value of applying Life Cycle Assessment to address different types of issues related to the textiles sector. Each of the four presentations in the session was based on case-studies, also highlighted the important challenges to be faced regarding methodological issues and market issues, to make LCA fully efficient for the sector. In particular, it has been demonstrated that one of the main concern for the sector is toxicity assessment, which is currently limited due to lack of data inducing weaknesses in characterisation of substances contributing to this impact. It also has been shown that in the objective of developing circular models, multiple issues must be addressed simultaneously. For example, to increase recycling of clothing unsuitable for reuse, markets must be developed at the same time that infrastructure is developed and collection mechanisms are put in place. It must also be tackled in a sustainable way, supported by Life Cycle Assessment and Life Cycle Costing.
\end{abstract}

\section{Introduction}

The textiles sector faces a number of challenges. Clothing is the eighth largest sector in the EU ranking of household expenditure, but the fourth largest when it comes to environmental impacts [1]. Only transport, housing and food have greater impacts. As part of the European Clothing Action Plan (ECAP), a Life+ funded programme, WRAP estimate that over 6.4 million tonnes of clothing were purchased across the EU in 2015, and that this clothing is associated with 195 million tonnes $\mathrm{CO}_{2} \mathrm{eq}, 46$ billion $\mathrm{m}^{3}$ of water and 11 million tonnes of waste across the whole life cycle of the clothing.

K. James $(\square)$

WRAP, Banbury, UK

e-mail: Keith.James@wrap.org.uk

J. Lings

The North Face, Stabio, Switzerland

(C) The Author(s) 2018

E. Benetto et al. (eds.), Designing Sustainable Technologies,

Products and Policies, https://doi.org/10.1007/978-3-319-66981-6_7 
However, these are just some of the challenges faced. At the LCM2017 conference, Sandra Roos from the Swedish research institute Swerea presented the toxicity footprint of Swedish Clothing Consumption [2]. Many environmental organizations have reported on the damage caused by dyeing and finishing of textiles, both humans and the environment. In addition, the use of pesticides in cotton production accounts for $25 \%$ of global pesticide use, despite the crop occupying only $2 \%$ of agricultural land. Roos et al. attempted to compare the toxicity impact of these three different stages. As part of the research Roos et al. identified only 7 out of 58 LCA studies focussed on textiles had included textile chemicals in the inventory, and of these only 4 studies had also developed LCIA to match these flows. It was therefore necessary to create additional inventories and develop characterisation factors. Characterisation factors are complex to develop; a data source selection strategy is recommended in order to keep consistency. The factors developed were combined with data on Swedish clothing consumption. The results suggest that eco-toxicity associated with wet treatment (dyeing and finishing) was one order of magnitude higher than for the cotton cultivation (all toxicity in the fibre production stems from cotton cultivation). This shows the value of Life Cycle Management and the need to understand life cycle stages to address priority issues.

\section{Framing and Responding to the Challenge}

The challenges may be framed and addressed in terms of a number of initiatives, such as the United Nations Sustainable Development Goals, the European Commission's Circular Economy Package, Product Environmental Footprint Initiative, and EU Garment Initiative, as well as Action Plans led by National Governments and Industry Associations.

Within each of these frameworks, different options are open to businesses across the clothing value chain. A key challenge is selecting the right strategy for the business, their products and their customers: they may consider increased durability, recyclability, or look more deeply at their business model to consider options such as subscription services, repair and advice on caring for clothing. Companies such as VF Corporation are seeking to address these challenges. Founded in 1899, $\mathrm{VF}$ is one of the world's largest apparel, footwear and accessories companies, incorporating over 30 brands such as North Face. Their sustainability mission is to protect our outdoor playgrounds and communities through the design and development of environmentally and socially responsible products and business practices. Through Life Cycle Assessment (LCA) they have determined that the majority of their environmental impact comes from materials processing, and product manufacturing. The North Face is now shifting the business away from finite materials, eliminating supply chain waste and developing closed loop solutions for post-consumer waste. 
Life Cycle Management can help to realise the potential environmental benefits of such changes and prioritise actions. Roos' work also identifies that, for climate change potential, there is little contribution from activity within Sweden, with the life cycle impacts dominated by yarn spinning, weaving and dyeing. However, in the UK, consumer care contributes significantly to the overall footprint. Regionalised tools, such as the ECAP Footprint tool, are therefore required to help businesses to make such changes based on representative information relevant for the appropriate supply chain and consumer.

Life Cycle Management is important at every decision point in the life cycle of clothing. Fierro [3] and Holmquist [4] both presented the role of life cycle management in choosing a raincoating, substituting out the most hazardous chemicals. Any substitution has the potential to lead to regrettable substitutions, and therefore needs to be holistic, and also ensure that the product functionality is maintained. Durable water and oil repellents (DWOR) are textile finishings which impart water (and oil) repellency, with three main chemistry types. Whilst the Long-chain perand polyfluoroalkyl substances (PFAS) are being phased out, the alternatives also give rise to emissions of hazardous substances.

Hanna Holmquist presented an ecotoxicity assessment of the three main chemistry types showing little difference between them, though USEtox does not capture PFAS hazards. A life cycle perspective however reveals that the ecotoxicity associated with the DWOR is dwarfed by the fabric manufacture, and the fabric and the membrane is important for Global Warming Potential. Change in the length of the product life therefore have the potential for large effects on the total potential ecotoxicity impact. This can be achieved through re-impregnation of DWOR, which has a relatively small contribution to ecotoxicity potential.

Julio Fierro presented the Life MIDWOR project and draft LCA results. DWORs have a complex structure (comb-like) with several side chains. Over time, perfluorinated chains are severed from DWOR structure and released to the environment. Consequently, PFC degradation compounds can be detected all over the world, with most originating from production sites. Their stability means the compounds can bio-accumulate, with concerns relating to potential mutagenic, carcinogenic and reprotoxic impacts. The MIDWOR project explores six different textile industries with a common finishing process. Two finishing products were considered: Short-chain DWOR and Silicone based DWOR. The draft LCA results suggest little difference between these when the impact of fabric production is also included, with differences only becoming apparent when considering finishing only. This is in line with Holmquist's conclusions.

In order to apply life cycle management principles to DWOR there is a need for further information on their production. The composition of DWORs is not always specified in Safety datasheets and is often a trade secret. These barriers must be overcome for life cycle management principles to be applied. In addition, life cycle methodologies do not reflect the impact of PFCs. Only the Ecological Scarcity methodology was identified as including the impact of PFCs, and this yields notably different results to other methodologies based on the same data. 
All frameworks identify a need to address multiple issues. Often these must be addressed simultaneously. For example, to increase recycling of clothing unsuitable for reuse, markets must be developed at the same time that infrastructure is developed, collection mechanisms are put in place and public communications occur; if any three of these occur without the fourth, then the system will not be able to change. Projects such as ECAP and RESYNTEX are seeking to address these complex challenges.

Harbi [5] presented the RESYNTEX project which addresses two objectives of a circular economy; to reduce waste and to reduce resource extraction through use of textile waste to generate secondary raw materials. RESYNTEX is a partnership between 20 organisations funded by Horizon 2020 to take the clothing currently sent to landfill or incineration and, through biochemical processing, generate resin, bioethanol, bottle-grade PET granulate and value added chemicals. The project will use both LCA and Life Cycle Costing (LCC) to assess the performance of the process, and will further explore biochemical treatment to understand the different contributions and optimise performance. The RESYNTEX system will also be compared with reference systems and global textile/chemical markets will be assessed.

\section{Future Perspectives}

The conference session achieved broad consensus on the scale of the challenge for the textiles sector, and the growth of these challenges if action is not taken. There is a recognition of the value of life cycle management in guiding organisations, and the need for fundamental change across the value chain.

There was consensus in the conference session that there is a need to consider multiple environmental issues, and that there are data barriers to understanding toxicity impacts. However, these are surmountable. There is a need for characterisation factors to reflect properly environmental impact of conventional and alternative DWORs and improvements in knowledge about DWOR production and composition. There is also a need to address multiple market challenges to realise a systemic change in the way clothes are managed at end of life and used as raw materials again. Further research and development in this area will be required.

\section{References}

1. EEA, Environmental Indicators Report 2014, pp 26, 114, 115.

2. Roos S, Arvidsson R, Jönsson C, Calculating the toxicity footprint of Swedish clothing consumption, LCM 2017 conference, Luxembourg, 2017.

3. Fierro J, Martínez C, Environmental analysis of the best available finishing products to provide water, oil and dirt repellency in the textile sector - A Life Cycle Assessment approach, LCM 2017 conference, Luxembourg, 2017. 
4. Holmquist H, Roos S, Jönsson C, Peters G, Choosing a rain coating - Life Cycle Assessment to guide substitution of hazardous durable water repellent chemistry, LCM 2017 conference, Luxembourg, 2017.

5. Magaud V, Dauriat A, Mzikian P, Troppenz V, Harbi S, Recycling textile waste into secondary material: Experience from the innovative RESYNTEX project using circular economy concepts, LCM 2017 conference, Luxembourg, 2017.

Open Access This chapter is licensed under the terms of the Creative Commons Attribution 4.0 International License (http://creativecommons.org/licenses/by/4.0/), which permits use, sharing, adaptation, distribution and reproduction in any medium or format, as long as you give appropriate credit to the original author(s) and the source, provide a link to the Creative Commons license and indicate if changes were made.

The images or other third party material in this chapter are included in the chapter's Creative Commons license, unless indicated otherwise in a credit line to the material. If material is not included in the chapter's Creative Commons license and your intended use is not permitted by statutory regulation or exceeds the permitted use, you will need to obtain permission directly from the copyright holder. 


\title{
Life Cycle Assessment of Organic, BCI and Conventional Cotton: A Comparative Study of Cotton Cultivation Practices in India
}

\author{
Pragnesh Shah, Abhishek Bansal and Rajesh Kumar Singh
}

\begin{abstract}
Cotton, the most important cash crop of India plays a dominant role in its agrarian and industrial economy. In India, the area under cotton cultivation is the highest in the world and industry provides livelihood to over seven million people. However, cotton productivity in India is low and farmers rely on heavy dosage of fertilizers and insecticides/pesticides to control insects, pests, weeds and growth regulators. Organic cotton farming is the process of growing cotton without the use of synthetic pesticides and chemical fertilizers. Better Cotton Initiative (BCI), is a concept to grow cotton with judicious use of water, chemical fertilizers and pesticides, to reduce the environmental footprint of cotton farming. The objective of the study was to quantify the environmental benefits associated with the production of organic seed cotton and BCI seed cotton compared to the conventional production of seed cotton, using Life Cycle Assessment approach. The aim was also to identify hotspots across the cultivation process. The study was based on primary data collected from farming sites managed by Arvind Group under contract farming model for BCI cotton and organic cotton cultivation in the state of Maharashtra, India. When compared with the conventional cotton system, the organic and BCI cotton show considerable advantage for several impacts categories.
\end{abstract}

\section{Introduction}

Cotton is an indispensable part of today's human life and India is now the second largest producer of cotton in the world, producing around $18 \%$ of the world's cotton [1]. It has the largest area under cotton cultivation in the world (8.9 Million hectare), representing about $25 \%$ of the world area under cotton cultivation [2]. For the

\footnotetext{
P. Shah · A. Bansal

Arvind Limited, Naroda Road, Ahmedabad, Gujarat, India

R. K. Singh $(\square)$

Thinkstep Sustainability Solutions Pvt. Ltd., 421, Sahar Plaza,

Andheri (E), Mumbai, Maharashtra, India

e-mail: Rajesh.Singh@thinkstep.com

(C) The Author(s) 2018

E. Benetto et al. (eds.), Designing Sustainable Technologies,

Products and Policies, https://doi.org/10.1007/978-3-319-66981-6_8
} 
financial year 2015-16 total production of cotton was 338 hundred thousand bales (1 Indian bale $=170 \mathrm{~kg}$ ), out of which $20 \%$ was exported [1]. Environmental sustainability of cotton fibres production has gained more and more attention from both suppliers and consumers in recent years due to the associated resources consumption and pollutant emissions. There are mainly three types of cotton cultivation practices: Conventional, Organic and BCI cotton seed farming. Conventional Cotton farming is the process of growing cotton with use of insecticides, pesticides, herbicides, defoliants, fertilizers, and lot of water. Many conventional systems use rain fed water instead irrigation thus conserving surface and groundwater and also limiting the use of agricultural inputs (fertilizers, pesticides, and machinery) [3]. Organic cotton farming is done without use of synthetic pesticides and chemical fertilizers and the only additives comes in the form of manures, while soil quality is controlled by crop rotation. The impact on the environment is therefore reduced drastically, producing clean and safe cotton while creating a sustainable cycle [4]. It is best suited for small and marginal farms and Arvind Limited is working closely with the farmers of Akola in the Vidarbha region of Maharashtra to grow organic cotton; initiative has helped to improve the livelihood of the farmers by increasing their per-acre income. BCI is a concept to grow cotton with judicious use of water, chemical fertilizers and pesticides. This technique works well in fertile and irrigated regions where organic farming is not economically viable. Since 2010, Arvind as implementation partners of BCI in India have been working with over four thousand farmers in the Akola region covering over 15,815 ha of farmland. In 2014, the total production of BCI cotton was 283,000 MT on 456,000 ha. Better Cotton is produced in ten states of India and year-on-year figures for BCI Farmers, Better Cotton lint produced, and hectares under cultivation have increased [5]. Several studies based on Life Cycle Assessment (LCA) [6] have been done on the environmental footprint of cotton cultivation which includes Murugesh and Selvadass [7], Khabbaz et al. [8], Barnes [9], Cotton Incorporated [10], Textile Exchange and PE International [4], Cotton Made in Africa [3]. Conventional seed cotton fibres have greater environmental impacts compared to the organic cotton fibres [7] and irrigation has the highest contribution for both conventional and organic seed cotton fibres [7]. Field emissions and fertilizers manufacturing contribute significantly to most of the environmental impacts categories [Global Warming Potential (GWP), Ozone Depletion Potential (ODP), and Primary Energy Demand (PED)] [9]. Cotton Incorporated, a pioneer research and marketing company, has done an LCA of cotton fibres with the help of thinkstep AG (former PE International) [10]. The foreground data have been collected from three countries U.S., China, and India; representing 67\% of the world cotton fibres production. GWP and PED for $1 \mathrm{~kg}$ of cotton fibres were estimated to be $15 \mathrm{MJ}$ and $0.268 \mathrm{~kg} \mathrm{CO}_{2}$ eq. According to other literature references, cotton fibres production induces an energy consumption of $46.40 \mathrm{MJ}$ per $\mathrm{kg}$ produced [8] to $67.1 \mathrm{MJ} / \mathrm{kg}$ [11]. 


\section{Methodology}

The LCA has been carried out following the ISO 14040 and ISO 14044 guidelines by modelling the LCI for each of the three different scenarios of cotton cultivation.

\subsection{Goal}

The goal of the study was to quantify the environmental benefits associated with production of organic seed cotton and BCI seed cotton compared to conventional seed cotton production, and also to identify the environmental hotspots over a range of environmental impacts categories. The audience of the study was internal management of Arvind Limited and critical review was not carried out. As such, Arvind Limited does not challenge the results.

\subsection{Scope of the Study}

The typical system under consideration is a cradle-to-gate Life Cycle Inventory including the cultivation of the cotton plant until farm gate. Figure 1 shows the system boundaries considered for the three farming practices under study.

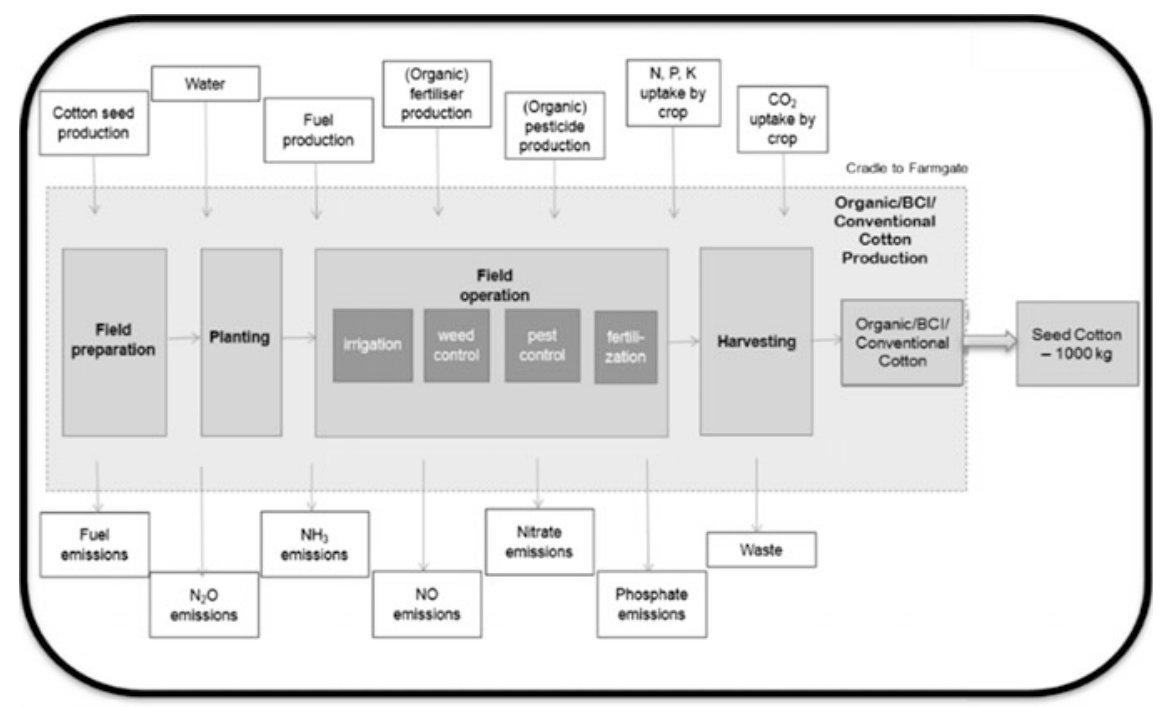

Fig. 1 System boundaries considered in this study 
Cotton cultivation includes four main tasks: field preparation, planting, field operations, and harvesting. Under the collective term field operations: irrigation, weed and pest control, and fertilization are included. These tasks consume energy (electricity and fuel), require inputs (seeds, fertilizers, water etc.) and produce wastes and emissions - all part of the present system.

\subsection{Functional Unit}

The functional unit for this study was 1 ton of seed cotton at the farm gate, for all the three systems, i.e. conventional cotton, BCI cotton and organic cotton production.

\subsection{Data Collection}

Primary data for BCI, organic and conventional cotton cultivation was provided by Arvind Limited for ten representative farming sites. Specifically adapted questionnaires were used to collect inventory data for agricultural systems. These questionnaires were filled in by representatives of producer groups and the data provided were subjected to quality check considering literature references and were compared to other primary cultivation data sources in order to ensure reliable results. The data from each cultivation site were modelled into ten different site specific LCI models and weighted average of those sites were used to create an LCI profile for BCI, Organic and conventional cotton production respectively. Table 1 shows the questionnaires that were used to collect primary inventory data for all three farming practices.

\subsection{Allocation}

During cotton production, two valuable co-products are produced, cotton fibres and cottonseed. Thus, the environmental burden is allocated to both the fibres and the seed. It has been determined that mass-based allocation is the most suitable method to use for this study.

\subsection{Inclusion and Exclusion}

Included in the study are all material and energy flows required for the cultivation phase, as well as all associated wastes and emissions. The study is not limited to 
Table 1 Questionnaires to collect inventory data of organic, BCI cotton and conventional cotton production stages

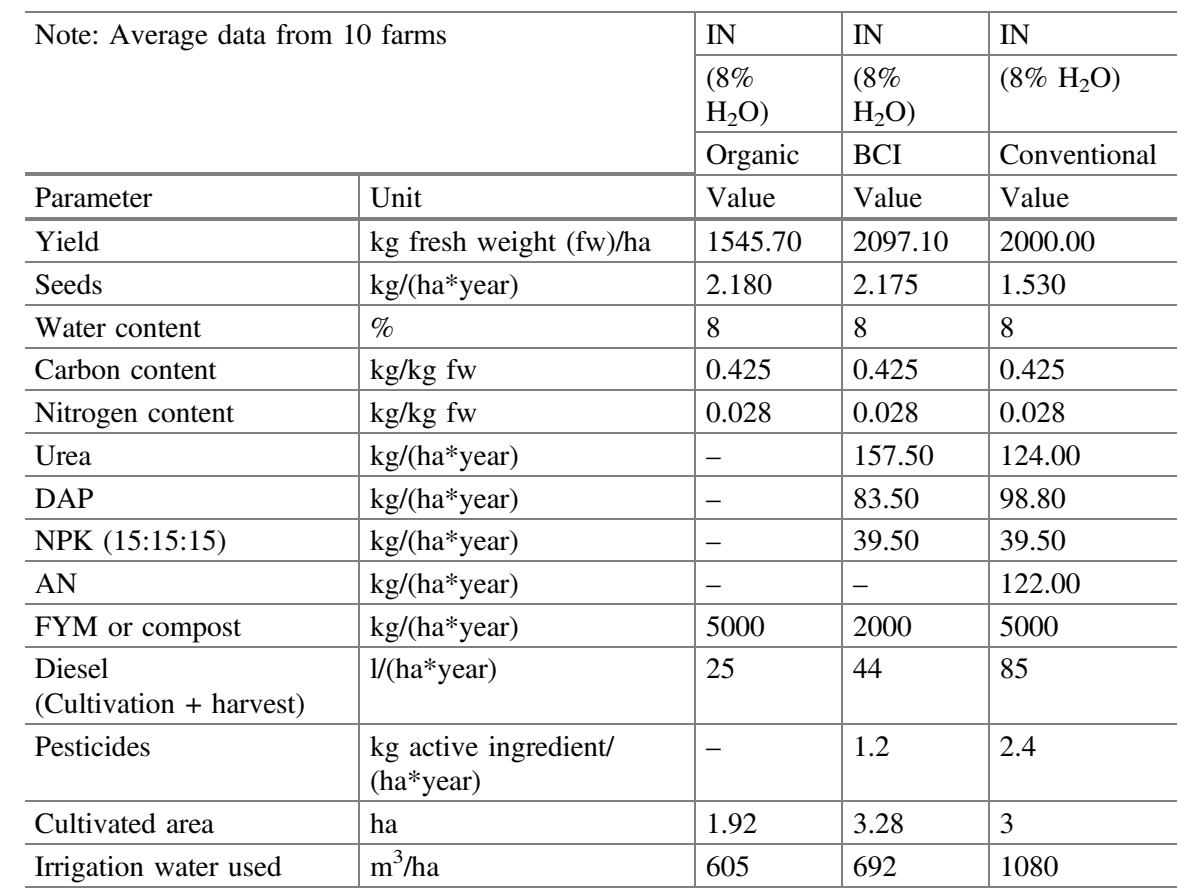

fertilizer and pesticide production but also includes field emissions (e.g. $\mathrm{N}_{2} \mathrm{O}$ ), electricity for pumps and all transports (fertilizer to the field). The provision of infrastructure is not included. The impact of provision of other capital goods such as buildings is also expected to be low, as for example storage takes place at producer groups, so that the scaling effect will result in very low impacts per $\mathrm{kg}$ final product.

\subsection{Software and Database}

The LCA model was created using the GaBi 6.4 Software system for life cycle engineering, developed by thinkstep AG. The associated Gabi database provided the background LCI data in order to characterise several of the raw materials and processes involved in the background system. 


\subsection{Life Cycle Impact Assessment (LCIA)}

The following impacts categories were considered of high relevance: GWP, PED, Acidification Potential (AP), Eutrophication Potential (EP) and Water Consumption (WC). The CML impact assessment methodology was used (Institute of Environmental Sciences of the University of Leiden framework, CML2001, 2013). The impact categories identified above are also considered by Cotton Inc. [8] and Textile Exchange [5].

\section{Modelling Approach-Agriculture Model}

Various factors like the variety of different locations, large number and diversity of farms, variety of agricultural management practices applied, lack of a determined border to the environment, complex and indirect dependence of the output (harvest, emissions) from the input (fertilizers, location conditions, etc.), variable weather conditions within and between different years, and variable pest populations (insects, weeds, disease pathogens, etc.) contribute to the complexity of agricultural modelling. Due to the inherent complications characterizing an agricultural system, a non-linear agrarian calculation model is applied displaying plant production (jointly developed by the LBP of the University of Stuttgart and thinkstep AG). This software model covers a multitude of input data, emission factors and parameters. The agricultural model accounts for the nitrogen cycle in agricultural systems. Specifically, the model includes emissions of nitrate $\left(\mathrm{NO}_{3}{ }^{-}\right)$in water and emissions of nitrous oxide $\left(\mathrm{N}_{2} \mathrm{O}\right)$, nitrogen oxide $(\mathrm{NO})$ and ammonia $\left(\mathrm{NH}_{3}\right)$ into air. The model ensures that emissions from erosion, the reference system (comparable non-cultivated land area) and nutrient transfers within crop rotations are modelled consistently. Carbon-based emissions such as $\mathrm{CH}_{4}, \mathrm{CO}, \mathrm{CO}_{2}$ are considered in foreground and background datasets. Background datasets include emissions resulting from production of fertilizer, pesticides, electricity, and diesel while foreground datasets contain direct emissions such as $\mathrm{CO}_{2}$ due to combustion of fossil fuels by the tractor or irrigation engines and application and decomposition of urea fertilizers in the soil.

\section{Results and Discussion}

Table 2 shows the comparative LCIA results on absolute basis for all three farming practices. It clearly reflects that LCA results of organic cotton is better than BCI cotton and conventional cotton production for all the environmental impacts categories. There has not been significant difference of yield between conventional and BCI cotton production in this study. However, if the yield of BCI cotton increases 
significantly, it will improve their environmental impacts considerably which will bring environmental performances closer to the one from organic cotton. It is anticipated that fertilisers consumption can further be optimised which would further result in mitigation of environmental impacts. Moreover, BCI cotton is a holistic approach to sustainable cotton production which covers all three pillars of sustainability: environmental, social and economic. The system is designed to ensure the exchange of good practices, and to encourage the scaling up of collective action. In case of organic farming, the only additives come in the form of manures, and the soil quality is controlled by crop rotation. The impact on the environment is reduced drastically. Organic farming is best suited for small and marginal farms. Overall, the region of India considered in the study has been battered by multiple droughts and considered amongst the least developed with farmers having poor socio-economic living conditions due to rising cost and declining production yield. With continuous efforts on implementation of BCI initiative and organic cotton farming, Arvind Limited brought around an economic transformation with thousands of farmers, enhancing their farm productivity. The details of environmental performances effects are discussed later in this chapter.

Figure 2 shows the LCIA contribution analysis for all three farming practices.

Acidification Potential (AP): Acidification Potential is calculated in terms of $\mathrm{kg}$ $\mathrm{SO}_{2}$-eq/1 ton of cotton seed. Nitrogen oxide, Sulphur dioxide and Ammonia are the major contributor for this impact category. Ammonia released from field emissions is the highest contributor for the three practices $(7.47 \mathrm{~kg}$ for conventional, $6.93 \mathrm{~kg}$ for BCI and $1.43 \mathrm{~kg}$ for organic cotton). BCI and organic practices shows some advantages compared to conventional cotton $\left(12.14 \mathrm{~kg} \mathrm{SO}_{2}\right.$-eq. for BCI, $3.34 \mathrm{~kg}$ $\mathrm{SO}_{2}$-eq. for organic and $14.06 \mathrm{~kg} \mathrm{SO}_{2}$-eq. for conventional/1 ton of seed cotton), because of reduced field air emissions of ammonia due to elimination of inorganic fertilizers application and less air emissions from tractor operation. Again, the difference is driven by agricultural inputs which are used to a lesser extent in the BCI systems (or not used at all for organic system), i.e. fertilizers and pesticides production, irrigation pumps and tractor operations. Here, the most relevant emissions (sulphur dioxide and nitrous oxides) are caused by power generation and in diesel combustion (transports and machinery use).

Table 2 Comparative analysis of LCIA for organic cotton, conventional cotton versus BCI cotton production (Per 1 ton seed cotton)

\begin{tabular}{l|l|l|l}
\hline Impacts categories & $\begin{array}{l}\text { Organic } \\
\text { cotton }\end{array}$ & $\begin{array}{l}\text { Better Cotton } \\
\text { Initiative } \\
(\mathrm{BCI}) \text { cotton }\end{array}$ & $\begin{array}{l}\text { Conventional } \\
\text { cotton }\end{array}$ \\
\hline Acidification potential $\left[\mathrm{kg} \mathrm{SO}_{2}\right.$-Equiv.] & 3.34 & 12.14 & 14.06 \\
\hline Eutrophication potential $\left[\mathrm{kg} \mathrm{PO}_{4}\right.$-Equiv.] & 0.46 & 2.49 & 7.07 \\
\hline $\begin{array}{l}\text { Global warming potential }(\mathrm{GWP} 100 \\
\text { Years) [kg CO}\end{array}$-Equiv.] & 295 & 435 & 731 \\
\hline Primary energy demand (PED) [MJ] & 1351 & 2510 & 5375 \\
\hline Blue water consumption (WC) $\left[\mathrm{m}^{3}\right]$ & 391.80 & 330.61 & 541.06 \\
\hline
\end{tabular}




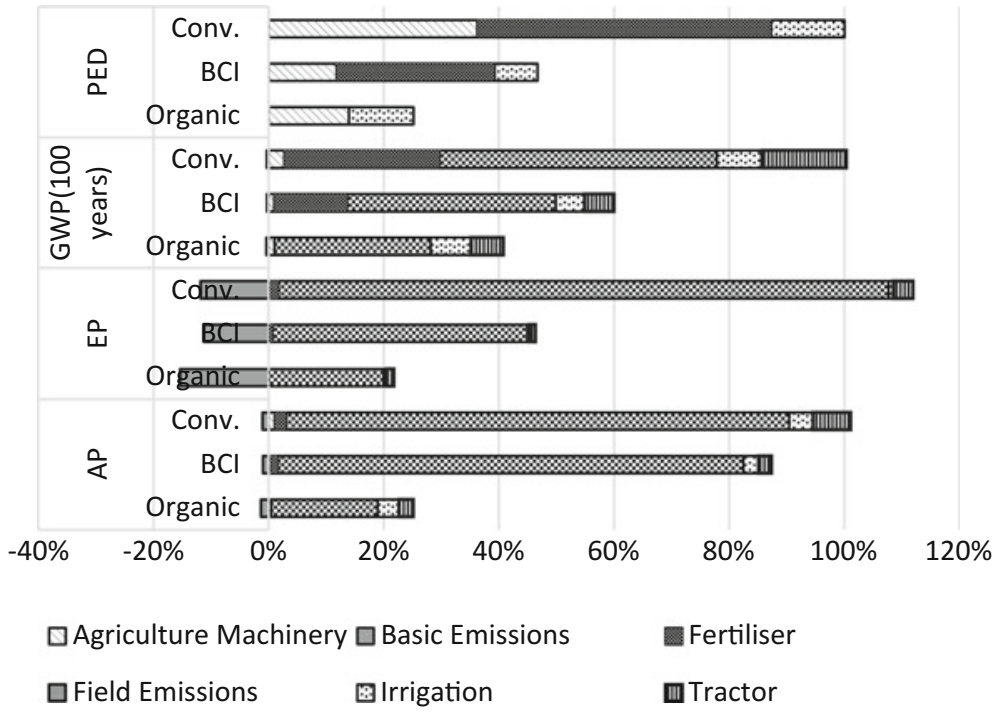

Fig. 2 Comparative analysis of the three cotton cultivation practices

Global Warming Potential (GWP 100 Years): Global Warming Potential is calculated in terms of $\mathrm{kg} \mathrm{CO}_{2}$ eq./1 ton of cotton seed. The combustion of fossil fuel for agricultural machinery, irrigation and other upstream processes are major contributors for GWP. Carbon dioxide emissions are mostly induced by irrigation and agriculture-machinery for all three practices and field emissions are due to $\mathrm{N}_{2} \mathrm{O}$ and $\mathrm{CH}_{4}$. Conventional cotton cultivation depicts the highest among the three practices for carbon dioxide emissions, followed by BCI and organic cultivation. The difference in results is mainly due to the contribution of agricultural inputs, i.e. fertilizer production, provision of pesticides, tractor operations and energy use for irrigation in the conventional system. For all three practices GWP from $\mathrm{N}_{2} \mathrm{O}$ emissions from field is the highest. This is mainly due to the various fertilizers used, water management system and soil use pattern. BCI and organic practices shows some advantages compared to conventional cotton because of reduced field air emissions of Nitrous oxide due to reduction of inorganic fertilizers use, savings of $\mathrm{CO}_{2}$ emissions due to production of less inorganic fertilizers and less $\mathrm{CO}_{2}$ emissions from tractor operation.

Eutrophication Potential (EP): Eutrophication Potential is calculated in terms of $\mathrm{kg} \mathrm{PO}_{4}$ eq./1 ton of cotton seed. Ammonia, nitrogen oxides, nitrous oxide are the major air emissions and nitrate, nitrogen and phosphate are major water emissions resulting in eutrophication potential. Ammonia emissions from field is the highest contributor for BCI and conventional cultivation. This is mainly due to the use of fertilisers in these practices. Nitrogen oxides are major contributors for Organic cultivation. When comparing with the eutrophication potential of all three pro-

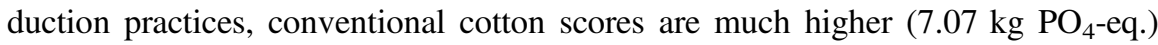


than BCI and Organic which scores $2.40 \mathrm{~kg} \mathrm{PO}$-eq. and $0.46 \mathrm{~kg} \mathrm{PO}_{4}$ eq. respectively. This can be explained by the fact that eutrophication in the BCI as well as conventional system is dominated by soil erosion, and soil erosion data refer to area and are not influenced by production yield. That means that the lower the yield per hectare, the higher the soil erosion per $\mathrm{kg}$ final product. BCI and organic practices shows some advantages compared to conventional cotton in this impact category because of reduced field air emissions of ammonia due to reduction of inorganic fertilizers application.

Primary Energy Demand (PED): PED represents an indicator for fossil resources. Conventional system shows highest PED followed by BCI and organic systems (Conventional-5375 MJ, BCI-2510 MJ and Organic-1351 MJ). BCI and organic practices shows some advantages compared to conventional cotton in this impact category because of savings of energy associated to the production of inorganic fertilizers used in a lower extent, reduced fuel requirement for tractor operation and low fuel requirement for irrigation process.

Water Consumption (WC): Water consumption is smaller for BCI Cotton ( $330 \mathrm{~m}^{3} / 1$ ton of seed cotton) and organic cotton $\left(391 \mathrm{~m}^{3} / 1\right.$ ton of seed cotton) than for the conventional Cotton $\left(541 \mathrm{~m}^{3} / 1\right.$ ton of seed cotton). BCI and organic practices shows some advantages compared to conventional cotton in this impact category because of less irrigation water requirement.

\section{Limitations}

At the level of primary inventory, considering average data over different years would improve their representativeness. Also, increasing the number of farm samples for all the cultivation systems would further enhance the quality and robustness of the inventory data. It is also important to note that for many relevant aspects (such as soil types, nutrient content of soils, soil erosion) primary data were very hard to obtain, there for some of the data proxy values were applied, not necessarily representative of local conditions. An important parameter is also the difference between manual and mechanised farming practices carried out by marginal farmers and rich farmers respectively. Then, Indian geography has different climatic conditions but the study focused only on the western part of the country. Aggregating data into regional averages is an additional challenge and can potentially lead to distortions in a model trying to represent a realistic cultivation system. Finally, some environmental impact routes (biodiversity, carbon sequestration in soils) are difficult to assess in a LCA framework and are not investigated in this study. Hence, some are omitted from the assessment where organic and BCI cotton Cultivation could potentially also show advantages over conventional production systems. 


\section{Conclusion}

To summarize, BCI cotton production system and organic system are extensive cultivation systems that are well adapted to available resources and geographical environment, as well as to existing social and socio-economic conditions. Organic cotton has shown the lowest impacts across all categories assessed, due to non-use of synthetic pesticides and chemical fertilizers, the only additives being manures, and the soil quality being controlled by crop rotation. BCI systems employ good cultivation practices like intercropping and reduce use of mineral fertilizers for cultivation. These systems intend to minimise the harmful impact of crop protection practices, use water efficiently, take care of soil health and conserve natural habitats while preserving the quality of the fibres. Farmers, traders, retailers and consumers, benefit from the economic, social and ecological advantages of responsible cotton projects.

\section{References}

1. < http://www.cotcorp.gov.in/index.aspx> (Accessed 30.04.2017).

2. <https://www.indiaagronet.com/indiaagronet/seeds/organiccottonarticle-seeds.htm> (Accessed 12.04.2017).

3. Cotton Made in Africa and PE International, Life Cycle Assessment of Cotton Made in Africa, Study report, 2014.

4. Textile Exchange and PE International, Life Cycle Assessment of Organic Cotton - A Global average, 2014.

5. <http://bettercotton.org/about-better-cotton/where-is-better-cotton-grown/india/> (Accessed 11.05.2017).

6. International Organization for Standardization, ISO Series 14040 and 14044, Environmental Management - Life Cycle Assessment - Principles and Framework, 2006.

7. Murugesh B.K, Selvadass M, Life Cycle Assessment for Cultivation of Conventional and Organic Seed Cotton fibres, International Journal of Research in Environmental Science and Technology 3(1): 39-45, 2013.

8. Khabbaz B. G, University of Southern Queensland Life Cycle Energy Use and Greenhouse Gas Emissions of Australian Cotton: Impact of Farming Systems, 2010.

9. Barnes E, The Cotton Life Cycle Inventory Project EDWARD BARNES, 2010.

10. Cotton Inc. and PE International, Life Cycle Assessment of Cotton Fibre and Fabric. A Project of the Cotton Foundation and managed by Cotton Incorporated, Cotton Council International and The National Cotton Council. 2012.

11. The Food and Environment Research Agency of UK, The role and business case for existing and emerging fibres in sustainable clothing, 2010. 
Open Access This chapter is licensed under the terms of the Creative Commons Attribution 4.0 International License (http://creativecommons.org/licenses/by/4.0/), which permits use, sharing, adaptation, distribution and reproduction in any medium or format, as long as you give appropriate credit to the original author(s) and the source, provide a link to the Creative Commons license and indicate if changes were made.

The images or other third party material in this chapter are included in the chapter's Creative Commons license, unless indicated otherwise in a credit line to the material. If material is not included in the chapter's Creative Commons license and your intended use is not permitted by statutory regulation or exceeds the permitted use, you will need to obtain permission directly from the copyright holder.

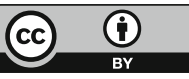




\title{
Life Cycle Management in the Pharmaceutical Industry Using an Applicable and Robust LCA-Based Environmental Sustainability Assessment Approach
}

\author{
Yasmine Emara, Marc-William Siegert, Annekatrin Lehmann \\ and Matthias Finkbeiner
}

\begin{abstract}
Despite growing concerns over the environmental impacts of pharmaceuticals, the use of Life Cycle Assessment (LCA) within the pharma-sector remains quite fragmentary. The aim of this paper is to present gaps and challenges, impeding a full adoption of LCA in the pharma-sector. A review of existing pharma-LCAs revealed a considerable degree of inconsistency and inhomogeneity in their methodological choices, highlighting the need for product category rules (PCRs) for the pharmaceutical industry to harmonize and facilitate the future use of LCA in that sector. Additionally, existing life cycle impact assessment (LCIA) methods fail to model several pharma-specific impact pathways (e.g. endocrine disruption). Preliminary thoughts on the development of pharma-PCRs and the inclusion of pharma-specific impact pathways into LCIA are presented, providing important stimulus for further research.
\end{abstract}

\section{Introduction}

A global rise in pharmaceutical consumption has come hand in hand with a parallel surge in environmental contamination with active pharmaceutical ingredients (APIs), their metabolites and transformation products [1, 2]. The eco-toxicological concerns regarding the presence of pharmaceuticals in surface waters, together with the pharmaceutical industry's resource- and energy-intensive environmental profile, have prompted the sector to increasingly integrate green chemistry and green engineering principles into its production processes [3-6].

Despite some drug companies recognizing the merits of utilizing Life Cycle Assessment (LCA) to measure their progress towards 'greener' production, LCAs

Y. Emara $(\bowtie) \cdot$ M.-W. Siegert · A. Lehmann · M. Finkbeiner

Chair of Sustainable Engineering, Institute of Environmental Technology,

Technische Universität Berlin, Berlin, Germany

e-mail: y.emara@tu-berlin.de

(C) The Author(s) 2018

E. Benetto et al. (eds.), Designing Sustainable Technologies,

Products and Policies, https://doi.org/10.1007/978-3-319-66981-6_9 
remain far from becoming common practice in the pharmaceutical industry [7, 8]. Furthermore, existing pharma-LCAs are quite inhomogeneous in multiple respects, e.g. the choice of functional unit or of impact categories. This, in turn, reduces the robustness of their results and their reproducibility and makes industry-wide insights with respect to typical environmental 'hotspots' or possible strategies for 'greener' drug designs practically futile. Naturally, the distinct goals of pharma-LCAs are partly responsible for the diversity of methodological choices among the studies. However, given the high degree of flexibility provided by the ISO 14040/14044 standards, it is fair to assume that even two studies of the same drug done by two different LCA-practitioners would show considerable disparity. It is exactly that realisation that has led over the last few years to the need - and consequently the parallel rise - of so-called 'product category rules' (PCRs), i.e. a set of harmonized rules on specific LCA modelling requirements for a given product category [9]. With the aim to develop PCRs for the pharma-sector, a three-year project, entitled 'Development of a sector-specific environmental sustainability assessment approach for pharmaceutical products and processes' (German abbreviation: SERUM), was launched last year at the Technische Universität Berlin.

The purpose of this paper is to present current gaps and challenges impeding a full adoption of LCA into the sustainability practices and product development processes of the pharma-sector and to provide preliminary recommendations on how to tackle some of those challenges. Following a short review of existing LCAs in the pharma-sector in Sect. 2, Sect. 3 will briefly describe the current state of Life Cycle Impact Assessment (LCIA) in pharma-LCAs and introduce some of the present constraints in modelling the toxicity of pharmaceutical compounds. Section 4 addresses early considerations on the way to developing PCRs for the pharmaceutical sector. Finally, a few concluding remarks and implications for future research within the LCA community will be presented.

\section{Review of LCAs in the Pharmaceutical Industry}

A comprehensive review on the state of LCA-application in the pharmaceutical industry, specifically human pharmaceuticals, was initially performed in order to identify common LCA-practices within the sector (e.g. choice of functional unit), regularly identified 'Hotspots' in the life cycle of drugs, as well as often encountered challenges and remaining gaps. Results of the review were meant to lay the ground for harmonized sector-specific rules-in the form of PCRs - and reveal thematic focal points for achieving greater LCA-application within the pharma-sector. Given the unique characteristics of the pharmaceutical industry (e.g. exceptionally high standards of cleanliness maintained during production) and the relatively young age of 'green pharmacy'-practices, the focus of the literature review was on LCA case studies of human pharmaceutical products (i.e. APIs or final drug, incl. packaging) or pharmaceutical processes, performed in or after the year 2000. LCAs of precursor chemicals (e.g. enzymes) were only included if 
downstream application in the pharmaceutical industry is clearly intended. The search thus excluded LCAs in the broader field of green chemistry and in the healthcare sector in general (e.g. medical equipment). Using search terms such as "Life Cycle Assessment", "LCA", and "footprint" in combination with "pharmaceutical*' or 'fine chemical*' on Google Scholar has so far yielded a notably limited number of 'pure' pharma-LCAs ( $<30$ studies) which have been published in peer-reviewed journals. These LCAs were conducted for a myriad of purposes, including comparative assessments of different synthesis routes, processing modes (e.g. batch vs. continuous processing), drug formulations, varying dosages and packaging options.

The LCA-studies examined thus far have with very few exceptions all performed a cradle-to-gate analysis, while often criticizing the lack of sufficient data beyond the production phase. Figure 1 shows a generic product system of a pharmaceutical product and different possibilities to set the system boundaries. A full life-cycle perspective is crucial in the context of pharmaceuticals, because firstly outsourcing certain synthesis or formulation steps (and with that the 'outsourcing' of impacts) is quite prevalent in the sector. Secondly, the environmental burden of upstream processes (e.g. production of input chemicals and 'background' energy production)

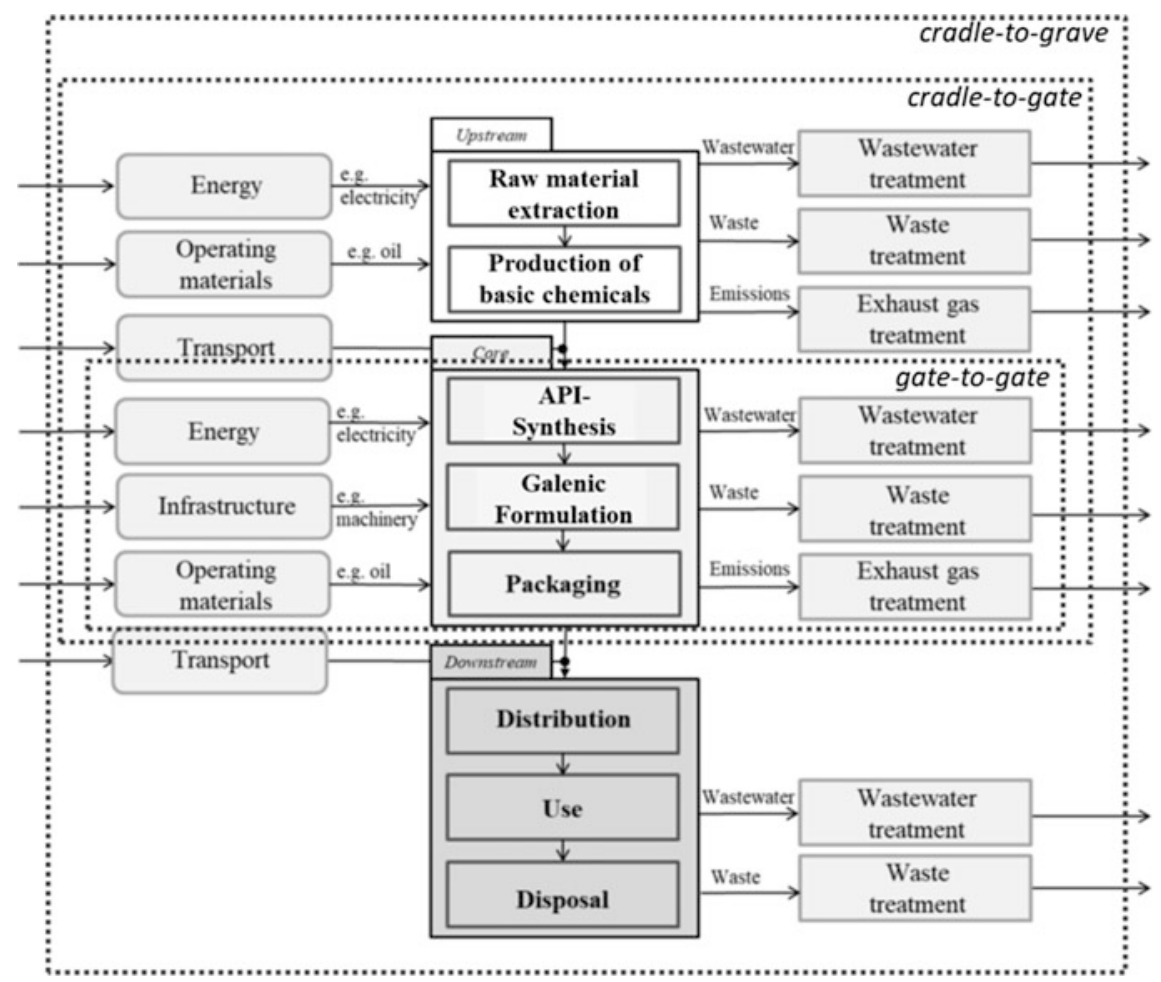

Fig. 1 Generic life cycle of a pharmaceutical product (excluding research and development) 
typically dwarfs impacts of the actual in-house synthetic processes [10, 11]. Furthermore, the use phase and end-of-life of a pharmaceutical product may as well bear considerable environmental impacts, especially if eco- and human-toxicological effects due to API-emissions into sewage systems (and eventually into surface waters) are to be included in the analysis (for further details see next section). Consequently, pharmaceutical corporations should strive towards complete cradle-to-grave analyses.

A closer examination of the existing pharma-LCAs quickly revealed that the individual studies are quite inhomogeneous in a number of respects: e.g. their choice of functional unit (FU), system boundaries setting, use of background databases and data quality, choice of impact assessment methods and the impact categories they consider. For instance, while Brunet et al. [12] set the FU at $20,840,000 \mathrm{~kg}$ of penicillin V produced over a time horizon of 20 years, De Soete et al. [13] chose 1 daily dosage of PREZISTA (anti-HIV medication) as their FU. The majority of reviewed studies though opted for $1 \mathrm{~kg}$ of API as FU. Similarly, while Wernet et al. [10] chose to assess sixteen impact categories (both at midpointand endpoint level) using 5 different impact assessment methods, Kim et al. [14] considered 5 impact categories using one method only.

In light of the different goals and scopes of the reviewed studies and the inherent uniqueness of individual APIs/drug formulations, a certain degree of methodological variation among pharma-LCAs is only logical, if not expedient. Nevertheless, lack of sufficient experience and guidance has led to large discrepancies in the application of LCA in the pharma-sector, often jeopardizing the coherence and reliability of pharma-LCAs. Consequently, there is an evident need for PCRs for the pharmaceutical industry to guide and facilitate future pharma-LCAs. Preliminary thoughts on pharma-PCRs are discussed in Sect. 4.

\section{Life Cycle Impact Assessment (LCIA) in Pharma-LCAs}

The LCIA phase of the reviewed pharma-LCAs was carried out using quite divergent impact categories and impact assessment methods. A streamlined LCA tool developed by the American Chemical Society Green Chemistry Institute (ACS-GCI) Pharmaceutical Roundtable (hereinafter 'the Roundtable') sets forth nine impact categories/indicators to be assessed in LCAs of drug synthesis routes [11]. Table 1 lists these nine impact categories, next to the top five assessed impact categories in the reviewed pharma-LCAs, as well as a preliminary selection of eight categories recognized by the authors as the most relevant for pharma-LCAs. The latter list was determined in consultation with the SERUM advisory committee, which comprises experts from academia, politics and the pharmaceutical industry.

Quite notably, impacts - especially toxicity-related impacts — which have been identified as relevant for the pharmaceutical industry within the SERUM project are not often considered in LCA studies nor recommended in the Roundtable's streamlined tool. Given the desired functionality of pharmaceuticals - e.g. to kill 
Table 1 Recommended impact categories for pharma-LCA

\begin{tabular}{|c|c|c|c|}
\hline Source & Streamlined LCA-tool [11] & $\begin{array}{l}\text { Top five in } \\
\text { pharma-LCAs }\end{array}$ & $\begin{array}{l}\text { Preliminary selection } \\
\text { SERUM-project }\end{array}$ \\
\hline \multirow{9}{*}{$\begin{array}{l}\text { Impact } \\
\text { categories }\end{array}$} & Climate change & Climate change & Climate change \\
\hline & Acidification & Acidification & $\begin{array}{l}\text { Human toxicity, cancer } \\
\text { effects }\end{array}$ \\
\hline & Eutrophication & Eutrophication & $\begin{array}{l}\text { Human toxicity, non-cancer } \\
\text { effects }\end{array}$ \\
\hline & $\begin{array}{l}\text { Net life cycle mass of materials } \\
\text { used }\end{array}$ & $\begin{array}{l}\text { Ozone } \\
\text { depletion }\end{array}$ & Eutrophication, aquatic \\
\hline & $\begin{array}{l}\text { Life cycle water usage, } \\
\text { exclusive of process water }\end{array}$ & $\begin{array}{l}\text { Cumulative } \\
\text { energy demand }\end{array}$ & Ecotoxicity, freshwater \\
\hline & Cumulative energy demand & - & $\begin{array}{l}\text { Ecotoxicity marine and } \\
\text { terrestrial }\end{array}$ \\
\hline & $\begin{array}{l}\text { Oil and natural gas depletion for } \\
\text { materials manufacture }\end{array}$ & - & $\begin{array}{l}\text { Resource depletion (fossil, } \\
\text { mineral and renewables) }\end{array}$ \\
\hline & Photochemical ozone creation & - & Resource depletion, water \\
\hline & $\begin{array}{l}\text { Total organic carbon load before } \\
\text { waste treatment }\end{array}$ & - & - \\
\hline
\end{tabular}

rapidly dividing cells (anticancer), affect the action of neurotransmitter chemicals in the brain (antipsychotic), kill or inhibit microorganisms (antimicrobial) - and the growing body of literature providing pertinent evidence of potential unanticipated eco-toxicological effects of APIs (reviewed in [3, 15-17]), it is concerning that none of the existing pharma-LCAs considered impacts related to the presence of pharmaceutical residues in the environment.

The discrepancy between practice, recommendation and (perceived) relevance of the categories 'human toxicity' and 'eco-toxicity' for pharma-LCAs is largely the result of a number of methodological constraints on toxicity modelling within LCIA, the most prominent of which are:

(1) Lack of characterization factors (CFs) for pharmaceutical compounds in existing toxicity models

(2) Several impacts or impact pathways associated with pharmaceuticals and their toxic mode of action are neglected in current impact assessment methods.

In an attempt to address the first constraint and enhance the assessment of pharmaceuticals' toxicity in LCIA, several studies have recently updated or calculated new CFs for APIs in the categories human toxicity, freshwater, marine or terrestrial eco-toxicity using mostly USEtox, but also EDIP97 and/or USES-LCA 2.0 [18-20]. Despite the mentioned efforts, the total number of covered pharmaceuticals within common toxicity models remains considerably low (at the current state of the authors' knowledge below 100 compounds).

The second methodological constraint relates to a wide variety of missing, (pharma-specific) effects such as: 
- endocrine disruption mediated by exposure to endocrine disrupting chemicals (EDCs) (reviewed in [21]),

- the development of antibiotic resistance [22] or

- behavioural alterations such as change in feeding behaviour or predator avoidance due to wildlife-exposure to antidepressants (reviewed in [23]).

Such examples of more subtle and sub-lethal toxic effects with possibly significant repercussions on species' ecological fitness and population-relevant endpoints are often not captured by endpoints that are typically assessed in regulatory ecotoxicology. Consequently, using toxicity data on traditional endpoints (e.g. mortality, growth and reproduction) as recommended by USEtox to calculate CFs for pharmaceutical compounds fails to reflect certain impact pathways of individual pharmaceuticals with specific toxic modes of action such as EDCs or antibiotics [24].

In an attempt to tackle the second methodological constraint, Larsen et al. [24] sought to integrate endocrine disruption into LCA by suggesting to use test results from fish laboratory tests with the endpoint sex ratio as effect data when estimating eco-toxicity characterization factors for estrogenic compounds [24]. Considering the complexity of the endocrine system - whether in humans or in wildlife - and the variety of EDCs' mechanisms of action (e.g. (anti)estrogenic/(anti)androgenic or interference with thyroid hormone pathways) more endpoints will have to be eventually included to capture and map the 'full' array of EDCs and their potential toxic effects.

When seeking to include other missing pharma-specific impact pathways such as the development of antibiotic resistance, it is less likely that the same approach, i.e. to broaden the endpoints used to calculate effect factors, would suffice to reflect the specific mechanisms in which bacteria develop or transfer antibiotic resistant genes. Consequently, a new indicator and characterization model for the inclusion of antibiotic resistance into LCIA may need to be developed.

\section{Product Category Rules (PCRs) for the Pharmaceutical Industry}

PCRs are usually defined for a group of products which have an equivalent or similar function, making them largely comparable. Despite the compelling case for a harmonized framework in the form of PCRs to guide LCAs of pharmaceutical products, so far there has only been one PCR developed for vaccines [25]. On the basis of this PCR, Pfizer conducted and published an Environmental Product Declaration (EPD) for IMPROVAC, an immunological product used as alternative to physical castration in pig management [26]. However the EPD for IMPROVAC is no longer valid since 2015 .

To develop new PCRs, there are no rules on how 'narrow' or 'broad' a product category should be defined. In other words, the 'granularity' of PCRs is entirely up 
to their developers. Based on feedback obtained in consultation with pharma-experts, developing PCRs at two different levels/granularities would prove practical for the pharmaceutical industry (see Fig. 2). First, a generic PCR for the pharmaceutical sector ('horizontal rules') should be developed, determining broad LCA-modelling provisions which capture some of the industry-wide characteristics (e.g. the importance of including treatment processes of solvent waste within the system boundaries). Such a generic "frame-PCR" is subject to a considerable degree of uncertainty/inaccuracy, as it intends to provide common modelling rules for quite distinct products of a given sector, while having to rely on numerous assumptions and a significantly simplified representation of the industry as a whole. Therefore, in a second step and in close alignment with the frame-PCR, specific PCRs ('vertical rules') should be cumulatively developed for:

(1) pharmaceutical products categorized into different drug classes according to the international Anatomical Therapeutic Chemical (ATC) Classification System (product-PCRs) and

(2) drug manufacturing processes (process-PCRs).

The product-PCRs are to be developed for the different drug classes available at the third level of the ATC-code, i.e. the different therapeutic/pharmacological subgroups of APIs, and would serve the objective of assessing drug alternatives according to the same harmonized scheme on the basis of their common functionality (therapeutic purpose) and pharmacological properties. Process-PCRs would guide LCAs which are primarily focused on process optimization. The existence of a generic 'frame-PCR' encircling product- and process-PCRs will prove particularly valuable at the early stages when PCRs have not yet been developed for all therapeutic/pharmacological subgroups of APIs.

A harmonized LCA reference framework for the pharmaceutical industry will inevitably guarantee better (qualitative) comparability of the results (especially

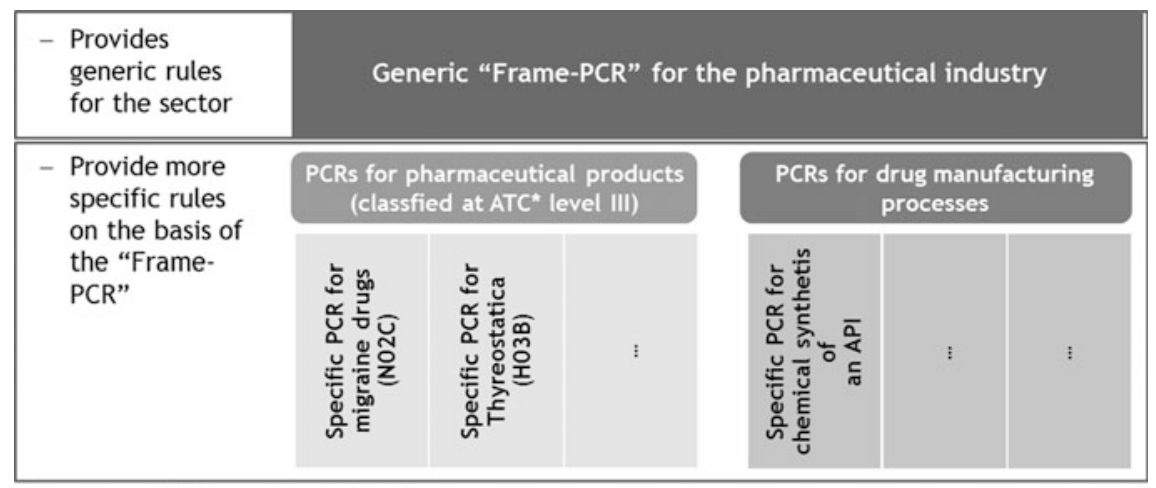

"ATC: Anatomical Therapeutic Chemical classification system

Fig. 2 Proposal for categorization ('granularity') of product category rules for pharmaceutical products and processes 
within one therapeutic/pharmacological subgroup using the same product-PCR) and improve the reliability of future pharma-LCAs. Additionally, it paves the way for EPDs to establish themselves within the sector, with potentially significant social implications (e.g. doctors/consumers opting for 'greener' drug alternatives).

\section{Conclusions and Outlook}

Despite recent efforts of the pharmaceutical industry to integrate green chemistry and green engineering principles into their production processes and drug designs, the utilization of LCA to monitor and measure progress towards 'greener' pharmaceutical products remains far from common practice. A review of available pharma-LCAs revealed a considerable degree of inconsistency and inhomogeneity in their modelling choices, often leading to quite unreliable results. The problem is compounded by the fact that existing life cycle impact assessment methods fail to include a variety of pharma-specific impact pathways within their toxicity modelling (e.g. endocrine disruption or antibiotic resistance) and provide a noticeably limited number of characterization factors for pharmaceutical compounds.

The development of product category rules for pharmaceutical products and processes is regarded as a necessary development to harmonize, facilitate and expand the future use of LCA in the sector. Additionally, calculating new CFs for pharmaceutical compounds within established toxicity models or developing new characterization models that reflect pharma-specific toxicological effects is imperative to delivering a comprehensive and accurate quantification of the environmental impacts of human drugs.

Only when an applicable and robust LCA-based environmental sustainability assessment approach is adapted to the needs and specificities of the pharmaceutical industry can life cycle management establish itself within the sector and truly guide eco-innovation towards 'green pharmacy'.

\section{References}

1. Taylor D, The Pharmaceutical Industry and the Future of Drug Development, In Pharmaceuticals in the Environment, Hester, R. E., Harrison, R. M., Eds., Issues in Environmental Science and Technology No. 41, The Royal Society of Chemistry, 2015, pp 1-33.

2. Christen V, Hickmann S, Rechenberg B, Fent K, Highly active human pharmaceuticals in aquatic systems: A concept for their identification based on their mode of action, Aquatic toxicology, Vol. 96, No. 3, 2010, pp 167-181.

3. Arnold, K. E, Brown A. R, Ankley G. T, Sumpter J. P, Medicating the environment: assessing risks of pharmaceuticals to wildlife and ecosystems, Phil. Trans. R. Soc. B, Vol. 369, 2014.

4. Caldwell D. J, Sources of Pharmaceutical Residues in the Environment and their Control, In Pharmaceuticals in the Environment, Hester, R. E., Harrison, R. M., Eds., Issues in Environmental Science and Technology No. 41, The Royal Society of Chemistry, 2015, pp 92-119. 
5. Voulvoulis N, Barceló D, Verlicchi P, Pharmaceutical Residues in Sewage Treatment Works and their Fate in the Receiving Environment, In Pharmaceuticals in the Environment, Hester, R. E., Harrison, R. M., Eds., Issues in Environmental Science and Technology No. 41, The Royal Society of Chemistry, 2015, pp 120-179.

6. Jiménez-González C, Constable D. C, Ponder C. S, Evaluating the "greenness" of chemical processes and products in the pharmaceutical industry-a green metrics primer, Chemical Society reviews, Vol. 41, No. 4, 2012, pp 1485-1498.

7. Jiménez-González C, Overcash M. R, The evolution of life cycle assessment in pharmaceutical and chemical applications - a perspective, Green Chem., Vol. 16, No. 7, 2014, p 3392.

8. Mata T. M, Martins A. A, Neto B, Martins M. L, Salcedo, R, Costa C, LCA Tool for Sustainability Evaluations in the Pharmaceutical Industry, Chemical Engineering Transactions, Vol. 26, 2012.

9. Minkov N, Schneider L, Lehmann A, Finkbeiner M, Type III Environmental Declaration Programmes and harmonization of product category rules: Status quo and practical challenges, Journal of Cleaner Production, Vol. 94, 2015, pp 235-246.

10. Wernet G, Conradt S, Isenring H. P, Jiménez-González C, Hungerbühler K, Life cycle assessment of fine chemical production: A case study of pharmaceutical synthesis, Int J Life Cycle Assess, Vol. 15, No. 3, 2010, pp 294-303.

11. Jiménez-González C, Ollech C, Pyrz W, Hughes D, Broxterman Q. B, Bhathela N., Expanding the Boundaries: Developing a Streamlined Tool for Eco-Footprinting of Pharmaceuticals, Org. Process Res. Dev., Vol. 17, No. 2, 2013, pp 239-246.

12. Brunet R, Guillén-Gosálbez G, Jiménez L, Combined simulation-optimization methodology to reduce the environmental impact of pharmaceutical processes: Application to the production of Penicillin V, Journal of Cleaner Production, Vol. 76, 2014, pp 55-63.

13. De Soete W, Boone L, Willemse F, Meyer E, Heirman B, van Langenhove H, Dewulf J, Environmental resource footprinting of drug manufacturing: Effects of scale-up and tablet dosage, Resources, Conservation and Recycling, Vol. 91, 2014, pp 82-88.

14. Kim S, Jiménez-González C, Dale B. E, Enzymes for pharmaceutical applications-a cradle-to-gate life cycle assessment, Int J Life Cycle Assess, Vol. 14, No. 5, 2009, pp 392 400 .

15. Taggart M. A, Richards N, Kinney C. A, Impacts of Pharmaceuticals on Terrestrial Wildlife, In Pharmaceuticals in the Environment, Hester, R. E., Harrison, R. M., Eds., Issues in Environmental Science and Technology No. 41, The Royal Society of Chemistry, 2015, pp 216-254.

16. Crane M, Watts $\mathrm{C}$, Boucard T, Chronic aquatic environmental risks from exposure to human pharmaceuticals, Science of the Total Environment, Vol. 367, 2006, pp 23-41.

17. Santos L. H. M. L. M, Araujo A. N, Fachini A, Pena A, Delerue-Matos C, Montenegro M. C. B. S. M., Ecotoxicological aspects related to the presence of pharmaceuticals in the aquatic environment, Journal of hazardous materials, Vol. 175, 1-3, 2010, pp 45-95.

18. Alfonsín C, Hospido A, Omil F, Moreira M. T, Feijoo G, PPCPs in wastewater - Update and calculation of characterization factors for their inclusion in LCA studies, Journal of Cleaner Production, Vol. 83, 2014, pp 245-255.

19. Ortiz de García S, García-Encina P. A, Irusta-Mata R, The potential ecotoxicological impact of pharmaceutical and personal care products on humans and freshwater, based on USEtox ${ }^{\mathrm{TM}}$ characterization factors. A Spanish case study of toxicity impact scores, The Science of the total environment, Vol. 609, 2017, pp 429-445.

20. Muñoz I, José Gómez M, Molina-Díaz A, Huijbregts M. A, Fernández-Alba A. R, García-Calvo E, Ranking potential impacts of priority and emerging pollutants in urban wastewater through life cycle impact assessment, Chemosphere, Vol. 74, No. 1, 2008, pp 37-44.

21. Bergman Å, Heindel J. J, Jobling S, Kidd K. A, Zoeller R. T, Eds., State of the Science of Endocrine Disrupting Chemicals - 2012: An assessment of the state of the science of endocrine disruptors, WHO; UNEP, 2013. 
22. Kümmerer K, Antibiotics in the aquatic environment-a review-part II, Chemosphere, Vol. 75, No. 4, 2009, pp 435-441.

23. Brodin T, Piovano S, Fick J, Klaminder J, Heynen M, Jonsson M, Ecological effects of pharmaceuticals in aquatic systems-impacts through behavioural alterations, Phil. Trans. R. Soc. B, Vol. 369, No. 1656, 2014.

24. Larsen H. F, Olsen S. I, Hauschild M. Z, Laurent A, New sustainable concepts and processes for optimization and upgrading municipal wastewater and sludge treatment: Deliverable 4.2. Methodology for including specific biological effects and pathogen aspects into LCA, NEPTUNE Contract-No. 036845 (FP6 project), 2009.

25. International EPD System, Vaccines for human or veterinary medicine, whether or not put up as medicaments: Product group classification: UN CPC 35270, prepared by Life Cycle Engineering, 2011.

26. Pfizer, Environmental Product Declaration for IMPROVAC: EPD No.: S-P-00261, BUREAU VERITAS Certification No. SE00250-1, 2012 (accessed May 5, 2017).

Open Access This chapter is licensed under the terms of the Creative Commons Attribution 4.0 International License (http://creativecommons.org/licenses/by/4.0/), which permits use, sharing, adaptation, distribution and reproduction in any medium or format, as long as you give appropriate credit to the original author(s) and the source, provide a link to the Creative Commons license and indicate if changes were made.

The images or other third party material in this chapter are included in the chapter's Creative Commons license, unless indicated otherwise in a credit line to the material. If material is not included in the chapter's Creative Commons license and your intended use is not permitted by statutory regulation or exceeds the permitted use, you will need to obtain permission directly from the copyright holder. 


\title{
Establishing LCA in the Healthcare Sector
}

\author{
Nanja Hedal Kløverpris
}

\begin{abstract}
Novo Nordisk has used Life Cycle Assessment (LCA) for many years and a few years ago the company took a major step forward and completed a mapping of the product carbon footprint of the company's key products. Through successful cross-organisational collaboration, technical LCA data and results have been translated into easy-to-understand messages that have helped the organisation to understand the LCA concept, drive improvements across the life cycle and to communicate about the environmental impact of products to external stakeholders such as patients, healthcare professionals, payers and policy-makers.
\end{abstract}

\section{Introduction}

Novo Nordisk is a global healthcare company with more than 90 years of innovation and leadership in diabetes care. Headquartered in Denmark, Novo Nordisk employs approximately 42,000 people in 77 countries and markets its products in more than 165 countries.

A key element in Novo Nordisk business philosophy is the triple bottom line principle, which is about balancing financial, social and environmental considerations. This is anchored in the Novo Nordisk Articles of Association. The company has a long history of optimising the environmental performance through ambitious strategies and partnerships. One example is the Kalundborg Symbiosis, where Novo Nordisk was one of the founders of an industrial symbiosis partnership. Another example is the 10-year long partnership between Novo Nordisk and DONG Energy, which has resulted in major energy savings and ensured that all Novo Nordisk production sites in Denmark are powered by renewable energy from an offshore wind farm.

Novo Nordisk has taken the next step in the journey towards environmental responsibility and intensified the focus on the environmental impact at the product

\footnotetext{
N. H. Kløverpris $(\bowtie)$

Novo Nordisk A/S, Bagsvaerd, Denmark

e-mail: nnhk@novonordisk.com

(C) The Author(s) 2018

E. Benetto et al. (eds.), Designing Sustainable Technologies,

Products and Policies, https://doi.org/10.1007/978-3-319-66981-6_10
} 
level. This allows Novo Nordisk to make choices in R\&D and production that minimise carbon emissions from products and at the same time, support the commercial part of the organisation with environmental messages. This article describes how Novo Nordisk has taken the lead in reducing carbon emissions at the product level.

\section{Building a Common Framework for Product Carbon Footprints in the Healthcare Sector}

For more than 25 years, Novo Nordisk has used LCA as the basic methodology to measure environmental impacts across the product life cycle. These LCAs follow the international LCA standards ISO 14040 and 14044.

In 2008, the UK NHS identified pharmaceuticals as one of the key contributors to the carbon footprint of the UK healthcare system [1]. This sparked the need for more detailed guidelines on how to calculate the carbon footprint of pharmaceutical products. Together with the NHS, Novo Nordisk and a handful of other healthcare companies founded the international Coalition of Sustainable Pharmaceuticals and Medical Devices (CSPM). The coalition initiated the development of a sector-specific guideline on product carbon footprint. In 2012, the Greenhouse Gas Accounting Sector Guidance for Pharmaceutical Products and Medical Devices [2] was published. It was reviewed by WRI for conformance with the GHG Protocol Product Life Cycle Standard and earned the "Built on GHG Protocol" mark.

Novo Nordisk was the first diabetes company to apply the guideline for product carbon footprint of pharmaceuticals and medical devices. The first two carbon footprint reports were carried out by an external consultant and finalised in 2013. To ensure compliance with the new guideline, a third party review was performed by the consultancy company ERM, who had prepared the sector guideline for the CSPM. Since 2013, Novo Nordisk has had internal LCA expertise and has continued to use the guideline.

The sector-specific guideline and the ISO 14040/44 LCA standards ensure a scientifically solid approach to calculating product carbon footprints. The sector-specific guideline gives clear guidance on scoping, data collection and reporting specifically focusing on the production processes and products in the healthcare sector. All product carbon footprints within the healthcare industry should comply with this guideline as it is considered best practice.

All Novo Nordisk product carbon footprint reports are third party validated according to the international guidelines by an internationally recognised accountancy company. The pharmaceutical industry has a large responsibility of ensuring patient safety, and the industry is strictly regulated to protect the patients. Therefore, third party validation of product carbon footprint ought to be standard in the healthcare industry. 


\section{Implementing LCA in Novo Nordisk}

Novo Nordisk has used LCA as a tool for decision support for more than 25 years. During these years, the LCA methodology and data availability has improved significantly. In 2011, LCA became an integrated part of Novo Nordisk environmental strategy, and a programme was launched with the aim of mapping the carbon footprint of the diabetes portfolio. Novo Nordisk was the first to calculate the carbon footprint of one year diabetes treatment, including the active pharmaceutical ingredient (API), device and needle.

The programme was anchored in an executive management committee and was driven by a cross-organisational team with representatives from production, $R \& D$, marketing and stakeholder engagement. The top management commitment and collaboration across organisational boundaries were essential to the success of the programme.

Achieving a sufficiently high quality of data is critical when LCA is used for supporting decisions. Novo Nordisk has a long history of environmental reporting, which focuses on resource consumption, emissions and waste amounts. Environmental data has for many years been monitored and reported on building/ site/company level and related to KPIs. However, it is a challenge to convert these datasets to the product level, and it requires close collaboration with local production experts. Novo Nordisk has proven that it is worth the effort: It provides the organisation with a completely new perspective on environmental impacts, which in the longer term allows for new approaches to setting targets and benchmarking across production units, e.g. by measuring energy consumption per unit produced instead of energy consumption per building.

LCA screenings have for several years been an integrated part of the device development process in Novo Nordisk. One of the keys to ensure that the screenings are carried out is the integration into the development manual, which means that the LCA screenings are obligatory. The LCA screenings are based on the same LCA model as is used for the marketed products, which ensures consistency across the organisation.

Life cycle thinking was introduced in the ISO14001 standard on environmental management systems in 2015. Product carbon footprint is a great method to help determine the environmental hotspots and focus environmental initiatives within production to create the most impact. Moreover, Novo Nordisk uses LCA and carbon footprint calculations as decision-support tool across the organisation, both in relation to initiatives and projects driven by the environmental organisation and in relation to projects and changes with a significant environmental impact.

Novo Nordisk has built an advanced LCA model for APIs, devices and needles and it creates a strong fundament for updating the carbon footprint of products and adding new scenarios. Since the LCA model covers the majority of Novo Nordisk products and relates to most production sites, new LCA calculations are made very effectively. This allows for providing quick answers to the organisation to support decision processes. 


\section{Communicating About LCA and Product Carbon Footprint in the Healthcare Sector}

Novo Nordisk experiences an increasing interest in the environmental impact of healthcare products from authorities, insurance companies, doctors and patients. Novo Nordisk believes that stakeholders have the right to know about the environmental impact of the products. This was one of the reasons why Novo Nordisk decided to map the carbon footprints across the diabetes portfolio.

The cross-organisational collaboration has been crucial in ensuring that the extensive work on the mapping of product carbon footprints is also translated into stories and messages that are understandable for non-experts.

Many LCA practitioners experience that it is difficult to communicate the relatively technical results of an LCA including the underlying assumptions. The details of LCA conflict with the need for clear and short statements that can be disseminated to a wide audience with no previous knowledge on product carbon footprint. Translating the technical results into easy-to-understand messages requires a close collaboration between environmental experts and communicators and that both parties have the resources and willingness to listen and learn from each other. This is a challenge that is common in many companies, and Novo Nordisk is actively seeking sparring with other companies on this and other issues related to the use of LCA in large companies. Novo Nordisk was one of four initiators of a Danish LCA and ecodesign network consisting of representatives from major companies and academia in Denmark working within the field of LCA. The purpose of the network is to share knowledge and learn from each other with focus on implementation of LCA in companies.

In Novo Nordisk, it became clear that communicating the specific carbon footprint in $\mathrm{kg} \mathrm{CO}_{2}$ equivalent did not hold value for the target audiences as they found it difficult to relate to this number. Therefore, we focus on comparisons with other consumables. An example of a message that has resonated well with the target groups is that daily diabetes treatment has a carbon footprint equivalent to a cup of tea [3]. We also use comparisons like driving a car and air travel to put product carbon footprints into perspective. It helps both internal and external stakeholders to understand, remember and communicate the size of the footprint.

A comparison with competing products is not relevant, as this requires that the carbon footprints are calculated using the same system boundaries and assumptions and that we would have access to competitor production data. As a patent-driven pharmaceutical company, most of our products are unique and therefore product-to-product comparisons e.g. per unit of insulin are not relevant.

Communication to doctors and other healthcare professionals is in many countries strictly regulated and marketing claims related to specific products must be based on peer reviewed publications that documents the clinical efficiency of a drug on the basis of clinical trials. There are no guidelines on the use of 
environmental messages in the marketing of pharmaceutical products. The Guidelines for Providing Product Sustainability Information being developed by UNEP and International Trade Centre points at third party validation as the most reliable assurance model for sustainability claims [4]. Klöppfer [5] points to the fact that an LCA study can only be claimed to be in accordance with the ISO 14040 and 14044 standards if a third party review has been performed, whereas an LCA published in a peer reviewed scientific journal cannot be claimed to be ISO compliant based on the peer review process alone as this does not follow the requirements for the review process set out in the ISO standards. A third party reviewer will often have access to more background data, LCA models etc. and have more time assigned for a review than peer reviewers appointed by a scientific journal. Therefore, Novo Nordisk supports the use of third party validation as the best way to ensure validity of the LCA results.

\section{The Future of LCA in Healthcare}

Novo Nordisk has demonstrated that product carbon footprints provide a basis for communicating with internal and external audiences about the environmental impact of pharmaceutical products. There is a great potential for driving further improvements across the product life cycle of Novo Nordisk current and future products and LCA will play an increasingly important role in ensuring that the environmental perspective is present in decision making across the organisation.

In the healthcare sector, LCA can bring valuable knowledge about the environmental impact of treating patients. However, it is important that pharmaceuticals are considered in a full care pathway perspective - or what Novo Nordisk calls the 'patient carbon footprint'. The impact from treating patients goes beyond pharmaceuticals and medical devices. Diabetes can lead to complications such as eye problems, kidney disease, amputations etc. that are also associated with carbon emissions from hospitalisation, surgeries, frequent visits to clinics etc. This means that more efficient treatment leads to a reduced carbon emissions related to complications. Methods for quantifying the environmental impact of the patient carbon footprint are currently being developed [6].

\section{References}

1. Stockholm Environment Institute (SEI), NHS Sustainable Development Unit, NHS England Carbon Emissions: Carbon Footprinting Report, 2008.

2. Penny T, Fisher K, Collins M, Allison C, Greenhouse Gas Accounting Sector Guidance for Pharmaceutical Products and Medical Devices, 2012. 
3. Kløverpris N.H, Jensen N.D, Product Carbon Footprint, FlexPen® incl. API and needle, 2017.

4. UNEP, ITC, Guidelines for Providing Product Sustainability Information, Draft for Global Stakeholder Consultation, 2016.

5. Klöppfer W, The critical review of life cycle assessment studies according to ISO 14040 and 14044, Int J Life Cycle Assess, Vol. 17, No. 9, 2002, pp. 1087-1093.

6. Penny T, Collins M, Whiting A, Aumônier S, Care Pathways: Guidance on Appraising Sustainability—Main Document, 2015.

Open Access This chapter is licensed under the terms of the Creative Commons Attribution 4.0 International License (http://creativecommons.org/licenses/by/4.0/), which permits use, sharing, adaptation, distribution and reproduction in any medium or format, as long as you give appropriate credit to the original author(s) and the source, provide a link to the Creative Commons license and indicate if changes were made.

The images or other third party material in this chapter are included in the chapter's Creative Commons license, unless indicated otherwise in a credit line to the material. If material is not included in the chapter's Creative Commons license and your intended use is not permitted by statutory regulation or exceeds the permitted use, you will need to obtain permission directly from the copyright holder. 


\title{
Improving the Life Cycle Performance of Chemical Products and Materials Through Data Exchange Along the Value Chain-Synthesis of LCM2017 Session Presentations
}

\author{
Guido Sonnemann and Carmen Alvarado
}

\begin{abstract}
This paper provides a summary of what has been presented and discussed at the 8th international conference on Life Cycle Management (LCM 2017 conference, Luxembourg), during the session entitled "Improving the life cycle performance of chemical products and materials through data exchange along the value chain". The purpose of the session was to demonstrate how to assess and manage the global sustainability of chemical products and materials, taking into account their whole life cycle, to achieve real improvements. Over the six oral presentations, the most challenging issues regarding availability and transparency of Life Cycle Inventory data from the chemicals industry were discussed. Some approaches developed in order to face these challenges were detailed, illustrated also with the presentation of some specific case-studies.
\end{abstract}

\section{Introduction}

Most companies are part of long and sometimes complex supply chains. Often the control over the environmental impacts along the value chain is limited for one single company. The purpose of the session was to demonstrate how to assess and manage the global sustainability of chemical products and materials, taking into account their whole life cycle, to achieve real improvements. This includes the identification of hot spots and work towards sustainable innovation. Radical improvement along the value chain is only possible with close collaboration with

\author{
G. Sonnemann $(\square)$ \\ University of Bordeaux, CNRS, UMR 5255, 33405 Talence Cedex, France \\ e-mail: guido.sonnemann@u-bordeaux.fr \\ C. Alvarado \\ Akzo Nobel Chemicals, 3818 LE Amersfoort, The Netherlands \\ G. Sonnemann \\ CNRS, ISM, UMR 5255, 33405 Talence Cedex, France \\ (C) The Author(s) 2018 \\ E. Benetto et al. (eds.), Designing Sustainable Technologies, \\ Products and Policies, https://doi.org/10.1007/978-3-319-66981-6_11
}


suppliers, for example, through sharing supplier specific data on environmental impacts of raw materials. There are many obstacles which need to be overcome before sharing data along the value chain: trust, data formats, methodology harmonization, etc. The PEF (Product Environmental Footprint), together with Sustainability and the WBCSD life cycle metrics for chemical products are good examples of platforms that can help companies share information in a safe, fair and efficient way to steer to real improvements towards sustainable development. Six presentations were selected for the session. The main outcomes of each of them are summarised in this paper.

\section{Rapid Estimation of Life Cycle Inventories}

Many regulations and company sustainability activities are based on the application of Life Cycle Assessment. A significant challenge is the amount of data that needs to be compiled, harmonized and modelled before obtaining meaningful and robust results.

In this context, the United States Environmental Protection Agency (U.S. EPA) developed an approach to rapidly generate more comprehensive and reliable life cycle inventories (LCI) than those currently available. The basic approach to generating such inventories is to combine four individual efforts:

(1) top-down data mining,

(2) bottom-up simulation (combining material and energy balances with emissions modelling),

(3) LCI chemical lineage (computationally tracing the ancestry of a chemical alternative), and

(4) LCI reconciliation of both the top-down and bottom-up approaches.

Life Cycle Inventories developed through this approach can be updated once new datasets are available [1].

\section{Current Models and Approaches for Data Matching and Modelling of Chemical Production}

Ecoinvent is a data provider for the Product Environmental Footprint (PEF) pilot phase, driven by the European Commission. In this context many of the data tenders are based on ecoinvent LCI data in the background and ecoinvent was selected as the direct data supplier for 270 products and over 800 datasets, on the production of chemicals. The challenge was to deal with significant data needs on chemical products on short notice (3 months), while delivering quality and integrating primary data. 
Around 80 chemicals needed completely new datasets. Around 200 new chemicals unit process datasets were created. The industry stakeholders group discussion involved in the PEF pilot phase was organised jointly with the creation of these datasets. The short timeframe made the collaboration difficult, but several sources provided data. A framework model that provides a unit process result, was created in a way that each part of the process is adaptable. Thanks to the "modular model" approach chosen, the datasets can be upgraded on specific topics when data becomes available. A stoichiometric model of the reactions was required, with reactants and other reagents. Average values for yield, heat electricity, water and supporting processes were available from industry reports. The air emission model was based on vapour pressure, filtering systems and toxicity. The waste water treatment model used determines the water emissions. Previous models existed but all aspects of the new model have been updated and extended for this project.

The project was an interesting combination of data format and data requirement issues and high efficiency data generation. The flexible model of chemical unit processes allows users to work with various data availabilities. The model is accessible, with all values and sources. The datasets list source types and acknowledge industry sources in coordination with the data providers. The data are now used in the PEF pilot projects, and many will also be published in the upcoming ecoinvent 3.4 [2].

\section{PlasticsEurope Experience in Managing Information Along the Value Chain for RA and LCA}

Risk assessments are carried out by PlasticsEurope members for each substance. It takes the form of lists of substances with maximum limit of concentration or migration. The full chemical composition of the plastics must be known, including traces of starting substances, additives, impurities at very low thresholds. The information must be transmitted in the supply chain so that the one putting the packed product on the market has the data on the plastics available. When there is no sectorial organisation, each actor interprets differently the requirements of information and sends questionnaires upstream. They are all different and tend to ask more than required. Suppliers hesitates to send information downstream since they are afraid of misinterpretation and misuse due to the fear of being challenged (substance ban, deselection...). There is a need for a common playing field in the supply chain with a common interpretation of regulation, mutual understanding, respect of confidentiality and trust.

The situation for life cycle inventory data is similar: confidentiality issues protection of competitiveness and know-how, competition law with a large grey zone of interpretation, fear of giving readability on costs and of being deselected, etc. That means also here it is primordial to build mutual understanding and trust. The global guidance principles on Life Cycle Assessment (LCA) databases are a good 
starting point. For the way forward an approach is proposed that differentiates averaged and individual datasets. For averaged datasets partial disaggregation is possible for commodities (large number of producers) but partial disaggregation is not possible when there are not enough producers ( 3 being an absolute minimum). For individual dataset, it is up to business to decide. Most often, it is confidential. Overall there is a need for common rules. As an example PlasticsEurope's recommendation on Steam Cracker allocation for the sake of comparability of petrochemical products was given [3].

\section{Biomass Balance Approach: An Innovative and Complementary Approach for Using Biomass in the Chemical Industry}

BASF recognises four drivers for including renewables in their portfolio: competitiveness, upcoming regulations, climate change and raw material diversification. BASF has developed a methodology to account and communicate the renewable content of his materials to customers. This approach is an intermediate situation on the way to dedicate production of materials with renewable content. The latter is often costly as the market is not matured yet.

The biomass balance approach is similar to that for "green" electricity: The fossil raw materials needed to produce a customer's products are replaced by renewable raw materials. Only the quantities of renewable raw materials requested by a customer are purchased up front by BASF and managed in the SAP system. When a customer places an order, the required quantities of renewable feedstock are allocated to the products purchased.

The advantages of this approach are the production of drop-in products, easy accountability and it can be implemented directly [4].

\section{Sustainability at Teijin: Customer Benefit Model and Case Study Tires}

Being eco-effective is important for Teijin and for this reason eco-efficiency assessments are often made to their products. With the help of this assessment Teijin is able to assess the real eco-performance of its products. The presented case study was about car tires, where $90 \%$ of the impact along the value chain is in the use phase, due to consumption of fuel. Therefore, products with lower weight and less rolling resistance turn out to show a better eco-efficiency performance [5]. 


\section{Life Cycle Assessment of Acrylic Fibre and Garment in Thailand}

A case study is presented that aims first at the quantification of environmental impacts by LCA according to ISO 14040/44 standard [6, 7], for one tonne of Acrylic Fibre manufactured at Thai Acrylic Fibre Co. Ltd, i.e. cradle to gate study, and then for one piece of Acrylic Garment of $450 \mathrm{~g}$ throughout the entire life cycle, i.e. cradle to grave study with 100 washes. Acrylonitrile, electricity and steam consumption were identified as major hot-spots in the value chain across the various identified environmental impacts. Use-stage electricity and water consumption are also hot-spots in cradle to grave study. This information is used for the development of a report for communication to stakeholders. Improvements are possible in the captive power plant efficiency, the steam and electricity consumption in polymerisation, the solvent recovery and the wet-spinning processes. Scenarios of different usages of acrylonitrile produced through the propane route will be analysed in the future to further understand the processes and improve them [8].

\section{Conclusions}

The presentations provided a good overview of how Life Cycle Assessment is used to assess chemical products and materials and which challenges exist with regard to the data exchange along the value chain. The session shows how companies use LCA results to improve the chemical products in their journey towards sustainability, in which they use life cycle management. Some presentations also gave an insight in the progress made in the quality and the possibilities of preparing LCIs of chemical products and materials.

Overall, including also poster presentations, it can be said that the chemicals industry has achieved an important degree of maturity in implementing life cycle management since the beginning of this century. In the future it can be expected that the data exchange along the value chain will be further improved, using as an example the established exchange of toxicity information stemming from risk assessment.

\section{References}

1. Smith R-L, Meyer D-E, Ruiz-Mercado G-J, Gonzalez M-A, Abraham J-P, Barrett W-M, Randall P-M, Niblick B, Mittal V-K, Rapid Estimation of Life Cycle Inventory, LCM 2017 conference, Luxembourg, 2017.

2. Wernet G, Levova T, Bourgault G, Current models and approaches for data matching and modeling of chemical production, LCM 2017 conference, Luxembourg, 2017. 
3. Castelan G, De Hults Q, PlasticsEurope experience in managing information along the value chain for RA and LCA, LCM 2017 conference, Luxembourg, 2017.

4. Krüger C, Kicherer A, Kormann C, Raupp N, Biomass Balance: an innovative and complementary approach for using biomass in the chemicals industry, LCM 2017 conference, Luxembourg, 2017.

5. Beers H, Bosman H, Kamer M, Huguet Ferran P, Sustainability at Teijin: Customer Benefit Model and Case Study Tires, LCM 2017 conference, Luxembourg, 2017.

6. ISO 14040: Environmental management-Life cycle assessment-Principles and framework, Geneva, 2006.

7. ISO 14044: Environmental management—Life cycle assessment—Requirements and guidelines, Geneva, 2006.

8. Barik J, Singh R, Life Cycle Assessment of Acrylic Fiber and Garment in Thailand, LCM 2017 conference, Luxembourg, 2017.

Open Access This chapter is licensed under the terms of the Creative Commons Attribution 4.0 International License (http://creativecommons.org/licenses/by/4.0/), which permits use, sharing, adaptation, distribution and reproduction in any medium or format, as long as you give appropriate credit to the original author(s) and the source, provide a link to the Creative Commons license and indicate if changes were made.

The images or other third party material in this chapter are included in the chapter's Creative Commons license, unless indicated otherwise in a credit line to the material. If material is not included in the chapter's Creative Commons license and your intended use is not permitted by statutory regulation or exceeds the permitted use, you will need to obtain permission directly from the copyright holder.

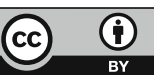




\title{
Biomass Balance: An Innovative and Complementary Method for Using Biomass as Feedstock in the Chemical Industry
}

\author{
Christian Krüger, Andreas Kicherer, Claudius Kormann \\ and Nikolaus Raupp
}

\begin{abstract}
Fossil resource and greenhouse gas savings can be claimed when certified biomass is co-fed with fossil raw materials into a highly efficient interlinked production network. BASF and partners have developed a novel biomass balance certification standard for chemical synthesis. It offers a reliable response to customers and end consumers who are increasingly interested in solutions that are based on renewable feedstock without compromising resource efficiency and performance. The new standard describes how renewable feedstock is attributed to a given sales product. Existing products can thus be derived from biomass and provided with third-party certification. Highest technical product standards can be maintained while fostering the strategic goals of a bio-economy, often requested from different stakeholders.
\end{abstract}

\section{BASF's Biomass Balance Approach}

The Circular Economy (CE) concept has gained a broad momentum in politics, industry, and society within the past few years [1]. One of the six levers is the shift to renewable energy and materials, which allow companies to reclaim, retain, and regenerate the health of ecosystems. For the chemical industry, it is still a big challenge to use renewable feedstocks. Bio-based chemistry is currently no solution to a rapid switch to renewable feedstocks because of fundamental limits. Only some products can be produced and large investments must be executed in R\&D and production assets. BASF found a concept to introduce biomass by using existing chemical pathways in a cost-efficient way. The sustainability of feedstocks and the mass balance between feedstocks and final products is proven by an independent certificate:

C. Krüger $(\bowtie) \cdot$ A. Kicherer · C. Kormann

BASF SE, Sustainability Strategy, 67056 Ludwigshafen, Germany

e-mail: christian.krueger@basf.com

N. Raupp

BASF SE, Global Sustainability Manager Dispersions \& Pigments,

67056 Ludwigshafen, Germany

(C) The Author(s) 2018

E. Benetto et al. (eds.), Designing Sustainable Technologies,

Products and Policies, https://doi.org/10.1007/978-3-319-66981-6_12 
This concept is called "The Biomass Balance Approach". This approach is applicable to almost all fossil-based products in the chemical industry (see Fig. 1, [2]).

Certified renewable feedstock (e.g. biogas derived from organic waste) replaces fossil resources at the beginning of the value chains in the chemical industry. Then the renewable feedstock is attributed verifiably to sales products in the corresponding quantities (see Fig. 2: comparison to bio-based chemistry). Third-party certification (TÜV SÜD) confirms to customers that BASF has used the required

Feedstock

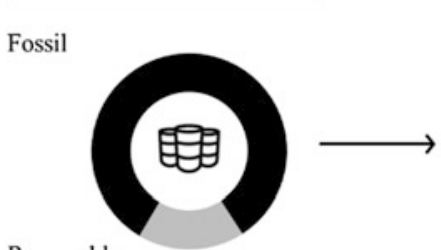

Renewable

Use of renewable feedstock in very first steps of chemical production (e.g. steam cracker)
BASF Production

\section{Verbund}

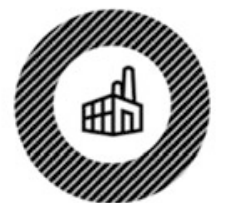

Utilization of existing Production Verbund for all production steps

\section{Products}

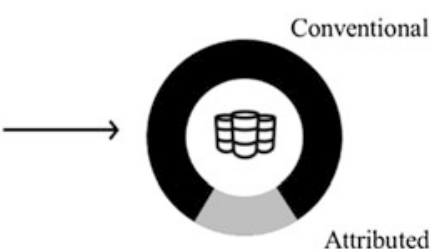

Attribution of renewable feedstock to selected products

Fig. 1 BASF's biomass balance approach

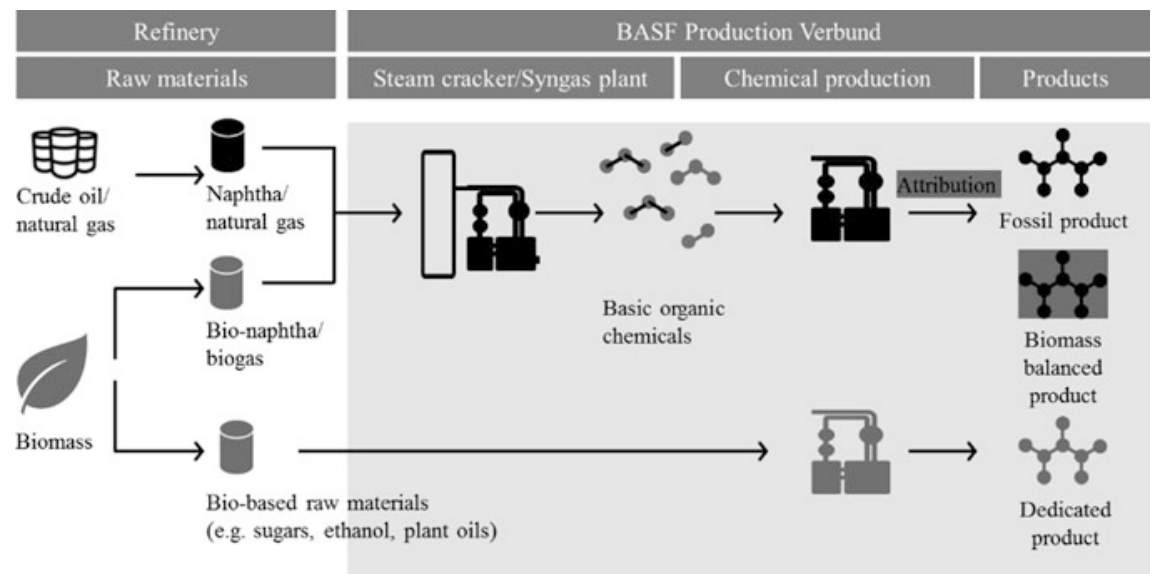

Fig. 2 Comparison of the biomass balance chain-of-custody method with dedicated production of bio-based materials 
quantities of renewable feedstock for the products they purchase [3]. The resulting products are called "Biomass balanced products". They are technically identical to their fossil comparatives but they are associated with quantifiably lower greenhouse gas (GHG) emissions due to the renewable feedstock used. The biomass balance chain-of-custody method is applicable to almost all products in BASF's portfolio, for example Acronal ${ }^{\circ}$ binders used for premium decorative paints. To date, more than 50 BASF products have already been certified, and several of them have been introduced into the market, thus contributing to sustainable development by saving fossil resources and reducing GHG emissions. BASF has received great attention thanks to the biomass balance method and has been selected as finalist for the "The Circulars 2017 Award" in the category "Multinational". The biomass balance method enables BASF to react quickly and flexibly to the increased interest to use renewable raw materials thus helping customers to differentiate in the market.

\section{Biomass Balance in Chemical Processes}

Comprehensive experience about mass balance methods is already available in other markets, especially in the biofuel, biogas and bioenergy markets [4]. Therefore, only minor adaptation to existing standards is needed for using BASF's biomass balance method in chemical production.

The system boundary is defined as the integrated chemical production system, which represents a physically interconnected arrangement of production sites at the same location, or the physical interconnection of operating facilities over different locations by means of dedicated transportation systems (e.g. pipelines, rolling pipelines).

Only sustainably sourced biomass or bio-based products are used to replace fossil comparatives to manufacture intermediates and final products. Bio-based renewable feedstock may strongly differ in value for a chemical synthesis. In order to make renewable feedstock of varying "chemical value" comparable to fossil feedstock, we introduced a correction factor for each raw material similar to the biofuel sector. BASF uses the lower heating value (LHV) as the most appropriate one. Therefore, each consignment of raw material entering the system boundary (i.e. the input) is assessed according to its LHV.

The exchange of fossil raw materials with bio-based feedstocks for production allows for new claims. For example, according to the TÜV SÜD standard [3] the following sales claim is certifiable for BASF and customers: "Fossil resource saving product. $100 \%$ of the fossil feedstock required for the manufacturing of this product was replaced in the production site by renewable raw materials."

Only renewable feedstock that enters in intermediates or products is eligible for the biomass balance chain-of-custody method. Raw materials needed for generating energy as steam or electricity are not counted. 


\section{Sustainability of Renewable Feedstocks}

Typically, renewable raw materials are usually not superior in all environmental categories and pros and cons have to be considered. Therefore, BASF only uses sustainable renewable feedstock, which is certified as sustainable according to the criteria of the Renewable Energy Directive of the EU Commission. Under this framework several standards (e.g. ISCC, REDcert) comply with principles such as:

(1) Sustainable production of feedstock

(2) Fulfilment of social standards in agriculture

(3) Protection of biodiversity

(4) Reduction of greenhouse gas emissions

(5) Minimized interference with local food supply

BASF strives to continuously improve the sustainability profile of purchased bio-based feedstocks and therefore it is important to keep the share of 1st generation renewables as low as possible. Thanks to new collections systems and innovative technologies, the production of renewable feedstocks based on wastes (e.g. kitchen or other residues) is getting more attractive, and BASF is cooperating with its suppliers to broaden the sustainable feedstock portfolio.

For the chemical industry, naphtha and natural gas are among the most important raw materials. BASF has identified certified bio-based comparatives without technical compromises. By replacing naphtha and natural gas at the beginning of the value chain at highly optimized Verbund sites, BASF can principally produce many thousands of biomass balance products, which can be used as drop-in solution for customers in various industries.

\section{Implementation in LCA}

Life Cycle Assessment (LCA) is an important tool to evaluate quantitatively the environmental impacts of products [5]. Therefore, BASF implemented the biomass balance concept into LCA by respecting the appropriate ISO 14040 series.

The calculation for BMB products is conducted in GaBi software on the basis of the existing model for the fossil-based product. A material flow analysis is used that enables calculating the feedstock use in the final product.

The respective BMB product is calculated on the basis of the fossil product by replacing fossil-based naphtha and natural gas with biofeedstock derived naphtha (= bionaphtha) and biofeedstock derived natural gas (= biogas). In Fig. 3 the calculation principle of the environmental burden B for biomass balance products is shown, which is the sum of the difference of the bio-based feedstocks and its fossil comparatives. 


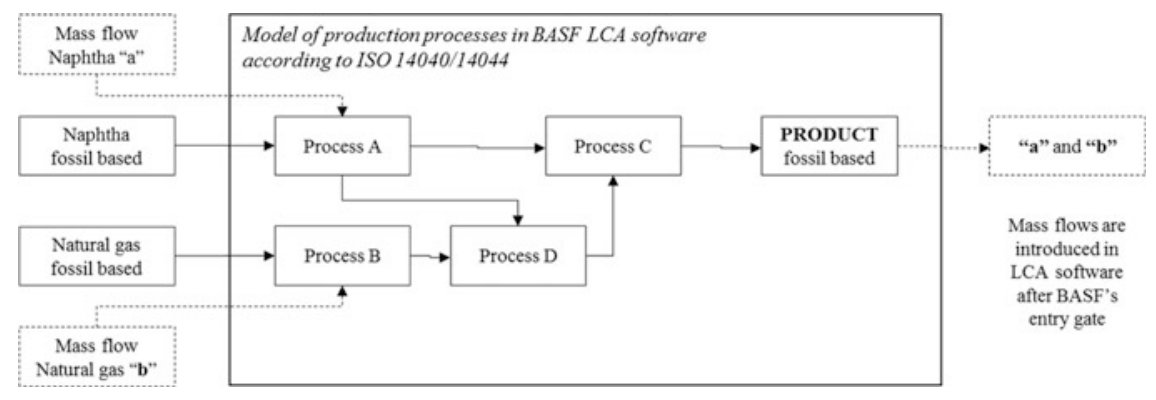

Fig. 3 Biomass balance calculation principle (example: bionaphtha and biogas)

The calculation of the life cycle inventory for BMB products is according to the equation as shown below:

$$
\mathrm{B}=\mathrm{B}_{\text {fossil }}+\mathrm{a} \times\left(\mathrm{cv}_{\mathrm{BN} / \mathrm{N}} \times \mathrm{B}_{\mathrm{BN}}-\mathrm{B}_{\mathrm{N}}\right)+\mathrm{b} \times\left(\mathrm{cv}_{\mathrm{BG} / \mathrm{NG}} \times \mathrm{B}_{\mathrm{BG}}-\mathrm{B}_{\mathrm{NG}}\right)
$$

where:

$\mathrm{B}, \mathrm{B}_{\text {fossil }}, \mathrm{B}_{\mathrm{BN}}, \mathrm{B}_{\mathrm{BG}}, \mathrm{B}_{\mathrm{N}}, \mathrm{B}_{\mathrm{NG}}$ : environmental burdens

a: amount of naphtha substituted by bionaphtha

b: amount of natural gas substituted by biogas

cv: "chemical value" factor (dimensionless): $\mathrm{cv}=\mathrm{LHV}_{\text {fossil feedstock (substituted) }}$ / $\mathrm{LHV}_{\text {biofeedstock }}$

LHV: lower heating value

Indices: $\mathrm{BN}=$ bionaphtha, $\mathrm{N}=$ naphtha, $\mathrm{BG}=$ biogas, $\mathrm{NG}=$ natural gas

\subsection{Chemical Value Factor (cv)}

Fossil feedstocks cannot always be replaced equally on a mass basis by bio-based comparatives, because of its different chemical nature in many cases. Sometimes bio-based feedstocks are used instead of fossil, where the carbon content is similar, but the energetic value is lower. Therefore, it is necessary to consider the "chemical value" of feedstock (the term coined by BASF in the context of the BMB approach, although it is not necessarily related to chemical parameters but is used to denote the use of feedstocks as chemicals rather than energy sources). The main feedstocks in the chemical industry are naphtha and natural gas, which are further processed either by cracking or oxidation, which takes place at high temperatures. The calorific value of those feedstocks can be used as an approximation of the chemical value, assuming lower heating value (LHV). The latter is also the determining factor for the design of the plant. 


\section{Conclusions}

BASF's biomass balance is an innovative approach to introduce biomass to the chemical industry, which is certified by an independent party. We believe that it is complementary to bio-based chemistry to further increase the consumption of renewable materials as feedstock for the chemical industry. Our customers acknowledge two main advantages:

(1) The customer can use a biomass balanced product as drop-in solution without any compromise in performance. For most existing fossil-based products we can provide biomass balanced alternatives by introducing sustainably sourced bionaphtha and biogas into our interconnected production sites.

(2) There is no need for investment into $R \& D$ and new assets (which will usually make new products more expensive).

A certification standard from TÜV SÜD requires the sustainability of the renewable feedstocks and its correct use for the production of biomass balanced products. BASF is able to easily calculate LCAs without building up the whole value chains separately from the fossil route in a LCA model by respecting existing ISO standards.

\section{References}

1. Peter Lacy et al., Waste to Wealth: The Circular Economy Advantage, Palgrave, 2015.

2. <https://www.basf.com/en/company/sustainability/environment/resources-and-ecosystems/ renewable-raw-materials/biomass-balance.html> BASF's biomass balance approach, website from BASF (Accessed DD:05.2017).

3. <http://www.tuev-sued.de/plants-buildings-technical-facilities/focal-topics/renewable-energies/ renewable-resources> Renewable Resources, website from TÜV SÜD (Accessed DD.05.2017).

4. <http://www.ble.de/SharedDocs/Downloads/DE/Klima-Energie/Nachhaltige-Biomasseherstellung/ LeitfadenNachhaltigeBiomasseherstellung.html?nn=8911410> Leitfaden Nachhaltige Biomasseherstellung, BLE 2010, Website from German Federal Office for Agriculture and Food (Accessed DD.05.2017).

5. Peter Saling, The BASF Eco-Efficiency Analysis-A 20-year success story, BASF, 2016. 
Open Access This chapter is licensed under the terms of the Creative Commons Attribution 4.0 International License (http://creativecommons.org/licenses/by/4.0/), which permits use, sharing, adaptation, distribution and reproduction in any medium or format, as long as you give appropriate credit to the original author(s) and the source, provide a link to the Creative Commons license and indicate if changes were made.

The images or other third party material in this chapter are included in the chapter's Creative Commons license, unless indicated otherwise in a credit line to the material. If material is not included in the chapter's Creative Commons license and your intended use is not permitted by statutory regulation or exceeds the permitted use, you will need to obtain permission directly from the copyright holder.

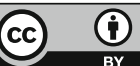




\title{
Greening Agri-food Value Chains in Emerging Economies
}

\author{
Matthias Stucki and Anél Blignaut
}

\begin{abstract}
Emerging economies play an increasingly important role in global food security. They often rely on fossil fuels, lag behind on food governance and are characterised by subtropical climates, often requiring energy intensive irrigation and refrigerated storage. Mitigation options for agri-food value chains in emerging economies are novel and have substantial sustainability potential. The session on Greening Agri-food Value Chains in Emerging Economies at the 8th International Conference on Life Cycle Management showed the manifold challenges of emerging economies on their transition path to contributing to a sustainable global food system. LCA can support this path by identifying specific environmental hotspots in food value chains as well as by evaluating and prioritising potential solutions from an environmental perspective.
\end{abstract}

\section{Introduction}

Emerging economies such as Brazil, India, China and South Africa play an increasingly important role in global food security since they are among the world's largest producers of crops. Recently, their economies have experienced rapid growth and increased integration into the global economy [1]. Sustainable development in agricultural value chains of emerging economies is thus of high relevance for global food security. While the reduction of environmental impacts of food value chains has been widely studied in industrialised countries, mitigation options for food value chains in emerging economies are new and have substantial sustainability potential. Many emerging economies rely heavily on fossil fuels, lag behind on food governance and are characterised by climate subtropical conditions,

\author{
M. Stucki (ه) \\ Institute of Natural Resource Sciences, Zurich University \\ of Applied Sciences, 8820 Wädenswil, Switzerland \\ e-mail: matthias.stucki@zhaw.ch
}
A. Blignaut
Blue North Sustainability, Stellenbosch, South Africa
(C) The Author(s) 2018
E. Benetto et al. (eds.), Designing Sustainable Technologies,
Products and Policies, https://doi.org/10.1007/978-3-319-66981-6_13 
often requiring energy intensive irrigation and refrigerated storage [2-5]. In order to green agri-food value chains in emerging economies, these specific conditions need to be taken into consideration. Therefore, the development and implementation of sustainable technologies and production methods along the entire agri-food value chain is a crucial step towards a global sustainable food system.

During the session on Greening Agri-food Value Chains in Emerging Economies at the 8th International Conference on Life Cycle Management in Luxembourg in September 2017 successful projects and options for reducing environmental impacts of agri-food value chains in emerging economies were discussed.

\section{Summary of the Session Presentations}

Within the session on Greening Agri-food Value Chains in Emerging Economies five presentations from four different continents were held. Subsequently, each presentation is referenced at the beginning of each section and therefore not repeated again.

\subsection{Rice, Sugarcane and Oil Palm Cultivation in Thailand}

Trakarn Prapaspongsa analysed technology choices by farmers within the rice, sugarcane and oil palm industries in Thailand [6]. An eco-efficiency assessment was performed using the ISO 14045:2012 standard, whilst the environmental assessment was performed using Life Cycle Assessment (LCA).

Overall, rice cultivation had the largest negative impact on terrestrial acidification and marine eutrophication, with land use change (LUC) playing the most important role. Prapaspongsa determined toxicity and marine eutrophication to be most significant in sugarcane production. Significant environmental impacts are related to burning land. In palm oil production, pesticide use has a high impact.

Financial incentives were recommended: for example, higher prices for sugarcane when the fields are not burnt. Environmental impacts in Thailand could be significantly lowered if pesticide use in palm oil production were reduced and if sugarcane producers refrained from field burning practises.

\subsection{Dairy and Maize Farming in South Africa}

Regula Keller from the Zurich University of Applied Sciences conducted a LCA on the South African dairy industry, where she identified environmental impacts and evaluated the environmental mitigation potential of cleantech options [7]. The study 
led to the development of recommendations for sustainable production and the inclusion of datasets in the ecoinvent database.

A methane inhibitor (3NOP) can be used to reduce the enteric emissions of dairy cows, leading to an $18 \%$ reduction in greenhouse gas (GHG) emissions, with no observable trade-off in the other impact categories. Variable Frequency Drives (VFD) that reduce the electricity demand for pumping water, led to a reduction of $34 \%$ of GHG emissions from maize cultivation. The environmental impacts arising from irrigated maize fodder production could be reduced by $47 \%$ by using solar powered irrigation compared to using South African grid electricity. The addition of a methane inhibitor only reduced the climate change impact category but did not have a significant effect on the other impact categories. Additionally, the long-term safety needs to be proven and public acceptance needs to increase.

It was clear that the irrigation of maize for feed production benefitted from the use of both VFD and, to an even greater extent, from solar photovoltaic energy use. All three options can generally be recommended and early implementation of cleantech could potentially have a significant effect. Different cleantech measures could also be combined to minimise the environmental impacts of milk production.

\subsection{Pork Meat Production in South Africa}

Valentina Russo from the University of Cape Town analysed the water footprint of the commercial pork value chain in South Africa [8]. Meat production in South Africa is on the rise and there is a perception that the water footprints of meat products are large. In addition, South Africa is a water stressed country with physical water scarcity predicted by 2040. The goals of this study were to assess the stress-adjusted blue consumptive water footprint in order to locate the environmental hotspots in meat production and to determine whether South Africa should be concerned about meat consumption in regards to water intensity.

The WSI \& AWARE methods were used to determine the water footprint. The water stress index (WSI [9]) relates water consumption to water scarcity in the same watershed and the AWARE method [10] indicates the available water remaining and quantifies the potential of water deprivation, either to humans or to ecosystems.

The study concluded that the centre of South Africa and the Western Cape are hotspots for meat production. Most of the water is used for feed production (43\%) and farming activities (54\%). It was recommended that feed production be located in water management areas that experiences less stress and that practices should move away from irrigation or toward more efficient irrigation systems. Water use, water stress and water efficiency need to be considered in feed optimisation for intensive animal finishing. 


\subsection{Hydrous Ethanol Production from Sugarcane in Brazil}

Monica Alcantara from Odebrecht Agroindustrial determined the carbon and water footprint of hydrous ethanol produced from sugarcane in Brazil [11]. During growth, sugarcane absorbs carbon dioxide at a rate of $28.3 \mathrm{~g} \mathrm{CO}_{2} / \mathrm{MJ}$. The cultivation and harvesting of sugarcane releases $17.8 \mathrm{~g} \mathrm{CO}_{2} / \mathrm{MJ}$, transport with diesel-powered trucks $4.5 \mathrm{~g} \mathrm{CO}_{2} / \mathrm{MJ}$, whilst processing releases $1 \mathrm{~g} \mathrm{CO}_{2} / \mathrm{MJ}$. Finally, burning in a car engine releases $0.6 \mathrm{~g} \mathrm{CO}_{2} / \mathrm{MJ}$. The results of the carbon footprint depend on the inclusion of LUC in the assessment. The water footprint was quantified at $18.41 \mathrm{of}$ water per litre of ethanol produced at the Odebrecht plant including the life cycle stages from agricultural production involving irrigation up to the operation of the Odebrecht plant. Water use was identified as a hotspot for the Odebrecht Agroindustrial company.

\subsection{Emissions from Global Land Use Change and Deforestation}

Jean-Baptiste Bayart from Quantis discussed how to embed LUC in corporate footprints [12], since it contributes around $18 \%$ of global GHG emissions, more than the direct contribution of the agricultural sector (around 13.5\%). The example of soybeans in Argentina showed that more than $5 \mathrm{~kg} \mathrm{CO}$-eq per $\mathrm{kg}$ soybean are released due to land use change. Corporations can only adopt meaningful strategies to avoid deforestation and other types of LUC in order to reach their GHG reduction targets if these effects can be measured in a harmonised way. However, current models have high uncertainty and there are many different models, which can influence the results. A corporate consortium led by Quantis aims at giving recommendations on how to quantify GHG emissions arising from land use change.

For the allocation of GHG emissions from LUC over time, Bayart proposed a model with linear discounting over 20 years. This means that the allocated emissions during the first crop cycle are higher but will reduce over time.

Bayart concluded that their study is a step forward in reaching a consensus on LUC modelling and that significant methodological improvements have been made. The next step for Quantis is the launch of the pilot phase where the model will be tested on products and commodities.

\section{Outcomes of the Session}

The session on Greening Agri-food Value Chains in Emerging Economies at the 8th International Conference on Life Cycle Management showed the manifold challenges of emerging economies on their transition path to contributing to a 
Sustainable global food system

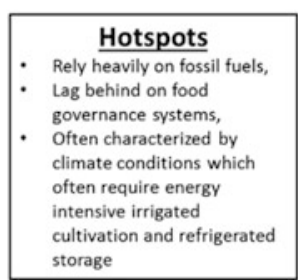
storage

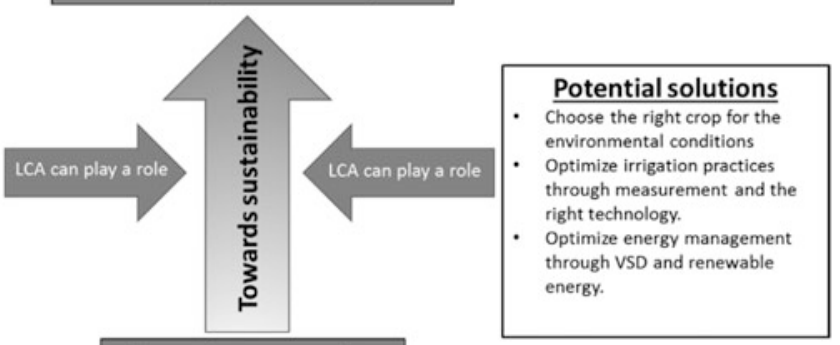

Emerging economies

Fig. 1 The role of LCA in supporting emerging economies on their path to contributing to a sustainable global food system

sustainable global food system. Figure 1 presents a framework on how LCA can support this path by identifying specific environmental hotspots in food value chains within emerging economies as well as by evaluating and prioritising potential solutions from an environmental perspective.

\section{Future Perspectives}

The contributors to the session highlighted a range of specific recommendations for agri-food value chains in emerging economies. These value chains could be made more environmentally sustainable by including environmental aspects when choosing crops for cultivation and when selecting cultivation systems, by avoiding the burning of residues, by reducing irrigation, by using renewable energy, by transforming degraded land and by avoiding practises, such as deforestation, that lead to land use change. From a LCM perspective, there is a need for more specific background data for life cycle inventories in emerging economies and for more harmonised methodologies to order to enhance comparability.

\section{References}

1. Fan S, Brzeska J, The Role of Emerging Countries in Global Food Security, International Food Policy Research Institute, 2010.

2. Asif M, Muneer T, Energy supply, its demand and security issues for developed and emerging economies, Renew. Sustain. Energy Rev., vol. 11, no. Journal Article, pp. 1388-1413, 2007.

3. Carbon Trust, The emerging Cold Economy. Sustainable solutions for rapidly increasing cooling needs, 2015.

4. Douben N, Ratnayake R.M.W, Characteristic data on river floods and flooding; facts and figures, in Floods, from Defence to Management: Symposium Proceedings of the 3rd 
International Symposium on Flood Defence, Book, Section vols., J. van Alphen, E. van Beek, and M. Taal, Eds. 2006, pp. 19-35.

5. IFPRI, 2014-2015 Global Food Policy Report, International Food Policy Research Institute, Washington, 2015.

6. Prapaspongsa T, Gheewala S.H, Right Product or Technology for Sustainable Agri-Food Value Chains? Lessons Learned from Rice, Sugarcane and Oil Palm Cultivation in Thailand, presented at the 8th International Conference on Life Cycle Management, Luxembourg, 06-Sep-2017.

7. Keller R.L, Eyman L, Wettstein S, von Blottnitz H, Scharfy D, Stucki M, Assessment of Cleantech Options to Mitigate Environmental Impacts of South African Dairy and Maize farming, presented at the 8th International Conference on Life Cycle Management, Luxembourg, 06-Sep-2017.

8. Russo V, et al., Life Cycle Inventories of South Africa Agricultural Products, ecoinvent centre in cooperation with University of Cape Town, Blue North, The Green House and Zurich University of Applied Sciences, Zürich, 2017.

9. Pfister S, Koehler A, Hellweg S, Assessing the environmental impacts of freshwater consumption in LCA, Env. Sci Technol, vol. 43, no. 11, pp. 4098-4104, 2009.

10. Boulay A.-M, et al., The WULCA consensus characterization model for water scarcity footprints: assessing impacts of water consumption based on available water remaining (AWARE), Int. J. Life Cycle Assess., pp. 1-11, Jun. 2017.

11. Sodré C, Alcântara M, Fernandes M, Koszka Kiss B.C, Carbon and water footprint of hydrous ethanol from Brazilian sugarcane produced by Odebrecht Agroindustrial, presented at the 8th International Conference on Life Cycle Management, Luxembourg, 06-Sep-2017.

12. Zollinger M, Dettling J, Rossi V, Humbert S, Bayart J.-B, How to embed land use change/ deforestation-related emissions from corporate supply chains into corporate footprints and corporate GHG targets in an accurate and credible manner, presented at the 8th International Conference on Life Cycle Management, Luxembourg, 06-Sep-2017.

Open Access This chapter is licensed under the terms of the Creative Commons Attribution 4.0 International License (http://creativecommons.org/licenses/by/4.0/), which permits use, sharing, adaptation, distribution and reproduction in any medium or format, as long as you give appropriate credit to the original author(s) and the source, provide a link to the Creative Commons license and indicate if changes were made.

The images or other third party material in this chapter are included in the chapter's Creative Commons license, unless indicated otherwise in a credit line to the material. If material is not included in the chapter's Creative Commons license and your intended use is not permitted by statutory regulation or exceeds the permitted use, you will need to obtain permission directly from the copyright holder.

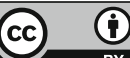




\title{
Assessment of Cleantech Options to Mitigate the Environmental Impact of South African Dairy Farming
}

\author{
Regula Keller, Lea Eymann, Sarah Wettstein, Deborah Scharfy \\ and Matthias Stucki
}

\begin{abstract}
Milk production in South Africa has increased substantially over the past ten years and is associated with various environmental impacts. These can be reduced by different means, four of which were analysed in this study: choice of breed, the use of methane emission reducing feed additives, solar power as well as variable frequency drive usage in fodder irrigation. The results showed that Holstein cows had a lower impact than Ayrshire cows per litre of milk, but that differences between farms were greater than between breeds alone. The feed additive 3-nitrooxypropanol (3NOP) led to an $18 \%$ reduction in the climate change impact category, and did not have negative effects in other categories. Using solar power for irrigation decreased the environmental impact by a larger degree than integrating a variable frequency drive to reduce the electricity demand of the water pump. All four are adequate means of reducing the environmental impact of milk.
\end{abstract}

\section{Introduction}

Agriculture is the main contributor to global anthropogenic non- $\mathrm{CO}_{2}$ greenhouse gas emissions [1] and also contributes considerably to air pollution, land, soil and water degradation, and the reduction of biodiversity [2]. As in most emerging economies, livestock is one of the fastest growing sectors of the agricultural economy in South Africa. Milk production increased by $26 \%$ in South Africa from 2004 to 2014 [3] and rising meat consumption could exacerbate water stress in South Africa [4]. Given the considerable environmental impacts caused by dairy production systems and the industry's growth, environmental mitigation strategies are required.

Enteric emissions are responsible for the largest share of greenhouse gas emissions of milk at farm gate [5]. Effective measures to reduce enteric methane

\footnotetext{
R. Keller $(\varangle) \cdot$ L. Eymann $\cdot$ S. Wettstein · D. Scharfy · M. Stucki

Zurich University of Applied Sciences, Institute of Natural Resource Sciences,

Life Cycle Assessment, 8820 Wädenswil, Switzerland

e-mail: Regula.Keller@zhaw.ch

(C) The Author(s) 2018

E. Benetto et al. (eds.), Designing Sustainable Technologies,

Products and Policies, https://doi.org/10.1007/978-3-319-66981-6_14
} 
emissions include the genetic selection of animals producing fewer emissions and having higher production efficiency (genetic approach), as well as management approaches, e.g. practices to reduce non-voluntary culling and diseases and improvements in nutrition [4]. In this paper, choice of breed as well as the use of feed additives are analysed.

The World Bank sees investing in more advanced technologies as an answer to the environmental problems caused by agriculture [6]. In a sustainability evaluation of 17 cleantech measures in agriculture, the two cleantech options analysed in this paper - solar electricity and the use of frequency converters - were rated among the five best approaches [7]. A joint research project of the University of Cape Town and the Zurich University of Applied Sciences was carried out, aimed at identifying environmental hotspots in the life cycle of South African agri-food products in order to determine the key intervention points for mitigating their environmental impacts. This paper specifically describes four clean technologies and their potential to reduce the environmental impact of South Africa's milk by applying life cycle assessment (LCA).

\section{Methods}

Data collection for the LCA of milk and maize were part of this research project: data on maize cultivation were collected from the major maize production corporations in South Africa (GWK AGRI, Grain SA). Manufacturing data, including fertiliser and pesticide use, diesel consumption, production area and yield are average values from the Grain SA planning models of three different regions (Eastern Highveld; North West and Central; Northern Free State) of maize production in South Africa from 2006 to 2013 [8]. The modelling is based on different methods of production (rainfed and irrigated) and three different maize varieties: genetically modified (GM)-insect tolerant trait (RR, only rainfed); GM-genetically modified herbicide tolerant trait and GM-free ( $\mathrm{Bt}$, only irrigated) and GM-free. Both multi-nutrient fertilisers (NPK-fertilisers) and cattle manure are applied.

Data for the milk model was collected in 2014 from five dairy farms in the province of KwaZulu-Natal (KZN) [9], one of the three main milk production areas South Africa [3].

Allocation between beef and milk was conducted according to the approach recommended by the International Dairy Federation (IDF) that reflects the underlying use of energy from fodder by the dairy animals and the physiological feed requirements of the animal to produce milk and meat [10]. To distribute the beef's environmental impact between calves and cull dairy cows, economic allocation was performed.

Based on the results of these LCAs, four different measures to reduce the environmental impact of raw milk were considered: choice of suitable breed: comparison of Ayrshire and Holstein cows (A); reduction of enteric emissions with feed-additives: feeding $3 \mathrm{NOP}$ to lactating cows (B) and producing maize feed with 


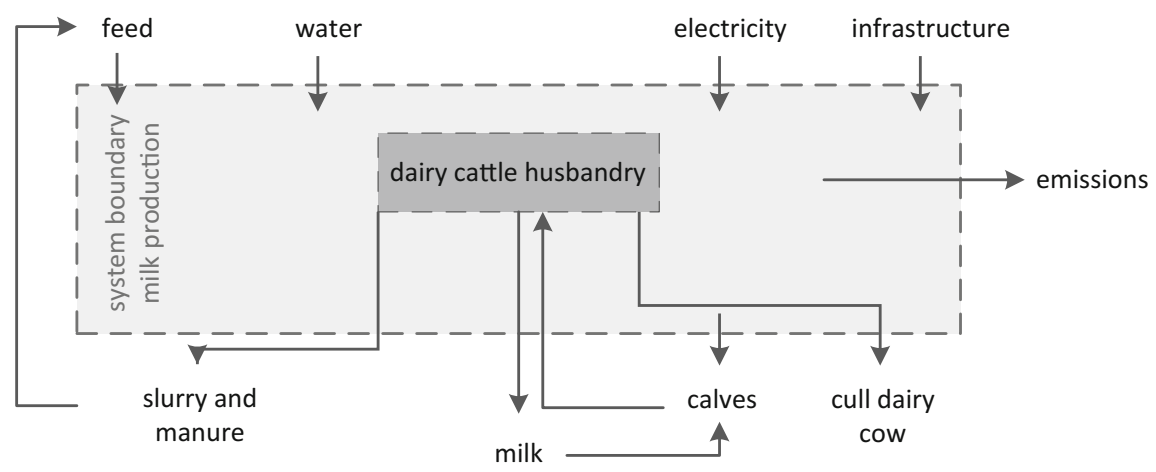

Fig. 1 System boundary of raw milk production

two types of cleantech for irrigation (C): solar power (C1) and integration of a variable frequency drive that reduces the electricity demand of the water pump (C2). The measures are described in more detail in Chapter "A Synthesis of Optimization Approaches for LCA-Integrated Industrial Process Modeling: Application to Potable Water Production Plants". All scenarios of clean technologies were based on the same raw milk LCA model [9] that includes infrastructure, water, electricity and feed input (see Fig. 1).

The functional unit was defined as one kilogramme of fresh milk at the farm gate in South Africa. For a sensitivity comparison of breeds, $1 \mathrm{~kg}$ of fat and protein corrected milk (FPCM) as well as price were included as additional functional units.

Ecoinvent v.3.3 data with the system model cut-off [11] were used as background data. The details on the foreground data are described in the sub-chapters. The results were calculated and analysed in SimaPro v8.3. To assess the environmental impacts associated with South African dairy farming and processing, five impact categories and respective methods were used:

(1) Climate change (abbr.: GHG emissions) with the method IPCC 2013, GWP 100a [1].

(2) Non-renewable energy (fossil + nuclear) (abbr: CED non-ren.) with the method Cumulative Energy Demand (CED) [12]. In this study, only fossil and nuclear energy resources were considered.

(3) Freshwater and marine eutrophication (abbr. Freshw./Marine Eutr.) with the EUTREND model as implemented in ReCiPe [13].

(4) Ecotoxicity (fresh water) (abbr. Ecotox.) with the USEtox model [14]. The version "USEtox (recommended + interim) v1.04" was used.

(5) Land use with the method Ecological Scarcity 2013 (global model) [15]. 


\section{Life Cycle Inventory}

This study uses the "Tier 2" approach described in the IPCC Guidelines to calculate methane emissions from enteric fermentation [16]. Thereby, the methane emissions from enteric fermentation of a dairy cow during its lifetime are calculated from the gross energy intake (GE), the methane conversion rate $(\mathrm{Ym})$ and the energy content of methane $(55.56 \mathrm{MJ} / \mathrm{kg})$. Based on the IPCC standard, the methane conversion rate $(\mathrm{Ym})$ was assumed to be $6 \%$, the value for dairy cows in developing countries. Further details of modelling are described for each measure individually.

\section{Different breeds (A)}

The four major dairy breeds in South Africa are Holstein, Jersey, Guernsey and Ayrshire [3]. This study analysed the environmental impact of raw milk from Ayrshire and Holstein breeds.

A production mix of the five farms where data was collected (see Chapter "Sustainability Performance Evaluation for Selecting the Best Recycling Pathway During Its Design Phase") with an equal share from each farm was modelled. Three farms keep only Holstein cows and two farms keep both Holstein and Ayrshire cows. The number of dairy cows per farm varied between 260 and 1345. Data on feed quantities were collected from each farm. Silage maize and grain maize in concentrated feed was modelled based on $\mathrm{kg}$ input and published inventories [8]. The quantity of hay, pasture grass and kikuyu silage was included based on the production area on each farm. Ryegrass was irrigated on all farms, whereas kikuyu grass was cultivated under rainfed conditions. For silage and grain maize, the share of irrigation was based on the average share in South Africa from 2006 to 2013 [17].

\section{Addressing enteric fermentation (B)}

Enteric fermentation was responsible for about $20 \%$ of the worldwide greenhouse gas emissions from agriculture, forestry and other land use (AFOLU) from 2000 to 2010, of which cattle contributed the largest share $(75 \%)$. The enteric emissions increased most in Africa during this period (by $2.4 \%$ per year) [1].

Due to the importance of bovine enteric methane emissions, research has been carried out to determine means of reduction. Feed supplements have been found to achieve a significant reduction in methane emissions from enteric fermentation. The effect of administering the methane inhibitor 3-nitrooxypropanol (3NOP) was analysed during a 12-week experiment in Pennsylvania (US): 48 Holstein cows were fed $60-80 \mathrm{mg}$ of $3 \mathrm{NOP}$ per $\mathrm{kg}$ dry feed [18]. The feed consisted mainly of maize silage $(42.2 \%)$ and alfalfa haylage $(18 \%)$. The rumen methane emissions were measured five times during this period. An average reduction of $30 \%$ in rumen methane emissions was observed for the cows that were fed with $3 \mathrm{NOP}$, while the milk yield was not affected.

To estimate the effect of the methane inhibitor $3 \mathrm{NOP}$ on the environmental impact on South African milk, 3NOP supplementation was modelled for one farm with 
Table 1 Input per litre of milk at Farm 5 with feed additive 3NOP

\begin{tabular}{l|l|l|l}
\hline Type of flow & Dataset & Origin & $\mathrm{mg} / \mathrm{kg}$ raw milk \\
\hline Input & Chemical, organic (3NOP) & Global & 47 \\
\hline Input & Silica sand & Global & 265 \\
\hline Input & Propylene glycol, liquid & Global & 265 \\
\hline Output & Hydrogen emissions & - & 25 \\
\hline
\end{tabular}

Holstein cows (Farm 5). For the model, the average methane reduction achieved using 60 and $80 \mathrm{mg}$ 3NOP per kg dry feed in the experiment was 30\% [18] was used.

The original input data of Farm 5 and modelled data for the theoretical administration of 3 NOP were evaluated. The 3NOP supplement contains $8.85 \%$ 3NOP mixed with silicon dioxide and propylene glycol [18]. The share of silicon dioxide and propylene glycol was assumed to be $50 \%$ each. The quantity of 3 NOP added to cow feed was calculated per litre of milk: based on the average concentration of 3 NOP used in the experiment $(70 \mathrm{mg} / \mathrm{kg}$ dry feed) and the total feed (the sum of maize and concentrated feed) per kilogram of milk. The input of 3NOP was modelled as the production of organic chemicals. Emissions of hydrogen increased 64 fold due to the treatment [18] and this was included in the model (see Table 1). The emission of hydrogen is not environmentally relevant and thus not assessed by any impact assessment method used in this study.

\section{Addressing animal feed production (C)}

Direct emissions play a major role in the greenhouse gas emissions of raw milk. All other impact categories are dominated by the production of concentrated feed and the housing system [9]. Figure 2 shows the contribution analysis of milk from a South African farm for the six impact categories.

Feed (i.e. silage maize, concentrated feed and grass) is responsible for 15-24\% of the climate impact of milk. In all other categories considered, feed dominates the impact (see Fig. 2). Measures reducing environmental impacts from the production of animal feed are therefore a way to decrease the life cycle impact of milk.

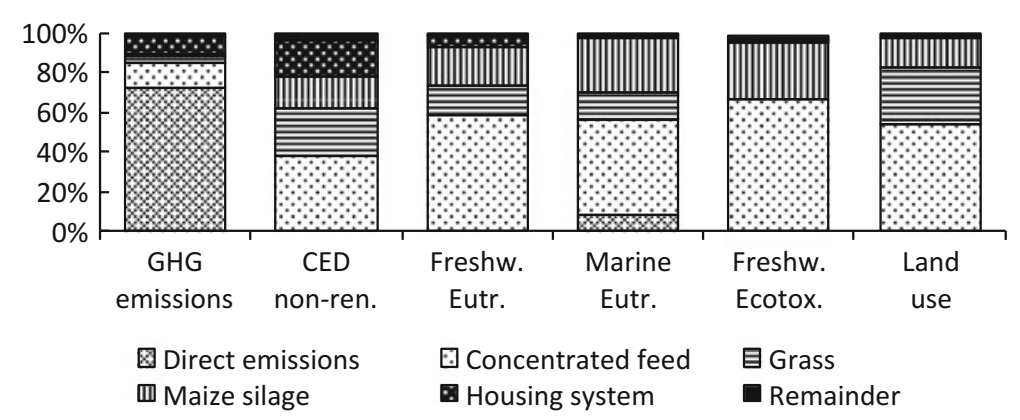

Fig. 2 Contribution analysis of raw milk from Holstein cows at a farm in KwaZulu-Natal analysed in our study (Farm 2) for six impact indicators 
In this study, two cleantech measures in the production of cow-feed were considered: the reduction of electricity demand by integrating a variable frequency drive for the electric motor of a centre pivot irrigation system ("VFD") and the use of solar electricity for irrigation ("solar").

To assess the influence of the cleantech on the environmental impact of milk, it was assumed that cleantech is used in all irrigated feed production, i.e. silage maize, grain maize (in concentrated feed) and grass irrigated on the farms. For purchased feed, the share of irrigated feed on total is based on the South African average share between 2006 and 2013 [17].

Electric motors are most efficient when they are running at their maximum capacity. Current irrigation systems running at their maximum capacity, regardless of the current water demand, use more electricity than needed for irrigation. Variable speed drives allow the regulation of speed and rotational force - or torque output — of the motor in accordance with the actual demand. The centrifugal pump on irrigation systems has particularly high potential to save power. A subcategory of variable speed drives are variable frequency drives: they combine a converter and an inverter with a control unit in between to allow adjustment of the frequency, which changes the speed of the motor. A $30 \%$ reduction in electricity use of the water pump [19] was used for this model.

Photovoltaic electricity for irrigation in South Africa was modelled based on a $570 \mathrm{kWp}$ open ground multi-crystalline silicon power plant in the ecoinvent database [11, p. 3]. The city Welkom, lying in the main maize production region (Northwest and Free State), was used for the estimation of the photovoltaic yield. An annual photovoltaic yield of $1770 \mathrm{kWh} / \mathrm{kWp}$ is expected for that city according to PVGIS of the Joint Research Centre [20]. For the calculations, a lifetime of 30 years for the photovoltaic modules was used, with a yield degradation of $0.7 \%$ per year. This corresponds to an average loss of $10 \%$ of the yield per year $(1593 \mathrm{kWh} / \mathrm{kWp}$, including degradation). These assumptions correspond to the recommendations of the IEA [21]. The module $\left(22.1 \mathrm{~m}^{2}\right)$ has an efficiency of $13.6 \%$ (module area of $7.4 \mathrm{~m}^{2} / \mathrm{kWp}$ ), resulting in an annual yield of $216 \mathrm{kWh} / \mathrm{m}^{2}$ of module. It was assumed that the total electricity demand for irrigation was met using solar electricity, replacing grid electricity from South Africa.

\section{Life Cycle Impact Assessment}

\section{Different breeds (A)}

The greenhouse gas emissions associated with the production of raw milk on the five farms in $\mathrm{KZN}$ varied between 1.2 and $2.0 \mathrm{~kg} \mathrm{CO}$-eq $/ \mathrm{kg}$. A study on milk from Western Cape supports these findings, with $1.0-1.6 \mathrm{~kg} \mathrm{CO}_{2}$-eq/kg raw milk [5]. The production mix for raw milk had a carbon footprint of $1.5 \mathrm{~kg} \mathrm{CO}_{2}-\mathrm{eq} / \mathrm{kg}$. 
Direct emissions (methane and dinitrogen monoxide) accounted for $67-71 \%$ of the overall greenhouse gas emissions.

On Farms 2 and 4, where both Ayrshire and Holstein cows are kept, the climate impact is lower for milk from Holstein cows (see Fig. 3). This is mainly a result of the higher milk yields of Holstein cows [22]. However, the variability between farms was greater than that between breeds. High variability is common in agriculture [23]. In addition to the yield, feed amount and type and longevity of the cows are crucial factors influencing the results.

Ayrshire milk is sold at a higher price and has a higher fat content than standard milk. The comparison of the two types of milk according to price (South African rand, ZAR) and fat and protein corrected milk (FPCM) does not alter the results: the milk from Holstein cows still had a lower impact on climate change than that from Ayrshire cows.

In other impact categories, milk from Ayrshire cows also had a higher impact than the milk from Holstein cows from the same farm (see Fig. 4). Here too, the differences between farms overshadow the differences between the two breeds.

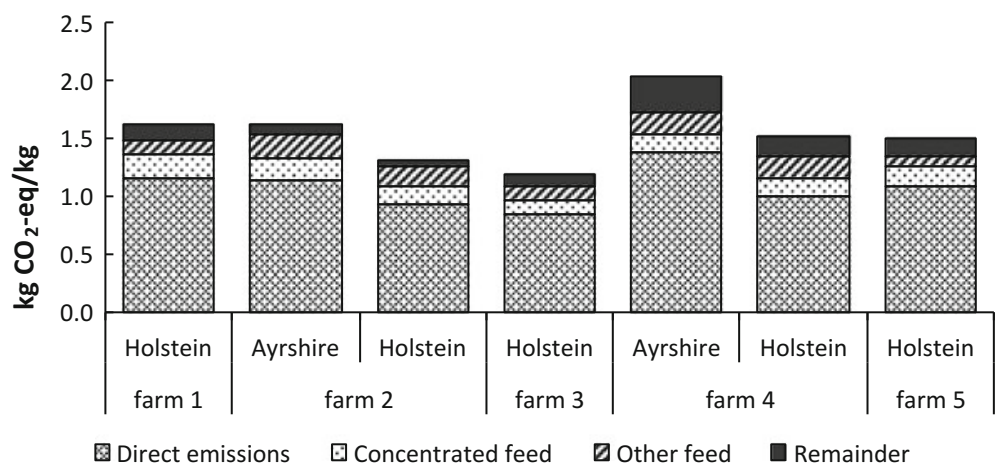

Fig. 3 Greenhouse gas emissions per kilogram of raw milk at five different farms, by breed

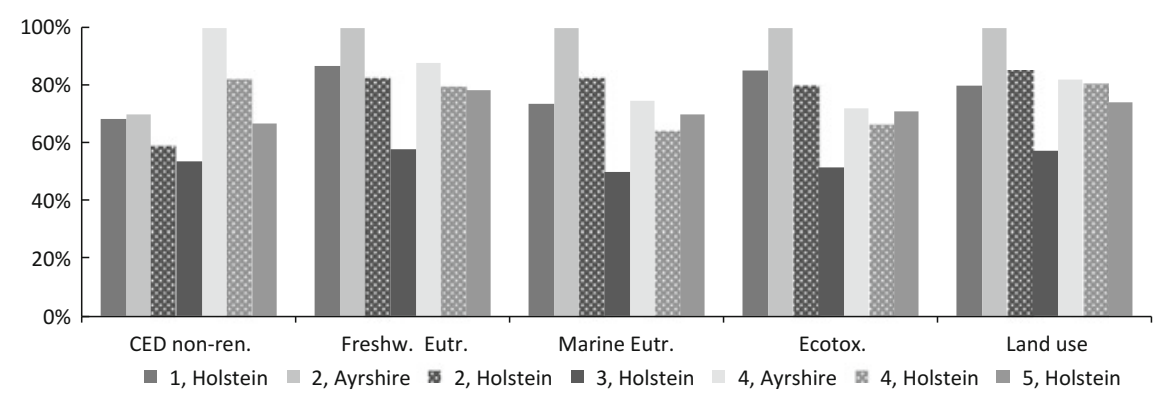

Fig. 4 Comparison of the environmental impact of raw milk at five farms per kilogram milk 


\section{Addressing enteric fermentation (B)}

Adding the methane inhibitor 3 NOP to feed led to an $18 \%$ reduction in the life cycle greenhouse gas emissions of milk, with only very small changes in the other impact categories (see Fig. 5).

These predicted reductions took the direct emissions of cows into account but not potential change in emissions from the manure. Long-term observations are necessary to rule out potential negative effects on the animals, the milk and the meat produced: the possible accumulation of hydrogen in the rumen, the potential nitrite toxicity and adaptation of the animals to the supplements have to be considered [24]. If there is no change in the emissions from the manure and if there are no negative effects on the cows' health, the administration of 3 NOP can be recommended to decrease the environmental impact of milk production.

\section{Addressing animal feed production (C)}

Figure 3 shows that between 8 and $13 \%$ of the climate impact of raw milk can be attributed to concentrated feed and 6-15\% to other feed such as grass, silage or milk powder. The sum of silage, grass and concentrated feed is responsible for 15-24\% of the climate impact.

Using cleantech in the irrigation of feed led to a reduction in the environmental impact of milk in the categories greenhouse gas emissions, non-renewable cumulative energy demand, freshwater eutrophication and freshwater ecotoxicity. No change was observed for the categories land use and marine eutrophication (see Fig. 6). The replacement of the electricity mix with solar electricity ("solar") leads to a larger reduction in environmental impacts than the implementation of a variable frequency drive ("VFD”).

The highest reduction occurred for the non-renewable cumulative energy demand, where a reduction of up to $4 \%$ (VFD) and $11 \%$ (solar) compared with average milk was reached. For freshwater eutrophication, an average reduction of $3 \%$ (VFD) and 10\% (solar) was achieved. Depending on the share of irrigated feed and electricity use per farm, the reduction for individual farms differed, ranging from 2 to $4 \%$ (VFD) and 6 to 14\% (solar) for freshwater eutrophication.

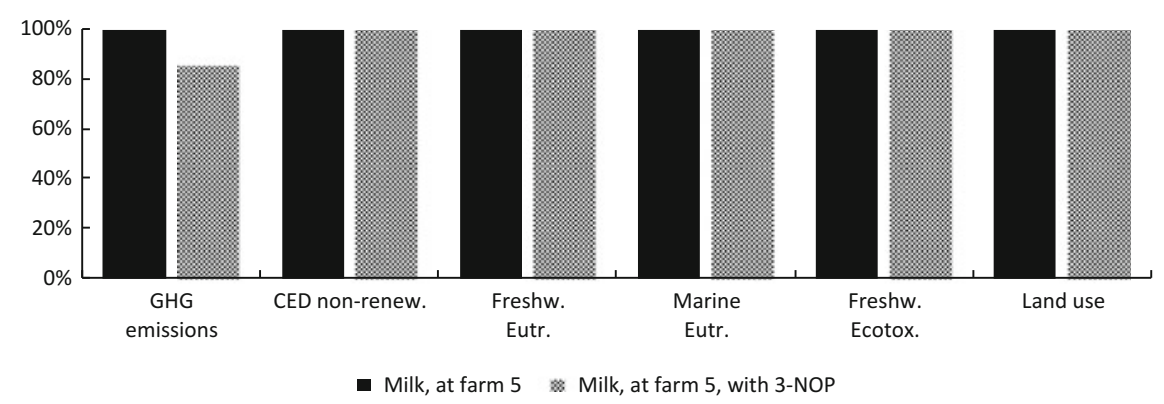

Fig. 5 Impact of feeding 3NOP to cows, calculated using Farm 5 as an example (South African farm with Holstein cows) 


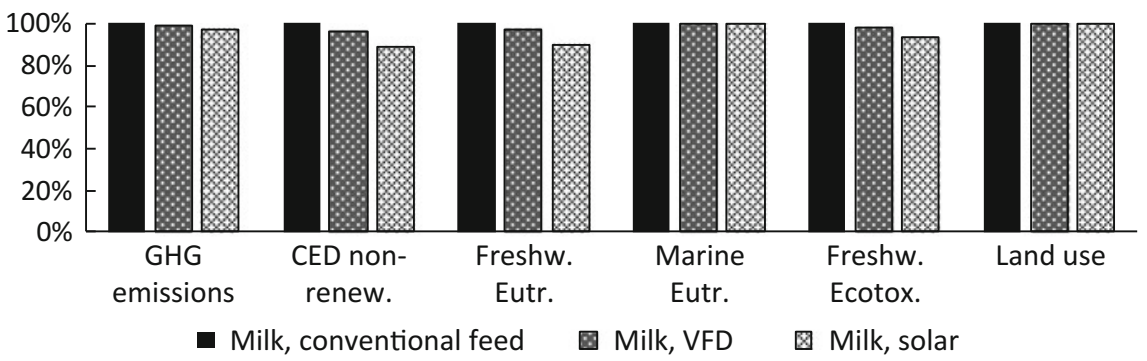

Fig. 6 Influence of cleantech in the irrigation of feed on the environmental impact of milk

Theoretically, a reduction in global warming potential of $34 \%$ is possible if grid electricity is replaced with photovoltaic-generated electricity [8]. Due to the high share of coal (92\%) in South Africa's electricity production [25], greenhouse gas emissions can effectively be decreased by reducing grid electricity demand. Since methane emissions of cows are responsible for the largest share of the global warming potential, the indirect effect of cleantech on the global warming potential of milk remained small.

\section{Conclusions}

On the five farms analysed, milk from Holstein cows had a lower impact than milk from Ayrshire cows. However, the influence of farm management was more relevant than the choice of breed. For climate change, enteric emissions are responsible for the highest share of impact. Therefore, the reduction of direct emissions of cows can generally be recommended. However, only the impact of one environmental category is reduced and the long-term safety of 3NOP has not yet been demonstrated. Reducing impacts associated with feed production may be a more suitable approach, as it dominates the other impact categories. Addressing animal feed production with the use of a variable speed drive or the production of solar electricity decreased the impact in three of the six impact categories considered while there was no trade-off in the other categories. These can therefore be recommended. Using solar electricity for irrigation reduced the impact to a higher degree than the integration of a VFD and is therefore more effective.

In view of the importance of agriculture for sustainable development and the rise in production and consumption of animal products in South Africa, early implementation of cleantech could potentially have a considerable influence on the state of the environment both in South Africa as well as worldwide.

Many measures are available that decrease the environmental impact of milk and different means should be combined to reach the goal of environmentally sound production. Both the cost of implementation of these technologies and potential monetary benefits for the farms, i.e. arising from the reduction of grid electricity 
consumption, have to be analysed to evaluate the financial sustainability of these measures. Since the lack of acceptance from consumers or farmers could be a potential barrier to the implementation of improvement strategies, the acceptance of these technologies also has to be investigated. Finally, the analysis of social effects would complete the sustainability evaluation of these measures.

\section{References}

1. IPCC, Climate Change 2013: The physical science basis. Contribution of working group I to the fifth assessment report of the Intergovernmental Panel on Climate Change, Cambridge University Press, 2013.

2. Steinfeld H, Gerber P, Wassenaar T, Castel V, Rosales M, Haan C, Livestock's long shadow environmental issues and options, FAO, Rome, 2006.

3. DAFF, A profile of the South African dairy market value chain 2014, Department of Agriculture, Forestry and Fisheries, 2015.

4. Harding G, Courtney C, Russo V, When geography matters. A location-adjusted blue water footprint of commercial beef in South Africa, Journal of Cleaner Production, 2017, Vol. 151, pp. 494-508.

5. Notten P, Mason-Jones K, Life cycle assessment of milk production in the Western Cape, 2011.

6. The World Bank, World development report 2008—overview, Washington D.C., USA, 2007.

7. Scharfy D, Boccali N, Stucki M, Clean technologies in agriculture-how to prioritise measures?, Sustainability, 2017, Vol. 9, No. 8, p. 1303.

8. Wettstein S, Muir K, Scharfy D, Stucki, M, The environmental mitigation potential of photovoltaic-powered irrigation in the production of South African maize, Manuscript submitted for publication, 2017.

9. Eymann L, Stucki M, Schwab S, Wettstein S, Environmental impact of South African milk: A comparison of Ayrshire and Holstein breeds, in 10th International conference on Life Cycle Assessment of Food, University College Dublin, Ireland, 2016, pp. 99-105.

10. IDF, A common carbon footprint approach for the dairy sector. The IDF guide to standard life cycle assessment methodology, International Dairy Federation, 2015.

11. Ecoinvent Centre, ecoinvent data v3.3, Swiss Centre for Life Cycle Inventories, Zürich, 2016.

12. Hischier R, Weidema B, Althaus H, Bauer C, Frischknecht R, Doka G, Dones R, Hellweg S, Humbert S, Jungbluth N, Köllner T, Loerinck Y, Margni M, Nemecek T, Implementation of life cycle impact assessment methods, Swiss Centre for Life Cycle Inventories, Dübendorf, $\mathrm{CH}, 2010$.

13. Goedkoop M, Heijungs R, Huijbregts M.A.J, De Schryver A, Struijs J, van Zelm R, ReCiPe 2008-A life cycle impact assessment method which comprises harmonised category indicators at the midpoint and the endpoint level. First edition. Report I: Characterisation, NL, 2013.

14. Rosenbaum R.K, Bachmann T.M, Gold L.S. Huijbregts M.A.J, Jolliet O, Juraske R, Koehler A, Larsen H.F, MacLeod M, Margni M, McKone T.E, Payet J, Schuhmacher M, van de Meent D, Hauschild M.Z, USEtox - the UNEP-SETAC toxicity model: recommended characterisation factors for human toxicity and freshwater ecotoxicity in life cycle assessment, International Journal of Life Cycle Assessment, 2008, Vol. 13, No. 7, pp. 532-546.

15. Frischknecht R, Büsser Knöpfel S, Swiss Eco-Factors 2013 according to the Ecological Scarcity Method. Methodological fundamentals and their application in Switzerland, Federal Office for the Environment, Berne, 2013.

16. IPCC, IPCC Good Practice Guidance and Uncertainty Management in National Greenhouse Gas Inventories-Chapter 4 Agriculture, 2006. 
17. Grain SA, Operational planning of white maize grain production in the production year 2011/ 2012 in Eastern Highveld, Northwest, and Central and Northern Free State, South Africa; Maize production in South Africa from 2006 to 2013, 2014.

18. Hristov A.N, Oh J, Giallongo F, Frederick T.W, Harper M.T, Weeks H.L, Branco A.F, Moate P.J, Deighton M.H, Williams S.R.O, Kindermann M, Duval S, An inhibitor persistently decreased enteric methane emission from dairy cows with no negative effect on milk production, Proceedings of the National Academy of Sciences, 2015, Vol. 112, No. 34, pp. 10663-10668.

19. Eskom, Variable Speed Drives: Reducing the energy cost of irrigation on farms, Eskom Integrated Demand Management, 2015.

20. European Commission, Photovoltaic Geographical Information System (PVGIS) - geographical assessment of solar resource and performance of photovoltaic technology, 2012.

21. Fthenakis V, Frischknecht R, Raugei M, Chul K.H, Alsema E, Held M, Scholten M.W, Methodology guidelines on life cycle assessment of photovoltaic electricity, Subtask 20 "LCA", IEA PVPS Task 12, 2011.

22. Moore R.K, Kennedy B.W, Schaeffer L.R, Moxley J.E, Relationships between age and body weight at calving and production in first lactation Ayrshires and Holsteins, Journal of Dairy Science, 1991, Vol. 74, No. 1, pp. 269-278.

23. Hersener J-L, Baumgartner D.U, Dux D, Zentrale Auswertung von Ökobilanzen landwirtschaftlicher Betriebe (ZA-ÖB)., Agroscope, Forschungsanstalt Agroscope ReckenholzTänikon ART, Zürich/Ettenhausen, 2011.

24. Hristov A.N, Oh J, Firkins J.L, Dijkstra J, Kebreab E, Waghorn G, Makkar H.P.S, Adesogan A.T, Yang W, Lee C, Special topics-Mitigation of methane and nitrous oxide emissions from animal operations: I. A review of enteric methane mitigation options, Journal of Animal Science, 2013, Vol. 91, No. 11, pp. 5045-5069.

25. International Energy Agency, South Africa: electricity and heat for 2014, 2017, https://www. iea.org/statistics/statisticssearch/report/?country=SouthAfric\&product=electricityandheat, (Accessed 25.05.2017).

Open Access This chapter is licensed under the terms of the Creative Commons Attribution 4.0 International License (http://creativecommons.org/licenses/by/4.0/), which permits use, sharing, adaptation, distribution and reproduction in any medium or format, as long as you give appropriate credit to the original author(s) and the source, provide a link to the Creative Commons license and indicate if changes were made.

The images or other third party material in this chapter are included in the chapter's Creative Commons license, unless indicated otherwise in a credit line to the material. If material is not included in the chapter's Creative Commons license and your intended use is not permitted by statutory regulation or exceeds the permitted use, you will need to obtain permission directly from the copyright holder.

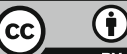




\title{
Food Waste Management (Sector) in a Circular Economy
}

\author{
Nicole Unger and Francesco Razza
}

\begin{abstract}
The presentations addressed the topic of food waste management in a circular economy from various angles. Understanding the amount of food waste generated and setting measures to prevent it should be the starting point. Any not prevented food waste should be valorised aiming at a positive triple bottom line (social, economic and environmental). The huge environmental and economic potential of co-digestion of dairy manure and food wastes by linking waste producers with costumers for derived products was demonstrated for the US. Further the link between bio-waste, compost and soil quality is highlighted which contributes to food security and the wider bio-economy. Looking forward research into the application of selected agri-food wastes to produce alternative sources of protein could result in more sustainable source of proteins able to compete with industrial chicken production. Overall, the presentations and discussions showed the multi-facetted nature of food waste management emphasising that a range of measures by many stakeholders are required to move towards a circular economy society.
\end{abstract}

\section{Introduction}

The Sustainable Development Goal 12.3, focuses on the food waste sector and sets the target to halve the per capita global food waste at the retail and consumer level by 2030 and to reduce food losses along production and supply chains, including post-harvest losses. The European circular economy package [1] explicitly aligns to this target and made food waste one of its priority areas. In the same document, the

\footnotetext{
N. Unger $(\bowtie)$

University of Natural Resources and Life Sciences,

Institute of Waste Management, Vienna, Muthgasse 107,

1190 Vienna, Austria

e-mail: nicole.unger@boku.ac.at

F. Razza

Novamont-Ecology of Product and Environmental Communication,

Piazz.le Donegani 4, 05100 Terni, Italy

(C) The Author(s) 2018

E. Benetto et al. (eds.), Designing Sustainable Technologies,

Products and Policies, https://doi.org/10.1007/978-3-319-66981-6_15
} 
EU focuses on boosting the market for secondary raw materials, which currently only account for a small proportion of materials used in the EU. With these ambitious targets, the need for science based guidance is eminent. Any approach tackling food waste needs to align to sustainable development, making environmental, social and economic sense. The aim of this session is to explore the role of waste management (sector) as industry and society move towards a circular economy model.

Four oral presentations by speakers from four countries addressed the session topic from different angles. Two presentations, by Sergiy Smetana and Francesco Razza have full papers accompanying them that are also part of this book.

\section{Food Waste Generation in Europe}

The management of food waste starts with understanding how much food waste is currently produced and what share of it can be prevented. Silvia Scherhaufer presented in this context outcomes of the EU FP7 funded project FUSIONS (http:// www.eu-fusions.org/) where this was evaluated on a European level.

Food waste and other side flows from the food supply chain include food and other, non-edible parts occur at every step of the supply chain. In total 88 Mio tonnes of food waste is generated in Europe per year, the majority (about 53\%) occurring during food preparation and consumption at home. Based on these figures, 31 Mio tonnes of food wastes would need to be reduced each year in order to meet the United Nations' Sustainable Development goal by 2030.

Silvia Scherhaufer then further expanded the procedure developed to estimate the impacts of the food supply chain and impacts which can be related to food waste including food waste management [2]. For example, in order to provide $1 \mathrm{~kg}$ of apples to consumers, $1.28 \mathrm{~kg}$ need to be produced (Fig. 1). Along the supply chain, those $0.28 \mathrm{~kg}$ of edible and inedible parts of apples are removed for various reasons. Environmental impacts related to these $0.28 \mathrm{~kg}$ of food waste occur during production, processing, retail and distribution, consumer activities (e.g. cooking, storing) as well as food disposal (e.g. composting, waste incineration).

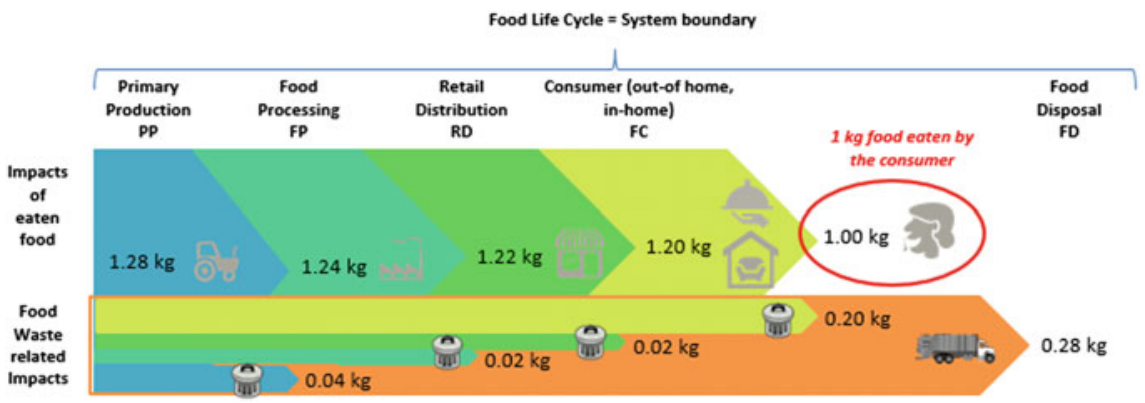

Fig. 1 Stakeholder interactions upstream and downstream of digesters [2] 
The later in the supply chain food is wasted the more environmental impacts are associated with this food waste. Food waste reduction by prevention would therefore avoid those impacts.

Concluding the presentation, she emphasised that taking measures to reduce food waste at the consumer level is key, also for industry. Governments have an important role to play in providing transparency on legal options for food redistribution. By preventing food waste at the consumer level, around 26 Mio tonnes of food can be saved from being wasted (assuming that $57 \%$ of food waste is avoidable). This would result in a reduction potential of 69 Mio tonnes $\mathrm{CO}_{2}$ eq. (corresponding to the level of Finland's total greenhouse gas emissions).

\section{Business Opportunity Through Co-digestion of Dairy Manure in the US}

Methane emissions from food waste landfill and dairy manure anaerobic lagoons are causing significant greenhouse gas emissions (GHG). This case study, presented by Ying Wang, shows how the introduction of a sustainable dairy anaerobic digester system can have co-benefits across the supply chain by turning waste into useful products through industry symbiosis. In this case study, Magic dirt, a trademark for digester-derived nutrient fibre, grew its business from 25 stores in 1 state to 2800 stores in 41 states from 2014 to 2017.

The success of the business model, including complex stakeholder interactions (Fig. 2), depends on multiple factors: (1) Dairy association set up sector GHG reduction targets that incentivises big food companies to "insetting" carbon emissions along their own supply chain; (2) third party investors who construct and operate anaerobic digesters take financial risks away from dairy farmers; (3) secured substrate supply agreements with manure providers and food waste producers, such as commercial, retail and processing sites, and also "off-take agreements" that secure future contracts of selling produced energy to utility companies, nutrient

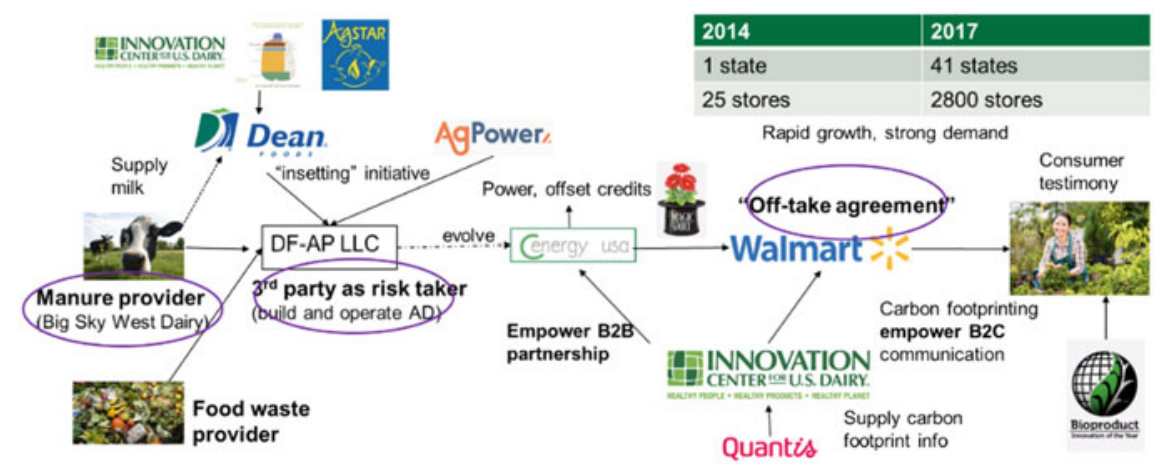

Fig. 2 Stakeholder interactions upstream and downstream of digesters 
fibres to retailers such as Walmart, as well as produced nitrogen and phosphorus to offset commercial fertilisers. Carbon reductions can be also sold as offsetting credits. In this business model, dairy farms and food companies achieved GHG reduction targets and investors profit by converting waste into product, which meet green supplier criteria. Target incentives, collaboration along supply chain, and a business model that balances business risk and opportunities largely contributed to the success. The AgSTAR project of the U.S. EPA [3] analysed the possibility of installing anaerobic digesters with energy generation and nutrient capture in confined animal feeding operations with 500+ cows in the US, suggesting 2647 digesters to be installed nationwide. By implementing the proposed business model to dairy operations and 3rd party digester companies, it could potentially generate 12 Mio MWh of electricity, and also 42 Mio metric tons of $\mathrm{CO}_{2}$ offsetting credits annually, mainly driven by reducing methane emissions from landfilling food waste and manure lagoons, and also avoiding grid electricity production.

\section{The Role of Composting in a Circular Economy}

A decline in soil organic matter (SOM) can be observed in Europe. It is one of the most important indicators for soil quality which hugely affects the agricultural sector and bio-economy. The sustainable management of SOM is an explicit requirement of several international standards and programmes e.g. the EC 16752 Bio-based product sustainability criteria. Food waste and garden waste are common feedstocks for the production of compost. The waste material is collected and biologically treated. The produced compost contains nutrients and humic substances which directly contribute to increase the SOM pool in the soil. Applying compost to agricultural land contributes to improved soil quality and thus bio-economy. Compost use also affects the GHG balance of crops since 58\% of SOM is carbon.

Francesco Razza reported on a case study where compost is applied in a cardoon cropping system in North-East Sardinia. The crop system consists of a rotation of 6 years of cardoon, 1 year of durum wheat and 1 year of field bean, analysed over a time frame of 22 years. One 'compost' scenario, where compost is applied during the 1 st and the 4th year of cardoon is compared to a 'no compost' scenario, where the only organic matter contribution was from above-ground biomass left in the soil after harvest. The trials showed clearly higher levels in SOM increasing steadily over the time frame of 22 years (carbon stock changes are generally calculated over a time frame of 20 years). A GHG balance of the above crop system also showed benefits for the application of compost: $\mathrm{CO}_{2}$ uptakes linked to the increase of SOM in soil have the same order of magnitude as the overall cardoon "Cradle to gate" GHG emissions. Consequently, taking into account SOM dynamics compost scenario shows a reduction of its carbon footprint of $70 \%$ compared to 'no compost' scenario.

Putting this research into the bigger European context shows that only about 1/3 of municipal bio-waste is collected and recycled into high quality compost and digestate while demand for compost clearly outstrips availability. A better 
acknowledgement of the link between bio-waste management, compost quality and the sustainable biomass production required for food security and the wider bio-economy is crucial.

\section{Agri-food Waste Streams Utilisation for Food Substitutes Development}

Food production and consumption systems are changing. This reflects not only on the nutritional need, but also on the popularity of diets such as vegan or flexitarian. Substitution of highly nutritional foods with analogs is gaining momentum. The research presented by Sergiy Smetana focused on the valorisation of agri-food waste streams for food substitutes design. A LCA approach (cradle to plate) was used to assess the environmental impact of different meat substitutes. It showed that depending on the impact or indicator (greenhouse gases, energy, land use or water use) different substitutes show advantages and disadvantages. The comparison was done per weight unit but it needs to be pointed out that different meat substitutes do not have the same nutritional profile. Further his research analysed the potential of waste-to-food application and Technology Readiness Level (TRL) for various biomass sources (e.g. insects, microalgae) in comparison against conventional animal derived products (e.g. chicken, whey concentrate) $[4,5]$.

Overall it was concluded that, traditional food substitution produced with alternative biomass sources is a necessity to design a more sustainable transition to the new food system. For those considerations of TRL, nutritional profile and environmental impact are important to better understand their potential application in the future. State-of-the-art production of meat analogs based on milk, mycoproteins, insects and microalgae biomass are currently not competitive in terms of environmental impact to the benchmark meat (chicken in this case). However, there is a high positive potential of agri-food waste and side streams (molasses, distilled grains, grain brans) application for insect and single cell protein products as food substitutes. The application of agri-food waste streams is complicated but possible for the cultured media production. Application of selected agri-food wastes to produce alternative sources of protein could result in more sustainable source of proteins able to compete with industrial chicken production.

\section{Discussion and Conclusions}

The presentations addressed the topic of food waste management in a circular economy from various angles. Prevention of food waste will be key to meet the UN's Sustainable development goals. Any food waste not prevented should be valorised in a manner such that there is a clear business case, aligning food waste supply with demand for the valorised products, as presented for the US by use of co-digestion. 
The role food waste can play to help mitigate agricultural soil quality losses is maybe not yet widely enough discussed but it is a key to ensure future food supply and input into the bio-economy. Lastly, using food waste as input into food substitute production is a promising technology for the future allowing to reduce pressures on the environment caused by food production. Overall, the presentations and discussions showed the multi-facetted nature of food waste management highlighting that a range of measures by many stakeholders are required to move towards a circular economy society. All of these will require changes in society, in the way we produce and consume food and how we best utilise generated side flows.

Acknowledgements This session was proposed based on work carried out in REFRESH project: 'Resource Efficient Food and Drink for Entire Supply Chain'. REFRESH is funded by the Horizon 2020 Framework Programme of the European Union under Grant Agreement no. 641933 (http:// www.eu-refresh.org).

Moreover, we want to express our honour and gratitude to His Royal Highness Prince Louis of Luxembourg who attended and contributed to this session.

\section{References}

1. European Commission, Closing loop-An EU action plan Circular Economy, Communication from the Commission to the European parliament, the Council, the European economic and social committee and the committee of the regions, C.O.M. 0614, 2015.

2. Scherhaufer S, Moates G, Hartikainen H, Waldron K, Obersteiner G, Environmental impacts of food waste in Europe. Journal of Waste Management. Submitted.

3. U.S. Environmental Protection Agency (USEPA), Market Opportunities for STAR Biogas Recovery Systems at U.S. Livestock Facilities, 2011.

4. Smetana S, Palanisamy M, Mathys A, Heinz V, Sustainability of insect use for feed and food: life cycle assessment perspective, Journal of Cleaner Production, 2016, 137, 741-751.

5. Smetana S, Sandmann M, Rohn S, Pleissner D, Heinz V, Autotrophic and heterotrophic microalgae and cyanobacteria cultivation for food and feed: Life Cycle Assessment. Bioresource Technology, 2017, in press.

Open Access This chapter is licensed under the terms of the Creative Commons Attribution 4.0 International License (http://creativecommons.org/licenses/by/4.0/), which permits use, sharing, adaptation, distribution and reproduction in any medium or format, as long as you give appropriate credit to the original author(s) and the source, provide a link to the Creative Commons license and indicate if changes were made.

The images or other third party material in this chapter are included in the chapter's Creative Commons license, unless indicated otherwise in a credit line to the material. If material is not included in the chapter's Creative Commons license and your intended use is not permitted by statutory regulation or exceeds the permitted use, you will need to obtain permission directly from the copyright holder. 


\title{
The Role of Compost in Bio-waste Management and Circular Economy
}

\author{
Francesco Razza, Lorenzo D'Avino, Giovanni L'Abate \\ and Luca Lazzeri
}

\begin{abstract}
The increase of separate collections of bio-waste, largely represented by food waste, and their biological treatment is an essential feature of the waste management strategy. The aim of this paper was to highlight the role of compost in the circular economy, and its use in the agricultural sector. An annual time-step model for estimating soil organic matter (SOM) stock dynamics in a 22-year time frame was developed and tested on cardoon cropping system. The model took into account few soil parameters, mean annual temperature, and the cultural systems management, in particular organic fertilizers and crop residues. This work indicates that compost use in agriculture would be beneficial both for SOM increase and GHG reduction. The results showed how high-quality compost could represents the actual driving force of this change able to connect food, waste, economy and environment.
\end{abstract}

\section{Introduction}

In the EU between 118 and 138 million tonnes of bio-waste are produced every year, of which about 88 million tonnes come from municipal waste [1] which corresponds at about $170 \mathrm{~kg}$ of bio-waste per capita per year and about $150 \mathrm{~kg}$ per capita per year of realistic potentials [2]. Of the overall bio-waste amount, only $25 \%$ (i.e. 30 million tonnes per year) is recycled into digestate or high-quality compost [3]. The latter must

\footnotetext{
F. Razza ( $\bowtie)$

Novamont S.p.A, 05100 Terni, Italy

e-mail: francesco.razza@novamont.com

L. D’Avino - G. L'Abate

Consiglio per la ricerca in agricoltura e l'analisi dell'economia agraria,

Centro di ricerca Agricoltura e Ambiente (CREA-AA), 50125 Florence, Italy

L. Lazzeri

Consiglio per la ricerca in agricoltura e l'analisi dell'economia agraria,

Centro di ricerca Cerealicoltura e Colture Industriali (CREA-CI), 40128 Bologna, Italy

(C) The Author(s) 2018

E. Benetto et al. (eds.), Designing Sustainable Technologies,

Products and Policies, https://doi.org/10.1007/978-3-319-66981-6_16
} 
meet several requirements like: allowable heavy metals contents, absence of pathogenic bacteriology and phytopathogens, absence of plastic materials $>10 \mathrm{~mm}$ etc.

Composting predominates over anaerobic digestion for the bio-waste separately collected, resulting over $90 \%$ of food and garden waste being processed into compost. For the most part, food waste is still landfilled within Europe, leading to the release of uncontrolled greenhouse gases [4].

The Circular Economy Package, published by the EU Commission in December 2015, paved the way for a resource-efficient society and sustainable recycling industry across Europe and it contains also proposals addressing the EU waste legislation with the aim of avoiding, reusing and recycling more waste in the future [4]. Of particular relevance, for bio-waste treatment in Europe, is the proposed changes to the EU Landfill Directive [5] whose aim is to reduce the landfill of municipal waste to $10 \%$ by 2030 . In this ambit food waste fraction plays an important role in recycling and in raising circular economy since up to $50 \%$ of municipal solid waste is biogenic. Therefore, the $10 \%$ landfill target can be only achieved through sustainable bio-waste management, including composting and anaerobic digestion. In this paper, it is addressed the valuable effects of compost use in agriculture on Soil organic matter (SOM) management and GHG emissions balance of an industrial oil crop pointing out its role in the circular economy.

The SOM is primarily composed of carbon (C), and in soil plays a role in providing four important ecosystem services: (i) resistance to soil erosion, (ii) soil water retention, (iii) soil fertility for plants and (iv) soil biodiversity. SOM is therefore the main indicator of soil quality. Even small changes of the soil $\mathrm{C}$ pool could have strong effects both on agricultural yield and on global greenhouse gas cycle. Maintaining organic C-rich soils, restoring and improving degraded agricultural lands and, in general terms, increasing the soil $\mathrm{C}$, could play a fundamental role in addressing food security and in mitigating the anthropogenic GHG emissions [6]. Organic matter (OM) in compost is rich in humifiable and humified materials and so it provides and improve SOM pool and consequently soil fertility. A specific SOM model has been developed and applied within BIT3G Italian project funded by MIUR (Ministry of Education, Universities and Research) as part of the National Technology Cluster of Green Chemistry SPRING with the aim of defining a predictive tool suitable for estimating the site- specific SOM dynamics in function both of pedoclimatic conditions and agricultural practices. Here are reported the experimental results defined on cardoon industrial crop (Cynara cardunculus var Altilis DC) cultivated in the North-West of Sardinia following two agricultural protocols: with and without compost application. In addition, this study shows how compost can help to reach the objective of the ' 4 per 1000' initiative launched at the COP21 that aspires to increase global soil organic matter stocks (SOMS) by $0.4 \%$ per year as a compensation for the global emissions of greenhouse gas (GHG) by anthropogenic sources [7].

The final aim is to point out the valuable role of compost which represents the bridge between bio-waste strategy targets and sustainable agriculture principles as qualitatively described through a virtuous circular economy model reported in the discussion. 


\section{Materials and Methods}

An annual time-step model for estimating SOM dynamics was developed and tested $[8,9]$ in a 22-year time frame. Indeed, $\mathrm{C}$ stock changes are generally calculated in a duration longer than 20 years. The model takes into account the main soil characteristics, annual mean temperature, and management of cropping systems and in particular organic fertilizers and crop residues. The analysed agricultural system consists in a not irrigated 8-years rotation repeated until 22 years: 6-year cardoon, one-year durum wheat (Triticum durum Desf.) one year field bean (Vicia faba cv Minor) and so on.

The model implements the Hénin-Dupuis equation [10-12] which apply two different kinetic constants $\left(\mathrm{k}_{1}\right.$ and $\left.\mathrm{k}_{2}\right)$ on annual step in the above complex cropping system.

$$
\Delta \mathrm{SOM}=\mathrm{k}_{1} * \mathrm{M}-\mathrm{k}_{2} * \mathrm{SOM}
$$

where $\mathrm{M}$ (dry matter, $\mathrm{Mg} / \mathrm{ha}$ ) stands for raw $\mathrm{OM}$, exogenous $\mathrm{OM}$ and/or crop residues, $\mathrm{k}_{1}(\% \mathrm{w} / \mathrm{w})$ as the humification constant (i.e. the organic matter that arrive to become humus) and $\mathrm{k}_{2}(\% \mathrm{w} / \mathrm{w})$ as the mineralisation constant (i.e. how much humus is mineralised in $\mathrm{CO}_{2}$ ). Soil type did not affect significantly the mineralisation behaviour of $\mathrm{M}$ according to Noirot-Cosson et al. [13], rather constant $\mathrm{k}_{1}$ is specific for each $\mathrm{M}$, and it is correlated to specific biological stability index (BSI, \% w/w), calculated after a laboratory measurement of biochemical fractions [14], and $\mathrm{OM}$, calculated as $\mathrm{M}$ without ashes content, by Eq. (2)

$$
\mathrm{k}_{1}=\mathrm{BSI} * \mathrm{OM} / \mathrm{M}
$$

One gross estimate of the mineralisation constant $\mathrm{k}_{2}$ depends on agricultural practices $(\mathrm{P}$, i.e. tillage frequency and depth, irrigation, crop residues and organic fertilizer frequency), clay content $(\mathrm{A}, \mathrm{g} / \mathrm{kg})$, total carbonates $\left(\mathrm{g} / \mathrm{kg} \mathrm{CaCO} \mathrm{Ca}_{3}\right)$, site mean annual air temperature $\left(\mathrm{T},{ }^{\circ} \mathrm{C}\right)$ :

$$
k_{2}=\frac{1200 * 0.2 *(T-5)}{(200+A) *\left(200 * 0.3 * \mathrm{CaCO}_{3}\right)}
$$

In addition, specific residual biomasses, agricultural practices (i.e. tillage frequency and depth, irrigation, crop residues and organic fertilizer frequency), were considered [8]. The SOM stock (SOMS, Mg/ha) in $30 \mathrm{~cm}$ topsoil (h, m) were estimated taking into account soil organic carbon (SOC, \% w/w), bulk density (BD, $\left.\mathrm{Mg} / \mathrm{m}^{3}\right)$, coarse materials $(\mathrm{CM}, \% \mathrm{v} / \mathrm{v})$ :

$$
S O M S=\frac{S O C}{0.58} * B D *(1-C M) * h
$$


Six soils cultivated with cardoon were sampled within $10 \mathrm{~km}$ of Porto Torres area, (Sassari, Sardinia Region, Italy) and assessed for required parameters to make possible the model running (Table 1).

The mineralisation coefficient $\left(\mathrm{k}_{2}\right)$ for a "derived soil" from parameter averages, was calculated according to Castoldi and Bechini [11], considering the lack of irrigation $(\mathrm{I}=1)$. The amount of resistant SOM fraction was estimated according to Boiffin et al. [15] or applying BSI [10,14] on cardoon above-ground residues: wheat 0.08 , bean 0.1 , and roots 0.15 ; above-ground cardoon was 0.18 experimentally estimated from compositional analysis. Even contribution of cardoon basal leaves and renewal of roots was calculated and computed in the model. This issue will be the subject of a specific forthcoming publication.

Cardoon residues were harvested leaving on the ground $10 \%$ of above-ground biomass (w/w as dry matter).

Two scenarios were considered: "compost" and "no compost". Compost scenario planned for $20 \mathrm{Mg} / \mathrm{ha}$ compost application before cardoon sowing and $15 \mathrm{Mg} / \mathrm{ha}$ on the crop during the 4th year of cultivation. Compost incorporation applied dry matter $(\mathrm{dm}) 50 \%(\mathrm{w} / \mathrm{w})$, Nitrogen $(\mathrm{N})$ content $1.8 \%(\mathrm{w} / \mathrm{w} \mathrm{dm})$, organic carbon $48 \%$ (w/w dm), BSI 0.53, $\mathrm{k}_{1} 0.25$ [16]. In "no compost" scenario the contribution of $\mathrm{OM}$ is represented only from the cardoon above-ground biomass (i.e. 10\%) that remains in soil after harvesting.

Cardoon C footprint was estimated using BioGrace tool [17] and IPCC [18] methodology applying 20-year time-horizon to assess global warming potential for $\mathrm{CO}_{2} \mathrm{CH}_{4}$ and $\mathrm{N}_{2} \mathrm{O}$ [19]. In particular following IPCC [18], GHG emissions fixed in the industrial production process of urea was taken into account along with direct and indirect $\mathrm{N}_{2} \mathrm{O}$ emissions due to $\mathrm{N}$-synthetic and organic fertilisers, crop residues

Table 1 Parameters implemented in the model for different soil samples (from 1 to 6) and if their contribution in increasing (+) or containing (-) the SOM stock mineralisation rate, according to the model

\begin{tabular}{l|l|l|l|l|l|l|l|l}
\hline Sample/parameter & $\begin{array}{l}\text { SOMS } \\
\text { correlation }\end{array}$ & 1 & 2 & 3 & 4 & 5 & 6 & $\begin{array}{l}\text { Mean } \pm \text { st. } \\
\text { dev. }\end{array}$ \\
\hline $\begin{array}{l}\text { Mean annual } \\
\text { temperature }\left({ }^{\circ} \mathrm{C}\right)\end{array}$ & + & 16.7 & 16.7 & 16.6 & 16.7 & 16.8 & 16.7 & $16.7 \pm 0.1$ \\
\hline $\begin{array}{l}\text { Soil organic matter }(\% \\
\text { w/w) }\end{array}$ & + & 2.10 & 2.45 & 2.03 & 2.59 & 2.76 & 2.38 & $2.39 \pm 0.31$ \\
\hline Bulk density $\left(\mathrm{g} / \mathrm{cm}^{3}\right)$ & + & 1.32 & 1.28 & 1.31 & 1.26 & 1.26 & 1.26 & $1.28 \pm 0.03$ \\
\hline Coarse materials $(\% \mathrm{v} / \mathrm{v})$ & - & 0.00 & 0.13 & 0.17 & 0.17 & 0.12 & 0.02 & $0.10 \pm 0.07$ \\
\hline Clay $(\mathrm{g} / \mathrm{kg})$ & - & 367 & 517 & 458 & 567 & 400 & 121 & $405 \pm 82$ \\
\hline Carbonates $(\mathrm{g} / \mathrm{kg})$ & - & 163 & 37 & 3 & 79 & 9 & 616 & $151 \pm 66$ \\
\hline
\end{tabular}


and $\mathrm{N}$ mineralisation associated with eventual SOM loss. Agricultural supplies were experimentally estimated for each year of cardoon cultivation and then averaged out; so, for example, the $\mathrm{CO}_{2}$ equivalents needed for seed production to grow one hectare of cardoon was divided by six.

\section{Results and Discussions}

The model was firstly applied to cardoon cropping system, but it could be applied even to any other crop system (i.e. food and non-food) [8, 9]. Assessed scenarios showed that SOM increased by the supply of exogenous OM rich in humifiable materials, such as high-quality compost. The magnitude of GHG emissions or sequestrations caused by SOM variation depended on soil characteristics (Table 1). The results obtained by the soil model with mean characteristic are reported in Fig. 1. Error bars show the variation due to the soil variability. For both compost and no-compost scenarios the upper SOMS values above the curves set a " $\mathrm{C}$ sink layout", built implementing a "derived soil" having values obtained by mean plus standard deviation of parameters that contain mineralisation and mean minus standard deviation of parameters that increase mineralisation (Eqs. 3 and 4). On the contrary, the lower SOMS values below the curves in Fig. 1 set a "C source layout", built considering the mean plus standard deviation of parameters that increase mineralisation and mean minus standard deviation of parameters that contain mineralisation (see Table 1).

Compost application allows to reach, on average, a $6.2 \mathrm{Mg} / \mathrm{ha}$ organic matter sequestration in 22 years, a value that corresponds to about $160 \mathrm{~kg}$ of $\mathrm{C}$ per year. This SOM improvement will corresponds to reach more or less a SOMS increase of around $0.4 \%$ per year requested by ' 4 per 1000 ' initiative [7]. Instead, in the "no compost" scenario, is expected to deplete $3.9 \mathrm{Mg} / \mathrm{ha}$ SOMS (around $100 \mathrm{~kg} \mathrm{C}$ lost per year).

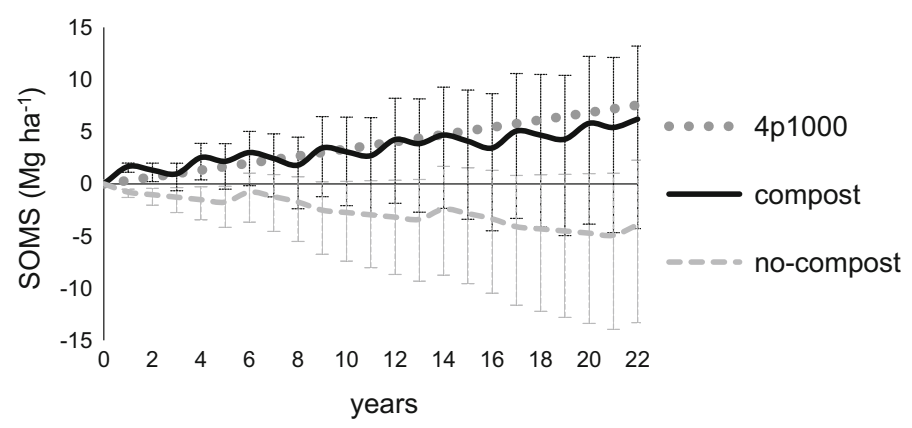

Fig. 1 Soil Organic Matter Stock (SOMS) variation on average soil (out of 6 soil samples) in 22-years cardoon-durum wheat-field bean cropping system simulation. Error bars shows $\mathrm{C}$ sink and source layout obtained by standard deviations of soil parameters. Compost application allows to increase, on average, the SOMS by the $\approx 0.4 \%$ per year 
In addition, the cropping system allows for maintaining a roughly high level of OM, mainly by virtuous release by cardoon roots decay at the end of every production cycle. Nevertheless, in soil with higher mineralisation (e.g. with lower content of clay and/or carbonates), the supply of appropriate compost amounts is necessary to maintain, or at least to minimise, SOMS losses. Indeed, an exogenous SOM supply increases biogenic $\mathrm{C}$ mineralisation, as well as pool of recalcitrant $\mathrm{C}$ in soil [13]. Therefore, compost amounts need to be appropriate also because the supply of excessive amounts of compost, i.e. $30 \mathrm{t} / \mathrm{ha}$ dry matter in Mediterranean condition, declines the $\mathrm{C}$ conversion efficiency [20], and some threats might occur (i.e. metals and excess nutrients into groundwater and increase in soil salinity) depending on compost quality [21].

SOC sequestrations or depletions can be fundamental in greenhouse gases (GHG) crop management as demonstrated by the Life Cycle Assessment focused on cardoon agricultural phase.

The GHG balance of one year for cardoon cultivation is reported in Fig. 2. Among the cardoon cultivation inputs, the main $\mathrm{C}$ footprint were due to the chemical fertilizers (i.e. urea) and diesel according to Cocco et al. [22]. Moreover, the use of compost cut down on $\mathrm{N}_{2} \mathrm{O}$ emissions according to Aguilera et al. [23]. However, the contribution of SOMS dynamic is preponderant. Carbon source and sink from soils play a fundamental role in $\mathrm{C}$ footprint assessment of agricultural phase: the net balance of GHG emissions in the "no compost" scenario accounts for $1710 \mathrm{~kg} \mathrm{CO}$ eq per hectare, whereas the same crop system where compost was applied resulted to be characterised by $490 \mathrm{~kg} \mathrm{CO}_{2}$ eq per hectare, with an over $70 \%$ reduction.

The promoting of a sustainable agriculture has a huge relevance since agricultural sector represents the ground of bio-economy. High quality compost availability, even assuming a notable increase of bio waste recycling (i.e. 60 million of metric tonnes per year of bio waste) would result much lower if compared to potential demand: according to a rough estimation of the authors, the annual amount of compost (potentially) produced would be enough to be used in the 1-3\% of the EU-28 agricultural land. Consequently, SOM management shall be pursued with the aim of innovative agricultural practices (e.g. intercropping, green manure, incorporation of higher amount of biomass in soil etc.). Nevertheless, the authors agree to the EU Landfill Directive proposal that will oblige EU Member States to introduce the separate collection of bio-waste as far as is technically, ecologically and economically feasible. This change, in fact, would activate a series of virtuous mechanisms whose benefits can be summarise as follows:

1. $10 \%$ landfill target achievement and reduction of GHG emissions from inappropriate disposal (i.e. landfill)

2. Increase labour market (i.e. food waste management and composting)

3. Reduction of greenhouse gas emissions from crops cultivation (i.e. $\mathrm{CO}_{2}$ uptake linked to SOM increase, Fig. 2)

4. Sustainable agriculture (i.e. no SOM depletion). 


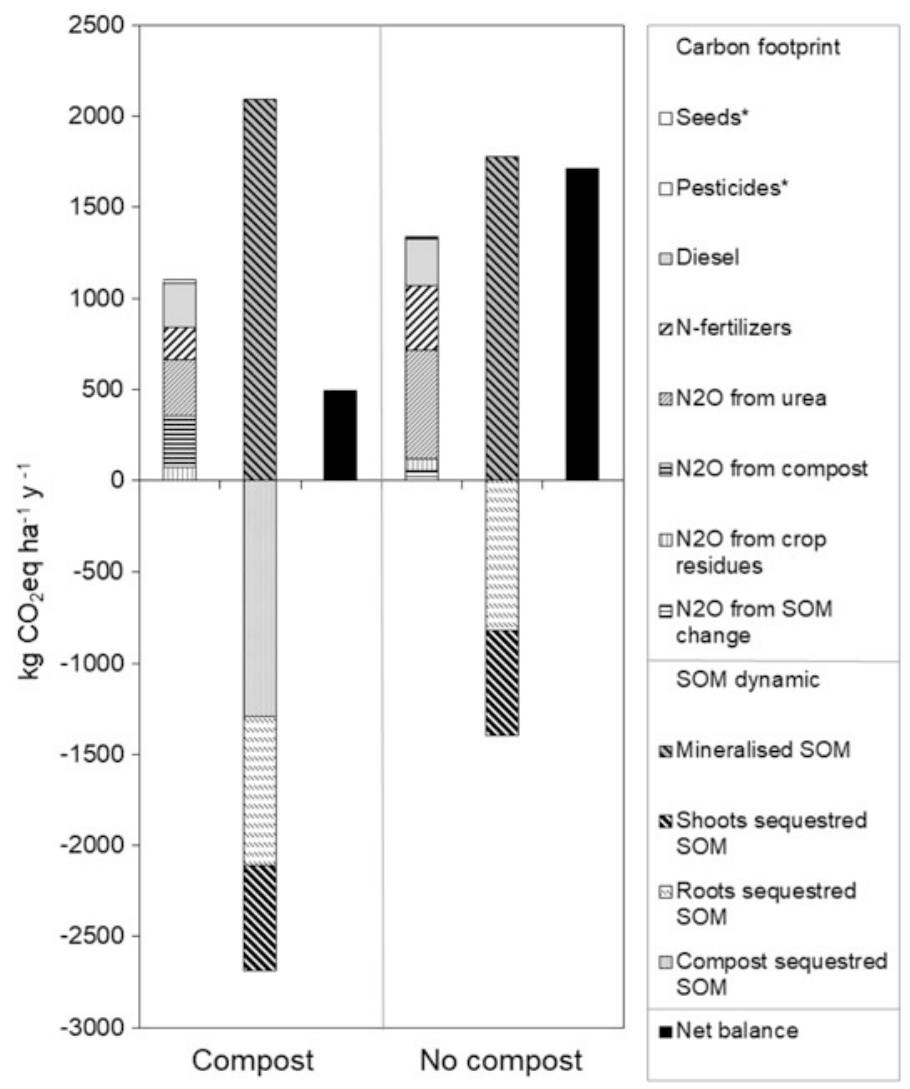

Fig. 2 Yearly average GHG balance for one hectare of cardoon cultivation, with or without compost, $*$ stands for negligible contribution

Figure 3 describes an ideal model of circular economy where food-waste are able to bring the benefits described in 1-4.

In order to put into practice this model and make real the social, economic and environmental benefit of bio-economy, specific incentives could be introduced for those farmers that apply compost in their agricultural fields. This would increase the demand of high quality compost which could/should be the driving force of the whole system. It is worth to point out that sustainable SOM management is a requisite of emerging standards on the sustainable biomass production like the European EN16751 [24].

Another important aspect for the success of this circular economy model is the execution of an appropriate separate collection of bio-waste by the consumers as a key prerequisite to (i) ensure high quality compost and (ii) reduce the bio-waste management costs of Citizen should be more and more informed about the importance of their behaviour on the success of bio waste management chain. This 


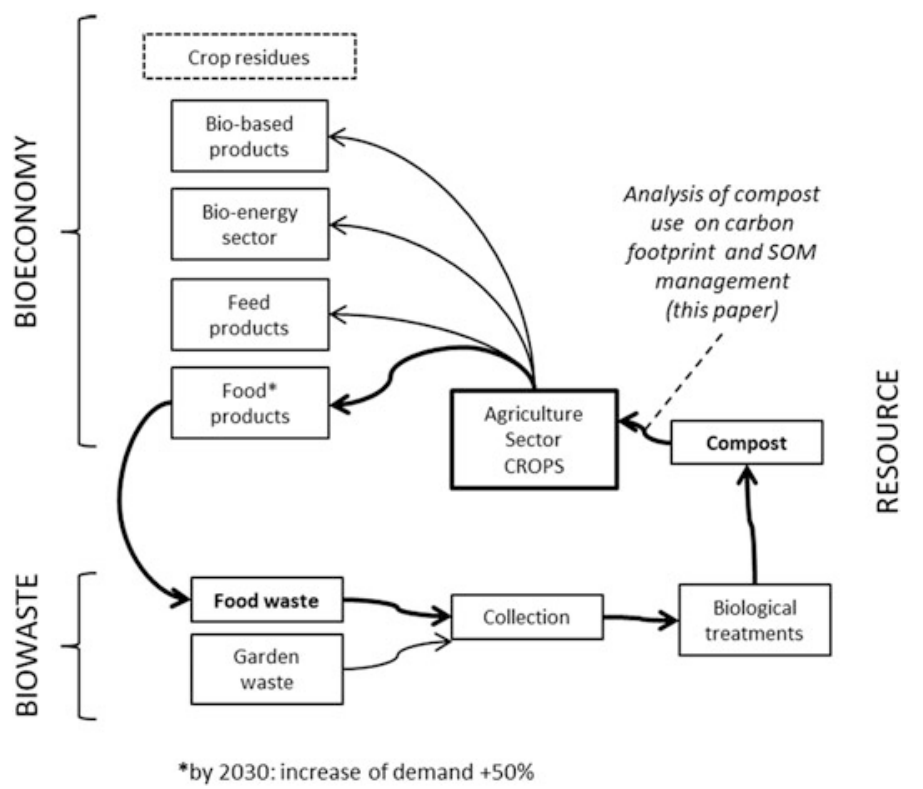

Fig. 3 Virtuous circular economy model for food waste (rows in bold). Above-ground biomass (crop residues) could be exploited to produce bio-based or bio-energy, or partially/totally returned to the soil

aim could be achieved through informative and formative campaigns performed at local level by municipal authorities, NGOs, scholar programmes etc. A real case study of a positive achievement is the experimental program introduced by Agenzia Milanese Servizi Ambientali (AMSA) [25] in 2016 related to the collection of biogenic waste produced at street markets level in Milan. The implementation of ad hoc organic waste collection system [26], along with an effective informative campaign have allowed to reach valuable results: in the five months of experimentation in the fifteen street markets passed from 60 metric tonnes (2015) to 260 metric tonnes (2016) in the same period, with an increase of around $+320 \%$ (AMSA, personal communication).

In reference to labour market increase linked to bio waste chain (point 2), it was estimated by the European Compost Network that up to 50,000 [4] new jobs in Europe could be created if additional 60 million tonnes of municipal bio-waste would be collected and composted/anaerobically digested across Europe.

\section{Conclusions}

It has become clear that globally the issue of food waste has significant social, economic and environmental impacts. The Circular Economy Package, published by the EU Commission in December 2015, contains specific proposals addressing 
the EU waste legislation with the aim of avoiding, reusing and recycling more waste in the future in order to save resources within Europe. One of the targets contained in the proposals is the maximum percentage of municipal solid waste to be disposed in landfill set equal to $10 \%$ by 2030 . Food waste management is therefore a key topic of the expected strategic action plan since up to $50 \%$ of municipal solid waste is biogenic. Biological treatments are well established in Europe with about 3500 treatment plants across Europe, nevertheless, still $25 \%$ of bio waste is recycled into high quality compost [3]. These figures suggest e considerable potential for expansion, especially in the southern Europe areas where the treatment capacity is still limited [4]. In this way, sustainable bio-waste management could also be used to strengthen the economy of rural areas and, at the same time, could benefit of compost application in agricultural fields. The SOM model developed within BIT3G Italian projects, suggested that compost use in agricultural sector is meaningful from a SOM management and carbon footprint perspectives as well. The SOMS after 22 years of cardoon cropping system with compost application resulted incremented of 6.2 metric tonnes per ha (first $30 \mathrm{~cm}$ of soil) whereas the scenario with zero inputs resulted decremented of 3.9 metric tonnes per hectare. In terms of GHG balance it was observed that average $\mathrm{CO}_{2}$ uptake has the same order of magnitude of the overall emissions coming from cardoon cultivation. In specific circumstances the $\mathrm{CO}_{2}$ uptake overcomes GHG emissions making cardoon crop cultivation GHG balance neutral or even negative.

The link between food waste management, compost production/use and food/ biomass sustainable production is therefore a key element of both for circular economy and bio-economy to be further investigated by decision makers for its valuable implications in the medium and long term on social, economic and environmental pillars.

\section{References}

1. EU, Communication from the Commission to the Council and the European Parliament on future steps in bio-waste management in the European Union, COM/2010/0235 final, 2010. http://eur-lex.europa.eu/legal-content/EN/TXT/?uri=CELEX:52010DC0235 (Accessed 30.05.2017).

2. Barth J, Amlinger F, Favoino E, Siebert S, Kehres B, Gottschall R, Bieker M, Löbig A, Bidlingmaier W, Final Report-Compost production and use in the EU, European Commission, DG Joint Research Centre/ITPS, 2008.

3. https://www.compost.it/materiali/Regolamento_Marchio_Qualita_CIC.pdf (Accessed 29.09. 2017).

4. Siebert S, Bio-Waste Recycling in Europe Against the Backdrop of the Circular Economy Package, 161024 ECN Biowaste Recycling in Europe, 2016. http://www.compostnetwork. info/download/bio-waste-recycling-europe-backdrop-circular-economy-package/ (Accessed 18.05.2017).

5. http://eur-lex.europa.eu/legal-content/EN/TXT/?uri=CELEX:52015DC0614 (Accessed 25.08. 2017)[6] COM (2015) 594 final Proposal for a Directive of the European Parliament and of the Council Amending Directive 1999/31/ EC on the landfill of waste. http://eur-lex.europa. eu/legal-content/EN/TXT/?uri=COM:2015:594:FIN (Accessed 29.09.2017). 
6. FAO, Unlocking the potential of soil organic carbon. Outcome document. Global symposyium on soil organic matter, Rome, Italy, 2017.

7. Minasny B, Malone B.P, McBratney A.B, Angers D.A, et al., Soil carbon 4 per mille. Geoderma, 292, 2017, pp. 59-86.

8. D'Avino L, Lazzeri L, Razza F, L'Abate G, Costantini E, Using SOM modelling outcomes to support the carbon footprint analysis of industrial crops: a case-study of perennial vs. annual oleaginous crops in Sardinia Region, in: abstract book of 5th International Symposium on soil organic matter session 3.1. C dynamics and sequestration under Global Change, 2015.

9. D'Avino L, L'Abate G, Chiarini F, Correale F, Morari F, Estimation of the soil carbon sequestration in a four year rotation managed with conventional and conservative methods. Proceedings of the Global symposium on soil organic carbon, Rome, Italy, 2017, pp. 304 307. http://www.fao.org/3/a-i7565e.pdf (Accessed 29.09.2017).

10. Fernandez-Tirado F, Parra-Lopez C, Calatrava-Requena J, A methodological proposal for Life Cycle Inventory of fertilization in energy crops: The case of Argentinean soybean and Spanish rapeseed, Biomass and Bioenergy 58, 2013, pp. 104-116. https://doi.org/10.1016/j. biombioe.2013.07.022.

11. Castoldi N, Bechini L, Agro-ecological indicators of field-farming systems, Rivista Italiana di Agrometeorologia, Vol. 1, 2006. pp. 19-31.

12. Mary B, Guérif $\mathbf{J}$, Intérets et limites des modèles de prévision de l'évolution des matières organiques et de l'azote dans le soil. Cah. Agric. 3, 1994, pp. 247-257.

13. Noirot-Cosson P.E, Dhaouadi K, Etievant V, Vaudour E, Houot S, Parameterisation of the NCSOIL model to simulate $\mathrm{C}$ and $\mathrm{N}$ short-term mineralisation of exogenous organic matter in different soils, Soil Biology and Biochemistry, 104, 2017, pp. 128-140. https://doi.org/10. 1016/j.soilbio.2016.10.015.

14. Tremblay M.E, Nduwamungu C, Parent L.E, Bolinder M.A, Biological Stability of Carbon and Nitrogen in Organic Products and Crop Residues using Fourier-Transform Near-Infrared Reflectance Spectroscopy, Communications in Soil Science and Plant Analysis, Vol. 41, Issue 8, 2010.

15. Boiffin J, Zagbahi J.K, Sebillotte M, Systèmes de culture et statut organique des sols dans le Noyonnais: application du modèle de Hénin-Dupuis. (in French.) Agronomie 6, 1986, pp. 437-446.

16. Houot S, Bodineau G, Rampon J.N, Annabi M, Francou C, Poitrenaud M, Agricultural use of different residual waste composts - current situation and experiences in France, Conference "The future of residual waste management in Europe", 2005.

17. Hennecke A.M, Faist M, Reinhardt J, Junquera V, Neeft J, Fehrenbach H, Biofuel greenhouse gas calculations under the European Renewable Energy Directive-A comparison of the BioGrace tool vs. the tool of the Roundtable on Sustainable Biofuels, Applied Energy, 102, 2013, pp. 55-62.

18. IPCC, Agriculture, Forestry and Other Land Use, Guidelines for National Greenhouse Gas Inventories, Vol. 4, Japan, 2006.

19. Myhre G, Shindell D, Bréon F-M, Collins W, Fuglestvedt J, Huang J, Koch D, Lamarque J.F, Lee D, Mendoza B, Nakajima T, Robock A, Stephens G, Takemura T, Zhang H, Anthropogenic and Natural Radiative Forc-ing, Climate Change 2013: The Physical Science Basis. Contribution of Working Group I to the Fifth Assessment Report of the Intergovernmental Panel on Climate Change, Cambridge University Press, 2013.

20. Morra L, Pagano L, Iovieno P, Baldantoni D, Alfani A, Soil and vegetable crop response to addition of different levels of municipal waste compost under Mediterranean greenhouse conditions, Agronomy for sustainable development, 30,3, pp. 701-709.

21. Hargreaves J.C, Adl M.S, Warman P.R, A review of the use of composted municipal solid waste in agriculture. Agriculture, Ecosystems \& Environment, 123(1), 1-14, 2008.

22. Cocco D, Deligios P.A, Ledda L, Sulas L, Virdis A, Carboni G, LCA study of Oleaginous Bioenergy Chains in a Mediterranean Environment. In: Energies, 7(10), 2014, pp. 62586281. https://doi.org/10.3390/en7106258. 
23. Aguilera E, Lassaletta L, Sanz-Cobena A, Garnier J, Vallejo A, The potential of organic fertilizers and water management to reduce $\mathrm{N}_{2} \mathrm{O}$ emissions in Mediterranean climate cropping systems. A review, Agriculture, Ecosystems and Environment, 164, 2013, pp. 32-52. https:// doi.org/10.1016/j.agee.2012.09.006.

24. CEN, EN16751 Bio-based products - Sustainability criteria, 2016.

25. http://www.amsa.it/gruppo/cms/amsa/multilingua/en (Accessed 30.05.2017).

26. http://www.ecodallecitta.it/notizie/384800/raccolta-differenziata-umido-nei-mercati-di-milanodal-20-febbraio-si-fa-in-15-mercati-su-94/ (Accessed 30.05.2017).

Open Access This chapter is licensed under the terms of the Creative Commons Attribution 4.0 International License (http://creativecommons.org/licenses/by/4.0/), which permits use, sharing, adaptation, distribution and reproduction in any medium or format, as long as you give appropriate credit to the original author(s) and the source, provide a link to the Creative Commons license and indicate if changes were made.

The images or other third party material in this chapter are included in the chapter's Creative Commons license, unless indicated otherwise in a credit line to the material. If material is not included in the chapter's Creative Commons license and your intended use is not permitted by statutory regulation or exceeds the permitted use, you will need to obtain permission directly from the copyright holder. 


\title{
Agri-Food Waste Streams Utilization for Development of More Sustainable Food Substitutes
}

\author{
Sergiy Smetana, Kemal Aganovic, Stefan Irmscher and Volker Heinz
}

\begin{abstract}
Substitution of food out of alternative biomass sources is aimed to supply consumers with food products similar in nutrition and with lower environmental impact compared to conventional products. At current state of development, meat substitutes are not competitive with chicken meat, except for plant based meat analogs (although they have weaker nutritional profile). Upscaling, further technological development and use of agri-food waste as main source substrate can assure the environmental benefits of insects $\left(2 \mathrm{~kW} \mathrm{~h}\right.$ of energy, $1 \mathrm{~kg} \mathrm{CO} \mathrm{CO}_{2}$ eq., $1.5 \mathrm{~m}^{2}$ of land and $0.1 \mathrm{~m}^{3}$ of water) and single cell products $\left(10 \mathrm{~kW} \mathrm{h,}-4 \mathrm{~kg} \mathrm{CO}_{2}\right.$ eq., $0.5 \mathrm{~m}^{2}$ of land and $0.25 \mathrm{~m}^{3}$ of water), making them more competitive compared to industrial chicken production. The results of the current research are preliminary and further studies are required to assure the industrial applicability of agri-food wastes use for food production.
\end{abstract}

\section{Introduction}

Food production is one of the most important human industries, which can be responsible for a majority of environmental impacts in developed countries [1]. The main causes for such impacts relate to overpopulation, growing demand for consumption of animal derived products and high rates of food waste. While some research targets development of solutions for separate issues, more recent sources indicate the need for a more holistic and systematic change of the complete food production chain $[2,3]$. The substitution of traditional foods with alternative analogs produced by non-traditional methods is foreseen as one example of sustainable transitions [4]. However, considering a complete life cycle of food substitutes, their performance is not always cost efficient and environmentally beneficial [5-7]. Therefore, a more sustainable status of food transitions should be confirmed with extensive research of economic efficiency, environmental advances and social acceptability.

S. Smetana $(\bowtie) \cdot$ K. Aganovic $\cdot$ S. Irmscher $\cdot$ V. Heinz

German Institute of Food Technologies, (DIL e.v.), Quakenbrück 49610, Germany

e-mail: s.smetana@dil-ev.de

(C) The Author(s) 2018

E. Benetto et al. (eds.), Designing Sustainable Technologies,

Products and Policies, https://doi.org/10.1007/978-3-319-66981-6_17 
Substitution of environmentally high-impacting meat with more sustainable alternatives is becoming not only necessary, but also popular through vegetarian, vegan and flexitarian diets. Numerous food products are under recent development to cover the requirement of consumers in more environmentally sustainable and yet nutritionally beneficial food analogs. Several scientific studies indicated that meat substitutes had a lower environmental impact than meat [8-17]. Even though the environmental benefits of texturized plant protein substitutes (soybean, pea, lupine, etc.) are well-known and proven [18, 19], their nutritional and organoleptic qualities require improvement $[20,21]$. Other analogs based on milk, mycoproteins, insects, microalgae, etc., are not environmentally beneficial in all of the cases [5, 6, 22, 23], but have improved nutritional profile, more similar to animal derived products [23-26]. Developments in cellular agriculture claim that creation of meat, milk, cheese and eggs without agricultural inputs is more environmentally friendly due to the avoidance of agricultural stage [7, 27]. Despite the complete nutritional identity between cellular products and animal derived foods, the environmental impacts of cultured food are usually higher due to low Technology Readiness Levels (TRL) and increased use of energy as well as biotechnological raw materials [5, 7, 28]. Although, few comparative studies of environmental performance and nutritional value of meat substitutes to animal derived products were published $[5,10,15]$, the analyses did not cover complete variety of food analogs and production technologies. Even though further environmental optimization measures for food substitutes are known, they are rarely analyzed and reported in literature. Upscaling of production, reduction of energy consumption, reduction of waste amounts and utilization of waste streams as raw materials are among the most known measures, which should be analyzed to identify future potential of food substitutes. Therefore, it is necessary to perform a holistic systematization of more sustainable production potential for "food of the future" based on innovative and emerging technologies of alternative protein products supply. Such systematization should provide an answer on more sustainable ways to produce food substitutes with existing industrial infrastructure and the potential of improvement with the use of agri-food waste.

\section{Methods and Materials}

The study included two main stages. First, it relied on a systematic review of research literature addressing the production of food substitutes and their associated environmental impacts. It was complied with own results on the LCA of food substitutes. Further, the authors assessed the technological potential of agri-food waste application for the design of food substitutes. This way, the first part of the study indicated the most promising options of food substitute's production from environmental perspective, while the second part estimated further potential for environmental impact improvement. The second part resulted in generalized comparison model (matrix) used for the assessment of environmental impact of food production scenarios with application of waste streams as a main source of raw materials. 
The study relied on the results of Life Cycle Assessment (LCA) according to ISO standards (ISO 14040-14044) [29, 30] present in literature (Part 1 of the study) and own assessments (Part 1 and 2).

The first part of the research included literature search on Google Scholar online platform with key words "environmental impact", "LCA" and "Life Cycle Assessment", "substitutes", "food products" for the period of 10 years (2007-2017) and returned with 580 publications identified. Further search was refined with additional key words: "meat substitutes" (64 results), "meat analogs" (7 results), "cultured meat" (21 results), "milk substitutes" (12 results), "dairy substitutes" (10 results), "alternative protein sources" (21 result). The resulted literature (135 references) was analyzed in detail to identify the environmental impacts of food substitutes. It was identified that 41 study presented results on various aspects of food substitutes LCA. We relied on the data from the literature with adaptation to the requirements of current study (attributional LCA with four impact categories of global warming potential, non-renewable energy use, land use and water footprint based on IMPACT 2002+ and ReCiPe methodologies) [31, 32]. It was aimed to answer the research question on identification of more sustainable food substitutes presented in literature. Environmental impact of food substitutes was compared in between and with conventional products.

The second part of the research addressed the potential of environmental impact improvement with the use of agri-food waste streams. The estimation of the potential required the assessment of current uses of agri-food wastes for food biomass production and further technological potential assessment for food design. The second part in a great degree relied on own data of industrial processing trials (DIL, Quakenbrueck) and background data available in literature and relevant databases (Agri-footprint and ecoinvent 3) [33, 34] with modelling in SimaPro 8 software. The results on Technology Readiness Level (TRL) and potential of agri-food waste use (includes all the types of food waste generated along the supply chain) was included into matrix (Table 1), which allowed overview of conventional foods substitution with potentially more sustainable alternatives. Furthermore, the analysis of agri-food waste potential included the comparison of nutritional profile of resulting products with benchmark food.

\section{Results and Discussion}

\subsection{Environmental Impact of Food Substitutes}

The analysis of environmental performance of meat substitutes included production and processing of protein sources based on plant proteins (soya, lupine, peas, gluten, etc.), dairy products, insect biomass, cultured meat, microalgae biomass, fungi, yeast and bacteria (Fig. 1). The analysis demonstrated that at despite certain comparability of the impacts for different sources of protein biomass, in many 
Table 1 Matrix of agri-food waste application potential for the production of food substitutes source biomass in relevance to technology readiness levels

\begin{tabular}{l|l|l|l|l}
\hline \multirow{2}{*}{ Source of biomass for substitutes } & \multicolumn{4}{l}{ Substituted animal derived products } \\
\cline { 2 - 5 } & Meat & Dairy & Eggs & Fat \\
\hline Plant & $\mathrm{IX} / 9$ & $\mathrm{IX} / 6$ & $\mathrm{VII} / 6$ & $\mathrm{IX} / 9$ \\
\hline Milk & $\mathrm{IX} / 0$ & $\mathrm{n} / \mathrm{a}$ & $\mathrm{VI} / 0$ & $\mathrm{IX} / 0$ \\
\hline Insect & $\mathrm{VI}-\mathrm{IX} / 7$ & $\mathrm{I}-\mathrm{VII} / 8$ & $\mathrm{VI}-\mathrm{VII} / 8$ & $\mathrm{IX} / 9$ \\
\hline Cultured & $\mathrm{VI} / 4$ & $\mathrm{VI} / 4$ & $\mathrm{VI} / 4$ & $\mathrm{VII} / 6$ \\
\hline Single cell protein & $\mathrm{VII} / 5$ & $\mathrm{VIII} / 5$ & $\mathrm{VIII} / 5$ & $\mathrm{IX} / 7$ \\
\hline Microalgae & $\mathrm{IX} / 6$ & $\mathrm{IX} / 5$ & $\mathrm{VIII} / 5$ & $\mathrm{IX} / 9$ \\
\hline Fungi & $\mathrm{VII} / 6$ & $\mathrm{VI} / 6$ & $\mathrm{VIII} / 6$ & $\mathrm{VI} / 6$ \\
\hline Y\&B &
\end{tabular}

Note I-IX-Technology readiness level (TRL) after [48] for the application of source biomass to substitute animal derived products; 0-9-potential of agri-food waste application for the source biomass production (with 0 -no potential identified to 9 confirmed industrial application possible); $\mathrm{Y} \& \mathrm{~B}$ - yeast and bacteria

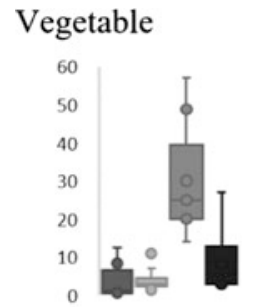

Microalgae

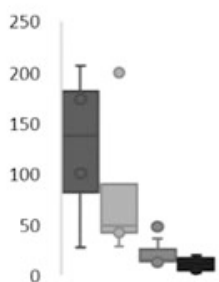

Dairy

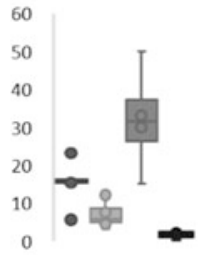

Fungi

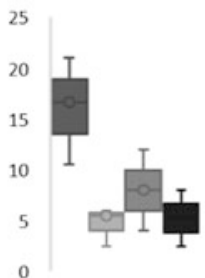

Insect

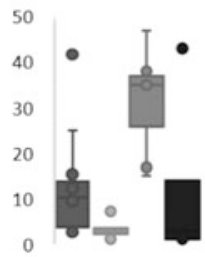

Y\&B

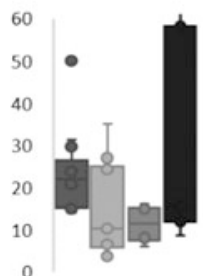

Cultured

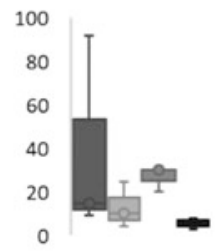

Legend:

Energy use, kWh

GWP, kg CO2 eq.

Land use, $\mathrm{m} 2 \mathrm{a} * 10$

Water use, $100 \mathrm{~L}$

GWP - global warming potential, Y\&B - yeast and bacteria, the assessment is based on $[5,6,10,11,14,18,19,35-40]$ and own calculations for insects, microalgae and yeasts and bacteria (Y\&B) according to the production data and inventories $[39,41]$

Fig. 1 Environmental impact of meat substitutes based on various alternative sources of proteins

cases, there were a few limitations for a reliable distinction between the comparable products. On the one hand, there were wide ranges of environmental impacts (e.g. due to land use for plant based substitutes, energy use for cultured meat and microalgae, water use for yeast and bacteria), and on the other hand there was a lack 
of available studies, data sources and relevant models in order to narrow down the ranges of environmental impacts (e.g. water use for dairy based, microalgae and cultured meat substitutes).

Despite mentioned limitations, it was possible to indicate that according to the current state of TRL the most promising sources of proteins in terms of energy consumption were plant and insect protein biomass (grown on commercial chicken feed). Meat substitutes based on biomass derived from dairy, yeast and bacteria had higher level of energy consumption, but also high potential for further development. Alternative sources of proteins had low impact on climate change (comparable with chicken 2-4 $\mathrm{kg} \mathrm{CO}_{2}$ eq. and pork 4-6 $\mathrm{kg} \mathrm{CO}_{2}$ eq. per kg of meat) for all of the sources, except for meat substitutes from microalgae (related to variety of production conditions). Land use impacts were comparable between alternative sources of proteins $\left(2-4 \mathrm{~m}^{2}\right.$ a per $\mathrm{kg}$ of product, vs. $5-7 \mathrm{~m}^{2}$ for chicken and 7-8 $\mathrm{m}^{2}$ for pork). Fungi, yeast and bacteria based sources of proteins were exception and had lower land use impact (up to $2 \mathrm{~m}^{2}$ a year ${ }^{-1}$ ). Water footprint was quite similar between different protein sources with lower impacts associated with microalgae and fungi biomass. Benefits of water consumption of dairy and insect based meat substitutes, as well as of cultured meat could not be demonstrated due to the lower data quality.

In terms of nutritional quality, the most identical substitute to meat was produced by cellular agriculture methods (cultured meat). However, due to muscle purity, meat substitute produced by cellular agriculture usually consists of lower fat content then traditional meat. Plant based meat substitutes are well researched and documented, with high presence on the market. At the same time, the diversity of vegetable protein sources and their unbalanced nutritional composition set difficulties for the complete substitution of animal derived products.

Insect and dairy based food substitutes are characterized with excellent nutritional qualities similar, or often more beneficial than traditional meat products. Milk based protein products usually have a moderate protein and fat content (10-14\% and $8-10 \%$ respectively). However, dairy based protein concentrates and isolates can serve as a high protein additive for human consumption. Insects can be considered as a good source of proteins (up to $77 \%$ dry weight), fats (up to $62 \%$ dry weight) and polyunsaturated amino acids [42]. Moist cooked products, based on whole mealworm and depending on the processing technology, resulted in products with $22-30 \%$ proteins and $2-20 \%$ fat (own data). However, the limiting factor of insects' application for food and feed could be low amounts of methionine [43].

Single cell production (fungi, algae, yeast and bacteria) can provide excellent sources of proteins (30-65\% in dry weight) and fats (up to $20 \%$ in microalgae), but they are also a source of nucleic acid (3-12\%), which can be a serious obstacle for the direct use in food, as it might cause health complications [44]. 


\subsection{Potential of Agri-Food Wastes Application for Production of Food Substitutes Biomass}

Application of agri-food waste can be foreseen as one of the key solutions to improve the sustainability of current food production. The main options of dealing with agri-food waste include prevention, creation of food banks (or storages), valorization for animal feed and other purposes (e.g. recovery of nutrients or extraction of highly valuable components, recycling of proteins for the new products), reuse (application for other purposes like generation of energy and heat), compositing and landfilling [45-47]. Current study dealt with a combined approach addressing prevention of food wasting and food waste valorization, when agri-food wastes are assessed from the position of feeding substrate or media for insect and single cell production, as well as for meat culturing and direct application as a source of plant protein biomass (Table 1).

TRL plays an important role for the identification of opportunities for the agri-food waste application. Low TRL do not allow for the effective modelling of food substitutes production based on agri-food waste. Nevertheless, it was identified that direct application of agri-food waste for the plant based food substitutes is very challenging, but possible. Most known examples of such application include the use of soybean meal and okara as components of composite foods for direct human consumption. Side streams of plants processing usually fall in this category and can be utilized as a source for food. Main challenges were associated with assurance of food safety and adequate biomass quality (as in case of waste from consumers). On the other hand, application of processing side-streams (e.g. soybean meal, gluten), is well realized to produce texturized vegetable protein foods (Fig. 1).

Cultured meat production could potentially rely on cultivation media generated by bacteria fed on agri-food side and waste streams, however, such potential is only highlighted as possible in literature [14, 27, 28, 49]. In this case, the environmental impact could be reduced and reach the lower levels of impact indicated in Fig. 1. Such results correspond well to the result of the study [14], where it was reported that cultivation of meat on the cultured media from cyanobacteria could be environmentally beneficial. It is a previous conclusion and confirmation to the applicability in industrial scale is required.

The application of agri-food waste streams for single cell and insect production is an upcoming development, taking into consideration current improvements in TRL, performed and planned trials (projects CORNET "ENTOMOFOOD”, ERA NET-LAC "EntoWaste"). The biggest challenge here is associated with proper selection of suitable agri-food waste, which would assure the high efficiency of production and therefore would have a beneficial nutritional composition. In many cases agri-food waste corresponding to the identified properties, is represented by side-stream of food processing, used for animal feeding. So, another challenge relates to the application of multiple agri-food wastes as animal feed. The need to replace such feed material could result in higher environmental impacts than benefits from alternative proteins productions. At the same time, multiple sources of 
agri-food wastes are not utilized as feed. In most of the cases the producer of a reference product covers the costs of transportation to the farms and thus pays for the utilization. Such precondition in combination with good nutritional qualities makes such agri-food wastes (e.g. mill brans, distiller's dried grains with solubles, brewery grains, milled pre-consumer waste) an excellent source for single cell and insect production. Successful application of agri-food wastes could reduce the environmental impact of food substitutes production with existing technologies (Fig. 1). Thus, the impact of insect-based food $(1 \mathrm{~kg})$ could be decreased to $2 \mathrm{~kW} \mathrm{~h}$ of energy use, around $1 \mathrm{~kg} \mathrm{CO} 2$ eq. of GWP, $1.5 \mathrm{~m}^{2}$ of land use and $0.1 \mathrm{~m}^{3}$ of water use. Single cell production can supply $1 \mathrm{~kg}$ of high quality protein food which would require around $10 \mathrm{~kW} \mathrm{~h}$, emit $2-4 \mathrm{~kg} \mathrm{CO}_{2}$ eq., occupy $0.5 \mathrm{~m}^{2}$ of land and consume $0.25 \mathrm{~m}^{3}$ of water. Such environmental impact results make insects and single cell products more sustainable alternatives to meat and other foods.

\section{Conclusions}

Traditional food substitution with alternative biomass sources is becoming a necessity to design a more sustainable transition to the new food system. The current analysis, which included comparison of alternative protein sources according to the technology readiness level, nutritional profile and environmental impact, indicated the potential for the substitution of traditional food. Alternative sources of biomass can be used as food substitutes if their environmental and economic benefits are assured. It could be done thought the application of agri-food wastes and further development of production technologies.

The results of the current study indicated that state-of-the-art production of meat analogs based on milk, mycoproteins, insects and microalgae biomass are not competitive in terms of environmental impact to the benchmark meat (chicken in this case). The application of waste and side streams from agri-food production (molasses, distilled grains, grain brans) could potentially decrease the impact of insect and single cell products for their use as meat substitutes. Improvement in technologies for biomass processing is vital for the optimization of analogs based on single cell products. Similarly, processing stages play high importance for cellular-based products. However, high impact was associated with the production of culture media for meat production and raw resources of yeast derived milk, cheese and eggs. Cellular meat production at current state of TRL has a higher environmental impact than traditional production meat. With scaling up approach, a competing potential with beef and pork was shown, but not with chicken. The application of agri-food waste streams is complicated due to the need of providing a sterile culture media, although is possible for the cultured media production. Application of selected agri-food wastes to produce alternative sources of protein (specifically insects and single cell products) could result in more sustainable source of proteins able to compete with industrial chicken production. 
The results of this study are preliminary and are characterized with high assumption uncertainties. However, it is possible to conclude that the application of circular economy principles to the production of traditional foods via innovative processing methods involving agri-food waste streams could allow for improvement of their environmental performances.

\section{References}

1. Tukker A, Goldbohm RA, de Koning A, Verheijden M, Kleijn R, Wolf O, et al, Environmental impacts of changes to healthier diets in Europe. Ecol. Econ. [Internet]. 70, 2011; 1776-88. Available from: http://linkinghub.elsevier.com/retrieve/pii/ S092180091100190X.

2. Nemecek T, Jungbluth N, Mila i Canals L, Schenck R, Environmental impacts of food consumption and nutrition: where are we and what is next? Int. J. Life Cycle Assess. [Internet]. 21, 2016; 607-20. Available from: http://link.springer.com/10.1007/s11367-0161071-3.

3. Sala S, Anton A, McLaren S.J, Notarnicola B, Saouter E, Sonesson U, In quest of reducing the environmental impacts of food production and consumption. J. Clean. Prod. [Internet]. 140, 2017;387-98. Available from: http://linkinghub.elsevier.com/retrieve/pii/ S0959652616313956.

4. van der Goot A.J, Pelgrom P.J.M, Berghout J.A.M, Geerts M.E.J, Jankowiak L, Hardt N.A, et al, Concepts for further sustainable production of foods. J. Food Eng. [Internet]. 168, 2016; 42-51. Available from: http://linkinghub.elsevier.com/retrieve/pii/S026087741500309X.

5. Smetana S, Mathys A, Knoch A, Heinz V, Meat alternatives: life cycle assessment of most known meat substitutes. Int. J. Life Cycle Assess. [Internet]. Springer Berlin Heidelberg; 20, 2015; 1254-67. Available from: http://dx.doi.org/10.1007/s11367-015-0931-6.

6. Smetana S, Palanisamy M, Mathys A, Heinz V, Sustainability of insect use for feed and food: Life Cycle Assessment perspective. J. Clean. Prod. [Internet]. 137, 2016; 741-51. Available from: http://linkinghub.elsevier.com/retrieve/pii/S0959652616310447.

7. Tuomisto H.L, Ellis M, Steer M, Could cellular agriculture reduce environmental impacts? Putt. LCA into Pract. Int. Conf. Life Cycle Assess. Food 2016. Dublin, Ireland; 2016. p. $1110-5$.

8. Raats J, Meat (substitutes) comparing environmental impacts. A Case study comparing Quorn and pork. Training thesis at Centre for Energy and Environmental Studies, University of Groningen. Retrieved from http://www.temoa.info/node/209029. University of Groningen; 2007.

9. Håkansson S, Gavrilita P, Bengoa X, Comparative Life Cycle Assessment Pork vs. Tofu. Stockholm; 2005.

10. Head M, Sevenster M, Croezen H, Life Cycle Impacts of Protein-rich Foods for Superwijzer. Delft; 2011.

11. Blonk H, Kool A, Luske B, de Waart S, ten Pierick E, Milieueffecten van Nederlandse consumptie van eiwitrijke producten. Gevolgen van vervanging van dierlijke eiwitten anno 2008.

12. Berardy A, A Consequential Comparative Life Cycle Assessment of Seitan and Beef. SSEBE-CESEM-2012-CPR-002 Course Project Report Series. 2012.

13. Finnigan T, Lemon M, Allan B, Paton I, Mycoprotein, Life Cycle Analysis and the Food 2030 Challenge. Asp. Appl. Biol. 102, 2010; 81-90.

14. Tuomisto H.L, de Mattos M.J.T, Environmental impacts of cultured meat production. Environ. Sci. Technol. [Internet]. 45, 2011; 6117-23. Available from: http://www.ncbi.nlm. nih.gov/pubmed/21682287. 
15. van Huis A, van Itterbeeck J, Klunder H, Mertens E, Halloran A, Muir G, et al, Edible insects: Future prospects for food and feed security. FAO Forest. Rome: FAO, United Nations; 2013.

16. Oonincx D.G.A.B, de Boer I.J.M, Environmental impact of the production of mealworms as a protein source for humans - a life cycle assessment. PLoS One [Internet]. 7, 2012 [cited 2014 Mar 19]; e51145. Available from: http://www.pubmedcentral.nih.gov/articlerender.fcgi? artid $=3526541 \&$ tool $=$ pmcentrez\&rendertype $=$ abstract.

17. Nonhebel S, Raats J, Environmental impact of meat substitutes: comparison between quorn and pork. Proc. 5th Int. Conf. LCA foods. Gothenburg, Sweden; 2007. p. 73-5.

18. Zhu X, van Ierland E.C, Protein Chains and Environmental Pressures: A Comparison of Pork and Novel Protein Foods. Environ. Sci. [Internet]. 1, 2004; 254-76. Available from: http:// www.tandfonline.com/doi/abs/10.1080/15693430412331291652.

19. Nijdam D, Rood T, Westhoek H, The price of protein: Review of land use and carbon footprints from life cycle assessments of animal food products and their substitutes. Food Policy [Internet]. Elsevier Ltd; 37, 2012 [cited 2013 Aug 9]; 760-70. Available from: http:// linkinghub.elsevier.com/retrieve/pii/S0306919212000942.

20. Sanders T.A.B, The nutritional adequacy of plant-based diets. Proc. Nutr. Soc. Cambridge Univ Press; 58, 1999; 265-9.

21. Kumar P, Chatli M.K, Mehta N, Singh P, Malav O.P, Verma A.K, Meat analogues: Health promising sustainable meat substitutes. Crit. Rev. Food Sci. Nutr. [Internet]. 57, 2017; $923-$ 32. Available from: https://www.tandfonline.com/doi/full/10.1080/10408398.2014.939739.

22. Halloran A, Roos N, Eilenberg J, Cerutti A, Bruun S, Life cycle assessment of edible insects for food protein: a review. Agron. Sustain. Dev. [Internet]. 36, 2016; 57. Available from: http://link.springer.com/10.1007/s13593-016-0392-8.

23. Sturtewagen L, De Soete W, Dewulf J, Lachat C, Lauryssen S, Heirman B, et al, Resource use profile and nutritional value assessment of a typical Belgian meal, catered or home cooked, with pork or Quorn ${ }^{\mathrm{TM}}$ as protein source. J. Clean. Prod. [Internet]. 112, 2016; 196-204. Available from: http://linkinghub.elsevier.com/retrieve/pii/S0959652615012275.

24. Draaisma R.B, Wijffels R.H, Slegers P, Brentner L.B, Roy A, Barbosa M.J, Food commodities from microalgae. Curr. Opin. Biotechnol. [Internet]. 24, 2013; 169-77. Available from: http://linkinghub.elsevier.com/retrieve/pii/S095816691200153X.

25. Poma G, Cuykx M, Amato E, Calaprice C, Focant J.F, Covaci A, Evaluation of hazardous chemicals in edible insects and insect-based food intended for human consumption. Food Chem. Toxicol. [Internet]. 100. 2017; 70-9. Available from: http://linkinghub.elsevier.com/ retrieve/pii/S0278691516304598.

26. Rumpold B.A, Schlüter O.K, Potential and challenges of insects as an innovative source for food and feed production. Innov. Food Sci. Emerg. Technol. [Internet]. 17, 2013; 1-11. Available from: http://linkinghub.elsevier.com/retrieve/pii/S1466856412001452.

27. Post M.J, Cultured meat from stem cells: challenges and prospects. Meat Sci. [Internet]. Elsevier Ltd; 92, 2012 [cited 2014 Feb 6]; 297-301. Available from: http://www.ncbi.nlm. nih.gov/pubmed/22543115.

28. Mattick C.S, Landis A.E, Allenby B.R, Genovese N.J, Anticipatory Life Cycle Analysis of In Vitro Biomass Cultivation for Cultured Meat Production in the United States. Environ. Sci. Technol. [Internet]. 49, 2015; 11941-9. Available from: http://pubs.acs.org/doi/10.1021/acs. est.5b01614.

29. ISO 14044, Environmental management—Life cycle assessment-Requirements and guidelines. International Organization for Standardization; 2006.

30. ISO 14040, Environmental Management-Life Cycle Assessment-Principles and Framework. 2006.

31. Jolliet O, Margni M, Charles R, Humbert S, Payet J, Rebitzer G, et al, IMPACT 2002+ : A New Life Cycle Impact Assessment Methodology. Int. J. Life Cycle Assess. 8, 2003; 324-30.

32. Goedkoop M, Heijungs R, De Schryver A, Struijs J, van Zelm R. ReCiPe 2008, A LCIA method which comprises harmonised category indicators at the midpoint and the endpoint level. Characterisation. Updated RIVM report. Bilthoven, Netherlands: RIVM. 2013. 
33. Blonk H, Ponsioen T, Kool A, Marinussen M, The Agri-footprint method; Methodological LCA framework, assumptions and applied data. Blonk Milieu Advis. Gouda. Version. 1, 2011.

34. Wernet G, Bauer C, Steubing B, Reinhard J, Moreno-Ruiz E, Weidema B, The ecoinvent database version 3 (part I): overview and methodology. Int. J. Life Cycle Assess. Springer; 21, 2016; 1218-30.

35. Tuomisto H.L, Roy A.G, Could cultured meat reduce environmental impact of agriculture in Europe ? 8th Int. Conf. LCA Agri-Food Sect. Rennes, Fr. 2-4 Oct. 2012. 2012.

36. Pelletier N, Arsenault N, Tyedmers P, Scenario modeling potential eco-efficiency gains from a transition to organic agriculture: life cycle perspectives on Canadian canola, corn, soy, and wheat production. Environ. Manage. [Internet]. 42, 2008 [cited 2014 Jan 26]; 989-1001. Available from: http://www.ncbi.nlm.nih.gov/pubmed/18574623.

37. Miglietta P, De Leo F, Ruberti M, Massari S, Mealworms for Food: A Water Footprint Perspective. Water [Internet]. 7, 2015; 6190-203. Available from: http://www.mdpi.com/ 2073-4441/7/11/6190/.

38. Halloran A, Hanboonsong Y, Roos N, Bruun S, Life cycle assessment of cricket farming in north-eastern Thailand. J. Clean. Prod. [Internet]. 156, 2017; 83-94. Available from: http:// linkinghub.elsevier.com/retrieve/pii/S0959652617307163.

39. Harding K, A generic approach to environmental assessment of microbial bioprocesses through life cycle assessment (LCA). University of Cape Town; 2008.

40. Smetana S, Sandmann M, Rohn S, Pleissner D, Heinz V, Autotrophic and heterotrophic microalgae and cyanobacteria cultivation for food and feed: Life Cycle Assessment. Bioresour. Technol. [Internet]. 2017; Available from: http://linkinghub.elsevier.com/retrieve/ pii/S096085241731427X.

41. Heinzle E, Biwer A.P, Cooney C.L, Development of sustainable bioprocesses: modeling and assessment. John Wiley \& Sons; 2007.

42. Rumpold B.A, Schlüter O.K, Nutritional composition and safety aspects of edible insects. Mol. Nutr. Food Res. Wiley Online Library; 57, 2013; 802-23.

43. Grau T, Vilcinskas A, Joop G, Sustainable farming of the mealworm Tenebrio molitor for the production of food and feed. Zeitschrift für Naturforsch. C [Internet]. 2017; Available from: http://www.degruyter.com/view/j/znc.ahead-of-print/znc-2017-0033/znc-2017-0033.xml.

44. Nasseri A.T, Rasoul-Amini S, Morowvat M.H, Ghasemi Y, Single cell protein: production and process. Am. J. food Technol. Academic Journals; 6, 2011; 103-16.

45. Xue L, Liu G, Parfitt J, Liu X, Van Herpen E, Stenmarck A, et al, Missing food, missing data? A critical review of global food losses and food waste data. Environ. Sci. Technol. ACS Publications; 2017.

46. Östergren K, Davis J, De Menna F, Vittuari M, Unger N, Loubiere M. Food supply chain side flows management through Life Cycle Assessment and Life Cycle Costing: a practitioner's perspective. Proc. Food Syst. Dyn. 2017; 300-3.

47. Davis J, De Menna F, Unger N, Östergren K, Loubiere M, Vittuari M. Generic strategy LCA and LCC: Guidance for LCA and LCC focused on prevention, valorisation and treatment of side flows from the food supply chain. 2017.

48. Mankins J.C, Technology readiness levels. White Pap. April. 6, 1995.

49. Mattick C.S, An Emerging Technology Assessment of Factory-grown Food. Arizona State University; 2014. 
Open Access This chapter is licensed under the terms of the Creative Commons Attribution 4.0 International License (http://creativecommons.org/licenses/by/4.0/), which permits use, sharing, adaptation, distribution and reproduction in any medium or format, as long as you give appropriate credit to the original author(s) and the source, provide a link to the Creative Commons license and indicate if changes were made.

The images or other third party material in this chapter are included in the chapter's Creative Commons license, unless indicated otherwise in a credit line to the material. If material is not included in the chapter's Creative Commons license and your intended use is not permitted by statutory regulation or exceeds the permitted use, you will need to obtain permission directly from the copyright holder.

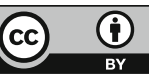




\title{
Implementation and Management of Life Cycle Approaches in Business- Challenges, Opportunities, Business Learnings and Best Practice
}

\author{
Lena Landström and Sara Palander
}

\begin{abstract}
This paper presents a summary of the session "Implementation and management of life cycle approaches in business-challenges, opportunities, business learnings and best practice". In the session, the audience got the opportunity to listen to examples from industry and ways to include life cycle approaches in their business decision making processes. The audience also attended presentations where learnings from the environmental footprint process were discussed. The conclusions from the presentations and the final panel discussion can be summarized in some key messages. To succeed we need to go together and that is why harmonization of e.g. methods and regulations are important. As a basis when developing the way forward it was concluded that the life cycle approach is an important tool.
\end{abstract}

\section{Introduction}

During the latest decades, companies have implemented life cycle assessment (LCA) into business management processes, e.g. in strategy, investment, procurement, and marketing, with the objective to increase the environmental performance of their products and services. In the latest update of the standard ISO 14001:2015 (environmental management systems, EMS), companies shall use a life cycle perspective to fulfil the requirements when for instance identifying the most important and relevant environmental impacts [1]. Also, the Product and

\footnotetext{
L. Landström

Vattenfall, Gothenburg, Sweden

L. Landström $\cdot$ S. Palander $(\square)$

Chalmers University of Technology, Swedish Life Cycle Center,

41296 Gothenburg, Sweden

e-mail: sara.palander@chalmers.se

S. Palander

Chalmers University of Technology, Centre for Environment

and Sustainability, 41296 Gothenburg, Sweden

(C) The Author(s) 2018

E. Benetto et al. (eds.), Designing Sustainable Technologies,

Products and Policies, https://doi.org/10.1007/978-3-319-66981-6_18
} 
Organisation Environmental Footprint process of the European Commission will affect the international market and business to apply the life cycle approach in decision making and communication [2]. So how does industry embed life cycle approaches into their business? What is best practice? How are ISO 14001:2015 and the Product/Organisation Environmental footprint process (respectively PEF and $\mathrm{OEF}$ ) being used and can they support the work of improving the environmental performance of products, services and organizations?

The session invited industries to present different ways to implement the life cycle perspective into their environmental management systems, learnings and best practices. The session also invited presenters to take up from results and learnings from the European PEF/OEF process. What is the next step for businesses, policy-makers and the life cycle management community in order to create a more sustainable industry and communicate results to customers, citizens and other stakeholders? What actions have been seen and will be seen? What are the opportunities and challenges? The session attracted many abstracts with different perspectives and the presenters were selected to reflect those perspectives with the hopes of a fruitful panel discussion at the end of the session.

\section{Main Messages from the Presentations}

Three oral presentations were selected to describe different ways of how life cycle approaches and life cycle management were incorporated into business strategies. At Procter \& Gamble, the OEF has been used to understand the corporate hot spots and identify sustainability actions based on these, quantify progress over time and effectively report to external programs such as CDP [3].

Aptar Italia's roadmap towards sustainability was presented to give an insight in the company's use of LCA as a basis for their business. The organization's most important strategic principle is the customers' awareness of the value of sustainability [4].

AkzoNobel has used monetization of externalities (expressing consequences of an economic activity experienced by unrelated third party in monetary/financial terms) as an approach to integrate life cycle management in decision making. The methods behind the "3D profit and loss account" were described and also the methods behind the economic, environmental and social capital for both positive and negative externalities, e.g. the Environmental Priority Strategies (EPS) [5].

The last two presentations were selected to give insights and perspectives on the environmental footprint process from the European Commission. The OEF process was discussed by one of the OEF pilots in the retail sector and from a communication effectiveness perspective. The pilot study shows both a potential and a value of OEF as it helps retailers to assess and improve their product portfolio and to make sure their sustainability strategy targets major issues first [6].

The communication effectiveness was studied through how human brains interpret environmental information on products and how to use this information in 
communication of life cycle based environmental labelling. The key message from the research group behind the presentation is education of consumers in all levels of the society [7].

\section{Discussion on Messages and Outcomes}

After their talk, the presenters were invited to be part of a panel discussion to raise the perspective of the individual company to a society level.

They were first invited to discuss the actions needed in order to make progress towards a more sustainable industry, what actions are needed.

Need for harmonization and collaboration were agreed on among the participants in the panel. According to Mr. Van Hoof, "International harmonization is a key word to drive sustainability actions". Mr. Van Hoof and Mr. Van Hemelryck also discussed the importance of communicating products' environmental impact and understand customers' needs and interest were also discussed. Mr. Sonnen added the perspectives on financial rewarding systems to the table, and Mrs. Lewandowska highlighted the importance of education and to educate employees, business partners, customers, students, children, advising the use of the life cycle perspective as a basis to give trust.

Then the panelists were questioned about that key aspects needed for the environmental footprint methodologies to be put into practice.

The panel highlighted important factors such as the need to develop some trainings for companies and non-LCA experts and guidelines translated into different languages (Mrs. Lewandowska), make it trustable for the consumers and not risk to lose trust within some years (Mr. Sonnen), create incentives on communication and make LCA tools and data accessible (Mr. Del Grosso).

Other questions raised by the audience resulted in a discussion about responsibilities for the consumers, retailers and policy makers. It was raised by Mrs. Schreiber and Mr. Van Hemelryck that some of the requirements, coming by any of the three types of stakeholders can drive the environmental performance of products or companies in the wrong direction and could be harmful. For instance, retailers are fighting to satisfy their customers and therefore they put requirements on farmers for e.g. limiting or prohibiting pesticides and herbicides use. Other requirements coming from the European Commission and/or national governments legislation also exist regarding these substances. Then farmers have to deal with so many different regulations that they do not know how to act. The panel also reflected on a market with only eco-labeled products, but as highlighted by Mr. Sonnen it seems to be a long way to go.

The panelists ended with key messages for the session. All agreed on the need for incentives for collaborations along the value chain, with other companies and with consumers. Life cycle based information appeared as the best way forward to gain trust in the market. As a scientific based method, life cycle based approached 
will give trust in the communication process. To succeed in this process, Mrs. Lewandowska once again highlighted education as one of the most powerful tools to succeed.

\section{Conclusion and Future Perspectives}

The session demonstrated that life cycle based approaches are being used among industry to identify where to act in the most efficient way when it comes to sustainability, in particular environmental sustainability. This session only gives a few perspectives on how the life cycle perspectives are being used for business management and their actions based on this information.

There was a consensus on the need to build a harmonized, scientifically based framework and methodology to produce life cycle based information one of the priority being to ensure the public trust. The European Commission works for a "Single market for green products" by developing guidelines and tools such as the PEF and OEF, aiming at harmonizing LCA practices, and Lewandowska et al. showed that eco-labelling is something that consumers pay attention to [8].

Harmonization came up and was discussed many times during the session as a mean to make a progress on the road towards sustainable businesses. From the authors' perspectives, and based on the experience of running the Swedish Life Cycle Centre, an increasing interest from government agencies to act for a harmonization at the society level has been noticed. This perspective needs to be raised to create an equal basis to operate within the market. One of the success factors for harmonization, agreed by the panelists and also identified by the Swedish Life Cycle Centre, is the important part of working together in cross-industry collaboration and not as an individual industry or organization.

The roles of ISO 14001:2015 and the PEF/OEF process on the market, and their effects at the societal level were brought up during the session. However, more discussions are required before finding a consensus on this question.

\section{References}

1. International Organization for Standardizations (ISO), ISO 14001:2015 Environmental management systems - requirements with guideline for use, ISO, 2015.

2. http://ec.europa.eu/environment/eussd/smgp/index.htm (Accessed 26.09.2017).

3. Van Hoof G, Smith C, Mcaneny J; Desylva J, Informing corporate sustainability strategies with life cycle assessment: the Procter \& Gamble footprint, LCM 2017 Conference, Luxembourg, 2017.

4. Del Grosso M, Simboli A, Cutarella N, Raggi A, Business strategies to improve the environmental performances: life cycle approaches and tools an Aptar Italia, LCM 017 Conference, Luxembourg, 2017. 
5. Sonnen M, Hallberg K, Integration of life cycle management in decision making: a monetisation approach, LCM 2017 Conference, Luxembourg, 2017.

6. Schreiber H, Humbert S, van Hemelryck S, Poivet R, Developing common rules to quantify the organisation environmental footprint in the retail sector with support of the chain-OEF approach, LCM 2017 Conference, Luxembourg, 2017.

7. Borusiak B, Jerzyk E, Kurczewski P, Lewandowska A, Sobierajewicz J, Witczak J, Dierks C, Giungato P; Suh S, How human brains interpret the environmental information on the products? A Polish case of neuro-marketing tools to assess a communication effectiveness of lite cycle based environmental labelling, LCM 2017 Conference, Luxembourg, 2017.

8. Swedish Life Cycle Centre, Life cycle based innovation-A research and innovation agenda for a leading position within industrial and societal development where the life cycle thinking and sustainability is used as a basis for innovation, CPM report no 2013:3, 2013.

Open Access This chapter is licensed under the terms of the Creative Commons Attribution 4.0 International License (http://creativecommons.org/licenses/by/4.0/), which permits use, sharing, adaptation, distribution and reproduction in any medium or format, as long as you give appropriate credit to the original author(s) and the source, provide a link to the Creative Commons license and indicate if changes were made.

The images or other third party material in this chapter are included in the chapter's Creative Commons license, unless indicated otherwise in a credit line to the material. If material is not included in the chapter's Creative Commons license and your intended use is not permitted by statutory regulation or exceeds the permitted use, you will need to obtain permission directly from the copyright holder.

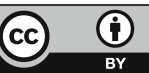




\title{
Neuro-marketing Tools for Assessing the Communication Effectiveness of Life Cycle Based Environmental Labelling- Procedure and Methodology
}

\author{
Anna Lewandowska, Barbara Borusiak, Christian Dierks, \\ Pasquale Giungato, Ewa Jerzyk, Przemyslaw Kurczewski, \\ Jagna Sobierajewicz, Sangwon Suh and Joanna Witczak
}

\begin{abstract}
The paper presents a procedure and a methodology of research which aimed at assessing and comparing the effectiveness of different variants of labels in communicating life cycle based environmental information (EU ecolabel, draft PEF labels). Based on a survey, an electroencephalography (EEG) and an eye-tracking, the information regarding consumers' ecological awareness, their neurological reaction and a visual attention is gathered and used for identifying the ecolabels' elements with the highest communication potential. A potential target audience of the project is not limited to the specialists in the environmental labelling, but includes also the readers involved in green marketing, Product Environmental Footprint and Life Cycle Assessment practitioners.
\end{abstract}

\footnotetext{
A. Lewandowska $(\square) \cdot$ B. Borusiak $\cdot$ E. Jerzyk $\cdot$ J. Witczak

Poznan University of Economics and Business, al. Niepodleglosci 10,

61-875 Poznan, Poland

e-mail: anna.lewandowska@ue.poznan.pl

C. Dierks

Fraunhofer-Institut für Silicatforschung, Brentanostrasse 2,

63755 Alzenau, Germany

P. Giungato

University of Bari "Aldo Moro", Taranto district-via Alcide de Gasperi,

Taranto, Italy

P. Kurczewski

Poznan University of Technology, Piotrowo 3, 60-965 Poznan, Poland

J. Sobierajewicz

Adam Mickiewicz University in Poznan, Wieniawskiego 1, 61-712 Poznan, Poland

S. Suh

Bren School of Environmental Science and Management, University of California,

Santa Barbara, CA 93106-5131, USA

(C) The Author(s) 2018

E. Benetto et al. (eds.), Designing Sustainable Technologies,

Products and Policies, https://doi.org/10.1007/978-3-319-66981-6_19
} 


\section{Introduction}

Information regarding the environmental performance of products or organisations plays a more and more important role in consumers' decisions. There are different communication vehicles and many strategies of green marketing which allow incorporating environmental information into the communication between various stakeholders representing both sides of the market (supply and demand). A way of performing this incorporation has come a long way from disclosing general, qualitative, unverified and single-issue information ("bio", "eco", "eco-friendly") to move toward verified, quantitative, specific and multi-issue statements. Sustainability has become an integral aspect of product quality and organisation image and it goes beyond a single environmental dimension. Today the products don't need to be declared as green, they just need to be green [1]. Consumers differ considerably in their sense of sustainability and ecological awareness. Some of them are not susceptible to greenwashing. They have a tendency to use verified product-related information taken not only from informal sources, but also from professionals and trusted third parties [2]. Green consumers-as early adopters and leaders - influence purchasing behaviour [1]. Also, the role of authorities and idols (e.g. celebrities) in the popularisation of "sustainable everyday" [3] can be significant [4]. From this perspective, an interesting issue is to ask about the importance of life cycle thinking (LCT) in communication between producers and consumers. Of among twenty new rules of green marketing listed by Ottman [1], at least seven can be directly or indirectly linked with life cycle thinking (LCT) and Life Cycle Assessment (LCA). However, the above question should not be limited to a potential role of LCA in marketing communication, but it should also address the issue of measuring the effectiveness of this communication. While papers on the former issue can be found in the literature, only single examples of work on the latter were found. Moreover, no information was found about the research on using neuroscience to measure the effectiveness of communication based on the results of LCA. For this reason, a core goal of this paper is to present a procedure and a methodology aimed at estimating the human neurological reaction as a result of exposure to life cycle based environmental labels and advertisements.

\section{Life Cycle Based Environmental Information as Part of Product-Related Communication}

A relationship between eco-design or LCA and green marketing has been discussed in literature. In 1993, Bhat [5] talked about the general strategies of eco-design (e.g. Design for Disassembly, Design for Recycling, and Design for Durability) as a basis for formulating marketing strategies. While there are many publications about eco-labelling and green marketing, only a few works can be found regarding a discussion narrowed to a role of LCA [6-10]. Some authors discussed this issue 
more generally by presenting good practice in communicating LCA results [11-13]. The discussion has been dominated by positive opinions, showing that eco-design and LCA are good fundaments for marketing claims. However, there are also some voices advising caution when using LCA results in marketing communications. The scepticism doesn't relate to the LCA as a method, but rather to its subjectivity and, especially, a high flexibility in formulating the initial assumptions [14, 15].

Besides being discussed in the literature, LCA is more and more present in business practices and communication. LCA results are usually released as a part of organisation-related sustainability report or product-related communication. The results can be disclosed in comprehensive, quantitative and third party verified forms (EPD declarations, the environmental certificates), but can also include more general content of advertising spots, brochures and websites. Some recent examples of using LCA in product advertising can be found. Life cycle based advertising video spots are published on mainstream media channels, for example, the ads published on YouTube by Beiersdorf AG (Nivea creams) [16], Toyota Motor Corporation (Toyota Prius) [17] and W. L. Gore \& Associates (Gore-tex ${ }^{\circledR}$ footwear) [18]. In these cases, the spots are based on a presentation of general information about life cycle thinking and LCA results.

A question regarding life cycle based communication is pivotal, not only because of an increase in the number of LCA-based ads, but also because of the European Commission Initiative on Product and Organization Environmental Footprints. PEFs and OEFs are intended for estimating and communicating the life cycle environmental performance of products and organisations [19]. The verification and assessment of various communication vehicles are currently important subjects for the European Commission and for related pilot working groups. Different variants of PEF labels, as one of the communication vehicles, are under consideration. A few years ago, in 2012, a study entitled "Different options for Communicating Environmental Information for Products" was launched by the European Commission-DG Environment. The aim of this study was to review and analyse the existing knowledge about different ways of providing final consumers with multi-criteria environmental information related to products [20].

As mentioned above, the papers about using LCA results as content of marketing communications can be found. However, no information was discovered in the literature about using neuroscience to verify the effectiveness of this kind of communication. In order to fill this gap, an initiative has been undertaken by the authors to use neuromarketing tools to assess the human reaction to products labelled with life cycle based environmental labels and to ads with life cycle environmental information included. Our project aims at answering the following questions:

(1) What, if any, are the differences in the neurological responses of consumers as a result of exposure to eco-labelled and non-eco-labelled products?

(2) Which elements of environmental labels and the eco-labelled products attract the visual attention most? 
(3) Is there any correlation between consumers' ecological awareness and their response?

(4) What kind of neurological response (in terms of activated brain regions and response time) follows the exposure to environmental information?

(5) Are the selected ecolabels convincing enough for consumers to persuade them to select the eco-labelled products?

\section{Project Procedure and Methodology}

The project consists of three parts intended to be performed independently: a survey, monitoring of purchasing behaviour (in a shopLAB) and monitoring of psychophysiological reactions (in an EEG laboratory). A description of the parts in terms of placing, tools, key elements and their relationship with the project goals (questions to be answered) is presented in Table 1. A first part-the survey-is aimed at identifying the types of participants involved, in terms of their environmental awareness and tendency to practice the principles of sustainable development in daily life. This survey is assumed to play a supplementary role for the whole project. The methodology and the consumer typology assessment tool have been described in [21]. The main purpose of the survey is to analyse the relationship between consumer types (Voluntary Simplifiers, Beginner Voluntary Simplifiers, Accidental Simplifiers, Non-Voluntary Simplifiers) [21, 22] and their reaction to environmental labels and environmentally labelled products. Attensee Software [23] is intended to be used to verify the visual attention of the surveyed participants. The international scope of the survey provides a chance to compare results obtained in different countries from the perspective of cultural and international differences. The same consumer assessment tool is intended to be used in the EEG laboratory. However, in this case, only Polish respondents will be involved and the focus is on finding a relationship between consumer types and their reaction which will be measured by electroencephalograph (EEG) and the eye-tracker. More detailed information regarding the research in EEG lab can be found in a Sect. 4.

Another part of the project is to analyse consumer behaviour in a shopping environment. This task is performed in a shopLAB - a laboratory where real shopping conditions are reflected. The research procedure is as follows: the products with and without environmental labels are placed on the shelves and the participants - equipped with an eyetracker-make decisions about selecting products. The participants are divided into three groups. All of them are provided with the same shopping list on which the products to buy are listed. The members of the first group are fully free in their decision making (the only condition is to follow the shopping list). The second group is recommended to buy only green products. They make decisions based only on their current knowledge and experience, without any prior training. The members of the third group take, before shopping, a short course 
Table 1 A description of the project parts

\begin{tabular}{|c|c|c|c|}
\hline & \multicolumn{3}{|l|}{ Project parts } \\
\hline & Survey & $\begin{array}{l}\text { Monitoring of purchasing } \\
\text { behaviour }\end{array}$ & $\begin{array}{l}\text { Monitoring of } \\
\text { neurological reaction and } \\
\text { visual attention }\end{array}$ \\
\hline Placing & $\begin{array}{l}\text { A remote survey via } \\
\text { internet scoped } \\
\text { internationally } \\
\text { (respondents from } \\
\text { various countries } \\
\text { included) }\end{array}$ & $\begin{array}{l}\text { The shopLAB reflecting } \\
\text { real shopping conditions } \\
\text { (shelves with products, } \\
\text { check out) (Poznan, } \\
\text { Poland) }\end{array}$ & $\begin{array}{l}\text { The laboratory equipped } \\
\text { with devices for } \\
\text { measuring the human } \\
\text { body's } \\
\text { psychophysiological } \\
\text { reactions (EEG, GSR, } \\
\text { OG) (Poznan, Poland) }\end{array}$ \\
\hline Tools & $\begin{array}{l}\text { - Consumer typology } \\
\text { assessment tool } \\
\text { - Attensee Software }\end{array}$ & $\begin{array}{l}\text { - Questionnaire } \\
\text { - Consumer typology } \\
\text { assessment tool } \\
\text { - Eyetracker }\end{array}$ & $\begin{array}{l}\text { - EEG } \\
\text { - Eyetracker } \\
\text { - Consumer typology } \\
\text { assessment tool } \\
\text { - Questionnaire } \\
\end{array}$ \\
\hline $\begin{array}{l}\text { Key } \\
\text { research } \\
\text { elements }\end{array}$ & $\begin{array}{l}\text { - Consumer types } \\
\text { - Environmental } \\
\text { awareness of the } \\
\text { analysed consumers } \\
\text { - Visual attention }\end{array}$ & $\begin{array}{l}\text { - Consumer types } \\
\text { - Purchasing } \\
\text { decision-making } \\
\text { - Purchase intention } \\
\text { - Visual attention } \\
\end{array}$ & $\begin{array}{l}\text { - Consumer types } \\
\text { - Visual attention } \\
\text { - The regions of the brain } \\
\text { activated }\end{array}$ \\
\hline $\begin{array}{l}\text { Research } \\
\text { objects }\end{array}$ & $\begin{array}{l}\text { - Environmental labels } \\
\text { - Environmentally } \\
\text { labelled products }\end{array}$ & $\begin{array}{l}\text { - Environmental labels } \\
\text { - Environmentally } \\
\text { labelled products }\end{array}$ & $\begin{array}{l}\text { - Environmental labels } \\
\text { - Environmentally } \\
\text { labelled products } \\
\text { - Print and video } \\
\text { advertising } \\
\end{array}$ \\
\hline $\begin{array}{l}\text { Exposition } \\
\text { mode }\end{array}$ & $\begin{array}{l}\text { Via computer screen } \\
\text { (only sight involved) }\end{array}$ & $\begin{array}{l}\text { Full and direct exposition } \\
\text { (sight, hearing and touch } \\
\text { involved) }\end{array}$ & $\begin{array}{l}\text { Via computer screen } \\
\text { (sight and hearing } \\
\text { involved) }\end{array}$ \\
\hline $\begin{array}{l}\text { Number of } \\
\text { participants }\end{array}$ & ca. 400 & ca. 100 & ca. 90 \\
\hline $\begin{array}{l}\text { Questions } \\
\text { to be } \\
\text { answered }\end{array}$ & Q3 & Q1, Q5 & Q1, Q2, Q3, Q4 \\
\hline
\end{tabular}

on LCA and environmental labels. From this, the effect and role of an information campaign can be noticed.

Four product categories have been selected for the research in all parts of the project: red meat, t-shirt, liquid detergent and TV-set. There were two criteria for selecting these product categories:

- they represent various modes of purchasing decision-making (meat-purchased emotionally with low commitment; t-shirt - purchased emotionally with high commitment; household detergent - purchased rationally with low commitment and TV-set - purchased rationally with high commitment),

- they have been included in a PEF pilot phase and a draft version of the PEFCR document is available and/or the EU ecolabel criteria exist. 
Two sorts of environmental labels are within the scope of the research: draft variants of PEF labels (own proposals inspired by Lewandowska et al. [20, 24]) and the EU ecolabel [25]. Additionally, ads with life cycle based environmental information are prepared.

\section{Measurement of Human Reaction to Environmental Labels and Advertising}

A core part of the project is to perform the research in the EEG lab. The neurological reaction of the participants is measured by using electroencephalography (EEG) and the visual attention by applying an eye-tracker. There are some metrics used in neuro-marketing practice, for example, NeuroMetrics developed by NeuroFocus Inc. [26]. Pradeep [26] mentions three primary metrics: an attention, an emotional engagement and a memory retention. The attention (visual and cognitive) and the emotional engagement can be described in terms of reaction location (the regions of the brain activated) and the parameters in the EEG/EOG recordings. For example, emotional engagement can be measured as a frontal asymmetry in the brain which can be used especially as a measure for a motivational direction [27]. Greater left-sided activation at baseline predicts dispositional tendencies toward approach. Greater right-sided asymmetry predicts dispositional tendencies towards avoidance [27, 28]. According to a dimensional model of emotions [27, 29], three emotion-related factors can be distinguished: an emotional valence (positive vs. negative), an emotional intensity (from high arousal to low) and a motivational direction (from approach to avoidance). Environmental information and environmental labels can be considered as minor emotional stimuli, so there is a risk of low emotional arousal as a result of exposure to analysed labels and the environmental information included in the ads. The research is intended to confirm or deny this assumption, also from the perspective of the consumers' typology. The assumptions related to the labels as an object of emotional stimulation are presented in Fig. 1.

Effective environmental communication (made via ecolabels or ads) occurs when a sensation activated by exposure to a label or information is recognised as interesting and/or easy to understand. As a next step, a sensation must be-for rational and/or emotional reasons-recognised as worth saving in a memory (Fig. 2).

The research participants in the EEG lab, equipped with an eye-tracking glasses and an EEG device, are intended to be exposed (via screen) to the following:

- the impact category names,

- the ecolabels (different draft variants of PEF labels and the EU ecolabel),

- the products (meat, t-shirt, TV-set, detergents) with and without ecolabels,

- the print ads with life cycle based environmental information included,

- the video ads of the eco-labelled products. 


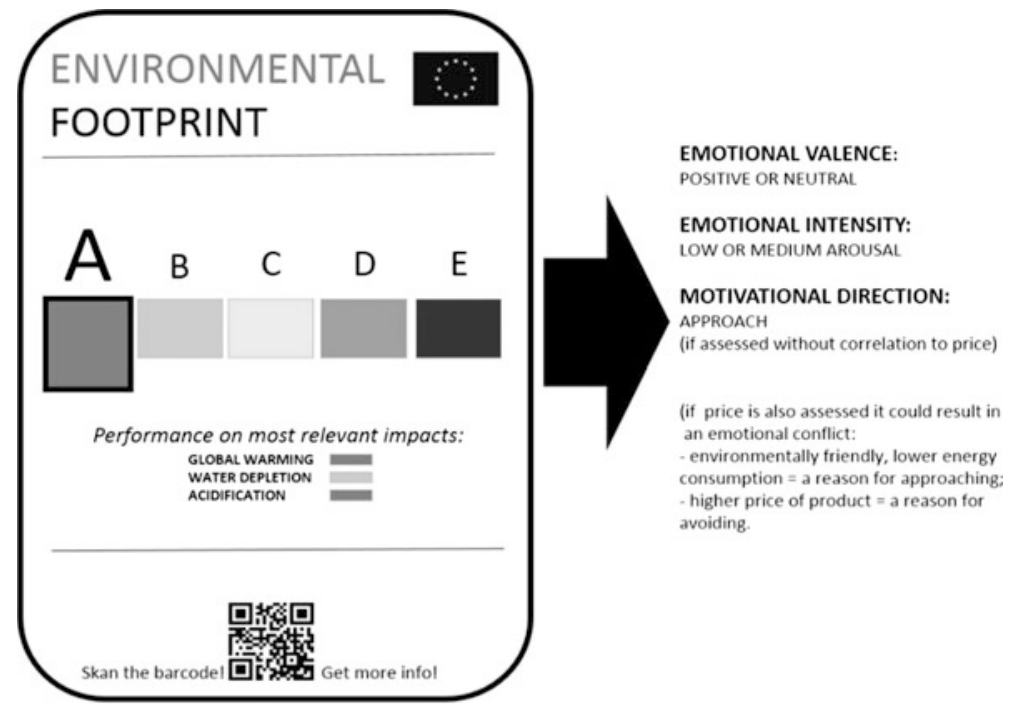

Fig. 1 The assumptions regarding environmental labels as emotional stimuli (draft version of PEF label as an example)

Step 1. Visual attention and percpetion - processes realised without involving higher cognitive processes

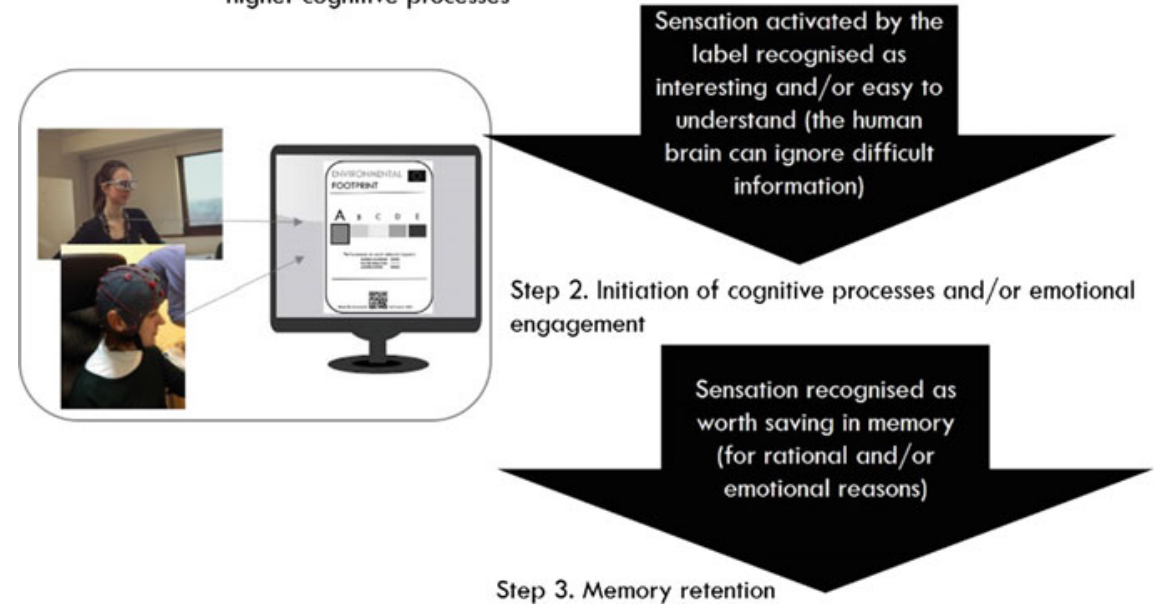

Fig. 2 The initiation of mental processes as a result of exposure to ecolabels or environmental information included in the ads

Figures 3 and 4 illustrate an example of the results which are expected from the project. Both show the eye-tracking statistics related to the EU ecolabel: displayed solely (Fig. 3) and with a product (Fig. 4). In both cases the perception area has been divided into predefined areas called "Areas Of Interest" (AOIs). Figure 3 


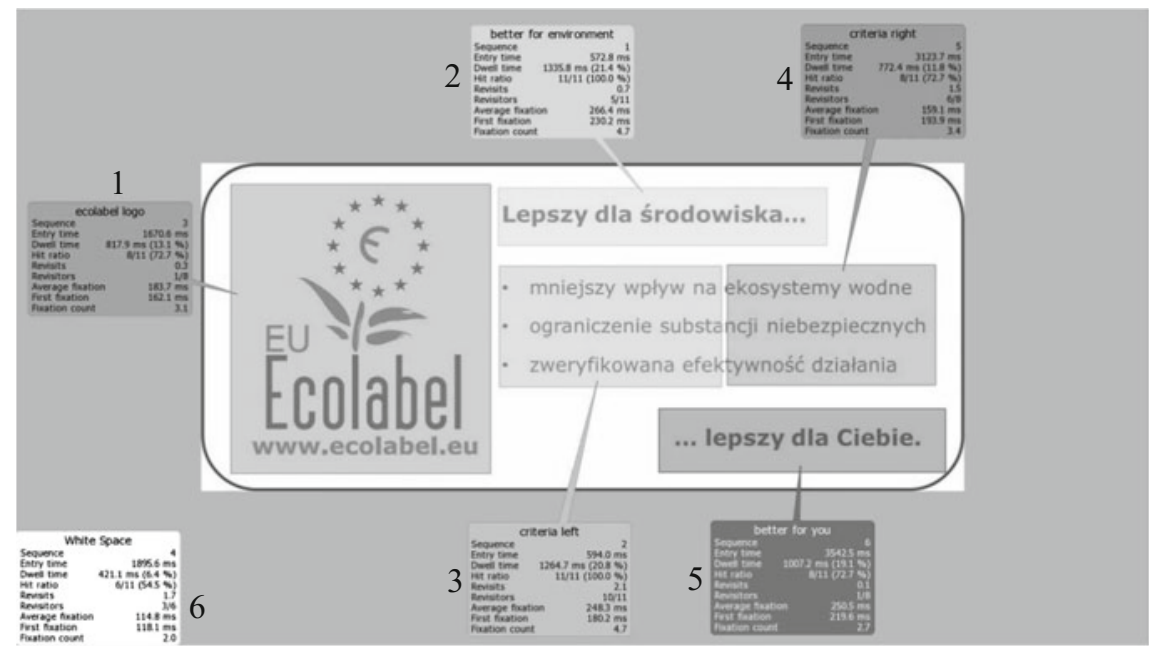

Fig. 3 An example of eye-tracking statistics for the EU ecolabel

includes six AOIs named: an "ecolabel logo" (1), "better for environment" (2), "criteria left" (3), "criteria right" (4), "better for you" (5) and a "white space" as a remaining area (6). Figure 4 presents seven AOIs: a "dark blue label" (1), and the six AOIs previously cited. For each AOI the following information is listed: a sequence (an order of gaze hits into the AOIs), an entry time (average duration from start of the trial to the first hit on an AOI), a dwell time (all fixations and saccades within an AOI for all selected participants), a hit ratio (how many participants out of

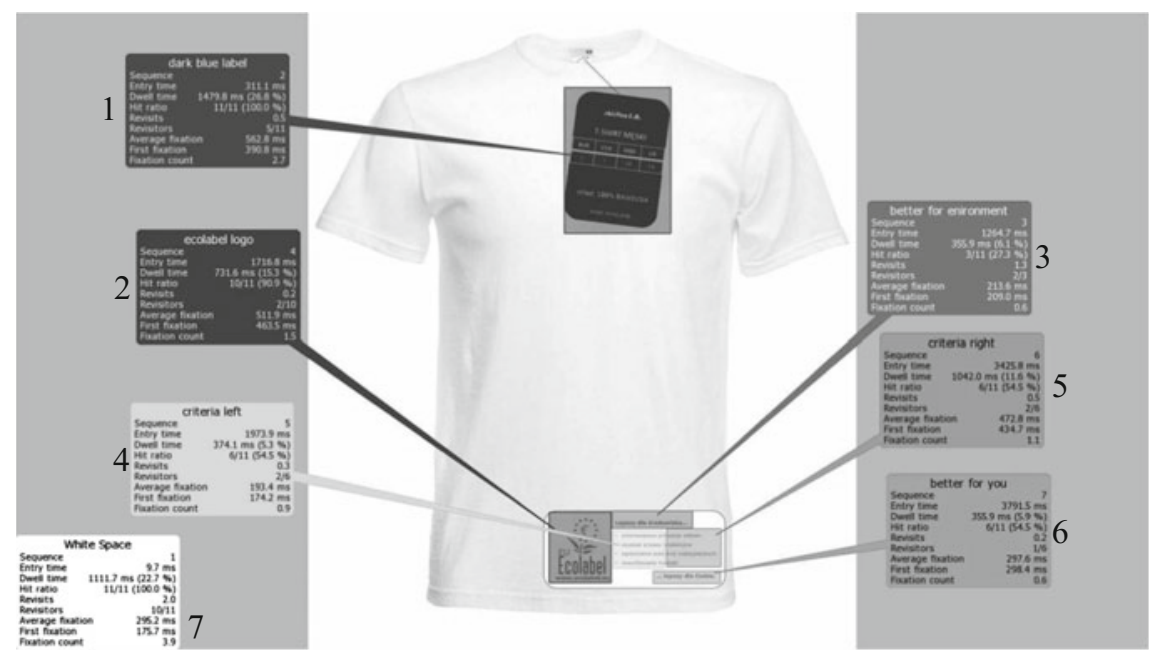

Fig. 4 An example of eye-tracking statistics for the eco-labelled product 
the selected ones looked at least once into the AOI), revisits (a number of revisits divided by a number of selected participants with at least one glance), re-visitors, average fixation (the sum of fixation times divided by a number of fixations) and first fixation (average duration of first fixation).

The final phase of research in the EEG lab is to assess the reactions of the participants as a result of their exposure to video advertising. For four selected products, the ads are prepared in two variants: emotional and rational. Additionally, the content of the ads is differentiated in terms of scope and in the way environmental information is presented. Due to the analysis of EEG and EOG recordings, reactions to the different ways of environmental information presentation can be compared.

\section{Final Conclusions}

The role of ecolabels for a product-related environmental communication and for the consumers' decision making is well-known and often discussed. A discussion on the same role of LCA and life cycle based environmental information is less evident, although some works on this subject can be also found in the literature. However, in most cases, a survey based on a questionnaire is used as the main way to get knowledge about consumer motivation and preferences. The use of neuro-marketing tools gives more room for interpretation and seems to be more objective than a traditional surveying. The eye-tracking results let follow a glance of the participants and identify these AOIs which attract their visual attention first and for the longest time. At the same time, the elements which are ignored by the participants can be found. It is important as the visual stimulation is a first step for potential activation of higher cognitive processes. From this point of view, a crucial point is to know the sequence and the fixation times for AOIs predefined for the ecolabels, the eco-labelled products and the ads. It can be helpful in designing the ecolabels and the ads in order to display the environmental information in a way which actually attracts the consumers' visual attention. The preliminary results showed that the elements of ecolabels which attract participants attention most are: EP scale (especially EP class); the words "environmental footprint", "better", "improved", "limited"; EU ecolabel flower; life cycle circle; a final improvement score; the sentence "better for you ... better for environment". The expected results and implications of our project go far beyond the environmental labelling and reach a more broadly scoped life-cycle based communication. It causes that a potential target audience of our project is not limited to the specialists in the environmental labelling, but also the experts in green marketing as well as LCA practitioners and $\mathrm{PEF} / \mathrm{OEF}$ society. 


\section{References}

1. Ottman JA, The new rules of green marketing: strategies, tools, and Inspiration for Sustainable branding, Greenleaf Publishing, 2011.

2. Oates C, McDonald S, Alevizoua P, Hwang K, Young W, McMorland LA, Marketing sustainability: Use of information sources and degrees of voluntary simplicity, Journal of Marketing Communications, 14 (5), 2008: 351-365.

3. Janikowski R, Imperative for Sustainable Everyday (org. Nachhaltigkeit Als Imperativ des Alltags). Humanities and Social Science 19 (XIX) 21 (3), 2014: 71-82.

4. Eren-Erdogmus I, Lak HS, Çiçek M, Attractive or Credible Celebrities: Who Endorses Green Products Better?, Procedia-Social and Behavioral Sciences 235, 2016: 587-594.

5. Bhat VB, Green Marketing Begins with Green Design, Journal of Business and Industrial Marketing (8)4, 1993: 26-31.

6. Nissinen, A., Heiskanen, E., Grönroos, J., Honkanen, A., Katajajuuri, J.-M., Kurppa, S., Mäkinen, T., Seppälä, J., Timonen, P., Usva, K., Virtanen, Y., Voutilainen P, Developing LCA-based benchmarks for sustainable consumption-for and with users $2005 \mathrm{http} / /$ orgprints.org/11268/1/LCA.pdf accessed: 21st May 2017.

7. Molina-Murillo SA, Smith TM, Exploring the use and impact of LCA-based information in corporate communications, International Journal of Life Cycle Assessment, 14, 2009:184194.

8. Lewandowska A, Witczak J, LCA in advertising, Ecomanager, 5, 2012:44-45 (in Polish).

9. Dahlbo H, Koskela S, Pihkola H, Nors M, Federley M, Seppälä J, Comparison of different normalised LCIA results and their feasibility in communication, International Journal of Life Cycle Assessment, 18, 2013:850-860.

10. Geyer R, DuBuisson MA, life cycle-based framework for environmental assessments of eco-entrepreneurship IN: Gary D. Libecap (ed.) FRONTIERS IN ECO-ENTREPRENEURSHIP RESEARCH (Advances in the Study of Entrepreneurship, Innovation \&amp; Economic Growth, Volume 20) Emerald Group Publishing Limited, 2015, pp. 53-78.

11. Klöpffer W, Heinrich AB, How to Communicate LCA Results, The International Journal of Life Cycle Assessment, 5 (3) 2000:125.

12. Heijungs R, Ten easy lessons for good communication of LCA, The International Journal of Life Cycle Assessment, 19 (3), 2014:473-476.

13. Koffler C, Reply to "Ten easy lessons for good communication of LCA" by Reinout Heijungs, The International Journal of Life Cycle Assessment, 19 (4), 2014:1170-1171.

14. Two sides facts. Are "Go Green-Go Paperless" claims supported by sound and verifiable evidence? http://www.twosides.info/download/Understanding_generic_calculators_Life_ Cycle_Assessments_and_risks_to_corporate_reputation.pdf (accessed: 21st May 2017).

15. Framework for Responsible Environmental Marketing Communications ICC DOCUMENT $\mathrm{N}^{\circ}$ 240-46/557-14 JANUARY 2010 Prepared by ICC Commission on Marketing and Advertising http://www.iccwbo.org/Advocacy-Codes-and-Rules/Document-centre/2011/ICC-frameworkfor-responsible-environmental-marketing-communications-(2011)/ (accessed: 21st May 2017).

16. https://www.youtube.com/watch? $\mathrm{v}=6 \mathrm{RNnzfUHw} Y 8$ (accessed: 21st May 2017).

17. https://www.youtube.com/watch?v=CVa4GBze3tU (accessed: 21st May 2017).

18. https://www.youtube.com/watch?v=iD-m6qBij8Q (accessed: 21st May 2017).

19. Commission Recommendation of 9 April 2013 on the use of common methods to measure and communicate the life cycle environmental performance of products and organisations.

20. BIO Intelligence Service, Study on different options for communicating environmental information for products. Final report prepared for the European Commission - DG Environment, 2012. 
21. Lewandowska A, Witczak J, Giungato P, Dierks C, Kurczewski P, Lemanska-Pawlak K, Inclusion of life cycle thinking in sustainability oriented consumer's typology — a proposed methodology and an assessment tool, 2018, Sustainability (under submission).

22. Mcdonald S, Oates C J, Alevizou P J, Young C W, Hwang K, Individual strategies for sustainable consumption. Journal of Marketing Management, 28 (3-4), 2012: 445-468.

23. http://www.attensee.com/method/ (accessed: 22nd May 2017).

24. http://ec.europa.eu/environment/eussd/smgp/pdf/infographic-env-info.pdf (accessed: 23rd May 2017).

25. Eu Ecolabel logo guidelines http://ec.europa.eu/environment/ecolabel/documents/logo_ guidelines.pdf (accessed: 23rd May 2017).

26. Pradeep AK, The Buying Brain: Secrets for Selling to the Subconscious Mind, Wiley, 2010 ISBN: 978-0-470-60177-8.

27. Mauss IB, Robinson MD, Measures of emotion: A review, Cognition and Emotion, February 1, 23(2), 2009: 209-237.

28. Keshava MGN, Zaved AK, Cognitive attention behaviour detection systems using Electroencephalograph (EEG) signals, Research Journal of Pharmacy and Technology, 7 (2), 2014:238-247.

29. Dabrowski A, What are emotions? Multicomponents theory of emotion, Analysis and Existence, 27, 2014:123-146.

Open Access This chapter is licensed under the terms of the Creative Commons Attribution 4.0 International License (http://creativecommons.org/licenses/by/4.0/), which permits use, sharing, adaptation, distribution and reproduction in any medium or format, as long as you give appropriate credit to the original author(s) and the source, provide a link to the Creative Commons license and indicate if changes were made.

The images or other third party material in this chapter are included in the chapter's Creative Commons license, unless indicated otherwise in a credit line to the material. If material is not included in the chapter's Creative Commons license and your intended use is not permitted by statutory regulation or exceeds the permitted use, you will need to obtain permission directly from the copyright holder.

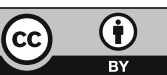


Part II

Innovation for Sustainable Production and Urban Systems 


\title{
LCM for Transport and Mobility
}

\author{
Stephan Krinke and Mara Neef
}

\begin{abstract}
LCM studies of industry and academia show the increasing importance of implementing life cycle thinking in the transport and mobility sector. Tools facilitating the use of LCA in product development processes increase the relevance of this topic in companies. OEMs use LCM as decision support for decarbonisation strategies and operationalization of greenhouse gas reduction targets. Next to environmental impacts, financial and social aspects are considered for a holistic assessment of vehicles. Regional impacts of alternative fuels and power trains need to be considered to support the development of sustainable mobility strategies in nation states. Regional specifics are also included in new data sets for modelling flat steel production along the value chain. Introducing voluntary credit transfer options in automotive legislation is proposed to incentivize low-carbon innovations throughout the whole life cycle of vehicles.
\end{abstract}

\section{Implementing Life Cycle Management in Transport and Mobility Industry}

In the Open Hybrid Lab Factory (OHLF) in Wolfsburg LCM is implemented throughout the design and manufacturing process of eco-efficient lightweight components. For this reason, an approach to interpret and apply LCA results in an effective way during different development and production steps is necessary. However, most of the involved design and production experts have no background in LCA. In order to facilitate the use Life Cycle Engineering (LCE) by non-LCE experts, a tool that allows for intuitive handling of inventory data and fast

\footnotetext{
S. Krinke $(\square)$

Volkswagen AG, Environmental Affairs, Letter Box 1896,

38436 Wolfsburg, Germany

e-mail: stephan.krinke@volkswagen.de

M. Neef

Volkswagen AG, Environmental Affairs Product, Letter Box 1774,

38436 Wolfsburg, Germany

(C) The Author(s) 2018

E. Benetto et al. (eds.), Designing Sustainable Technologies,

Products and Policies, https://doi.org/10.1007/978-3-319-66981-6_20
} 
interpretation of results was developed [1]. The integrated engineering process is visualized on a number of screens to show system interdependencies. Like this, material choices or manufacturing processes can be compared and evaluated fast and effectively from a life cycle point of view. In the future, environmental hotspots and trade-offs are visualized via spatial representation and cluster heat maps.

Likewise, Henkel set up an LCA tool (Henkel Easy LCA) to facilitate the use of LCA by non-LCA experts in the development process of new products [2]. The goal was to develop a streamline LCA tool with the highest level of detail necessary to make substantiated decisions in a first-tier assessment. For example, with the help of the tool, used raw materials can be assessed in less than two minutes. For higher-tier assessments, LCA specialists can be consulted for more detailed analyses. Until now, only greenhouse gas (GHG) emissions are considered in the raw materials assessment though water consumption will be included next. Henkel Easy LCA is also used to model and assess the logistics of products. It includes intermodal shifting, warehousing and capacity utilization.

At BMW, Life Cycle Sustainability Assessment (LCSA) on a component level was developed and adopted [3]. The three sustainability dimensions are assessed in an integrated way by combining Life Cycle Costing (LCC), Social-LCA (S-LCA) and LCA. Criticality points of up to 100 represent the respective impacts in each dimension caused by a specific component. The dimensions are equally weighted so that as a result, the average of criticality points of all dimensions show the relative performance of the assessed component in relation to all other components used. When applying LCSA it is possible to generate a higher environmental or financial impact by choosing a material that causes least negative social impacts. The goal, however, is to improve the vehicle's impacts in all of the sustainability dimensions.

At Volkswagen, different powertrains and fuels are assessed with LCA considering current circumstances and developments until 2030 to provide long-term support for the Group's decarbonisation strategy [4]. Different power trains are compared via the Performance Feel Index that assures a comparison of vehicles with similar user experiences in terms of overall driving dynamics. The whole life cycle is assessed to avoid burden shifting between life cycle phases when altering the fleet's power train and fuel composition. Like this, main drivers for efficiency improvements are analysed. The study showed that all power train systems still have significant improvement potentials. Special effort should be put into reducing the carbon dioxide burden of lithium-ion battery production, e.g. via energy efficiency, green energy supply and closed-loop recycling.

Similarly, at Toyota a life cycle approach is pursued to reach their 2050 target of reducing carbon emissions by $90 \%$ compared to 2010 [5]. Toyota will therefore focus on hybrid and fuel-cell vehicles powered with renewable energy and alternative fuels. Their LCAs further show that wrought alloy aluminium has a comparatively low environmental impact and should therefore be used for light-weight materials. The supply of needed quantities of wrought alloy aluminium is problematic though. Cooperation between academia, OEMs and other industries is proposed to solve the supply problem and develop more detailed data sets on the re-use of aluminium in the automotive industry. 
An LCA study by the Zurich University of Applied Sciences focused on synthetic power-to-gas as fuel for transportation with a Volkswagen Golf as reference car [6]. It showed that if synthetic power-to gas is produced with renewable energy, it emits less GHG per kilometre than fossil gas. Though at the moment, the Swiss grid mix includes a high share of nuclear power so that the electricity consumption of the electrolysis causes $51 \%$ of the overall impact. Additional recommendations are improving the electrolysis efficiency and using industrial waste gases as carbon dioxide source rather than atmospheric carbon dioxide to be competitive with conventional natural gas production.

The Japanese National Institute of Advanced Industrial Science and Technology assessed whether hydrogen is a suitable automotive energy carrier in Japan [7]. It was assumed that the hydrogen would be produced in Australia and subsequently transported to Japan. The LCA study showed that the hydrogen source, producer and transport need to be evaluated regionally in order to give meaningful results. Generally, liquid hydrogen should be produced with renewable energy to reduce its environmental impact. Within the assessed scenarios, hybrid vehicles cause the overall lowest environmental impact followed by hydrogen-powered and gasoline-powered vehicles.

The German Stahlinstitut VDEh developed regional specific datasets for flat steel products that facilitate a detailed analysis along the value chain [8]. The motivation for realizing the project was providing suitable data for customers and governments interested in modelling steel recycling. As steel producers either use iron ore or scrap to produce flat steel, both strategies are available in the datasets. In order to model steel recycling, the multiple recycling approach was applied. As a result, after including six recycling steps, the carbon dioxide burden of $1 \mathrm{~kg}$ of steel is reduced by $50 \%$.

\section{Policy Framework}

A study presented by the Technical University Berlin proposes an approach to foster sustainable mobility by integrating a life cycle perspective in automotive legislation [9]. Although policies concerning the automotive sector are becoming more relevant they do not include an LCA point of view yet but mainly focus on regulating tailpipe emissions. As the introduction of BEVs is shifting the environmental burden from the use to the production phase, a life-cycle approach in policies is needed to display this development. Instead of introducing a single life-cycle based policy tailored to vehicles, voluntary credit options should be operationalized within existing systems. Until now, automotive OEMs have no policy advantage if they save emissions in the production. Therefore, a credit transfer should be facilitated that values low-carbon innovations in the manufacturing phase. Hence, improving the environmental burden of vehicles throughout the whole life cycle becomes more attractive for OEMs. 


\section{Conclusions}

The session on LCM in transport and mobility underlined the necessity of including a life cycle perspective in the development of sustainable solutions in the sector. Companies and governments use a life cycle approach to develop decarbonisation strategies and operationalize targets. LCM is applied by industry to foster climate-friendly innovations from the design to end-of-life phase of materials, components and final products. Streamline LCA tools help disseminating life cycle thinking and effective implementation of LCA results by different occupational groups. By shifting the current focus on tailpipe emission regulations to a life cycle perspective in automotive legislation, the use of LCM by OEMs could further be consolidated.

\section{References}

1. Kaluza A, Kleemann S, Walk W, Vietor T, Herrmann C, Integrating life cycle engineering in the development of eco-efficient lightweight concepts.

2. Otte N, Singh R, Engelmann K, Tolls J, Nawaz M, Parametric LCA for a first tier assessment of products by the example of logistics.

3. Tarne P, Wagner V, Finkbeiner M, Adopting Life Cycle Sustainability Assessment in the Automotive Industry.

4. Plaga B, LCA comparison of power trains and fuels today and 2030 .

5. Denis R, The Toyota 2050 Environmental Challenges.

6. Wettstein S, Stucki M, Synthetic PtG as Fuel for Transportation.

7. Kudoh Y, Kitagawa N, Muramatsu R, Ozawa A, Genchi Y, Environmental Footprint Profiles of Hydrogen as Automotive Energy.

8. Hatscher N, Kroop S, Mayr R, Meincke A, Mohr L, Redenius A, Schuster S, Regional specific LCA data of flat steel products for detailed analysis along the value chain.

9. Lehmann A, Berger M, Finkbeiner M, Life cycle-based $\mathrm{CO}_{2}$ emission credits - an approach to foster sustainable mobility by integrating a life cycle perspective into automotive legislation.

Open Access This chapter is licensed under the terms of the Creative Commons Attribution 4.0 International License (http://creativecommons.org/licenses/by/4.0/), which permits use, sharing, adaptation, distribution and reproduction in any medium or format, as long as you give appropriate credit to the original author(s) and the source, provide a link to the Creative Commons license and indicate if changes were made.

The images or other third party material in this chapter are included in the chapter's Creative Commons license, unless indicated otherwise in a credit line to the material. If material is not included in the chapter's Creative Commons license and your intended use is not permitted by statutory regulation or exceeds the permitted use, you will need to obtain permission directly from the copyright holder. 


\title{
Conceptual Development of Hybrid Structures Towards Eco-Efficient Vehicle Lightweighting
}

\author{
Alexander Kaluza, Tim Fröhlich, Sebastian Kleemann, \\ Wolfgang Walk, Christoph Herrmann, Stephan Krinke \\ and Thomas Vietor
}

\begin{abstract}
Lightweight design is a major strategy in automotive development. The dominant motivation is a reduction of use-phase energy demands while retaining or improving technical performance. The application of new materials is the prevalent lightweighting strategy. Modern vehicle concepts extend material substitution up to the combination of different materials on a component level, so called hybrid designs. While engineering processes, methods and tools in design and production engineering are well established for conventional designs, hybrid designs pose new challenges. Lightweight materials as well as new manufacturing and recycling processes may cause increased environmental impacts. In order to achieve eco-efficient lightweight structures, energy savings from the vehicles' use phase need to compensate additional burdens in other lifecycle stages. The current work presents findings gained in a public-private research collaboration. Its starting point is the understanding of the role of life cycle engineering towards its impact on overall sustainability goals. Based on derived key requirements, an integrated life cycle engineering approach is developed. Activities and interfaces between life cycle engineering, component design and manufacturing are elaborated. A special focus is set on the conceptual design stage, as emerging materials and manufacturing technologies lead to a broader concept variety. This stage presents also a major lever for shaping the life cycle environmental impact of components.
\end{abstract}

\footnotetext{
A. Kaluza $(\bowtie) \cdot$ T. Fröhlich · S. Kleemann · W. Walk · C. Herrmann

S. Krinke · T. Vietor

Open Hybrid LabFactory e.V., 38440 Wolfsburg, Germany

e-mail: a.kaluza@tu-braunschweig.de
}
A. Kaluza · C. Herrmann
Chair of Sustainable Manufacturing and Life Cycle Engineering, Institute of Machine Tools and Production Technology (IWF),
Technische Universität Braunschweig, 38106 Brunswick, Germany
T. Fröhlich · S. Kleemann · T. Vietor
Institut for Engineering Design, Technische Universität Braunschweig, 38106 Brunswick, Germany
W. Walk · S. Krinke
Volkswagen AG, 38440 Wolfsburg, Germany
(C) The Author(s) 2018
E. Benetto et al. (eds.), Designing Sustainable Technologies,
Products and Policies, https://doi.org/10.1007/978-3-319-66981-6_21 


\section{Introduction}

Transport is accounting for a quarter of global greenhouse gas emissions with road transport being responsible for three thirds of this share. Towards increasing the environmental sustainability of the transport sector, use phase energy demands gained significant importance in the development of mass-produced vehicles. This is induced by legal requirements targeting greenhouse gas emissions for manufacturers' vehicle fleets. Several measures enable to improve the use phase efficiency, i.e. drivetrain adaptions or optimised aerodynamics. Introducing lightweight structures reduces energy demands during driving situations with frequent acceleration and deceleration as well as elevations. There are different strategies to achieve lightweight design. The predominant approach in vehicle engineering is material substitution. For instance, the application of high strength steels is widespread in the current vehicle generation. As those designs reach their limits in achieving further weight reductions, new approaches take the stage. While multi-material designs assign different materials to each component, hybrid designs combine different materials on a component level.

The integrated framework by Hauschild et al. is presented (see Fig. 1) in order to provide a perspective on the overall effect of lightweight structures regarding sustainability goals as well as the role of life cycle engineering (LCE) in this context [1]. Within the framework an environmental impact axis and a temporal axis span spaces of different scopes. Those scopes set a frame for separate optimisation with spatial/ geographical, organisational and technological implications. It is linked to the IPAT thinking that relates central driving forces to the production and consumption patterns in a future sustainable society. The total environmental impact is presented (I) as a function of the human population (P), the human affluence (A) and the technology factor $(\mathrm{T})$. While this represents a top-down approach within the above mentioned framework that is strongly interrelated with absolute sustainability limits, the publication on hand focuses on a complementary bottom-up approach. Here, product development plays a central role as a large share of the later environmental impact is already decided at this stage. Methods and tools should help to support decision making towards the upper scopes of concern, orienting it towards absolute sustainability. Within the framework, LCE is defined as sustainability-oriented product development activities within the scope of one to several product life cycles. The methods and tools used in LCE must support reducing the total environmental impact associated with technology change. Life cycle management (LCM) has to support this understanding on a company level [2]. As LCE activities relate to an integrated product and process life cycle planning, the interface between LCM and LCE is explained as a floating transition zone [3].

The overarching goal of the presented research is the understanding and adaption of engineering processes in conceptual design towards eco-efficient lightweight structures. The term eco-efficiency in general is defined according to [4] as environmental impact per unit of product. The current research is focusing on a rather technological perspective of that definition. Findings from executing development 


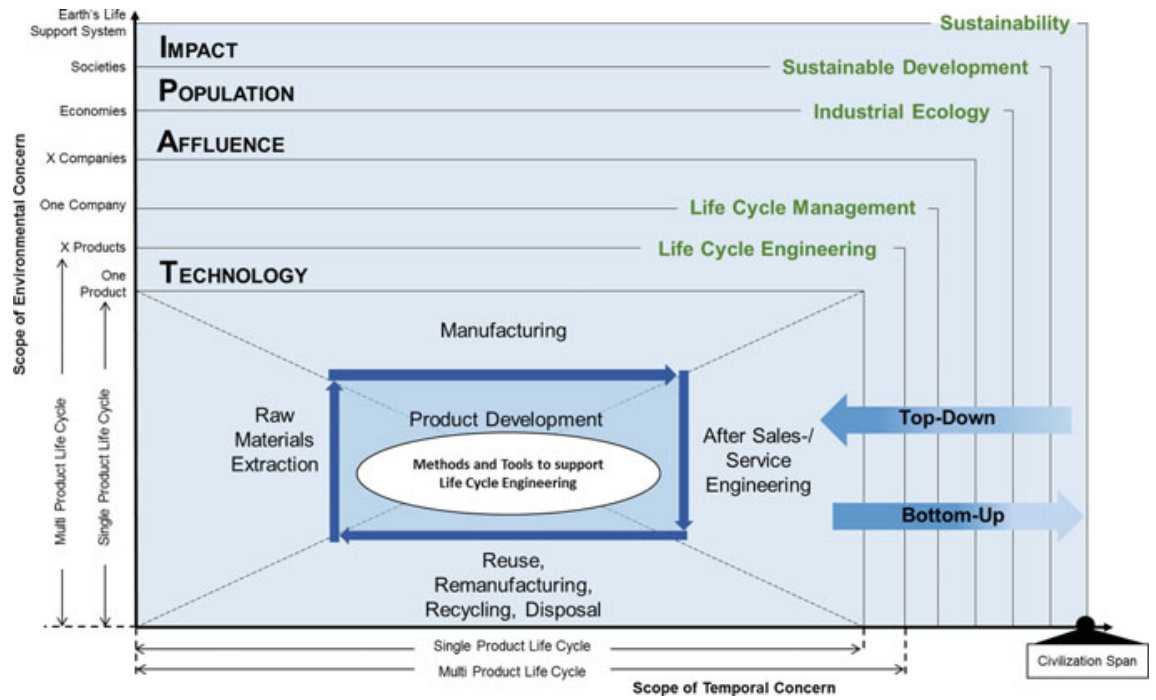

Fig. 1 Life Cycle Engineering (LCE) framework placing manufacturing within the context of planetary boundaries and absolute sustainability [1]

projects on lightweight structures in collaboration between research and large-scale industry production are reflected. This encompasses strengths and weaknesses of current exchange between the involved domains and the impacts of derived decisions. A generalization and aggregation to key requirements guides future research in LCE for lightweight structures. Further activities require to focus on the linkage of technology-based decisions towards a macro-level eco-efficiency enabling a broader sustainability performance, as e.g. promoted by Huppes \& Ishikawa [5].

\section{Automotive Lightweight Structures and LCE}

\subsection{Design of Automotive Lightweight Structures}

Lightweight design (LWD) is an engineering approach intending to reduce a product's weight without downgrading its performance. From a mechanical engineering point of view, the intention is to improve load-bearing capacities without increasing weight [6]. Lightweight design as a discipline is a combination of product development, material science as well as mechanics. Different lightweight strategies are distinguished. Material LWD promotes the substitution towards materials that show an improved technical performance for the specific application. Form LWD targets the shape and topology of structures according to the required load case. Manufacturing $L W D$ enables the manufacturing of lightweight structures through new processes. Constraint LWD subsumes external requirements that 
influence product weight, e.g. maximum payloads in civil engineering. Concept $L W D$ targets the reflection on component boundaries, e.g. aiming for functional integration [7].

LWD requires the joint consideration of all lightweighting strategies. The interplay between material selection and geometrical conceptualization is a major interface in LWD. Material selection based on material performance indicators, e.g. specific stiffness, shows major drawbacks. Those approaches often assume large cross-sections and thin-walled structures without any space restrictions. This is why the theoretical weight reduction numbers are hard to achieve in practice. In contrast to that, Wanner considered the boundary condition of limited installation space [8]. Additionally, automotive component development requires to strictly meet cost restrictions. Kleemann et al. as well as Fröhlich et al. discuss the dependencies of mechanical performance, weight and costs for an automotive roof structure [9]. The manufacturing of lightweight structures requires adaption of existing and qualification of new manufacturing processes, as summarized e.g. in [10]. Manufacturing routes that enable the realization of hybrid designs combine process technologies from the metals, plastics and textile industry. The integration of new processes in current process chains requires the development towards competitive cycle times, the qualification for sensitive process steps, e.g. the cathodic dip painting, as well as the consideration of additional invest volumes as exemplary shown in [11]. The variety and complexity of LWDs and manufacturing suggests to jointly elaborate the potentials and obstacles of a large-scale manufacturing from a design and manufacturing perspective.

\subsection{LCE for Automotive Lightweight Structures}

Several research papers discuss the topic of LCE in automotive engineering. Three constituting aspects of LCE are distinguished as derived from [12]. First, the need for an evaluation method, which enables to assess environmental impacts of products and processes, second the incorporation of a life cycle perspective and third the methods and tools required to achieve the consideration of those aspects in the engineering of products and processes. Life Cycle Assessment (LCA) according to ISO 14040 serves as an established evaluation method enabling the analysis and interpretation regarding different environmental impact categories. As illustrated in Fig. 2, the consideration of the entire product life cycle is crucial within the engineering of lightweight structures. Compared to conventional (steel) designs, lightweight structures often show higher environmental impacts from raw materials extraction. In order to enable eco-efficient LWDs on a product level, use phase energy savings need to compensate those burdens. In addition, potential efforts in the end-of-life phase occur, as conventional processes are not suitable for hybrid components and new processes lack maturity compared to the steel reference. Figure 2 schematically shows the influence of two lightweight alternatives on the environmental impacts of a vehicle component manufacturing in comparison to a 


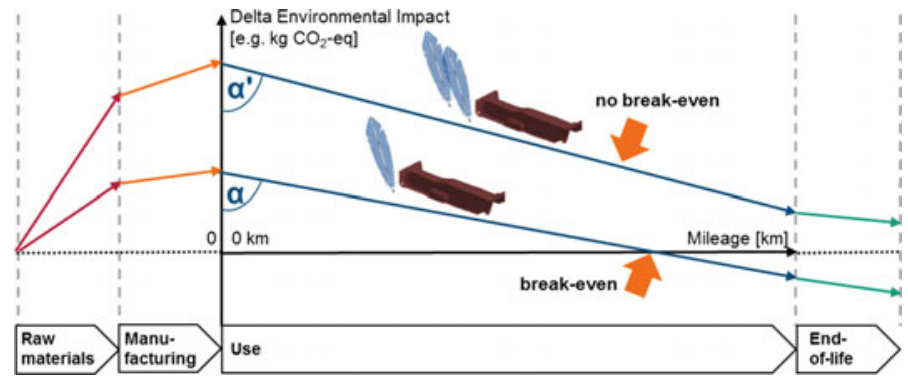

Fig. 2 Life cycle perspective of the influence of lightweight designs on vehicle environmental impacts. Relative representation compared to reference design (base line)

reference (y-intercept). The gradient (angle alpha; alpha') represents benefits due to a decreased energy consumption during use. The break-even point indicates the required driving distance to achieve an environmentally benign alternative. The end-of-life covers the effort to recover material, the avoided landfill and avoided primary material consumption due to the availability of secondary material. In the given example, two components are evaluated. One might reach the break-even during the predicted vehicle use, while the other lightweight alternative does not compensate additional burdens from raw materials and manufacturing during use.

Available methods and tools in LCE reach from qualitative tools to quantitative approaches [13]. Broch emphasises the importance of robust LCE decisions in automotive product development [14]. This includes considering parameters originating from the foreground and relevant background systems as described in [15]. The application of LCE methods and tools is widespread in industry-either taking a prospective or retrospective view. In automotive engineering, full LCA studies help to evaluate the impact of innovative technologies. Furthermore, the assessment of full vehicles is a common application. The integration of LCA results in product development is assisted through elaborated modelling tools. These enable an acceleration of the assessment. Detailed environmental evaluations are typically upstream and downstream activities. A detailed LCA in predevelopment would serve as an input for requirements definition. A downstream assessment of the final product ensures the meeting of requirements. In upstream application, challenges often occur in the adequate prediction of engineering parameters [16]. In order to enable LCE for the engineering with new materials, Ashby extends the property-driven material selection methodology through the consideration of eco-properties, e.g. the impact of raw materials extraction on climate change [17]. Shortcomings might occur as background system parameters are fixed to a certain extent. For example, regional effects in raw materials extraction might significantly influence resulting environmental impacts. Poulikidou et al. propose a method, which integrates design and life cycle assessment in automotive component development. The applied geometry retains for different material alternatives and only monolithic designs are discussed [18]. Lindner \& Schmitt provide a 
methodology that assists in the prediction of energy and resource flows for innovative composite manufacturing chains [19]. In summary, approaches covering single aspects within the LCE support of lightweight vehicle structures are present. However, a comprehensive methodology supporting the design and LCE of lightweight structures is missing for the automotive application. Experiences from aviation applications could serve as a fruitful input for the automotive case. However, the benefits of lightweight structures and consequently the environmental break-even differ due to longer use periods.

\section{Key Requirements for Effective LCE in the Development of Lightweight Concepts}

The current research builds on findings from a public-private collaboration comprising expertise from industrial practice and academic research. The joint development is executed in a lab environment that is designated for developing and providing methods and tools towards developing eco-efficient lightweight concepts for large-scale vehicle manufacturing. The engineering on a product and technology level is in the focus of the current research. This follows the bottom up approach of LCE as described in [1]. The collaboration between experts from design and production engineering as well as life cycle evaluation during the development of various hybrid vehicle lightweight structures enables the derivation of five key requirements. These build the basis for shaping methods \& tools that enable adequate LCE support on the technology level (see Fig. 3).

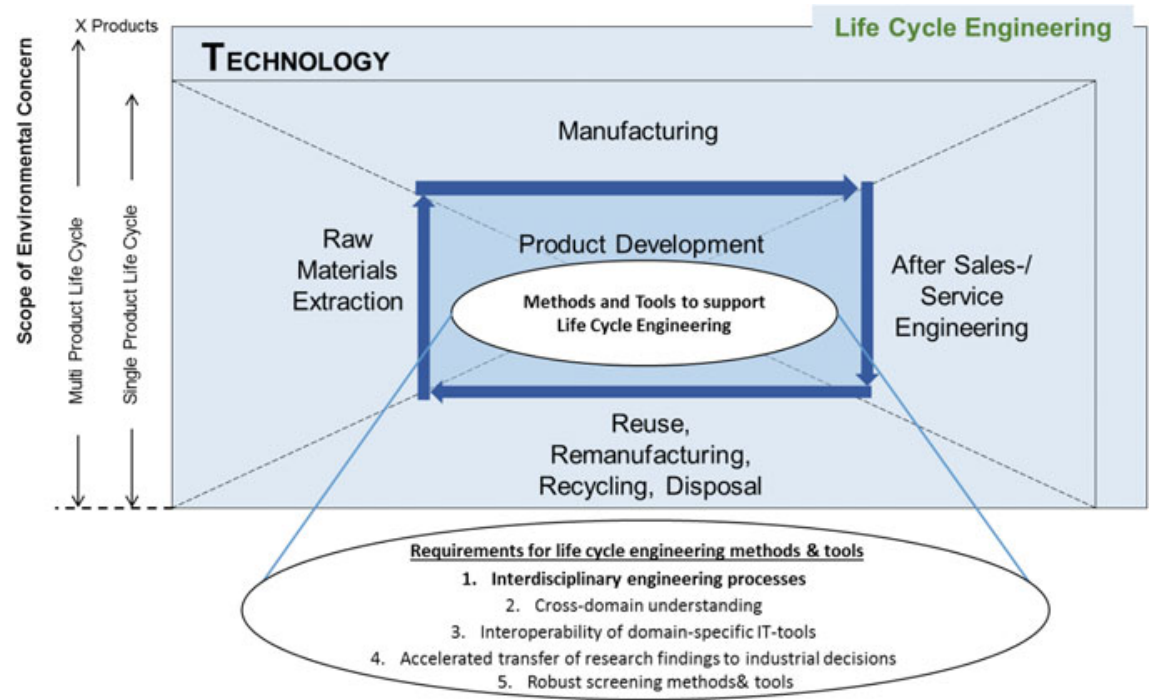

Fig. 3 Key requirements for methods and tools to support LCE of lightweight structures 
1. Interdisciplinary engineering processes: Concurrent engineering approaches are an established concept in the development of vehicle structures. When it comes to the design of hybrid or multi-material concepts, one has to face some specific challenges. Lightweight structures based on hybrid or multi-material approaches require new designs compared to established materials, comprehensive experience is currently missing. Manufacturing technologies for hybrid designs are developing rapidly with implications for parts' design and life cycle evaluation. A particularly high number of variations and gaps in LCA databases, e.g. manufacturing processes, disqualify click-and-rush LCA studies composed of standard modules. All of the considered engineering disciplines have to deal with high uncertainties and engineering processes that are even more dynamic than in conventional vehicle development. Especially product development and manufacturing technology development are to a certain extent parallel activities in the given case.

$2 \& 3$. Cross-domain understanding and interoperability of domain-specific IT tools: All domain-specific engineering activities require the assistance of advanced methods and tools, e.g. numerical simulation regarding crash behaviour or manufacturing process simulations. In addition, the modelling of energy and resource demands relies on complex models incorporating different data sources-from static life cycle inventories to the simulation of different use patterns or the consideration of live manufacturing data. Typically, simplified key performance indicators and visualization techniques are used to exchange information between disciplines. Their benefit lies in the easy interpretability. At the same time, the understanding for cross-disciplinary interlinkages decreases. As the decision scope shifts from the gate-to-gate perspective towards the whole product life cycle as well as across life cycles, domain-specific methods and tools should allow an information flow across disciplines.

4. Accelerated transfer of research findings to industrial decisions: In the context of lightweight structures, but similarly concerning technologies, innovation cycles become shorter including a dynamic innovation network. Thus, academic and industrial research to a certain extent parallel the development itself. Higher dynamics in the interaction of stakeholders are the result.

5. Robust screening methods \& tools for the acceleration of engineering processes: The introduction hybrid or multi-material lightweight structures significantly increases the solution space during the development of vehicle components. This leads to suspension of traditional geometries, component boundaries or installation spaces creating new challenges for methods and tools in the design domain. Cost assessment faces new challenges due to the fast changing designs and the evaluation of new materials, processes and geometries. For both environmental assessment and cost assessment, one major challenge is the scaling up of the applied models in order to predict the behaviour in vehicle mass production. This increased complexity in all disciplines, from engineering design over manufacturing planning up to LCE, needs to be reflected in developing evaluation tools that build on the described complexity, but enable to derive reliable decisions. 


\section{Interdisciplinary Engineering Processes}

In the following, an interdisciplinary engineering process for the conceptual development of lightweight vehicle structures is presented. This addresses the first key requirement as described in the previous section. The presented process extends prior work that focused on a functional modelling level [15]. Building on that, a holistic view on the conceptual development process of hybrid lightweight structures is in the focus of the current work. In this course, a detailed description of interfaces between component design, production engineering as well as life cycle evaluation is provided. Current component development processes consider the disciplines in various depths. Technical, economic and environmental performance are key targets in component development. While a constant tracking of the first two is established, environmental evaluation for new technologies is performed as upstream and downstream activity. The increasing complexity and concept variety through the introduction of lightweight structures, e.g. regarding their materials or joining technologies, suggests a constant tracking of environmental performance within the vehicle development process $[14,20]$. Each of the disciplines rely on own established methods and tailored tools. The proposed process interprets integrated LCE as a close and agile interlink between the disciplines (see Fig. 4).

Within the process, different columns represent the engineering disciplines, whereas four lanes describe the sequence of engineering activities in the respective

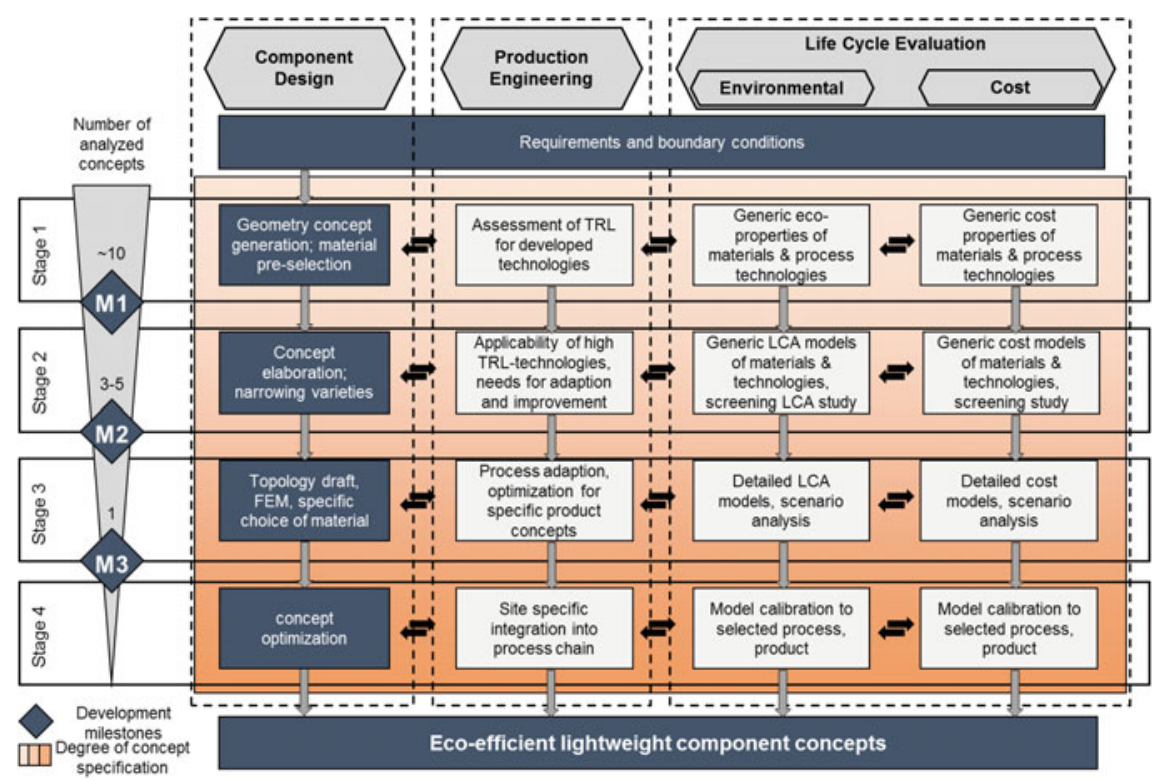

Fig. 4 Interaction between component design, production engineering and life cycle evaluation in an integrated LCE approach 
disciplines (stage 1 to stage 4). Based on a requirements definition by each of the disciplines, a generic component engineering process is followed. The component design provides the pace of the process (blue boxes). This complies with the established situation in vehicle manufacturers, where product development represents the key business process. Contrary to the current practice, production engineering and environmental life cycle evaluation are included as continuous and synergetic activities (white boxes). The sequence of engineering activities and interplays between the disciplines is broken down into sub-activities and interchanges. All activities on a horizontal level are executed as concurrent activities. At each stage, an interdisciplinary review is performed within defined milestones. This does not only result in a stepwise narrowing of uncertainties and the identification of interdisciplinary trade-offs, but also helps to sort out succeeding options while dropping other alternatives as represented by the funnel and respective milestones in Fig. 4. The main outcome of the engineering process are concepts for lightweight components that comply to the disciplines' requirements.

Stage 1 of the proposed process opens up a broad solution space for achieving the desired technical parameters through different geometries, materials and manufacturing routes. This stands in opposition to current component development processes, that rely on a strict breakdown of vehicle targets and thus limit variations for the developed concepts as described in [20]. The second stage represents a concept realisation with the assistance of analytic and simplified numeric calculations. As a result, further processing regards only a small number of concepts. The third stage covers numeric studies as measures to enable a detailed design with final specifications on material compositions as well as manufacturing routes for only one or few concepts. The fourth stage deals with the optimization of the concept in favour.

\section{Discussion and Outlook}

The introduction of hybrid lightweight structures poses new challenges on the support of LCE in automotive development. While the effect of lightweight structures on a broader sustainability perspective defines the overarching research demands, the scope of the current research focuses on a technology perspective. This enables to understand engineering activities and decisions on a micro-level to allow a further integration into a broader LCE context. An increasing variety of materials and manufacturing processes significantly widens the solution space in conceptual development of hybrid structures. In result, LCE methods \& tools need further integration side-by-side to engineering design and production engineering. Key requirements towards the adaption of LCE methods \& tools have been derived and the introduction of integrated engineering processes is elaborated. The approach targets the requirements in a double sense: Firstly, the process ensures that 
non-succeeding options are eliminated in early stages and efforts can be focused. Secondly, the process enables an evolutionary building on established methods and tools.

Further activities are required to enhance the capabilities of the presented approach. The elaboration of technical interfaces between domain-specific tools will accelerate the information exchange in the proposed manner. Coincidently, the identification of further interdisciplinary trade-offs will be enabled. Yet a small number of case studies have confirmed the presented approach. Within the public-private partnership Open Hybrid LabFactory further experience through implementation and adaption will be generated. The communication between disciplines could be enhanced by providing visualizations that enable a low barrier accessibility of the research results. One key facility at the Open Hybrid LabFactory is the newly established 'Life Cycle Design \& Engineering Lab', a workspace to explore modes of cross-discipline cooperation and visualisation techniques in order to support and promote integrated LCE.

Acknowledgements This research is funded by the German Federal Ministry of Education and Research (BMBF) within the research campus "Open Hybrid Lab Factory" and managed by the Project Management Agency Karlsruhe (PTKA). The authors are responsible for the contents of this publication.

\section{References}

1. Hauschild M. Z, Herrmann C, Kara S, An Integrated Framework for Life Cycle Engineering. Procedia CIRP, 61, 2-9, 2017, https://doi.org/10.1016/j.procir.2016.11.257.

2. Herrmann C, Ganzheitliches Life Cycle Management. SpringerVerlag Berlin Heidelberg, 2010, https://doi.org/10.1007/978-3-642-01421-5.

3. Umeda Y, Takata S, Kimura F, Tomiyama T, Sutherland J.W, Kara S, Duflou J. R, Toward integrated product and process life cycle planning-An environmental perspective. CIRP Annals-Manufacturing Technology, 61(2), 681-702, 2012, https://doi.org/10.1016/j.cirp. 2012.05.004.

4. Huppes G, Ishikawa M, A framework for quantified eco-efficiency analysis. Journal of industrial ecology, 9(4), 25-41, 2005, https://doi.org/10.1162/108819805775247882.

5. Huppes G, Ishikawa M, Eco-efficiency guiding micro-level actions towards sustainability: Ten basic steps for analysis. Ecological Economics, 68(6), 1687-1700, 2009, https://doi.org/ 10.1016/j.ecolecon.2009.01.007.

6. Wiedemann J, Leichtbau. Berlin, Heidelberg: Springer Berlin Heidelberg, 2007, https://doi. org/10.1007/978-3-540-33657-0.

7. Friedrich H.E, Leichtbau in der Fahrzeugtechnik, 2013, https://doi.org/10.1007/978-3-83482110-2.

8. Wanner A, Minimum-weight materials selection for limited available space. Materials \& Design, 31(6), 2834-2839, 2010, https://doi.org/10.1016/j.matdes.2009.12.052.

9. Kleemann S, Fröhlich T, Türck E, Vietor T, A Methodological Approach Towards Multi-material Design of Automotive Components. Procedia CIRP, 60, 68-73, 2017, https:// doi.org/10.1016/j.procir.2017.01.010. 
10. Taub A.I, Luo A.A, Advanced lightweight materials and manufacturing processes for automotive applications. MRS Bulletin, 40(12), 1045-1054, 2015, https://doi.org/10.1557/ mrs.2015.268.

11. Eckstein L, Ickert L, Goede M, Dölle N, Lightweight Floor Structure with Reinforcements of CFRP and GFRP. ATZ Autotechnology, 11(2), 48-52, 2011.

12. Jeswiet J, Life Cycle Engineering. In CIRP Encyclopedia of Production Engineering (pp. 757-758), Berlin, Heidelberg: Springer Berlin Heidelberg, 2014, https://doi.org/10.1007/ 978-3-642-20617-7_6609.

13. Bovea M.D, Pérez-Belis V, A taxonomy of ecodesign tools for integrating environmental requirements into the product design process. Journal of Cleaner Production, 20(1), 61-71, 2012. https://doi.org/10.1016/j.jclepro.2011.07.012.

14. Broch F, Warsen J, Krinke S, Implementing Life Cycle Engineering in Automotive Development as a Helpful Management Tool to Support Design for Environment. In G. Sonnemann \& M. Margni (Eds.), Life Cycle Management (pp. 319-329). Springer, 2015, https://doi.org/10.1007/978-94-017-7221-1.

15. Kaluza A, Kleemann S, Fröhlich T, Herrmann C, Vietor T, Concurrent Design \& Life Cycle Engineering in Automotive Lightweight Component Development. Procedia CIRP, 66, 16-21, 2017, https://doi.org/10.1016/j.procir.2017.03.293.

16. Broch F, Integration von ökologischen Lebenswegbewertungen in Fahrzeugentwicklungsprozesse. Springer, 2017.

17. Ashby M.F, Introduction. In Materials Selection in Mechanical Design (pp. 1-13). Elsevier, 2011, https://doi.org/10.1016/b978-1-85617-663-7.00001-1.

18. Poulikidou S, Schneider C, Björklund A, Kazemahvazi S, Wennhage P, Zenkert D, A material selection approach to evaluate material substitution for minimizing the life cycle environmental impact of vehicles. Materials \& Design, 83, 704-712, 2015, https://doi.org/10. 1016/j.matdes.2015.06.079.

19. Lindner F, Schmitt R, Goal-oriented life cycle investigations for composite manufacturing chains. Procedia CIRP, 29, 438-443, 2015, https://doi.org/10.1016/j.procir.2015.02.043.

20. Kaluza A, Kleemann S, Broch F, Herrmann C, Vietor T, Analyzing decision-making in automotive design towards life cycle engineering for hybrid lightweight components. Procedia CIRP, 50, 825-830, 2016, https://doi.org/10.1016/j.procir.2016.05.029.

Open Access This chapter is licensed under the terms of the Creative Commons Attribution 4.0 International License (http://creativecommons.org/licenses/by/4.0/), which permits use, sharing, adaptation, distribution and reproduction in any medium or format, as long as you give appropriate credit to the original author(s) and the source, provide a link to the Creative Commons license and indicate if changes were made.

The images or other third party material in this chapter are included in the chapter's Creative Commons license, unless indicated otherwise in a credit line to the material. If material is not included in the chapter's Creative Commons license and your intended use is not permitted by statutory regulation or exceeds the permitted use, you will need to obtain permission directly from the copyright holder.

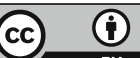




\title{
LCA in Strategic Decision Making for Long Term Urban Transportation System Transformation
}

\author{
Florian Ansgar Jaeger, Katrin Müller, Cornelia Petermann \\ and Eric Lesage
}

\begin{abstract}
The paper provides an overview of how Siemens uses LCA methodology and tools to support cities in the decision-making process to promote sustainable urban transportation systems. It focuses on GHGs and local air pollution. Determining the cause of GHG emissions and air pollution requires flexible scopes and a highly parameterized, hierarchical model, which can be adapted to any city's transportation system. Emission forecasting capabilities are very important since motorized transportation modes quickly change properties over time. The model screens a large set of infrastructure improvement measures by the click of a button and analyses their impact on KPIs for different years. Applicability, challenges and limits of LCA to the specific application of urban transportation system modelling are discussed.
\end{abstract}

\section{Introduction}

LCA methodology and tools have proven useful for assessing products or even companies in organizational LCA. Having used LCA-based methods and tools to model transport systems in more than 20 cities, we can say that this applies to mobility related infrastructure solutions in cities as well. This report shows how it was done and that the variety of indicators and flexibility LCA offers, not only matters to different products and product categories, but is also crucial for cities. The cities in the world have different focuses on environmental, economic or social

\footnotetext{
F. A. Jaeger $(\bowtie) \cdot$ K. Müller · C. Petermann

Research in Energy and Electronics, Energy Systems,

Sustainable Life Cycle Management and Environmental

Performance Management, Siemens Corporate Technology,

13629 Berlin, Germany

e-mail: florian_ansgar.jaeger@siemens.com

E. Lesage

Business Development and Strategy, Mobility Consulting,

Siemens Mobility, Berlin, Germany

(C) The Author(s) 2018

E. Benetto et al. (eds.), Designing Sustainable Technologies,

Products and Policies, https://doi.org/10.1007/978-3-319-66981-6_22
} 
aspects/KPIs. These differ by region, in scope (Carbon Accounting Scope 1-3) and in time (in terms of target setting, short term for air pollutants, long term for GHG-emissions). This diversity and the fact that there are vast differences in the level of maturity of cities with regard to data availability, setting targets and developing plans on how to achieve them, sets the requirements for any community scale model.

The aim of the transportation model is to support cities in creating climate action plans or clean air plans and to support cross-department activities in city administrations. The model is embedded in a process that supports city administrations in building alternative future infrastructure scenarios. It reflects all generally accepted/ assumed changes in technology, fleets and national policy over time by default and focus on the cities' core indicators.

\section{Method}

The methods and tools are a combination of conventional LCA, consequential LCA, greenhouse gas accounting for cities and futurology. Even though the software and modelling concept such as the functional units are common LCA-based, the model is aligned with the GPC-Protocol [1]. Forecasting elements are included from HBEFA [2] for market and technology development. Scenario workshops enable the inclusion of the political agenda during the projects process.

\subsection{Scope and Indicators}

The scope of the transport system model follows the basic rules of the GPC-Protocol [1], since greenhouse gas accounting is a quite common procedure for municipalities. It is a comparably well-defined process. The majority of cities with which we have worked have experts with a good understanding of the GPC-Protocol methodology and relevant stakeholders in cities are likely to know how to interpret its results.

With regard to the scope of activities, traffic in a territory is included, while emissions from airports and liquids or gases in pipelines are excluded. All processes are separated into the three scopes: direct, energy related indirect and other indirect emissions. By the click of a button, the model enables the inclusion and exclusion of different lifecycle phases or infrastructure categories and activities such as e.g. maintenance.

Despite these options being available, all cities we worked with decided to balance the direct operation of vehicles and direct emissions of infrastructure such as street lighting only. In addition to the standard activity data necessary to establish a baseline, the model also requires data to determine the level of maturity of the transportation system and its individual components. This is necessary in order to 
calculate the impact of levers at a later stage. Levers are new technologies or measures that are introduced to the system. Available levers either shift transport volume from a high to a low-emitting mode of transport or increase the efficiency of an individual or several modes.

The transportation system model supports the common material flows and indicators, covered by the ecoinvent database, but its focus is on greenhouse gases and regulated air pollutants. For levers only, the social dimension is addressed with the KPI local job creation potential of project related infrastructure build-up. As economic figures, CAPEX and OPEX of newly built infrastructure are calculated. The social and economic KPIs are not discussed in the following.

\subsection{Structure of the Model}

The model breaks down the general transportation demand within the city boundaries, with the functional units being passenger kilometres and ton kilometres. The main mode categories are rail based, road based and non-motorized transportation. The two functional units are broken down into vehicle types, where they are converted into actual vehicle kilometres, regardless of whether the mode is mass rapid transit by metro or individual passenger transport by car. The individual vehicle types are then specified in a parameterized process.

The same structure applies to the power model, which is connected to all processes, consuming electricity in the urban transportation system. The power is drawn on different grid levels, reflecting different T\&D losses and the grid mix is specified by fuel type and region.

Along with the transportation demand-oriented processes, a second stream quantifies basic road infrastructure such as lighting. The lighting demand is assessed based on lamp types and energy consumption and converted into lumen*h as a functional unit.

In order to model the set of 38 transportation and some energy levers, additional processes are modelled or the changes to the infrastructure are directly implemented in baseline processes. The calculations are each triggered by only one parameter and modelled on global (project) level or process level, depending on rather the induced change effects several processes or just one process.

\subsection{Parameterization}

The baselining process starts out with the model of a default city and adjusts it step by step to the actual city. The process can be compared to the fitting process of a tailor, making a dress on demand. Just like the dress, which starts with a certain size, but somewhat standard proportions, the city's transportation system model is assembled from different parts that are pre-configured with default values and 
assembled. In two to X fittings, data is adjusted to perfectly fit the city's transportation system or match its official reporting. The necessary fitting parameters are not hard coded, but part of questionnaire and fed to the model via calculation setups. The level of parameterization is the result of constant adaptations to the needs of numerous cities. In order to avoid having to feed thousands of parameters to the model for each calculation, most likely cases are defined, which can be combined and controlled with much fewer parameters. The hard-coded cases in the model consist of extracts from external databases such as HBEFA, which were imported via EcoSpold format previous to actual projects. This way, the number of commonly necessary data points was reduced to just 250 (not including forecasting parameters). Global project parameters define the scope of the model, which observation year to balance or the "flux" of transportation volumes of individual processes. Local process parameters are set to adapt individual processes. As one of the most relevant process categories, the mode type passenger cars for example are categorized by fuel type and conversion technology (combustion, hybrid...). Parameterization includes the average fuel consumption or tank to wheel GHG emissions, the EURO-Class, the difference in yearly vehicle mileage between diesel and petrol vehicles, as well as newer versus older vehicles and the average journey distance for cold start emission determination.

In addition to the parameters necessary for baselining, lever specific parameters are defined to obtain the current development or state of an individual infrastructure. Others identify the maximum applicability of a lever. One Parameter is set for each lever, to trigger it and set the degree to which it is implemented at a certain time.

\subsection{Forecasting}

The model is built as a decision support too for city administrations. When making long term infrastructure decisions, it is of little use to know impacts of a lever if it was in place today, if it takes 10 years in real world to construct the necessary infrastructure. The cause therefore requires forecasting options to determine the future baseline situation and lever impacts during the years the levers will actually be in place.

Forecasting elements are separated into three categories and treated differently:

- Forecasting parameters which are mainly influenced by the market development or can only be impacted by national or state union level governance are implemented by default or by case selection. This concerns technology improvements and fleet turnovers.

- Example 1: Average ages of vehicle technology are automatically forecasted based on vehicle fleet distributions in EURO classes. 
- Example 2: Future vehicle fleet distributions in EURO classes are automatically forecasted based on the current city's fleet distribution and matched with forecasts of the German national fleet.

- Parameter forecasts, which are the result of local phenomena such as the population increase or decrease and the travel behaviour, are extracted from reports or discussed in BAU-Scenario workshops with the city and have to be adjusted manually. MS Excel-based tools are provided to help with the effects of cross assumptions.

- Parameters impacted by local politics, facilitated in projects, policy and regulation are discussed in BAU-Scenario workshops and have to be modelled upfront in an iterative process, using levers, to determine input parameters.

- Example: If the city started constructing a new metro line, it is modelled in the transportation model and the resulting modal split in future years is used as inputs for the BAU-Scenario.

\subsection{Target Setting}

After the baseline is agreed in the form of a BAU scenario for the urban transportation system, the targets for different emissions at different target years are set. Carbon emission targets are commonly published, whereas air pollutant emission targets have to be retrieved from a separate process, converting limitations for concentrations of air pollutants at hot spot measurement stations into emission targets for transport-related emissions.

\subsection{Levers}

In order to reach their emission reduction targets, cities need to take measures. These measures are modelled as scenarios in the transportation system model and are referred to as levers. The levers aim at reducing transportation demand, shifting transportation volume to less polluting modes (modal shift), shifting to other fuels, increasing the energy/carbon efficiency of one or several modes of transport or adding pollution control devices. The levers can be of different natures (see the following list with examples):

- Technology based levers: advanced traffic light management

- Policy based: the implementation of a low emission zone

- A combination of policy and technology based: city tolling

- Behaviour change: eco-efficient driver training

- Generic: an X percent reduction of car use. 
The levers are implemented in the model as scenarios, with a ramp up phase, followed by another phase where they constantly deliver benefits. The level of possible implementation is defined by an implementation rate and depends on the current state and the maximum applicability. As an example: For the Lever Automated train operation (ATO) metro, the current state is the share of metro lines that have an ATO already and the maximum applicability would be $100 \%$ unless there are historic lines that shall keep human drivers.

The efficiency of each lever has to be quantified depending on the city specific conditions. And these conditions are assessed with individual parameters. In the case of the ATO for metros, a simulation in FALKO [3] (a simulation tool for train scheduling and simulation of energy consumption) was run for a typical metro line to check which parameters have the most significant impact on energy consumption. It turned out that the distance between two consecutive stations has the most significant correlation with the energy saving potential of an ATO. The city transport system model is therefore fed with the average distance between stations.

All levers model relative changes and can be combined in scenarios, also reflecting impacts of one lever on another lever. Some levers address the same saving potential and are therefore modelled combined in one process or on global/ project level.

\section{Results}

The following section states first the results in terms of how appropriate and practically feasible the above described methodology shows for the specific cause. Afterwards, the relevance of individual methodological aspects is illustrated in the form of actual modelling results from city projects.

\subsection{Scope}

The Scope of the model is in line with city's requirements. Two large cities requested two separate analyses for their inner city and outer city. Three cities, two of them in Scandinavian countries, requested the exclusion of Scope 3 GHG emissions in the energy upstream. Only one city in the south of Germany had Scope 3 GHG emissions from the energy upstream in their carbon accounting (WTW). All but two cities could be convinced to include Scope 3 GHG emissions for this relative comparison between levers. It allows a better symmetry between systems for indicators that are only of relevance in their global context. Since the studies did not aim to compare cities among each other, they did not have to fear an unfair competition.

It was a common request that for air pollutants, Scope 1 emissions should be reported individually. Especially traffic related urban emissions have a very high 
local impact due to the fact that they are emitted in very densely populated locations. Cities have strict local targets for these pollutants. Reporting on Scope 1-3 air pollution emissions is misleading in an urban context. In some cities for example in Austria, an increased share of photovoltaic (PV) power in the forecasted electricity mix lead to higher overall particulate matter emissions. These Scope 3 particulate matter emissions from PV panel production are of little relevance for the city where the panels are installed, since the emissions occur far away in much less densely populated areas and have little global impact.

\subsection{Structure and Parameterization}

The hierarchical structure of the model in combination with the strong parameterization involves difficulties in modelling practice, since a parameter calculated in a process is not passed down to processes in the lower hierarchies (upstream processes) in LCA-Software. Any parameter, used by more than one process, therefore has to be calculated on a project level (global parameter). LCA-software does not provide a folder structure on this level.

The most difficult parameters to derive are the overall transportation volume, especially for freight, and passenger kilometre or ton kilometre based modal split data. All cities were able to provide journey shares. But this data can't be used to create a passenger kilometre-based inventory without additional assumptions and data. Central databases such as Urban Audit asses KPIs, which are only a cutout of the overall transportation system. A typical example is the "[s]hare of journeys to work by car $-\%$ " [4].

\subsection{Forecasting \& Target Setting}

The first important information, the modelling delivers the cities, is how the overall sum of future emissions will develop over time in the BAU-Scenario. This serves as the baseline. Each lever calculation is compared with this BAU-Scenario to provide relative saving potentials. This is most relevant for $\mathrm{NO}_{2}$ (Fig. 1). It implies, that the distance to the city's target is significantly reduced before any lever is applied.

Secondly, the model delivers a contribution analysis in time slices. For PM2.5, emission shares of private vehicles increase compared to those of trucks (Fig. 2 vs. Fig. 3). 


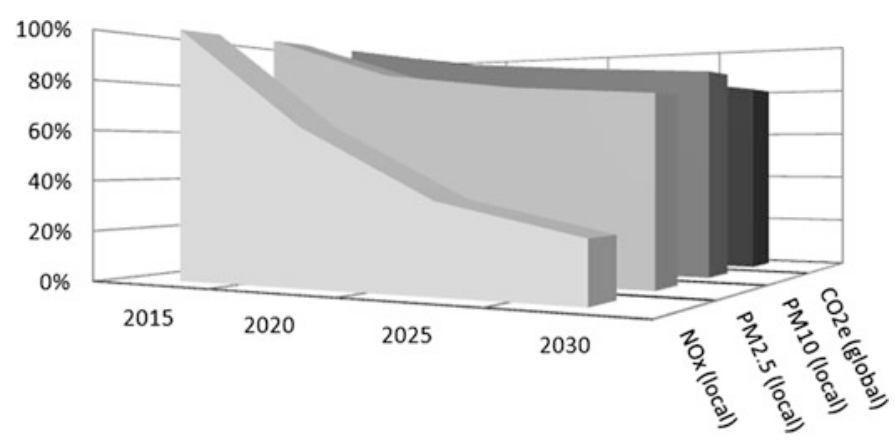

\begin{tabular}{|l|c|c|c|c|}
\cline { 2 - 5 } \multicolumn{1}{c|}{} & 2015 & 2020 & 2025 & 2030 \\
\hline m NOx (local) & $100 \%$ & $61 \%$ & $34 \%$ & $23 \%$ \\
\hline m PM2.5 (local) & $100 \%$ & $83 \%$ & $77 \%$ & $73 \%$ \\
\hline m PM10 (local) & $100 \%$ & $91 \%$ & $87 \%$ & $84 \%$ \\
\hline a CO2e (global) & $100 \%$ & $92 \%$ & $87 \%$ & $80 \%$ \\
\hline
\end{tabular}

Fig. 1 Relative reduction of four different transport related emissions in a city (results from a medium size city in the south of Germany)

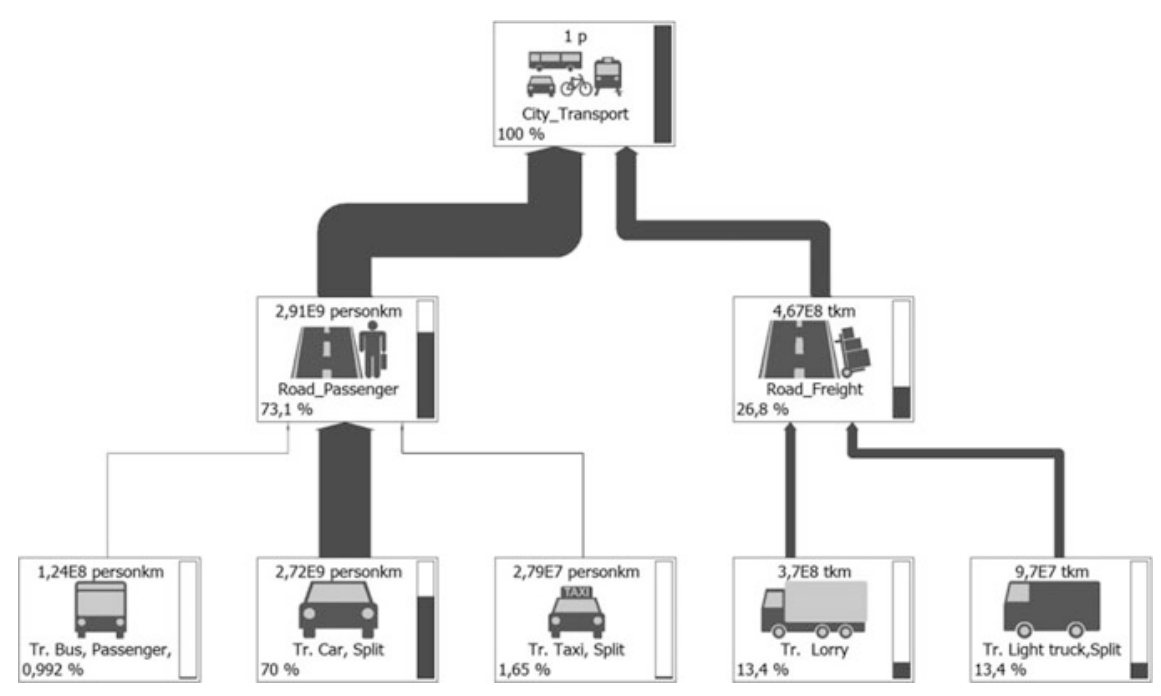

Fig. 22015 PM2.5 contribution tree (results from a medium size city in the south of Germany)

\subsection{Levers}

The third type of finding provides the lever analysis. For every lever, the new system performance of the city's transportation system at selected future years is calculated for GHG-Emissions (global) and for several air-pollutants (local). 


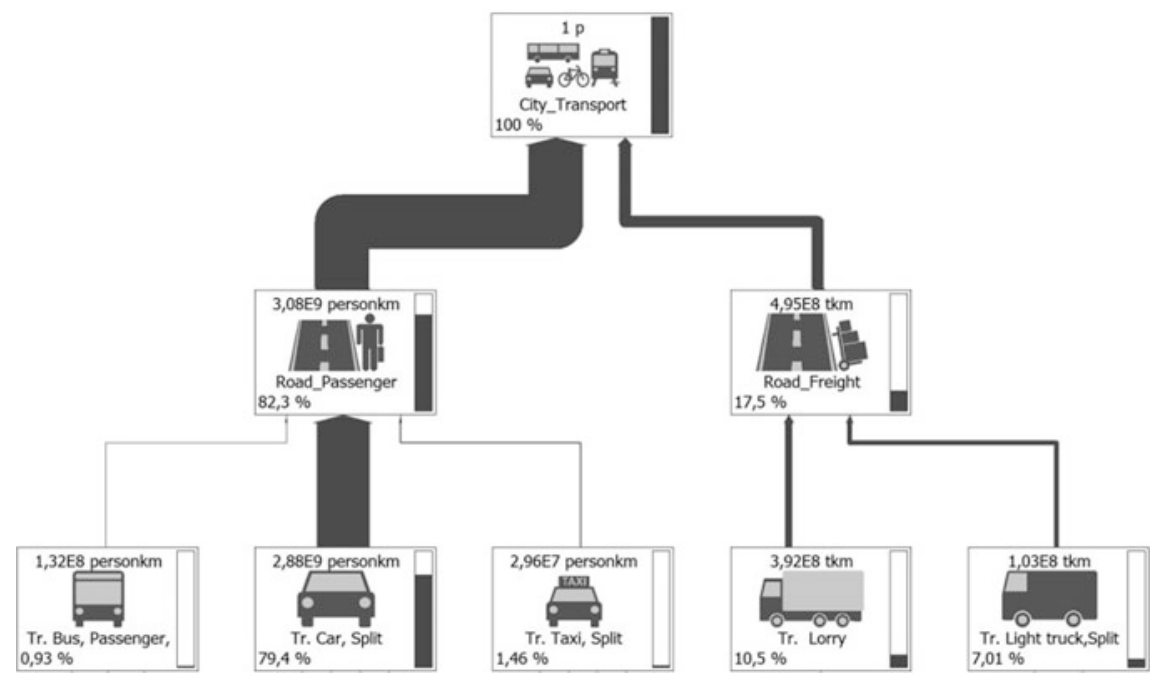

Fig. 32025 PM2.5 contribution tree (results from a medium size city in the south of Germany)

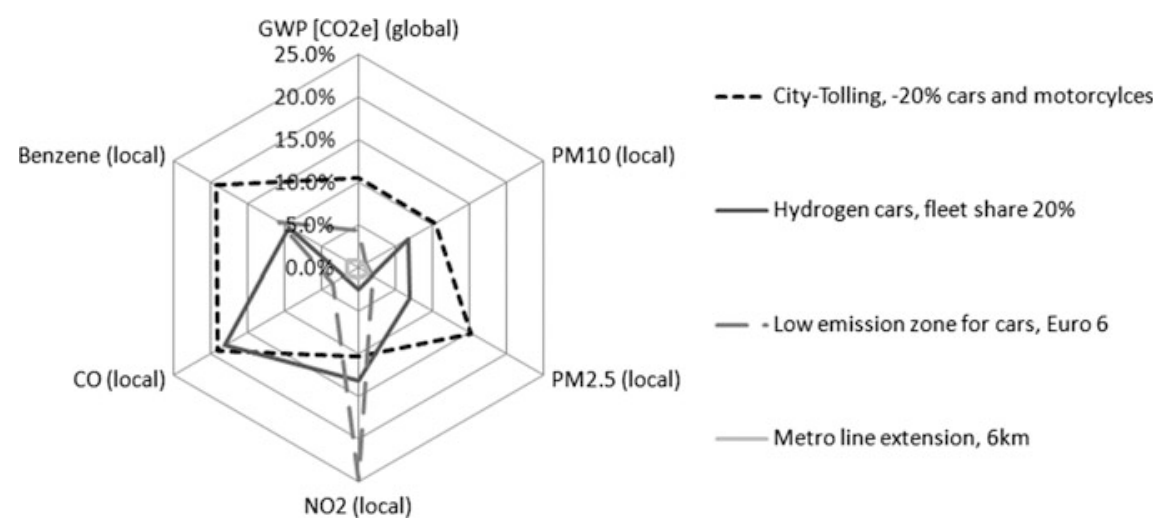

Fig. 4 Saving potentials of four levers for 6 different emissions at observation year 2025 (results from a medium size city in the south of Germany)

The following graph shows a spider diagram of a small selection of levers (Fig. 4). It clearly shows how diverse the impacts of a single measure can be on different indicators. 


\section{Interpretation}

\subsection{Scope, Structure and Parameterization}

The scope of the model must be flexible enough to adapt to any city's carbon reporting guidelines or methodology. It creates trust in the model, if results turn out similar to what the city expects to see for a carbon baseline. Based on this trust, additional scopes can be included for lever comparison. Since the target of this model isn't to compare cities, but levers within a city, the majority of all cities showed little reluctance to include additional scopes and therefore emissions.

The structural options of current LCA-Software provide limits to the complexity of models. Since parameters, calculated in one process can't be passed down to processes of subordinate processes, any cross-functionality between processes has to be modelled outside the processes on a project level, where it can't be properly managed. Since processes of urban transportation systems influence each other inherently, an increasing size of a model will eventually make it unmanageable in practice.

The overall transportation volume in pkm or tkm is not a KPI that is tracked by most cities. The determinations of the very basis of any urban transportation system balance, therefore becomes a tedious process.

\subsection{Forecasting and Target Setting}

The bottom up forecasting, based on commonly available emission databases, that have to be imported into the LCA-software, provide a good indication how emissions will develop in the future. For carbon, this is sufficient for target setting for lever selection and scenario definition, since carbon targets are expressed in annual emissions. For Air quality, it is a good indication, but hard to draw a final conclusion. A distance to political target/threshold judgment can only be made in combination with a contribution analysis of an individual air pollution measurement station, allowing an emission to concentration conversion. Some cities have this available already [5].

The contribution analysis of different time slices identifies which transportation mode to address with levers at what time, to get the maximum benefit. As an example: if a lever reduces the PM2.5 emissions of light trucks by 50\%, the effect for the overall transportation system related emissions will be almost twice as high in 2015 than in 2025 (see Figs. 2 and 3). 


\subsection{Levers}

The variety of measures that can be taken to improve an urban transportation infrastructure is very broad. Therefore, the impacts differ significantly on the chosen set of indicators, making a wider set of KPIs recommendable as a decision basis.

\section{Conclusion}

LCA-Methodology and tools are suitable to assess the transportation systems of a city and provide strategic decisions relevant information about alternative future developments.

A model, which is flexibility in scope and can adapt to any city's carbon accounting, is crucial in order to match existing reporting at least during the validation phase. This is true even if the final study later includes additional scopes. It is much easier and faster to check where differences are between the assessments. In addition, it allows the analysis of local effects such as air pollution.

Efficient model structuring and parameterization capabilities of LCA-software could be significantly improved if processes were capable of passing on parameters to subsequent processes.

Forecasting is necessary, when assessing systems that quickly change properties which effect target indicators and if the target is to compare systems that last long or take long to build. Both are the case for urban transportation systems, especially when focusing on air pollution. Otherwise absolute system emissions, as well as individual process contributions, have a high inaccuracy and might lead to wrong decisions.

The diversity in performance of a lever on different KPIs (Fig. 4) clearly shows that making an infrastructure decision for sustainable urban transport requires multi-KPI-modelling. This is a strength of LCA-methodology and software.

To significantly improve the applicability of LCA for urban transportation systems, KPI systems that cities report on should be adapted to assess indicators which cover the system as a whole and not only segments and can be used to create inventories.

\section{References}

1. Fong W, Sotos M, Doust M, et al., The Global Protocol for Community-Scale Greenhouse Gas Emission Inventories (GPC), 1st Ed., Greensouse Gas Protocol, 2014. < http://ghgprotocol. org/greenhouse-gas-protocol-accounting-reporting-standard-cities>, (Accessed 18.04.2017).

2. Keller M, Wuethrich P, Ickert L, Schmied M, Stutzer B, et al., Handbook Emission Factors for Road Transport, 3.2 Ed., INFRAS AG, 25.7.2014. 
3. Falko: Fahrplan-Konstruktion und -Validierung Optimierung betriebliche Planung und Steuerung, Siemens AG, 2007

4. Urban Audit, Eurostat, European Commission, 2016, < 2017http://appsso.eurostat.ec.europa. eu/nui/show.do?dataset=urb_ltran\&lang=en>, (Accessed 17.05.2017).

5. Rusp A, Verursacheranalyse 2013 für NOX, NO2 und PM10 für den Nürnberger LÜB-Standort Von-der-Tann-Straße, TÜV, 2014

Open Access This chapter is licensed under the terms of the Creative Commons Attribution 4.0 International License (http://creativecommons.org/licenses/by/4.0/), which permits use, sharing, adaptation, distribution and reproduction in any medium or format, as long as you give appropriate credit to the original author(s) and the source, provide a link to the Creative Commons license and indicate if changes were made.

The images or other third party material in this chapter are included in the chapter's Creative Commons license, unless indicated otherwise in a credit line to the material. If material is not included in the chapter's Creative Commons license and your intended use is not permitted by statutory regulation or exceeds the permitted use, you will need to obtain permission directly from the copyright holder.

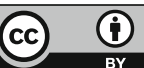




\title{
Management of Construction Waste: LCA and Complex System Modeling
}

\author{
Anne Ventura and Maxime Trocmé
}

\begin{abstract}
Construction and demolition waste (CDW) generation, identified as a priority stream by the European Commission, accounts for approximately 25 to $30 \%$ of all waste generated in the European Union. According to local specificities (e.g. regulations, waste management organization) best environmental options may differ for transforming waste into new resources. Five oral presentations were given in the session which focused on innovative modelling initiatives combining LCA with complex models in order to improve knowledge for more sustainable urban construction waste management. During the discussions, all participants agreed that re-use or recycling mass performance is a weak and unsufficient indicator for assessing waste management systems. There is an important need for better characterizing stocks and predicting nature and quality of output flows. Geospatialized data combined with Material Flow Analysis was the methodology identified and used by the research community.
\end{abstract}

\section{Introduction}

Construction and demolition waste (CDW) generation, identified as a priority stream by the European Commission, accounts for approximately $25-30 \%$ of all waste generated in the European Union. Recent political consciousness about circular economy has focused the spotlight on the construction sector, seen as a real

\footnotetext{
A. Ventura (ه)

Materials and Structures Department (MAST), Aggregates and Materials

Processing Laboratory (AMPL), IFSTTAR, Nantes, France

e-mail: anne.ventura@ifsttar.fr

A. Ventura

GeM, Institut de Recherche En Génie Civil et Mécanique, CNRS UMR

6183-Chaire Génie Civil Eco-Construction,

Université de Nantes, Nantes, France

M. Trocmé

Vinci Construction, Rueil-Malmaison, France

(C) The Author(s) 2018

E. Benetto et al. (eds.), Designing Sustainable Technologies,

Products and Policies, https://doi.org/10.1007/978-3-319-66981-6_23
} 
opportunity to generate new resources and local employment based on urban mining. Behind this will, many challenges arise. Indeed, reaching best environmental performances often relies on many interrelated aspects such as relationships between: territorial waste management, sorting practices and possible reuse or improvements of recycled materials' qualities; existing or innovative treatment technologies, regulations and standards, and possible markets for future recycled products; disturbance of markets' equilibrium by the introduction of recycled materials and possible avoided environmental impacts; environmental effects of scale changing from small innovative experience to large territorial applications, etc. According to local specificities (e.g. regulations, waste management organization) best environmental options may differ. Finding them is finding favorable combinations of solutions all along the waste treatment chain for sustainable urban systems, rather than ready-made preconceived ones. Introducing economic, technological, or social mechanisms into LCA calculations provides knowledge that can be adapted in various contexts.

Five oral presentations were given in the session which focused on innovative modelling initiatives combining LCA with complex models in order to improve knowledge for more sustainable urban construction waste management.

\section{Prospective and Regional Modelling of Construction Material Flows}

The session started with a presentation from Niko Heeren [1] providing an overview of a bottom-up dynamic MFA model he developped and applied for Switzerland, and the relevance of construction material flows for the environmental impact of buildings. Future possible scenarios were compared and potentials for closed loop recycling analysed [1]. The development is based on a GIS-based building stock model to determine material flow of the Swiss building stock. By merging a national building register with a three-dimensional dataset, it is possible to quantify the material volumes for all residential buildings. In order to perform a prospective assessment, element and building service life are determined using a stochastic approach. Therefore, the model accounts for the dynamics due to material dwelling time. Life cycle assessment is used to assess the environmental impacts of future material flow. 


\section{Guidelines for Effective and Sustainable Recycling of Construction and Demolition Waste}

Serena Giorgi presented an analysis of actual re-use and recyling practices in European countries [2]. Starting from a screening of current percentage of reuse and recycling, types of waste and recycling destinations, as well as incentive policies in member states of European Union-28, she explained that the objective of the directive 2008/98/EC on waste that imposes a minimum of $70 \%$ re-use or recycling by weight within 2020 , can be easily reached or even already achieved by more than half of European countries. Research data of the average material composition of CDW percentage shows that $60-70 \%$ (by weight) is composed of concrete and masonry, followed by minor percentages of asphalt, wood, metal, gypsum and plastic. Aggregates are therefore the largest amount of CDW. Based on these facts Serena Giorgi reported that since the Directive 2008/98/CE expresses the objective in percentage of weight, it doesn't value the best material to be recycled in terms of effectiveness and sustainability (e.g. recycling of plastic parts in Italy is only 21\%), but heavier ones. Also she argued that there isn't any assessment of the "quality" of recovery, i.e. differentiation between reuse, upcycling/downcycling or energy recovery. In this way, it was found that in many countries, aggregates are mainly recycled for road foundations, earth works and backfilling. A further issue is the assessment of the effectiveness of recycling sustainability, especially for inert waste. A study of the ecological balance of the concrete produced with recycled aggregates shows that there is a sharp increase of environmental impact, first due to the increase of cement content used with recycled aggregates and second due to the transport from supply points to the production facilities. Finally the speaker defined ways to improve legislation and proposed some guidelines, aimed at making the recycling of CDW management more effective and sustainable through Life Cycle Management.

\section{Adaptive Reuse of Buildings and Its Life Cycle Sustainability Benefits}

Harn Wei Kua then presented a case study of primary school refurbishment strategies in Singapore, based on a life cycle based approach [3]. The environmental and economic assessment was performed for alternate designs for school buildings, aiming at reducing the need or extent of future renovations and demolitions. This analysis lead to design guidelines, in which alternate designs are compared in terms of potential net amount of $C \& D$ wastes that could be saved as well as net decrease in life cycle impacts and resources requirements. Also, for each alternate design, the potential of each kind of generated C\&D wastes for recycling or reuse, and the life cycle costs of the buildings were estimated, as well as the social and cultural effects of building preservation based on similar projects elsewhere in Singapore. 
Based on the life cycle sustainability values of adaptive reuse, policy strategies were recommended to promote and incentivize this alternate design approach in Singapore.

\section{Evaluating the Construction and Demolition Waste Management System Implemented in Lombardy Region (Italy) Through Life Cycle Assessment}

Among Italian regions, Lombardy was the largest producer of CDW in 2014, with a rough equivalent of 1 ton of CDW per inhabitant during the year 2014. Hence, the establishment of an effective and sustainable CDW management is essential to ensure environmental protection and enhance waste valorisation towards a resource-efficient system. Lucia Rigamonti presented the results of the overall environmental performances of the CDW management system currently implemented in the region, based on LCA [4]. The quality of secondary products obtained from CDW and their actual use has been considered. A focus has been made on the technical characteristics and the actual market of the recycled aggregates, allowing to estimate the type and amount of "avoided natural materials" that can be replaced by recycled products. The environmental benefits due to the "avoided impacts" have been calculated. Based on LCA results and a literature state-of-the-art of treatment technologies, alternative scenarios for CDW management strategies, innovative processes and/or alternative recovery solutions (i.e. "alternative scenarios"), have been defined. LCA results for the alternative scenarios were compared to the results of the current situation to quantify the benefits arising from the suggested improving actions. Results have been used to provide recommendations to the regional authorities to enhance the CDW recycling chain.

\section{Alternatives for Materials from an End-of-Life Building, Evaluated with Life Cycle Costing and Life Cycle Assessment}

Valentina Prado-Lopez presented a study derived from the EU Horizon 2020 HISER research and innovation program [5]. Two scenarios of demolition waste management were compared, a 'best practice' (BP) and a 'business as usual' (BAU) scenario, from an environmental and financial perspective. An integrated Life Cycle Costing and Life Cycle Assessment was performed to quantify and compare the environmental implications and cost-effectiveness of the scenarios. The scenarios are based on field data collected in the BP demolition of a building located in Almere, the Netherlands. The BP alternatives favor the reuse of retrieved building components and the upcycling of concrete rubble, over general recycling 
and final disposal. In the BP scenario, the reuse of ceiling tiles, wooden doors and stairs, gypsum walls, and metal beams are win-win situations, compared to the treatments in the BAU scenario, which are more expensive and more pollutant. In both scenarios, the metal beams are the largest contributors to the environmental burden. If metal beams are reused, the cost of onsite construction of a new building drops dramatically. The overall BP scenario is cheaper than the BAU scenario. As further recommendations, the impact of waste management of each material flow can considerably decrease by shortening the transport distances. Selective dismantling and demolition, and the reuse of components should be promoted by including a phase prior to dismantling, in which reusable components and materials are inventoried and potential buyers are contacted.

\section{Conclusions}

The whole session showed that the construction and demolition waste (C\&D waste) management is a complex system to be modelled because several types of information must be considered and collected at different scales: at material, building and processes levels, defining the possibilities in terms of technical valorisation, and at regional level in order to consider regulations and urban planning policies. The temporal aspects are also crucial because today's decisions on construction engage consequences for the next 100 years, therefore with some potential important repercussion for the waste management system despite its strong inertia to changes. All participants agreed that re-use or recycling mass performance is a weak and unsufficient indicator for assessing waste management systems. There is an important need for better characterizing stocks, to predict nature and quality of output flows. Geospatialized data combined with Material Flow Analysis was the methodology identified and used by the research community. Predictions are based on scenarios comparison, mainly comparing recycling technologies and possible substituted materials. However, the link between sorting processes and quality of output material is not enough investigated, and possible changes in building design methods that are expected to avoid waste by favoring better adaptability of buildings should also be investigated. It should support the assessment of waste prevention strategies efficiency. Finally, including economic market mechanisms, making the link with availability of material flows responding to the future materials demand would also be relevant in the framework of the sustainable assessment of C\&D waste management. 


\section{References}

1. Heeren N, Hellweg S, Prospective and regional modelling of construction material flows, LCM2017 conference, Luxembourg, 2017.

2. Giorgi S, Lavagna M, Campioli A, Guidelines for effective and sustainable recycling of construction and demolition waste, LCM2017 conference, Luxembourg, 2017.

3. Kua H.W, Adaptive reuse of buildings and its life cycle sustainability benefits, LCM2017 conference, Luxembourg, 2017.

4. Rigamonti L, Pantini S, Evaluating the construction and demolition waste management system implemented in Lombardy Region (Italy) through Life Cycle Assessment, LCM2017 conference, Luxembourg, 2017.

5. Miranda-Xicotencatln B, Ita-Nagy D, Hu M, Prado-Lopez V, Alternatives for materials from an end-of-life building, evaluated with Life Cycle Costing and Life Cycle Assessment, LCM2017 conference, Luxembourg, 2017.

Open Access This chapter is licensed under the terms of the Creative Commons Attribution 4.0 International License (http://creativecommons.org/licenses/by/4.0/), which permits use, sharing, adaptation, distribution and reproduction in any medium or format, as long as you give appropriate credit to the original author(s) and the source, provide a link to the Creative Commons license and indicate if changes were made.

The images or other third party material in this chapter are included in the chapter's Creative Commons license, unless indicated otherwise in a credit line to the material. If material is not included in the chapter's Creative Commons license and your intended use is not permitted by statutory regulation or exceeds the permitted use, you will need to obtain permission directly from the copyright holder.

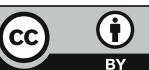




\title{
Guidelines for Effective and Sustainable Recycling of Construction and Demolition Waste
}

\author{
Serena Giorgi, Monica Lavagna and Andrea Campioli
}

\begin{abstract}
Directive 2008/98/EC on waste (WFD) provides that, within 2020, the preparing for re-use and recycling of non-hazardous construction and demolition waste shall be increased to a minimum of $70 \%$ by weight. Beginning from a screening of the current percentage of reuse and recycling, type of recycling (types of waste and destinations) and incentive policies in Member States of European Union-28, the research aims to evaluate the effectiveness of the Directive and possible ways of improvement through a Life Cycle based approach. In this paper the incentive policies and some critical issues regarding current regulations are analysed. Further ways to improve legislation are proposed as well as guidelines, which would have an effect on a local level and are aimed at making the recycling of CDW management more effective and sustainable through Life Cycle Management.
\end{abstract}

\section{Introduction}

Construction and demolition waste is receiving more attention recently following new circular economy policies [1, 2]. Action plans primarily move towards reduction of waste by recycling, promoting the by-products exchange among companies and supporting digital technology platforms in order to stimulate business opportunities. In this situation, the Waste Framework Directive (2008/98/EC) [3] regains a vital role. It establishes the waste hierarchy, defines the meaning of by-product and the consequent cessation of waste classification. Furthermore, the WFD sets the target for reuse and recycling of non-hazardous construction and demolition waste by $70 \%$ in terms of weight, within 2020 . In order to define the main critical aspect of the Directive, it is important to highlight that the Article 11 set out, does not include stone and soil. Moreover the percentage of recycling includes backfilling operations, which use waste to substitute other material.

S. Giorgi $(\bowtie) \cdot$ M. Lavagna $\cdot$ A. Campioli

ABC Department, Politecnico Di Milano, Milan, Italy

e-mail: serena.giorgi@polimi.it

(C) The Author(s) 2018

E. Benetto et al. (eds.), Designing Sustainable Technologies,

Products and Policies, https://doi.org/10.1007/978-3-319-66981-6_24 
In the following section, at first, limitations in the statistical database are shown, causing difficulties in monitoring waste management. In spite of the diversity of data, in the second section, this paper gives an overall review of the amount of construction and demolition waste in EU-28 and the material composition of waste, based on the more recent available statistical data, such as Eurostat data. Then, the third part analyses Member States' recycling and recovery rates. This information is based on data provided by Bio Intelligence Services investigations [4] and the research "Resource Efficient Use of Mixed Waste" [5]. These sources give articulated data and information already analysed and corrected, in comparison with Eurostat data that are not so much detailed. Moreover, the destination of waste in recycling processes is analysed. The fourth section analyses policies and incentives of some Member State, with regards to legislative instrument and non-legislative instrument. Finally, in the last section, this paper discusses the main strategies to improve the effect of WDF in order to make it more effective and sustainable within the circular economy vision. The application of Life Cycle Management is the strategy toward sustainability.

\section{Sources and Data Quality on CDW}

In the countries of EU-28, to manage waste recycling and reuse, it's necessary to know CDW flows, but the first critical issue is that it isn't monitored well. Indeed, in the reports published by European Commission and each Member State [4, 5] and in statistical data (e.g. Eurostat, ISPRA, etc.), it has been always reported that the data based on waste flow is uncertain and sometimes needs adjustments and estimations. For example, in Italy, data collection is difficult: the national production of special waste has been quantified from the information contained in the MUD statements (Modello Unico di Dichiarazione ambientale), specifically the data presented in the ISPRA Report [7]. It should be noted that Legislative Decree $152 / 2006$ provides for several exemptions from the obligation to declare, therefore, MUD database processing cannot provide complete information on the production of waste [6]. In addition, the reprehensible practice of the abandonment of waste is not possible to estimate. Production data must therefore be correctly estimated and this constitutes a major limitation of the analysis [7].

Another limit of the available data is that construction part and demolition part are not separated, even if demolition waste stream is larger by weight than construction waste stream, they are different. Construction waste (originated from new construction) is less mixed, less contaminated and its recovery potential is higher than demolition waste because of these characteristics. Demolition waste stream is more contaminated (with painting, adhesives etc.) and more mixed due to the integration of different elements. Furthermore, there is very little information on waste material type. CDW are composed by different quantities of concrete, cement, bricks, gypsum products (e.g. plasterboard, building plaster and gypsum block), ceramic products (e.g. tiles and consumer products), glass and asphalt, each 
one with different recycling potential. Finally, the inclusion of excavated material does not seem to be systematic in national reporting. As this flow represents a big quantity in respect of the total amount in construction, demolition and excavation waste (e.g. $80 \%$ in France): uncertainty about their inclusion in national CDW statistic is a major source of doubt in the data on CDW. Data limitations make it difficult and complex to compare the amount of waste made by the Member States, and it is the first obstacle to identify efficient waste management.

\section{Amount of Waste and Material Composition of Waste}

The most recent data regarding the amount of waste production in EU-28 and each Member States, is represented by Eurostat Data (Construction-Code F-that includes general construction and specialized construction activities for buildings and civil engineering works). According to the statistical data in EU-28, construction is the main activity that produces waste, contributing to $33.5 \%$ of the total waste generated by all economic activities and households in 2014 in the EU-28. The construction sector creates the biggest amount of waste followed by mining and quarrying (29.8\%), manufacturing $(9.8 \%)$, households $(8.1 \%)$ and energy $(3.7 \%)$ and other economic activities (15\%) mainly including waste and water services and services [8]. It is important to highlight that the value specified for the construction sector includes the quantity of soil. On a European level, the $98 \%$ of CDW is non-hazardous waste, that represents the percentage pointed out by WFD.

By observing the statistical data from Eurostat (Fig. 1), there are big differences in total CDW yearly produced among the Member States: France (224 million tons), Germany (199 million tons) and United Kingdom (119 million tons) are the major producers of CDW, followed by Netherland (88 million tons) and Italy (51 million tons). Greece (479 thousand tons), Latvia (453 thousand tons) and Lithuania (425 thousand tons) are the minor producers.

From the total CDW generated, $51 \%$ is divided into soil waste and $32 \%$ mineral waste. Metal waste is almost $2 \%$, and wood waste around $1 \%$. Other types of waste (such as glass, paper and cardboard, rubber wastes) are less than $1 \%$. The $13 \%$ missing from the total CDW represents heterogeneous, mixed and undifferentiated wastes. By making a comparison with other research data, it is shown that when excluding the soil portion, 60-70\% (by weight) of CDW is composed of concrete and masonry, followed by minor percentages of asphalt, wood, metal, gypsum and plastic [4]. Aggregates, therefore, are the largest amount of CDW. Asphalt constitutes another major share of the stream but it is usually treated separately, as this fraction is largely collected unmixed with other CDW and it is often recovered immediately on-site. 


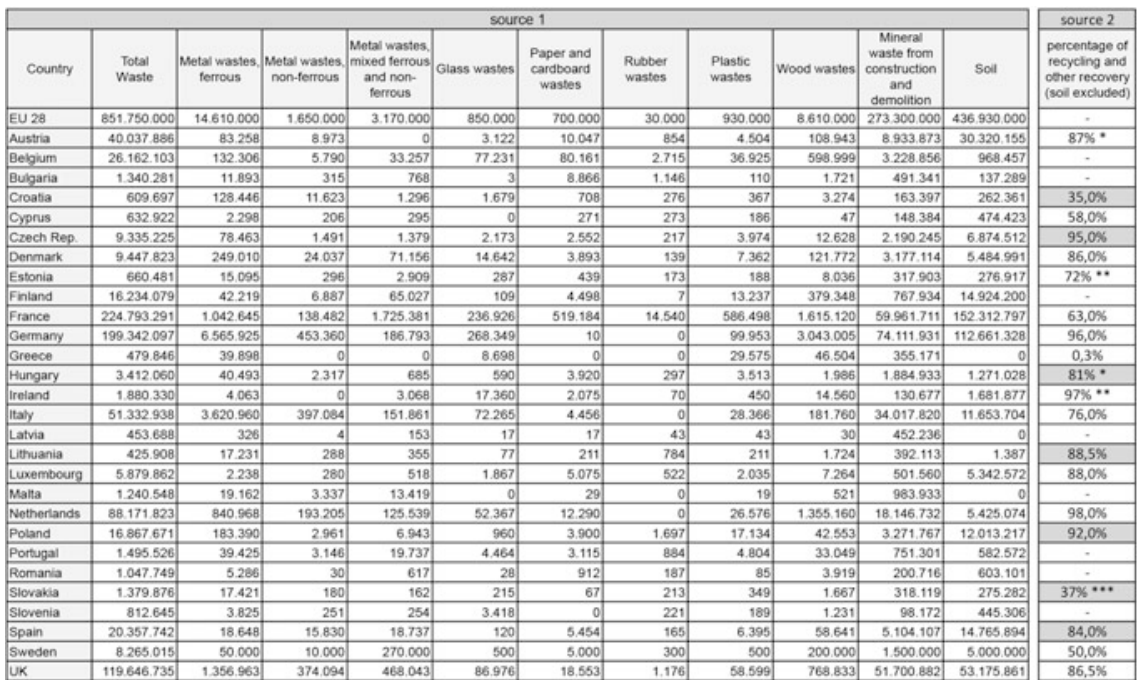

\begin{tabular}{|l|l|l|l|l|l|l|l|l|r|r|}
\hline Inpe of material \% & 1,72 & 0,19 & 0,37 & 0,10 & 0,08 & 0,00 & 0,11 & 1,01 & 32,09 & 51,30 \\
\hline
\end{tabular}

Fig. 1 Amount of waste, material composition and recovery and recycled rate. Sources Eurostat (source 1) data and Resource Efficient Use of Mixed Waste, Member State factsheet (source 2). Note Table represents the total construction waste, and the main portion of material waste. The table does not report $13 \%$ of heterogeneous, mixed and undifferentiated wastes. The percentages of recycling to each Member States are related to year 2012. Percentages with *are related to 2013, with **are related to 2011 , and with $* * *$ are related to 2009 . Data in grey cells in last column may contain soil parts

\section{Recovery and Recycling Rate and Destination of Waste}

The recovery and recycling rate of CDW is an important piece of data to define the current state of Member States, with regards to the $70 \%$ recovery goal and recycling rates set by the WFD. Nevertheless, this information is very difficult to find and the associated data are not reliable yet. The European "Resource Efficient Use of Mixed Waste" research program [5] tried to fill the gaps and inconsistencies by collecting data from each Member State, based on statistics provided by national agencies. This paper gives an overview of the collected data in Table 1 last column (Fig. 1).

It is possible to observe that the objective of the WFD is already abundantly achieved by many European countries and can be easily reached by many other (Table 1). In the Netherlands around $98 \%$ of CDW is recovered (recycling, energy recovery and other recovery), of which $95 \%$ is recycled. The amount of landfilled CDW corresponds to only $2 \%$ of all CDW. In Germany $96 \%$ of CDW are recovered or recycled, and the percentage of landfilled waste is only $4 \%$. Less advanced is the construction and demolition waste management in Italy, where in 2012 the recovery operations accounted for $76 \%$ (even if recycling rate has been steadily growing 
from 2010, when it stood at 68.4\%) and landfilling remains 24\% (however, recovered is over than 70\%). However, according to WFD, the recycling rate includes reuse, recycling (and other forms of material recovery), as well as backfilling.

Moreover, there are still differences in national calculations: some countries' percentage still contains soil; others do not distinguish between hazardous and non-hazardous CDW and consider total amount. One other main point is the destination of waste recycled to understand if the practices follow measures to promote high quality recycling, as promoted by WFD.

An important overview of the trade market of CDW and hence the destination of waste is given by the final report of European Commission, written by IDEA Consult [9]. This report shows the general situation of waste trade in the EU, in particular the main stream of mineral waste, completed on collected data from many sources (Eurostat 2010, Koumpanis 2008, EAPA 2010, ANPAR 2011, UEPG 2014). The $10 \%$ of waste is from new construction, $75 \%$ from demolition and $15 \%$ from road construction. The IDEA Consult report [9] demonstrates that after collection, around $11 \%$ of CDW is sent to backfilling, and $18 \%$ is brought to a landfill. The remaining $71 \%$ is separated into different materials. Metal, plastic and wood, which are a small percentage with respect to the total amount, come out of the process as recyclable material or energy recoverable. The remaining big amount of inert is recycled (on-site, in order to produce secondary aggregates that are used again in the production of new construction materials, or off-site in stationary recycling plants, where it is separated and crushed) or, when the waste is not contaminated, it is reused, after the cleaning or transforming operation. The $71 \%$ includes either reused, recycling, recovery and backfilling activities, such as the use of recycled aggregates (secondary material) to restore mining works. The secondary material goes to the industries in a small portion and the remaining goes to the backfilling (the percentage is unknown).

Concrete, masonry, and mixed demolition debris, after crushing (and certifying the quality) become recycled aggregates, constituting: Recycled Concrete Aggregates (with a large concrete content), Recycled Masonry Aggregates (with a large bricks and mortar content) or Mixed Recycled Aggregates [10].

All of the recycled aggregates types can be used as low performance application in sub-base layers in roads, ground improvements and building foundations. Aggregate use in concrete production requires high density and cleaner fraction, and the use of recycled aggregates in asphalt is limited due to mechanical characteristics, as regulated by standards (e.g. EN 12,620, Aggregates for concrete; EN 13,043 Aggregate for bituminous mixtures and surface treatments for roads, airfields and other trafficked areas). In fact, in civil construction and industrial product (such as gypsum, concrete, ceramic and bricks productions) only $6 \%$ of material used is from recycled material, while $94 \%$ come from virgin materials [9]. It is possible to understand that the major applications of recycling aggregate are downcycling. This issue is also favoured by the fact that in most countries the production of natural aggregates dominates [9]. In the Flemish Region, the main CDW are recycled granulates, mainly used for road construction and similar 
activities. In Italy, ANPAR (National Association of Recycled Aggregates) reports the recovery percentages about the portion of recycled aggregates: these secondary materials are widely used in roadworks for substrates and fillings (almost $60 \%$ of the total recycled in 2013); the remaining is used for pavements and backfilling [11]. In Italy the use of recycled aggregates into new concrete production is not widely practiced, due to the regulations (DM January 14, 2008). This regulation indicates the maximum percentage use of recycled aggregates into new concrete, compared to "concrete strength classes". For example, concrete class C30/37 allows a maximum $30 \%$ of inert derived from concrete or reinforced concrete waste. Buildings demolition rubble are only allowed for non-structural concrete C8/10 [12]. In addition, the regulation imposes mandatory laboratory tests about aggregates compliance. Through interviews obtained, ANCE (National Association of Builders) states that the tests are too expensive and require too much time.

Price is another point that obstructs the use of secondary materials in new industrial products. A defining factor is transport costs. Secondary materials are, in general, not much cheaper than primary resources and can even be more expensive. The distances among natural aggregates buyers, suppliers, sellers and consumer cannot be too large. Interviews evidence that the costs of transport could double for every additional 30 kilometres travelled [9]. As a result, this characteristic makes the markets for mineral CDW very local.

\section{Policies and Incentives}

Waste prevention and sustainable recovery strategy is achieved through a defined legislative framework, favouring conditions for a sustainable management of CDW among operators and consumers (designers, planning supervisors, public administration, construction companies). Overall policies that incentive the recycling and recovery rate growth are related to waste disposal (landfill taxes) and exploitation of natural resources reduction (aggregates levies or taxes). Each of these actions can enhance price differences between recycled materials and virgin materials, towards the economic attractiveness of secondary materials. The largest obstacles for recycling CDW is cheap availability of low cost raw materials. Therefore, there is not a large economic incentive for business. A solution could be to increase (through taxation) the price of raw material goods [13]. Aggregates levies or taxes is considered more effective to make economically advantageous secondary materials. It concerns a direct relation with the price of virgin aggregates, instead landfill taxes could implicate illegal disposal risks [12]. The final report of Bio Intelligence Service [14] provides an overview between the CDW tax rate and the percentage of CDW recycled. It shows that there is no significant relationship between the tax rate and the amount of waste recycled. To reduce significantly illegal traffic, Belgium (Flanders) introduced a mandatory pre-demolition inventory of the types/quantities of materials present in 
buildings (for non-residential buildings with an enclosed volume over $1000 \mathrm{~m}^{3}$ ) to identify hazardous and other waste fractions [15].

To explore other main guidelines to incentive the recycling percentage, it is interesting to analyse the countries that have the highest percentage of recycling, based on the country screening factsheet of the research "Resource Efficient Use of Mixed Waste". Analysing Germany and The Netherland's policies, it is noted that both countries had waste regulation before the WDF.

Germany took the initiative in 1996 through a voluntary commitment to cut in half the amounts of CDW landfilled. The high recycling rates were achieved although there is no national ban on landfilling of CDW material. The initiative "Kreislaufwirtschaft Bau" (Circular Economy in Building) has been documenting mineral construction and demolition waste arising and treatment since 1995, setting waste reduction targets. It can be seen as one of the main reasons for the high recovery rates that are observed today (96\%).

With regard to The Netherlands, since January 1st, 1994, the Dutch legislation on waste can be found primarily in the Environmental Management Act (Wet Milieubeheer; Wm), which already sets the waste hierarchy (used before the WFD). However, a large number of issues was not in the law itself, but was regulated at local level: provincial environmental regulations or municipal waste regulations (such as landfills and waste bans).

Furthermore, these countries have many legislative instruments that follow the WFD. In Germany the Ordinance on the Management of Municipal Wastes (Gewerbeabfallverordnung) contains national obligations for selective demolition. It defines on a national level, separation and requirements regarding the pre-treatment of CDW.

In The Netherland, in order to implement Directive 2008/98, the Minister of the Environment submitted a proposal in 2010 to amend the Environmental Protection Act $(\mathrm{Wm})$, the Law on environmental taxes and the Law on economic offenses.

The main strategies present in the countries with high level of recycling, (differently to country with low level of recycling) are non-legislative instruments. They contribute to create good conditions for the management of CDW recycling. In Germany several municipalities already integrate the preferred use of recycled construction material in their calls for tenders (even if this is not a standard). Demolition has to be performed by authorized companies, which need to be certified (Regional level). Moreover, different regional standards for recycled CDW exist and are defined in the respective waste management plans. In Germany, Sustainable Building Certificate is an important driver. A voluntary scheme run by the German Sustainable Building Council (DGNB) encourages the practice of recycling. It sets particular criteria regarding CDW.

Also in The Netherland, the role of non-legislative instruments is strong. Relating to pre-demolition audits, it is mandatory to have a demolition license for every demolition with more than $10 \mathrm{~m}^{3}$ of waste (Model Bouwverordening MBV). Selective demolition (Kaderrichtlijn Afvalstoffen) companies must be registered in order to carry, collecting or trade waste. Moreover, a "passport" for buildings was developed which contains what substances are in the materials of the building. 
Tools are used to understand what is possible to do in construction and demolition in order to be sustainable. Eventually it leads to obtaining the BREEAM certificate. The Netherlands promotes initiatives focused on trying to get the quality of reusable concrete as high as possible and offering sustainable wood.

In Italy, there is the legislative instrument Decree 205/2010 as implementation of WFD. In legislative framework the drive of Green Public Procurement (GPP) requirements, that incentive waste recovery, has important role. Indeed, the use of recycled materials becomes mandatory and strategic in the assignment of the tender. The project must contain second raw materials, at least $15 \%$ by weight on all materials used (with $5 \%$ in non-structural materials). This percentage must be documented. Moreover GPP requirements promotes the application of Environmental Product Declaration. Instead, non-legislative instruments (such as pre-demolition audit, demolition license etc.) are not so much promoted.

Overall, in countries where non-legislative instruments are not highly used, the recycling percentage is low.

\section{Guidelines for Sustainable Strategies}

Given that the Directive 2008/98/CE shows the percentage in weight, and the 60$70 \%$ (by weight) of CDW is composed of concrete and masonry, as a result the directive target is achieved in the majority of Member States. Indeed, as a result, it was found that in many countries, the target is achieved through the recycling of aggregates for road foundations, earth works and backfilling, even if these works are not "high quality recycling" as required by Article 11 of WFD (and resulting in a loss of economic value of inert).

To achieve a sustainable recycling management, it is important to consider material recycling with the approach of Life Cycle Management, as an integrated approach, based on technology and process defined considering LCA and LCC results.

This approach can be useful to support the waste hierarchy of WFD, to make a decision about the best way to reuse, recycle or dispose waste materials. It is clear that since the CDW has high "embodied" environmental impacts (in terms of the investment done to produce what turn into waste), the re-use or recycling can eliminate the need for further investment in primary production [16]. Obviously prevention is often the best possible solution for the environment avoiding the replacement and prolonging the service life of components.

A life cycle approach is important to move towards an upcycling of waste, to improve the economy and creating an effective sustainable market.

To make CDW recycling management more effective and sustainable it is important to apply a LCT in non-legislative instruments.

The objective of this paper is to suggest sustainable guidelines to improve work of legislative operators, and local authority action. Following, the main important 
point made by analysing the best practice and the application of Life Cycle approach.

Regarding legislative improvements, it is important to suggest:

(1) to look at quality rather than quantity: value the best material to be recycled in terms of effectiveness and sustainability, not heavier ones; through the separation of recycling targets (percentage well-defined for every type of waste material) related to the quality target;

(2) to improve the waste hierarchy: support decisions of the type of recovery with an assessment of quality of recovery, in term of environmental and economical sustainability (support by LCA and LCC). As well as to promote upcycling directions;

(3) to reassess the inclusion of some actions considered in the percentage (70\%) of recycling rate: "backfilling" (is a low quality recycling) and "preparing for re-use" (this is only storage of material to check, clean or for repair recovery operations, until the real recovery stage).

With regard to non-legislative action of local authorities, that is useful to improve the sustainable local waste management, they should follow these guidelines:

(1) to improve selective demolition, which helps prepare for the reuse process;

(2) to use restrictive pre-demolition audit in order to have the information about the consistency and quality of material; to evaluate the environmental impact with LCA and the market opportunities with LCC approach before deconstruct a building. This information is important to make a decision of end-of-life stage, to drive resource efficiency and effectiveness of refurbishment and demolition projects. Thus preventing unnecessary waste and maximizing the value and sustainable use of construction and demolition waste;

(3) to use mandatory building passport and material passport (for new construction): encouraging the use of Environmental Product Declaration (EPD) in order to facilitate the assessment of construction products and material sustainability, promoting recycled material. One of the current problems is the high application of composite materials (not recyclable at end-of-life) used due to their high thermal performance in energy-efficient buildings or due to their recycled content.

(4) to apply mandatory environmental criteria in GPP on waste management: to improve waste recovery and sustainability of buildings through building benchmark LCA based in the assignment of public procurements.

To overcome the obstacles created by the lack of consumer and operator awareness is fundamental:

(1) to increase skills and knowledge: to eliminate the lack of confidence in the quality of materials from recycled construction and demolition waste;

(2) to support professional training of the operators within the demolition sector; 
(3) to use environmental labelling of recycled (and non-recycled) products to encourage awareness and stimulate a sustainable waste market.

\section{Conclusion}

In this paper, an analysis of CDW recycling and recovery was conducted, with the objective to find possible applications of a life cycle approach in support to recycling practices policies. The first obstacle to a sustainable waste flow management is the gaps in the database for monitoring CDW quantities. CDW is the main stream of waste in EU-28, and almost $60 \%$ of the weight is composed of concrete and masonry materials. It is possible to observe that many countries have already abundantly achieved the target set by WFD of $70 \%$ recycled waste. The main recycling practice focuses on inert waste. It is used as low performance application (downcycling) of sub-base layers in roads and backfilling, since aggregate is used in new concrete production or asphalt it requires high performance (following standard regulations).

Regarding the policies and incentives of some Member States, the analysis shows that the most exemplary countries have many legislative instruments and many non-legislative instruments (e.g. criteria in public tenders). Non-legislative instruments play a crucial role in the waste management target. The Life Cycle approach is needed in order to set more effective, for the environment, and more cost-efficient, for stakeholders, policy targets. Policies at regional level should be encouraged to move towards a Life Cycle Management, therefore achieving a sustainable management of waste within a new definition of circular economy.

\section{References}

1. European Commission, COM 398 Towards a circular economy: A zero waste programme for Europe. Brussels, 2014.

2. European Commission, COM 614 Closing the loop-An EU action plan for the Circular Economy. Brussels, 2015.

3. Directive 2008/98/EC on waste (Waste Framework Directive). Official Journal of the European Union. L 312/3.

4. Bio Intelligence Service, Service Contract on management of construction and demolition waste SR1, 2011.

5. Member State, Resource Efficient Use of Mixed Waste, CDW Factsheet Final, 2015.

6. Pantini S, Rigamonti L, Evaluation of the mass balance of the construction and demolition waste management system in Lombardy Region (Italy), CRETE 2016 Conference Proceedings, 2016.

7. ISPRA, L'Italia del Riciclo, 2013, pp. 196-177.

8. Eurostat, Key figures on Europe, Belgium, 2016, pp. 161-164.

9. IDEA Consult (lead partner), Analysis of certain waste streams and the potential of Industrial Symbiosis to promote waste as a resource for EU Industry, Final Report, Brussels, 2015. 
10. Silva R.V, De Brito J, Dhir R.K, Properties and composition of recycled aggregates from construction and demolition waste suitable for concrete production, Construction and Building Materials, 65, 2014, pp. 201-217.

11. ISPRA, L'Italia del Riciclo, 2014, pp. 176-185.

12. Moriconi G. Aggregati riciclati nel calcestruzzo: le Norme, l'evoluzione in Italia e in Europa e i pregiudizi da superare, in Concreto, December, 2013, pp. 1-8.

13. [13] Dahlbo H., Bachér J., Lahtinen K. et al., Construction and demolition waste management: a holistic evaluation of environmental performance, Journal of Cleaner Production, 107, 2015, pp. 333-341.

14. Bio Intelligence Service, Use of Economic Instruments and Waste Management Performances, Final Report, 2012.

15. Resource Efficient Use of Mixed Waste, Minutes of the workshop-Task 5, Improving management of construction and demolition waste, 2016.

16. JRC, Supporting Environmentally Sound Decisions for Construction and Demolition (C\&D) Waste Management, European Union, 2011.

Open Access This chapter is licensed under the terms of the Creative Commons Attribution 4.0 International License (http://creativecommons.org/licenses/by/4.0/), which permits use, sharing, adaptation, distribution and reproduction in any medium or format, as long as you give appropriate credit to the original author(s) and the source, provide a link to the Creative Commons license and indicate if changes were made.

The images or other third party material in this chapter are included in the chapter's Creative Commons license, unless indicated otherwise in a credit line to the material. If material is not included in the chapter's Creative Commons license and your intended use is not permitted by statutory regulation or exceeds the permitted use, you will need to obtain permission directly from the copyright holder. 


\title{
Environmental Assessment of Energy Related Products and Energy Systems Across Their Life Cycle
}

\author{
N. Espinosa and Y. J. Suh
}

\begin{abstract}
In a global context, where several international and national policies attempt to define strategic energy plans that address environmental sustainability, it is necessary to adopt a holistic perspective. In this session, we want to stimulate inputs on how Life Cycle Assessment (LCA) models can capture the complex management challenges in the whole energy sector. In that sense, the various sectors related to energy (namely heat, power, etc.) will become more interrelated, which will be challenging to deal with in Life Cycle Management (LCM). The next decades, LCM modelling of energy systems will have to be quite innovative in order to create realistic models. Furthermore, if LCA wants to do real LCM, the methodology should be widened to e.g. include long-term environmental implications.
\end{abstract}

\section{Introduction}

In the global energy context, where several international and national policies attempt to define strategic energy plans that address environmental sustainability, it is necessary to adopt a holistic perspective.

A comprehensive quantification of environmental impacts in the energy sector can be done by means of Life Cycle Assessment (LCA). Current developments in energy systems and energy products are limited in their scopes with strong focus on greenhouse gas accountings with disregard for other environmental problems like the impacts of chemical pollution and particulate matters on human health and ecosystems. The session aims to address the importance of rooting environmental sustainability assessments with broad impact coverage into energy planning to

\footnotetext{
N. Espinosa $(\square)$

European Commission, Joint Research Centre, Inca Garcilaso s/n,

41092 Seville, Spain

e-mail: nieves.espinosa@ec.europa.eu

Y. J. Suh

Samsung Electronics Co., Ltd., Suwon, Korea

(C) The Author(s) 2018

E. Benetto et al. (eds.), Designing Sustainable Technologies,

Products and Policies, https://doi.org/10.1007/978-3-319-66981-6_25
} 
prevent or minimise problem shifting and ensure an environmentally-sound energy transition.

Other than environmental factors such as costs, politics, regional interdependencies, risks, or social issues, an energy transition will have to be furthermore addressed in true Life Cycle Management (LCM) models of future energy supply.

The topics included in the session were the following:

- How to address emerging energy technologies in LCA, e.g. carbon capture and storage (CCS), etc.

- Integration of the ecodesign directive in energy-related products and technologies

- Inclusion of foresight and dynamic aspects in applications of LCA to energy systems

- Barriers and solutions in application of LCA to energy systems, e.g. modelling challenges such as temporal and geographical resolution versus data availability and level of detail of results needed

- Handling of bioenergy (poses specific methodological challenges, e.g. iLUC)

- Relevance of transportation in LCA energy systems

The objective of the session was to discuss and advance the implementation of Life Cycle approach and Circular Economy along the businesses value chain, supporting environmental, social and economic sustainability related to the development of industrial technologies, products, services and policies.

The session was proposed in synergy with a parallel session conducted at the LCM 2017 conference on LCM of energy and energy transitions. In this session, we wanted to stimulate inputs on how LCA models can capture the complex management challenges in the whole energy sector. In that sense, the various sectors related to energy (namely heat, power, etc.) have become more interrelated, which will be challenging to deal with in LCM. Therefore, the next decades, LCM modelling of energy systems will have to be quite innovative in order to create realistic models. Furthermore, if LCA wants to do real LCM, the methodology should be widened to e.g. include long-term environmental implications.

\section{Summary of the Session Presentations}

The session proposed had the aim to highlight the importance of rooting environmental sustainability assessments with broad impact coverage into energy planning.

The presentations had in general an outstanding quality. Data availability and level of detail of results needed was a topic proposed. Life cycle inventories of good quality are essential to conduct any study and there were a number of presentations dealing with that topic.

Oberschelp et al. [1] deal in their work with other case of data availability: the emission of particulate matter from the production of electricity in China. A detailed 
inventory for Chinese power production from fossil fuels was developed and combined with characterization factors for Chinese particulate matter emission impacts.

Moreover, the geographical resolution of the data may indeed reveal big differences in environmental impacts of energy production, and this is precisely presented in a contribution targeting the LCI data for electricity production at smaller geographic regions of both China and India by Levova et al. [2]

The full coverage of impacts and the comprehensiveness of LCA for energy systems were two topics dealt with in the work presented by Chatzsisideris et al. [3] about quantifying the importance of comprehensive life cycle and impact coverage for photovoltaic systems.

In the same field, the PV systems, Weyand et al. [4] show in their work for a portable solar charger how consequential LCA modelling can extend the boundaries to consider indirect impacts associated with the materials and energy use and the function the product delivers, which has so far been neglected in assessment efforts for an emerging technology such as organic photovoltaics.

The LCA study of a mobile phone charger incorporating $20 \%$ recycled plastic made by $\mathrm{Heo}$ et al. from Samsung Electronics [5], covers each life cycle stage including pre-manufacturing, manufacturing, use and disposal phase. The study shows that the use phase is the most significant life cycle stage due to the impact on global warming resulting from charging the phone.

For a sustainable production of plastics Himmelreich [6] illustrates the development of $\mathrm{CO}_{2}$ based plastics and the implementation into consumer products as well as discuss the influence of different $\mathrm{CO}_{2}$ sources and their impact dependent on the valuation method for $\mathrm{CO}_{2}$.

Van Nieuwenhuyse et al. [7] propose a work related to resource efficiency and the development of extended producer responsibility schemes that are fostering the development of updated and accurate Life Cycle Inventories (LCI) data on end-of-life operations. A consortium of companies have joint efforts to develop a LCI database of Waste Electrical and Electronic Equipment (WEEE).

About the usage of energy consuming product, Heslouin et al. [8] propose to improve the modelling of the use scenario to improve the environmental footprint. The challenge here is the data availability to model specific scenarios for each use case.

Improving energy efficiency is one of the main topics of the European 2020 strategy [9]. The building sector consumes $40 \%$ of the total energy whereby the major part is being used for space heating where the energy is quickly lost to the environment. This loss could be significantly counterbalanced in the future by the development of multi-functional energy-efficient windows or façades, designed to produce energy from sunlight and to store heating energy. New energy efficient window design accompanying LCA (and cost considerations) are proposed to be integrated in the overall development process right from the start by Schmidt et al. [10]. 


\section{Analysis and Outcomes of the Session}

The problem of particulate matter impacts from energy production in China had not been assessed on a high regional resolution due to a lack of a site-specific technology-linked inventory for emissions. Oberschelp et al. have developed a detailed inventory for Chinese power production derived from the International Energy Agency's coal power atlas and the PLATTS World electric power plant (WEPP) database. Remaining gaps in the inventory were filled with global US Geological Survey (USGS) fossil fuel data. A combustion model was moreover applied to quantify outputs of the main pollutants contributing to particulate matter formation ( $\mathrm{PM} 2.5, \mathrm{NO}_{\mathrm{x}}, \mathrm{SO}_{2}$ ). Flue gas cleaning is included by taking into account technology-specific cleaning rates. These emissions were multiplied by regional characterization factors for all global cities with more than 100,000 inhabitants and characterization factor recommendations by the UNEP SETAC Life Cycle Initiative. Results and conclusions have been drawn from the country-level PM characterization factors provided by LC-Impact. Impacts were put into perspective by comparing them with other sectors (transport, industry, domestic coal combustion) in China [1].

With regard to the comprehensiveness of a LCA study, the ISO 14040 principles establish that such a study should consider the entire life cycle of the system and assess all relevant impacts on the natural environment, human health and resources. This is important in order to be able to identify and evaluate potential shifting of environmental burdens or trade-offs between life cycle stages or impact categories. However, according to Chatzsisideris et al. existing review papers of LCA on photovoltaic systems tend to exclusively focus on greenhouse gas assessments disregarding other impacts on human health and ecosystems and eluding an explicit description of which parts of the PV life cycle were considered by the LCA studies under review. Hotspots may not be properly identified if there is not a full coverage of impact categories. The analysis of Chatzsisideris showed that only a third of the analysed contributions were including the balance of system (BOS) components (i.e. power electronics, wiring, mounting structures) in their assessments, which has been proved to contribute significantly to most environmental impact categories [3].

The use of consequential LCA modelling was performed by Weyand et al. [4], to evaluate the environmental response of a phone charger made of an emerging technology such as organic photovoltaics. The impacts of an OPV charger associated with "an additional $10 \mathrm{Wh}$ electricity generation for charging phones" as functional unit, were calculated and compared them to possible substituted conventional energy generation technologies and country-specific electricity mixes. Based on the assumptions, that charging the mobile phone with an OPV charger reduces electricity at the grid, substitution scenarios of several European countries are analysed. The environmental responses of these scenarios are modelled within the software OpenLCA and analysed for the impact categories using the ReCiPe Midpoint $(\mathrm{H})$ method. Current substitution scenarios of the electricity mixes of France and Germany assume that the substituted electricity is nuclear power and 
electricity from lignite - mainly due to the high share of these energy sources in the electricity mixes. The preliminary results indicate considerable environmental benefits in the case of the OPV charger in 7 of the 18 ReCiPe impact categories. In particular, the reductions take place in categories like toxicity (freshwater, human, and marine) and eutrophication (freshwater, marine). In 8 of the 18 impact categories, the electricity generated by the OPV charger has lower impacts than one of the country-specific electricity mixes, mostly than the German scenario. For example, the OPV charger mitigates the climate change potential compared to German scenario significantly, whereas the French scenario still has the lowest impacts due to the high share of nuclear energy. Just in three impact categories, the OPV charger seems to have higher impacts than both scenarios.

For a conventional phone charger, however, key components were identified in the pre-manufacturing stage by Heo et al. [5]. These are the printed circuit board and the plastic case composed from $20 \%$ recycled poly carbonate (PC). The study compared the global warming potential (GWP) for both recycled PC and virgin PC. The result shows that recycled PC is clearly beneficial: the GWP is $96 \%$ less. The amount of GHG emission of the mobile charger containing $20 \%$ recycled plastics is $1.9648 \mathrm{~kg} \mathrm{CO}_{2}$ which is $0.089 \mathrm{~kg} \mathrm{CO}_{2}$ less than a conventional charger containing virgin plastics. A $10 \%$ increase of recycled PC contents in the charger results in $0.3 \%$ GHG-reduction across the total life cycle.

After launching the first industrial-scale production plant for polyols containing about 20 percent $\mathrm{CO}_{2}$, possible thanks to the development of a catalyst which was able to reduce the activation energy of $\mathrm{CO}_{2}$, Himmelreich did an LCA on the process. The evaluation the environmental impact of these new polyols showed that the result will be dependent on the chosen source of $\mathrm{CO}_{2}$ (e.g. biogenic, natural sources, power plants or ammonia plants) and the assumed burden of the carbon dioxide [6].

Eco-systèmes and other leading companies in take-back schemes approved by the French authorities are working on collecting and recycling WEEE. They represent several thousand producers. The database on WEEE management by Van Nieuwenhuyse et al. may contribute to solve several challenges faced by producers to adopt a circular approach, from an environmental assessment perspective, notably by:

- reducing the dissymmetry between production and end-of-life modelling, in terms of reliability and granularity of the data used;

- facilitating the modelling of a life-cycle stage which is generally not managed by material producers nor product manufacturers, and involves multi-step and multi-actor pathways;

- taking into account, on the basis of field data, the environmental benefits of material and energy recovery.

A LCI database has been developed to couple material with WEEE categories. Thereby, the database allows to take the complete recycling chain into account and 
to adapt the assessment specifically to the product design, without requiring any additional hypothesis or settings than the product material composition [7].

The usage of products is frequently modelled by average scenarios of use in LCAs. One challenge of the modelling is the data availability to model the specific scenario of each case of use. The modelling needs to collect several data can be expensive and time consuming to collect a lot of specific data to improve the modelling of the use phase. The case study of the work from Heslouin et al. is a truck refrigeration unit for which the most impactful phase is the use phase. The energy consumption depends on good and bad practice of use [8].

In the framework of the European collaborative project LAWIN, a transparent heat transfer fluid is integrated in a newly designed glass module to make use of temperature differences for energy generation. This energy shall be used directly for heating or cooling processes in the building. Optimization of the overall concept ensures that additional costs in the manufacturing phase and thus, the selling price, are kept in the range of established window or façade elements in order to ensure a quick and broad acceptance of the product building. Selected outcomes of this anticipatory evaluation comparing, e.g., different positions of the LAWIN module in windows or facades, its application as collector or as heat exchanger for heat pumps, or its use for heating or cooling show a strong dependence from the regional location in the use phase [10].

\section{Future Perspectives in Light of the Presentations}

The industrialized and densely populated Eastern parts of China around the provinces Hebei and Shandong and the municipalities Beijing, Tianjin, and Shandong heavily suffer from PM emissions. Despite recent efforts in the introduction of strict emission limits, further upgrade of flue gas treatment in power plants is necessary to reduce health issues. The closure of inefficient power plants, fuel replacement and relocation of emissions are promising options for improvement [1].

The assessment of the results for the different regions in China and India proved that there are indeed big differences in environmental impacts of energy production. Therefore, further discussion can be thus initiated whether more regions should be sub-divided or whether there is a tipping point at which further detail will not bring any additional value [2].

In order to make a comprehensive study and be able to identify hotspots with lower uncertainty, it is recommended that LCA practitioners performing LCA on PV systems consider the entire PV life cycle, including the BOS, and all relevant impact categories. In that way ecodesign opportunities can be identified without risking potential shifting of environmental burden from one part of the life cycle to another or from one impact category to another [3]. 
There is a significant potential in using OPV chargers to mitigate impacts that dominate number of impact categories, especially in regions heavily dependent on coal powered electricity such as Germany. Further analysis needs to be done to include also electricity mixes from other European countries and to understand the reasons behind the differences [4].

Whether to include recycled materials in a conventional mobile phone charger or not has been shown as clearly beneficial according to Heo et al. [5].

More environmentally sustainable plastics could further reduce the results before [5]. Himmelreich presented her work a successful use of $\mathrm{CO}_{2}$ as an alternative building block for various plastics, replacing a proportion of the petrochemical precursors otherwise used [6].

The specific data need of the producers, can thus be satisfied with the provision of a LCI on WEEE; where material efficiency is linked to the different life-cycle stages: raw material extraction, manufacturing, end-of-life [7].

Specific end-user LCAs and well defined average scenarios are recommended by Heslouin et al. [8] to improve the global environmental footprint. The work also highlights how far we have to go in the collection of data for users' behaviour.

Energy savings of the building sector can be enhanced in the future if LCA studies are accompanying the development of multi-functional energy-efficient windows or façades, designed to produce energy from sunlight and to store heating energy [10].

\section{References}

1. Oberschelp C, Pfister S, Hellweg S, Reduction of site-specific electricity generation particulate matter impacts in China, Life Cycle Management Conference, Luxembourg, 2017.

2. Lévová T, Valsasina L, Geographical resolution of LCI data on electricity production - the level of detail needed, Life Cycle Management Conference, Luxembourg, 2017.

3. Chatzisideris M.D, Espinosa N, Laurent A, Krebs F.C, Quantifying the importance of comprehensive life cycle and impact coverage for photovoltaic systems, Life Cycle Management Conference, Luxembourg, 2017.

4. Weyand S, Glogic E, Sonnemann G., Schebek L,Young S.B, Consequential life cycle assessment of an organic photovoltaic portable solar charger applied in the context of European electricity mixes, Life Cycle Management Conference, Luxembourg, 2017.

5. Heo Y, Rana S, Suh Y, Life Cycle Assessment of a Mobile Charger containing Recycled Plastics, Life Cycle Management Conference, Luxembourg, 2017.

6. Himmelreich B, A Dream comes true- Use of $\mathrm{CO}_{2}$ for the production of plastics, Life Cycle Management Conference, Luxembourg, 2017.

7. Van Nieuwenhuyse T, Assimo, P, Morice L, Lesage R, Hugrel C, Palluau M, Osset P, An innovative LCI Database on WEEE management, Life Cycle Management Conference, Luxembourg, 2017.

8. Heslouin C, Pourcheresse L, Stumpf A, Bernardet V.P, Cornier A, Perry N, Is it useful to improve modeling of usage scenario to improve the environmental footprint of energy consumption product? Life Cycle Management Conference, Luxembourg, 2017. 
9. A European strategy for smart, sustainable and inclusive growth, 2010, Brussels: EUROPEAN COMMISSION.

10. Schmidt A, Su L, Fraass M, Wondraczek L, Design accompanying Life Cycle Assessment for the development of new energy-efficient window concepts, Life Cycle Management Conference, Luxembourg, 2017.

Open Access This chapter is licensed under the terms of the Creative Commons Attribution 4.0 International License (http://creativecommons.org/licenses/by/4.0/), which permits use, sharing, adaptation, distribution and reproduction in any medium or format, as long as you give appropriate credit to the original author(s) and the source, provide a link to the Creative Commons license and indicate if changes were made.

The images or other third party material in this chapter are included in the chapter's Creative Commons license, unless indicated otherwise in a credit line to the material. If material is not included in the chapter's Creative Commons license and your intended use is not permitted by statutory regulation or exceeds the permitted use, you will need to obtain permission directly from the copyright holder.

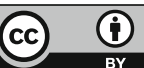




\title{
Is It Useful to Improve Modelling of Usage Scenarios to Improve the Environmental Footprint of Energy-Using Product?
}

\author{
Charlotte Heslouin, Véronique Perrot-Bernardet, \\ Lionel Pourcheresse and Nicolas Perry
}

\begin{abstract}
When considering the Life Cycle Assessment of an energy-using product, usage is often modelled by average scenarios of use. One challenge of modelling is the availability of data to model the specific scenario in each case. This type of modelling requires the collection of data from several inputs. Also, it can be expensive and time-consuming to collect the specific data to improve the modelling of the use phase. This case study examines a truck refrigeration unit, for which the most environmentally impactful phase is the use phase. The energy consumption of the unit depends on usage. We highlight the importance of modelling a detailed usage scenario specific to each user and examine if it is enough to consider an average usage scenario. This study shows how a specific end-user Life Cycle Assessment and customized recommendation can contribute to improving the global environmental footprint. This is demonstrated by using the energy consumption life cycle inventory analysis of specific end-user behaviour based on experimental data and average scenarios. The results show how far we have to go in the collection of data.
\end{abstract}

\section{Introduction}

Energy-using products are commonly known to have their main environmental impact in the use phase [1-3], hence the importance of accurately modelling the usage of this kind of products.

\footnotetext{
C. Heslouin · L. Pourcheresse

Carrier Transicold Industries, 76520 Franqueville Saint Pierre, France

C. Heslouin · V. Perrot-Bernardet

Arts et Métiers Paristech, Institut de Chambéry, Savoie Technolac,

73375 Le Bourget du Lac, France

e-mail: veronique.perrot-bernardet@ensam.eu

C. Heslouin · N. Perry $(\square)$

Arts et Métiers Paristech, I2M, UMR 5295, 33400 Talence, France

e-mail: nicolas.perry@ensam.eu

(C) The Author(s) 2018

E. Benetto et al. (eds.), Designing Sustainable Technologies,

Products and Policies, https://doi.org/10.1007/978-3-319-66981-6_26
} 
Life Cycle Assessment (LCA) is recognized to be one of the most reliable tools for environmental analysis. LCA is ruled by the ISO 14040 [4] and ISO 14044 [5] standards. LCA methods are described in more detail in the ILCD Handbook [6]. However, none of these references specifically describe how to model the use phase. In fact, the relationship between the usage of product and its environmental performance is rarely considered in LCA. Usually an average usage scenario is used which does not take into account the effects of the usage context on environmental performance, which can be positive or negative. The effect of usage context and its modelling has been recognized as a priority by LCA researchers and practitioners. Telenko and Seepersad [7] proposed to model usage context by using Bayesian network models. Among the usage context factors considered were human (who? skills or habits?), situational (where? when? for what task?) and product (design and specification influencing the use of the product) ones. Ma and Kim [8] proposed a time usage model in which the lifespan of the product was proven to have a strong impact. Egede et al. [9] analyzed the influence of internal and external factors such as vehicle characteristics, location of use and user influence.

In this paper, we consider the case of a LCA of truck refrigeration units (TRUs). It has been shown that TRUs' environmental footprint is mainly due to use of refrigerant and the energy needed during their lifetime [10-12]. However, the use phase it depends on several factors which are influencing the energy consumption. The latter include: trailer specification, size and packaging of product loaded, outside climate (temperature, hygrometry), operating mode (continuous run vs. start/stop), start/stop parameters, type of product (fresh or frozen), type of transport (urban distribution vs. long haul), speed of engine, coefficient of performance (COP) of the unit and refrigerant efficiency [13-18].

In the ecoinvent database V3.2 [19], the fuel consumption of truck refrigeration systems is modelled as an average scenario, assuming a $20 \%$ increase as compared to conventional truck transport without refrigeration [20]. In this scenario, none of the aforementioned parameters that can influence the energy consumption have been taken into consideration.

This paper shows:

- The potential benefits of modelling the usage phase in detail as compared to an average scenario and how the results can be used to provide specific recommendations to end-users that may significantly improve the environmental impact of TRUs.

- The difficulties of collecting reliable and real-time data to perform a LCA of the use phase.

- The kind of data that can be used and how to collect it.

- The potential influence of the use phase life cycle inventory, using an example of a TRU with an average scenario of energy consumption versus different specific scenarios.

- The potential benefits of using specific energy consumption data to improve product design in order to promote sustainable behaviour when using energy-using products. 


\section{Modelling a Product Usage Scenario}

\subsection{Source of Data Collection}

In order to model a usage scenario, it is necessary to gather data from the use phase. Different kinds of data can be collected in order to build usage scenarios. In this paper, four sources of data are identified: real-time data, experimental data, numerical simulation data and average scenario data. They are analysed based on four criteria: (i) time needed to collect data, (ii) cost to collect data, (iii) reliability of data and (iv) accuracy of real usage data (Table 1).

Real-time data are collected directly with the help on sensors while the product is running. It is the most reliable kind of data and allows the analysis of the actual usage specific to each user. However, data collection and analysis can be time-consuming and/or costly, as it is necessary to use multiple sensors and allocate resources to analyse data. In addition, it implies that the environmental footprint must be evaluated continuously or only at the end of the product life. The environmental footprint for a specific set of data will be accurate for a specific usage but rather specific to it, i.e. not necessarily pertinent for another. It can be helpful to build an average scenario, specific to each type of business (e.g. fresh or frozen product, international long haul, national long haul delivery or urban delivery).

Experimental data are collected during punctual measurement on the product in order to recreate real condition of use. Again, collection can be time-consuming and costly, depending on the resources used. However, it requires determining precise parameters to analyze in order to have reliable data. It does allow for analysing the influence of single parameters or for mixing the influence of different parameters. It does allow for creating specific scenarios that can be accurate with real usage.

Data from numerical simulations are based on mathematical routines recreating real-world use conditions. The approach to data collection requires a thorough knowledge of the different parameters to set up the simulation and interpret the results. In some cases, especially with products requiring the integration with another system, it can be difficult to control all the external parameters influencing

Table 1 Proposal of quotation of four sources of data to evaluate the environmental performance of the use phase ${ }^{\mathrm{a}}$

\begin{tabular}{l|l|l|l|l|l}
\hline$\#$ & Solution & $\begin{array}{l}\text { Time } \\
\text { needed }\end{array}$ & Cost & $\begin{array}{l}\text { Reliability of } \\
\text { data }\end{array}$ & $\begin{array}{l}\text { Accuracy of real } \\
\text { usage data }\end{array}$ \\
\hline 1 & Real-time data & -- & - & ++ & ++ \\
\hline 2 & Experimental data & - & - & + & + \\
\hline 3 & $\begin{array}{l}\text { Numerical } \\
\text { simulation data }\end{array}$ & + & + & - & + \\
\hline 4 & $\begin{array}{l}\text { Average scenario } \\
\text { data }\end{array}$ & ++ & ++ & -- & -- \\
\hline
\end{tabular}

${ }^{\mathrm{a}}$ Quotation ++ very good; + good; -bad; -- very bad 
the energy consumption, which can have a detrimental effect on the accuracy of the results. Numerical simulations have the advantage to not to require resources and materials in order to be performed.

Average data are collected from literature reviews, customer statements or expert knowledge. Compared to the previous kinds of data, average data are usually less reliable and, in particular when only a few data are required to build a scenario, can lead to an usage model very far from the actual one. However, this type of data is very easy to gather and at low cost. Average scenario can also be built from the meta-analysis of literature sources or from expert opinions.

\subsection{Data Collected}

In order to identify which data to collect for building usage scenarios for LCA and sensitivity analysis, the important parameters that influence the environmental footprint of the use phase must be identified (such as the technology, the accessories including curtains, partition wall, etc., the setting from the end-user as the setting of the temperature, the operating mode, etc.). These parameters can be further characterized using expert and/or user feedback and/or literature sources. Based on graph theory (which is used to model pairwise relations between parameters), a directed acyclic graph (i.e. a directed graph with no loops or cycles, as e.g. a hierarchy) is built in order to model all the parameters that can influence energy consumption of a TRU (Fig. 1) based on literature review [10-18] and expert knowledges from Carrier Transicold. As explained in the introduction, many

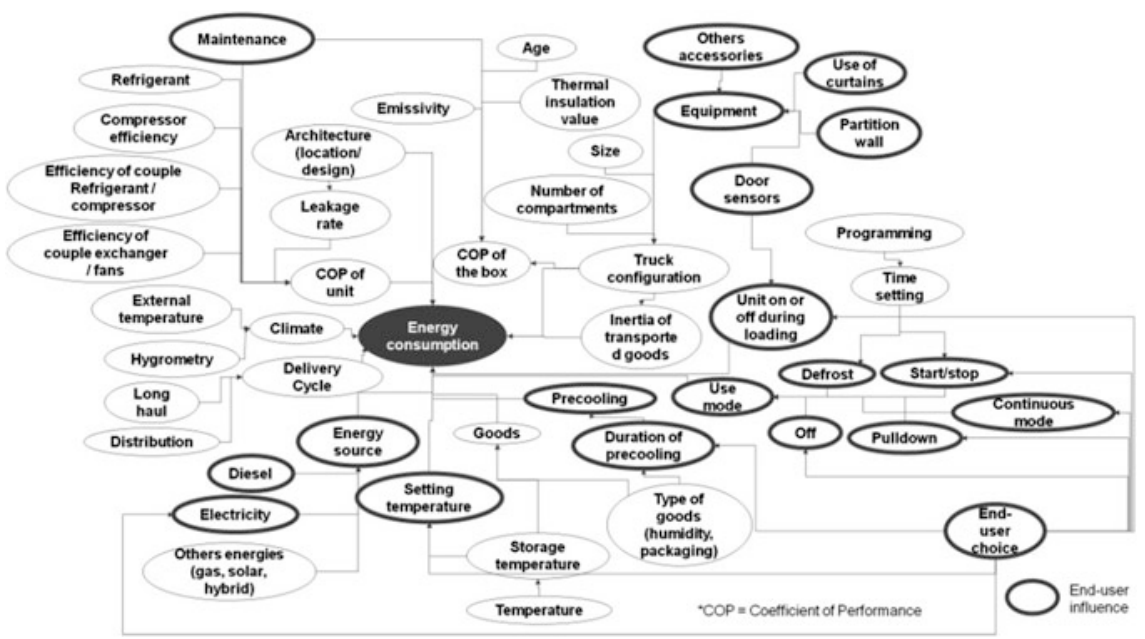

Fig. 1 Directed acyclic graph of energy parameters influencing energy consumption of TRU based on literature review [10-18] and expert knowledge's from Carrier Transicold 
internal and external parameters can influence the energy consumption of the system. The graph shows the complexity of energy consumption modelling and the number of parameters that have to be considered to obtain a realistic usage scenario. As a result, only experimental data with specific parameters were chosen. Many parameters are directly chosen by the customer or the end-user (in this case the driver). Consequently, setting these parameters implies a thorough understanding of end-user behaviours, which can be influenced by different factors (social and personal norms, awareness, habitual processes as routine, intentional processes as willingness for environmental habits and situational influences as surrounding environment) [21]. In this study, we have chosen a few parameters to analyse based on expert knowledge of end-user behaviour and the most commonly used parameters in scientific literature.

\subsection{Scenario Studied}

In order to define the usage scenario, two collection methods were chosen based on the four kinds in Table 1: average scenario data and experimental data.

First a European usage scenario based on average data was modelled and then nine specific scenarios were modelled from experimental measurements using different end-user behaviours and usage settings.

Although it is known that refrigerant leakage [12] significantly contribute to the total environmental impact of the sue phase of TRUs, in this paper we focus only on the influence of energy consumption.

\subsubsection{Average Usage Scenario}

The European average usage scenario was selected. It is a combination of the business activity and one TRU setting:

- Temperature of transportation $\left(0{ }^{\circ} \mathrm{C}\right.$ for fresh product or $-20{ }^{\circ} \mathrm{C}$ for frozen product). This parameter is directly linked to the business activity of the customer.

- Operating mode (start/stop or continuous run). The parameter is chosen by the end-user. It is selected depending on transported product (sensitive or not) but there is no obligation from the manufacturer.

This results in an average scenario of use (Table 2) with an average energy consumption associated of $1 \mathrm{l} / \mathrm{h}$ (this value is defined as a normalized value; it is not the raw value). A weighting factor, obtained from the analysis of $150 \mathrm{TRU}$ usage data sets based on time, has been defined for each combination of parameters and it is indicated here to better illustrate the average European sharing. 
Table 2 Average European usage scenarios of TRUs depending on the main parameters (Carrier Transicold data)

\begin{tabular}{l|l|l}
\hline Operating mode & Temperature of transportation $\left({ }^{\circ} \mathrm{C}\right)$ & Weighting $(\%)$ \\
\hline Continuous run & 0 & 20 \\
\hline Start/stop & 0 & 49 \\
\hline Continuous run & -20 & 0 \\
\hline Start/stop & -20 & 15 \\
\hline Full capacity & 0 or -20 & 16 \\
\hline
\end{tabular}

\subsubsection{Specific Scenarios of Use from Experimental Measures}

The focus here is on the influence of end-user behaviour on the energy consumption. Data for specific use scenarios were collected from experimental measures in external condition, with two similar trailer refrigeration units (in terms of unit model, obsolescence and hours of running), which are actually used by customers. In this case, energy consumption was measured depending on four parameters of use linked to the setting of the TRU (setting point, operating mode) and end-user behaviour (on/off during door openings, use of curtains for door openings). For the setting point, the two most common temperatures $\left(0\right.$ and $\left.-20{ }^{\circ} \mathrm{C}\right)$ were selected from the European average usage scenario and one temperature $\left(-25^{\circ} \mathrm{C}\right)$ was arbitrarily selected to show the influence of a setting temperature that is not optimal but does not affect the cold chain integrity. For each measure, the standard deviation has been calculated. Finally, eleven scenarios of use were built (Table 3). Other possible scenarios are either not representative of a real situation or too resource-expensive considering all the possible combinations of parameters.

\subsection{Results of Energy Consumption Modelling}

In order to assess the potential differences between average and specific usage scenarios, the global energy consumption has been calculated based on $1500 \mathrm{~h}$ of use of a TRU (Fig. 2).

Concerning door-openings, we assumed one 5-min period and one 10-min period during the experimental time of $3 \mathrm{~h}$.

Results show that, depending on the end-user behaviour, energy consumption can increase or decrease compared to the average European scenario. The environmental footprint is directly correlated with diesel energy consumption.

Energy consumption in specific scenarios ranges from -82 to $+9.6 \%$ of the average European scenario when considering $1500 \mathrm{~h}$ of use of the TRU (Fig. 2).

Results also show the influence of different parameters, especially the influence of end-user behaviour. The use of start/stop operating mode for frozen product is a recommended setting to ensure a good quality of cold chain and less energy 
Table 3 Specific scenario from experimental data depending on parameters and energy consumption results (from experimental measures by Carrier Transicold)

\begin{tabular}{l|l|l|l|l|l|l}
\hline$\#$ & $\begin{array}{l}\text { Setting } \\
\text { point }\left({ }^{\circ} \mathrm{C}\right)\end{array}$ & $\begin{array}{l}\text { Operating } \\
\text { mode }\end{array}$ & $\begin{array}{l}\text { On/off } \\
\text { during door } \\
\text { openings }\end{array}$ & $\begin{array}{l}\text { Use of curtains } \\
\text { for door } \\
\text { openings }\end{array}$ & $\begin{array}{l}\text { Energy } \\
\text { consumption } \\
\text { Mean }[1 / \mathrm{h}]\end{array}$ & $\begin{array}{l}\text { Standard } \\
\text { deviation }\end{array}$ \\
\hline 1 & 0 & Continuous & & & 0.58 & 0.09 \\
\hline 2 & 0 & Start/stop & & & 0.18 & 0.06 \\
\hline 3 & 0 & Continuous & On & No & 0.56 & 0.19 \\
\hline 4 & 0 & Continuous & Off & No & 0.55 & 0.11 \\
\hline 5 & 0 & Continuous & Off & Yes & 0.54 & 0.03 \\
\hline 6 & -20 & Start/stop & & & 0.69 & 0.52 \\
\hline 7 & -20 & Continuous & & & 0.78 & 0.18 \\
\hline 8 & -20 & Start/stop & On & No & 0.96 & 0.62 \\
\hline 9 & -20 & Start/stop & Off & No & 0.90 & 0.15 \\
\hline 10 & -20 & Start/stop & Off & Yes & 0.71 & 0.42 \\
\hline 11 & -25 & Start/stop & & & 1.10 & 0.09 \\
\hline
\end{tabular}

${ }^{\mathrm{a}}$ Because of confidentiality reasons, raw energy consumption data cannot be disclosed. Instead we calculated the energy consumption for each scenario as follows: (specific scenario raw energy consumption/European usage scenario raw value)

${ }^{\mathrm{b}}$ Standard deviation is calculated for discrete random variables

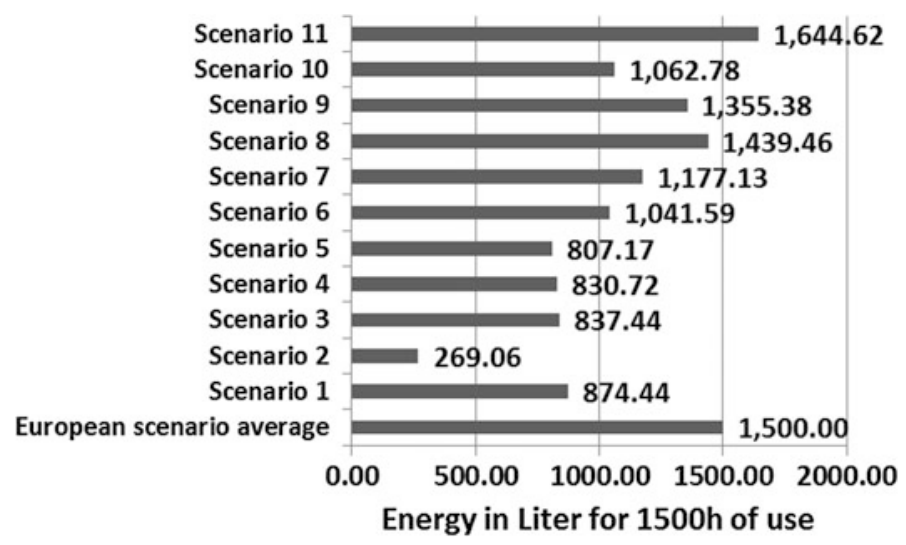

Fig. 2 Energy consumption for $1500 \mathrm{~h}$ of use of the TRU depending on the scenario of use (from experimental data by Carrier Transicold)

consumption. In the European scenario, the use of continuous run for frozen product hardly happens; it can occur if an end-user accidentally sets it or the setting is demanded by a customer. Curtains are optional and they are not always used by end-users. The use (or not) of curtains is not included in the European average scenario. 
Results demonstrate that even when the business of the company strongly influences the scenario of use (e.g. it is not possible to compare fresh products with frozen products), end-users can affect the energy consumption e.g. by setting the temperature (comparison of scenarios 6 and 11). A variation of $36.7 \%$ of energy consumption for a delta of $5{ }^{\circ} \mathrm{C}$ in the setting of the temperature is shown.

When comparing scenario 6 and 7 (influence of operating mode), the energy consumption varies by $11.5 \%$ for $1500 \mathrm{~h}$ of use for frozen products.

When comparing scenario 8 and 9 (TRU turns on or off when doors open) energy consumption varies by $6.2 \%$.

The influence of the use of curtains when doors open (scenario 9 and 10) involves a change of energy consumption of approximately $27.5 \%$.

As a result, the environmental footprint of the use phase can be significantly influenced by the end-user behaviours (setting of the temperature, choice of operating mode, the operation of the TRU when doors are opened and use of accessories such as curtains).

Results also show the importance of taking into consideration the business activity of the customers. The business activity mainly concerned the type of transported products and the type of delivery (long haul transportation or urban delivery with multiple door openings). The business activity will also influence other parameters linked with end-user behaviours. So, the global energy consumption for the whole lifespan of the product and the specific recommendation to end-users will not be the same.

\section{Discussion}

These results highlight how a specific usage scenario can change the lifecycle inventory when considering energy consumption (from +9.6 to $-82 \%$ ). One difficulty in the use of specific energy modelling is to find a good compromise between the need for reliable data and the resources (human, financial, time) that must be allocated to the collection. The difference between the average usage scenario data and the specific usage data is significant.

The study of experimental data took almost two months to be performed due to the size of the system (trailer refrigeration box) and the number of parameters to test. Also, refrigeration measure experimentation had to be conducted over a longer period of time in order to ensure the thermal stabilization of the system. This allowed to check the influence of different parameters on energy consumption in order to provide specific recommendations to the end-user to the aim to guarantee the continuity of the cold chain while reducing energy consumption. Experimental measures can provide as well interesting feedback to engineers for the ecodesign of the units and recommendations to the end-user to enforce sustainable behaviours. It can show the potential benefit of having an innovative design helping to reduce unsustainable end-user behaviours. However, it has to be checked that the 
additional environmental impact generated by the change of design is lower than the avoided impacts allowed during the use phase in order to have a net benefit.

This study finally showed that it is possible to make beneficial specific usage recommendations but the data results are not enough to give specific usage recommendations for each user and their specific usage. In fact, depending on the business of the customer, the recommendations could be adapted. It could be useful to have a specific tool in order to analyse the behaviour of each end-user and to estimate the potential savings by identifying undesirable usage practices. The savings can be estimated based first on experimental data, then on the real data feedback from the end-user. Based on this tool, an "eco-feedback" design strategy to encourage sustainable behaviour can be used. Eco-feedback involves informing the end-users of their energy consumption and associated environmental footprint. This requires real-time data processing analysis and environmental footprint calculation. However, the potential savings of energy consumption and thus the reduction of the environmental footprint (up to $-41.7 \%$ between an optimal and a sub optimal practice of use) can be worth the effort.

\section{Conclusions}

In this study, we compared average fuel consumption, based on the use of a TRU to transport goods, to specific energy consumption, depending on different parameters. The study aimed at showing the influence of energy consumption modelling on the assessment of the environmental performance of the product under study. The hypothesis was that energy consumption modelling based on experimental data provides a greater understanding of the influence of the different parameters useful to model energy consumption.

The results have proven the importance of giving specific usage recommendations to the customer to improve the environmental footprint of TRUs.

It is therefore important to improve the modelling of usage scenarios and of the lifecycle inventory of energy consumption, especially for energy-using products, for which the energy consumption is the major source of environmental impact during the lifespan of the product. However, the gain in using specific scenario modelling must be compared to the additional resources needed to collect data.

\section{References}

1. Brezet $\mathrm{H}$, Van Hamel C, Ecodesign: A promising approach to sustainable production and consumption, Paris: UNEP, 1997.

2. Lockton D, Harrison D, Neville S, Making the user more efficient: Design for sustainable behaviour, International Journal of Sustainable Engineering, Vol. 1, No. 1, 2008, pp. 3-8.

3. Wever R, Van Kujik J, Bock C, User-centred design for sustainable behaviour, Procedia CIRP, Vol. 40, 2016, pp. 469-474. 
4. ISO, Environmental management-Life cycle assessment-Principles and framework, ISO 14040, 2006.

5. ISO, Environmental management -Life cycle assessment - Requirements and guidelines, ISO 14044, 2006.

6. European Commission - Joint Research Centre-Institute for Environment and Sustainability, International Reference Life Cycle Data System (ILCD) Handbook-General guide for life cycle assessment-Detailed guidance, 1st Ed., Luxembourg: Publications Office of the European Union, 2010.

7. Telenko C, Seepersad C.C, Probabilistic graphical modeling of use stage energy consumption: a light vehicle example, Journal of Mechanical Design, Vol. 136, 2014, pp. 1-11.

8. Ma J, Kim H.M, Predictive usage mining for life cycle assessment, Transportation Research Part D, Vol. 38, 2015, pp. 125-143.

9. Egede $\mathrm{P}$ et al, Life cycle assessment of electric vehicles-A framework to consider influencing factors, The 22nd CIRP Conference on Life Cycle Engineering, Sydney, Australia, 2015, Vol. 29, pp. 233-238.

10. Barnabe F, Bensafi A, LCA of a transport refrigeration unit and development of an HVAC\&R equipment ecodesign tool, International congress of Refrigeration, Washington, D.C, 2009.

11. James S J, James C, The food cold-chain and climate change, Food Research International, Vol. 43, 2010, pp 1944-1956.

12. Heslouin C, et al, Effects of product usage scenarios on environmental performance for road transport refrigeration units, 4th International Conference on Sustainability and the Cold Chain, Auckland, 2016.

13. Repice C, Stumpf A, Energy efficiency in transport refrigeration, International Congress of Refrigeration, Beijing, 2007.

14. Tassou S A, De-Lille G, Ge Y.T, Food transport refrigeration-Approaches to reduce energy consumption and environmental impacts of road transport, Applied Thermal Engineering, Vol. 29, 2009, pp 1467-1477.

15. Barnitt R.A, et al, Emissions of transport refrigeration units with CARB Diesel, Gas-to-liquid Diesel, and Emissions Control Devices, SAE Powertrain, Fuels and Lubricants Meeting, San Antonio, Texas, 2009.

16. Cavalier G, Stumpf A, Measurement and simulation of energy consumption of transport refrigerated equipment, 1st Cold Chain Conference, Cambridge, 2010.

17. Fitzgerald W.B et al, Energy use of Integral refrigerated containers in maritime transportation, Energy Policy, Vol. 39, 2011, pp 1885-1896.

18. Wu X, Hu S, Mo S, Carbon footprint model for evaluating the global warming impact of food transport refrigeration systems, Journal of Cleaner Production, Vol. 54, 2013, pp 115-124.

19. <http://www.ecoinvent.org/>, (Accessed 01.03.2017).

20. Levova T, Freight transport with intermodal shipping containers and transport of goods in need of atmosphere control, Zürich, Ecoinvent Center, 2015.

21. Klöckner A.C, Blöbaum A, A comprehensive action determination model: Toward a broader understanding of ecological behavior using the example of travel mode choice, Journal of Environmental Psychology, Vol. 30, 2010, pp 574-586. 
Open Access This chapter is licensed under the terms of the Creative Commons Attribution 4.0 International License (http://creativecommons.org/licenses/by/4.0/), which permits use, sharing, adaptation, distribution and reproduction in any medium or format, as long as you give appropriate credit to the original author(s) and the source, provide a link to the Creative Commons license and indicate if changes were made.

The images or other third party material in this chapter are included in the chapter's Creative Commons license, unless indicated otherwise in a credit line to the material. If material is not included in the chapter's Creative Commons license and your intended use is not permitted by statutory regulation or exceeds the permitted use, you will need to obtain permission directly from the copyright holder.

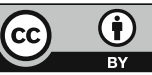




\title{
Life Cycle Management of Energy and Energy Transitions-Managing the Complexity of Todays and Future Energy Systems with a Life Cycle Focus: Challenges and Methodological Solutions
}

\author{
Karin Treyer, Roberto Turconi and Alicia Boyano
}

\begin{abstract}
To meet climate and sustainability goals a transition of the system of energy supply and use is needed. However, energy transitions are complex long-term processes and require a variety of methodologies to steer their direction. For this purpose, the combination of environmental, social, economic and technical assessments together with prospective energy scenario modelling is very promising but there are several challenges that need to be addressed to fully benefit from these methodologies. This paper presents the discussions held during a conference session on this issue. The solutions proposed facilitate the combination of energy system modelling frameworks and environmental and social assessments aimed at developing comprehensive prospective studies and feeding information to decision making processes for energy transition toward a low-carbon economy.
\end{abstract}

\section{Introduction}

In order to reach the pledges made under the Paris Agreement on climate change it is clear that an ambitious energy transition towards low-carbon solutions involving every part of the economy is needed [1]. Energy transitions, defined as structural changes in the way energy services are delivered and used, are inherently complex, uncertain and difficult to evaluate. In this context, it is increasingly acknowledged

\author{
K. Treyer $(\bowtie)$ \\ Paul Scherrer Institute, 5232 Villigen, Switzerland \\ e-mail: karin.treyer@psi.ch \\ R. Turconi \\ ArcelorMittal Global R\&D Sustainability, 57283 Maizières-lès-Metz, France
}

A. Boyano

Joint Research Center, Directorate B5 Circular Economy and Industrial

Leadership, 41003 Seville, Spain

(C) The Author(s) 2018

E. Benetto et al. (eds.), Designing Sustainable Technologies,

Products and Policies, https://doi.org/10.1007/978-3-319-66981-6_27 
that mixes of methodologies and modelling frameworks are required to address the various market and system challenges associated to such transition [2].

The available literature on the importance of assessing current and future energy systems and the associated transitions ranges from Life Cycle Assessments (LCA) to energy modelling with economic optimization or policy analyses and recommendations. One focal area has been environmental studies, among which LCA is one of the most established methods. LCA makes it possible to quantify potential impacts to the environment and human health of a product or/and system over the whole life cycle, to identify and discuss areas of improvement, and to conduct fair comparison of selected products or services. Another focal area concentrates on the economic perspective of energy systems, where energy systems optimization models are used to generate future scenarios and evaluate key parameters such as electricity production, related emissions and/or the system costs in the long-term future.

In recent years authors identified the need of combining the best aspects of several modelling frameworks. In particular, the combination of LCA and energy systems optimization models was studied in depth [3-5]. The motivation behind this combination is clear: LCA is a detailed and comprehensive, but static and environment-focused approach whereas modelling of future scenarios of the energy system includes multiple factors such as economic situation without considering environmental issues in detail. As most of the authors point out this combination of methodologies looks very promising, but challenges to its applications remain. Several (case) studies that address different challenges and propose possible solutions are presented and discussed in Sect. 2. The paper closes by providing concluding comments in Sect. 3.

\section{Challenges Identified and Possible Solutions}

A selection of challenges which need to be worked out to realize the full potential of integrating LCA and energy systems optimization models is presented in this section.

\subsection{Prospective Background Data}

Energy conversion technologies are likely to dramatically change in the coming decades not only as a consequence of the energy transition but also as a natural change due to economic, social and technological reasons. Nearly all prospective LCA studies include changes to the energy system when modelling foreground processes. However, in the vast majority of the prospective LCAs the background processes are not modified. The work presented by Cox et al. [6] attempted to use the outputs of the IMAGE model to create future versions of the ecoinvent 
database. According to the authors the use of IMAGE results as a source of future projections greatly simplifies the data collection stage and allows the systematic and consistent modification of ecoinvent background processes and market mixes. The authors pointed out the importance of a standard format of the results to allow to a large degree the automatization of future background databases to reflect different time horizons or scenarios.

\subsection{Lack of Data and Difficulties in the Integration of the Results}

The integration of energy system models and life cycle methods is an approach widely used to facilitate the modelling of prospective scenarios including environmental and economic aspects as well as political constraints, enabling sound decision making and reducing the risk of burden-shifting. Two of the most overwhelming challenges are the lack of data (e.g. power plant operation and emission factors) and the coupling of the results of the energy system models with life cycle inventories. Astudillo et al. [7] identified data gaps related to the energy supply and demand by analysing time-series and literature on emission factors and developed a screening algorithm enabling database integration.

\subsection{Capturing the Interplay of Changes in the Heat Sector and the Electricity Sector in LCM Models}

Bertrand et al. [8] developed a mixed integer linear programming model for the regional valorisation of industrial waste heat from manufacturing, energy production and waste incineration industries. Using waste heat would save resources and reduce emissions, as highlighted by the European Union in 2012 with the Energy Efficiency Directive [9]. By applying their modelling to a case study involving steel plants as heat sources and industries/towns as sinks, they demonstrated the economic profitability of using waste heat for all stakeholders involved.

\subsection{Management and Integration of Stochastic Renewables in LCM Models}

A growing share of renewable intermittent electricity such as wind and solar power leads to a fluctuating feed-in of electricity which might not correspond to electricity demand in a temporal dimension, leading to regional electricity surpluses. Seier et al. [10] proposed "temporal electricity purchase shifting" (TEPS) as one possible 
solution to absorb these electricity surpluses. The authors proposed a wastewater treatment plant (WWTP) as one actor for applying TEPS and demonstrated that TEPS led to decreased electricity purchase from fossil fuel fired power plants and reduced $\mathrm{CO}_{2}$ emissions for the WWTP operator.

\subsection{Integration of Prospective, Economic, Social and Other Issues in LCM Models}

Løkke et al. [11] carried out a socio-technical investigation of the decision process in a policy discussion, through critical review of the tools used and the scenarios developed. This study identified major barriers for the successful application of LCA to major infrastructure projects, provided learnings on designing scenarios and conducting LCA of large infrastructures. The authors demonstrated that a critical design of the LCA can help avoid biased decision-making and proposed LCA-based guidance for projects characterized by a highly political context.

\section{Concluding Comments}

This work has pointed out the importance of the integration of methodologies and modelling for assessing future energy systems. The necessity of using energy system models in combination with different environmental or social assessment tools was highlighted, particularly in prospective assessments. The difficulties in the integration of the results, the lack of data of the emerging energy technologies, the prospective background databases, the relationships between different energy sectors and the stochastic energy production from renewable energies have been highlighted as major challenges. The research work being carried out by the presenters in the session contributed toward finding possible solutions to these challenges and will be crucial to understand and facilitate a smooth energy transition towards a low carbon economy.

\section{References}

1. Baron R, Energy Transition after the Paris Agreement: Policy and Corporate Challenges, OECD, 2016.

2. OECD, Aligning Policies for a Low-carbon Economy, OECD, 2015.

3. Garcia-Gusano D, Garrain D, Dufour J, Prospective life cycle assessment of the Spanish electricity production, Renewable and sustainable Energy Reviews. 75 (2017) 21-34.

4. Röder A, Integration of life-cycle assessment and energy planning models for the evaluation of car powertrains and fuels (Thesis Diss ETH no 14291], Zurich (Switzerland), Swiss Federal Institute of Technology, 2001. 
5. Lund H, Mathiesen B.V, Christensen P, Schmidt J.H, Energy system analysis of marginal electricity supply in consequential LCA,. Int. J. Life Cycle Assess 2010, 15, 260-71.

6. Cox B, Mutel C, Mendoza Beltran A, van Vuuren D. Using integrated assessment model results to develop background databases for prospective LCA, LCM 2017.

7. Astudillo M, Vaillancourt K, Pedinotti-Castelle M, Pineau P.O, Ben Amor M, Using energy system models integrated with life cycle thinking to inform energy transitions in cold regions, LCM 2017.

8. Bertrand A, Mian A, Aggoune R, Maréchal F, Regional waste heat valorisation: a mixed integer linear programming method for energy service companies, LCM 2017.

9. European Parliament and Council, Energy Efficiency Directive 2012/27/EU.

10. Seier $\mathrm{M}$, Schebek $\mathrm{L}, \mathrm{CO}_{2}$ effects of temporal electricity purchase shifting on German wastewater treatment plants, LCM 2017.

11. Løkke S, Pizzol M, Distance to change: LCA as conserving/innovative tool-the case of the green prestigious project "Amager Bakke". LCM 2017.

Open Access This chapter is licensed under the terms of the Creative Commons Attribution 4.0 International License (http://creativecommons.org/licenses/by/4.0/), which permits use, sharing, adaptation, distribution and reproduction in any medium or format, as long as you give appropriate credit to the original author(s) and the source, provide a link to the Creative Commons license and indicate if changes were made.

The images or other third party material in this chapter are included in the chapter's Creative Commons license, unless indicated otherwise in a credit line to the material. If material is not included in the chapter's Creative Commons license and your intended use is not permitted by statutory regulation or exceeds the permitted use, you will need to obtain permission directly from the copyright holder.

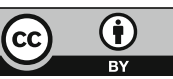




\title{
Integrating Energy System Models in Life Cycle Management
}

\author{
Miguel F. Astudillo, Kathleen Vaillancourt, Pierre-Olivier Pineau \\ and Ben Amor
}

\begin{abstract}
The energy supply chain is the backbone of industrialised societies, but it is also one of the leading causes of global environmental burden. Life cycle management (LCM) and life cycle assessment (LCA) are increasingly being used in combination with energy system optimisation models (ESOM) to better represent the energy sector and its dynamics, and facilitate better decision-making. The integration of ESOM and LCA can enable powerful analyses, but not without difficulties. In this chapter, we review studies linking a well-known bottom-up ESOM (TIMES) with LCA databases and identify the principal challenges and how they have been addressed. One of the main integration challenges is the identification of equivalent processes between life cycle inventories and ESOM databases: the mapping problem. Other concomitant issues such as double counting and parameter consistency have been identified and are also investigated.
\end{abstract}

\section{Introduction}

The economic growth observed in the last century has been heavily correlated with a sharp rise in energy use, improving living conditions for many, but also resulting in large environmental damage [1]. For instance, the energy sector is responsible for nearly two-thirds of global greenhouse gas emissions [2]. Therefore, there is an urgent need to transform the energy supply chain, decarbonising electricity supply

\footnotetext{
M. F. Astudillo $(\bowtie) \cdot$ B. Amor $(\square)$

Department of Civil Engineering, Université de Sherbrooke,

LIRIDE, Sherbrooke, Canada

e-mail: miguel@astudillo.com

B. Amor

e-mail: ben.amor@usherbrooke.ca

K. Vaillancourt

Esmia Consultants, Montreal, Canada

P.-O. Pineau

HEC Montreal, Chair in Energy Sector Management, Montreal, Canada

(C) The Author(s) 2018

E. Benetto et al. (eds.), Designing Sustainable Technologies,

Products and Policies, https://doi.org/10.1007/978-3-319-66981-6_28
} 
and electrifying services traditionally reliant on fossil fuels [2]. Many stakeholders have a role in this transition, from businesses to governments and consumers. Stakeholders need tools to understand how the energy system is likely to evolve and how it may react to various decisions made within the system.

In this journey, Life Cycle Management (LCM) can help to prevent burden shifting, but using it alone ignores the various energy dynamics. The use of energy system optimisation models (ESOM) together with life cycle thinking has the potential to underpin comprehensive understanding of the energy supply chain and its influence on sustainable production. There is a small but increasing number of studies combining life cycle assessment (LCA) and ESOM [3-12], ESOM best practices start to include LCM practices, such as goal and scope definition or the use of data quality indicators as a way to quantify epistemic uncertainty (i.e. the uncertainty associated with data quality) [13]. However, combining both models is not a simple task. In this chapter, we cover the challenges identified in the integration of ESOM and LCA, as well as existing approaches to address them and their limitations. For brevity, we focus on inventory level and do not cover impact assessment issues. Details of the criteria used to select articles are detailed in Sect. 1.3.

\subsection{Energy System Models: Origin and Strengths}

Energy infrastructure requires substantial investments and governments have used mathematical models for a long time to support policy analysis. The use of mathematical models can help understand the complex interactions that occur in the energy system, formalising the scattered knowledge about its dynamics [14]. Models of the energy sector grew in importance in the aftermath of the oil crisis of the 1970s. During that period the International Energy Agency (IEA) was founded and started developing its ESOM. Broadly speaking, energy models follow two different paradigms, they either provide scenarios of how the system could evolve from a normative standpoint (optimisation models), or they attempt to forecast how the system is likely to change (simulation models) [14]. Optimisation models are better suited to analyse long-term scenarios [15] and are therefore consistent with the common long-term temporal scope of LCA studies. This chapter focuses on bottom-up technology rich models, in particular the MARKAL/TIMES (The Integrated Markal Efom System) optimisation model generator developed by the IEA. TIMES is possibly the most widely used general purpose ESM [7, 14]. Alternative models following a similar structure such as TEMOA [16] or OSeMOSYS [17] are also considered.

TIMES models are based on cost minimisation and provide consistent possible evolutions of the energy system under a set of user-defined constraints. These models provide insights to businesses and policymakers, as they allow exploring potential interactions which are difficult to foresee without a formal mathematical framework. TIMES models are "bottom-up" models, with often thousands of 
technologies competing to provide a given level of demand for energy services. The solution of the optimisation problem provides potentially useful results, such as capacity additions, activity levels or material and emissions flows for each technology. TIMES models use simplifying assumptions, such as perfect competition. Perfectly competitive markets maximise the total surplus, which is an indicator of social welfare [15]. Thus, although real situations deviate from perfectly competitive markets, the solutions can be used as a benchmark. Deviations from perfect competition conditions can be studied through specific constraints such as pre-defined market-shares or myopic foresight [13, 15, 18]. In contrast with general-equilibrium models, TIMES models assume partial equilibrium, which means that sectors outside the system's boundary are assumed not to be affected by changes in the system. For instance, prices of imports and exports outside the boundaries of the system are exogenously defined and not determined by the model.

\subsection{Advantages of Integrating ESOM and LCA}

The advantages can be seen as an improvement in data quality aspects [19, 20]. TIMES models tend to have a relatively limited scope for assessing environmental burden, often only tracking primary pollutants from operating energy-related infrastructure [7, 18]. Most of them ignore up-stream emissions associated with imports [8] or consumption of resources linked to energy use, such as freshwater (of importance for the growing literature on the so-called water-energy nexus [21, 22]). Moreover, they lack the detailed impact assessment methodologies used in LCM. The absence of a comprehensive environmental assessment can result in burden shifting and fail to identify potential co-benefits of environmental policies. Life cycle thinking has been instrumental in addressing burden-shifting in the energy sector [23]. For example, it was through LCA studies that the environmental impact of biofuels was better understood [23].

TIMES models are also extremely useful for advanced life cycle studies, both for consequential and attributional approaches. TIMES explicitly model the future changes in the energy system, one of the major limitations of LCA [22]. The integration improves the temporal and technological representativeness, completeness and precision of inventories. TIMES also integrates economic considerations, which are fundamental in decision-making [19].

\subsection{Literature Review}

The integration challenges identified in this book chapter stem from our efforts linking the North American TIMES Energy Model (NATEM) with life cycle inventories (LCIs) [24, 25] and reading of associated literature. The existing approaches to address these challenges are based on a literature review. To identify 
potential articles, we used the search terms "LCA", "life cycle assessment", in combination with one of the following keywords: MARKAL, "TIMES model", OSeMOSYS, TEMOA, ESOM in the search engine Web of Science, covering publications of the last ten years. The keyword "TIMES" is too generic to be used as a meaningful filter. However, most of the publications mention the keyword MARKAL, the TIMES predecessor. To complete the review, we revised the annual summaries of the IEA Energy Technology Systems Analysis Program (ETSAP), covering publications from 2005 until 2013 [26]. ETSAP's reviews include over 250 references to articles using TIMES models in peer-reviewed journals. Studies deriving life cycle emissions from environmentally extended input-output (EEIO) data are included, despite having a different level of technological detail [8-10]. Only works published in peer-reviewed journals were considered. Studies that used LCA data for comparative purposes but did not attempt to integrate inventories (e.g. [27, 28]) or that had unclear methodology with unreachable authors [29] are not included in the review. Overall, ten articles are included in our analysis (Table 1). We note that prospective LCA of energy systems has also been done with other model paradigms such as general equilibrium models or power models [19]. However, linking such models entails different problems and is out of the scope of this chapter.

\section{LCA-TIMES Integration Challenges}

In TIMES models the energy supply chain is defined as a set of energy resources, conversion technologies and end-use demands connected by energy commodities in what is called the reference energy system (RES). The RES provides a complete

Table 1 Scope and number of technologies mapped in studies integrating TIMES models and LCA

\begin{tabular}{l|l|l|l|l}
\hline Sectors & $\begin{array}{l}\text { No of tech. } \\
\text { mapped }\end{array}$ & Scope & Database & Ref. \\
\hline Norwegian electricity sector & 9 & Attributional & ecoinvent 3 & {$[3]$} \\
\hline US electricity sector & 9 & Attributional & $\begin{array}{l}\text { US LCI and } \\
\text { ecoinvent } 2.2\end{array}$ & {$[4]$} \\
\hline $\begin{array}{l}\text { Multisector (end-uses) and } \\
\text { electricity sector (Switzerland) }\end{array}$ & 43 & Attributional & ecoinvent 2.2 & {$[5]$} \\
\hline Electricity and oil mining sector & 7 & Attributional & ecoinvent 1.1 & {$[7]$} \\
\hline Multisector & 192 & Consequential & ecoinvent 2.2 & {$[6]$} \\
\hline Spanish electricity sector & 26 & Consequential & ecoinvent 3 & {$[12]$} \\
\hline UK energy supply & 250 & Not stated & UK EE-MRIO & $\begin{array}{l}{[8,} \\
9]\end{array}$ \\
\hline EU electricity sector & 146 & Not stated & $\begin{array}{l}\text { Exiobase and } \\
\text { ecoinvent }\end{array}$ & {$[10]$} \\
\hline French biofuel sector & 35 & Not stated & mixed & {$[11]$} \\
\hline
\end{tabular}


description of the system boundaries and the level of technological detail of a model [30]. The RES is the equivalent to the product system in LCA [20]. The level of technological detail can be similar to process-based LCI databases. Thus, similar descriptions of the energy supply chain - from resource extraction to final use - can be found in both models. Linking ESM and LCI may seem straightforward, as both models share a similar structure, but these models are conceived to be used independently, and overlapping features can easily result in problems such as double counting [5, 6] or incomplete inventories. Faced with redundant information, the modellers need to choose which information prevails. The methodological choices may involve a trade-off between data quality aspects [19] which should be considered in the goal and scope definition.

Linking implies associating elements of the two models. We will refer to the problem of associating elements of two different models as the "mapping problem". Several issues complicate the mapping problem. First, TIMES models often include thousands of technologies, making a one-to-one linking between LCI and TIMES processes almost infeasible. There are no general name conventions or standard codes that can be used to automate the linking, which still heavily relies on manual identification. Second, both models can track the same emissions (e.g. greenhouse gases (GHG)), and some linkages between processes are not explicitly modelled in TIMES (e.g. cement production and infrastructure development). Adding LCI in the model can easily result in double counting [5]. Third, consistently introducing life-cycle emissions in the optimisation problem often requires a one-to-one mapping of processes in TIMES and LCIs. For example, emissions from end-of-life treatment could be included in TIMES, but these are potentially different for each process. In most of the cases, a one-to-one mapping would be excessively time-consuming. Fourth, key parameters of processes such as efficiency or emission factors may differ between models, which can result in inconsistencies. Finally, if multifunctional processes are within the system boundary, the allocation should be avoided using system expansion [20]. However, this is hardly discussed in the literature.

\section{Existing Approaches to Address the Integration Challenges}

\subsection{Mapping TIMES-LCA Processes}

The "too many processes" issue preventing a one-to-one mapping is one of the most complicated integration problems. This issue has been addressed using two simplifications: limiting the scope of the assessment to specific parts of the energy supply chain and representing sections of the supply chain by their aggregated LCI or LCA indicator. 
The integration efforts reviewed used multisector TIMES models, but most of the studies limit their scope to attributional studies of the electricity sector (Table 1) and do a partial integration. Several studies use market mixes from TIMES for prospective assessments, improving temporal representativeness [3, 4, 12], while others integrate life cycle emissions in TIMES models [7-11]. Limiting the boundaries to a particular sector reduces the number of technologies that need to be mapped, but it could result in a loss of completeness, questioning the suitability of the system boundary. For example, Ref. [4] used multisector MARKAL model to update a prospective electricity mix in the US for different scenarios such as cap and trade or $\mathrm{CO}_{2}$ taxes. However, such policies affect more than just the electricity sector, and induced changes in other sectors of the model should be considered to make a fair comparison of different scenarios. Refs. [7-11] added life cycle emissions to several processes of a MARKAL/TIMES model to internalise environmental emissions. It was a first step towards the integration of externalities, but incomplete mapping may be problematic as it may induce a bias against the mapped technologies (see Sect. 3.3). Reference [5] improved completeness, assigning life-cycle impact scores to all end-uses of all sectors. In this case, there is a wider range of processes that deliver the energy services (heat, transportation, etc.), raising the number of equivalent processes required (Table 1). The study used end-use technology mixes and energy demands from TIMES. However, the technology mixes and efficiencies for "non-end-use" processes (such as electricity generation) were selectively updated. The authors recommended using a more consistent approach in future research [5]. Indeed, upstream processes may also change over time or between scenarios (e.g. switch from conventional to unconventional gas or feedstock from biofuels). These changes should be identified in a systematic manner.

The approach of limiting the boundaries to a particular sector is more difficult to justify in consequential studies since all the processes that are expected to change should be included [19, 24]. Changes can be induced by market or policy effects. Our experiments using a TIMES model of a relatively small region (Quebec, Canada) indicate that a large proportion of the processes change their output to some extent [24]. The best example found of a consequential study using TIMES and LCA was an analysis of the effects of introducing biodiesel from biomass in France [6]. In this case, all technologies in the TIMES model were mapped (192). This approach produced a complete mapping, but it may be unfeasible with larger TIMES models, which can easily contain thousands of technologies.

We have recently proposed to use a cut-off criterion, that is, to exclude a percentage of the material and energy flows based on their contribution to an indicator measured in TIMES (e.g. $\mathrm{CO}_{2}$ eq emissions) [24]. The cut-off can help to discern the most relevant changes, reducing exponentially the number of processes that need to be mapped [24]. Nonetheless, it introduces some other problems, such as the possibility of omitting processes with high impacts in other areas of concern but low $\mathrm{CO}_{2}$ eq emissions. 


\subsection{Double Counting}

Double counting problem is hardly discussed in the literature of TIMES-LCA linking [5]. Part of the energy and emissions considered in TIMES models is used to deliver intermediate products that are already accounted in LCI, resulting in double counting $[5,6]$. For example, the output of the cement sector is typically considered independent of other demands in TIMES models but will be used to build the infrastructure that is already included in LCIs. As pointed by Ref. [6], this can be mitigated by presenting relative results between scenarios, where double counting effects cancel out, to some extent. This limitation is not exclusive of TIMES models. A recent review of integrated assessment models stated that most of them miss linkages related to infrastructure and ignore material cycles, which are the fundamental characteristics of the life cycle perspective [22]. Constructing more "circular" RES in TIMES models is challenging, as the models need to be calibrated using national statistics, and these do not necessarily have a high level of detail of material flows leaving each sector. Studies linking TIMES with EEIO data have more thoughtfully addressed double counting, erasing manually repeated areas of the inventory [8-10]. Reference [10] used fixed input-output coefficients to adjust the exogenously defined demands (e.g. the steel demand from energy technologies is used to adjust the energy demand of steel production). Ideally, these demand corrections would be calculated endogenously by the TIMES model, but that would require substantial additional linking of processes.

\subsection{Integrating Life Cycle Emissions in the Optimisation Problem}

Several of the reviewed publications integrated LCA data into the optimisation algorithm, but only for some parts of the energy system [3,7-11], with the risk of introducing a bias. A consistent integration of life-cycle emissions into the optimisation problem is challenging, as it will bias the assessment towards sectors where accounting of emissions is less complete. Reference [3] noted that imposing limits on LCA scores for electricity generation greatly affected trade balances against local production, as trade processes did not have emissions associated. Consistently including emissions for all processes in a TIMES model (e.g. end-of-life burdens) would require a one-to-one mapping, which is extremely time-consuming in large TIMES models. Alternatively, large TIMES models can include emissions if it is for a limited number of processes. For example, a model dealing with operating GHG emissions could include emissions from gas distribution or refrigerant leaks or electricity imports (e.g. [25]) without losing consistency. Ultimately, TIMES results should be interpreted taking into account potential inconsistencies and lack of completeness of the inventories. References $[8,10]$ are 
good examples, as they provide guidance on how to interpret results and what kind of conclusions could not be drawn.

\subsection{Technological Representativeness}

TIMES inventories represent future supply chains, while most of the LCI data are from current supply chains. Parameters such as efficiency, capacity factors or emission factors of the same technology may therefore differ. Unless a harmonisation step is introduced, the integration could lead to inconsistencies. The level of consistency in the different integration is difficult to evaluate because, with some exceptions (e.g. [5, 8]) it is not well documented and rarely discussed. Studies using EEIO data acknowledge that sectoral aggregation is an additional problem, as sectors with different emission intensities can be grouped together, eroding data representativeness [8]. References [3,30] suggested learning from the experiences linking bottom-up and general equilibrium models. Some of the concepts developed during other integration efforts can be applied to the integration of TIMES models and LCA databases. For example, the introduction of common measurement points (i.e. points where both models have the same result) would be useful to formalise the linking approach.

\subsection{Dealing with Multifunctional Processes}

Multifunctional processes should be handled in a way that is consistent with the scope of the analysis and if possible, avoiding the allocation using system expansion [20]. However, except Ref. [6], studies do not specify how allocation has been conducted when it could not be avoided (e.g. one of the outputs being outside the system boundary).

\section{Discussion and Conclusions}

The TIMES-LCA integration is clearly in expansion, with several recent publications on the topic. Integration can range from a very simple level (e.g. identification of future average or marginal technologies in a particular energy market), to more detailed ones, capturing market and policy interactions and transformations in the entire energy supply chain. More comprehensive approaches also imply a substantial additional effort, particularly solving the mapping of processes between the two bottom-up models.

To date, the existing literature has focused on attributional studies of electricity generation, although some multisector and consequential studies exist. Limiting the 
scope to part of the system may miss important changes outside the chosen system boundaries. The potential lack of completeness needs to be considered in the definition of the goal and scope. The truncation of the system boundary is especially unwarranted for consequential studies, where limiting the scope to specific sectors would result in an incomplete inventory.

TIMES and LCA often have overlapping representations of the supply chain, and modellers have to choose which data prevail, substituting parts of the TIMES representation by LCA counterparts. Direct substitution entails the risk of missing important changes across the supply chain. Therefore, the need for systematic approaches to prioritise which LCI data should be updated. The ordering of processes and application of a cut-off based on a criterion such as $\mathrm{CO}_{2}$ eq emissions can help to both identify relevant changes and reduce the number of processes to be mapped.

Consistent linking requires also updating parameters such as efficiency and emission factors. Integration efforts outside the LCA field suggested already in 1996 to use common measurement points, unambiguous measurement points where the two models should yield identical results [30]. The formalisation of these points implies a harmonisation of parameters, and the assessment of the extent by which both models measure the same phenomena and the same future [30]. The author also pointed out the need to share a common formalised language between models [30]. The specification of such conceptualisation is called ontology, a field of growing interest in industrial ecology and recently discussed in the LCM conference [31]. The need for more traceable and transparent workflows that go beyond the common reporting on scientific articles was also stressed [31]. We agree, as articles often don't offer sufficient explanation to understand the details of how the linking was done.

The process of linking models goes beyond solving the implementation problem [30]. It is also an opportunity to learn about the system and the implications of different perspectives, which are essential to interpret results. LCA modellers should consequently keep in mind the underlying assumptions and values of ESOM.

\section{References}

1. Smil V, World history and energy. Encycl Energy 2004;6:549-61.

2. IEA, Energy, climate change and environment, 2016 insights 2016. http://www.iea.org/ publications/freepublications/publication/ECCE2016.pdf (accessed September 23, 2017).

3. García-Gusano D, Iribarren D, Martín-Gamboa M, Dufour J, Espegren K, Lind A, Integration of life-cycle indicators into energy optimisation models: The case study of power generation in Norway. J Clean Prod 2016;112:2693-6.

4. Choi J-K, Friley P, Alfstad T, Implications of energy policy on a product system's dynamic life-cycle environmental impact: Survey and model. Renew Sustain Energy Rev 2012;16: $4744-52$. 
5. Volkart K, Weidmann N, Bauer C, Hirschberg S, Multi-criteria decision analysis of energy system transformation pathways: A case study for Switzerland. Energy Policy 2017;106: 155-68.

6. Menten F.M, Tchung-ming S, Lorne D, Bouvart F, Lessons from the use of a long-term energy model for consequential life cycle assessment: the BTL case. Renew Sustain Energy Rev 2015;43:942-60.

7. Pietrapertosa F, Cosmi C, Macchiato M, Salvia M, Cuomo V, Life Cycle Assessment, ExternE and Comprehensive Analysis for an integrated evaluation of the environmental impact of anthropogenic activities. Renew Sustain Energy Rev 2009;13:1039-48.

8. Daly H.E, Scott K, Strachan N, Barrett J, Indirect $\mathrm{CO}_{2}$ Emission Implications of Energy System Pathways: Linking IO and TIMES Models for the UK. Environ Sci Technol 2015;49:10701-9.

9. Scott K, Daly H, Barrett J, Strachan N, National climate policy implications of mitigating embodied energy system emissions. Clim Change 2016;136:325-38.

10. McDowall W, Solano Rodriguez B, Usubiaga A, Acosta Fernández J, Is the optimal decarbonization pathway influenced by indirect emissions? Incorporating indirect life-cycle carbon dioxide emissions into a European TIMES model. J Clean Prod 2018;170:260-8.

11. Hugues P, Assoumou E, Maizi N, Assessing GHG mitigation and associated cost of French biofuel sector: Insights from a TIMES model. Energy 2016;113:288-300.

12. García-Gusano D, Garraín D, Dufour J, Prospective life cycle assessment of the Spanish electricity production. Renew Sustain Energy Rev 2016;75:21-34.

13. DeCarolis J, Daly H, Dodds P, Keppo I, Li F, McDowall W, et al, Formalizing best practice for energy system optimization modelling. Appl Energy 2017;194:184-98.

14. Pfenninger S, Hawkes A, Keirstead J, Energy systems modeling for twenty-first century energy challenges. Renew Sustain Energy Rev 2014;33:74-86.

15. Loulou R, Remme U, Kanudia A, Lehtila A, Goldstein G, Documentation for the TIMES Model Part I. IEA Energy Technol Syst Anal Program 2005:1-78. https://iea-etsap.org/docs/ Documentation_for_the_TIMES_Model-Part-I_July-2016.pdf (accessed September 23, 2017).

16. Hunter K, Sreepathi S, DeCarolis J.F, Modeling for insight using Tools for Energy Model Optimization and Analysis (Temoa). Energy Econ 2013;40:339-49.

17. Howells M, Rogner H, Strachan N, Heaps C, Huntington H, Kypreos S, et al, OSeMOSYS: The Open Source Energy Modeling System. Energy Policy 2011;39:5850-70.

18. Glynn J, Fortes P, Krook-Riekkola A, Labriet M, Vielle M, Kypreos S, et al, Informing energy and climate policies using energy systems models. Springer International Publishing; 2015.

19. Astudillo M.F, Treyer K, Bauer C, Pineau P.O, Amor M Ben, Amor B, Life cycle inventories of electricity supply through the lens of data quality: exploring challenges and opportunities. Int J Life Cycle Assess 2016;3:374-86.

20. ISO, ISO 14044 Environmental management-Life cycle assessment-Requirements and guidelines. vol. 3. 2006.

21. Kapsarc, Energy Systems Modeling to Support Policy Making 2014. https://www.kapsarc. org/wp-content/uploads/2015/10/KS-1401-WB01B-Energy-Modeling-Workshop-PolicyBrief.pdf (accessed September 19, 2017).

22. Pauliuk S, Arvesen A, Stadler K, Hertwich E.G, Industrial ecology in integrated assessment models. Nat Clim Chang 2017;7:13-20.

23. Hellweg S, Milà i Canals L, Emerging approaches, challenges and opportunities in life cycle assessment. Science 2014;344:1109-13.

24. Astudillo M.F, Vaillancourt K, Pineau P, Amor B, Simplifying the integration of energy system models and LCA. Setac Brussels 2017, Brussels: 2017, p. 3-4. https://osf.io/cxzra (accessed September 19, 2017).

25. Astudillo M.F, Vaillancourt K, Pineau P.O, Amor B, Can the household sector reduce global warming mitigation costs? sensitivity to key parameters in a TIMES techno-economic energy model. Appl Energy 2017;205:486-98. 
26. ETSAP, Other. Annex Final Reports 2013. https://iea-etsap.org/index.php/applications/other (accessed September 19, 2017).

27. Frenette E, Bahn O, Vaillancourt K, Meat, Dairy and Climate Change: Assessing the Long-Term Mitigation Potential of Alternative Agri-Food Consumption Patterns in Canada. Environ Model Assess 2016.

28. Levasseur A, Bahn O, Beloin-Saint-Pierre D, Marinova M, Vaillancourt K, Assessing butanol from integrated forest biorefinery: A combined techno-economic and life cycle approach. Appl Energy 2017:1-13.

29. Tokimatsu K, Endo E, Murata A, Okajima K, Nomura N, An Integrated Assessment by Models for Energy Systems Analysis and Life-Cycle Assessment with a Case Study of Advanced Fossil-Fired Power Plants in China. Environ Model Assess 2015:291-305.

30. Wene C.O, Energy-economy analysis: Linking the macroeconomic and systems engineering approaches. Energy 1996;21:809-24.

31. Vandepaer L, Gibon T, 2018. The integration of energy scenarios into LCA: LCM 2017 Conference Workshop, Luxembourg, September 5, 2017. Int J Life Cycle Assess 23:970-977.

Open Access This chapter is licensed under the terms of the Creative Commons Attribution 4.0 International License (http://creativecommons.org/licenses/by/4.0/), which permits use, sharing, adaptation, distribution and reproduction in any medium or format, as long as you give appropriate credit to the original author(s) and the source, provide a link to the Creative Commons license and indicate if changes were made.

The images or other third party material in this chapter are included in the chapter's Creative Commons license, unless indicated otherwise in a credit line to the material. If material is not included in the chapter's Creative Commons license and your intended use is not permitted by statutory regulation or exceeds the permitted use, you will need to obtain permission directly from the copyright holder.

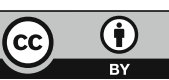




\title{
LCM at the Urban Scale: BIM and Nature Based Solutions
}

\author{
Antonino Marvuglia, Benedetto Rugani and Germain Adell
}

\begin{abstract}
Access to data on built environment databases makes nowadays possible generating models of the urban spaces to facilitate visualization and analysis of information and synthesize it in sustainability indicators to support urban planning decisions. Life Cycle Assessment (LCA) can greatly benefit from this wealth of potentially available information. The use of LCA data in models developed in Building Information Modelling (BIM) platforms is likely to facilitate the implementation of quantitative environmental assessment in the construction field and their extension, from the building to the city level. Within sustainable urban planning and management, also Nature-Based Solutions (NBS) play a potentially important role, although benefits, co-benefits and costs associated with NBS projects still remain not sufficiently understood. All those aspects have been discussed via the presentation of case studies, proofs of concept and experts' visions within this session.
\end{abstract}

\section{Introduction and Scope of the Session}

An assessment of energy and environmental performances of building stocks at large spatial scales is increasingly needed for decision support in sustainable urban planning and policy making. While current bottom-up building stock models have mainly focused on the operational energy use up to the last decade, a more holistic environmental assessment of the different stages of buildings life cycle is now required. However, extending a Life Cycle Assessment (LCA) model from the

\footnotetext{
A. Marvuglia $(\bowtie) \cdot$ B. Rugani

Environmental Research and Innovation (ERIN) Department, Luxembourg Institute of Science and Technology (LIST), 4422 Belvaux, Luxembourg

e-mail: antonino.marvuglia@list.lu

G. Adell

Nobatek/INEF4 Institute for the Energy Transition, 67 rue de Mirambeau, 64600 Anglet, France

(C) The Author(s) 2018

E. Benetto et al. (eds.), Designing Sustainable Technologies,

Products and Policies, https://doi.org/10.1007/978-3-319-66981-6_29
} 
building to the district or city scales entails a number of methodological and operational challenges.

LCA as inventory and environmental impact assessment tool can largely benefit from datasets and knowledge base frameworks based on Building Information Modelling (BIM) and Nature-Based Solutions (NBS). The use of BIM has the aim to help engineers to design digital models and share building information in an interoperable and reusable way [1]. However, firstly to apply BIM concepts designers create 3D models of buildings hinging upon built-in databases, which are not interoperable with the analysis tools used in LCAs of buildings. This hampers the quantification of the potential environmental impacts of buildings using those materials databases to support the decision-making process [2, 3]. Secondly, the modelling of a detailed BIM is time-consuming and especially in early design phases detailed information is usually not yet available. To this end, basic 3D models can be used to do simplified LCAs of buildings and neighbourhoods and compare variants in early design, when the optimization potential is high. Thirdly, a number of standards and data formats for 3D modelling and visualization of buildings and cities have been developed, e.g. CityGML [4, 5]; however, these models are often not used in planning and operation, partly because of problems with interoperability and partly due to the efforts to keep the models up to date [6].

Finally, BIM-based sustainability in the construction sector relates, at higher scales than single buildings, with the sustainability of urban planning and management, and therefore to the efficient use of resources and impact mitigation measures in cities. In this regard, it is worth observing that the improvement of cities' sustainability is nowadays supported through the explicit implementation of Nature-based Solutions (NBS) [7, 8] in urban planning. Those represent, among others, a means to activate impact mitigation measures in cities [7, 9]. How to harness NBS to promote well-being in urban areas does represent another challenging and timely research theme $[10,11]$, which is not explored in the LCA context. NBS strictly belong to the sustainable management of natural resources, urban spaces and technological know-how. Their introduction in urban contexts (at the spatial level of building, neighbourhood, or whole city) is recommended for several reasons, such as to reinforce the social cohesion and reduce poverty, and enhance the provision of urban ecosystem services and biodiversity. However, the impact of NBS projects, in terms of benefits, co-benefits and costs is still not sufficiently understood. Hence, the study of NBS related impacts, based on an LCA approach, could facilitate the interpretation of the sustainability dimensions underpinning NBS.

This LCM2017 conference session explored the challenges mentioned above via some case studies, proofs of concept and a discussion based on different experts' visions. The objective was to offer an overview on some methodological and applied advances that, from building to city scales, may open up concrete opportunities for: (i) a better exploitation of urban resources, data and know-how (i.e. integration between BIM and LCA), and (ii) a smooth transition towards sustainable and resilient cities (i.e. assessment/implementation planning of NBS with a life cycle thinking approach). 


\section{Summary of the Session's Presentations}

\subsection{Building Information Modelling Applications}

Impacts embodied in the building components (materials, etc.) have a more and more important role in buildings, considering the transition process from traditional buildings to energy-efficient buildings. In order to trace these embodied impacts efficiently, tools like BIM help in keeping track of inventory data for building construction elements, as well as technical installations, such as ventilation systems.

This was the subject of the first presentation, "Life Cycle Assessment of a Ventilation System in and Office Building in Trondheim", by Alexander Borg.

In this study, a BIM model was used to find inventory data on a ventilation system. A supplied dataset was used to create the inventory for the embodied impacts in the building components, and energy simulations allowed the calculation of the energy demand throughout the use phase of the building. A dynamic methodology was applied to better encompass the long lifetime of buildings. The results showed that energy use accounts for the majority of the impacts for most impact categories, but embodied impacts have a larger share of emissions than previously shown in literature, although total impacts coincide with literature data.

From the presented study emerges how BIM-LCA integration needs a collaborative process and data sharing. In order to benefit from BIM not only in the advanced stages of a project, but also in its early stages, there is a need for a simplified, design-integrated method based on early BIM models with limited information. The presentation of Alexander Hollberg ("Design-integrated LCA using early BIM") dealt with the potential of BIM technology to facilitate the application of LCA in the early design stage of a building. Early design stages are in fact very important for building's environmental impacts mitigation. Building's life-cycle impact can be significantly reduced by choosing materials with low embodied impacts at early design stages [12, 13]. However, the number and complexity of the decisions to be taken in this phase and typically the lack of knowledge on which decisions have the highest impacts on a building's environmental performance, often lead designers to postpone decisions to later stages of the design process. A high potential to facilitate this early stage decision process is seen in the use of BIM [14-16]. However, while BIM is more and more applied in detailed design stages, simple 3D models are typically used to compare design variants in early stages. A simplified, design-integrated method based on these early BIM models with limited information was thus introduced, which uses simple 3D geometry and a parametric LCA model. The method was applied to the conception design of a residential neighbourhood.

The integration between BIM and LCA should in any case hinge upon a mutual transfer of information: BIM data in the LCA framework and LCA results back into the BIM database. However, this full integration poses interoperability issues. The presentation by Adélaïde Mailhac ("A proposition to extend CityGML and ADE Energy standards for exchanging information for LCA simulation at urban 
scale") addressed the potential of BIM to reduce efforts during the data acquisition phase in order to facilitate the challenging task of extending LCA studies from single buildings to complex systems as districts, urban facilities and territories. To date, LCA data requirements have not been fully integrated into the CityGML format nor its Application Domain Extensions (ADE). The presenter proposed an extension of CityGML and Energy-ADE standards for exchanging information for LCA simulation at urban scale. The scope of the study was limited to the integration of information necessary for LCA of buildings' construction and renovation.

\subsection{Nature-Based Solutions}

When looking at the whole scale from building to city, the application of an LCA perspective can help disclosing the real benefits of NBS for the renaturing and sustainability of urban systems. The study entitled "Dynamic Assessment of Nature Based Solutions through Urban Level LCA”) and presented by Özge Yılmaz anticipated the basis to obtain a comprehensive environmental assessment of NBS to address urban challenges. The H2020 project Nature4Cities [11] was introduced and its overarching approach for modelling urban ecosystems, which in one work package integrates LCA and urban metabolism (UM), was described. Performing a dynamic assessment with time series data was suggested as a way to promote the identification of hotspots within a determined time frame, allowing the assessor to categorise and mitigate extremes and make informed decisions on desired temporal patterns. In the authors' view, data needs for the dynamic assessment is supported by two simulation methodologies: BIM for the built environment and Agent Based Modelling (ABM) for social behavioural patterns.

Finally, the last session's presentation complemented the previous ones by exploring whether or not different spatial distributions of possible NBS implementations in the city (as a function of the topological structure of the urban system itself) can make a difference in terms of quantification of the desired beneficial effect (such as carbon uptake). This was the topic of Marlène D.F. Boura ("How does the spatial distribution of green within cities impact carbon flows? A European scale analysis").

\section{Issues Discussed and Future Perspectives Identified}

The case study discussed by Alexander Borg has shown how the use of an external tool, like Excel, as an intermediate step for inventory modelling is very time consuming and not optimal to ensure a convenient use of a BIM-LCA model. There is potential for automation of this process, but it is important to first being able to ensure the reliability and transparency of the data used in an automatic data transfer 
process. The automation of information transfer from external data sources to BIM is one of the current challenges that BIM-LCA integration has to face.

Ongoing research shows that ventilation systems can contribute significantly to increase GHGs emissions, especially considering that these systems do not always work properly from the start, and faulty equipment must be replaced, thus generating additional emissions through materials and labour. The presentation showed that, given the role they play in buildings performances, embodied impacts of ventilation systems should be reduced, via increased recycled and recyclable materials content, and taking into account also alternative ventilation methods like hybrid natural ventilation.

The "early BIM" approach presented by Alexander Hollberg raised a lot of interest in the audience. A question was asked about the comparison between the impacts of a building where environmentally sound decisions are taken at the early stage and one where these decisions are taken only at later stages of the design. The comparison has not been done so far by the authors, but the same systems boundaries were used in the early stages as they would apply to later stages. As pointed out by one of the attendees, the approach presented is similar to the one taken by other tools used for LCA in building design, especially Tally ${ }^{\mathrm{TM}}$. However, as Alexander Hollberg highlighted, the main difference lays in the full integration into the design process. Their approach provides instant feedback while changing parameters, which is a unique feature in the current state of the art. Furthermore, they combine operational energy demand calculation and the embodied impact calculation in one method. This kind of approach appeared slightly different from a "conventional" BIM approach. The authors call it "early BIM", because they link information to the geometric 3D model. Conventional BIM, such as Revit models, contain more detailed information stored in the $3 \mathrm{D}$ model. This results in a more complex model which cannot be adapted as quickly as simple 3D models. Therefore, they use the simple models in early stages, which can then ideally be enriched with detailed information throughout the design process.

The topics discussed by Adélaïde Mailhac gave an interesting overview of the potential of BIM use in an LCA framework, explaining that BIM can be used in every phase of the LCA, not just in the early conception stage. However, data availability in the right format (e.g. CityGML compliant data) still remains a problem, since data is not provided easily by every actor, public or private.

Finally, the approach presented by Özge Y1lmaz intrigued the audience due to its modular structure, which integrates different approaches. It also raised concerns about the seamless incorporation of ABM, which is a dynamic simulation tool, with a rather static LCA approach. The presenter recognized that LCA provides a snap-shot of environmental impacts over a given period of time and a shift from static LCA to more dynamic LCA is certainly necessary for successful integration of ABM and LCA. A second observation concerned the suggestion to include not only the behavioural patterns, but also consumption patterns in the ABM simulations. This aspect will be taken into account in conjunction with an urban metabolism approach within the Nature4Cities project. 
Another question was asked about the actual sustainability assessment of NBS. In other words, NBS are nowadays often sought because they are capable of improving a certain element of an urban system, improve its performances with respect to a specific aspect (e.g. green facades to improve the thermal comfort in buildings and reduce heating and cooling loads) or supposedly increase people well-being. But is the lifecycle sustainability of NBS systematically assessed? Are we ready to provide holistic assessments of NBS, or are we rather running the risk of swooping on NBS without evaluating any possible side effect? The answer to this question can be found in the consistent application of holistic (lifecycle based) assessment of NBS, as proposed in Nature4Cities.

\section{References}

1. Vanlande R, Nicolle C, Cruz C, IFC and building lifecycle management, Automation in Construction, Vol. 18, 2008, pp. 70-8.

2. Schlueter A, Thesseling F, Building information model based energy/exergy performance assessment in early design stages. Automation in Construction, Vol. 18, 2009, pp. 153-63.

3. Jrade A, Jalaei F, Integrating building information modelling with sustainability to design building projects at the conceptual stage, Building Simulation; Vol. 6, 2013, pp. 429-44.

4. Stoter J, Beetz J, Ledoux H, Reuvers M, Klooster R, Janssen P, et al., Implementation of a National 3D Standard: Case of the Netherlands. In: Pouliot et al. (eds). Prog. New Trends 3D Geoinformation Sci., Berlin, Heidelberg: Springer Berlin Heidelberg; 2013, pp. 277-98.

5. van den Brink L, Stoter J, Zlatanova S, Establishing a national standard for 3D topographic data compliant to CityGML. International Journal of Geographical Information Science, Vol. 27, 2013, pp. 92-113.

6. Zlatanova S, Itard L, Kibria MS, van Dorst M. A user requirements study of digital 3D models for urban renewal. Open House International, Vol. 35, 2010, pp. 37-46.

7. Faivre N, Fritz M, Freitas T, de Boissezon B, Vandewoestijne S. Nature-Based Solutions in the EU: Innovating with nature to address social, economic and environmental challenges. Environmental Research, Vol. 159, 2017, pp. 509-518.

8. European Commission, EC Towards an EU Research and Innovation policy agenda for Nature-Based Solutions \& Re-Naturing Cities, 2015.

9. Eggermont H, et al., Nature-based Solutions: New Influence for Environmental Management and Research in Europe. GAIA-Ecological Perspectives for Science and Society, Vol. 24(4), 2015, pp. 243-248.

10. Maes J, Jacobs S, Nature-Based Solutions for Europe's Sustainable Development. Conservation Letters, Vol. 10(1), 2017, pp. 121-124.

11. <https://www.nature4cities.eu/>, (Accessed 24.09.2017).

12. Anand C K, Amor B, Recent developments, future challenges and new research directions in LCA of buildings: A critical review, Renewable and Sustainable Energy Reviews, Vol. 67, 2017, pp. 408-416.

13. Zabalza Bribián I, Capilla A V, Aranda Usón A, Life cycle assessment of building materials: Comparative analysis of energy and environmental impacts and evaluation of the eco-efficiency improvement potential, Building and Environment, Vol. 46, 2011, pp. 1133-1140.

14. Eleftheriadis S, Mumovic D, Greening P, Life cycle energy efficiency in building structures: A review of current developments and future outlooks based on BIM capabilities, Renewable and Sustainable Energy Reviews, Vol. 67, 2017, pp. 811-825. 
15. Shoubi M V, Shoubi M V, Bagchi A, Barough A S, Reducing the operational energy demand in buildings using building information modeling tools and sustainability approaches, Ain Shams Engineering Journal, Vol. 6, 2015, pp. 41-55.

16. Shadram F, Johansson T D, Lu W, Schade J, Olofsson T, An integrated BIM-based framework for minimizing embodied energy during building design, Energy and Buildings, Vol. 128, 2016, pp. 592-604.

Open Access This chapter is licensed under the terms of the Creative Commons Attribution 4.0 International License (http://creativecommons.org/licenses/by/4.0/), which permits use, sharing, adaptation, distribution and reproduction in any medium or format, as long as you give appropriate credit to the original author(s) and the source, provide a link to the Creative Commons license and indicate if changes were made.

The images or other third party material in this chapter are included in the chapter's Creative Commons license, unless indicated otherwise in a credit line to the material. If material is not included in the chapter's Creative Commons license and your intended use is not permitted by statutory regulation or exceeds the permitted use, you will need to obtain permission directly from the copyright holder.

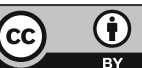




\title{
Design-Integrated LCA Using Early BIM
}

\author{
Alexander Hollberg, Julia Tschetwertak, Sven Schneider \\ and Guillaume Habert
}

\begin{abstract}
Life Cycle Assessment (LCA) is increasingly used for buildings, however, mostly for post-design evaluation of the environmental impact. To use the results for optimization, LCA has to be integrated in the early design stages. While Building Information Modelling (BIM) is more and more applied in detailed design stages, simple 3D models are typically used to compare design variants in early stages. The objective of this paper is to introduce a simplified, design-integrated method based on these early BIM models with limited information. The early BIM-LCA method uses simple 3D geometry and a parametric LCA model. Methodological simplifications are introduced and a single indicator based on the certification system of the German Sustainable Building Council (DGNB) is used to provide an intuitive real-time feedback for the designer. The method is applied to the conceptional design of a residential neighbourhood. The results highlight the great potential of using simplified LCA to quantify environmental performance for decision-making in early design stages.
\end{abstract}

\section{Introduction}

Life Cycle Assessment (LCA) is increasingly gaining importance for environmental evaluation of buildings, mostly in the form of building certification labels. The application of LCA at a late stage of the planning process for post-design evaluation of the environmental impact is not sufficient on its own if the results are not used to improve the design [1]. The highest optimization potential lies within the early design stages where the decisions made have the biggest influence on energy

\footnotetext{
A. Hollberg ( $\square) \cdot$ G. Habert

Chair of Sustainable Construction, IBI, ETH Zürich, Stefano-Franscini-Platz 5, 8093 Zurich, Switzerland

e-mail: hollberg@ibi.baug.ethz.ch
}

A. Hollberg · J. Tschetwertak · S. Schneider

Bauhaus University Weimar, Geschwister-Scholl-Straße 8, 99423 Weimar, Germany

(C) The Author(s) 2018

E. Benetto et al. (eds.), Designing Sustainable Technologies,

Products and Policies, https://doi.org/10.1007/978-3-319-66981-6_30 
demand [2] and environmental impact [3] while featuring the smallest costs for changes to the design [4]. Optimization in these early design stages can only be achieved by generating and comparing design variants. To allow for design-integrated assessment of the environmental performance a simplified and time-efficient method for LCA is needed. The additional effort for designers has to be kept to a minimum to avoid barriers for assessment. Therefore, tools for LCA should ideally be integrated in the usual design environment. Recently, various solutions to integrated LCA using BIM have been developed [5]. However, the conventional BIM is typically used in detailed design stages [6]. In early design stages, conceptional mass models are employed to quickly modify and generate variants at a low level of detail. Therefore, this paper discusses the use of simplified digital building models - referred to as early BIM-for LCA. The objective is to allow for time-efficient improvement of the environmental performance in early design at a point where many parameters needed for conventional BIM-LCA approaches are still unknown. The proposed approach is applied to a case study of the conceptional design of a neighbourhood.

\section{Early BIM-LCA Using Parametric Models}

The early BIM-LCA approach uses simplified digital building representations. These 3D models only consist of surfaces instead of volumetric components, see Fig. 1. This approach is also referred to as "shoebox" models [7] and is commonly employed in thermal models for energy demand calculation. The input of interior walls can be further simplified by using a global factor instead of modelling each individual wall, provided in Minergie guidelines [8], for example.

For a simplified input of materials, a building component catalogue of typically employed materials and heating, ventilation, and air conditioning (HVAC) systems

Fig. 1 Simplified building representation using surfaces only

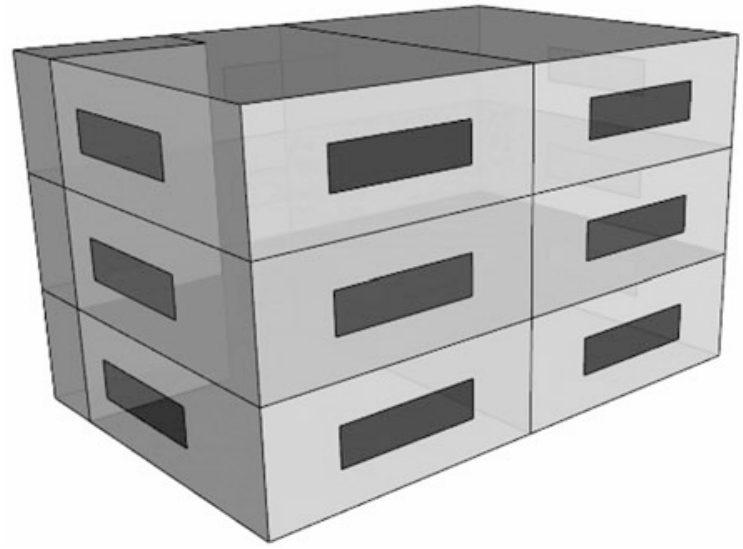


can be used. A variety of typical building components including LCA data can be found in many national catalogues, such as Bauteilkatalog [9] for Switzerland, Milieuprofiel van gebouwelementen [10] for Belgium, or Baubook [11] for Austria.

The calculation of the LCA is carried out using a parametric model [12]. The designer can typically influence three categories of parameters of a building: geometry, materials and HVAC systems [12]. Each category consists of a number of parameters, such as orientation, dimensions, window area, etc. for the geometry.

The parametric LCA approach combines the calculation of the operational and embodied environmental impact. The operational impact results from the use phase of the building (life cycle module B6 according to EN 15978 [13]) and is based on an energy demand calculation using monthly energy balancing [14]. The embodied impact results from the material production, replacements and the end-of-life (life cycle modules A1-A3, B4, C3, C4, and D). The implementation of the parametric LCA in a design tool such as Grasshopper [15] allows for a closed workflow of input, calculation, output without requiring any importing/exporting [16]. This is necessary for comparing variants automatically and is the basis for computational optimization approaches.

The parametric approach allows an advanced user to define and adjust their own weighting factors in order to consider the individual goals of the LCA study [16]. Furthermore, it allows for employing different predefined weighting factors, such as those from building certification systems. The DGNB system provided by the German Sustainable Building Council employs two criteria based on LCA and awards points for each [17]. These are weighted and combined into one indicator called life cycle performance (LCP) [18]. This single indicator ranges from 0 to 1 , with 1 being equal to $100 \%$ of the DGNB points related LCA criteria.

\section{Case Study}

In the following case study, the method is applied to the early design stages of a hypothetical residential neighbourhood providing a total gross floor area (GFA) of $2500 \mathrm{~m}^{2}$. Between two and four buildings are located on a rectangular site in Potsdam, Germany. The storey height of all apartments is $3 \mathrm{~m}$, and the buildings do not have basements. The floor area ratio (FAR) is set to 0.6 and minimum and maximum dimensions are set to ensure reasonable sizing of the building volumes. The buildings can have two to four floors and the glazing area is constantly $30 \%$ of the exterior wall area. It is assumed that the net floor area (NFA) equals $0.8 \times$ GFA. The functional unit is $1 \mathrm{~m}^{2}$ NFA for 1 year, and the reference study period is 50 years. Both climate and user data are taken from DIN V 18599-10:2011 [19].

It is assumed that the design process occurs in two stages, first definition of geometry, and second choice of materials. The HVAC systems are not modified parametrically but fixed. The heating system is set to a gas-condensing boiler with an efficiency of $98 \%$, no cooling is considered and the ventilation occurs naturally. 
For the first stage of defining the geometry, an algorithm positions buildings on the site. Due to the parametric positioning, it is likely that the buildings intersect initially. The algorithm resolves the intersections and maintains a pre-set minimum distance between the buildings [20]. A solar analysis is integrated to account for shading of the different buildings. Additionally, cores including the main circulation spaces within the buildings are inserted. In order to enable natural lighting and ventilation, they are only positioned at exterior walls, preferably in shaded areas to keep the solar gains to the usable floor spaces. If a building exceeds a defined building size, further circulation cores are added. The interior walls are not included in the 3D model, but instead inserted numerically based on a overall average factor of $0.4 \mathrm{~m} / \mathrm{m}^{2}$ GFA following the Swiss Minergie regulation [8]. All of those model features are parametrically controlled and each combination of the parameter values generates different design variants. The algorithm can quickly generate thousands of possible variants, which can be used for optimization [21]. The focus of the case study is the application of the early BIM-LCA method. Therefore, nine variants showing different geometric characteristics, such as number and position of buildings are chosen for stage 2 .

In the second stage, the resulting building volumes are combined with six different construction types. For each construction type four building componentsexterior walls, interior walls, ceilings, roofs - are manually generated, see Table 1. The individual materials of the constructions can be found in Table 2. The windows and the slab are the same for each construction type. The slab is made of reinforced concrete and polyurethane insulation; the windows consist of a PVC frame with double-glazing. The U-values for the building components of different construction types are the same and follow the minimum U-value of the 2014 German Energy Efficiency Regulation (EnEV) [22]. As such, the operational energy demand is only influenced by the geometry and not the thermal quality of the building envelope. Furthermore, all components possess a fire resistance of at least $60 \mathrm{~min}$. LCA data for the materials are taken from the German oekobau.dat database [23]. For each geometric variant, the calculation algorithm automatically runs a loop over the six different possible material combinations [18]. Finally, the LCP values for each variant are calculated.

Table 1 Different construction types

\begin{tabular}{l|l|l|l|l}
\hline Construction type & Exterior wall & Roof & Ceiling & Interior wall \\
\hline ETICS & ETICS & Concrete & Concrete & $\begin{array}{l}\text { Lime-sand } \\
\text { stone }\end{array}$ \\
\hline Brick & Insulated brick & Concrete & Concrete & Brick \\
\hline Concrete & Concrete & Concrete & Concrete & Concrete \\
\hline Wood & Wood frame & $\begin{array}{l}\text { Wood } \\
\text { beams }\end{array}$ & $\begin{array}{l}\text { Wood } \\
\text { beams }\end{array}$ & Wood frame \\
\hline Ventilated facade & Ventilated facade & Concrete & Concrete & $\begin{array}{l}\text { Lime-sand } \\
\text { stone }\end{array}$ \\
\hline $\begin{array}{l}\text { Double shell } \\
\text { masonry }\end{array}$ & $\begin{array}{l}\text { Double shell } \\
\text { masonry }\end{array}$ & $\begin{array}{l}\text { Wood } \\
\text { beams }\end{array}$ & Concrete & Wood frame \\
\hline
\end{tabular}


Table 2 Materials of the different building elements

\begin{tabular}{|c|c|c|c|}
\hline & Construction type & Material & $\begin{array}{l}\text { Thickness } \\
(\mathrm{cm})\end{array}$ \\
\hline \multirow{34}{*}{$\begin{array}{l}\text { Exterior } \\
\text { walls }\end{array}$} & \multirow{7}{*}{$\begin{array}{l}\text { External thermal insulation composite } \\
\text { systems (ETICS) }\end{array}$} & Synthetic plaster & 0.20 \\
\hline & & $\begin{array}{l}\text { Fibre glass reinforcement } \\
\text { grid }\end{array}$ & 0.05 \\
\hline & & Synthetic resin & 0.04 \\
\hline & & EPS & 13.00 \\
\hline & & Synthetic resin & 0.04 \\
\hline & & Lime-sand stone & 24.00 \\
\hline & & Gypsum plaster & 1.50 \\
\hline & \multirow[t]{3}{*}{ Brick } & Plaster & 2.00 \\
\hline & & Insulated brick & 26.00 \\
\hline & & Plaster & 2.00 \\
\hline & \multirow[t]{6}{*}{ Conrete } & Plaster & 0.20 \\
\hline & & $\begin{array}{l}\text { Fibre glass reinforcement } \\
\text { grid }\end{array}$ & 0.05 \\
\hline & & Synthetic resin & 0.04 \\
\hline & & EPS & 13.00 \\
\hline & & Synthetic resin & 0.20 \\
\hline & & $\begin{array}{l}\text { Concrete C20/25, } 2 \text { vol\% } \\
\text { reinforcement }\end{array}$ & 15.00 \\
\hline & \multirow[t]{6}{*}{ Ventilated facade } & Wood cladding & 2.40 \\
\hline & & Wood laths $40 / 60 \mathrm{~mm}$ & $0.048^{\mathrm{a}}$ \\
\hline & & Sheathing membrane & 0.08 \\
\hline & & Rock wool & 11.00 \\
\hline & & Lime sand stone & 24.00 \\
\hline & & Plaster & 1.50 \\
\hline & \multirow[t]{8}{*}{ Wood frame } & Wood cladding & 0.20 \\
\hline & & Wood laths $40 / 60 \mathrm{~mm}$ & $0.048^{\mathrm{a}}$ \\
\hline & & Wood fibre board & 3.00 \\
\hline & & Gypsum plaster board & 0.95 \\
\hline & & Wood beam $12 / 18 \mathrm{~cm}$ & $2.16^{\mathrm{a}}$ \\
\hline & & $\begin{array}{l}\text { Wood fibre insulation } \\
\text { board }\end{array}$ & 11.00 \\
\hline & & OSB board & 1.80 \\
\hline & & Gypsum plaster board & 1.50 \\
\hline & \multirow[t]{4}{*}{ Double shell masonry } & Facing brick & 12.50 \\
\hline & & Cellulose insulation boards & 6.00 \\
\hline & & Brick & 24.00 \\
\hline & & Gypsum plaster & 1.50 \\
\hline \multirow[t]{3}{*}{ Roofs } & \multirow[t]{3}{*}{ Concrete } & Gravel 2/32 & 4.00 \\
\hline & & Bitumen sheeting & 0.80 \\
\hline & & XPS & 18.00 \\
\hline
\end{tabular}


Table 2 (continued)

\begin{tabular}{|c|c|c|c|}
\hline & Construction type & Material & $\begin{array}{l}\text { Thickness } \\
(\mathrm{cm})\end{array}$ \\
\hline & & Vapor barrier PA & 0.30 \\
\hline & & $\begin{array}{l}\text { Concrete C20/25, } 4 \text { vol\% } \\
\text { reinforcement }\end{array}$ & 20.00 \\
\hline & & Gypsum plaster & 1.50 \\
\hline & Wood beams & Bitumen sheeting & 0.80 \\
\hline & & $\begin{array}{l}\text { Wood fibre insulation } \\
\text { board }\end{array}$ & 18.00 \\
\hline & & Vapor barrier PA & 0.30 \\
\hline & & Wood planking & 2.40 \\
\hline & & Wood beam $12 / 18 \mathrm{~cm}$ & $2.16^{\mathrm{a}}$ \\
\hline \multirow[t]{14}{*}{ Cellings } & \multirow[t]{6}{*}{ Concrete } & Tiles & 1.00 \\
\hline & & Tile adhesive & 0.80 \\
\hline & & Cement screed & 6.00 \\
\hline & & Vapor barrier PE & 0.02 \\
\hline & & $\begin{array}{l}\text { Concrete C20/25, } 4 \text { vol\% } \\
\text { reinforcement }\end{array}$ & 18.00 \\
\hline & & Gypsum plaster & 1.50 \\
\hline & \multirow[t]{8}{*}{ Wood beams } & Parquet floor & 2.00 \\
\hline & & Dry screed & 2.50 \\
\hline & & $\begin{array}{l}\text { Wood fibre footstep sound } \\
\text { insulation }\end{array}$ & 2.00 \\
\hline & & Chipboard & 2.50 \\
\hline & & Wood beam $12 / 18 \mathrm{~cm}$ & 2.16 \\
\hline & & Vapor barrier PE & 0.02 \\
\hline & & Wood laths $30 / 60 \mathrm{~mm}$ & $0.036^{\mathrm{a}}$ \\
\hline & & Wood cladding & 2.40 \\
\hline \multirow{13}{*}{$\begin{array}{l}\text { Interior } \\
\text { wallls }\end{array}$} & \multirow[t]{3}{*}{ Concrete } & Gypsum plaster & 1.50 \\
\hline & & $\begin{array}{l}\text { Concrete C20/25, } 4 \text { vol\% } \\
\text { reinforcement }\end{array}$ & 10.00 \\
\hline & & Gypsum plaster & 1.50 \\
\hline & \multirow[t]{3}{*}{ Lime-sand stone } & Gypsum plaster & 1.50 \\
\hline & & Lime sand stone & 11.50 \\
\hline & & Gypsum plaster & 1.50 \\
\hline & \multirow[t]{3}{*}{ Brick } & Gypsum plaster & 1.50 \\
\hline & & Brick & 11.50 \\
\hline & & Gypsum plaster & 1.50 \\
\hline & \multirow[t]{4}{*}{ Wood frame } & OSB board & 1.80 \\
\hline & & Wood beam $6 / 8 \mathrm{~cm}$ & $3.00^{\mathrm{a}}$ \\
\hline & & Rock wool & 7.50 \\
\hline & & OSB board & 0.90 \\
\hline
\end{tabular}


Table 2 (continued)

\begin{tabular}{l|l|l|l}
\hline & Construction type & Material & $\begin{array}{l}\text { Thickness } \\
(\mathrm{cm})\end{array}$ \\
\hline \multirow{2}{*}{ Slabs } & \multirow{2}{*}{ Concrete } & Tiles & 1.00 \\
\cline { 3 - 3 } & & Tile adhesive & 0.80 \\
\cline { 3 - 3 } & Cement screed & 6.00 \\
\cline { 3 - 3 } & Vapor barrier PE & 0.02 \\
\cline { 3 - 3 } & XPS & 10.00 \\
\cline { 3 - 3 } & Bitumen sheeting & 0.40 \\
\hline & $\begin{array}{l}\text { Concrete C20/25, 4 vol\% } \\
\text { reinforcement }\end{array}$ & 25.00 \\
\cline { 3 - 4 } & Lean concrete & 8.00 \\
\hline
\end{tabular}

${ }^{\mathrm{a}}$ Equivalent thickness

\section{Results}

Combining nine geometric variants with six construction types results in 54 solutions. The results are visualized in a tree with two stages, see Fig. 2. The LCP show a high range of 0.376 between the minimum value of 0.580 (variant 7 , concrete) and the maximum value 0.956 (variant 3 , wood). The median of the six different construction types is calculated to evaluate the geometry independently from the choice of material. Variant 3 performs best according to the median LCP. Variant 3 also achieves the maximum overall performance in stage 2, when the wood-based construction variant is chosen. Variant 7 achieves least LCP according to the median and performs worst in both cases, when choosing wood or concrete.

The difference between the median of worst and best geometric variant is 0.17 showing that the influence of the geometry is significant. With a range of $0.22 \mathrm{LCP}$, the average range of the different material combinations is even higher. This clearly shows the great importance of the choice of material. The best solutions can be achieved with the wooden construction for all geometries in this case study. Even the worst geometric variant with wooden construction performs better than the best geometric variant built in ETICS, brick, concrete or a ventilated façade, see Fig. 3.

\section{Discussion}

The results highlight the importance of materials choices. In this case study, the median proves to be a robust indicator of the environmental performance in stage 2 . It can be used to evaluate and rank design solutions in stage 1 without having defined the exact material. This is important for application in practice, because usually there is no time for elaborate studies, such as presented here. A simple measure to indicate the environmentally best performing variants to the design team 


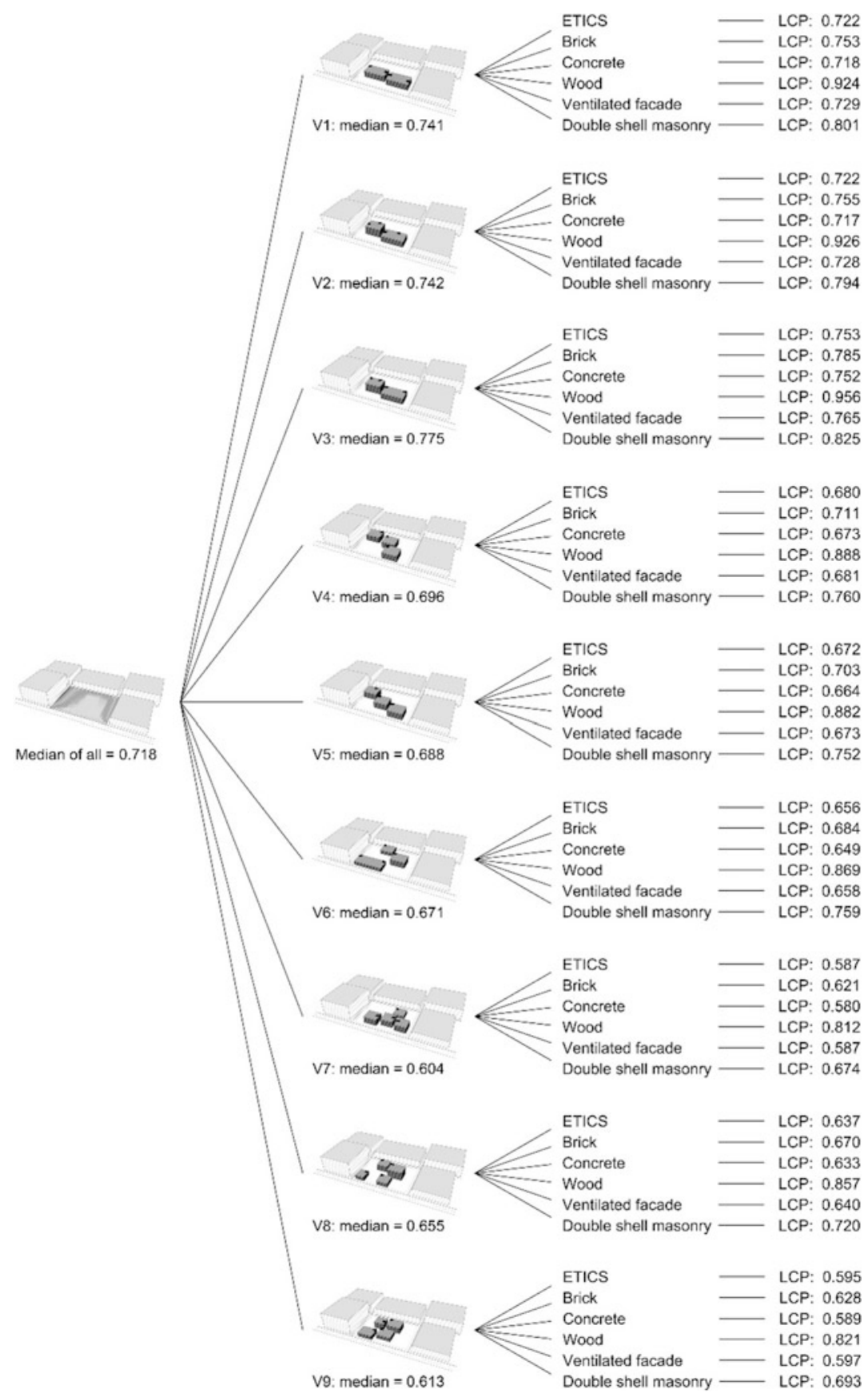

Fig. 2 LCP results for design tree 
Fig. 3 Ranges of material choice

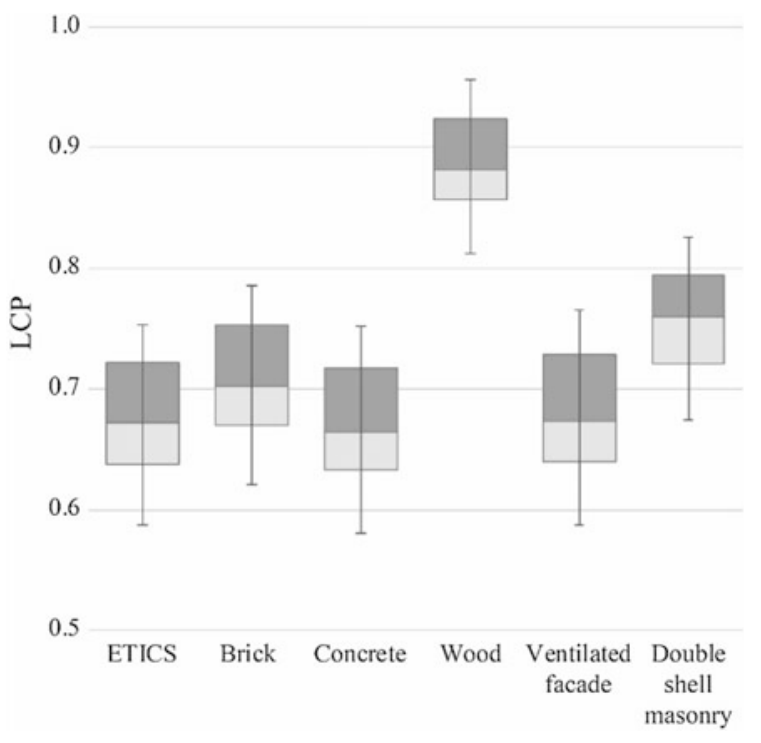

is needed. By automatically looping through a number of material variants and calculating the median this simple measure can be provided easily and time-efficiently. In the case study, each calculation took less than $0.1 \mathrm{~s}$, which allows to calculate the median within less than $0.6 \mathrm{~s}$. According to Nielsen [24] a response time of $1 \mathrm{~s}$ is acceptable for designers to not feel interrupted in their flow of thoughts. As such, the computer can loop through typical materials and provide an LCP before materials have been assigned. Using this measure, the designer can optimize the geometry without having information usually required for an LCA. Ideally speaking, this method frees the designer from worrying about HVAC systems and building materials in early design stages and allows focusing on the geometry.

In the case study presented here, the influence of the geometry was slightly smaller than the influence of the choice of material. However, the design space for the geometric variants was rather constrained. For simplification purposes, only cubic buildings with a fixed window to wall ratio and between two and to four floors have been generated. Increasing the variability of the geometry would clearly result in a higher influence of the geometry on the LCP. This would further highlight the strength of the presented approach of a combined assessment of geometry and material decisions in early design stages. 


\section{Conclusion and Outlook}

Design-integrated, simplified LCA based on digital building models can help to make informed environmental decisions during conceptual design. The early BIM-LCA approach using a parametric model presented here proves to be valuable for quick variant comparison of neighbourhoods in early design stages. The simplified visualization and the use of a single indicator can help designers to make informed decisions based on the LCA results. These simplified approaches are necessary to move from the current post-design evaluation of buildings to a design-integrated assessment. This finally allows designers to improve the environmental performance of their buildings from the very beginning of the planning process.

Through the development towards nearly zero energy buildings, buildings produce more and more of the energy they need for operation themselves. As such, the integration of local energy production should be integrated in the assessment. This aspect could be easily integrated in the parametric LCA model. Furthermore, the link to further analysis methods, such as daylight availability could provide a means to extend the early BIM-LCA to a more holistic performance evaluation tool in the future.

\section{References}

1. Wittstock B, Albrecht S, Makishi Colodel C, Lindner J. P, Hauser G, Sedlbauer K, Gebäude aus Lebenszyklusperspektive - Ökobilanzen im Bauwesen, Bauphysik, vol. 31, 2009.

2. Hegger M, Fuchs M, Stark T, Zeumer M, Energie Atlas: Nachhaltige Architektur. Birkhäuser, 2007.

3. Schneider C, Steuerung der Nachhaltigkeit im Planungs- und Realisierungsprozess von Büround Verwaltungsgebäuden. 2011.

4. Paulson Jr., B.C, Designing to Reduce Construction Costs, J. Constr. Div., vol. 102, no. 4, pp. 587-592, 1976.

5. Soust-Verdaguer B, Llatas C, García-Martínez A, Critical review of bim-based LCA method to buildings, Energy Build., vol. 136, pp. 110-120, 2017.

6. Köhler M, Rhinos, Hoppers, CAD-Monkeys, Competition online, pp. 85-90, 2016.

7. Dogan T, Reinhart C.F, Automated conversion of architectural massing models into thermal 'shoebox' models, BS2013 13th Conf. Int. Build. Perform. Simul. Assoc., pp. 3745-3752, 2013.

8. Minergie, Berechnung der Grauen Energie bei MINERGIE-A®, MINERGIE-ECO ${ }^{\circledR}$, MINERGIE-P-ECO® UND MINERGIE-A-ECO® BAUTEN, pp. 1-12, 2016.

9. Holliger Consult, Bauteilkatalog, 2002. [Online]. Available: http://www.bauteilkatalog.ch/ch/ de/Bauteilkatalog.asp. [Accessed: 08-Aug-2017].

10. Allacker K, et al., Environmental Profile of Building elements, Towards an integrated environmental assessment of the use of materials in buildings, 2013.

11. IBO, "baubook," 2014. [Online]. Available: www.baubook.at.

12. Hollberg A, Ruth J, LCA in architectural design — a parametric approach, Int. J. Life Cycle Assess., vol. 21, no. 7, pp. 943-960, Jul. 2016. 
13. CEN, EN 15978: Sustainability of construction works - Assessment of environmental performance of buildings - Calculation method. 2011.

14. Hollberg A, Lichtenheld T, Klüber N, Ruth J, Parametric real-time energy analysis in early design stages: a method for residential buildings in Germany, Energy, Ecol. Environ., Mar. 2017.

15. Rutten D, Grasshopper3D, 2015. [Online]. Available: http://www.grasshopper3d.com/. [Accessed: 08-Aug-2017].

16. Hollberg A, Parametric Life Cycle Assessment - Introducing a time-efficient method for environmental building design optimization. Bauhaus-Universitätsverlag, 2016.

17. German Sustainable Building Council, DGNB system, 2015. [Online]. Available: http://www. dgnb-system.de/en/. [Accessed: 08-Aug-2017].

18. Hollberg A, Klüber N, Schneider S, Ruth J, Donath D, A Method for Evaluating the Environmental Life Cycle Potential of Building Geometry," in Systems Thinking in the Built Environment. Sustainable Built Environment (SBE) Regional Conference Zurich 2016, 2016, pp. 590-595.

19. DIN, DIN V 18599-10:2011 Energetische Bewertung von Gebäuden - Berechnung des Nutz-, End- und Primärenergiebedarfs für Heizung, Kühlung, Lüftung, Trinkwarmwasser und Beleuchtung - Teil 10: Nutzungsrandbedingungen, Klimadaten. DIN Deutsches Institut für Normung e. V., pp. 1-102, 2011.

20. Tschetwertak J, Schneider S, Hollberg A, Donath D, Ruth J, A Matter of Sequence Investigating the Impact of the Order of Design Decisions in Multi-Stage Design Processes, in Computer-Aided Architectural Design Future Trajectories 17th International Conference, CAAD Futures 2017 Istanbul, Turkey, July 12-14, 2017 Selected Papers, G. Çağdaş, M. Özkar, G. Ethem, and L. F. Gül, Eds. Springer Nature Singapore Pte Ltd., 2017, pp. 100-120.

21. Tschetwertak J, Schneider D, Hollberg A, Donath D, Ruth J, Does the sequence matter? Investigating the impact of the order of design decisions on the Life Cycle Performance, in World Sustainable Built Environment Conference 2017 Hong Kong, 2017, no. June, pp. 1933-1938.

22. Bundesregierung, EnEV 2014: Energieeinsparverordnung - Nichtamtliche Lesefassung zur Zweiten Verordnung zur Änderung der Energieeinsparverordnung vom 18. November 2013. Germany: Bundesanzeigerverlag, 2013, pp. 1-90.

23. BBSR, ökobau.dat. Bundesministerium für Umwelt, Naturschutz, Bau und Reaktorsicherheit, 2015.

24. Nielsen J, Usability Engineering. Academic Press, 1993.

Open Access This chapter is licensed under the terms of the Creative Commons Attribution 4.0 International License (http://creativecommons.org/licenses/by/4.0/), which permits use, sharing, adaptation, distribution and reproduction in any medium or format, as long as you give appropriate credit to the original author(s) and the source, provide a link to the Creative Commons license and indicate if changes were made.

The images or other third party material in this chapter are included in the chapter's Creative Commons license, unless indicated otherwise in a credit line to the material. If material is not included in the chapter's Creative Commons license and your intended use is not permitted by statutory regulation or exceeds the permitted use, you will need to obtain permission directly from the copyright holder.

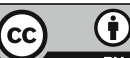




\title{
A Proposition to Extend CityGML and ADE Energy Standards for Exchanging Information for LCA Simulation at Urban Scale
}

\author{
Adélaïde Mailhac, Emmanuelle Cor, Marine Vesson, Elisa Rolland, \\ Pascal Schetelat, Nicoleta Schiopu and Alexandra Lebert
}

\begin{abstract}
Environmental performances assessment of urban projects is essential to meet the current challenges of urban sustainable development. In recent years, Life Cycle Assessment (LCA) has been applied to urban scale to assess complex systems such as districts, urban facilities and territories. Yet, application of LCA to large scale is challenging in terms of data modelling. To overcome this challenge, integration of Building Information Model (BIM) to LCA could reduce efforts during the data acquisition, as well as allowing the feedback of LCA results into BIM. To ensure interoperability e.g. with energy simulation tools, digital mock-up using an open information standard must be preferred. To answer this need, the Open Geospatial Consortium (OGC) developed the open standard CityGML which is an XML-based data model that defines classes and relations for 3D object in cities (e.g. buildings, roads, water bodies etc.). This format also provides for domain-specific extension to other objects or attributes using Application Domain Extensions (ADE). To date, LCA data requirements have not been fully integrated into the CityGML format nor its ADE. The aim of this paper is to propose extension of CityGML and Energy-ADE standards for exchanging information for LCA simulation at urban scale. The scope of the study is limited to the integration of information necessary for LCA of buildings' construction and renovation. First, data requirements are listed and then compared to CityGML and Energy-ADE
\end{abstract}

\footnotetext{
A. Mailhac $(\varangle) \cdot$ E. Cor $\cdot$ M. Vesson $\cdot$ E. Rolland $\cdot$ P. Schetelat

N. Schiopu - A. Lebert

Scientific and Technical Center for Buildings (CSTB),

University Paris-East, Champs-sur-Marne, France

e-mail: adelaide.mailhac@cstb.fr
}

\section{A. Mailhac}

Laboratoire Matériaux et Durabilité Des Constructions (LMDC), INSA/UPS

Génie Civil, Federal University of Toulouse Midi-Pyrénées, Toulouse, France

\author{
A. Mailhac $\cdot$ E. Cor · P. Schetelat · N. Schiopu $\cdot$ A. Lebert \\ ITE Efficacity, Champs-Sur-Marne, France \\ (C) The Author(s) 2018 \\ E. Benetto et al. (eds.), Designing Sustainable Technologies, \\ Products and Policies, https://doi.org/10.1007/978-3-319-66981-6_31
}


structures to identify missing information. Finally, propositions and recommendations are presented to fully integrate information needed for LCA at urban scale into CityGML and Energy-ADE. This paper paves the way for further integration of LCA models with CityGML.

\section{Introduction}

In the construction sector, LCA has been used since the 1990s to evaluate construction products and buildings [1]. For few years, LCA has extended to urban scale, and is more and more used to assess urban precincts and building stocks at large scales [2]. Performing LCA at building and urban scale requires to collect large amount of information -data- on the foreground system to complete the life cycle inventory (LCI). This routine of data collection is time and effort consuming if repeated for different simulations (e.g. energy, cost, environment simulations) [3]. On the other hand, the integration of Building Information Model or Modelling (BIM) to building and urban LCA can reduce efforts during data acquisition, as well as allowing feedback of LCA results into BIM for results visualization.

Building Information Modelling (BIM) is a working process where digital representations of physical and functional characteristics of building and civil engineering objects are processed and managed. Building information models (BIMs) are data stocked in files which can be exchanged or networked to support expert analysis and decisions about a building or other built asset. They contain information regarding objects, geo-localization, geometry and semantic data [4].

In order to ensure interoperability with different software (e.g. with energy simulation tools), digital mock-up using an open information standard must be preferred.

\subsection{Open BIM Standards at Building Scale}

BIM is often associated with open data structures for representing information such as the Industry Foundation Classes (IFC) and Green Building XML (gbXML) formats at building scale and LandXML and CityGML formats at urban scale. Such data structures are intended to describe building and civil engineering construction industry data in a neutral and open manner i.e. they are open file formats that are not controlled by a company or a group [4].

At building scale, IFC format has a wide scope and is compliant with data requirements of building LCA [5] while gbXML format is specific to export data used by energy tools [6]. Some building specific LCA tools are already compatible with the IFC and gbXML formats. These international open formats ensure that any Architecture/Engineering/Construction (AEC) software understand and operate properly technical and geometrical information from BIM [3]. They greatly enhance interoperability between AEC software. 


\subsection{The CityGML Format and Its Application Domain Extensions (ADE)}

At urban scale, the international open standard CityGML is an XML-based data model that defines classes and relations for 3D object in cities (e.g. buildings, roads, water bodies etc.). This format also provides for domain-specific extension to other objects or attributes using Application Domain Extensions (ADE) [4].

The Energy-ADE extends the CityGML Standard by features and properties necessary to perform urban energy simulation and to store the corresponding results [7, 8]. It contains information concerning construction, materials, building occupancy, energy and systems. Moreover, developers of this ADE highlight that "the Energy ADE is structured modularly in order to potentially reuse and extend some of its modules in other domains and applications" [8]. In other words, the data model developed in this ADE can be potentially extended to integrate data required for urban scale LCA.

To date, no LCA tools or studies were identified that developed a link with CityGML files to perform urban scale LCI. An important issue related to the integration of BIM and LCA at urban scale is that LCA data requirements have not been fully integrated into the CityGML format nor its ADE.

\subsection{Aims and Approach}

The aim of this paper is to propose ways for extension of CityGML and Energy-ADE standards in order to exchange information for LCA simulation at urban scale. The scope of the study is limited to the integration of information necessary for LCA of buildings' construction and renovation. First, data requirements are listed and then compared to CityGML and Energy-ADE structures to identify missing information. Finally, propositions and recommendations are presented to fully integrate information needed for LCA at urban scale into CityGML and Energy-ADE.

\section{Identification of Data Needs for LCA at Urban Scale}

\subsection{LCA Methodology at Urban Scale}

To perform LCA at urban scale, the physical boundaries of the urban project under study have to be defined. All objects and flows located within these physical boundaries are identified. This includes in particular the following objects: 
Table 1 Example of aggregated results of LCA at urban scale

\begin{tabular}{l|l}
\hline Indicators & Value \\
\hline Total primary energy consumption $(\mathrm{kWh} /$ year) & $147,706,783$ \\
\hline Total non-renewable primary energy consumption $(\mathrm{kWh} /$ year) & $141,642,624$ \\
\hline Global warming $(\mathrm{kg} \mathrm{CO}$ eq/year) & $4,463,791$ \\
\hline Water consumption (L/year) & $317,981,008$ \\
\hline Hazardous waste disposed (kg/year) & 2553 \\
\hline Non-hazardous waste disposed (kg/year) & $2,024,629$ \\
\hline Radioactive waste disposed (kg/year) & 7222 \\
\hline Atmospheric acidification $\left(\mathrm{kg} \mathrm{SO} \mathrm{S}_{2}\right.$ eq/year) & 22,399 \\
\hline Photochemical ozone creation $\left(\mathrm{kg} \mathrm{C}_{2} \mathrm{H}_{4}\right.$ eq/year) & 839 \\
\hline
\end{tabular}

- Buildings;

- Energy and water flows related to building consumption, during use;

- Transport infrastructure (roads, cycle tracks, pedestrian paths, parking, rails);

- Network infrastructure (electricity, heat, water, lighting);

- Other (activity resources, green areas).

The scope of the LCA is set to include input and output flows of materials (including water) and energy related to each life phases of the project (production and provision of construction materials, construction phase, use phase and end of life), in accordance with a functional unit and an evaluation period [9].

The environmental impacts are then calculated from these flows, thanks to environmental databases that can be specific to the construction sector; such as the German ökobau database [10] or the INIES database (French national reference database of environmental declarations for products, equipment, and services in the construction sector) [11].

Depending on the objectives of the LCA and the nature of the urban project (e.g. renovation operation, new construction), the environmental impacts of each object are $100 \%$ allocated to the project or allocation rules can be identified. The LCA result is a set of environmental indicators that reflect the environmental burden of the urban project. These results can be aggregated or presented for each life cycle phase of the project, or for each object. In Table 1, results obtained for a LCA study in the Parisian region are presented.

\subsection{Data Needs for LCA at Urban Scale}

To provide these results, a considerable amount of data related to each object under study has to be collected (general information, materials and products used, quantitative data ...). Some information contained in the CityGML standard can be used to reduce data acquisition efforts. However, data requirements for LCA have not yet been fully integrated into the CityGML standard. An important issue related 
to the integration of LCA data into the CityGML is related to the diversity of data needed, and to its quality, which can affect the precision of the results.

Data needs have been identified based on our experience from the development of the building LCA tool Elodie [12] and based on data requirements of the future French building regulation 2020 initiated by the "Energy- Carbon" labelling process [13]. Depending on the objective of the LCA study, and the data availability, a screening, simplified or complete/detailed LCA approach can be applied [14]. In this paper, we focus on data structuring for the environmental evaluation of building objects and energy consumption during use. Data structuring for environmental evaluation of other objects such as transport or utility infrastructures are not addressed in this work. Data needs considered in this work for environmental evaluation at urban scale of building objects can be structured into three categories, which are described below.

\subsubsection{Building Object: General Data}

General information, related to each building object inside the area under study is included into this module of data. The geometry of the building, height, number of floors and floor area are used for the quantification of construction materials when detailed data is not available. The building typology (e.g. individual house, collective dwelling, office building ...) is also needed when no detailed data about construction materials is available. This will mainly help the application of environmental indicators ratios for the evaluation depending on the typology. Other general data, such as construction year, number of occupants, housing units, type of operation (new construction or renovation) has to be collected. Table 2

Table 2 Data needs for building object, general data

\begin{tabular}{l|l}
\hline Data needs & Type (unit if specified) \\
\hline Building typology & CodeList \\
\hline Floor area & Decimal $\left(\mathrm{m}^{2}\right)$ \\
\hline Geometry & Geometry \\
\hline Construction year & Date \\
\hline Number of storeys & Integer \\
\hline Height & Decimal \\
\hline Type of operation & CodeList \\
\hline Number of occupants & Integer \\
\hline Housing units & Integer \\
\hline Glazing ratio & Decimal \\
\hline Renovation actions & String \\
\hline Main structure type & CodeList \\
\hline Main structural material & CodeList \\
\hline Main filling material in facade & CodeList \\
\hline Foundation type & CodeList \\
\hline &
\end{tabular}


recapitulates all building's general data needed and their related types and unit. Data type CodeList is used when the data can be selected from a list.

\subsubsection{Building Object: Envelope, Products and Systems}

This module of data contains information related to the walls, roofs and floors as well as information related to products and energy systems installed in each building. Data in bold in Table 3 is the minimum data set to collect to perform a simplified LCA. It describes surface components type such as roofs, floors, walls etc. for the building. To perform a simplified LCA, each surface component in the building has to be further described by information on its constructive system (e.g. for a wall type component, it can be a frame wall, a masonry wall ...) and by its main material (concrete, wood ...).

To perform a detailed LCA, the quantity and service life of any construction product and system has to be collected. Other information, such as construction package corresponding to the product or system and a field to describe its technical characteristics is needed. This information is necessary to link an environmental ID to the product or system.

\subsubsection{Building Object: Energy Consumption During Use}

This module of data contains the information concerning the energy consumption of the building object during its use phase. It can be connected with the information contained within the Energy ADE. For LCA at urban scale, final energy consumption of each end use type (heating, cooling, hot water for domestic use, lighting ...) must be quantified. This information is then linked to specific data with an environmental ID number, to evaluate the environmental impacts of energy

Table 3 Data needs for building object: envelope, systems and products

\begin{tabular}{l|l}
\hline Data needs & Type and unit \\
\hline Construction package & CodeList \\
\hline Description field & String \\
\hline Quantity & Decimal \\
\hline Service life & Integer \\
\hline Component type (wall, roof, floor ...) & CodeList \\
\hline Construction system (e.g. flat roof if the component type is roof, ....) & CodeList \\
\hline Main material (concrete, wood ...) & CodeList \\
\hline U value (for thermal boundaries) & Decimal \\
\hline Nominal power (for energy systems) & Decimal \\
\hline Environmental identifier & Database \\
& Name + Id \\
\hline
\end{tabular}


Table 4 Data needs for building object: energy consumption during use

\begin{tabular}{l|l}
\hline Data needs & Type \\
\hline Type of energy (electricity, gas, biomass ...) & CodeList \\
\hline End use (heating, air conditioning ...) & CodeList \\
\hline Final energy consumption, for each energy type and end use type & Decimal $(\mathrm{kWh})$ \\
\hline Environmental ID & Database Name + Id \\
\hline Primary energy factor & Decimal \\
\hline
\end{tabular}

consumption, for each end use type and for each type of energy (electricity, natural gas, fuel, pellets, wood, coal ...) (Table 4).

\section{The CityGML and Energy-ADE}

The building physics module (represented in Fig. 1) of the Energy-ADE extends the building class with new attributes and defines entirely new concepts suited for the energy simulation tools. A building is thus composed of thermal zones which serves as space units for the building heating and cooling calculation. Those thermal zones are bounded by thermal boundaries that can be optionally linked to geometrical boundary surfaces (e.g. Roof Surface, Wall Surface ...) of the CityGML 3D building description. Thermal boundaries are described by a construction object which specifies global thermal properties at the wall scale and optionally describes a sequence of layered material, themselves described by their physical properties.

At first, one could try to extend the material description with an environmental product declaration (EPD) database identifier. This is not satisfactory for two reasons. First, this would only allow to specify the environmental properties of thermal boundaries, and making it dependent to the arbitrary thermal zoning of the building. Second, this would not allow to account for other components such as stairs, HVAC systems and network. Thus, new propositions are needed in order to integrate environmental data into CityGML.

\section{Proposal}

In order to overcome the Energy-ADE limitations, new attributes are added to the building class. Those attributes are:

- The Energy consumption attribute, which describes the data needed to perform LCA related to energy consumption. This attribute gives the final energy consumption value $(\mathrm{kWh})$ for each end use (domestic hot water, electrical 


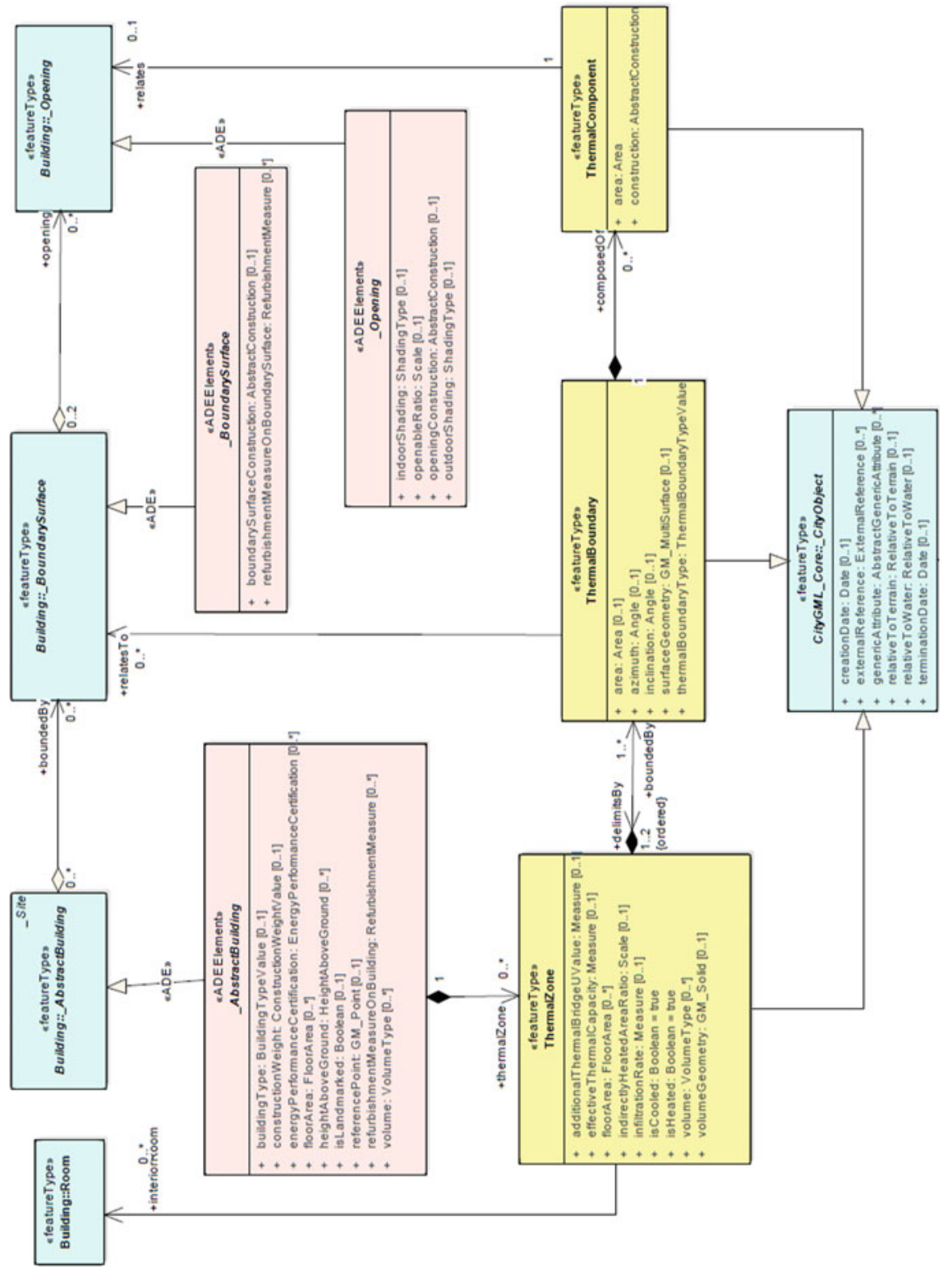

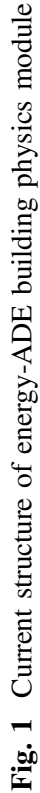


appliances, lighting, space cooling, space heating, ventilation or auxiliary) and energy source (coal, chilled water, electricity, fuel oil, hot water, natural gas, propane, steam, wood chips, wood pellet). An environmental ID is associated to the data for the calculation of environmental indicators.

- Generic attributes such as Main structure material (Concrete, Cellular concrete, Wood, Reconstituted wood, terracotta ...) are added to the building class to perform screening LCA when specific data is not available (cf. Fig. 2).

- We propose then to create a new concept class Component. This new class can be added to any city object to specify an Environmental Product Declaration identifier (environmental ID) and a quantity. A service life attribute provides information to calculate the component replacement rate during the city object life time. This class is then declined into four children classes: Surface Component, Network Component, System Component, and Other Component.

- The Surface Component class describe components such as walls, floors, roofs ... when a detailed characterization of products and materials composing the building is not available. The construction system and main material attributes

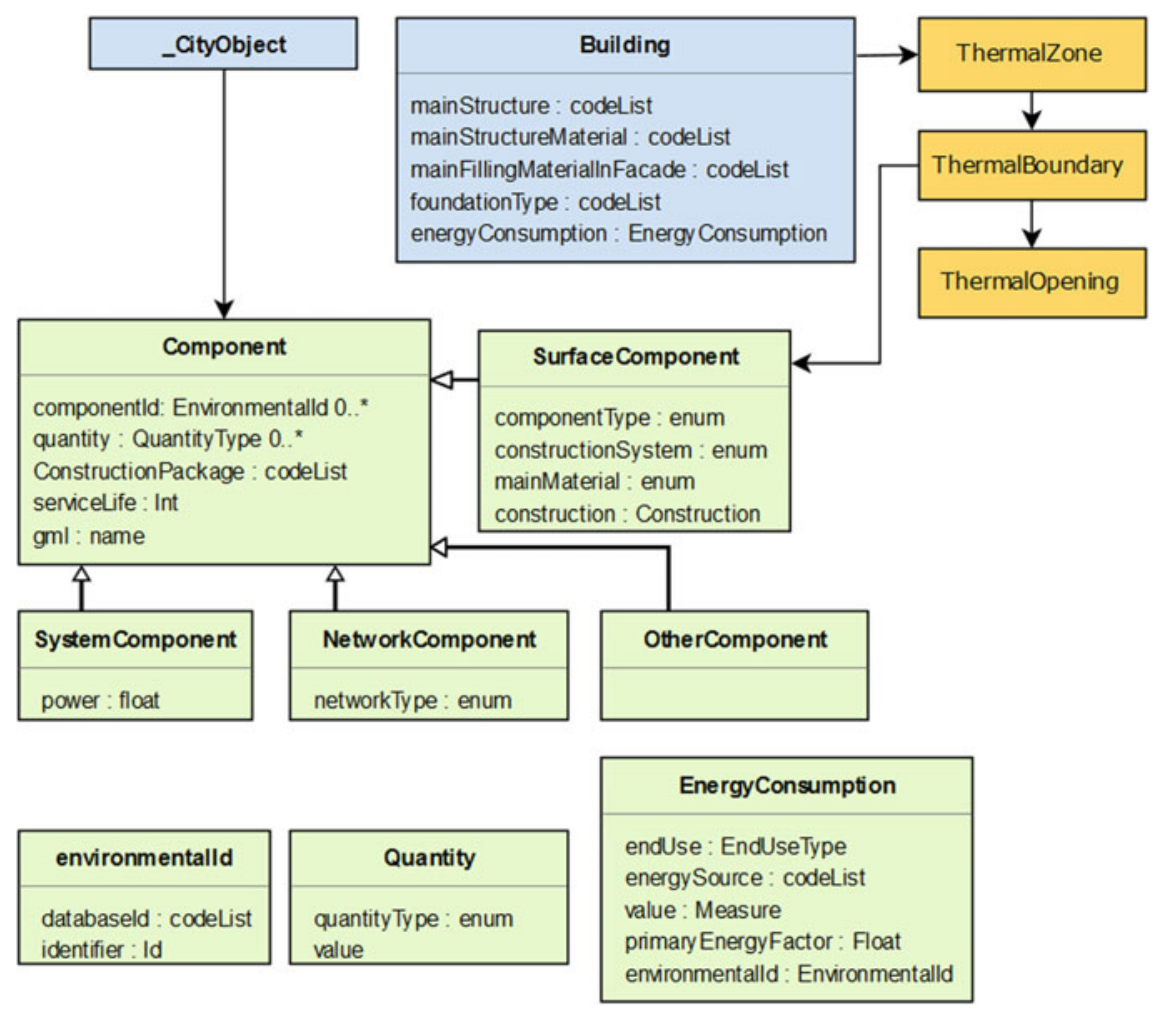

Fig. 2 Proposal for the integration of LCA data requirements into CityGML and energy-ADE standards. New concepts proposed appear in green 
give a generic description of the surface component, which supports simplified LCA. Information contained in the construction attribute can be used to specify more precisely the surface component.

- The three other children classes (Network Component, System Component, and Other Component) describe individual products, systems and networks in the building when detailed information is available. A gml name is defined to characterize the component (e.g. Concrete stairway, Individual boiler etc.). Through this description, the link to an environmental ID related to the product, system or network can be done. Specific information for the systems such as nominal power for system components and network type for network components has to be recorded. A Construction Package is allocated to any component. This allows the aggregation of LCA results for each construction packages such as:

- Roads and networks,

- Foundations and infrastructures,

- Superstructure/Masonry,

- Roofing/Frame/Waterproofing,

- Partitioning/Doubling/Interior carpentry,

- Facades/Exterior Carpentry,

- Coverings (floor, wall, ceilings)/Decoration products,

- HVAC,

- Plumbing-sanitary,

- Energy networks (high voltage) and communication networks (low voltage)

- Equipment for local electricity production.

Figure 2 recapitulates the proposed structure for environmental data.

\section{Conclusion and Perspectives}

In this paper, propositions and recommendations are presented to integrate information needed for building LCA at urban scale into CityGML and Energy-ADE. The structure proposed is meant to be suitable for screening, simplified or detailed LCA of the building. Validation of this new developments will be achieved through an iterative process. During the next months, a test phase will allow confronting the proposed data structure with real projects and software integration issues. In addition, the structure and the completeness of the schema should be discussed within the LCA practitioners' and Energy-ADE developers' communities.

Moreover, this work is limited to the description of building components, systems and energy consumption. To perform full life cycle assessment of building as defined by the European standard EN 15978 [15], further work is required to integrate information on water consumption, waste production, mobility needs and worksite. Finally, to perform urban scale LCA, information required on utility networks, transport infrastructures and public spaces should be listed and integrated into the CityGML standard. 


\section{References}

1. Khasreen M. M, Banfill P. F. G, Menzies G.F, Life-Cycle Assessment and the Environmental Impact of Buildings: A Review, Sustainability, vol. 1, no 3, p. 674-701, 2009.

2. Mastrucci A, Marvuglia A, Leopold U, Benetto E, Life Cycle Assessment of building stocks from urban to transnational scales: A review, Renew. Sustain. Energy Rev., vol. 74, p. 316332, 2017.

3. Jrade A, Abdulla R, Integrating building information modeling and life cycle assessment tools to design sustainable buildings, in International Conference of CIB W, 2012, vol. 78, p. 29.

4. Lebègue E, Celnik O, BIM \& maquette numérique: Pour l'architecture, le bâtiment et la construction, Paris; Marne-la-Vallée: Eyrolles, 2014. (in French).

5. Bazjanac V, Crawley D.B, The Implementation of Industry Foundation Classes in Simulation Tools for the Building Industry, Lawrence Berkeley Natl. Lab., 1997.

6. <http://www.gbxml.org/>, Accessed 27-06-2017.

7. Nouvel R, et al., Genesis of the citygml energy ADE, in Proceedings of International Conference CISBAT 2015 Future Buildings and Districts Sustainability from Nano to Urban Scale, 2015, p. 931-936.

8. Nouvel R, et al., Development of the citygml application domain extension energy for urban energy simulation, in 14th international conference of the international building performance simulation association (IBPSA). Retrieved from <http://www.bs2015.in/files/ BS2015Proceeding.pdf>, 2015.

9. Mailhac A, et al., LCA applicability at district scale demonstrated throughout a case study: shortcomings and perspectives for future improvements, in Sustainable Built Environment Conference, Zurich, 2016.

10. «Informationsportal Nachhaltiges Bauen». <http://www.nachhaltigesbauen.de/>, Accessed 21-09-2017.

11. INIES, Les données environnementales et sanitaires de référence pour le bâtiment, <http:// www.inies.fr/accueil/>, Accessed 27-06-2017.

12. ELODIE, <http://www.elodie-cstb.fr/>, Accessed 21-08-2016.

13. Bâtiment à Énergie Positive \& Réduction Carbone, <http://www.batiment-energiecarbone.fr/ evaluation/cadre/>, Accessed 25-07-2017.

14. Wittstock B, et al., EEBguide Operational Guidance for Life Cycle Assessment Studies of the Energy Efficient Buildings Initiative, 2012, Available on http://www.eebguide.eu/.

15. CEN, European standard EN 15978 Sustainability of construction works-Assessment of environmental performance of buildings - Calculation method, 2011.

Open Access This chapter is licensed under the terms of the Creative Commons Attribution 4.0 International License (http://creativecommons.org/licenses/by/4.0/), which permits use, sharing, adaptation, distribution and reproduction in any medium or format, as long as you give appropriate credit to the original author(s) and the source, provide a link to the Creative Commons license and indicate if changes were made.

The images or other third party material in this chapter are included in the chapter's Creative Commons license, unless indicated otherwise in a credit line to the material. If material is not included in the chapter's Creative Commons license and your intended use is not permitted by statutory regulation or exceeds the permitted use, you will need to obtain permission directly from the copyright holder. 


\title{
Dynamic Assessment of Nature Based Solutions Through Urban Level LCA
}

\author{
Duygu Başoğlu, Emre Yöntem, Seda Yöntem, Beril Şenyurt \\ and Özge Yılmaz
}

\begin{abstract}
Planning Nature Based Solutions (NBS) to address urban challenges requires an approach that embeds the multiple dimensions of NBS to effectively portray their use and impact. Life Cycle Assessment (LCA) provides a comprehensive assessment methodology in this regard. This paper discusses the environmental assessment of NBS via LCA using urban metabolism as an overarching approach to model the urban ecosystem. Performing a dynamic assessment with time series data is suggested as a way of identifying hotspots of the indicators studied within a determined time frame, allowing the assessor to observe and mitigate extremities and make informed decisions on desired temporal patterns. Dynamic assessment is supported by two simulation methodologies: Building Information Modelling for the built environment and Agent Based Modelling for social behavioural patterns.
\end{abstract}

\section{Introduction}

Cities consume $75 \%$ of the world's resources, generate around $75 \%$ of greenhouse gas emissions globally, and are responsible for $60-80 \%$ of energy consumption around the world [5]. Poverty and urban decay are rising problems. Yet urbanization is also inevitable as a necessity to accommodate the growing world population in limited land, and as hubs that provide economic, social and environmental potential. Over half of the world's population lives in cities, with more than $20 \%$ in cities with a population over 1,000,000, while cities occupy less than $2 \%$ of the surface area of the earth. Urban areas generate around $80 \%$ of the global economic output [1-5]. This is made possible by the critical density provided by cities as arenas of agglomeration economies and sharing [6].

\footnotetext{
D. Bașoğlu $(\bowtie) \cdot$ E. Yöntem · S. Yöntem · B. Șenyurt · Ö. Yı1maz

Ekodenge Consultancy Engineering Architecture Inc., 06800 Ankara, Turkey

e-mail: duygu.basoglu@ekodenge.com

(C) The Author(s) 2018

E. Benetto et al. (eds.), Designing Sustainable Technologies,

Products and Policies, https://doi.org/10.1007/978-3-319-66981-6_32
} 
Rapid urbanization following the industrial revolution has shaped cities as consumers of resources, pictured in a frame of antagonism with nature. However, UN HABITAT points that today cities stand out as land use efficient accommodations for large populations: they actually need four times less land and ten times less local road than rural areas [1]. Yet this also indicates a concentrated demand for various resources, which may exceed local supply and require consumption of commodities produced all around the world and transported in long distances. Improving the quality, resilience and resource efficiency of urban space is the key to maintain them as sustainable alternatives to sprawl which consumes agricultural land and the wilderness.

Many opportunities exist for cities to tackle the challenge of sustainability. Cities need to follow the example of natural ecosystems and their metabolisms to be reformulated as balanced, circular, and to whatever possible extent, self-sustaining systems. Whether man-made or based on natural ecosystems, different alternatives should be evaluated and prioritized with regards to their potential to achieve sustainability goals by means of a sound assessment methodology. A way of designing natural elements as a tool for sustainability in cities is called Nature Based Solutions (NBS). NBS are means of bringing nature back into cities for the provision of ecosystem services and related environmental, social and economic benefits as a tool for achieving urban sustainability.

This paper studies urban scale life cycle assessment (LCA) as a comprehensive assessment methodology and its application to assessing NBS. Considering that achieving grounded results through LCA is highly dependent on data, the main challenge is modelling urban space efficiently to portray the network of resources, environmental impact, and the behavioural impact on citizens. Opportunities brought by the capability to generate and process more data lead to discussions regarding adding dynamic elements to LCA, which is inherently static. The urban metabolism approach seeks to model the city as a system of flows that creates inputs and outputs, while generating results from the interactions of flows, as in the metabolism analogy. Further discussions focus dynamic modelling to comprehend the changing trends of flows, and on integrating time series data for this. The concept of agent-based modelling (ABM) is discussed for the social layer of the urban area and behavioural changes. The use of building information modelling (BIM) is finally discussed to provide time series data for the built environment, enabling a more precise estimation of flows related to energy, consumption and pollution.

\section{Nature Based Solutions}

NBS are defined as interventions inspired or supported by nature and suit many of the dynamic challenges cities face as they respond to multiple purposes. They are often resilient, adaptable, resource efficient, locally adjustable and optimized [7-9]. The term "Nature Based Solutions" entered scientific literature in the agricultural 
context and migrated into design as the use of nature as a component or a subject of study for solutions inspired by it. Following their use in ecosystem-based adaptation for reducing climate-based risks, the use of NBS for urban planning has been referred to by the UN Secretary General in 2013 [10] and is currently on the research agenda of the EU [9]. They respond to the complex problems of cities with multiple purpose solutions, and enrich the urban environment by re-naturing.

To illustrate the applicability of NBS across scales for multiple challenges, two examples can be used. The Cheong Gye Cheon Restoration Project in Korea is an example of large scale urban generation by NBS, where an overpass was removed and the exposed river channel was landscaped. This intervention generated benefits to the whole urban area, including those related to biodiversity, economic growth, tourism, and urban revitalisation while mitigating urban heat island by a $3.6^{\circ} \mathrm{C}$ thermal relief measured on July 27 th [11].

A smaller scale example of NBS could be the green façade implemented on the Vienna Magistrate MA48 Building (Fig. 1), which has provided aesthetic, biodiversity and thermal advantages for its surroundings and benefited the building with up to $50 \%$ reduction in heat loss in winter and a cooling effect in summer that is equivalent to 45-3000 W air conditioning units [12]. Both examples have targeted multiple issues related to different scales in changing conditions. Even when an NBS is selected for a focused motive, such as mitigating the surface temperature of a building, side benefits occur as a result of the complex and multi-layered aspect of

Fig. 1 "Climate façade" on the MA48 building, Vienna

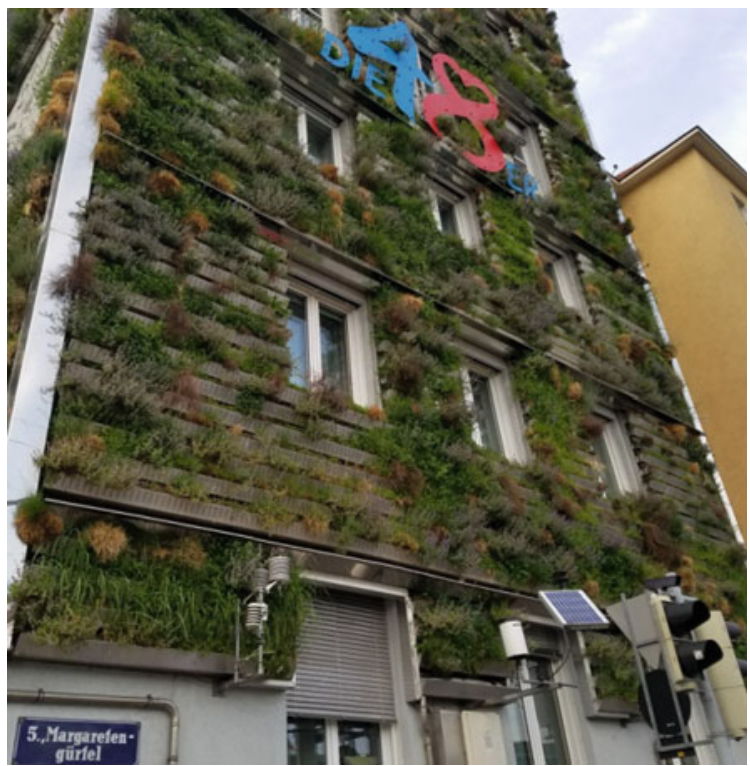


natural elements. This portrays the need for assessment and decision support tools that can model complex relations and integrate the multiple benefits of NBS to effectively support their use in addressing urban challenges.

\section{Urban Scale LCA for the Evaluation of NBS}

\subsection{Life Cycle Assessment (LCA) and Urban Metabolism (UM)}

As stated earlier, urban activities are among the major sources of sustainability issues, in particular global climate change; yet, they are also highly vulnerable to the effects of such issues. Through utilization of various assessment tools ranging from indicators to complex models, urban sustainability assessment provides insight to the current state of the environment, causes of environmental impacts, hotspots among these causes, which ultimately lead to performance evaluation of initiatives and policies for sustainable urban development [13]. Consequently, it is imperative to conduct sustainability assessment for monitoring and measuring the past or current environmental pressures, states, or impacts of urban areas. Urban assessments help cities to identify main bottlenecks regarding resilience, vulnerability to climate change, food, water and energy security issues and develop sound strategies or policies to tackle them.

LCA is a holistic methodology capable of revealing a broad range of environmental impacts in a systematic way and has been widely used for urban sustainability assessment [17]. Through LCA, it is possible to go beyond inventorying direct consumption and emissions, and consider transboundary or cross media effects embedded in urban flows and stocks. Of course, this is only possible by means of reflecting the complexity of urban systems, which are comprised of layers of sub-systems in LCA system models.

NBS are among the strategies that can benefit from such a holistic assessment due to their utility under themes including greenhouse gas emissions, biodiversity, water, urban food, air and health [14]. Moreover, LCA is also suitable to address the multi layered nature of NBS as it covers various environmental mechanisms revealing information on different impacts at different scales that apply to mid-point and end-point results.

To be able to reach the desired level of representation of complex urban systems, the conceptual models developed during system boundary definition stage of LCA can benefit from the urban metabolism (UM) approach. This is a good foundation for identifying the relevant urban flows within the system boundary and their interactions. The UM approach is based on an analogy for the resource consumption and waste generation of cities, resembling the requirement for nutrient 
intake, energy demand, consequent growth and production of metabolic wastes of a living organism [17]. It is defined as "a broad range of quantitative methods that attempt to conceptualize urban areas as organisms, requiring goods and energy to maintain functionality and support growth, while emitting waste as a by-product" [15]. Due to the complexity of urban systems, they can be considered to be more similar to ecosystems encompassing a group of multiple individuals, located within their environment, interacting among themselves and in a place [16] rather than single organisms. UM supports LCA by allowing the practitioner to analyse interlinked urban sub-systems from a holistic point of view.

UM of cities can be studied alternatively by different approaches, one of which is Odum's emergy approach, which describes the accounting of UM flows in terms of (solar) energy equivalents, thus translating city's flows of material, nutrient, services and wastes in one common unit of measurement [17]. Beyond conceptual system model definition, UM also supports LCA through the identification of urban metabolic flows. This identification entails two basic types of cycles within the cities; the operating cycle and investment cycle. Operating cycle is comprised of the continuous flows within the system boundary, whereas the investment cycle contains the flows that turn into stock, which has a non-continuous and accumulative character [18]. By analysing these different types of urban cycles, UM approach allows us to monitor the temporal changes within cities and supports dynamic urban LCA studies. This can enable the assessor to identify hotspots of the indicator studied within the determined period of time, allowing to identify and mitigate extremities, make decisions based on desired performance patterns over time and optimize the life span of the study.

Although LCA is a methodologically well-established assessment with applicable ISO standards 14040 and 14044, data availability and quality can become important bottlenecks for an LCA study. This limitation imposed can be more pronounced for complex urban systems, where without proper inclusion of all relevant data on flows, benefits of sustainability strategies with multiple target improvements such as NBS cannot be revealed. Furthermore, the evaluation of resilience issues and decisions on adaptation and mitigation strategies for cities is highly dependent not only on the current status of the environment but also on future trends. Finally, the environmental performance of cities, their vulnerability to extreme events and the availability of resources to all citizens in fairness has direct implications on social structure. Therefore, sustainability assessment needs to consider problems of urban planning interlinked to its scope and cover social aspects.

The question here is how to amend and support environmental sustainability assessment through LCA with additional tools. Opportunities in achieving data for such a tool lie in supplementing LCA with BIM and dynamic social assessment. 


\subsection{A Data Centric Approach for Urban Level Complex Assessments}

Just like the accuracy and precision of an LCA study being highly dependent on data quality, urban metabolism models also rely on obtaining and generating realistic data as pointed earlier. This leads the practitioner to a data centric approach for urban level complex assessments which can be based on a trend that builds upon business intelligence towards a smart city approach operating via large quantities of complex data.

In their comprehensive literature review study, Beloin-Saint-Pierre et al. have categorised the urban metabolism modelling strategies into Black Box, Grey Box and Network approaches. Starting from the basic simplified input and output data of the Black Box Approach, the Grey-Box Approach studies further level of inner flows and the Network Approach defines the links on all inner components of the studied system. The main drawback of the Network Approach is identified to be the challenging implementation of analyses due to the big amount of data. This fact can be easily accepted as a reason of complexity yet still being the solution towards answering multi-dimensional analyses that can be very beneficial for urban level decision making [19].

The handling of such complex data can be studied through the business intelligence approach, which has roots in the database management field and benefits from various data collection, extraction, and analysis technologies [20]. Development in data analytics technologies has led to the Internet as a platform and eventually cloud-based data hosting and analyses capabilities. Business intelligence has evolved because the amount of data generated through the internet and smart devices has grown exponentially altering how organizations and individuals use information [21]. Current technological advancements in the data analytics fields and accessibility of complex data analytics algorithms within the cloud services offer a promising and reliable platform to realize data centric assessments.

The age of "big data" has also led to the transparency, accessibility and interoperability of data repositories. EU has initiated and mobilised the INSPIRE directive [22], identifying standards for data sets, and their distributed services. Urban level data management and referencing standards have also been emerging. One of these standards is the BSI Guide to establishing a model for data interoperability, which aims to look beyond the current use of data to facilitate city services, and encourage decision-makers to explore the reuse of data as a resource to innovate the future direction of systems and services. This standard identifies four key types of insight to be required when sharing data in a city; operational insight examining the characteristics of urban elements, critical insight for real time monitoring, analytical insight for exploring the data ecosystem to determine patterns, and strategic insight for examining outcomes related to strategic objectives [23]. 
Open data and data sharing in cities regarding various urban systems from transportation to communication make various multi-dimensional and interrelated urban level assessments possible. Relying on this background, supported with the enhanced analytical assessment capabilities decision makers can be served with complex interdependent analyses such as LCA, social and economic analyses.

This data centric trend in urban analysis makes the generation of data central to further studies. Big data capabilities bring forth an opportunity as they can now integrate detailed forecasts such as dynamic assessment into assessment procedures and details down to building level can be considered for urban analyses. This paper will proceed to explore some of these opportunities.

\subsection{Dynamic Social Assessment}

As pointed earlier, the significance of cities comes mainly from the fact that they accommodate most of the world's population, and even more, provide a sharing and meeting ground for their activities. Social wellbeing is thus a major goal of urban space. As citizens and related actors which can be studied as agents are continuously adapting their behaviours, it is crucial for the decision maker to understand the interaction of citizens with the studied urban system.

UM provides a detailed view on the physical space of the city, even incorporating inter-boundary relations as flows and allowing temporal results. Yet the social layer is different than any other flow: its agents, individual people, are more complex. The question is whether it is possible to include them in a model of UM.

A research held by UCL classifies debates on UM and reflects on the social implications of urban metabolism by pointing that even though urban space is modelled in units of spaces, materials and physical systems, social consequences occur and this layer is also a part of the metabolism [24]. Studies in terms of class and inequalities in relation to infrastructure networks define the term "social metabolism" proposed by Martinez-Alier as "a manner in which human societies organize their growing exchanges of energy and materials with the environment" $[24,25]$. These studies point at UM as a driver for major social movements and changes descriptively. However, an approach such as ABM that quantifies changes in behaviour over time and in relation to flows and trends of the UM is needed to complement the dynamic assessment approach this paper discusses.

$\mathrm{ABM}$ is a computational tool that is capable of examining social systems with the perspective of a complex adaptive system. It studies how macro effects emerge from micro scale behavioural patterns among heterogeneous social agents, which are evolving, autonomous and interacting [26]. 
ABM simulations handle the mathematically abstracted environment and the interactions with the involved entities in time series iterations, where the frequencies and time span can be selected to best represent the studied system. This can be citizens in an urban system, aligned with the time patterns of flows. This approach can directly benefit from data sources, where the related parameters effecting the agents' behaviours are made available in the identified resolution in terms of iteration frequencies and quality. To provide the required background for the simulations at the urban ecosystem, multidimensional data needs to be utilised. This includes the spatial urban data in the form of GIS, BIM and CIM (City Information Model) models, material and energy flow data in the form of UM approach, weather and climate related time series data.

\subsection{Developing an Integrated Assessment Platform for NBS}

The assessment method proposed for Nature4Cities, a flagship HORIZON2020 project on NBS, aims to integrate multiple tools that incorporate modelling abilities at different scales and techniques such as remote sensing for a platform that covers economic, social and environmental aspects of NBS. The environmental assessment module is planned to handle the relation between the UM and social well-being through ABM along with LCA for the assessment of environmental impacts. The project aspires for a holistic assessment of NBS, making the discussed data needs critical. This approach shall look into the relations of urban flows' distribution, intensity, services and impacts with the behaviour of citizens as agents. Dynamic assessment covers the changing nature of this interaction: supply of flows influences the decisions and behaviours of the population, resulting in changes in the settlement pattern, creating or reducing demand in flows over time.

The EPESUS City platform will be part of the technologies that contribute to the integrated assessment platform of Nature4Cities along with other novel technologies. It is an ICT tool that performs life cycle assessment at the urban scale in a time series approach [30]. This enables the assessor to identify hotspots of the indicator studied within the determined period of time, allowing to identify and mitigate extremities, make decisions based on desired performance patterns over time and optimize the life span of the study.

\subsection{Integrating BIM and New Opportunities}

Integrating BIM to urban assessment suggests new opportunities in improving the precision of data sources for LCA and the scalability of assessment at different 
levels. BIM is basically digitally representing a building or built object, attributing physical and functional properties gathered from all contributors to the design and implementation. A major benefit of using BIM is enabling collaboration in-between different stakeholders through the interoperable, shared representation of the object $[27,28]$ and this applies to the different tools and approaches proposed for assessment here. For NBS, this perspective of object level modelling is promising as many solutions offer different impacts at object level, such as regulating functions related to thermal comfort, while contributing to the urban environment's cumulative natural assets, such as the carbon storage of a city.

EPESUS software has been using BIM to generate detailed data for Sustainable Energy and Carbon Action Plans (SECAP) for local governments [30]. DesignBuilder software is used as a building energy modelling interface for Energy + to generate time series energy consumption data for the building models. Building types are determined based on available data and surveys held in the subject area of the SECAP. These types are then attributed to building masses drawn on a GIS interface of the city and their building energy model data is extrapolated in EPESUS. Results are calibrated to achieve the closest alignment with real bill data and spatial distribution based on neighbourhood characteristics. Upon obtaining a baseline, scenarios are studied based on proposed interventions such as envelope specifications and heating and cooling systems.

Such use of BIM for allocation to GIS units has been referred to as a City Information Model (CIM): incorporating object models that generate data to decision support tools using GIS [29]. What EPESUS does (Fig. 2) is using BIM and generating assumptions, aligning them with real data and achieving scenarios on a GIS in this regard. EPESUS is currently capable of modelling energy consumption, carbon emissions and life cycle impact, also delivering hotspots in the impact area. EPESUS can also visualize impacts like pollution as heat-maps.

These capabilities provide a good base to integrate NBS into an assessment method that imports time series data and delivers a dynamic assessment, with an ability to use BIM to consider micro scale impacts. Having detailed information at object level allows EPESUS to display heat-maps over a GIS interface to visualize local conditions. NBS can be part of the BIM stage of this assessment method. Such an LCA tool with GIS capabilities is functional to see some of the most desired effects of NBS including:

- Urban heat island mitigation in areas of reflective surfaces and dense population,

- Pollution control based on heat-maps resulting from energy systems and density,

- Areas' desirability for urban agents and behavioural impact,

- Other local ecosystem services such as biodiversity support. 

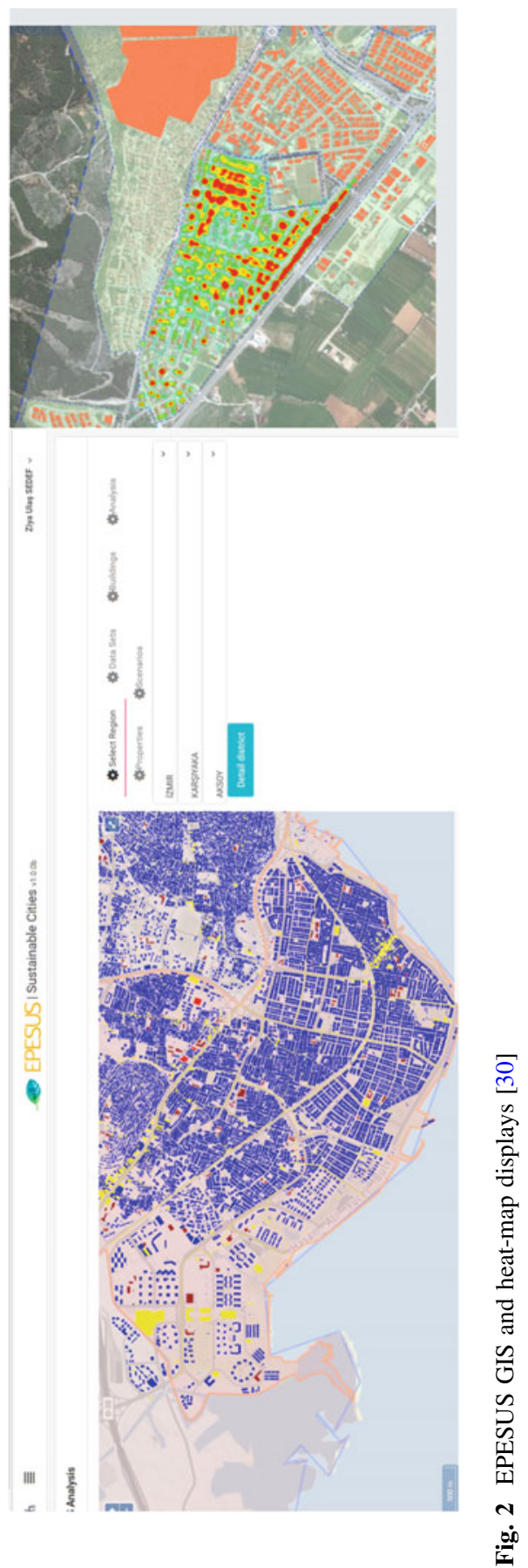


\section{Conclusions}

The Environmental Assessment of NBS Understanding the environmental impacts of NBS requires different levels of results: urban level, object and area level, and the interactions and temporal patterns for different scales. While LCA is a wellestablished environmental assessment method guided by ISO standards 14040 and 14044 , the results it generates are only as good as the data in the hands of the practitioner. Therefore, a major challenge in rendering the multi benefit and interlinked nature of NBS is to model the urban system efficiently. This requires collecting reliable data for this model, and estimating temporal patterns via suitable modelling approaches.

The UM approach illustrated in Fig. 3 is viable for understanding the links between flows influenced by NBS interventions. However, for a detailed understanding of these flows, additional parameters of the urban system model are necessary. Today, ABM provides opportunities in calculating dynamic results regarding the impacts of NBS in behavioural patterns to cover social aspects that are integral to urban assessment. In addition, established BIM tools are capable of providing time series data, which is a promising area for enhancing dynamic urban level assessment. Together, these approaches provide a state-of-the-art solution to obtaining detailed dynamic data for an LCA study of NBS in urban assessments.

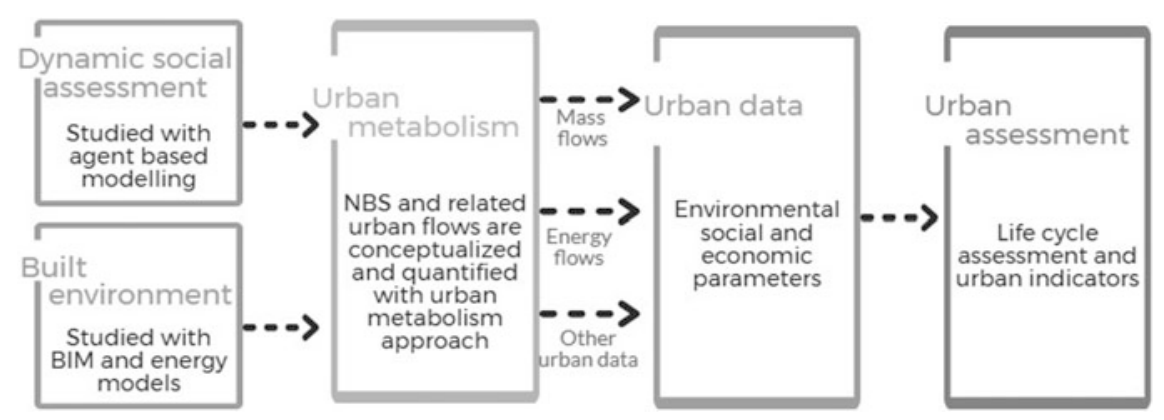

Fig. 3 Environmental assessment of NBS using the UM approach 
Acknowledgements Nature4Cities project has received funding from the European Union's Horizon 2020 research and innovation programme under grant agreement No. 730468.

\section{References}

1. UN, World Economic and Social Survey 2013. 2013.

2. UN Population Division, The World's Cities in 2016: Data Booklet., p. 29, 2016.

3. EC and UN-Habitat, The State of European Cities 2016. 2016.

4. UNDESA, World Urbanization Prospects. 2014.

5. BRE, Cities as systems: BRE solutions for urban environments, 2015.

6. Keivani R, A review of the main challenges to urban sustainability, Int. J. Urban Sustain. Dev., vol. 1, no. 1-2, pp. 5-16, 2010.

7. De Boissezon B, Smart and sustainable cities with nature-based solutions, no. September, pp. 1-15, 2015.

8. Yeroyanni M, Commission E, Innovation Renaturing cities with nature based solutions ENERO Workshop: Fostering future integration of Environment in European research \& innovation no. October, 2014.

9. European Commission, Towards an EU Research and Innovation policy agenda for Nature-Based Solutions \& Re-Naturing Cities. 2015.

10. Potschin M, Kretsch C, Haines-Young R, Furman E, Berry P, Baro F, Nature-based solutions, OpenNESS Synth. Pap., no. 18, pp. 1-5, 2014.

11. Lee I.K, Cheong Gye Cheon Restoration Project, in ICLEI, 2006.

12. Naumann S, Kaphengst T, McFarland K, Stadler J, Nature-based Approaches to Climate Change Mitigation and Adaption, pp. 1-27, 2014.

13. Yigitcanlar T, Dur F, Dizdaroglu D, Towards prosperous sustainable cities: A multiscalar urban sustainability assessment approach, Habitat Int., vol. 45, no. P1, pp. 36-46, 2015.

14. Xing X, Jones P, Donnison I, Characterisation of Nature-Based Solutions for the Built Environment, Sustainability, vol. 9, no. 1, Jan. 2017.

15. Goldstein G, Birkved M, Quitzau M.B, Hauschild M.Z, Quantification of urban metabolism through coupling with the life cycle assessment framework: concept development and case study, Environ. Res. Lett., vol. 8, p. 35024, 2013.

16. Chrysoulakis N, et al., Sustainable urban metabolism as a link between bio-physical sciences and urban planning: The BRIDGE project, Landsc. Urban Plan., vol. 112, no. 1, pp. 100-117, 2013.

17. Kennedy $\mathrm{C}$, Pincetl $\mathrm{S}$, Bunje $\mathrm{P}$, The study of urban metabolism and its applications to urban planning and design, Environ. Pollut., vol. 159, no. 8-9, pp. 1965-1973, 2011.

18. SUME, Urban Metabolism: Sustainable Urban Metabolism for Europe (SUME) Project website. [Online]. Available: http://www.sume.at/urban_metabolism. [Accessed: 01-June-2017].

19. Beloin-Saint-Pierre D, et al., A review of urban metabolism studies to identify key methodological choices for future harmonization and implementation, J. Clean. Prod., 2015.

20. Chaudhuri S, Dayal U, Narasayya V, An overview of business intelligence technology, Commun. ACM, vol. 54, no. 8, p. 88, 2011.

21. Larson D, Chang V, A review and future direction of agile, business intelligence, analytics and data science, Int. J. Inf. Manage., vol. 36, no. 5, pp. 700-710, 2016.

22. EC, INSPIRE Legislation, 2014. [Online]. Available: http://inspire.ec.europa.eu/index.cfm/ pageid/3. [Accessed: 01-Jul-2017].

23. BSI, BSI Standards Publication Smart city concept model - Guide to establishing a model for data interoperability, pp. 1-56, 2014.

24. Rapoport E, CastánBroto V, Allen A, Interdisciplinary Perspectives on Urban Metabolism, J. Ind. Ecology, 2011. 
25. Martinez-Alier J, Kallis G, Veuthey S, Walter M, Temper L, Social metabolism, ecological distribution conflicts, and valuation languages, Ecol. Econ., vol. 70, no. 2, pp. 153-158, 2010.

26. Holland J.H, Complex Adaptive Systems, Daedalus, vol. 121, no. 1, A New Era in Computation, pp. 17-30, 1992.

27. National Institute of Building Sciences (NIBS), Frequently Asked Questions About the National BIM Standard-United States, 2014. [Online]. Available: http://www.national bimstandard.org/faq.php\#faq1.

28. O'Donnell J.T, et al., Transforming BIM to BEM: Generation of Building Geometry for the NASA Ames Sustainability Base BIM, Bim, no. January, p. 26, 2013.

29. Gil J, Almeida J, Duarte J. P, The backbone of a City Information Model (CIM), 29th eCAADe Conf., no. Cim, pp. 143-151, 2011.

30. Ekodenge, EPESUS City. [Online]. Available: http://www.ekodenge.com/epesus/epesus-city/ [Accessed: 01-June-2017].

Open Access This chapter is licensed under the terms of the Creative Commons Attribution 4.0 International License (http://creativecommons.org/licenses/by/4.0/), which permits use, sharing, adaptation, distribution and reproduction in any medium or format, as long as you give appropriate credit to the original author(s) and the source, provide a link to the Creative Commons license and indicate if changes were made.

The images or other third party material in this chapter are included in the chapter's Creative Commons license, unless indicated otherwise in a credit line to the material. If material is not included in the chapter's Creative Commons license and your intended use is not permitted by statutory regulation or exceeds the permitted use, you will need to obtain permission directly from the copyright holder.

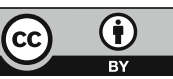




\title{
Role of Carbon Capture and Storage (CCS) or Use (CCU) on Climate Mitigation
}

\author{
Jan Bollen
}

\begin{abstract}
ArcelorMittal, world leader in steel production, is working together with industrial partners from the cement and chemical industries, other energy intensive sectors, for potential synergies between the respective manufacturing processes and how these could contribute to the reduction of overall $\mathrm{CO}_{2}$ emissions. Based on this project, the session aimed at demonstrating the place of LCM methods and tools in support of regional and/or local policy frameworks. Industrial representatives and policy makers involved in the panel presented and discussed the technical and political challenges faced when developing and implementing disruptive technologies and associated business models.
\end{abstract}

\section{Synthesis of Presentations and Discussions}

Eric De Conick (ArcelorMittal) presented the reuse of various process gases as feedstock for producing low carbon fuels and chemical feedstocks are under development or study at ArcelorMittal. The latter will allow producing plastics which can be ground at the end of life, and be re-injected as a C-agent in the blast furnace. That way a circular economy will emerge from the re-use of the second-hand carbon in industrial gasses. Even if, in a first stage of this development, the gasses would be converted in fuels for transport, and the carbon would be burnt and emitted in the atmosphere, a significant reduction of GHG emissions worldwide will be realized by the replacement of fossil oil and gas fuels. The replacement of the electricity, currently produced from these process gasses, should be done with renewable electricity. Minerals or steel slags can be further carbonated

\footnotetext{
J. Bollen ( $\square)$

ArcelorMittal Europe, Brussels, Belgium

e-mail: jan.bollen@arcelormittal.com

(C) The Author(s) 2018

E. Benetto et al. (eds.), Designing Sustainable Technologies,

Products and Policies, https://doi.org/10.1007/978-3-319-66981-6_33
} 
and converted into building materials with surplus $\mathrm{CO}_{2}$. A third of overall steel production related $\mathrm{CO}_{2}$ emissions of today could potentially be diverted for reuse. For the remaining $\mathrm{CO}_{2}$ volumes, underground permanent storage becomes an option once such infrastructure is being built.

Scale up of carbon reuse projects without a specific regulatory frame work is unlikely as the cost of products from carbon reuse will be higher than for the fossil equivalent.

Manuela Ojan (HeidelbergCement) introduced an installation that HeidelbergCement is piloting in Moroccan cement plant that produces micro algae for fish feed. This application allows this stand-alone plant to capture $10 \%$ of its total $\mathrm{CO}_{2}$ emissions.

Tom Bradley (Narec Distributed Energy_UK) presented the InteSusAl project in Portugal, which is producing algae for conversion into fuel. To enable comparisons of different process set-ups, the LCA methodology for assessing the various algae technologies were harmonized.

Colin Hills (UK Centre for Environment Research and Innovation) explained that technologies are being implemented in 2 UK plants ( 5 by 2021) to mineralize $\mathrm{CO}_{2}$ with reactive waste (APCr). However, other wastes (e.g. red mud, ...) can also be processed into aggregates for construction. From mapping EU available wastes, a conservative potential of $6 \mathrm{Mt}$ carbon abatement exists while producing $122 \mathrm{Mt}$ of aggregate, covering $6 \%$ of EU consumption.

Finally, Anders Hammer Strømman (Norwegian University of Science and Technology) stated that methodologies assessing the abatement potential of CCU technologies should consider their beneficial effect on anthropogenic perturbation of global carbon cycle. Attention should be given to whether these are in the domains of either the slow else the fast carbon cycle.

Some conclusions were drawn from discussion between panel and audience after the Q\&A session:

- Without a price on carbon, these new technologies cannot economically compete with their fossil-based alternatives.

- A regulatory support is required to enable them a market.

- To maximize their impact on carbon abatement, the technologies will require renewable energy as input for operation.

- Assessment methodologies shall reflect an evolution to more renewable energy to enable the initial viability of the new $\mathrm{CCU}$ technologies.

- Regulation framework has to reward synergies created by combined production systems rather stimulate the market based on benchmarking of the individual products as the latter will raise allocation issues. 
Open Access This chapter is licensed under the terms of the Creative Commons Attribution 4.0 International License (http://creativecommons.org/licenses/by/4.0/), which permits use, sharing, adaptation, distribution and reproduction in any medium or format, as long as you give appropriate credit to the original author(s) and the source, provide a link to the Creative Commons license and indicate if changes were made.

The images or other third party material in this chapter are included in the chapter's Creative Commons license, unless indicated otherwise in a credit line to the material. If material is not included in the chapter's Creative Commons license and your intended use is not permitted by statutory regulation or exceeds the permitted use, you will need to obtain permission directly from the copyright holder.

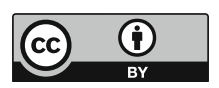


Part III

Development of LCM Methods and Tools 


\title{
Potentials and Limitations of Combined Life Cycle Approaches and Multi-dimensional Assessment
}

\author{
Johanna Berlin and Diego Iribarren
}

\begin{abstract}
The use of life cycle approaches - purely or in combination with non-life cycle methods - to assess product systems from a multi-dimensional perspective arises as a current need in the path towards actual sustainability. While pure life cycle approaches involve mainly life cycle sustainability assessment, a wide range of novel approaches are currently under study in order to overcome the limitations typically linked to multi-dimensional assessment and multi-criteria decision analysis. This is done through the synergistic combination of life cycle (mainly, life cycle assessment) and non-life cycle (techno-economic assessment, data envelopment analysis, energy systems modelling, agent-based modelling, land change and ecosystem services modelling, urban metabolism analysis, interdisciplinary policy assessment, material flow analysis, etc.) methods. The main potentials, fields of application and pending issues associated with these tools are summarised herein.
\end{abstract}

\section{Introduction}

While life cycle methodologies such as life cycle assessment (LCA), carbon footprinting (CFP) and emergy analysis focus on the evaluation of the environmental realm of systems, other approaches such as life cycle costing (LCC), social life cycle assessment (SLCA) and life cycle sustainability assessment (LCSA) attempt to extend life cycle thinking to other spheres of sustainable development [1]. Though valuable and useful, the life cycle-based approaches proposed to date have not yet succeeded in providing a well-accepted and sound evaluation of sustainability issues. In this respect, it is acknowledged that the combined use of life

\footnotetext{
J. Berlin ( $\square)$

RISE Research Institutes of Sweden, Eklandagatan 86,

41261 Göteborg, Sweden

e-mail: johanna.berlin@ri.se

D. Iribarren $(\square)$

Systems Analysis Unit, IMDEA Energy, 28935 Móstoles, Spain

e-mail: diego.iribarren@imdea.org

(C) The Author(s) 2018

E. Benetto et al. (eds.), Designing Sustainable Technologies,

Products and Policies, https://doi.org/10.1007/978-3-319-66981-6_34
} 
cycle and other system-based or non-life cycle methodologies might offer interesting potentials towards a robust and practical assessment with a multi-dimensional perspective [2]. Nevertheless, scarce and scattered information is available on this type of integrative approaches.

LCA and other system-based approaches (e.g., technological innovation systems analysis, policy and market analysis, and design and analysis of user needs) provide clues about the environmental, economic and social nature of current and future industrial systems. Material flow analysis, cost-benefit analysis, energy systems modelling and multi-criteria decision analysis (MCDA) tools such as data envelopment analysis (DEA) are also among the methodologies that have already been used in combination with life cycle approaches [3]. However, because each of the methods separately is likely to provide different observations and conclusions, the development of harmonised approaches and guidance for technical innovation is required. Within this context, further discussion on both potentials and limitations concerning the use of hybrid approaches is needed.

\section{Current Activities in Life Cycle-Based Multi-dimensional Assessment}

During the LCM2017 conference held in Luxembourg, a number of multi-dimensional approaches including a life cycle perspective were addressed. The main methodological frameworks are summarised below:

- Life cycle sustainability assessment (LCSA) to comprehensively assess the relation and trade-offs between environmental, social and economic impacts under circular economy and industrial ecology principles. Current advances include the use of open space workshops and the combination with agent-based modelling.

- Environmental techno-economic assessment through the integration of LCA and techno-economic assessment. For instance, this could be applied to thoroughly assess a technology from the first stages of development until its industrial commercialisation.

- Development of new LCA tools for environmental optimisation and implementation in biddings, thereby integrating environmental impact budgeting into decision-making processes.

- Combination of life cycle approaches with DEA for sustainability benchmarking of multiple similar entities. The so-called LC + DEA concept benefits from the availability of different life cycle approaches (LCA, CFP, emergy analysis, etc.) and numerous DEA models. In this respect, the novel use of the SBM-Max model combined with LCA has recently been proposed for the gradual operational and environmental benchmarking in terms of continuous improvement. 
- Highly trans-disciplinary frameworks examining the system from multiple perspectives. For instance, this can involve technical (e.g., laboratory cultivation and mechanical testing), social (e.g., actor analysis, policy analysis, market analysis and social acceptance), resource (e.g., material flow analysis) and environmental (e.g., LCA) evaluations.

- Other relevant methodological frameworks referred to the integration of land change modelling and ecosystem services modelling into LCA, the use of urban metabolism analysis, interdisciplinary policy assessment, and environmental, health and safety screening.

Finally, regarding the field of application of these hybrid methods, a wide range of case studies were presented during the LCM2017 conference. An increasing interest in bioeconomy-related case studies is observed, involving e.g. algae-based biorefineries, bio-based high-density polyethylene, microbial production of succinic acid from mixed food waste, and other biomass value chains. Nevertheless, many other sectors and topics could be highlighted, such as road planning, food industry, fishing sector, nanomaterials, and residential buildings.

\section{Lessons Learned}

It is seen that the development and application of methodological frameworks involving different approaches, scopes and methods leads to a useful holistic perspective in the evaluation of product systems. In this sense, this type of nuanced evaluation can offer a more robust foundation for decision-making processes than one perspective or method in isolation. Nevertheless, with the aim of guaranteeing the provision of consistent and sound results, a robust methodological harmonisation is of paramount importance, but often underdeveloped in many cases, e.g. when defining benchmarks in MCDA studies. This arises as a key issue to be addressed when developing and applying combined methods for multi-dimensional assessment.

A key strength of the proposed frameworks is the enriched interpretation and communication of the results, often providing easy-to-report results in line with the demands of the industry and other relevant stakeholders (policy-makers, society, etc.). Trans-disciplinary approaches can inform an arena of research and industrial partners with different perspectives to facilitate discussion and more well-informed decisions. Furthermore, combined methodological frameworks can even shorten the time-to-market for new technologies. However, achieving an extended use of multi-dimensional approaches is still a pending issue. In addition to research efforts in the field of systems analysis, a higher level of stakeholder involvement (e.g., through open space workshops) could help in this direction. 


\section{Conclusions and Perspectives}

The combination of life cycle approaches such as LCA with other life cycle (e.g., LCC and SLCA) or non-life cycle (e.g., techno-economic assessment, DEA, interdisciplinary policy assessment, etc.) approaches offers singular advantages for the multi-dimensional assessment of product systems. The enrichment of decision-making processes and the enhancement of the discussion, interpretation and communication of the results arise as key strengths of these hybrid methods. Nevertheless, further efforts are still required in order to guarantee the consistency of the results through methodological harmonisation, as well as to involve stakeholders and achieve an extended use of these approaches.

\section{References}

1. Guinée J.B, Heijungs R, Huppes G, Zamagni A, Masoni P, Buonamici R, Ekvall T, Rydberg T, Life cycle assessment: past, present, and future, Environmental Science \& Technology, Vol. 45, 2011, pp. 90-96.

2. Jeswani H.K, Azapagic A, Schepelmann P, Ritthoff M, Options for broadening and deepening the LCA approaches, Journal of Cleaner Production, Vol. 18, 2010, pp. 120-127.

3. Martín-Gamboa M, Iribarren D, García-Gusano D, Dufour J, A review of life-cycle approaches coupled with data envelopment analysis within multi-criteria decision analysis for sustainability assessment of energy systems, Journal of Cleaner Production, Vol. 150, 2017, 164174.

Open Access This chapter is licensed under the terms of the Creative Commons Attribution 4.0 International License (http://creativecommons.org/licenses/by/4.0/), which permits use, sharing, adaptation, distribution and reproduction in any medium or format, as long as you give appropriate credit to the original author(s) and the source, provide a link to the Creative Commons license and indicate if changes were made.

The images or other third party material in this chapter are included in the chapter's Creative Commons license, unless indicated otherwise in a credit line to the material. If material is not included in the chapter's Creative Commons license and your intended use is not permitted by statutory regulation or exceeds the permitted use, you will need to obtain permission directly from the copyright holder.

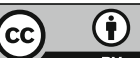




\title{
Propagating Uncertainty in Life Cycle Sustainability Assessment into Decision-Making Problems: A Multiple Criteria Decision Aid Approach
}

\author{
Breno Barros Telles do Carmo, Manuele Margni and Pierre Baptiste
}

\begin{abstract}
Life cycle sustainability assessments (LCSA) are a comprehensive source of information on product performance to support decision-making processes toward sustainable production and consumption. Multiple criteria decision aid (MCDA) approach provides a structured decision modelling that considers the value judgments of the decision-makers and it has been proved to be useful to support decision-making based on LCSA results. We proposed an approach able to take into account LCSA performances when making decisions. We applied our approach through a case study of tire life extension scenarios selection. The scenario with retreading is the solution that offer the best compromise between the three sustainable dimensions with more than $63 \%$ probability to rank first for Weighted sum, Topsis and Prométhée II MCDA methods.
\end{abstract}

\section{Introduction}

Supporting sustainable decision-making problems involves taking into account environmental, social and economic performance assessments into mathematical decision models, in order to communicate results to decision-makers. However,

B. B. T. do Carmo ( $\bowtie) \cdot$ M. Margni · P. Baptiste

Canada Mathematics and Industrial Engineering Department,

Polytechnique Montreal, 2900 boul. Édouard-Montpetit, Montreal,

QC H3T 1J4, Canada

e-mail: breno.carmo@polymtl.ca

B. B. T. do Carmo · M. Margni

Ciraig, 3333, Chemin Queen Mary, Office 310, Montreal,

QC H3C 3A7, Canada

B. B. T. do Carmo

Engineering Centre, Federal University of Semi Arid (UFERSA),

Avenida Francisco Mota, 572, Mossoró, Rio Grande do Norte

59.625-900, Brazil

(C) The Author(s) 2018

E. Benetto et al. (eds.), Designing Sustainable Technologies,

Products and Policies, https://doi.org/10.1007/978-3-319-66981-6_35 
Martín-Gamboa et al. [1] highlights the need to develop frameworks able to integrate sustainability indicators and decision-makers preferences.

One of the challenges in assessing these potential impacts is the dispersion of them throughout product life cycle, making complex the assessment process. Life cycle approach is able to deal with it. As such, Life Cycle Sustainability Assessment (LCSA) analysis takes into account all stages of a product's lifecycle, when assessing performances, being considered a holistic tool used to measure product sustainability. Therefore, LCSA refers to environmental, social and economic assessments of product systems from a life-cycle perspective in order to promote product sustainability [2, 3].

However, the use of this type of result in organizational decision-making is not obvious. Halog and Manik [4] highlight three characteristics that increase the complexity of decision-making through LCSA results: (i) the indicators are multidimensional (each one is expressed in different units), (ii) the objectives are contradictory for the majority of decision-making problems (it is impossible to maximize the performance of a product system in all indicators) and (iii) performance evaluation is uncertain.

In this type of decision-making problem, decision makers must choose according to different criteria (LCSA indicators), leading to multiple criteria decision problem. Multiple criteria decision analysis (MCDA) approach aims to recommend an ideal solution, which is not necessarily optimal in all criteria, but a compromise solution according to the value judgment of decision-makers. The main advantage of this approach is its ability to consider a relatively large number of criteria when making a decision [5]. Laurin et al. [6] remark this approach when comparing product systems through the analysis of compromise recommendations.

LCSA results could be too disaggregated and, as such, too difficult to understand and interpret by decision-makers [7]. The use of these indicators in combination to support sustainable decision-making can be done through MCDA methods, as proposed by several authors [8-11].

We found few scientific researches discussing the use of the multiple criteria decision analysis approach into decision-making comprising LCSA results for sustainable decision-making ([9, 12-15, 7]).

The researches carried out by Vynies et al. [9] and Keller et al. [12] rely on arbitrary and unjustified aggregation procedures and neglect uncertainty when selecting a product system between a set of alternatives based on LCSA performances. Myllyvitta et al. [13] used MCDA approach to identify and weight relevant impact categories when assessing the environmental impacts of biomass production. Traverso et al. [7] provide a tool for comparing LCSA performance-based product systems. However, it is not clear how they defined the procedure for establishing the weighting factors and they do not take into account the uncertainty associated to LCSA performances. Finally, Hanandeh and El-Zein [14] adapted Electre III method to account for the uncertainty associated with preference data as weighting factors when choosing between alternatives based on LCA performance.

As such, we did not find many studies carrying out analyses including LCSA uncertainties, or the implications of choosing among the various MCDA methods 
existing in the scientific literature. This paper aims to propose an approach able to support sustainable decision-making where the considered indicators are defined as LCSA performances.

\section{Methodology}

The assessment of the performance of products in each pillar of sustainability is uncertain and can vary widely due to the parameters of the impact assessment model and input data. Different sources of uncertainty are present in environmental, social and economic studies, considering life cycle analysis. It is therefore necessary to take these uncertainties into account when deciding.

As such, our methodology includes three phases: (i) assessing uncertain LCSA performances for the three pillars of sustainability (ii) extending LCSA performances uncertainty to MCDA methods and (iii) interpreting stochastic rankings provided by the MCDA methods implemented, followed by conclusions.

The achievement of uncertain performance followed the ReCiPe method [16] for the environmental component and Social Hotspot database results for the social pillar. For the economic pillar, life cycle cost was considered. We applied "environmental life cycle costing (LCC)" according to Hunkeler et al. [17] to calculate the sum of private costs supported by all stakeholders involved throughout entire product life cycle, i.e. beyond the costs of the producer. Please note that in environmental LCC, no externalities are monetized (e.g. health care costs due to air pollution from trucks). Because of the strong inflation in Brazil in recent years, costs from year 2012 were have been adjusted with national inflation rates. No discounting was considered because of the relatively short duration of the tire life cycle.

The sources of uncertainty for environmental, social and economic dimensions are related to reference flows (number of tires required-considering the lifetime and the fuel consumption during use). For environmental dimension, we also considered the uncertainty related to ecoinvent, the end-of-life benefits, tire wear, transport distances, land use change and yield for hevea and soybean agriculture and emission factors. On the other hand, we did not consider the uncertainty associated to prices for social and economic dimensions and the ones associated to environmental and social characterization factors.

To represent these sources of uncertainty in LCSA performances, we performed a Monte Carlo simulation for all indicators comprised in the three pillars of sustainability.

In order to propagate the uncertainty on LCSA performance scores to decision-making problem, we applied three MCDA models (Weighted sum, Topsis and Prométhée II). These models were chosen according to the type of results provided (multiple criteria methods able to provide full rankings), the ease of being implemented without specific software package (ease of use in generic software, for example Microsoft Excel) and the type of parameters requested from decision 


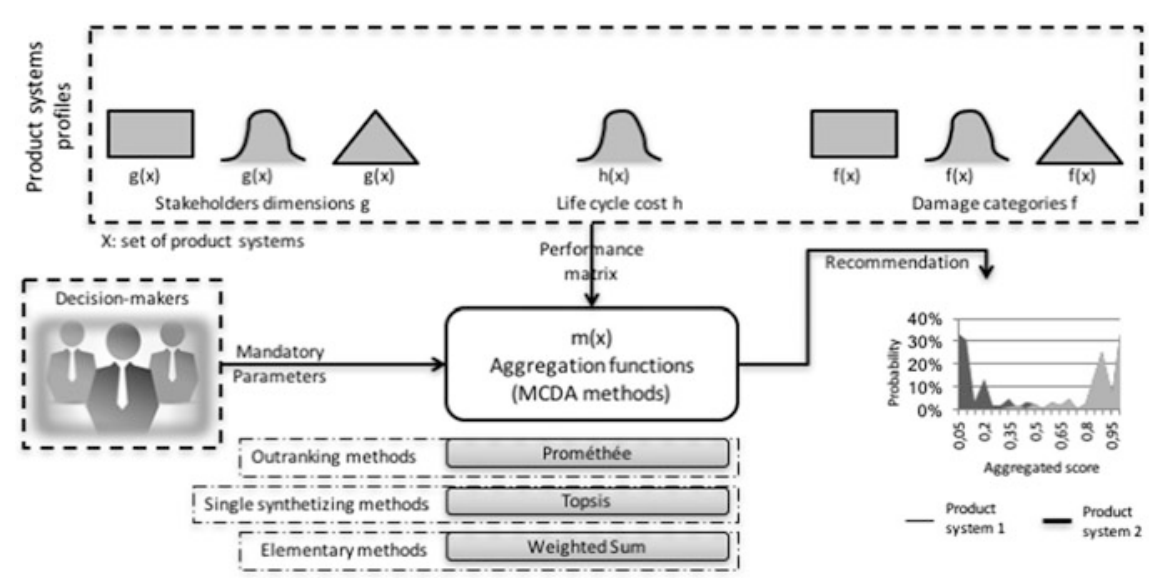

Fig. 1 Methodology proposed for support sustainable decision-making through LCSA uncertain performances

makers (parameters needed must be easy to be understood by decision makers through direct elicitation process).

Each method requires different parameters, for example the weights of each criterion (all methods) and the equivalence, weak preference and strict preference zones (for the Prométhée II method), as remarked by Carmo et al. [18]. These parameters were defined from an interview (elicitation process) with decision makers (three company's truck tire development experts). We run each model 1000 times, equivalent to the amount of Monte Carlo simulations we have carried out to generate the overall environmental, social and economic performances. As such, we got 1000 comparisons between product systems, each of which gives an order of preference.

In the last step, we analysed the probabilities for a product system to rank in a given position. This generates the level of confidence of the general ranking. Figure 1 illustrates the proposed method.

Our approach can be applied to all decision problems where uncertain social, environmental and economic life cycle performances are used as decision criteria when ranking products according to LCSA performances.

\section{Results}

\subsection{Case Study Description}

The focus of this case study is the life cycle of truck tires in Brazil. More specifically, this study compares, from a life cycle perspective, the potential 


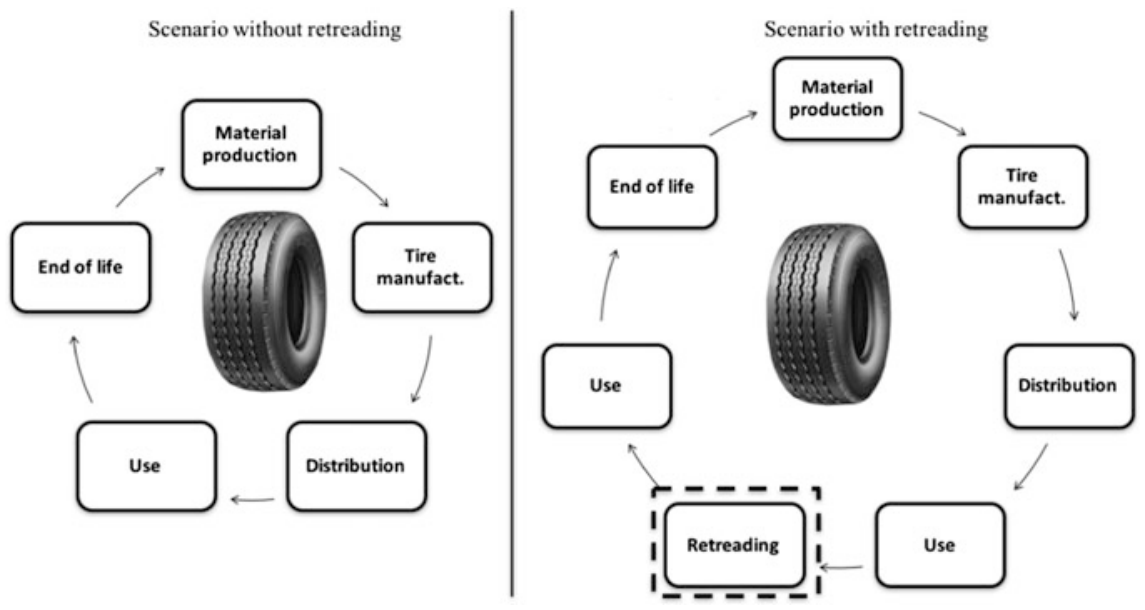

Fig. 2 Life cycle phases included into life cycle sustainability assessment

environmental and social impacts; together with life cycle costs of used tire management scenarios associated with different life extension options.

As such, this case study aims to analyse environmental, social and cost performances obtained through life cycle assessment approach in order to support the choice between two scenarios:

Scenario 1, without retreading - the tire reaches its final end-of-life once its original tread reaches maximum wear.

Scenario 2, with retreading - when the tread reaches maximum wear, the tire is retreaded and reused for the same freight transport.

The functional unit is the same for the three sustainability dimensions: "providing tires for truck transport with a payload of 32 metric tons over $600,000 \mathrm{~km}$ in Brazil in 2012 and managing used tires". It offers an impact evaluation based on the kilometres travelled rather than the number of units sold. Figure 2 illustrates the product system considered in our analysis.

\subsection{Case Study Results}

For the first phase of our methodology, assessing uncertain LCSA performances for the three pillars of sustainability, the performances were obtained from 2 successive studies conducted in 2015 and 2016 [19, 20]. Both studies are not publicly disclosed and include confidential information. The indicators considered in our Case study are illustrated in Fig. 3.

Figure 4 presents the performances for each indicator considered for the three pillars of sustainability. 


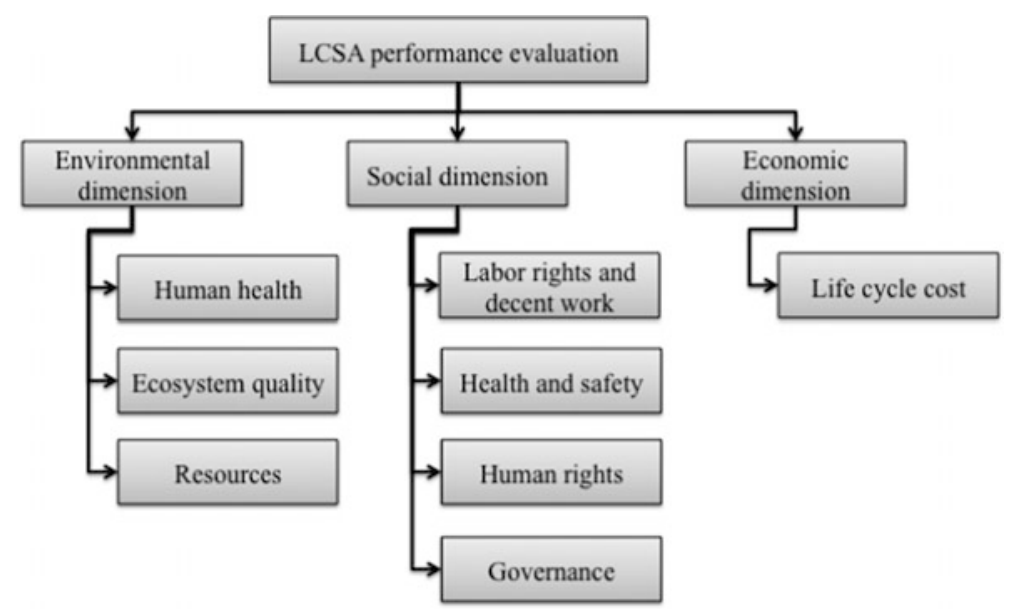

Fig. 3 Indicators adopted for life cycle sustainability assessment

Considering the second phase of our methodology, extending LCSA performances uncertainty to MCDA methods, we applied the three MCDA methods through two sets of weighting factors, as presented in Table 1.

Finally, for the third phase of our method, Fig. 5 presents the probability that the scenario with retreading scores better than the scenario without retreading, considering the three MCDA methods, two sets of weighting factors provided by decision makers (Table 1) and the probabilistic environmental, social and economic performances obtained by LCSA approach (Fig. 3). The confidence level is obtained by the counts of simulations where a scenario ranks higher than the other and normalized the count by total number of simulations.

Scenario with retreading is the preferred solution compared to scenario without retreading with more than $80 \%$ probability for the weighted sum and Topsis methods. The preference of the retreading scenario is reduced down up to $60 \%$ with the Prométhée II method, because this type of approach (outranking) takes into account the indifference and preference thresholds, creating the zones of equivalence and weak preference. As such, scenario with retreading seems to be a strong compromise solution for our case study for all combinations of MCDA methods and sets of weighting factors.

\subsection{Discussion}

This research highlights that it is feasible to account for the uncertainty associated with LCSA indicators in a decision-making process when applying MCDA methods. We were able to generate ranking about the preference of an option compared to the other whilst informing the decision-maker on the level of 

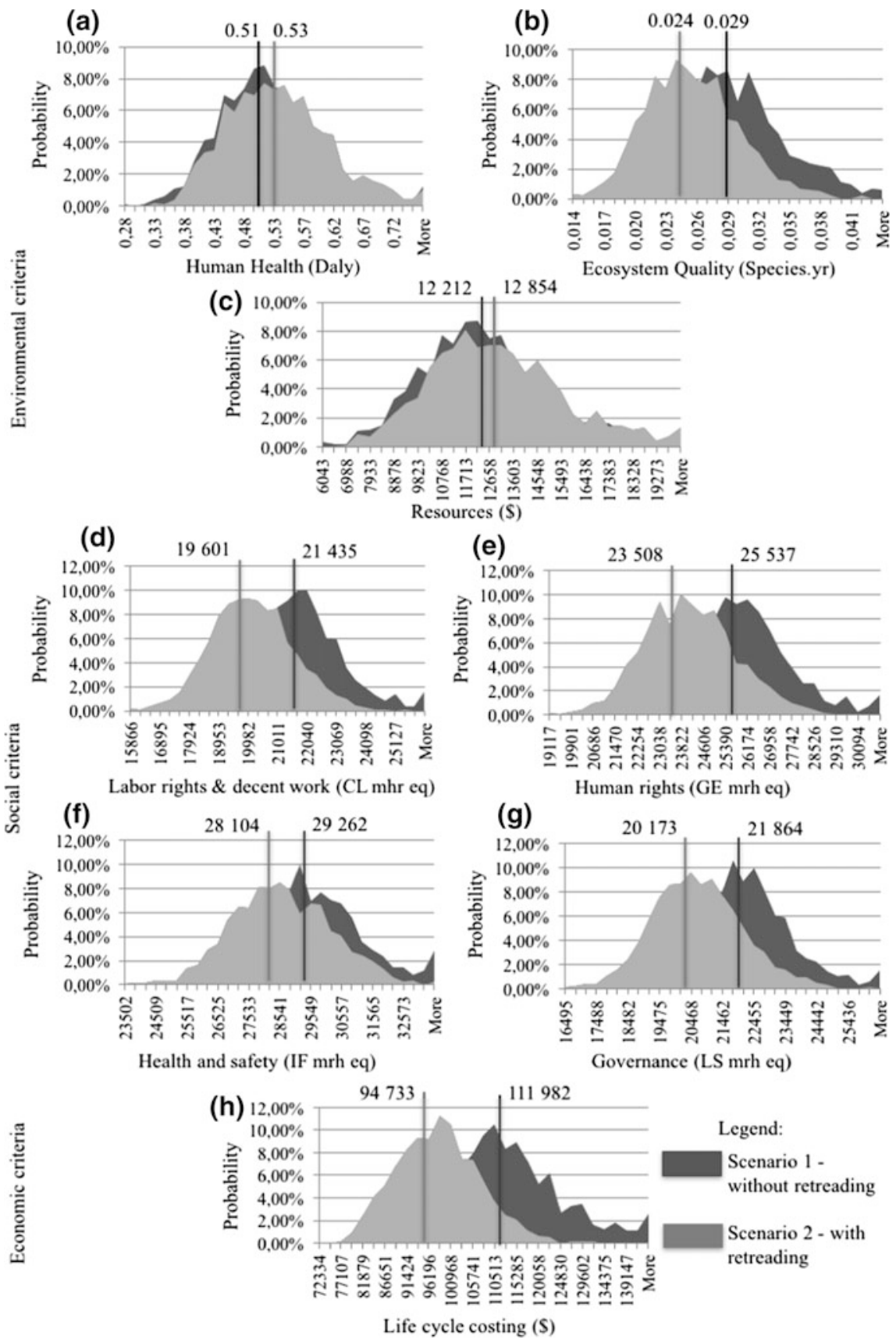

Fig. 4 LCSA performances for scenarios with retreading and without retreading 
Table 1 Sets of weighting factors considered for MCDA methods

\begin{tabular}{l|l|l|l}
\hline \multicolumn{2}{l|}{ Decision variables } & \multicolumn{3}{l}{ Weighting factors } \\
\hline \multirow{2}{*}{ Sustainability dimensions } & Decision criteria & Set 1 $(\%)$ & Set 2 $(\%)$ \\
\hline & Labour rights and decent work & 8 & 8 \\
\cline { 2 - 4 } & Health and security & 8 & 8 \\
\cline { 2 - 4 } & Human rights & 8 & 8 \\
\cline { 2 - 4 } & Governance & 8 & 3 \\
\hline \multirow{2}{*}{ Environmental } & Human health & 11 & 8 \\
\cline { 2 - 4 } & Ecosystem quality & 11 & 5 \\
\cline { 2 - 4 } & Resources & 11 & 13 \\
\hline \multirow{2}{*}{ Economic } & Life cycle cost & 34 & 50 \\
\hline
\end{tabular}

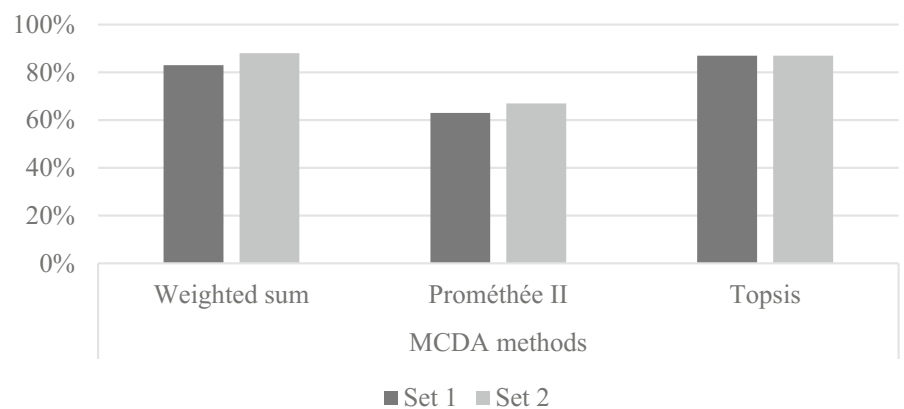

Fig. 5 Probability that Scenario with retreading scores better than Scenario without retreading for the two sets of weighting factors

confidence in such final ranking. Stochastic results provide a measure of the robustness of the ranking and conclusions. Scenario with retreading show a higher probability to reflect the best compromise solution according the value judgments of the decision-makers.

Our approach is very useful when ranking products considering their uncertain LCSA performances. It be also applied in cases where many scenarios are compared.

\section{Conclusions}

As all sustainable decision-making problems based on LCSA approach, choosing a scenario to supply tires for road transportation involves trade-offs when including the three pillars of sustainability into decision-making process. We used the MCDA approach to rank the potential scenarios and recommended a solution offering the better compromise in the view of decision-makers value judgments. 
The implementation of three MCDA methods through two sets of weighting factors and uncertain LCSA performances allowed analysing the robustness of the recommendation. From the results, we conclude that scenario with retreading is preferred than the one without retreading. The stochastic results revealed this scenario as a strong compromise solution.

This case study presented the importance of taking into account the following three elements when supporting decision-making process through the MCDA approach applied to LCSA performances: (i) implement different MCDA methods with different aggregation characteristics; (ii) vary the MCDA mandatory parameters and (iii) take into account the uncertainty of the LCSA performances.

The first element allowed analysing the similarity among the compromise recommendations from each method. Secondly, using different sets of mandatory parameters allowed incorporating the imprecision associated to the preference elicitation process, improving the representativeness of the decision-maker's value judgment. Finally, taking into account the uncertainty of the performances increased the robustness of the compromise ranking provided by each method.

\section{References}

1. Martín-Gamboa M, Iribarren D, García-Gusano D, Dufour J, A review of life-cycle approaches coupled with data envelopment analysis within multi-criteria decision analysis for sustainability assessment of energy systems, Journal of Cleaner Production, vol. 150, 2017, pp 164-174.

2. Zamagni A, Pesonen H-L, Swarr T, From LCA to life cycle sustainability assessment: concept, practice and future directions, The international journal of life cycle assessment, Vol. 18, 2013, pp 1637-1641.

3. Heijungs R, Huppes G, Guinée J.B, Life cycle assessment and sustainability analysis of products, materials and technologies. Toward a scientific framework for sustainability life cycle analysis, Polymer Degradation and Stability, Vol. 95, 2010, pp 422-428.

4. Halog A, Manik Y, Advancing integrated systems modeling framework for life cycle sustainability assessment, Sustainability, Vol. 3, 2011, pp. 469-499.

5. Shärlig A, Décider sur plusieurs critères: panorama de l'aide à la décision multicritère, Presses polytechniques et universitaires romandes, 1985.

6. Laurin L, Amor B, Bachmann T.M, Bare J, Koffler C, Genest S, Preiss P, Pierce J, Satterfield B, Vigon B, Life cycle assessment capacity roadmap (section 1): decision-making support using LCA, The international journal of life cycle assessment, Vol. 21, 2016, pp 443-447.

7. Traverso M, Finkbeiner M, Jorgensen A, Schneider L, Life cycle sustainability dashboard, Journal of industrial ecology, Vol. 16, No. 5, 2012, pp 680-688.

8. Finkbeiner M, Schau E M, Lehmann A, Traverso M, Towards life cycle sustainability assessment, Sustainability, Vol. 2, 2010, 3309-3322.

9. Vinyes E, Oliver-Solà J, Ugaya C, Rieradevall J, Gasol C.M, Application of LCSA to used cooking oil waste management. The international journal of life cycle assessment, Vol. 18, 2013, 445-455.

10. Lora E.E.S, Palacio J.C.E, Rocha M.H, Reno M.L.G, Venturini O.J, Olmo O.A, Issues to consider, existing tools and constraints in biofuels sustainability assessments, Energy, Vol. 36, 2011, pp 2097-2110. 
11. Bachmann T.M, Towards life cycle sustainability assessment: drawing on NEEDS project's total cost and multi-criteria decision analysis ranking methods, The international journal of life cycle assessment, Vol. 18, 2013, pp 1698-1709.

12. Keller H, Rettenmaier N, Reinhardt G.A, Integrated life cycle sustainability assessment - a practical approach, Applied Energy, Vol. 154, 2015, pp 1072-1081.

13. Myllyviita T, Holma A, Antikainen R, Lähtinen K, Leskinen P, Assessing environmental impacts of biomass production chains - application of life cycle assessment (LCA) and multi-criteria decision analysis (MCDA), Journal of Cleaner Production, Vol. 29, 2012, pp 238-245.

14. Hanandeh A.E, El-Zein, The development and application of multi-criteria decision-making tool with consideration of uncertainty: The selection of a management strategy for the bio-degradable fraction in the municipal solid waste, Bio resources Technology, Vol. 101, 2010, pp 555-561.

15. Benetto E, Dujet C, Rousseaux P, Fuzzy multicriteria approach with uncertainty analysis for Life Cycle Assessment. Environmental Software and Modelling, Vol. 23, Issue 12, 2008, pp 1461-1467.

16. Goedkoop M.J, Heijungs R, Huijbregts M, De Schryver A, Struijs J, Van Zelm R, ReCiPeA life cycle impact assessment method which comprises harmonised category indicators at the midpoint and the endpoint level; First edition Report I: Characterisation; 6 January 2009, http://www.lcia-recipe.net.

17. Hunkeler D, Lichtenvort K, Rebitzer G (eds.) Environmental life cycle costing. SETAC, Pensacola, FL (US) in collaboration with CRC Press, Boca Raton, FL, USA, 2008.

18. Carmo B.B.T, Margni M, Baptiste P, Ranking product systems based on uncertain life cycle sustainability assessment: A stochastic multiple criteria decision analysis approach, Journal of Cleaner Production, submitted.

19. Imbeault-Tétreault H, Carmo B.B.T, Life cycle sustainability assessment of tires retreading. Montréal, $51 \mathrm{p}, 2017$.

20. Imbeault-Tétreault H, Beaulieu L, Fallaha S, Russo Garrido S, Samson R., Revéret J-P, ACV environnementale et sociale d'options de prolongation de la vie utile de pneus de poids lourds au Brésil. Montreal, 294 p, 2015.

Open Access This chapter is licensed under the terms of the Creative Commons Attribution 4.0 International License (http://creativecommons.org/licenses/by/4.0/), which permits use, sharing, adaptation, distribution and reproduction in any medium or format, as long as you give appropriate credit to the original author(s) and the source, provide a link to the Creative Commons license and indicate if changes were made.

The images or other third party material in this chapter are included in the chapter's Creative Commons license, unless indicated otherwise in a credit line to the material. If material is not included in the chapter's Creative Commons license and your intended use is not permitted by statutory regulation or exceeds the permitted use, you will need to obtain permission directly from the copyright holder.

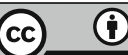




\title{
The Value of Transdisciplinary Perspectives During Transition to a Bio-based Economy: The Prospect for Converting Mixed Food Waste into Bio-based Chemicals
}

\author{
Birgit Brunklaus, Emma Rex, Johanna Berlin, Frida Røyne, \\ Johanna Ulmanen and Graham Aid
}

\begin{abstract}
Within the current political and industrial transition to a bio-based economy, food waste can be an alternative resource for biobased chemicals. This chapter describes a case study that evaluates the prospect for Swedish production of biobased chemicals such as succinic acid from food waste. The evaluation is addressed from multiple systems perspectives. From a technical and resource system perspective, the results of the case study show that production seems possible. However, from a social system perspective succinic acid production currently lacks institutional support and actor commitment and alignment for realizing development in Sweden. From an environmental and life cycle perspective, the scoping of the analysis is decisive for the results. The study shows that multiple perspectives complement each other when seeking a nuanced evaluation of technical innovation and give insights for the intended value chain.
\end{abstract}

\section{Introduction}

In line with current political and industrial ambitions for a transition into a bio-based economy, food waste can be an alternative to agro- and forest-based resources. The amount of food waste generated every year is about 1.3 billion tons globally [1]. This wasted food has caused problems related to climate change and resource depletion throughout their value chains, as well as increasing economic challenges related to waste disposal.

\footnotetext{
B. Brunklaus $(\bowtie) \cdot$ E. Rex $\cdot$ J. Berlin $\cdot$ F. Røyne $\cdot$ J. Ulmanen

Energy and Circular Economy, RISE Research Institutes of Sweden,

41261 Gothenburg, Sweden

e-mail: birgit.brunklaus@ri.se

G. Aid

R\&D Engineer and Group Strategy, Ragn Sells AB, Stockholm, Sweden

(C) The Author(s) 2018

E. Benetto et al. (eds.), Designing Sustainable Technologies,

Products and Policies, https://doi.org/10.1007/978-3-319-66981-6_36
} 
Some of these problems could be eased by converting food waste into valuable products like bio-based chemicals. Succinic acid (SA) is one such chemical with high market potential [2]. Today, it is produced from fossil sources but also partly from cultivated biomass on a commercial basis [3]. Several initiatives are now underway to examine the use of food waste as feedstock for SA production. Most of these initiatives are limited to homogenous industrial waste streams, but a research project in Sweden is looking at a more novel and complex feedstock — mixed food waste (MFW) [4].

The purpose of this project is to evaluate the prospect for realizing Swedish production of bio-based chemicals from MFW. In this chapter, we present the evaluation results of one possible production route, where microbial production of SA from MFW in Sweden is used as a case study.

The evaluation of SA from MFW is addressed from multiple perspectives: technical and social system structures are explored, in addition to resource and environmental implications. Several perspectives and methods are thus involved in the evaluations, such as the technical (laboratory cultivation and mechanical testing), the social (actor analysis, policy analysis, market analysis, and societal acceptance), the resource based (waste flow analysis and scenario analysis), and the environmental (life cycle assessment of current and future valorization techniques for MFW). The project thus uses a transdisciplinary approach and offers an arena where both research partners and industrial partners meet and discuss possible options for MFW-based SA production in Sweden.

\section{Multiple Perspectives and Evaluation Methods for Converting Mixed Food Waste into Bio-based Chemicals}

The case study chosen here involves microbal growth on mixed food waste derived from municipal solid waste to produce bio-based SA in Sweden. The mixed food waste was sourced from Ragn Sells AB's full-scale waste management facility in the area of Stockholm, Sweden. In Sweden, the current value pathway for MFW involves anaerobic digestion to produce biogas [5]. The future production could involve the cultivation of Escherichia coli bacteria to produce succinic acid as a raw material for further polymerisation. The case study was chosen together with academic and industrial partners in a multidisciplinary research project on how mixed food waste can be used as a resource for the production of bio-based chemicals and higher value products than current biogas (FORMAS 211-2012-70). Research questions derived from different partners included different disciplines, such as molecular biology (natural science), biotechnology (bio-based engineering), as well as social systems perspectives (social science) and environmental systems science (resource and life cycle perspective). Multiple perspectives, scales and methods were thus involved in the different evaluations presented in Table 1, which lead to different results presented in the following sections. 
Table 1 Multiple perspectives, scale and methods involved in the evaluation

\begin{tabular}{l|l|l}
\hline Multiple perspectives & Scale of analysis & Multiple methods \\
\hline Technical evaluation & Lab scale & $\begin{array}{l}\text { Elementary analysis } \\
\text { Laboratory cultivation } \\
\text { Mechanical property }\end{array}$ \\
\hline Social evaluation & $\begin{array}{l}\text { Innovation systems } \\
\text { National and EU scale }\end{array}$ & $\begin{array}{l}\text { Policy analysis } \\
\text { Societal acceptance } \\
\text { Screening of market }\end{array}$ \\
\hline Resource evaluation & Plant and national scale & $\begin{array}{l}\text { Material flow analysis } \\
\text { Scenario analysis }\end{array}$ \\
\hline Environmental evaluation & Plant and life cycle scale & $\begin{array}{l}\text { Life cycle assessment } \\
\text { Current and future system }\end{array}$ \\
\hline
\end{tabular}

\subsection{Technical Evaluation Performed on Lab Scale}

Several research questions and methods are involved in the technical evaluation e.g. laboratory cultivation and mechanical testing. By the use of elementary analysis, the composition of substances/molecules in mixed food waste was identified. The results show that mixed food waste contains required $\mathrm{N}$ and $\mathrm{C}$ for E.coli growth, although additional $\mathrm{N}$ would increase $\mathrm{C}$ use. Enzymatic hydrolysis and carbohydrate analysis was then used to show how the carbon sources included could be made more accessible for E.coli uptake [6]. Through laboratory cultivating tests it was assessed if E.coli could grow on mixed food waste. The results show that the liquid fraction of the waste supports growth and the solids can be used as carbon source. To determin if the water content of MFW was optimal for E.coli growth, mechanical property tests were made. The results show that the water content was too high for industrial production, described in Rex et al. [7].

\subsection{Social Evaluation Based on Innovation Systems}

Several research questions and methods were also involved in the social system evaluation, e.g. policy analysis, analysis of societal acceptance, actor and market analyses. A policy analysis was performed to assess how existing rules and regulations influence the possibility to realize SA production from mixed food waste in Sweden. The main source of data for the analysis were documents on policy directives and instruments complemented with workshops and interviews with focus groups. A key result is that there are hardly any policy instruments supporting bio-based chemical development on national and EU level. So far there exists only declarations of intent in various policy documents and reports and limited R\&D subsidies. Another key result is that subsidies and investments in the development of biofuels, in particular biogas, for the transport sector influence the access of 
feedstock for the production of bio-based chemicals and might contribute to increased competition in the future.

Societal acceptance is essential for the legitimacy of an innovation and to assess how norms and values in society influence the prospects of producing SA from mixed food waste. The main source of data for the analysis were interviews and workshops complemented with analysis of discussions in related areas, such as GMO and food versus fuel (e.g. through newspaper articles, NGO webpages, and research reports). The results show that the perceptions of circularity of biomass and bio-based chemicals and related products are positive. However, public resistance towards genetically modified organisms has previously hampered the realization of a bio process production facility and may do so in the case of SA as well.

A screening of market data and scenarios was carried out to judge the future demand of increased SA production. The main source of data for the analysis were documents and literature on future market. The results indicate that there is a demand for bio-based SA and that it is expected to grow in the future considering bio-based SA can substitute a range of fossil chemical components, not only fossil SA. Also, there is an interest of producing and buying bio-based chemicals and related products in a more resource efficient manner, e.g. using waste instead of the currently used food crops.

Finally, a mapping of current and future actors was made to determine if the right type of actors can be mobilized to realize a SA value chain to enable the production facility. The results show that although technical competences exist and market demand is expected in the future, actors are not yet fully committed or aligned to develop bio-based chemicals production such as SA from food waste. Further details can be found in related work of Ulmanen et al. [8].

\subsection{Resource Evaluation Based on Waste Flows}

Several research questions and methods were involved in the resource evaluation e.g. material flow analysis and scenario analysis. The question of which capacity is needed for production of bio-based SA was approached using benchmark of existing and planned production sites for bio-based SA production. The results show that a capacity of at least 10,000 tons bio-based SA/year is likely to be needed for a commercial plant. Material flow analyses were used to determine if there is sufficient mixed food waste in Sweden for that production capacity. The results show that there is sufficient mixed food waste in Sweden as a whole, but not in individual facilities. Further, scenario analysis (including material flow analysis and policy analysis) was used to assess if there is sufficient mixed food waste in Sweden for the production capacity, also given political goals to minimize avoidable food waste. The results show that there are sufficient flows in Sweden, although distributed among sites in a large geographical area. Further details can be found in related work of Rex et al. [7]. 


\subsection{Environmental Evaluation Based on the Life Cycle}

In the environmental evaluation, a life cycle assessment of current and future systems was performed. Life cycle assessment with focus on valorisation options was used to analyse if the current biogas production or the future production of SA had the lowest environmental impacts as valorisation option for mixed food waste. The process description of biogas is based on an existing biogas facility in Heljestorp [9]. The process description used of food waste to succinic acid is based on lab-scale data with mixed food waste [10]. The results show that current biogas production has a better environmental performance on all main impacts categories used (global warming potential, energy use, acidification potential, eutrophication potential) than future production of SA with mixed food waste. In parallell, a life cycle assessment with focus on feedstocks was performed to adress the question: Do mixed food waste have a better environmental performance than current feedstocks for SA production? The process description of Corn to succinic acid has been chosen based on Reverdia Direct Crystallization process described in Cok et al. [11] and Smidt et al. [12]. The results show that a future use of mixed food waste likely would have a better environmental performance than current corn-based feedstocks. Further details on the comparison and evaluation can be found in related work of Carlson [13] and Brunklaus et al. [14].

\subsection{Multiple Evaluations Show Complementing Results}

As seen from above, multiple perspectives and methods are involved in the different evaluations and show complementing results. The results of the case study show that, even though from a technical and resource system perspective, production seems possible, from a social system perspective SA production currently lacks institutional support and actor commitment and alignment for realizing development in Sweden. From an environmental and life cycle perspective, the scoping of the analysis is decisive for the results: SA production from mixed food waste is environmentally beneficial if compared with the SA production from corn, but from a pure waste handling perspective, it is environmentally better to produce biogas than SA from mixed food waste.

\section{Potentials and Limitations of Combined Systems Approaches}

Different systems approaches provide guidance about current and future industrial systems. Each of the method approaches are based on different questions and provide different answers. To take this further, one has to understand some general 
approaches in systems science: What are the differences in goal and scope? What are the potentials and limitations of combined systems approaches?

\subsection{Differences in Goal and Scope}

Systems approaches are based on the goals and the scope of the study. To say something about how valid the different results of the multiple perspectives are in relation to each other, it is important to understand the object and the scope of analysis of each assessment, presented in Table 2.

In the technical evaluation, the object of analysis is the biotechnological possibilities and the scope of the study lies on the molecular scale and the laboratory scale. Results have to be seen per scale and technical indications can be made limited to the scale. The social evaluation was made as part of of a technological innovation systems analysis on national and EU scale. Here the scope and boundaries of the study is based on so called structural components, that includes technology, actors, networks and institutions. Institutions refers to formal policy rules and regulations as well as informal norms and values related to societal acceptance. In the resource evaluation, material flows are in focus and the scope and boundaries the study is based on the production scale and geographical scale. In the environmental evaluation, the object of analysis is physical flows of material, resources, waste and energy, and the scope and boundaries of the study is based on processes that are part of the life cycle of handling food waste and producing SA, respectively. Results for resource and environmental evaluations have to be seen per scale and indication have to be seen as well in temporal scale of current and future value chains.

Systems approaches as used in the example above require significant time and efforts, as well as expertise in different scientific areas. These are essential for complex problem solving, and are thus system dependent as described in Flood and Calsson [15]. The potential in relation to systems perspectives lies in knowledge creation. Knowledge is built on like building blocks, which together forms the basis for decision-making and the next step in the innovation process.

Table 2 Multiple perspectives, object and scope of the analysis involved

\begin{tabular}{l|l|l}
\hline Multiple perspectives & Object of the anlaysis & Scope of the analysis \\
\hline Technical evaluation & Biotech possibilities & Molecular scale, lab scale \\
\hline Social evaluation & Innovation systems & National and EU scale \\
\hline Resource evaluation & Material and waste flows & Production scale \\
\hline Environmental evaluation & Current and future process & Life cycle, temporal scale \\
\hline
\end{tabular}




\subsection{Potentials in Relation to Life Cycle Management}

Taken together, the studies differ in both the objects of analysis and the scope and boundaries in which the results are valid. Moreover, methods used derive from different research traditions and method approaches such as the analytical, the system and the social approaches described in Arbor and Bjerke [16] and Wolf [17]. Due to different research traditions and method approaches and their fundamental discrepancies, and the related amount of work and efforts made, it might be questionable to relate answers from these perspectives in the same assessment, as is the case in the example above. Yet we argue that for Life Cycle Management, assessing multiple perspectives and transdiciplinary approaches is a valuable way of working. Life Cycle Management is about decreasing the negative impact of physical flows in society, by approaching the acts and practices of actors in the system. In this respect, there is a need to understand the premises for success in everything from technological possibilities in lab scale to the amount of materials available in a region, and the norms, values and intentions of actors needed to develop the system. Thus, combined systems studies should, with careful respect to the differences in goal and scope, be seen as complementary studies to guide technology innovation. Insights form these multiple perspectives can be of value for any actor aiming to manage these systems for the advances of less environmental impact from a life cycle perspective. Further research on evaluating various actors might give some insights on of how useful the various systems studies were in the collaboration, and toward decision making. To this end, the above perspectives are seen as complementary, yet not exhaustive. An important perspective not included in the above is an assessment of the economic feasibility of SA from MFW. Also combining SA with other products in a bio-refinery has not been evalutated, to give some examples.

\section{Conclusion}

Combined-systems approaches and multiple methods contribute with a useful holistic perspective to evaluate and guide technology innovation from a life cycle perspective. Results from a case study on bio-based SA does not provide a unanimous answer on whether the prospects for transforming MFW into bio-based chemicals are favorable or not, but gives a nuanced evaluation that offers a more firm foundation for decision and further development than one perspective or method in isolation. The transdisciplinary approach can inform an arena of research and industrial partners with different perspectives to facilitate discussion and more well-informed decisions. Despite time and efforts, the case thus illustrates the benefits of applying a transdisciplinary approach with multiple perspectives when assessing and evaluating potential routes to a more bio-based economy, and when seeking to manage the progress of sustainable value chains. 
Achnowledgements This research is financed by FORMAS and this paper builts on earlier studies including different perspectives: "systems perspective" [7], "technological innovation system" [8] and "life cycle assessment" [13, 14]. The authors greatfully acknowledge the Swedish Research Council Formas for financing this research (FORMAS project no 211-2013-70), as well as Ragn Sells AB for kindly providing the mixed food waste and production facility data.

\section{References}

1. FAO, Global food losses - Extent, causes and prevention. Food and Agriculture Organization of the United Nations FAO, ISBN 978-92-5-107205-9, Rome, 2011.

2. US Department of Energy, Top value assed chemicals from biomass. Energy Efficiency and Renewable Energy, US Department of Energy, 2004.

3. Choi S, Song C.W, Shin J.H, Lee S.Y, Biorefineries for the production of top building block chemicals and their derivatives. Journal of Metabolic Engineering, Vol. 28, 2015, pp. 223-239.

4. FORMAS, Swedish Research and Innovation Strategy for a Bio-based Economy. Report No 3, Swedish Research Council Formas, 2012.

5. Avfall Sverige, Hushållsavfall i siffor-Kommun- och länsstatistik. Rapport no 33. Avfall Sverige, 2016.

6. Rosander E, Svedendahl Humble M, Veide A, Municipal solid waste as carbon and energy source for Escherichia coli. Journal of Advances in Waste Management and Recycling, Vol. 1, Issue 2, 2016, pp. 2-8.

7. Rex E, Rosander E, Royne F, Veide A, Ulmanen J, A systems perspective on chemical production from mixed food waste: The case of bio-succinate in Sweden. Journal of Resource, Conservation and Recycling, Vol. 125, 2017, pp 86-97.

8. Ulmanen J, Rex E, Torén J, Prospecting development of sustainable innovation in a context of multiple technology systems: The case of high platform chemicals from food waste. IST Conference at SPRU, Brighton, 2015.

9. RagnSells, Technical information and data on the Heljestorp biogas facility. Internal document. Ragnsells AB, 2016.

10. Sun Z, Li M, Qi Q, Gao C, Lin C.S.K, Mixed food waste as renewable feedstock in succinic acid fermentation. Journal of Applied Biochemistry and Biotechnology, Vol. 174, Issue 5, 2014, pp. 1822-1833.

11. Cok B, Tsiropoulos I, Roes A.L., Patel M.K., Succinic acid production derived from carbohydrates: An energy and greenhouse gas assesment of a platform chemcial towards a bio-based economy. Journal of Biofuels, Bioproducts and Biorefining, Vol. 8, 2014, pp. 16-29.

12. Smidt M, Den Hollander J, Bosch H, Xiang Y, Van Der Graaf M, Lambin A, Duda, J-P, Life Cycle Assessment of Bio-based and Fossil Based Succinic Acis. In: Sustainability Assessment of Renewables-Based Products: Methods and Case Studies. Chichester, UK: John Wiley \& Sons, Ltd., 2015.

13. Carlsson E., The future of food waste-LCA of existing and emerging routes to bio-based chemicals. Master thesis at Energy and Environment, Report nr 2016:8, Chalmers University of Technology, 2016.

14. Brunklaus B, Rex E, Berlin J, The future of Swedish food waste: An environmental assessment of existing and prospective valorization techniques. Accepted in Special Issue: Food waste-Challenges and Opportunities for the emerging Bio-Economy, Journal of Cleaner Production, 2017. 
15. Flood R.L, Carson E, Dealing with complexity: An introduction to the theory and application of systems science. Springer Science \& Business Media, 2013.

16. Arbnor I, Bjerke B, Företagsekonomisk metodlära. Electronic Eition. Studentliteratur, 2008.

17. Wolff R, Beyond environmental managemnet—perspectives on environmental and management research. Journal of Business Strategy and the Environment, Vol. 7, Issue 5, 1998, pp. $297-308$.

Open Access This chapter is licensed under the terms of the Creative Commons Attribution 4.0 International License (http://creativecommons.org/licenses/by/4.0/), which permits use, sharing, adaptation, distribution and reproduction in any medium or format, as long as you give appropriate credit to the original author(s) and the source, provide a link to the Creative Commons license and indicate if changes were made.

The images or other third party material in this chapter are included in the chapter's Creative Commons license, unless indicated otherwise in a credit line to the material. If material is not included in the chapter's Creative Commons license and your intended use is not permitted by statutory regulation or exceeds the permitted use, you will need to obtain permission directly from the copyright holder.

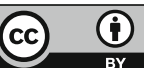




\title{
Improving Interpretation, Presentation and Visualisation of LCA Studies for Decision Making Support
}

\author{
Serenella Sala and Jessica Andreasson
}

\begin{abstract}
Interpretation, presentation, and visualization of life cycle assessment (LCA) results are key steps for ensuring proper decision support. Despite the interpretation being a crucial step, it is often not performed in a systematic way. For example, sensitivity analysis, variability and uncertainties analyses, comparison with results coming from different disciplines and domains are not properly done. So far, numerous LCA studies have been published and are often used by decision makers (both in business and policy contexts) to support the identification of hotspots or for drawing conclusions from meta-reviews while missing a good interpretation. Moreover, improving current practices in the visualisation of the results may help both the interpretation and the communication of LCA by a broader audience.
\end{abstract}

\section{Introduction}

According to ISO 14040, interpretation is the phase of LCA in which the findings from the inventory analysis and the impact assessment are considered together. It should deliver results that are consistent with the defined goal and scope of the study and which reach conclusions, explain limitations and provide recommendations. ISO 14044 further specifies that interpretation comprises the following elements: (i) identification of the significant issues based on the results of the life cycle inventory (LCI) and life cycle impact assessment (LCIA) phases of LCA; (ii) completeness, sensitivity and consistency checks; (iii) conclusions, limitations, and recommendations.

Despite the interpretation is a crucial step for supporting decision making, this step is often not performed in a systematic way. For example, sensitivity analysis from the

\author{
S. Sala $(\bowtie)$ \\ European Commission, Joint Research Centre, Directorate D-Sustainable Resources, \\ Bio-Economy Unit, via Enrico Fermi 2749, 21027 Ispra, VA, Italy \\ e-mail: serenella.sala@ec.europa.eu \\ J. Andreasson \\ Volvo Car Corporation, Gothenburg, Sweden \\ (C) The Author(s) 2018 \\ E. Benetto et al. (eds.), Designing Sustainable Technologies, \\ Products and Policies, https://doi.org/10.1007/978-3-319-66981-6_37
}


inventory step (e.g. between different source of secondary data) [1] up to the impact assessment (e.g. between different methods for impact assessment [2,3] and normalisation references [4]), variability and uncertainties analyses, comparison with results coming from different disciplines and domains are not properly done. So far, numerous LCA studies have been published and are often used by decision makers (both in business and policy contexts) to support the identification of hotspots or for drawing conclusions from meta-reviews while missing a good interpretation. In 2016, a guidance on interpretation has been published by the European Commission, Joint Research Centre [5], aiming at fostering a discussion on the critical role of interpretation and visualization of results. Besides, very different audiences, having different backgrounds and expectations, may use LCA results: decision-makers, scientists, public authorities, consumers, etc. The way LCA results are presented is too often generic instead of being tailored to their needs. Moreover, LCA deals with numerous environmental impact indicators, large amount of data, uncertainties, spatial differentiation etc. LCA has evolved from a simple carbon and energy accounting to the assessment of large and complex consequential systems. Still, the way the results are presented has not changed a lot, i.e. mainly bar-charts, pie-charts, simple tables and Sankey diagrams. In the meantime, data visualization and analysis has evolved, with new interactive ways of displaying results, which may both help interpretation and communication of the results.

\section{Interpretation, Presentation and Visualisation}

The session on "Improving interpretation, presentation and visualisation of LCA studies for decision making support" at LCM 2017 aimed at discussing how to promote a transition toward more robust interpretation, presentation, and visualization of the LCA results. This includes the identification of key elements and criteria for knowledge capitalization, with regard to existing studies.

Life cycle-based methodologies as LCA but also other integrated assessment approaches are used in decision-making processes, product development and marketing activities. LCA methodology may be used as a methodology for supporting the identification of 'hot spots' in the value chain and measuring progress towards sustainability targets. It is important for companies to find common ground on how to implement these approaches, which data assessments and impact assessments to be used and how results should be interpreted. ISO rules give a good basis for that work, though is not sufficient.

\subsection{Identifying Target Audience for LCA Results}

Within a company or an institution, before any successful adaptation and visualization of life cycle information, it is important for internal life cycle experts to 
identify and understand how other functions of the company or institution perceive and value life cycle information in their specific working situations and decision contexts. To get a better understanding of these internal users of life cycle information, life cycle experts in four multinational companies (Akzo Nobel, Vattenfall, Volvo Cars, Volvo Group) have joined forces with researchers in Life Cycle Management and behavioural science to create a graphical map of how life cycle information is spread and used in different parts of an organization. The map is constructed by combining (a) inventories on how quantitative data seeks its way to internal users through databases, reports and Key Performance Indicators (KPIs), with (b) qualitative interviews on goal framing and decision weights of e.g. environmental and economic information. As a result, the map illustrates both the "physical" flows of life cycle information and the "cognitive logics" of this information for different users (e.g. how values, attitudes and norms influence the target groups' likelihood of including life cycle information in their decision processes). Based on the map, each company can identify and discuss who the main users of life cycle information are and what premises for life cycle thinking these users have. In what decision-making situation is, or can, life cycle information be used? How is the information understood? What other sources of information and rationales for decisions are used in parallel to, or in conflict with, LCA results? Initial analyses on the usefulness of the map point to a better understanding of how life cycle experts can tailor information for decisions in different parts of the company, as well as on its usefulness in illustrating to people outside of the environmental departments the widespread use of life cycle information that already exist in the company.

Moreover, LCA results may be used to influence design solution for products, needing an interaction between LCA experts, products designer and consumers. This often happens is a context with certain legislative constraints or requirement. A model for supporting life cycle design of food and packaging has been presented at the LMC conference. Packaging designer cannot give packaging all functions, because it is impossible to grasp all relations among demands and functions, and there are trade-off relations among them. An activity model visualizing information flows among stakeholders was constructed to design an environmentally conscious product, having a broad life cycle perspective. This allowed to optimise design parameters (e.g., packaging material and weight, filling rate) and functions (e.g., permeability, usability) along the life cycle of food products.

\subsection{Tailoring the Presentation of LCA Results and Promoting Best Practices}

Several challenges are faced by industrial sustainability experts and practitioners when communicating and visualising LCA results in different decision-making contexts. The project SAMT (Sustainability assessment methods and tools to support decision-making in the process industries) [6], was focusing on 
communication of LCA results in different decision-making contexts addressing all those challenges. Experiences and good practices from the cement, oil, metal, water, waste and chemical industry were collected using interviews, workshops and practical case studies. Crucial aspects identified during the project were, among others: (i) communicating uncertainty; (ii) explaining the proximate and underlying causes of variability in results; (iii) integrating results representing different impact categories and defining proper benchmarks for comparison. Challenges related to interpretation and communication are faced especially in situations in which a decrease in one indicator or impact category is offset with an increase in another category. The findings of the project indicate that many of the challenges related to communication are similar despite of the sector in question. There is also a need to harmonise the way results are presented, in order to increase transparency and improve comparability of the studies.

For exchanging experiences, updating or adopting methods, the "International Sustainability Practitioners Network" (ISPN) was created since 2012. The ISPN is an exchange forum for LCA methodology in the context of industry and comprises sustainability experts from a range of different industry sectors. Examples of good practices of this cross-sectoral initiative and opportunities for improving sustainability assessments within of companies are the focus of the exchange between experts. The ISPN members address challenges and solutions in terms of data availability and uncertainty, streamlining and using standardization processes as well as communication of results with non-LCA-experts.

\subsection{Improving Visualisation of LCA Results}

Given the perceived complexity of LCA, improving the presentation and visualisation of results and the uncertainties thereof is crucial for decision support. New features have been presented for two softwares, namely Open LCA [7] and RangeLCA [8].

OpenLCA has expanded its interpretation component in order to better present the hotspots analysis using new visualisation features from the open source: radar charts, sun burst graphs, contribution trees, geographical display of regionalized results, and tables with the values of inventory, impact results and impact factor per elementary flow contributing to the hotspot towards a structured interpretation of the results. Moreover, an assessment and effective handling of the data quality is also key for the interpretation especially if the study is used for decision support. OpenLCA proposes an innovative way to calculate, aggregate and display data quality information along the life cycle, to make the detection of data quality hotspots easier, i.e. to detect e.g. whether hotspots appear in the case study to some extent because of weak data quality, or whether hotspots call for immediate action because they are based on reliable information. The calculated data quality is shown along with the LCI and LCIA results in the different visualisations of openLCA, including impact analysis tables and Sankey diagram. 
Besides, the LCA software RangeLCA is proposing new ways of visualizing comparative LCA results for representing the diversity of individual cases and the uncertainty. RangeLCA, automatically integrates a full sensitivity analysis by associating probability distributions or discrete ranges of values to each variable parameter. LCIA results are calculated for a large number of cases, each corresponding to a possible combination of variable parameters. For each impact category, results of all cases are plotted as a "cloud of points" in function of the most influential parameters. When comparing two systems, common variable parameters take common values for a same case. Results can hence be compared two by two and a delta between systems can be calculated for each case. Plotting these delta values in "delta graphs" allows the common variability to be eliminated and the interpretation to concentrate on the remaining variability. A colour code distinguishes positive and negative deltas. These graphs allow identifying areas of relevance where conclusions can be drawn for certain ranges of values of two main parameters. This type of graphs allows acquiring a more complete vision of a complex system, reliability of conclusions in comparative LCA by showing whether differences are significant or not in terms of sign and amplitude. Delta graphs and areas of relevance help LCA studies to answer much broader questions, such as: "In which range of situations is a system rather better than another one? "

\section{Conclusion and Outlook}

The last phase of a LCA, namely the interpretation of the life cycle inventory and impact assessment results, is especially important in LCM as it is key for decision-making and communication of results. Ability to create meaningful and easily understandable results would be essential in order to increase the acceptability and applicability of the methods, and to mainstream their use in different decision-making situations, that may include for example product and process development, supply chain management, investment decisions, marketing and responding to stakeholder requests. Future effort should be both on the side of improving the analytical power of LCA tools adopted by practitioners and in the understanding of the diversified communication needs of a wide range of stakeholders both in business and institutional context. From the analytical aspects point of view, a systematic approach in the interpretation and hotspots analyses, including methods for sensitivity analysis, variability and uncertainty analysis, is needed. From the communication point of view, there is the need of improving the identification of the target audience of the results, for tailoring the communication, including the rationale on how to choose visualization and graphical tools depending on the key messages to show and on the audience. This could be facilitated by the adoption of new ways of visualizing LCA results, using interactive and advanced visualization tools for both external and internal stakeholders. 


\section{References}

1. Corrado S, Zampori L, Castellani V, Sala S, Systematic analysis of secondary Life Cycle Inventories when modelling agricultural production: a case study for arable crops, Journal of Cleaner Production, Vol. 172, 2018, pp. 3990-4000.

2. Rigamonti L, Falbo A, Zampori L, Sala S, Supporting a transition towards sustainable circular economy: sensitivity analysis for the interpretation of LCA for the recovery of electric and electronic waste, International Journal of Life Cycle Assessment, Vol. 22, No. 8, 2017, pp. $1278-1287$.

3. Castellani V, Lorenzo B, Sala S, Hotspots analysis and critical interpretation of food life cycle assessment studies for selecting eco-innovation options and for policy support, Journal of Cleaner Production, Vol. 140, No. 2, 2017, pp. 556-568.

4. Benini L, Sala S, Integrated assessment of environmental impact of Europe in 2010: uncertainty and sensitivity of the normalisation factors to methodological assumptions, International Journal of Life Cycle Assessment, Vol. 21, No. 2, 2016, pp. 224-236.

5. Zampori L, Saouter E, Schau E, Cristobal Garcia J, Castellani V, Sala S, Guide for interpreting life cycle assessment result, JRC technical report, Publications Office of the European Union; 2016.

6. https://www.spire2030.eu/samt, (Accessed 05.09.2017).

7. http://www.openlca.org/, (Accessed 05.09.2017).

8. http://www.rdcenvironment.be, (Accessed 05.09.2017).

Open Access This chapter is licensed under the terms of the Creative Commons Attribution 4.0 International License (http://creativecommons.org/licenses/by/4.0/), which permits use, sharing, adaptation, distribution and reproduction in any medium or format, as long as you give appropriate credit to the original author(s) and the source, provide a link to the Creative Commons license and indicate if changes were made.

The images or other third party material in this chapter are included in the chapter's Creative Commons license, unless indicated otherwise in a credit line to the material. If material is not included in the chapter's Creative Commons license and your intended use is not permitted by statutory regulation or exceeds the permitted use, you will need to obtain permission directly from the copyright holder. 


\title{
Visualizing the Effects of Parameter Variability on Comparative LCA Results
}

\author{
Céline Alexandre, Elisabeth van Overbeke, Maxime Dupriez, \\ Johan Lhotellier and Bernard De Caevel
}

\begin{abstract}
Bar charts and other usual ways of presenting LCA results depict one average or typical situation, lacking to represent the diversity of individual cases and the uncertainties associated to input data or modelling assumptions. This paper presents ways of visualizing variable results in comparative LCA. The main concept is to perform at once calculations representing this variability. Based on Monte Carlo analysis, the approach is enabled by the LCA software RangeLCA, developed by RDC Environment. Results of all simulations can be plotted in function of one or two influential parameters. A clear and complete view can hence be obtained as well as more reliable conclusions. Instead of answering the question "Is a system better than another one in specific cases?", the presented graphs help LCA studies to answer a much broader question: "In which range of situations is a system better than another?".
\end{abstract}

\section{Introduction}

In most LCAs, some parameters are better characterized by variable values than by fixed "typical" values. The reasons for the variability can be of two types: uncertainty and diversity of situations. The uncertainty can be either systematic (e.g. linked to imprecision of the measuring instrument), stochastic (e.g. fluctuation of a parameter with time) or epistemic (e.g. modelling required in the absence of measurement) [1]. The diversity of potential situations within the studied system also leads to parameter variability, e.g. the transport to various customers is best modelled by a range of distances or a waste can be either incinerated or landfilled.

The way the input data variability influences the results can be analysed. The uncertainty analysis is the "systematic procedure to quantify the uncertainty introduced in the results of a life cycle inventory analysis due to the cumulative effects of model imprecision, input uncertainty and data variability" [2]. It is

C. Alexandre · E. van Overbeke $(\varangle) \cdot$ M. Dupriez $\cdot$ J. Lhotellier · B. De Caevel

RDC Environment, 1160 Brussels, Belgium

e-mail: elisabeth.vanoverbeke@ rdcenvironment.be

(C) The Author(s) 2018

E. Benetto et al. (eds.), Designing Sustainable Technologies,

Products and Policies, https://doi.org/10.1007/978-3-319-66981-6_38 
currently mostly performed by Monte Carlo analysis, which is available in several commercial LCA software tools [1].

Sensitivity analysis is defined as a "systematic procedure for estimating the effects of the choices made regarding methods and data on the outcome of a study" [2]. It helps identifying the most influential parameters, for prioritizing data refining and reinforcing conclusions. Sensitivity analyses are in current practice mainly carried out by varying one parameter at a time or by studying alternative scenarios [1].

Wei et al. [3] propose some guidelines on how to perform sensitivity analysis when some data and modelling parts are correlated. Groen et al. [4] discuss several approaches to conduct a sensitivity analysis. They analyse their capacities and drawbacks. They determined that the disadvantage of the method of elementary effects (MEE), using ranges of values from upper to lower boundary of an input parameter, is that "the results are not an estimation of the actual variance decomposition" but MEE can be used as a precursor to the more computationally demanding sampling methods as regression. They see as a main drawback of the standardized regression coefficients (using Monte Carlo sampling) that many runs are needed to calculate the variance decomposition.

In fact, the advantages of using ranges of values for input parameter and Monte Carlo sampling can be combined and the analysis is made easier to interpret by using some fast calculation methods and visual interpretation.

This paper describes the use of Monte Carlo analysis for depicting a large range of possible situations. Emphasis is put on presenting innovative ways of visualizing result variability, which helps interpretation and decision making. The discussion is based on a real case study.

\section{Description of the Case Study}

The selected case study aims at comparing two systems of packaging: a corrugated box versus a reusable folding plastic crate. The functional unit is defined as: the packaging, transport and delivery of goods in one case from the good manufacturer to the distributor or repacker. It is assumed that the cases have similar dimensions in both systems and contain the same number of goods.

For the purpose of this paper, results are only presented for the impact category global warming potential (GWP, with biogenic carbon taken into account). The study is divided into two parts: (i) the "average comparison" scenario, i.e. investigation of the impacts of two average cases, each representative of one system; (ii) the sensitive analysis, i.e. calculation and interpretation of results covering a large range of situations defined as possible for each system. 


\section{RangeLCA Software}

RangeLCA is the own LCA software developed by RDC Environment since the early 90s. It is used here to perform the sensitivity analysis and to propose innovative ways of presenting the variable results.

For every parameter, a probability distribution can be defined to express the parameter variability (either originating in uncertainty or in diversity of situations). Normal, lognormal, uniform, triangular and discrete distributions are available in the software, as well as any mathematically defined distribution. In the case study, a uniform distribution of probability is modelled within the ranges defined by the minimum and maximum values (cf. Table 1). In this example, variability is considered only for modelling parameters but it could also be applied to elementary flows.

RangeLCA performs at once a large number of simulations (3000 in the case study). For each simulation, it selects a random value for each variable parameter, based on its defined probability distribution. This Monte Carlo analysis allows all existing cases to be statistically modelled in a single LCA model. When common to several systems, a parameter takes at each simulation the same value for all the systems (as what is done in other LCA software tools). With RangeLCA, the parameters can be classified by decreasing order of sensitivity of the results to the parameters. Furthermore, the software allows the user to access the results and the parameter values for each Monte Carlo simulation. The variable results can then be plotted against selected parameters.

\section{Presentation of the Results}

\subsection{Average Results}

Results obtained for the defined average cases are presented in Fig. 1 for both compared systems.

Table 1 Case study - values of the main variable parameters

\begin{tabular}{l|l|l|l|l|r}
\hline Parameter & System & Unit & $\begin{array}{l}\text { Average } \\
\text { case }\end{array}$ & Min & Max \\
\hline Height of box & Both & $\mathrm{cm}$ & 20 & 3.5 & 35 \\
\hline Number of uses per crate & Crate & $\#$ & 71 & 1 & 100 \\
\hline Washing rate & Crate & $\%$ & 8.3 & 0 & 100 \\
\hline $\begin{array}{l}\text { Recovery transport (Sending back the crates to a } \\
\text { new good producer) }\end{array}$ & Crate & $\mathrm{km}$ & 200 & 0 & 1000 \\
\hline $\begin{array}{l}\text { Collection transport (Sending the dirty/damaged } \\
\text { crates to service centre) }\end{array}$ & Crate & $\mathrm{km}$ & 200 & 0 & 1000 \\
$\begin{array}{l}\text { Relocation transport (Dispatching the washed } \\
\text { crates) }\end{array}$ & Crate & $\mathrm{km}$ & 200 & 0 & 1000 \\
\hline
\end{tabular}

The total logistic transport for crates combines recovery, collection and relocation transports 


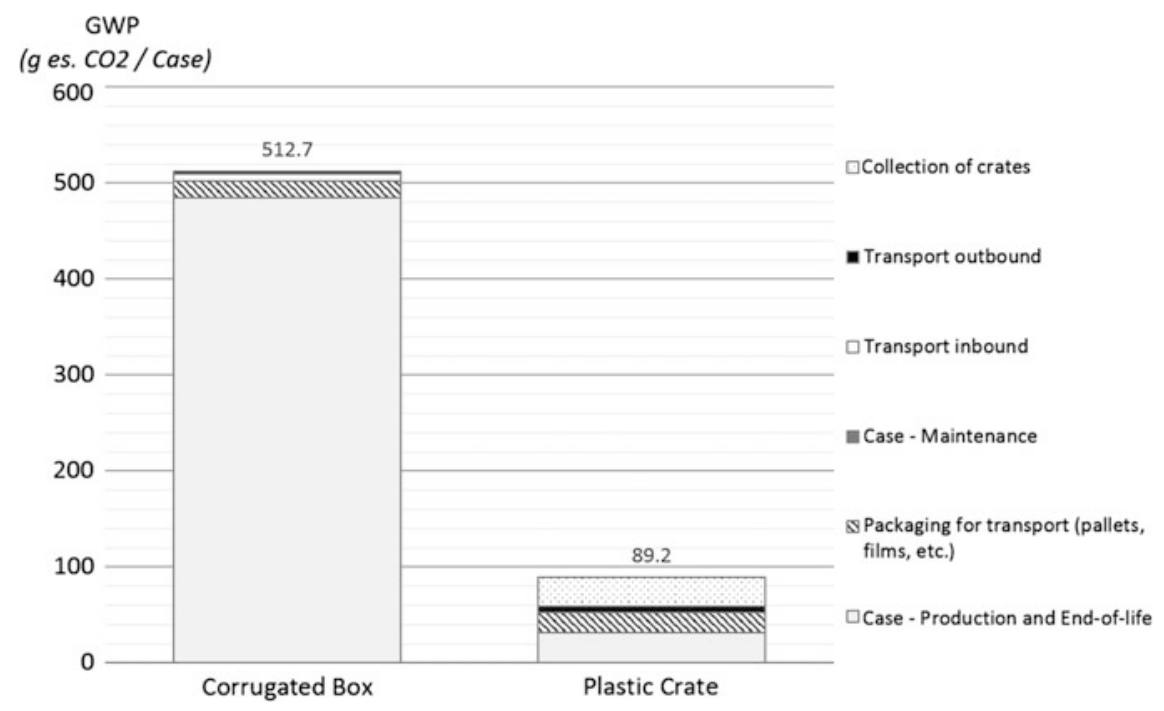

Fig. 1 GWP results for the comparison of average cases - detail per life cycle steps

This graph allows concluding on the lower impacts of the plastic crate versus the corrugated box for the average scenario. It also highlights the most contributing phases. However, such result presentation does not reflect the uncertainty nor provides indication on how would the results evolve in case of a modification of a main parameter value.

\subsection{Results with Variability}

\subsubsection{Range and Delta Graphs}

In Fig. 2, the GWP results of the 3000 simulations are plotted for the compared systems against the total logistic distance, i.e. the most influencing parameter for the crate system. In this "Range graph", a cloud of points is represented for each system. The slope of the trendline indicates how much the results depend on the parameter taken as x-as. The height of the cloud of points reflects the remaining variability, i.e. the variability that is not explained by this main parameter.

For comparing two systems, the relative positions of both clouds of points is analysed. In Fig. 2, the clouds overlap partially. Hence, it is difficult to determine in which situations the corrugated boxes have higher impacts than the crates.

In order to eliminate the common variability of the compared systems, the results of each simulation can be compared two by two. The differences between both systems calculated for all simulations can be plotted in a "Delta graph", represented in Fig. 3. The analysis of delta results indicates that corrugated boxes are better in 


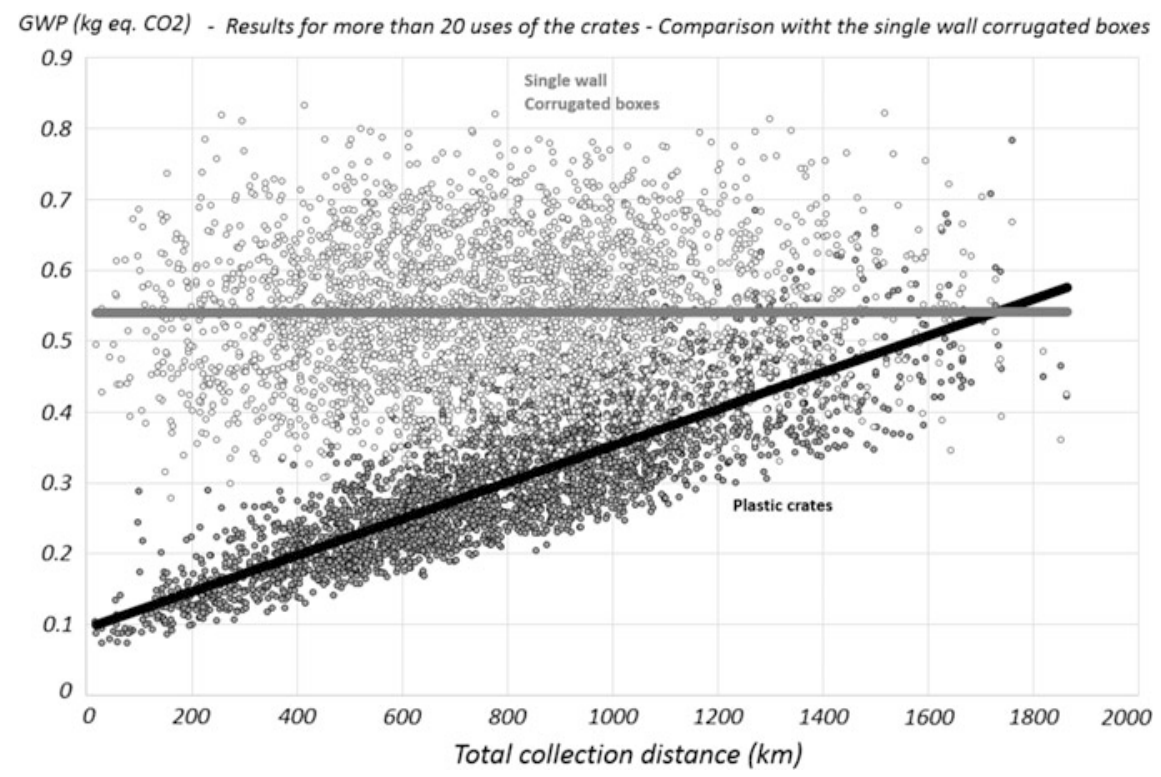

Fig. 2 GWP-variation of results for corrugated boxes and crates in function of the total collection distance

$3 \%$ of the cases studied, as can also be calculated by other software tools. However, the delta graph provides much richer information. It allows drawing specific conclusions for defined ranges of parameter values. In Fig. 3, it appears that up to a distance of $900 \mathrm{~km}$, the corrugated boxes always have higher GWP impacts than plastic crates ("always" means that the conclusion is drawn while keeping all parameters variable or uncertain).

\subsubsection{Areas of Relevance}

The residual variability, beside the one related to the most influential parameter, can be further analysed by looking at a second parameter, for example here the height of the cases. Small incremental ranges of values are defined for both variable parameters (for example, ranges of $1 \mathrm{~cm}$ of height). The results obtained for each combination of height and distance ranges are averaged and compared for both systems. The obtained impact ratios (or indexes) can be represented for all situations, as in Fig. 4, with the level of grey being related to the index (number inside the cells). Conclusions can be drawn within areas of relevance. With an index lower than 1, the light grey cells signify that the plastic crates have a lower impact (the crate is the best solution). The darker the cell, the higher the index. With index above 1, the corrugated boxes have a lower impact (the box is the best solution). A buffer zone is represented in intermediate 


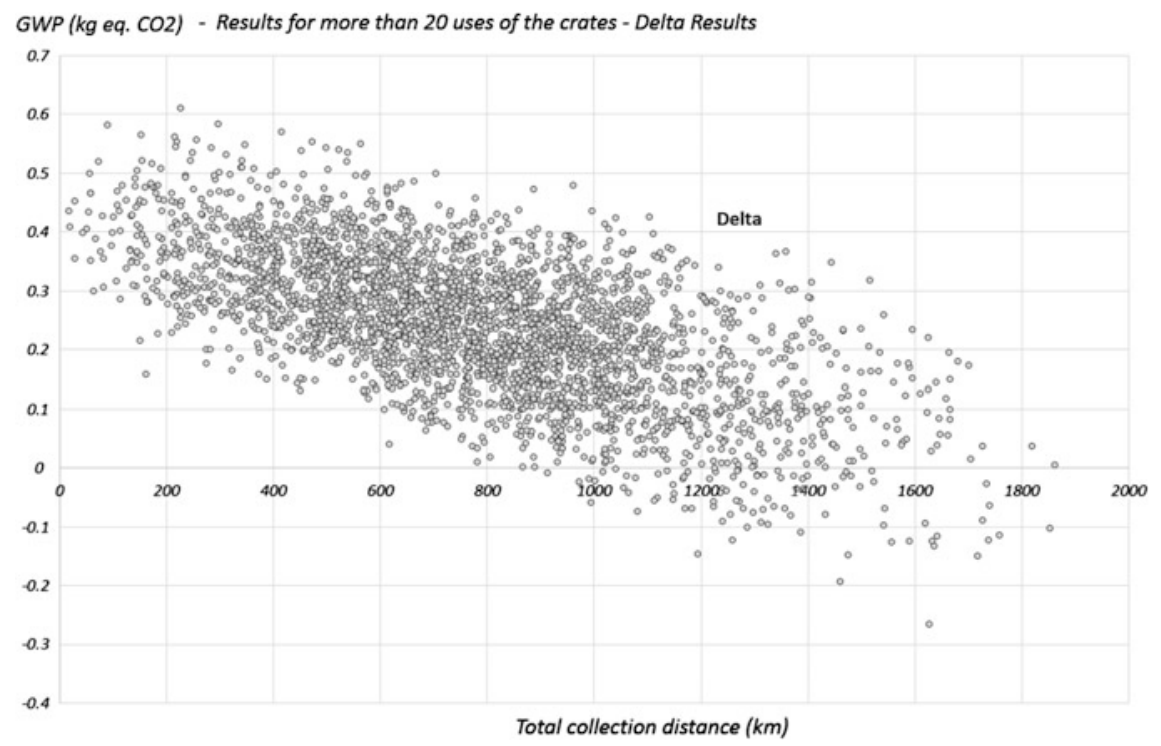

Fig. 3 GWP-variation of the differences between results for corrugated boxes and crates in function of the total collection distance

grey level when the index is around 1. For those cells, it is no clear whether it is the crate or the box that provides the best solution.

\subsection{Discussion}

The GWP results presented for the average cases reveal only that, in the defined specific scenario, crates provide a better solution than boxes, regarding this indicator. Instead, considering the variability of parameters like logistic distance and case height allows identification of the range of situations where this conclusion applies. 


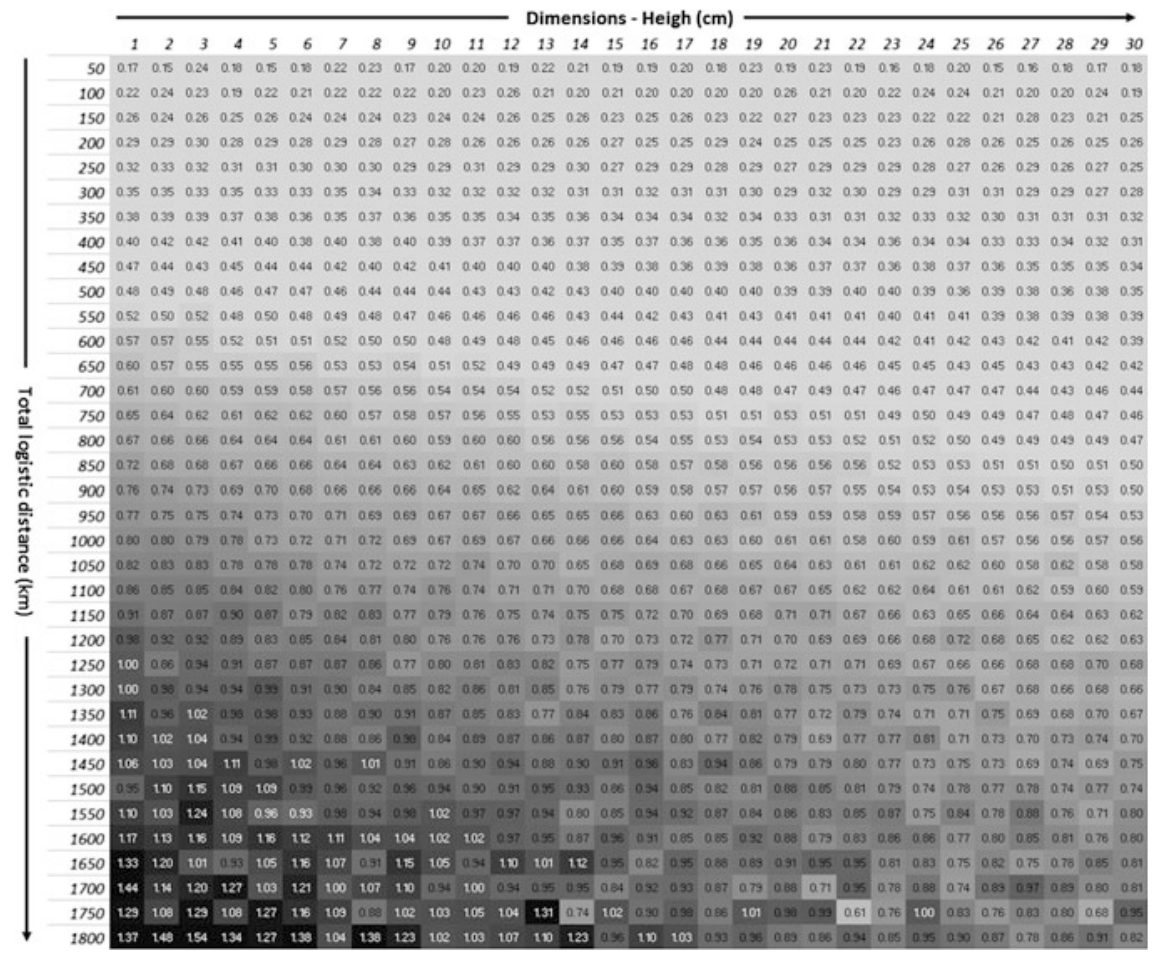

Fig. 4 Ratio of GWP impacts between crates and boxes considering a combined variation of the total logistic distance and the dimension of the cases. An index is visible for each simulation; it is calculated as the ratio between the crate impact and the box impact

\section{Conclusions}

When performing Monte Carlo analysis, it is very helpful to have access to the results of each simulation as well as to the corresponding values of the variable parameters. Thanks to these datasets, made available here by the software RangeLCA, valuable ways of presenting the results have been proposed in the paper. They have been illustrated for one impact category, with the help of a real case study.

Range graphs represent the variable results of each system studied as clouds of points. The sensitivity of the results to a parameter can be highlighted and the amplitude of the variability can be visualized through the cloud shape. This approach plays an important role in iterative LCA in defining priorities for data and model refining. In case of comparative LCA, result differences between two systems can be plotted for all simulations, either against one parameter (delta graphs) and by combining the variability of two parameters (areas of relevance). 
These types of graphs allow acquiring a more complete vision of a complex system. They enhance reliability of conclusions in comparative LCA by showing whether differences are significant or not in terms of sign and amplitude. Instead of answering the question "Is a system better than another one in defined specific cases?", delta graphs and areas of relevance help LCA studies to answer a much broader question: "In which range of situations is a system better than another one?". This is of great help to decision makers.

\section{References}

1. ScoreLCA 2014-03, Igos E, Benetto E, Les différentes sources d'incertitudes en ACV, leurs modes de calcul et impacts sur l'interprétation. June 2015.

2. ISO 14044:2006.

3. Wei W, Larrey-Lassalle P, Faure F, Dumoulin N, Roux P, Mathias J.D, How to conduct a proper sensitivity analysis in life cycle assessment: taking into account correlations within LCI data and interactions within the LCA calculation model, Environ Sci Technol., Vol. 49(1), 2015, pp. 377-85.

4. Groen E.A, Heijungs R, Bokkers E.A.M, de Boer I.J.M, Sensitivity analysis in life cycle assessment, Proceedings of the 9th International Conference on Life Cycle Assessment in the Agri-Food Sector, San Francisco, 2014, http://lcafood2014.org/papers/196.pdf (Accessed 12.06.2017).

Open Access This chapter is licensed under the terms of the Creative Commons Attribution 4.0 International License (http://creativecommons.org/licenses/by/4.0/), which permits use, sharing, adaptation, distribution and reproduction in any medium or format, as long as you give appropriate credit to the original author(s) and the source, provide a link to the Creative Commons license and indicate if changes were made.

The images or other third party material in this chapter are included in the chapter's Creative Commons license, unless indicated otherwise in a credit line to the material. If material is not included in the chapter's Creative Commons license and your intended use is not permitted by statutory regulation or exceeds the permitted use, you will need to obtain permission directly from the copyright holder.

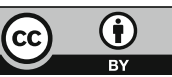




\title{
Life Cycle Management in Industry- Supporting Business with Life Cycle Based Assessments
}

\author{
Andreas Bednarz, Julia Beier, Thomas Grünenwald, \\ Birgit Himmelreich, Bärbel Hundt, Florian A. Jaeger, \\ Martin Kirchner, Stephan Krinke, Ulla Létinois, Christina Merz, \\ Lisa Mohr, David Morris, Nikolaj Otte, Gerald Rebitzer, Peter Saling, \\ Urs W. Schenker, Diederik Schowanek, Gerd Vollmer, Niklas von der \\ Assen and Alain Wathelet
}

\begin{abstract}
Sustainability is becoming more and more a strategic growth driver for numerous companies. In this context transparency on the environmental strengths and weaknesses of products and processes and related opportunities and risks is crucial. Accordingly, the assessment of sustainability aspects is gaining importance for companies and their customers along the value chain. Life cycle-based methodologies as Life Cycle Assessment (LCA) but also other assessment systems
\end{abstract}

\footnotetext{
A. Bednarz · B. Himmelreich

Covestro AG, 51373 Leverkusen, Germany

J. Beier

Beiersdorf AG, Unnastraße 48, 20245 Hamburg, Germany

T. Grünenwald · P. Saling ( $\square)$

BASF SE, 67056 Ludwigshafen, Germany

e-mail: peter.saling@basf.com

B. Hundt $\cdot$ N. von der Assen

Bayer AG, 51368 Leverkusen, Germany

F. A. Jaeger

Siemens AG, 80333 Munich, Germany

M. Kirchner · C. Merz

Evonik Technology \& Infrastructure GmbH,

Rellinghauser Straße 1-11, 45128 Essen, Germany

S. Krinke

Volkswagen AG Group Research Environmental Affairs, 38440 Wolfsburg, Germany

U. Létinois
}

DSM Nutritional Products, Process Research, Wurmisweg 576,

4303 Kaiseraugst, Switzerland

L. Mohr

Thyssenkrupp Steel Europe AG, 47166 Duisburg, Germany

(C) The Author(s) 2018

E. Benetto et al. (eds.), Designing Sustainable Technologies,

Products and Policies, https://doi.org/10.1007/978-3-319-66981-6_39 
are used in decision-making processes, product development and marketing activities. Many companies have a public corporate sustainability policy backed up with commitments in the form of quantitative targets. LCA methodology may be used as a tool supporting the identification of 'hot spots' in the value chain and measuring progress towards sustainability targets. In practice, however, common issues and challenges stand in the way of a full deployment of LCA methods in industry. It is important for companies to find common ground on how to implement these approaches, which data and impact assessments to be used and how results should be interpreted. ISO rules give a good basis for that work, though it is not sufficient for several questions. For exchanging experiences, updating or adopting methods, and generating data the International Sustainability Practitioners Network (ISPN) was created in 2012. The ISPN is an exchange forum for LCA methodology in the context of industry and comprises sustainability experts from a range of different industry sectors. To share experiences from the different activities, examples of good practices of this cross-sectoral initiative and to discuss opportunities for improving sustainability assessments within the companies are introduced. This article highlights challenges and solutions in terms of data availability and uncertainty, streamlining and using standardization processes as well as communication of results with non-LCA-experts.

\section{Introduction}

The International Sustainability Practitioners Network (ISPN) is a voluntary and loosely associated network of sustainability experts and/or managers from large industry organizations. It represents diverse product portfolios and its members occupy very different positions in the value chain. The intention of ISPN is to

D. Morris

DSM, Het Overloon 1, 6411 TE Heerlen, Netherlands

N. Otte

Henkel AG \& Co. KGaA, Henkelstrasse 67, 40589 Düsseldorf, Germany

G. Rebitzer

Amcor, Thurgauerstrasse 34, 8050 Zurich, Switzerland

U. W. Schenker

Nestlé Research, Vers-chez-les-blanc, 26, 1000 Lausanne, Switzerland

D. Schowanek

Procter \& Gamble, Brussels Innovation Centre, Temselaan 100, B-1853 Strombeek-Bever, Belgium

G. Vollmer

Merck KGaA, Frankfurter Straße 250, 64293 Darmstadt, Germany

A. Wathelet

Solvay, Research \& Innovation, B-1120 Brussels, Belgium 


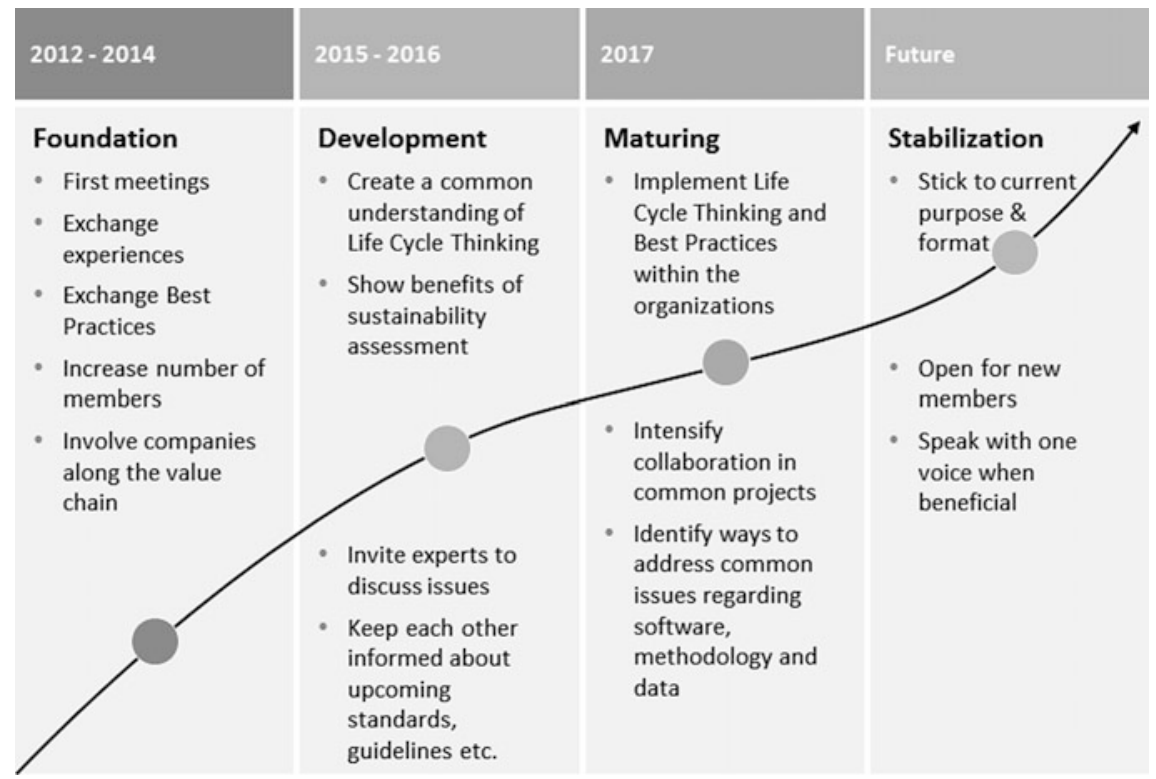

Fig. 1 Development and main focusses of the International Sustainability Practitioners Network (ISPN)

exchange information and successful practices around common aspects in sustainability assessment methodologies, lifecycle thinking and management consulting. In addition, the ISPN aims at fostering Life Cycle Assessment (LCA) as a tool to support sustainable innovation and measurement. The development and common understanding of the ISPN and its function is illustrated in Fig. 1. Participating in the ISPN serves as source of inspiration for its members and helps them to implement and disseminate Life Cycle Thinking and Best Practices regarding sustainability assessment within their organization.

Since its start the International Sustainability Practitioner Network (ISPN) gained more and more members and currently consists of 15 companies from various sectors and countries as shown in Fig. 2.

\section{LCA and Other Approaches for the Assessment of Sustainability}

Although each member company uses slightly different approaches to assess its sustainability performance, the basic idea of applying LCA in decision making and as communication tool is very similar across companies and industrial sectors. Simplified LCA approaches as well as Hotspot Analysis are of particular interest within the early research and development phase or during the Eco-design of a product, service or process. In addition, LCA is used in marketing and 


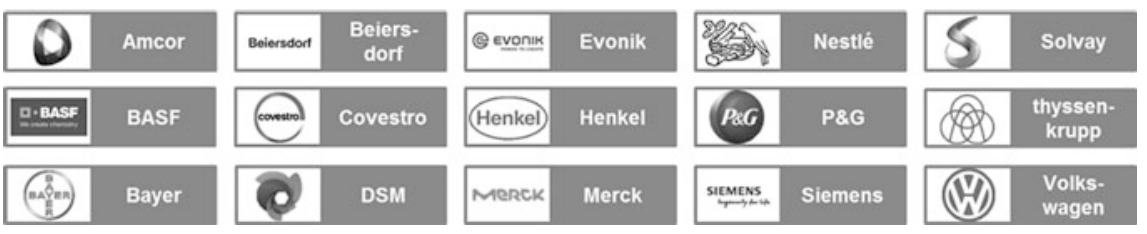

Fig. 2 ISPN members 2017

communication to inform customers, consumers, regulatory authorities or other stakeholders about the sustainability performance of a product, service or process. However, the areas of LCA applications are not strictly separated, i.e. a simplified LCA or a Hot-Spot Analysis needed in a decision-making process can be extended to a full LCA study for marketing and communication or a full LCA study performed for marketing can support decision making processes.

One example of such a process is shown in Fig. 3 where a sustainability evaluation is included in the Covestro stage-gate process for decision making and documentation in research and development projects. The methodology for sustainability assessment shown in Fig. 3 presents the increasing depth in sustainability evaluation in the advancing process design.

During the process design in the initial step a "Hotspot Identification" is carried out. It helps $R \& D$ departments to steer their activities in the direction of more sustainable product solutions in early development phases and without too much needs for information.

In the next phase the obtained Hotspots can lead to a more detailed analysis. Based on the results, the conduction of a Screening LCA or an update of the

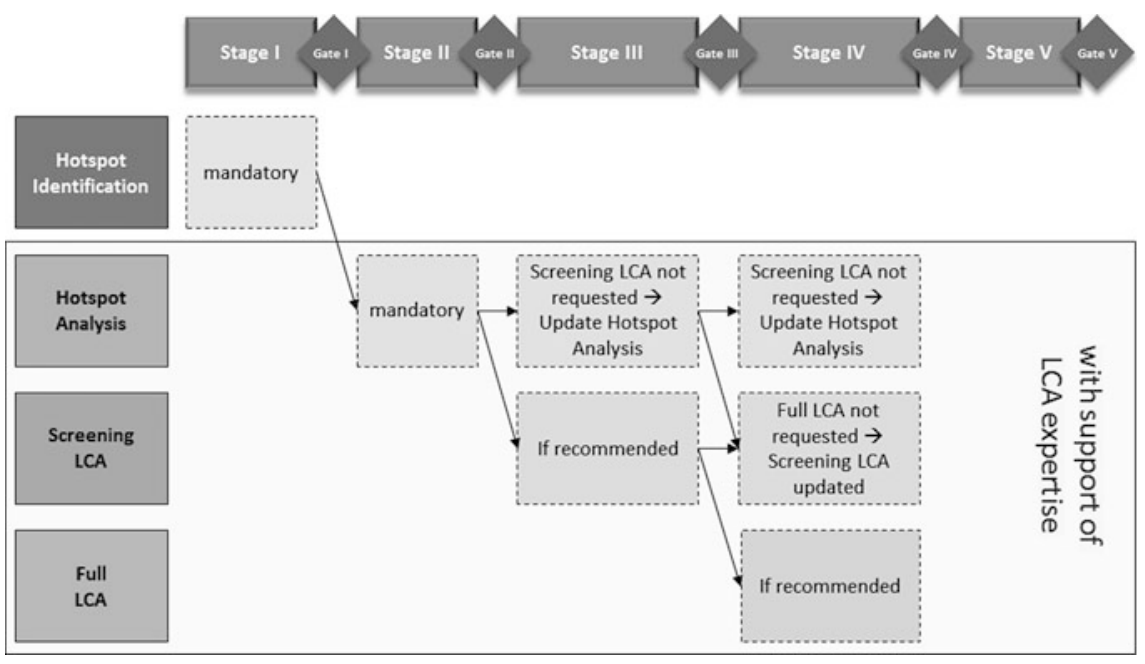

Fig. 3 Covestro stage-gate process for a systematic sustainability assessment during process design 
"Hotspot Analysis" is recommended for the next stage. A reason for such a recommendation can be unclear or probably negative results during the Hotspot Analysis to enhance the decision making. This might lead to the cancellation of such projects if they are not sustainable. On the other hand, a hotspot analysis may also reveal a potential unique selling point, which must be confirmed by a full LCA prior to market launch.

Successfully applied to the innovation process, LCA approaches help to steer towards given sustainability targets and can form a valuable basis for the companies' sustainability strategy. Such methods enable an overall assessment and the determination of the most significant processes and products for a successful development. Examples for a successful implementation of such methodologies in industry are the Sustainable Solution Steering ${ }^{\circledR}$ by BASF [1, 2], the Sustainable Portfolio Management by Solvay [3], the Company Footprint by $\mathrm{P} \& \mathrm{G}$ and $\mathrm{I} 2 \mathrm{P}^{3 \circledR}$ (Idea-to-People-Planet-Profit) by Evonik [4]. BASF assessed more than 65,000 product solutions to identify the most sustainable solutions and to promote those "Accelerators" in the business. Evonik's innovation unit, Creavis, manages its

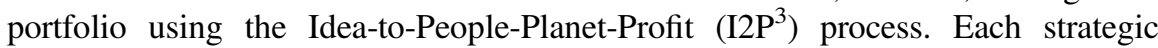
research project is assessed on the basis of environmental influences (planet) and societal aspects (people) as well as economic criteria (profit).

Solvay Sustainable Portfolio Management (SPM) tool enables a global and systematic assessment of more than $80 \%$ of the business portfolio revenue every year, a fact based and robust compass to steer Solvay's portfolio toward better business because it is more sustainable with 9\% growth, year-to-year, for Sustainable Solutions. Similar to the Solvay approach, P\&G's Company Footprint identifies 'Hotspots' for the whole company by multiplying the life cycle impacts (such as energy demand, $\mathrm{CO}_{2}$-emissions, water use or eutrophication) for a given product category and a life cycle stage with the sales. The results are then used to decide about the biggest opportunities for environmental improvement, and about the key actors along the value chain who have the biggest impact on the improvement, e.g. suppliers, production plants, transportation or consumers. This knowledge can be used in general to steer the product portfolio of the entire company. Since $P \& G$ found out that the biggest footprint is the energy used when consumers heat the water for their laundry, they expanded and improved their cold washing products. In the logic of this portfolio steering approach, a small change on a mega brand or category can be overall more effective for the environment than major design changes in small brands.

\section{Challenges of Data Exchange in the Value Chain}

To improve the quality of sustainability assessments and to close data gaps along the value chain in situations where no industry averages from associations are available an inter-company data exchange is needed. Thus, member companies already started to collaborate on common LCA projects which involve exchange of 
data and information along the value chain. Several formats for data exchange that allow for the protection of Intellectual Property (IP) already exist, but still the data exchange across different software tools and the harmonization of methodologies remain issues which will be further outlined here.

Each company in a value chain generally owns accurate information about processes bound to its own operations, but faces a lack of information when it comes to manufacturing steps or application of products outside of its operations. Commercial or public LCA databases, providing generic information are one valuable source to overcome data gaps for the coverage of all relevant life cycle stages. Depending on the requirements for data quality this source of generic information may not be sufficient as the sheer number of existing raw materials, intermediates, processes and applications in reality makes it unlikely that good quality data will be available for most specific product systems. This challenge grows when proceeding in the value chain.

Thus, an exchange of data among partners in a value chain seems a viable solution in terms of data coverage and quality. Two specific problem areas were identified for a successful data exchange, both discussed in the following sections.

One issue relates to data format to be used for exchanges, another one to the exchange process and harmonization in the data exchange itself.

\subsection{Data Formats}

The application of LCA depends on the availability of data to build reliable inventories. Data- demand and dependency are determined by the position of a company in the value chain as discussed above. For cradle-to-gate assessments, early value chain users are less dependent on external data and can invest their time into the analysis of internal processes, while late value chain users have to complement their own data with pre-catalogued values from databases or from their suppliers to cover the purchased raw material and utility inputs. To avoid using generalized datasets (proxies) from commercial databases, exchange of LCA information between companies is considered as an added value for the improvement of data quality in LCA. Nevertheless, this exchange is not a standard process by now.

As an important obstacle in exchanging LCA data the need for protection of IP has been identified. LCA data has sometimes been suspected to enable retro-analysis of the underlying processes, thus violating the requirements of IP protection.

Depending on how data is to be exchanged this problem might be alleviated. LCA data could be exchanged in three different formats:

- Unit process data

- Aggregated process data

- Impact indicator results 
Unit process data make the production processes of an enterprise completely transparent. To that end there will be confidentiality issues for companies who employ (A) sensitive processes or (B) wish to keep their supply chain confidential. Traceability will play a role in the future for some sectors such as the energy sector. Unit process data are one way of transferring such information, while keeping flexibility.

Aggregated process data provides a reasonable level of confidentiality, as it converts the mass and energy balance for the production of the evaluated product to elementary flows which don't allow any back tracking to company specific data. The aggregated data may be used by other LCA practitioners to calculate any given impact indicator in the scope of the dataset. To that end aggregated process data provide flexibility in the assessment and protect IPs. As additional information, this aggregated dataset could be completed (or not) with metadata describing some parts of the process or modelling hypothesis like allocation rules, etc.

Impact indicator results contain the least detailed information about any process when exchanged between value chain partners. If a "fingerprint" set of indicators was defined, this set of indicators would enable calculation of all relevant environmental impacts of derived products, but mainly outside of commercial LCA tools. Since the indicators are not so transparent, a detailed guideline in the calculation of such indicators is required. The advantage of impact indicators is the application of unambiguous assessment once rules for scope have been defined. Their easy tabulation enables rapid transfer and data warehousing. The ideal format for data exchange does not exist and depends on the demands of the involved parties. If a standardized set of indicators is used such as those recommended by the Product Environmental Footprint (PEF), then exchanging these indicator results may provide sufficient information. Such exchange would not allow further investigation of environmental flows or calculation of customized impact indicators. Also, current software tools typically run on aggregated process data for unit process data. Implementing a combination of impact indicators in conjunction with process data in LCA software is not easy to handle and would not work to be a currently viable solution.

Difficulties occurring during the exchange process are often caused by different LCA software used and are therefore somehow out of the direct influence of such companies.

\subsection{Data Exchange}

Companies that want to exchange LCI or LCA data often do not run the same LCA software or data storage platform. Several examples were experienced where such an exchange failed or led to imprecise results due to inconsistency in environmental flow definitions or different implementation of LCIA methods. 
Examples of issues that lead to such problems:

- Naming of environmental flows in different datasets varies

- Amount and naming of compartments for flows differ between databases/ software systems

- Application of substance specific characterization factors in different software is not the same

Matching of flows between different software systems is not precise, since one software type calculates with five different characterization factors (CF's) for eight (sub-) compartments, whereas the alternative software only considers three different CF's for three compartments. Thus, for example, the eco-toxicity impact of the same substance can vary significantly depending on the type of software/database used (Table 1). Unfortunately, this problem also appears for publicly available industry averages which are evaluated with different software tools.

From a comprehensive value chain perspective, it is desirable to have a common database format facilitating the exchange of data between different LCA systems. In the meantime, a continuous effort by an independent arbitrator could help perpetuate consistency of the data format and increased coverage of flows (typically, "mapping file") which should be implemented in all common LCA software tools to enable harmonized data exchange.

\section{Methodological Issues}

Methodological issues of Life Cycle Assessment are one of the main reasons why LCA is not more widely applied for sustainability assessments in industry and policy today. On the one hand, methodological issues sometimes make it difficult to identify and properly apply the most appropriate way of assessing a given product

Table 1 Example eco-toxicity of chromium VI: differences for the same flow in different databases $(\mathrm{CF}=$ characterization factor)

\begin{tabular}{l|l|l|l|c}
\hline Element & Compartment & Sub-compartment & CF's software 1 & \multirow{2}{*}{ CF's software 2 } \\
\hline \multirow{3}{*}{ Chromium (+VI) } & Air & Low population & 42,140 & \multirow{2}{*}{41,972} \\
\cline { 2 - 4 } & Air & High population & 41,800 & \\
\cline { 2 - 4 } & Air & Unspecified & 41,970 & \\
\cline { 2 - 4 } & Soil & Unspecified & 52,560 & 52,555 \\
\cline { 2 - 4 } & Water & Ground-, long-term & 52,560 & \multirow{2}{*}{104,644} \\
\cline { 2 - 4 } & Water & River & 104,600 & \\
\cline { 2 - 4 } & Water & Ground & 52,560 & \\
\cline { 2 - 4 } & Water & Unspecified & 104,600 & \\
\hline
\end{tabular}


or system. On the other hand, different methodological choices between actors (or stakeholders) in a supply chain or product system make it difficult to find consensus and agree on common metrics, which decreases the confidence level of the end user and decision maker in LCA as a tool.

Many review studies have compiled the most important methodological issues that remain open in LCA today. That was done e.g. in the program Sustainable Process Industry through Resource and Energy Efficiency "SPIRE" with the specific projects Sustainability assessment methods and tools project "SAMT" and Metrics for Sustainability Assessment in European Process Industries "MEASURE" in 2016, with ISPN members contribution [5]. For every industry sector, the key methodological issues are different: for example, while allocation between co-products is a key issue in the food industry and the waste sector, methods to assess (eco-) toxicity are of paramount importance for the chemicals or pharmaceuticals sector. The methodological issues of high relevance for industry can be positioned in the four phases of LCA (as defined by the ISO standards 14040 and 14044-Table 2).

The development of novel or improved guidance or standards on methodological issues needs a proper governance model; including stakeholder outreach and consultation (cf. the process applied to the development of new ISO standards). This cannot be done in the context of an informal network such as the ISPN, and also needs a proper process by which stakeholders outside the industry can be reached. Therefore, ISPN has not attempted to provide guidance or develop standards on methodological issues of LCA. Instead, a selection of key external initiatives was identified which published suggestions for it (see Fig. 4), and have decided to follow and engage in those processes. Standards and guidance processes are often industry-specific, the ISPN offers an opportunity for a given industry sector to better understand the working of other industry sectors.

While ISPN as an organization cannot take credit for the methodological advancements that have been achieved over the past years in LCA, most of the members have been involved in the development and consultation processes of influential guidance documents and standards. In ISPN, those experts have presented the most relevant methodological advancements, and ISPN has discussed the implications of these for the respective companies and industry sectors that we

Table 2 Examples of key environmental issues in LCA, currently being worked by industry, classified as per the ISO 14040 \& 14044 standards

\begin{tabular}{|c|c|c|c|}
\hline Goal and scope & LCI & LCIA & $\begin{array}{l}\text { Interpretation } \\
\text { (communication) }\end{array}$ \\
\hline $\begin{array}{l}\text { - EU PEF [6] } \\
\text { horizontal } \\
\text { methodological } \\
\text { issues (e.g. } \\
\text { granularity) }\end{array}$ & $\begin{array}{l}\text { - Data } \\
\text { availability/ } \\
\text { access } \\
\text { - Data quality } \\
\text { - EU PEF } \\
\text { horizontal } \\
\text { issues }\end{array}$ & $\begin{array}{l}\text { - Consensus on impact } \\
\text { methods e.g. (Eco-) } \\
\text { Toxicity, near-far } \\
\text { field impacts/ } \\
\text { nutritional benefits, } \\
\text { Land-use/biodiversity } \\
\text { aspects, Water } \\
\text { consumption) } \\
\text { - Normalization and } \\
\text { weighting }\end{array}$ & $\begin{array}{l}\text { - Hot spot analysis } \\
\text { recommendation } \\
\text { - Suitable B2B/B2C } \\
\text { approaches }\end{array}$ \\
\hline
\end{tabular}




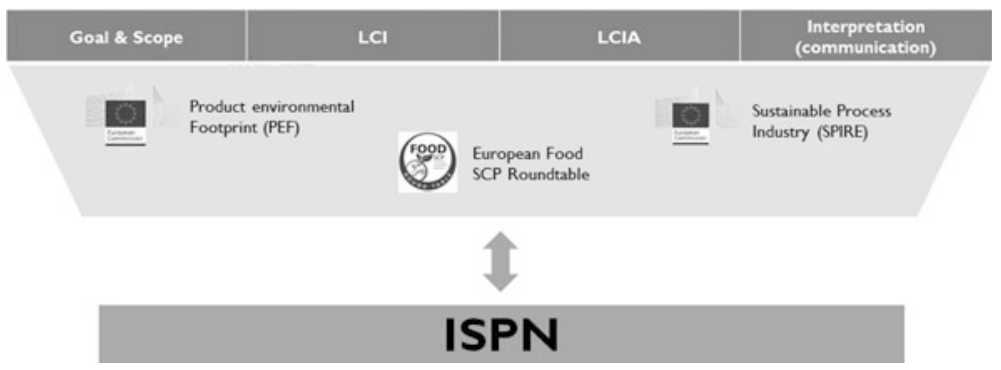

Fig. 4 Schematic overview of relevant organizations working on standardization and guidance for different parts of a LCA

represent. In that sense, the ISPN plays an important role for the methodological advancement of LCA and for the dissemination of methodological developments among experienced industry practitioners.

\section{$5 \quad$ Vision and Mission}

The members of the ISPN have concluded that there are commonalities and differences in the interests and approaches of the participants, due to the diversity of the group and the respective industry sectors. The ISPN conceptualized why already the understanding and thus concepts and practical approaches can be different. However, we consider a common understanding to be the key to the success of a sustainability life cycle thinking in large corporations. We believe the ISPN platform serves as a good starting point to foster such a common understanding of methodologies $[7,8]$.

For exchanging experiences, updating or adopting methods, and generating data the ISPN was created several years ago. The ISPN is an exchange forum for LCA methodology in the context of industry and comprises sustainability experts from a range of different industry sectors. To share experiences from the different activities, the presentation will give examples of good practices of this cross-sectoral initiative and discuss opportunities for improving sustainability assessments within of companies.

To give this basic approach a more precise frame and guidance for interested parties on how this network works together and how others can benefit from the ISPN, we started to develop a "Vision and Mission" section linked with a frame that show which aspects will be covered by the work of ISPN.

It can be summarized in the following overview:

\section{Vision}

- Leadership of cross-sectoral industry LCA experts

- High recognition by academia, government and industry

- Effective platform for collaboration on methods, tools and data 


\section{Mission}

- We facilitate exchange platforms of LCA experts discussing aspects of methods, tools and data

- We identify and prioritize relevant topics of cross-sectoral challenges in the field of sustainability assessments

- We collaborate on projects or method developments with academia, authorities, NGOs and other industry partners

- We extend the collaboration platform to new sectors taking their experiences and needs into account

- We recommend and suggest changes in methodologies, data management and tools

- We discuss and communicate our experiences with other practitioners' groups

- We work together effectively and with smart solutions

- We intensify networking activities and offer a broad cross-sectoral expertise

To cluster different work packages and to find out, which opportunities can be achieved by the ISPN, different topics and activities were identified. The cross-sectoral approach of ISPN allows the link of different experiences in the field of the assessment of sustainability as a starting point. The single companies work together and act as one organization defining needs and opportunities for a successful sustainability measurement. It gives guidance and defines good practices for the integration of the findings into the business and into business decision-making $[9,10]$.

Four activities were identified as a basis for suggesting changes and improvements of tools, methods and data systems. These four activities are using the extended expertise of the members, the exchange of experiences over the last decade, modes of collaboration in defining needs, requirements, pathways for improvement etc. The recognition by other practitioners, academia or NGOs for the ISPN will support the transportation and communication of important needs of different fields for further improvements. It shows that leading companies in the field of sustainability assessment can initiate and foster the development of new, modern, easy to use, applicable tools, methods and data sets [11, 12].

With this approach, the ISPN can support funded projects, standard generation, definition of basic requirements for sustainability assessments and of common practices. These findings will be publicly available and can be used for further improvement. Based on these findings, the ISPN can improve its way of collaboration, the definition of further topics and areas of improvements. In collaboration with other groups, new developments can be assessed, checked and be proven for applicability in different sectors (Fig. 5). 


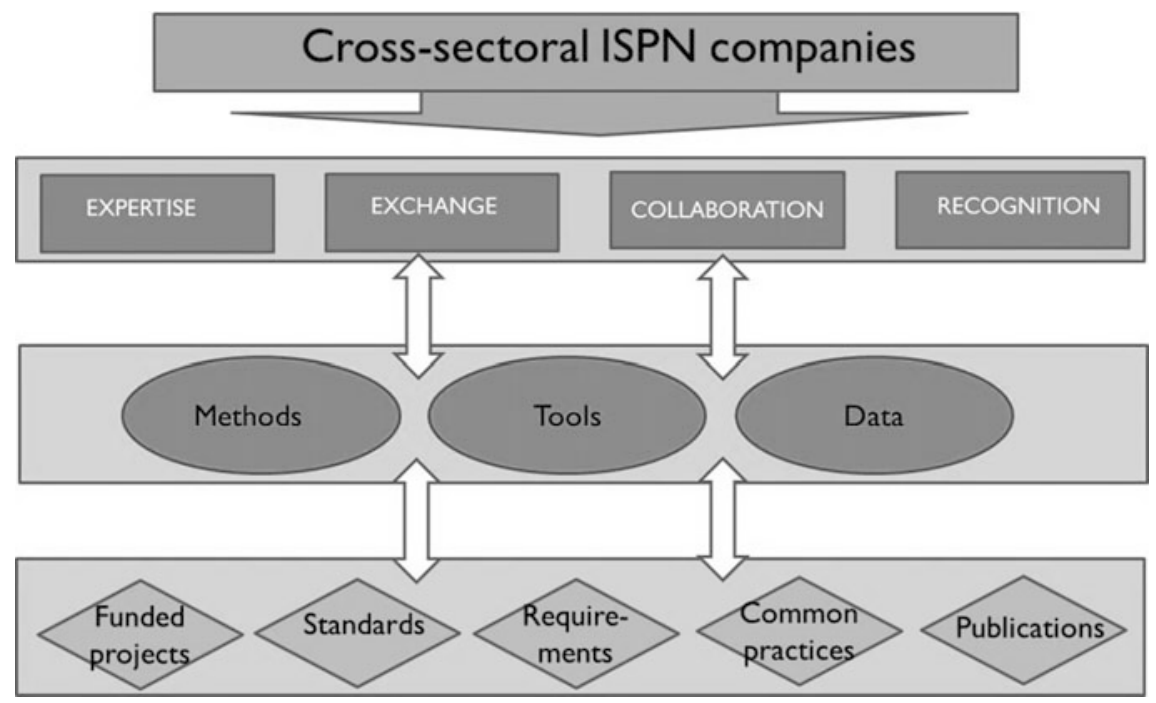

Fig. 5 Overview of processes and activities of ISPN

\section{References}

1. http://rd.springer.com/chapter/10.1007/978-94-017-7221-1_10\#page-1 (Accessed 31.05. 2017).

2. http://eman-eu.org/wp-content/uploads/2016/04/Proceedings_EMAN_2015_BridgingCorporate-and-Academic-Contributions.pdf\#page=42 (Accessed 31.05.2017).

3. http://www.solvay.com/en/company/sustainability/sustainable-portfolio-management/index. html (Accessed 31.05.2017).

4. http://corporate.evonik.de/_layouts/Websites/Internet/DownloadCenterFileHandler.ashx? fileid=3683 (Accessed 31.05.2017).

5. https://www.spire2030.eu/sites/default/files/users/user355/SAMT_D.1.1_final_updatedlinks_ Dec2016.pdf (Accessed 31.05.2017).

6. http://ec.europa.eu/environment/eussd/smgp/product_footprint.htm (Accessed 30.05.2017).

7. Herrmann C, Ganzheitliches Life Cycle Management: Nachhaltigkeit und Lebenszyklusorientierung in Unternehmen, VDI Buch, 1st Ed., Springer-Verlag Berlin Heidelberg, 2010.

8. Baitz M, Albrecht S, Brauner E, Broadbent C, Castellan G, Conrath P, Fava J, Finkbeiner M, Fischer M, Fullana i Palmer P, Krinke S, Leroy C, Loebel O, McKeown P, Mersiowsky I, Möginger B, Pfaadt M, Rebitzer G, Rother E, Ruhland K, Schanssema A, Tikana L, LCA's theory and practice: like ebony and ivory living in perfect harmony? J Life Cycle Assess (2013), Vol 18, 5-13.

9. WRI, WBCSD, Corporate Value Chain (Scope 3) Accounting and Reporting Standard: Supplement to the GHG Protocol Corporate Accounting and Reporting Standard, World Resources Institute and World Business Council for Sustainable Development, 2011.

10. Ehrlenspiel K, Kiewert A, Lindemann U, Mörtl M, Kostengünstig entwickeln und konstruieren: Kostenmanagement bei der integrierten Produktentwicklungen, 7th Ed., Springer-Verlag Berlin Heidelberg, 2014. 
11. Russo M.V, Fouts P.A, A Resource-Based Perspective On Corporate Environmental Performance And Profitability, Academy of Management Journal, Vol. 40, No. 3, 1997, 534559.

12. Hart S.L, Ahuja G, Does it pay to be green? An empirical examination of the relationship between emission reduction and firm performance, Business Strategy and the Environment, Vol. 5, 1996, 30-37.

Open Access This chapter is licensed under the terms of the Creative Commons Attribution 4.0 International License (http://creativecommons.org/licenses/by/4.0/), which permits use, sharing, adaptation, distribution and reproduction in any medium or format, as long as you give appropriate credit to the original author(s) and the source, provide a link to the Creative Commons license and indicate if changes were made.

The images or other third party material in this chapter are included in the chapter's Creative Commons license, unless indicated otherwise in a credit line to the material. If material is not included in the chapter's Creative Commons license and your intended use is not permitted by statutory regulation or exceeds the permitted use, you will need to obtain permission directly from the copyright holder.

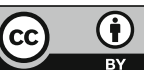




\title{
Sustainable Design of Complex Systems, Products and Services with User Integration into Design
}

\author{
Nicolas Perry and Julien Garcia
}

\begin{abstract}
This is the synthesis of the presentation of the session entitled "Sustainable design of complex systems, products and services with user integration into design" of the LCM-2017 conference. This session was interested in sustainable design which is of high interest for a large range of stakeholders. Indeed, customer's expectations, regulations and market pressure are some drivers that moves design teams towards the consideration of sustainable dimensions over the life cycle of an industrial system. Integration of users into the product design development becomes more and more crucial, for example, through service oriented offers, involving the users as effective actors of the product design. One of the weak point associated being that users may have bad or unexpected behaviour during use phase, inducing then increased impacts and placing the use phase as the main contributor. New approaches, methods, and tools used by or for industrial stakeholders in order to face such challenges were presented and discussed. The session started with product oriented presentations and proposals, and slightly moved to the concepts of service oriented design and integration of human into the loop.
\end{abstract}

\section{Product and Process}

Martin Kirchner (Evonik Technology \& Infrastructure), presented the PODeST (Process Optimization and Development through Sustainability) project in which sustainability becomes a parameter for process development and optimisation. Early identification of the potential opportunities and risks associated with new products and processes becomes of high importance, and Evonik's Life Cycle Management

\footnotetext{
N. Perry $(\bowtie)$

Arts et Métiers, I2M, CNRS, Bordeaux, France

e-mail: nicolas.perry@ensam.eu

J. Garcia

Groupe PSA, Vélizy-Villacoublay, France

(C) The Author(s) 2018

E. Benetto et al. (eds.), Designing Sustainable Technologies,

Products and Policies, https://doi.org/10.1007/978-3-319-66981-6_40
} 
group implements the standardized process called "PODeST". It aims at comprehensively optimising processes from a technical, economic as well as environmental perspective while exploiting the high flexibility regarding process design in early process development phases concerning all aspects of process relevance. PODeST is not striving for the highest level of accuracy but rather robustness of results (e.g. right order of magnitude). PODeST is benefiting from synergy effects by making use of mass and energy balance data already acquired during the development and planning phase.

This presentation highlighted the link between environmental evaluation of processes and product design cycle. It also opened the discussion on the Environmental Technology Verification. One key message to succeed in the integration of sustainability in design relied on the usability of tools and methods by engineers and designers, with support of LCA expert if needed.

The presentation of Julien Garcia (Groupe PSA), described the implementation of environmental requirements in the innovation process of complex systems applied to the automotive. The automotive design is restricted by environmental regulations, mainly focusing on reducing emissions of $\mathrm{CO}_{2}$ and other pollutants emitted by vehicle exhaust pipes. The eco-design strategy of Groupe PSA moves toward a more holistic point of view and experiences life cycle methodology to make environmentally virtuous design choices since seven years. A methodology and a tool have been developed for integrating the Evaluation of the Environmental Performance of Innovative Complex Sub-systems (EEPICS). It allows to integrate the requirements of Groupe PSA's environment department into the innovation procedures, to extend the environment dimension to the notions "life cycle" and "multi-criteria", and to generate an organizational learning for both innovation teams and environment/eco-design team. The eco-design framework is the central and structuring framework of environmental requirements integration, allowing the coordination of the development of a set of tools. Indeed, specific evaluation tools are customised and used, including one for evaluating the influence of innovation on vehicle recyclability, and another one for the evaluation of innovation environmental impacts. The outcomes of such analyses support the global learning. An application on the "Stop \& Start" technology was carried on, leading to three significant key results involving the innovation teams from the beginning of the eco-design based innovation process facilitates the learning phases, its validation through the quality procedure is essential for the approach to be sustainable; using generic models of vehicle is sufficient to evaluate quantitatively innovative automotive sub-systems.

The discussion, based on questions from the audience focused on the involvement of suppliers and subcontractors in the approach, since innovation process, focusing at products and suppliers, manufacturing systems or end of life solutions highlighted the need of a tool like eco-design at the early stage product development. 


\section{From Eco-design to Circular Economy}

Emma Rex, (RISE), addressed in her presentation the Sustainability of design for circularity, with integration of user and its behaviour into the design choices that can be made to shift from linear to circular business models in the Swedish furniture industry. Circular business models, promoting resource productivity and pollution prevention, are currently supported and encouraged by various policy agendas, resulting in an increased interest in design for modularity, adaptability and remanufacturing. The expected environmental benefits such circular business models offer are usually perceived as obvious, and seldom evaluated. Nevertheless, different design choices will have different impacts on the amount of material used, expected lifetime of products and possibilities for recycling. User behaviour patterns are neither commonly examined, even though user acceptance has been identified as a key enabler for the transition to a circular economy. The study combines interviews with potential users of pre-owned furniture, and customer experiences from furniture producing companies. Five pieces of furniture from three different producing companies are analysed. For each type of application, the environmental impacts of linear versus circular business models are compared through the use of LCA, and different user behaviour patterns are included as scenario analyses. User interviews pointed to the importance of functionality over novelty, and indicated a potential to increase the service content in existing business offers. LCA results further show that the environmental impacts of a shift from linear to circular business models both can decrease and increase, from -30 to $+50 \%$, depending on scenario settings, type of impact categories assessed, and assumptions made. Notably, results depend on expected user behaviour following decisions taken in business development and product design. The study demonstrated the importance of including user perspectives when designing for circularity, as well as when evaluating the effects of circular design offers. Also, whilst the calculations in this study revealed the potential direct environmental effects of a circular economy shift in existing industry, they also open up for discussions on indirect and broader sustainability effects following circular design attempts.

The question of multiple cycles arisen in the discussion, as well as how to build scenarios representative of the users' behaviour, and include them to the environmental impacts. Emma Rex explained that user behaviours have been decomposed into several typologies, allowing a scenario-based analysis. The discussion also touched on the service oriented solutions.

Venkata Krishna Kumar Upadhyayula (Umeå University) presented a concept where Life Cycle Assessment is used as a Decision Making Tool for Designing Products and Engineering Systems contributing to Circular Economy. Implementation of circular economy largely depends on three factors: (a) development of industrial infrastructure capable of enhancing the resource productivities on one hand and minimizing environmental externalities on the other, (b) understand the market dynamics \& consumer behaviour to promote sustainable consumption practices, (c) building sustainable cities and communities with smaller ecological 
footprints. However, in reality transformation leading from liner to circular economy faces multiple challenges. For example, (a) limitations of existing engineering practices which results in leakage of hazardous pollutants into biosphere, (b) introduction of new materials into economy with uncertain life cycle implications, or (c) effect of unsustainable consumer behaviours etc., are some factors that hamper implementation of circular economy. To overcome these, the future products and engineering systems must be designed for "circular economy" so to ensure circulation of technical nutrients within technosphere, return of biological nutrients safely to biosphere and decoupling economic growth from social and environmental externalities can be easily facilitated. The presentation detailed a structured framework for using Life Cycle Assessment as a tool to design new products or engineering systems for circular economy and supporting case studies were also shown.

The presenter opened on social issues to be addressed to ensure sustainability and was questioned about the reduction of the environmental footprint without reducing social and economic values.

\section{User Integration}

Birgit Brunklaus (RISE) discussed on LCA learnings from service design, therefore focusing on the influence of user behaviour in the design of sustainable systems, with the case-study of the leisure sector. The development of services in replacement of the traditional product selling model seems to be one solution for reducing resources consumption and emissions of pollutants. Similarly to products, services are designed to fulfil customers' needs, despite they are seldom involved in the design of products and they usually are in the design process of services.

A literature review of service design and LCA studies of leisure services like the opera and theatre, tourism related services, and movies, have been performed from the supplier and consumer perspectives respectively, in order to (a) identify similarities and differences between service design and Life cycle based approaches, and (b) to derive some propositions to further develop the Life Cycle based approaches (LCA, S-LCA, LCC) in order to integrate user into the design process.

The discussion referred to the integration of users into the LCA methodology, especially on the translation of users' behaviours characteristics into quantified items.

Finally, Gregor Waltersdorfer (University of Luxembourg) discussed in his presentation a meaning-based perspective of potential users on the implications of products and services for sustainability. He proposed a design method called "MeaningMap" and a sequence for meaning-based reasoning in design, which can support designers in considering meanings by potential users. He illustrated the integration of the user perspective into design of service systems, involving sharing bikes or washing machines. Integrating the perspective of potential users into design allow to take into account their limits in the perception of the economic, 
social and environmental impacts induced by products and services, due to their different personal values. More precisely, capturing users' meanings enables designers to analyse different aspects: (a) what is perceived by individual potential users, (b) how do potential users conceptualize their evaluations, and(c) which evaluations show low or high levels of agreement. The presenter argued that this knowledge can inform designers on which aspects of products and services they need to prioritise in order to improve its perceived implications for sustainability, and which aspects they need to further clarify in order to improve their communicative potential. Considering the meaning-based perspective of potential users can complement more traditional product-centred approaches such as LCA in early phases of the design process.

This proposal questions the robustness of the users feedback quantification, and how to turn these information into designers choices and contribute to product evolution.

\section{Conclusion}

The topics addressed in this session were also completed with nearly twenty posters, addressing and illustrating the will to integrate environmental considerations early in the product design phase fitting the circular economy paradigm.

Considering user centred design within a life cycle thinking approach in order to ensure the development of sustainable systems open numerous questions and requires new conceptual and methodological developments, consensus based and scientifically consistent in order to assess quantitatively users' behaviours. User oriented sustainable design intuitively leads to develop service-based models instead of product-based solutions. Then sustainable design methods and tools should be integrated with the Product Service Systems design method, which remains challenging.

Open Access This chapter is licensed under the terms of the Creative Commons Attribution 4.0 International License (http://creativecommons.org/licenses/by/4.0/), which permits use, sharing, adaptation, distribution and reproduction in any medium or format, as long as you give appropriate credit to the original author(s) and the source, provide a link to the Creative Commons license and indicate if changes were made.

The images or other third party material in this chapter are included in the chapter's Creative Commons license, unless indicated otherwise in a credit line to the material. If material is not included in the chapter's Creative Commons license and your intended use is not permitted by statutory regulation or exceeds the permitted use, you will need to obtain permission directly from the copyright holder.

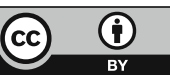




\title{
Implementation of Environmental \\ Considerations in the Innovation \\ Process of Complex Systems: \\ Groupe PSA Case Study
}

\author{
Julien Garcia, Pierre Tonnelier and Sophie Richet
}

\begin{abstract}
Car manufacturer's innovation teams are mainly focusing on decreasing automotive consumption and may lead to risks of transfers of environmental impacts. So, engineers in innovation should be able to evaluate their innovations in a life cycle and multi-criteria perspective. In this paper, Groupe PSA's approach for integrating the environmental dimension during the innovation phase is presented. An eco-design framework of reference has been developed in a collaborative manner by the environment team together with innovation leaders. It tackles both the issues of how to deploy environmental requirements in the innovation process, and how to generate an organizational learning. This methodology is explained through the example of the Stop and Start technology. The validation in the quality procedure is essential to make the approach sustainable.
\end{abstract}

\section{Introduction}

Climate change is the result of an increase in man-made greenhouse gas (GHG) emissions. The main GHG is carbon dioxide $\left(\mathrm{CO}_{2}\right) \cdot \mathrm{CO}_{2}$ is mainly emitted from fossil combustion. The transport sector is highly dependent on fossil fuels: 93\% of the 2013 world transport sector energy supply came from oil products [1]. European Union has tightened European rules on $\mathrm{CO}_{2}$ 's emission of vehicles. By 2020, car manufacturer's fleet will have to emit less than $95 \mathrm{~g} \mathrm{CO}_{2} / \mathrm{km} \mathrm{[2].}$

To reach this goal, car manufacturer's innovation teams are mainly focusing on decreasing automotive consumption. Four strategies can be highlighted: light-weighting, hybridization or electrification of the powertrain, improvements in aerodynamics and rolling resistance, and improvements in energy consumption of the vehicle (air conditioning, radio...). While innovations may contribute to reduce fuel consumption, they also may change the material composition of vehicles and

J. Garcia $(\square) \cdot$ P. Tonnelier · S. Richet

Groupe PSA, Vélizy-Villacoublay, France

e-mail: julien.garcia1@mpsa.com

(C) The Author(s) 2018

E. Benetto et al. (eds.), Designing Sustainable Technologies,

Products and Policies, https://doi.org/10.1007/978-3-319-66981-6_41 
lead to risks of transfers of environmental impacts: as the contribution of the use stage to the impacts is decreasing, the contribution from the manufacturing stage could relatively increase.

In order to prevent transfer of pollution, engineers in innovation should be able to evaluate their innovations in a life cycle and multi-criteria perspective. Environmental evaluation is generally carried out at the end of the product development process and results cannot be used during the design process itself. On the contrary, during innovation, less data on the future vehicle are available, but there are more time and more levers for making changes.

In order to integrate an eco-design approach within a company, tools must be suited to the company, its products, and the corresponding design phase and innovation process. Indeed, both the technical and organizational aspects need to be taken into account simultaneously [3] to ensure (1) the progressive expansion of the environmental field from a technical point of view, and (2) from an organizational point of view, a step by step integration of the environment in all organizational departments to ensure that users are more fully acquainted with the tools and to formalize environmental recommendations that are more accessible.

This article presents Groupe PSA's approach for integrating eco-design method in the innovation process, known as E3PICS (Methodology of an Evolutive integration of the Evaluation of the Environmental Performances of Innovative Complex Sub-systems). E3PICS is based on an eco-design framework of reference that incudes, in particular, a checklist for innovation leaders, and quantitative tools for simulating the effects of the innovations on the future vehicles.

\section{Eco-designing in the Automotive Sector's Innovation Process}

Groupe PSA's desire was to initiate an eco-design method during the innovation phase. For car manufacturers, most of the time, innovations have little impact on the vehicle architecture because of their incremental nature and the fact that such innovations often concern subsystems or components [4]. A set of innovations is generally proposed by the innovation team to the team in charge of the routine design of the vehicle, which Beaume represents as an "innovative feature" flow from the innovation department to the vehicle program department, during the "contextualization phase" [5]. Both structures are completely separated (see Fig. 1).

This study focuses on evaluating innovations during the "contextualization phase", i.e. between Technology Readiness Level (TRL) 5 and TRL 6. This phase aims to validate the connection of an innovation to the vehicle program and contains three milestones: TRL 5: innovation project's commitment; TRL 6: innovation project's connection to a vehicle program; and TRL $51 / 2$ : innovation project's mid-term review. 


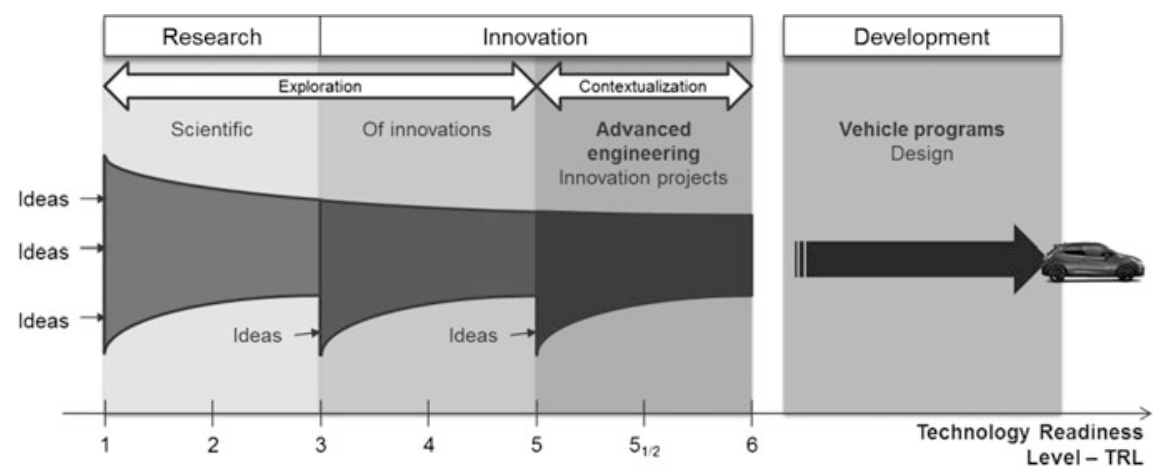

Fig. 1 Groupe PSA's innovation process

The automotive industry has developed several eco-design methods, e.g. Ford's Product Sustainability Index [6], Volvo's Environmental Priority Strategies [7] and Environmental Effect Analysis [8] methods, or Toyota's Eco-Vehicle Assessment System [9]. With the study on the life cycle design of an air intake collector, Keoleian and Kar [10] conclude that evaluating the results of integrating the innovative sub system should be carried out at the future complex system level. But in order to do this, environmental profiles of that complex system are needed. As there is very few data available during the innovation phase, complex systems must be represented by models. Neither the majority of tools developed by car manufacturers, nor the majority of tools available for eco-designing subsystems in the innovation phase [11-13] make it possible to use models to simplify the evaluation of innovative subsystems.

\section{Groupe PSA's Method for Integrating the Environmental Dimension into the Innovation Phase}

The role of Groupe PSA's "Environment" service within the "Materials" department is related to the material composition of the vehicles, and is to control a set of environmental requirements, mostly from regulations. The "Environment" service does not deal with reducing $\mathrm{CO}_{2}$ and pollutants emissions in use. It also hosts an "Eco-design" team dedicated to eco-design process and Life Cycle Assessment (LCA). The requirements driven by this service are already well integrated in the vehicle development programs, but not formalized in the innovation phase. The objective of the "Environment" service is to integrate these requirements during the innovation phase.

E3PICS methodology is based on the definition of an evolving eco-design framework of reference and its use during the innovation phase. It contains all the environmental requirements driven by the car manufacturer's "Environment" 
service. This eco-design framework of reference corresponds to writing the environmental requirements into the product specifications, one of the essential criteria for integrating environment in the design process [14].

E3PICS methodology is shaped by technical and organizational constraints consisting of four steps. The first one concerns the iterative design of the evolving eco-design framework of reference with the "Eco-design" team and with innovation leaders thus generating crossed learning. The second step consists in the "Eco-design" team's support for all innovation leaders so that they systematically use the eco-design framework of reference. The third step is the development of an analytical tool to assess the impact of innovations on vehicles' end-of-life recyclability rate (OSIRIS) [15]; models of vehicles must be created to solve the problem of insufficient data on the complete system during the design phase and to anticipate impacts on the future vehicle. Finally, the fourth step deals with developing a second analytical tool to evaluate the impact of innovations on vehicles' environmental life cycle performance (TEEPI). This tool also requires a method for developing environmental models of vehicles [16].

\section{The Eco-design Framework of Reference}

The eco-design framework of reference constitutes a methodological framework which contains the nine environmental requirements (see Table 1) that the "Eco-design" team wishes to integrate in the innovation process. It is based on a checklist called "Recycling \& Environment checklist" (R\&E checklist) containing requirements with specific deliverables and tools (prescriptive or analytical); a standard mail for the suppliers is also available to retrieve the missing data. Environmental requirements can be divided into two categories:

Table 1 Environmental requirements of Groupe PSA's eco-design framework of reference in the innovation process

\begin{tabular}{l|l}
\hline Nature & Environmental requirement on innovations \\
\hline Intrinsic & Restriction of use of lead, mercury, cadmium, and chromium VI \\
\cline { 2 - 2 } & Ban on the use of chemical substances \\
\cline { 2 - 2 } & Remove the risk of exposure to a chemical in a product \\
\cline { 2 - 2 } & Monitor the use of rare earths \\
\cline { 2 - 2 } & Ability to be pre-treated and/or dismantled at the end-of-life phase \\
\cline { 2 - 2 } & $\begin{array}{l}\text { Ban of component parts which must not be reused in the construction of new } \\
\text { vehicles }\end{array}$ \\
\cline { 2 - 2 } & $\begin{array}{l}\text { Encourage the use of recycled materials, natural organic modified materials and } \\
\text { natural fibres, and biopolymers }\end{array}$ \\
\hline Extrinsic & Evaluate the effects on the vehicle to recyclability and recoverability \\
\cline { 2 - 2 } & Evaluate the effects on the vehicle in terms of environmental impacts \\
\hline
\end{tabular}


(1) The "intrinsic" requirements: An environmental requirement for an innovation is "intrinsic" if it is linked to the function and elements that constitute the innovation; e.g. heavy metal ban, chemical risk elimination, or end-of-life treatment obligations are requirements that are directly related to the innovative element and do not require information on the system that will welcome it.

(2) The "extrinsic" requirements: An environmental requirement for an innovation is called "extrinsic" if it applies to the entire vehicle system; e.g. the ELV Directive requires approval for the recyclability of the vehicle system as a whole and not for the component parts of the vehicle taken separately.

The R\&E checklist aims to evaluate both intrinsic and extrinsic requirements in a "Go/No go" way. It has been co-developed by the eco-design leader and a sample of 11 voluntary innovation leaders. Moreover, for an evolutionary integration of the environmental requirements, the $\mathrm{R} \& \mathrm{E}$ checklist has been integrated in three steps: first, only intrinsic requirements; second, addition of the recyclability effect evaluation; and third, addition of the environmental impact evaluation. The co-development and the evolutionary integration have led to two major results:

(1) Beginning of a cross learning process: the "Eco-design" team has learned on innovation process, and innovation leaders have learned on environmental requirements.

(2) Systematic use of the $R \& E$ checklist in the innovation process: The $R \& E$ checklist was submitted to the department in charge of the quality in innovation; it has validated the inclusion in the milestone reviews of a paragraph relating to the environmental requirements requiring an approval from the "Eco-design" team. To obtain this approval and to validate the TRL 5, TRL 51/2, and TRL 6 milestones, the paragraph prescribes the completion of the R\&E checklist by the innovation pilots.

\section{Focus on the Environmental Impact Evaluation Requirement}

Both extrinsic requirements need an analytic evaluation tool to propagate the effects of the innovation to the complete future vehicle. Here is presented TEEPI, a tool for evaluating the environmental performance of innovations.

\subsection{TEEPI Operation}

TEEPI has been developed to evaluate the impact of innovations on vehicles' environmental life cycle performance. This tool also requires a method for developing environmental models of vehicles. Indeed, in a previous paper [16] we 
developed a method to create Evolving Models for the Environmental Evaluation of Complex Systems (EMEECS). EMEECS are supported by Hierarchical Agglomerative Clustering (HAC) [17] for processing vehicles' available LCA results followed by an algorithm for creating optimal clusters relative to a fixed uncertainty threshold.

For building EMEECS, available results of previous LCA are collected over a fixed number of life cycle stages and of environmental indicators. The life cycle stages are:

- Manufacturing: including raw materials extraction, manufacturing processes; excluding upstream and downstream logistics; the assembly plants are considered separately;

- Use: including well-to-wheels fuel consumption, tank-to-wheels emissions of $\mathrm{CO}_{2}$ and pollutants ( $\left.\mathrm{CO}, \mathrm{NOx}, \mathrm{HC}\right)$, maintenance;

- End of life: no credit has been taken into account because of recycled material provision.

Four environmental indicators are included in the study. These indicators are generally used by car manufacturers to communicate, or by scientific papers in the automotive sector. They are calculated using the CML 2001 method:

- Global warming potential, GWP, [kg CO 2 -Equivalent];

- Eutrophication potential, EP, [kg Phosphate-Equivalent];

- Photochemical ozone creation potential, POCP, [kg Ethen-Equivalent];

- Abiotic depletion potential, ADP, [kg Sb-Equivalent].

LCAs are processed with a HAC in order to classify the vehicles' environmental indicators values in dendrograms per life cycle stage and per environmental impact category. HAC is chosen because it is one of the most common methods to make clusters from a statistical population. As a result, each dendrogram is processed for extracting the "optimal" clusters, i.e. clusters whose error relative to real values of vehicles within the cluster is inferior to a fixed threshold. Each cluster has a value (the average value of vehicles' environmental indicators that form the cluster) and an uncertainty (the standard deviation of those same values). The EMEECS are environmental impact archetypes, calculated on several life cycle stages, of complex system clusters, i.e. the user of the proposed method would directly use the environmental impacts values of the initial and innovative solutions, and of the global system.

\subsection{Case Studies: Micro-Hybridization Systems}

TEEPI was tested on two micro-hybridization systems: fuel-electricity (Inno_1) and fuel-compressed air (Inno_2). The reduction in fuel consumption is achieved through supplying energy, complementary to traditional fossil energy to move the 
Table 2 EMEECS characteristics of Inno_1 and Inno_2

\begin{tabular}{l|l|l}
\hline EMEECS & Inno_1 & Inno_2 \\
\hline Type & B & B \\
\hline Shape & Sedan & Sedan \\
\hline Finish & Average & Average \\
\hline Gearbox & Manual & Manual \\
\hline Fuel & Diesel & Gasoline \\
\hline Consumption $[L / 100 \mathrm{~km}]$ & 3,8 & 4,5 \\
\hline Assembly plant & AP_A & AP_A \\
\hline
\end{tabular}

car. Both micro-hybrid systems require extra parts to be added to the vehicle. For innovative solution Inno_1, the hybridization requires a lead battery heavier than the normal one, an electronically-controlled gearbox instead of the manual gearbox, a reversible starter-alternator ensuring the Stop and Start function, and an ultracapacitor to supply the required power. The innovative solution is $42 \%$ heavier than the initial solution; nevertheless, a diesel consumption reduction of $11 \%$ was measured on the NEDC homologation test. Concerning innovative solution Inno_2, the gearbox and the powertrain adaptation are largely affected, an ultracapacitor is also added, while the lead battery mass is reduced. The innovation solution is $151 \%$ heavier than the initial solution; nevertheless, a 35\% reduction in gasoline consumption was measured on the NEDC homologation test. Table 2 is a synthesis of the vehicle characteristics for selecting the EMEECS, which are available to the "Eco-design" team during the innovation phase. On Fig. 2, TEEPI results are compared to the reference LCAs of Inno_1 and Inno_2.

We observe that the signs of the growth rates given by TEEPI are the same as those of the reference LCAs. Concerning the values of growth rates, the maximum

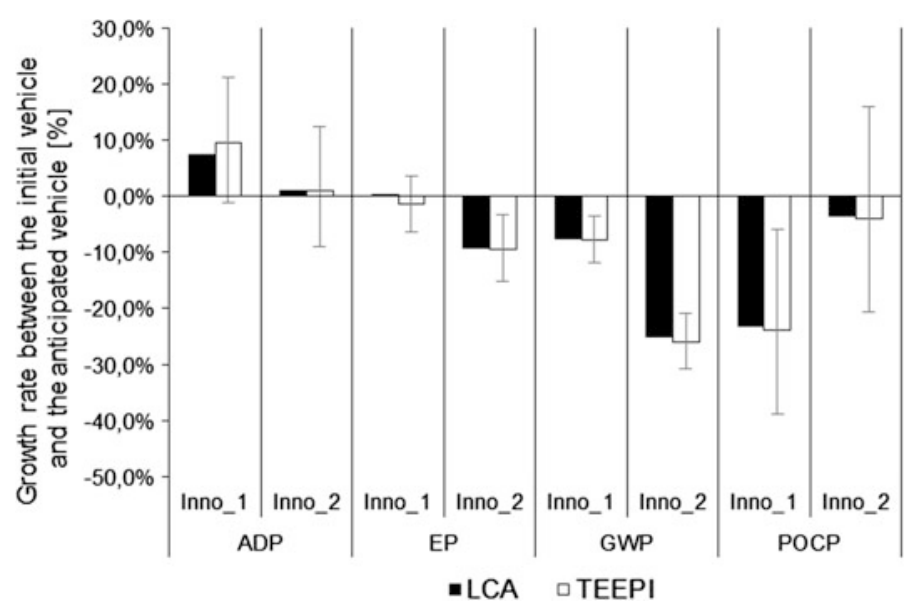

Fig. 2 Comparison between the results from TEEPI and the results from reference LCA for micro-hybrid systems Inno_1 and Inno_2 
error of TEEPI on the five innovations compared to reference LCAs is $2.1 \%$; this is obtained for the ADP indicator of Inno_2. We can also notice that the uncertainties of POCP are significantly higher than those of the other indicators. The length of uncertainty interval of the POCP indicator is directly linked to the use phase, for which the POCP depends on the emissions of three pollutants (carbon monoxide, nitrogen oxides, and hydrocarbons). These emissions vary randomly from one vehicle to another and from one powertrain to another.

\section{Conclusion}

E3PICS methodology has led to a successful integration of the "Environment" service's requirements in the innovation phase. Through the validation of the eco-design framework of reference in the quality procedures, the R\&E checklist is systematically used for all innovations from TRL 5 to TRL 6.

TEEPI was developed to take into account one of the "Environment" service's requirements of the eco-design framework of reference. Validating the eco-design framework of reference within the innovation process implies systematic use of TEEPI for the environmental evaluation of innovations that are selected according to three criteria defined by the eco-design leader: mass of the innovative solution, material change between initial and innovative solution and impact on vehicle fuel consumption.

TEEPI was not designed for generating eco-designed innovative concepts; therefore, it would be relevant to link this tool to a decision-making design tool such as those using the problem resolution by constraint satisfaction algorithm [18].

From an organizational point of view, TEEPI has been approved for being used during the innovation process; it is currently used by the LCA expert. Each innovation is saved in a database that could be used as a case-base in order to use knowledge management systems and generate good practices in eco-designing products. Lastly, to make it easier for innovation leaders to take the environmental dimension into account, they could use a version of TEEPI integrated into a company's intranet platform administrated by the LCA expert. This would help innovation leaders to simulate several innovation options directly. However, a necessary condition would be that innovation leaders have a good knowledge of life cycle issues. The current version of TEEPI is the learning vector of this knowledge.

\section{References}

1. International Energy Agency, Key World Energy Statistics 2015, 2015.

2. European Parliament Council, Regulation No. 443/2009 of the European Parliament and of the Council of 23 April 2009 setting emission performance standards for new passenger cars 
as part of the Community's integrated approach to reduce $\mathrm{CO}_{2}$ emissions from light-duty vehicles, 2009.

3. Johansson G, Greif A, Fleischer G, Managing the design/environment interface: studies of integration mechanisms, International Journal of Production Research, Vol. 45, No. 18-19, 2007, pp. 4041-4055.

4. Oltra V, Saint Jean M, Sectoral systems of environmental innovation: An application to the French automotive industry, Technological Forecasting and Social Change, Vol. 76, No. 4, 2009, pp. 567-583.

5. Beaume R, Maniak R, Midler C, Crossing innovation and product projects management: A comparative analysis in the automotive industry, International Journal of Project Management, Vol. 27, No. 2, 2009, pp. 166-174.

6. Schmidt W-P, Life Cycle Tools within Ford of Europe's Product Sustainability Index. Case Study Ford S-MAX \& Ford Galaxy (8 pp), The International Journal of Life Cycle Assessment, Vol. 11, No. 5, 2006, pp. 315-322.

7. Karlsson R, Steen B, Wendel A, EPS as a Life Cycle oriented System Assessment Tool to Facilitate Industrial Learning about Relations to the Environment, The 15th International Conference of the System Dynamics Society, Istanbul, Turkey, 1997, pp. 61-68.

8. Tingström J, Karlsson R, The relationship between environmental analyses and the dialogue process in product development, Journal of Cleaner Production, Vol. 14, No. 15-16, 2006, pp. 1409-1419.

9. Nakano K, Hirao M, Collaborative activity with business partners for improvement of product environmental performance using LCA, Journal of Cleaner Production, Vol. 19, No. 11, 2011, pp. 1189-1197.

10. Keoleian G.A, Kar K, Elucidating complex design and management trade-offs through life cycle design: air intake manifold demonstration project, Journal of Cleaner Production, Vol. 11, No. 1, 2003, pp. 61-77.

11. Bocken N.M.P, Allwood J.M, Willey A.R, King J.M.H, Development of an eco-ideation tool to identify stepwise greenhouse gas emissions reduction options for consumer goods, Journal of Cleaner Production, Vol. 19, No. 12, 2011, pp. 1279-1287.

12. Gasafi E, Weil M, Approach and application of life cycle screening in early phases of process design: case study of supercritical water gasification, Journal of Cleaner Production, Vol. 19, No. 14, 2011, pp. 1590-1600.

13. Poudelet V, Chayer J-A, Margni M, Pellerin R, Samson R, A process-based approach to operationalize life cycle assessment through the development of an eco-design decision-support system, Journal of Cleaner Production, Vol. 33, 2012, pp. 192-201.

14. Hatcher G.D, Ijomah W.L, Windmill J.F.C, Integrating design for remanufacture into the design process: the operational factors, Journal of Cleaner Production, Vol. 39, 2013, pp. 200-208.

15. Garcia J, Millet D, Tonnelier P, A tool to evaluate the impacts of an innovation on a product's recyclability rate by adopting a modular approach: automotive sector application, International Journal of Vehicle Design, Vol. 67, No. 2, 2015, pp. 178-204.

16. Garcia J, Millet D, Tonnelier P, Construction of evolving models for the environmental evaluation of innovative sub-systems based on a hierarchical agglomerative clustering, International Journal of Sustainable Engineering, Vol. 8, No. 6, 2015, pp. 386-404.

17. Hastie T, Tibshirani R, Friedman J.J.H, The elements of statistical learning, Springer New York, 2001.

18. Tchertchian N, Yvars P-A, Millet D, Benefits and limits of a Constraint Satisfaction Problem/ Life Cycle Assessment approach for the ecodesign of complex systems: a case applied to a hybrid passenger ferry, Journal of Cleaner Production, Vol. 42, 2013, pp. 1-18. 
Open Access This chapter is licensed under the terms of the Creative Commons Attribution 4.0 International License (http://creativecommons.org/licenses/by/4.0/), which permits use, sharing, adaptation, distribution and reproduction in any medium or format, as long as you give appropriate credit to the original author(s) and the source, provide a link to the Creative Commons license and indicate if changes were made.

The images or other third party material in this chapter are included in the chapter's Creative Commons license, unless indicated otherwise in a credit line to the material. If material is not included in the chapter's Creative Commons license and your intended use is not permitted by statutory regulation or exceeds the permitted use, you will need to obtain permission directly from the copyright holder.

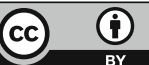




\title{
Development of an Environmental Evaluation Tool in the Transport Sector and Its Impact on Decision-Making in the Early Stages of Design
}

\author{
Sergio A. Brambila-Macias, Lisbeth Dahllöf, Karin Eriksson \\ and Tomohiko Sakao
}

\begin{abstract}
Due to government policies and regulations as well as customer and societal demands, organizations around the world are looking for ways to manage their economic, environmental and social sustainability. One of the most frequently used standards for organizations seeking to manage their environmental responsibilities is ISO 14001. This framework, however, is generic because it can be used by any organization irrespective of sector, activity or core values. Therefore, implementation of generic guidelines might result in the use of alternative tools that respond better to specific organizational needs and that provide outcomes that can be useful for decision-making. Through case study methodology, this paper shows how Volvo Group, a world-leading producer of transport solutions, developed an internal environmental evaluation tool called Environmental Screening (EnvS) to improve the environmental performance of its solutions.
\end{abstract}

\section{Introduction}

The degradation of the environment through resource extraction, manufacturing, use and disposal of products, as well as the provision of services has been one of the main drivers for societies and governments to look for more sustainable alternatives. The United Nations Conference on the Human Environment in 1972 in Stockholm was a milestone for raising awareness of the actions needed to tackle environmental deterioration. Currently, sustainable initiatives can range from global efforts, such as the UN Sustainable Development Goals [1], to regional directives

\footnotetext{
S. A. Brambila-Macias $(\bowtie) \cdot$ T. Sakao

Department of Management and Engineering, Division of Environmental

Technology and Management, Linköping University, 58183 Linköping, Sweden

e-mail: sergio.brambila@liu.se

L. Dahllöf

Swedish Environmental Institute, 41133 Gothenburg, Sweden

K. Eriksson

Volvo Group Trucks Technology, 41715 Gothenburg, Sweden

(C) The Author(s) 2018

E. Benetto et al. (eds.), Designing Sustainable Technologies,

Products and Policies, https://doi.org/10.1007/978-3-319-66981-6_42
} 
like the WEEE directive for electronic waste [2] and EVL for the end-of-life of vehicles in Europe [3] to local environmental objectives (e.g. Sweden's 16 environmental quality objectives) [4]. Furthermore, participatory initiatives and standards for companies and organizations such as the Global Reporting Initiative (GRI) and ISO standards demonstrate efforts undertaken towards sustainability. With regard to the environment, ISO 14001 exemplifies the participatory action that companies may take to reduce their impact on the environment. However, this standard is general and differences still remain, for example, organizations differ in how they are organized, what they offer, their business model and their core values. These differences may lead to the adoption and adaptation of tools that support decision-making in contexts suitable to the organizations and their specific circumstances $[5,6]$. A problem area that is frequent in product development organizations is that environmental awareness initiatives are based at the manufacturing level and not at the product level [7], resulting in narrow coverage by an environmental program. Through case study methodology, this paper offers a unique view on how Volvo Group developed Environmental Screening (EnvS), a tool aimed at improving the environmental performance of its products and solutions. In this regard, three research questions are postulated.

- How can an environmental evaluation tool towards sustainability be used more in practice at a large company?

- How can internal organizational needs be identified and addressed in order to develop an environmental evaluation tool towards sustainability?

- How could an environmental evaluation tool support decision-making in the early stages of design towards sustainability?

\section{Background Research}

The scientific literature provides several examples of how organizations can adopt and adapt tools to reduce the environmental impact of their operations. This is especially important in the early stages of design, given the greater potential there to reduce the environmental impact of products and services [8]. The early stages of design seem to benefit most from qualitative and easy-to-use tools. This could be partially explained by the lack of information, risk, uncertainty and subjectivity found during the early stages [7]. Schöggl et al. [9] suggest that qualitative tools are preferred at this stage, due to the lack of information and difficulty in quantifying environmental impacts. Tools and methods that address environmental impacts usually fall under the umbrella of Design for the Environment or Ecodesign [7], which can be described as an approach that addresses the environmental impact of the entire life of a product or service, from the early stages of design [6]. Tools following this approach are plentiful. Pigosso et al. [10] identified 350 publications between 1993 and 2015 dealing with tools and methods related to Ecodesign. However, Rousseaux et al. [11] suggest that there is still low uptake of such tools. 
Reasons for this can be due to lack of knowledge about the tools, lack of specialized staff that can use such tools, company size and low cooperation between departments. Poulikodou et al. [12] further identify obstacles to the use of environmental tools; for instance, tools may be too vague, many checklists are already available, tools need detailed information, the competence of users is low and there are delays from suppliers. Bovea and Elis [13] identified checklists, matrices and rules or guidelines (e.g. 10 golden rules of ecodesign) among the most often used qualitative and semi-quantitative tools. Knight and Jenkins [14] also suggest that some of the most common or preferred ecodesign tools are in the form of guidelines and checklists. An example of this can be seen in the work by Lindahl and Tingström [15], who adapted Failure Mode and Effect Analysis (FMEA) to develop a checklist for environmental considerations called Environmental Effect Analysis (EEA). EEA requires qualitative information as opposed to Life Cycle Assessment (LCA), which requires quantitative data and which users may find lengthy and difficult to use [13].

More recent tools not only addressing environmental aspects but also social and economic ones have also been proposed in the literature. For instance, Hallstedt [16] proposes a sustainability criteria matrix targeting the early stages of product development and a qualitative measure called sustainability index for decision support. Schöggl et al. [9, 17] propose the Checklist for Sustainable Product Development (CSPD) based on questions divided into four sustainability principles and 9 categories where a sustainability expert is crucial for the use of the tool.

Furthermore, Fitzgerald et al. [9] suggest that guidelines and checklists need to be company-specific and integrated systematically in the product development process, and that using standalone and generic tools may not be effective. Hence, specific procedures should be determined to avoid confusion on when and how to use such tools. Fitzgerald et al. [9] divide decision-making in new product development into two broad types: design decisions and management decisions. Design decisions address questions like what should be designed and determine shape, size, materials, processes and components. Management decisions address questions that attempt to ensure the design will result in a successful product. What will be doneand when and who will do it-are some management questions. It is therefore important to be clear about the purpose of the tool as well as who is going to use it, the necessary inputs and the beneficial outputs. The following case study further supports the use of an environmental checklist.

\section{Case Study}

Since 1989, Volvo Group has pledged to minimize the environmental impact of its operations through adopting a life cycle approach in its operations [18]. Today, Volvo's White, Grey and Black Lists for materials selection to substitute, caution or avoid materials, respectively [19-21], are an example of its commitment to reduce environmental impacts. With regard to environmental standards, Volvo Group 
began using the Environmental Failure Mode Analysis (E-FMEA) in 1997 in order to fulfil requirements in ISO 14001 for product development. However, since FMEA was a protected name the group changed it to the one described by Lindahl and Tingström, Environmental Effect Analysis (EEA) [22]. After some time, Volvo Group renamed the tool as Environmental Impact Analysis (EIA), which was later standardized within the group. Due to internal and external needs EIA was further developed, customized and replaced in some projects by the Environmental Screening (EnvS) tool.

The EIA as well as EnvS are based on forms to be filled in at cross-functional meetings for Product Development (PD) projects where representatives from PD, production including logistics, aftermarket and purchasing (when relevant) participate. A facilitator who has prepared and also has knowledge of the method and environmental aspects leads the meeting and fills in the forms. At the meeting, actions are determined and followed up on afterwards. The EIA has most frequently been used to evaluate new design of powertrain components since these components may impact the environment, especially during the use phase. The tool has also to a great extent been used to evaluate the design of packaging materials, since these are often volume products and easy to assess. It has, however, not been used for all PD projects. A reason for this is that users considered the tool to be too time consuming, and with little benefit since there already are checklists for this (e.g., chemical use and standards for marking of parts, facilitation of recycling) and environmental demands are already in place for technical requirements in PD projects. Another reason for the lack of use of the EIA tool has been that it is also highly dependent on the knowledge and experience of the meeting participants, increasing the possibility to miss environmental risks and making it more dependent on the previous knowledge and experience of the users. Figure 1 partially shows the EIA tool.

In comparison, the format with a checklist in the EnvS ensures that the environmental risk factors considered most important are covered. Furthermore, several external factors have influenced the need for EnvS as a new tool that could incorporate new demands. For instance, in 2015 a revised ISO 14001 places more emphasis on products, services and a life cycle perspective. The standard states in its Sect. 6.1.2: Within the defined scope of the environmental management system,

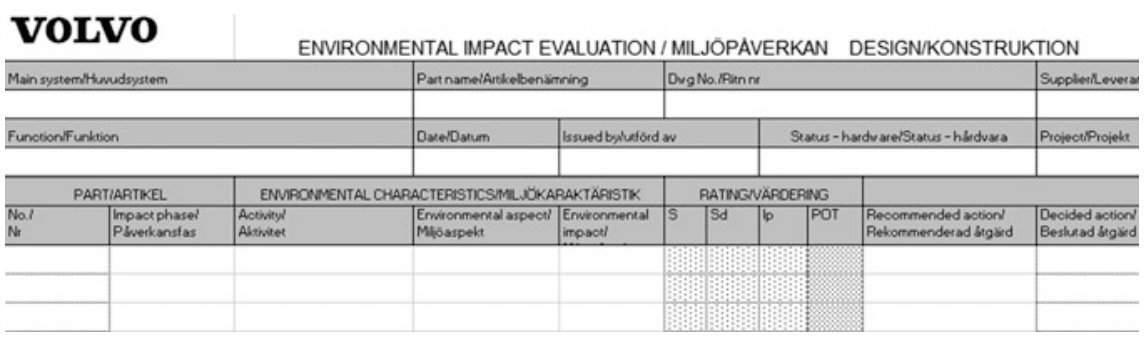

Fig. 1 A selected section of the environmental impact evaluation form 
the organization shall determine the environmental aspects of its activities, products and services that it can control and those that it can influence, and their associated environmental impacts, considering a life cycle perspective. p. 9 [23]. Additionally, resource efficiency has increasingly become the focus of European initiatives (e.g., the EU's circular economy package) [24].

Therefore, it had been identified that the environmental analysis had to be considered in all PD projects, but to be able to make this happen, the tool had to be easier and faster to use. A reference group was put in place to develop and approve the environmental screening tool. It was also acknowledged that the connection between PD and production could be improved. The improvement work was mainly done during 2015, together with the reference group with participants for PD and production from the different parts of Volvo Group. This reference group was important, since they had an opportunity to influence the development process of the environmental design tool. As opposed to EIA, EnvS will need to be regularly reviewed and updated to secure that the most important environmental risks are covered.

Moreover, in the effort to adapt ease of use and beneficial outcomes, two Environmental Screening (EnvS) tools were developed, one for PD and one for production, with a link in-between, so if a new component affected Volvo Group production, the checklist for production would be filled in. The EnvS for PD is a tool for collecting answers to all known, relevant environmental questions that are important for PD. It may be so that another tool needs to be used to be able to answer a question, [e.g., if a question regards Design for Recycling (DfRecycling)]. To be able to answer if a new part will be designed for recycling, the DfRecycling checklist may have to be completed. The EnvS for production is a checklist on its own, with no extra checklists to consider for answering the questions. For PD, it is important that the start-up meeting is held early in the development process. If it is held before a supplier is chosen, then requirements can more easily be added to the supplier. The two EnvS tools have been tested and further developed in pilot studies. During the pilot studies the enthusiasm from engineers was also crucial in developing the tool, since they expressed their desire to make a difference.

\section{Discussion}

The first research question can be answered by how Volvo Group established a reference group from different departments that also included users of the tool. The case study also highlighted the importance of carrying out a pilot study for useful feedback and refinement of the tool.

Regarding the identification of organizational needs and the determination of how to manage them in order to adapt or create a tool to address environmental performances, the case study showed that the context plays an important role not only in shaping company's core values, but also in its operations, use of tools and ultimately decision-making. In this case study, the organisational needs were 
identified by the manufacturing department as well as the engineers wanting to make a difference. It also became clear that communication between design and manufacturing had to be enhanced, and that a tool in the form of a checklist could help in strengthening that communication.

Finally regarding the identification of users, involved actors, inputs and useful outputs that tools need to consider and/or provide in order to support decision-making in the early stages of design, the EnvS tool(s) at Volvo Group identified the users of the tool as personnel from the manufacturing, engineering, purchasing, aftermarket and styling departments. The inputs for the tool came from external and internal sources, an example being the increasing awareness of environmental impact and enthusiasm from the engineering department to change the status quo.

The outputs are then useful for analysing decisions and acting on them. Information from a Design for Remanufacturing (DfReman), Design for Recycling (DfRecycling) or LCA study are some of the inputs to the EnvS checklist then providing outputs for actions such as choice of concept improvement of supplier demands and compliance with ISO 14001. It also illustrates that a checklist can serve the purpose of addressing both design and management decisions.

In order to illustrate how the checklist supports decision-making, the following diagram shows the different inputs, outputs, purpose and decision-making (actions) of the checklist. This is depicted by using the PDCA quality cycle, which stands for Plan-Do-Check-Act [25] (Fig. 2).

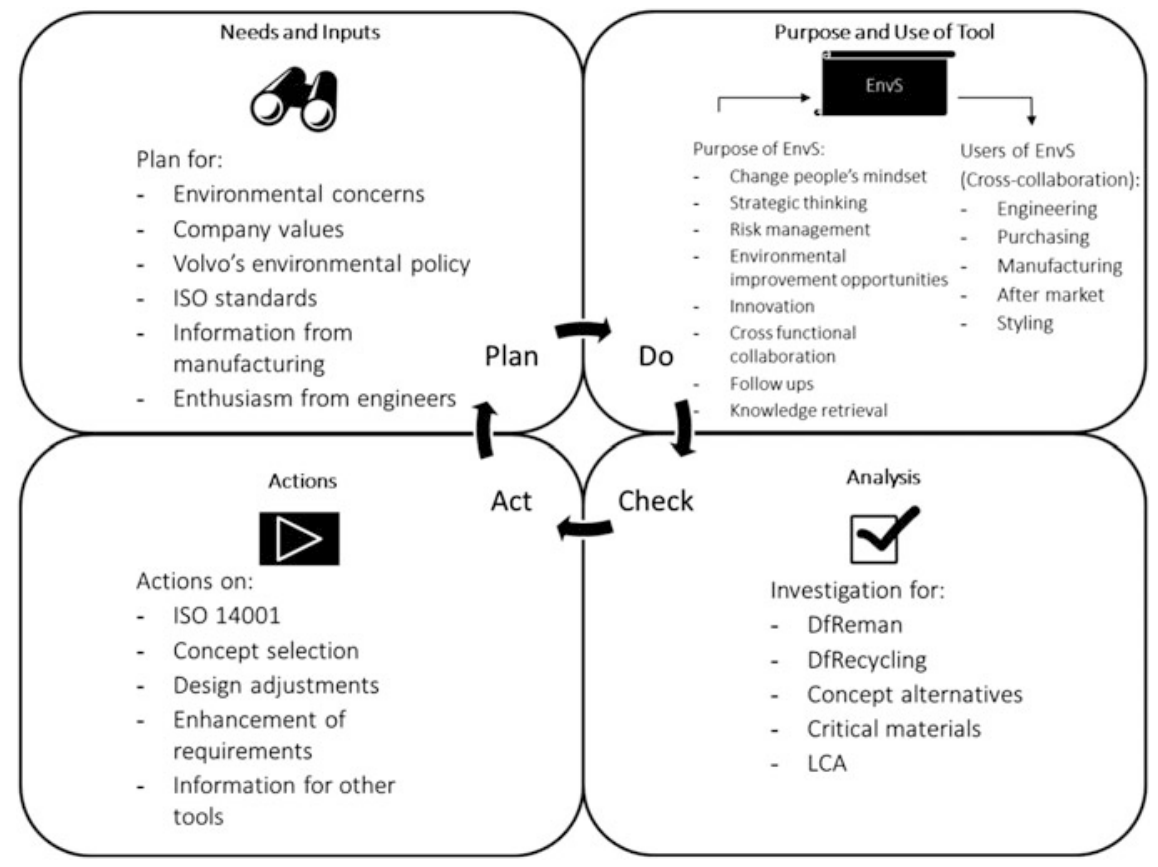

Fig. 2 The impact of environmental screening on decision-making 


\section{Conclusion}

This methodology development, presented through a case study, showed how an organisation in the transport sector has developed a checklist named Environmental Screening (EnvS) in production and product development. From the analysis of the case study, important conclusions can be drawn.

These can potentially help interested readers in their own design and implementation of environmental tools, and can be summarised as follows (in no specific order):

- Carrying out a pilot project in order to get feedback and refine the usefulness of the tool is advisable.

- Enthusiasm from workforce for buy-in and feedback can help in developing the tool.

- A facilitator with deeper knowledge can operate the tool and simplify complexity arising from multiple stakeholders and levels of knowledge.

- A reference group consisting of relevant people from different departments and potential users of the tool are important to consider.

- A tool should match and reflect company values for easier incorporation into every day operations.

It is also important to mention that this research has some limitations. The use of a single case study presents a particular example in industry. However, this research can stimulate discussion and an exchange of experiences from organizations facing similar challenges towards sustainability.

The results of this research can be insightful for companies that want to incorporate environmental tools into their design, operations and product development. They could potentially use this research when developing tools of their own. Academics that do research on design and implementation of methods can also benefit from this study. They can find and compare similar needs in other organizations and identify factors to be considered when developing tools and validating them. Finally, future work could address tools for earlier stages in design (fuzzy front end) and other sustainability aspects that can provide an insight into how needs are identified and addressed.

Acknowledgements This research was supported by the Mistra REES (Resource Efficient and Effective Solutions) program, funded by Mistra (The Swedish Foundation for Strategic Environmental Research). 


\section{References}

1. Griggs D, et al., Policy: Sustainable development goals for people and planet, Nature, 2013, 495(7441): pp. 305-307.

2. Zoeteman B.C, Krikke H.R, Venselaar J, Handling WEEE waste flows: on the effectiveness of producer responsibility in a globalizing world, The International Journal of Advanced Manufacturing Technology, 2010, 47(5): pp. 415-436.

3. Johnson M, Wang M, Sustainability and management of end-of-life vehicles (ELVs) within the European Union's Directive on ELVs, 2001, SAE Technical Paper.

4. Naturvårdsverket, Steering towards the environmental objectives: The Swedish Environmental Protection Agency's in-depth evaluation of the environmental objectives 2015, T.S.E.P. Agency, Editor, 2017.

5. Martín-Peña M.L, Díaz-Garrido E, Sánchez-López J.M, Analysis of benefits and difficulties associated with firms' Environmental Management Systems: the case of the Spanish automotive industry, Journal of Cleaner Production, 2014, 70: pp. 220-230.

6. Yin H, Schmeidler P.J, Why do standardized ISO 14001 environmental management systems lead to heterogeneous environmental outcomes? Business Strategy and the Environment, 2009, 18(7): pp. 469-486.

7. Fitzgerald D.P, et al., Design for environment (DfE): strategies, practices, guidelines, methods, and tools, 2007, Wiley, Hoboken, NJ. p. 1-24.

8. Lindahl M, Ekermann S, Structure for categorization of ecodesign methods and tools, Re-engineering manufacturing for sustainability, 2013, Springer, pp. 117-122.

9. Schöggl J.P, Baumgartner R.J, Hofer D, Improving sustainability performance in early phases of product design: A checklist for sustainable product development tested in the automotive industry, Journal of Cleaner Production, 2017, 140: pp. 1602-1617.

10. Pigosso D.C.A, McAloone T, Rozenfeld H, Characterization of the state-of-the-art and identification of main trends for Ecodesign Tools and Methods: Classifying three decades of research and implementation, Journal of the Indian Institute of Science, 2016, 95(4): pp. $405-428$.

11. Rousseaux P, et al., "Eco-tool-seeker": A new and unique business guide for choosing ecodesign tools, Journal of Cleaner Production, 2017, 151: pp. 546-577.

12. Poulikidou S, Björklund A, Tyskeng S, Empirical study on integration of environmental aspects into product development: processes, requirements and the use of tools in vehicle manufacturing companies in Sweden, Journal of Cleaner Production, 2014. 81: pp. 34-45.

13. Bovea M, Pérez-Belis V, A taxonomy of ecodesign tools for integrating environmental requirements into the product design process, Journal of Cleaner Production, 2012, 20(1): pp. 61-71.

14. Knight P, Jenkins J.O, Adopting and applying eco-design techniques: a practitioners perspective, Journal of Cleaner Production, 2009, 17(5): pp. 549-558.

15. Lindahl M, Tingström J, En liten lärobok om miljöeffektanalys, 2000: Institutionen för teknik, Högskolan i Kalmar.

16. Hallstedt $\mathrm{S}$, et al., An approach to assessing sustainability integration in strategic decision systems for product development, Journal of Cleaner Production, 2010, 18(8): pp. 703-712.

17. Schöggl J, Baumgartner R.J, Hofer D, A Checklist for sustainable product development: Improving sustainability performance in early phases of product design, 2014.

18. Louis S, Wendel A, Life cycle assessment and design-experience from Volvo Car Corporation, 1998, SAE Technical Paper.

19. Nordkil T, Volvos vita lista, Volvo Corporate Standard, 1998.

21. Nordkil T, Volvos vita lista, Volvo Corporate Standard, 1998. 
22. Tingström J, Karlsson R, The relationship between environmental analyses and the dialogue process in product development, Journal of Cleaner Production, 2006, 14(15): pp. 1409-1419.

23. ISO, ISO 14001: 2015 Environmental Management Systems-Requirements with Guidance for Use. 2015. Retrieved from https://www.iso.org/iso-14001-environmental-management.html.

24. European Commission Press release, Closing the loop: Commission adopts ambitious new Circular Economy Package to boost competitiveness, create jobs and generate sustainable growth, 2015.

25. International Organization for Standardization, ISO 14001: 2015. Quality management systems - Requirements.Geneva: International Organization for Standardization, 2015, p.viii.

Open Access This chapter is licensed under the terms of the Creative Commons Attribution 4.0 International License (http://creativecommons.org/licenses/by/4.0/), which permits use, sharing, adaptation, distribution and reproduction in any medium or format, as long as you give appropriate credit to the original author(s) and the source, provide a link to the Creative Commons license and indicate if changes were made.

The images or other third party material in this chapter are included in the chapter's Creative Commons license, unless indicated otherwise in a credit line to the material. If material is not included in the chapter's Creative Commons license and your intended use is not permitted by statutory regulation or exceeds the permitted use, you will need to obtain permission directly from the copyright holder.

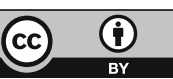




\title{
Discussion Panel-Assessment of Externalities: Monetisation and Social LCA
}

\author{
Stéphane Morel, Marzia Traverso and Philipp Preiss
}

\begin{abstract}
This paper aims to explore the topic of monetisation of environmental and social aspects. The content is issued from a discussion panel held in Luxembourg during the LCM2017 conference. It first explores historical and methodological aspects. The recommendations are made for companies who intend to monetise their impacts. Three industrial companies presented their contribution on their day to day use of monetisation. The key conclusions are twofold. In one hand, many challenges remain. The implementation is still not always feasible for all issues and an iterative approach is highly recommended. New methods on social LCA show a growing interest on this topic from various parties. ISO standards work is progressing and will support companies and government in their use of monetisation. From the company point of view, monetisation is a bridge between environmental and social assessment, and economic evaluation, while it provides a common unified unit (money). By using a common unit for financial and non-financial aspects, monetization allows the direct comparison of different aspects and therewith a more informed materiality assessment. By looking at impacts, a new perspective on current and future business risks and opportunities is provided. A better understanding of interdependencies enables more informed decision making. The monetary language is a door opener to access new stakeholder groups such as finance and accounting to raise awareness for sustainability.
\end{abstract}

\footnotetext{
S. Morel $(\bowtie)$

Renault, Alliance Technology Development, Guyancourt, France

e-mail: stephane.s.morel@ renault.com

M. Traverso $(\bowtie)$

RWTH Aachen University, Aachen, Germany

e-mail: marzia.traverso@inab.rwth-aachen.de

P. Preiss

Business School, Institute for Industrial Ecology, Sustainable Technology and Innovation Management, Pforzheim University, Pforzheim, Germany

(C) The Author(s) 2018

E. Benetto et al. (eds.), Designing Sustainable Technologies,

Products and Policies, https://doi.org/10.1007/978-3-319-66981-6_43
} 


\section{Introduction}

Social and environmental impacts occur along the product life cycle and are measured by different indicators. To assess the sustainability performance of a product life cycle, a social, economic and environmental impact assessment is needed.

Despite the absence of scientific agreement on a set of indicators to be considered in the framework of Life Cycle Sustainability Assessment (LCSA), several implementations have been already realised at companies and product levels.

However, the results of a sustainability assessment are often expressed through out several indicators, making their interpretation and the identification of the relative trade-offs challenging. A possibility to facilitate the interpretation is monetizing all impacts indicators. However, monetisation induced a set of methodological issues and choices that are debatable and that must be known before implementation. The aim of the session was to clarify the state of the art of related methodological approaches, towards a guidance for businesses and governments in order to correctly use monetisation in portfolio assessment and decision making.

Based on the outcomes of the session held at LCM 2017 conference, this paper first presents the most important elements from the methodological state of the art, and then some general guidance as well as concrete feedback from industries.

\section{State of the Art of the Methodology}

Monetisation is nowadays growing from research to standardisation. Monetary valuation has been used in policy making for decades mostly in cost-benefit analyses, since the early 30s in the United States. More recently in the European Union where this approach is observed since the 90s, in directives such as the National Emission Ceilings Directive (NEC directive (2001/81/EC), EU research projects such as the National Energy Education Development project (NEED (2007)), and many others. By the way, an umbrella of different concepts stays under the monetization term such as: market price, abatement costs, societal costs \& benefits etc.

Mr. Philipp Preiss clarify the definition as follow, based on ExternE (2005) [1, 2] researches:

- Externalities arise, when the social or economic activities of a participant have negative or positive impacts on another participant and these impacts are not fully accounted for or compensated by the first participant.

- External costs are externalities that are transformed into monetary values. They are the share of damage costs which is not internalised.

Monetisation is promoted for several reasons. In the first hand, with monetisation of externalities the monetary values are shown explicitly. However, without 
monetisation the impacts are implicitly monetised by the difference of the internal costs of corresponding alternatives (i.e. a decision between alternative technologies or policies). Or in other words, if impacts are not monetised the external costs are taken into account as if they were zero.

On the other hand, monetisation offers an additional indicator but must not be the only basis for decision-making. At member state level, environmental agencies are involved, such as the German Federal Environmental Agency (UBA): [3] or the French ministry of environment (MEEM) who held a specific workshop on monetisation of goods and environmental services through life cycle assessment in 2017.

Currently two ISO documents (ISO 14007 and ISO 14008) are under construction in order to provide guidance and allow a wider use of monetisation.

Mr. Preiss points out some limits regarding the comparability of results of various studies. Studies are different regarding their comprehensiveness. There is a lack of transparency if the underlying assumptions and models are not displayed. And generally, there is the lack of a widespread state of the art approach.

Mr. Chanoine (Deloitte) proposed a practical guidance issued from a study carried out with the industrial consortium SCORELCA in 2012 [4] and followed by a second study also driven by SCORELCA in 2016 of which the results are not published yet. It is based on operational recommendations on how to use monetary valuation to monetize LCA results. The proposal is an iterative approach with a go/ no go process in two steps. First, a set of questions allows to determine the necessity to carry out monetisation, and how it should be done. Then nine information sheets provide explicit implementation guidance.

As a first conclusion, monetary valuation in LCA should be used when identified as necessary, depending on the objectives of the study. It has been presented as an approach to solve trade-offs. As a counterpart monetisation remains an approach potentially complex to implement due to the set of parameters to be considered in correlation to set monetary values, moreover considering the potential subjectivity of those parameters. Also, it remains especially complex when it is required to develop specific monetarisation factors, which may request time, funding, some specific competences investments and political decisions.

\section{Feedback from the Industry on the Application of Monetisation}

This section provides feedback from three chemical companies.

Mr. Sonnen (Ecomatters), in collaboration with AkzoNobel, provided insights on how a company can monitor its global activity (downstream, upstream and themselves) on economic [5], environmental costs [6, 7] and social capitals [5]. It allows the company to identify business risks, opportunities, stimulate innovation 
and cooperation with value chain partners. This also permits to reduce the negative and strengthen existing positive externalities.

Since 2013, BASF calculates it 'real' contribution to a sustainable future. With the Value-to-Society approach, BASF assesses its positive and negative economic, social and environmental impacts on society along the value chain in $€$. The results are used in positioning and communication as well as progress monitoring. Application in decision making and goal setting is piloted. However, due to data accuracy, maturity level of methods as well as conceptual challenges, for a systematic embedment further research and standardization is required. These are under construction. Mr. van Gelder stated for BASF "With Value-to-Society we have a new macro perspective on benefits and costs of our economic, environmental and social impacts along the value chain".

Mr. Wathelet (SOLVAY) introduced the Sustainable Portfolio Management (SPM) map [7, 8]. There, the products are presented on a map according to two criteria: Operations Vulnerability (monetized environmental impact of production/ sales price) and Market Alignment (analyse of a product in an application through the lens of sustainability-benefits or roadblocks). This allows to categorized the products under three categories: Solutions (outstanding sustainability contribution for the society), neutral, and challenges (Strong negative signals in sustainability, to be improved or abandoned).

Those three companies are using monetarisation of LCA results to express the environmental impacts in terms of costs. Methods to evaluate social benefits remain very diverse. They all three agree that monetarisation of social and environmental aspects is very intuitive, allowing to gather positive and negative aspects of a system on a unique scheme, and can be useful to engage with top management. They also acknowledge the needs for further research.

\section{Discussion and Conclusion}

External cost calculation constitutes a bridge between economic decisions and environmental and social assessment while it can provide both types of results in a common unit, and is relevant to support three levels of decision: technical solution, product portfolio and the company strategy.

It can also be a support to engage with top management, allowing to align with materiality assessment and stakeholder's expectations.

Nevertheless, many challenges remain to improve the applicability of monetisation methodologies, and company acceptance. To be implemented, it is required to collect raw information from the supply chain and consumers. This starts with the life cycle inventory collection from the direct supplier to the very first one. Then the definition of monetization factor for each impact is necessary and crucial, for this last task a customer' survey can held in order to define the wiliness to pay. In alternative, monetization factors presented in literature can be used for the first implementations. 
The panel recommendations were to adapt the effort according to the pursued goal of the study. An iterative approach is highly recommended in order to first conduct a streamlined assessment, and then implement more complex methods when necessary.

The company acceptance relies on the communication of monetized results since monetization could be used at various level in companies, the targeted audience is diverse and the media shall be adapted in consequence. An exhaustive state of the art on methodological issues related to monetary valuation and on the way to interpret the monetised indicators would be valuable to the practitioners.

One remaining challenge also stands on the capability to introduce these cost and social information in decision making and performance monitoring. This raises here a new question grounded in the financial accounting. How to integrate costs and revenues which are not paid or perceived directly? Shall external cost performance be a key indicator and for whom? How to transfer the benefits and positive impact to the contributors?

As a final conclusion, despite these raising questions the panel underlined the remaining researches to be carried on, and agreed that environmental and social cost assessment, already today, have a major ability to reinforce decision making.

Acknowledgements We would like to thank the panel for their contribution: Mr. Chanoine (Deloitte), Mr. Sonnen (Ecomatters), Mr. Van Gelder (BASF) and Mr. Wathelet (SOLVAY).

\section{References}

1. ExternE is the acronym for "External Costs of Energy" and a synonym for a series of projects starting from early 90s till 2005, http://www.externe.info/externe_d7.

2. De Nocker L, Panis L, Torfs R, ExternE: A European accounting framework for Life Cycle Impact Assessment and external costs of transport, SAE Technical Paper, No. 2000-01-1480, 2000.

3. German Federal Environmental Agency (UBA), 2007: "Economic Valuation of Environmental Damage-Methodological Convention for Estimates of Environmental Externalities", 2013: update. http://www.umweltbundesamt.de/publikationen/economic-valuation-of-environmentaldamage-02018/19:\%202nd\%20update.

4. Ministère de l'Environnement, de l'énergie et de la mer, Monétarisation des biens et services environnementaux: L'analyse de cycle de vie, questions et enjeux autour de sa monétarisation, 2017.

5. Ecomatters, 2016. Ecomatters, Human capital creation along the value chain, 2016), www. ecomatters.nl/human-capital.

6. Weidema B-P, Pizzol M, Brandão M, The Use of Monetary Valuation of Environmental Impacts in Life Cycle Assessment: State of the art, strengths and weaknesses, SCORE-LCA report $\mathrm{Nb} 2012-03,2013$.

7. Steen B, 2015. Environmental Priority Strategy (EPS) system, Chalmers University, 2015), http://www.ivl.se/english/startpage/pages/focus-areas/environmental-engineering-and-sustainableproduction/lca/eps.html.

8. SOLVAY 2017 http://www.solvay.com/en/binaries/Solvay-SPM-Guide-154709.pdf. 
Open Access This chapter is licensed under the terms of the Creative Commons Attribution 4.0 International License (http://creativecommons.org/licenses/by/4.0/), which permits use, sharing, adaptation, distribution and reproduction in any medium or format, as long as you give appropriate credit to the original author(s) and the source, provide a link to the Creative Commons license and indicate if changes were made.

The images or other third party material in this chapter are included in the chapter's Creative Commons license, unless indicated otherwise in a credit line to the material. If material is not included in the chapter's Creative Commons license and your intended use is not permitted by statutory regulation or exceeds the permitted use, you will need to obtain permission directly from the copyright holder.

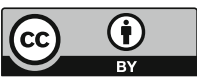




\title{
Applying LCA to Estimate Development Energy Needs: The Cases of India and Brazil
}

\author{
Narasimha D. Rao, Alessio Mastrucci and Jihoon Min
}

\begin{abstract}
This paper illustrates the use of life cycle assessment (LCA) methods to link human wellbeing to resource consumption. Based on a previously developed framework of the material requirements for human well-being, we use LCA and Input-Output (I/O) analysis, as appropriate, to estimate the life-cycle energy needed to meet the gap in living standards in two emerging economies, India and Brazil. We illustrate the relative contribution of different living standards components to energy requirements, as well as the uncertainty and trade-offs between upfront and long-term operating energy costs, and how these factors differ in the two countries. This analysis provides insights on how LCA analysis can be used to inform energy planning and its links to development goals.
\end{abstract}

\section{Introduction}

There is much potential for life cycle assessment (LCA) to inform public policy beyond its traditional use to address environmental objectives [1]. This paper illustrates how LCA can be used to inform development and energy policies related to improving living standards in emerging economies. Our motivation is that technology, material and design choices in the build-up of infrastructure can present important trade-offs between short-term (construction) and long-term (operating) energy needs, as well as different net energy requirements. These differences matter in emerging economies such as India and Brazil that expect high future economic growth and significant infrastructure investments. The Indian government anticipates 1.5 trillion dollars of infrastructure investments over the next decade [2]. Furthermore, almost a quarter of the building stock ( $\sim 50$ million units), requires upgrading [3], not counting population growth and future migration to urban areas.

N. D. Rao $(\varangle) \cdot$ A. Mastrucci · J. Min

International Institute for Applied Systems Analysis (IIASA),

Energy (ENE) Program, 2361 Laxenburg, Austria

e-mail: nrao@iiasa.ac.at

(C) The Author(s) 2018

E. Benetto et al. (eds.), Designing Sustainable Technologies,

Products and Policies, https://doi.org/10.1007/978-3-319-66981-6_44 
Brazil is a useful contrast, as it faces similar social challenges, such as high inequality, but a very different scale and with different resource endowments.

Based on a previously developed account of decent living standards ("DLS"), which define the material requirements for human wellbeing [4], we use LCA and Input-Output (I/O) analysis, as appropriate, to estimate the life-cycle energy required to meet the gap in, and sustain, DLS for all. We consider the energy associated with delivering DLS goods and services, including food production and preparation, clothing, housing, the extension of networks for water and sanitation, electricity, social infrastructure (health and education), communications, roads and transport systems. Our emphasis in this study is on methodology, not on empirical findings. That is, rather than supplying comprehensive estimates on the energy requirements for providing DLS, we aim to illustrate how LCA methods can be used to provide insights into such a quantification exercise. We make three contributions in this regard: (a) we illustrate the relative contributions of living standard components to the estimation of energy requirements for a DLS; (b) we show how uncertainty can be systematically quantified and attributed to these choices; and (c) we show the trade-offs in construction and operating energy inherent in these choices.

\section{Methods}

The methodology includes three steps: estimation of the gaps in DLS; calculation of life-cycle material and energy requirements; uncertainty analysis.

First, we estimate the gaps in DLS (Sect. 3) in material terms, such as the number of housing units for shelter, toilets for sanitation, minimum water consumption for water supply, basic appliances, etc. based on national and international sources. The key components of the DLS can be inferred from the normative standards in Table 1. A more comprehensive description can be found in Rao and Min [4].

These normative standards are converted into material requirements, the process of which is straightforward except for social dimensions of wellbeing (health and education), whose material needs are not easily determined. For these, we rely on previous empirical analysis [4] to determine the national expenditure levels required at a minimum to achieve standards of primary education and life expectancy respectively. We use a multi-region input-output (MRIO) to estimate the energy requirements associated with this expenditure. We also use MRIO for food production, whose heterogeneity across the economy is best aggregated through an MRIO.

For material items in the DLS, such as housing, appliances and related infrastructure, we use traditional LCA calculations [5]. For housing, we identify building archetypes representative of different climatic conditions, regions (e.g. depending on material availability) and urban/rural areas and we calculate construction and operational energy using a material inventory and a dynamic energy model. Using 
Table 1 Sources of uncertainty in assessing decent living standard (DLS) energy requirements

\begin{tabular}{|c|c|c|c|c|}
\hline Sector & DLS normative standard & Technology & Behavior & Geography \\
\hline $\begin{array}{l}\text { Food (production, } \\
\text { preparation, } \\
\text { conservation) }\end{array}$ & $\begin{array}{l}\text { Calorie requirement, } \\
\text { modern } \\
\text { cook stoves, refrigerators }\end{array}$ & $\begin{array}{l}\text { Efficiency of } \\
\text { refrigerators and } \\
\text { cook stoves }\end{array}$ & Diet & \\
\hline Clothing & Minimum clothing & & & \\
\hline Housing & $\begin{array}{l}\text { Minimum floor space, } \\
\text { safety, thermal and visual } \\
\text { comfort }\end{array}$ & $\begin{array}{l}\text { Materials } \\
\text { Lighting } \\
\text { technology }\end{array}$ & $\begin{array}{l}\text { Cooling } \\
\text { schedules }\end{array}$ & $\begin{array}{l}\text { Climatic } \\
\text { zone }\end{array}$ \\
\hline Water/Sanitation & $\begin{array}{l}\text { Minimum water, in-house } \\
\text { sanitation }\end{array}$ & $\begin{array}{l}\text { Water supply } \\
\text { system } \\
\text { configuration }\end{array}$ & & $\begin{array}{l}\text { Water } \\
\text { source } \\
\text { distance }\end{array}$ \\
\hline Health & $\begin{array}{l}\text { Life expectancy, number } \\
\text { of hospital beds }\end{array}$ & Expenditure/cap & & \\
\hline Education & $\begin{array}{l}\text { Primary/secondary } \\
\text { attainment }\end{array}$ & Expenditure/cap & & \\
\hline Communication & Access to TV & Efficiency & TV hours & \\
\hline $\begin{array}{l}\text { Mobility (transport, } \\
\text { roads) }\end{array}$ & $\begin{array}{l}\text { Access to motorized } \\
\text { transport }\end{array}$ & $\begin{array}{l}\text { Mode shares, } \\
\text { vehicle } \\
\text { efficiency, fuels }\end{array}$ & & \\
\hline
\end{tabular}

Entries in bold are illustrated in this study

this bottom-up simulation model [6] we are able to assess the influence of material, technology and design choices on both construction and operating energy requirements and finally extrapolate results to the national level. For some of the sectors, e.g. water supply and sanitation, where building a comprehensive LCA model would be out of the scope of this study, we rely instead on a set of international studies to assess the range of construction and operational energy intensities corresponding to different technologies and contextual conditions. Illustrative results of the energy requirements in different sectors for India and Brazil are shown in Sect. 4.

Uncertainty in energy estimates can arise from a number of inputs, which to yield total uncertainty. Table 1 sketches out the key dimensions of uncertainty: the extent of the gap in DLS; technology performance; and human behaviour. There may be cases where multiple estimation approaches can be used, which contribute model uncertainty. However, we do not include such cases in this study.

As indicated in the table, we use the housing and water supply analysis as the primary vehicle to illustrate the different contributions to uncertainty (Sect. 5). For each case, we varied one parameter at a time according to suitable ranges of values to obtain the total range of variation in life-cycle energy. In all other cases, we use 'status quo' conditions to quantify energy needs, to reflect present-day habits and culture and commercially available technologies. 


\section{Gaps in Decent Living}

Figure 1 compares the gaps in DLS between India and Brazil. Indians lag in all dimensions by a significant margin. Over $90 \%$ of Brazilians have access to most components of DLS, while access to DLS in India ranges from as low as $17 \%$ (refrigerators) to $100 \%$ having primary education. The main implication of this for LCA and its value to energy planning is that in India a more significant component of future energy requirements stem from the construction of new infrastructure. The assessment of the cost-performance trade-offs of either leapfrogging to new technologies at a significant cost as opposed to scaling up living standards quickly and at relatively low cost but 'locking-in' to less efficient technology becomes critical in countries like India. The uncertainty in energy demand may also be higher, which depends to a greater extent on the nature and extent of population growth, migration to cities, and the evolution of technology.

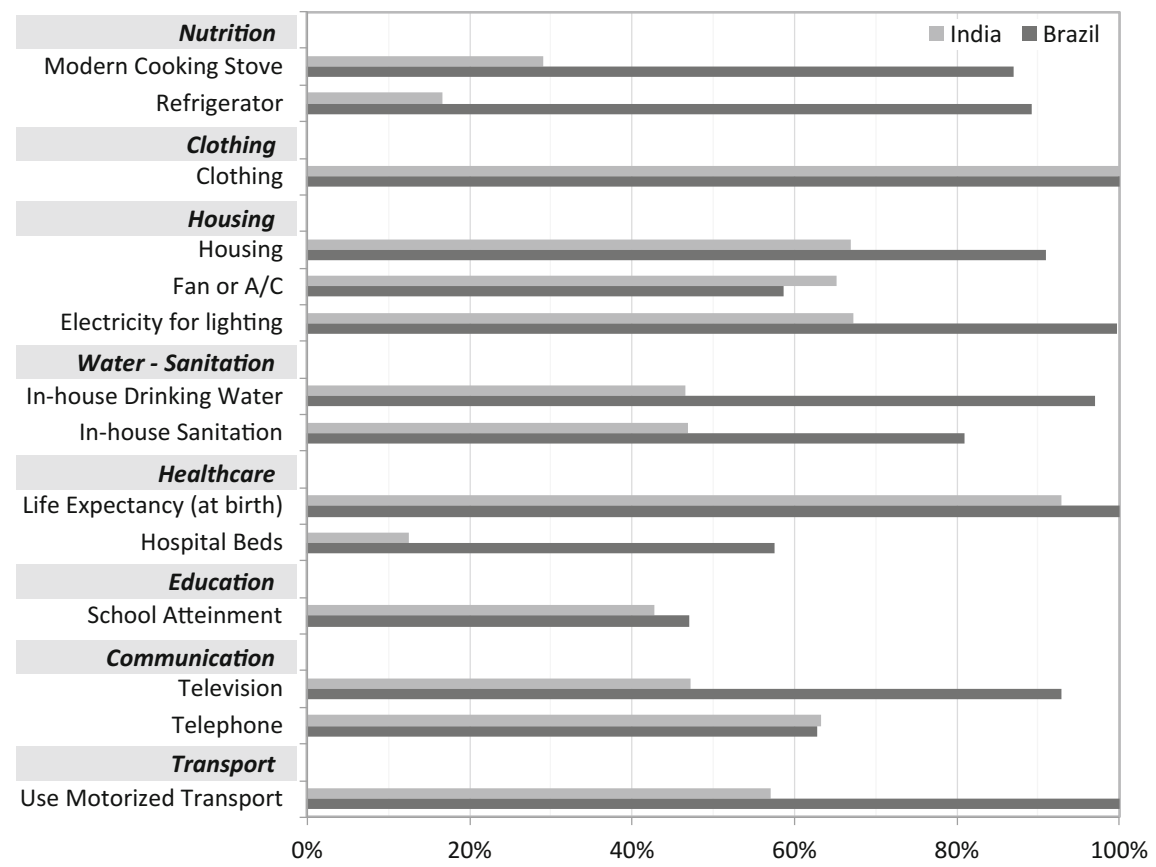

Fig. 1 Share of population with decent living conditions in India and Brazil. Notes life expectancy is a national average, shown as a proportion of 72 years $(100 \%)$; hospital beds are number per 1000 people, shown as a proportion of 2.2 (100\%). Sources IEA World Energy Outlook (2016); Census India (2011); UNICEF; National Health Profile 2010 (India); CIA The World Factbook; UNESCO; Seventh All-India Education Survey; NSSO 11-12 survey (India); POF 08-09 survey (Brazil); World Bank-Rural access index 


\section{Trade-off Between Embodied and Operational Energy}

Figure 2 illustrates the per capita operational, capital turnover, and total energy required for each DLS component in India and Brazil respectively. Note that both the operational and capital turnover energy represent a future scenario where
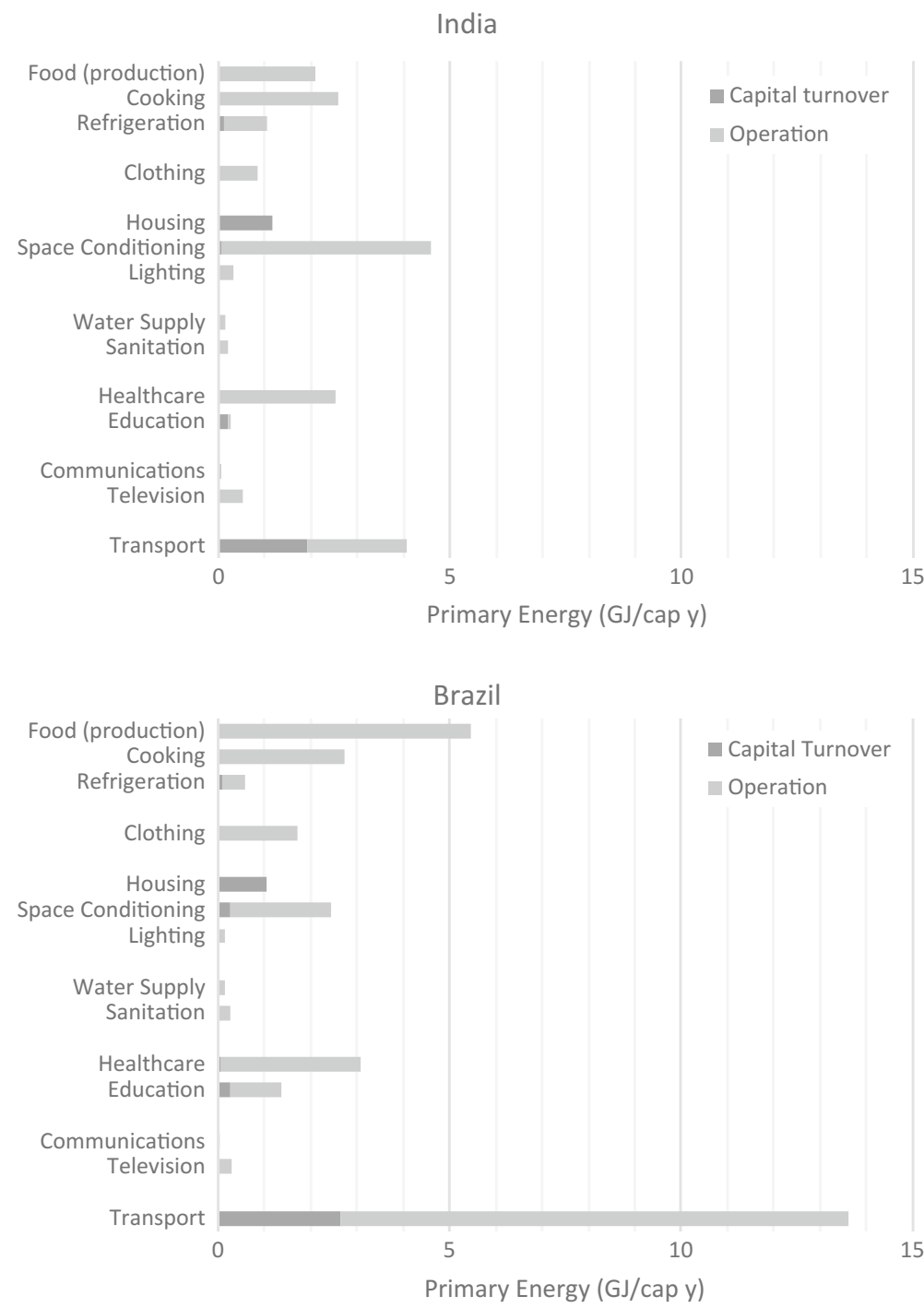

Fig. 2 Illustrative energy requirements to provide decent living standards in India and Brazil, showing capital turnover and operating energy. Note Space conditioning includes hot water production 
infrastructure gaps to manufacture and deliver the DLS products/services have been filled. Many of these results are still preliminary, and not meant to convey an empirical finding. Rather, they illustrate the kinds of insights such analysis enables. For instance, the relative energy requirements for different DLS components differ widely. Food in Brazil is far more energy intensive than in India, most likely due to the preponderance of meat consumption in Brazil compared to India. The housing energy requirement in India has a moderately higher share of capital turnover from the expansion of the existing stock. However, the energy required to heat and cool (largely cool) buildings is far greater in India due to, among other things, severe climatic conditions (high temperatures and humidity). In both countries, energy for overall capital expansion is dominated by housing and transportation.

Some caveats are in order. In general, in using existing country-specific data on material and energy intensities to meet a single standard, we can't discern whether differences arise from different levels of service quality, differences in energy needs, or differences in efficiency. For instance, health and education energy requirements in Brazil are higher than in India, because energy intensity levels in Brazil are significantly higher. This may imply that quality is higher, or that existing conditions (e.g., population density) may necessitate higher energy intensities in Brazil, or that energy use is less efficient in Brazil. In subsequent work, some discernment will be possible, based on deeper analysis of the health and education sectors.

Another caveat is that the electricity demand growth assumes power plants already exist (that is, the embedded energy associated with building new electric power plants to meet incremental electricity demand has not as yet been included). This is, however, typically very small in comparison to operating energy.

\section{Uncertainty Analysis}

An important aspect of energy demand estimation is uncertainty and its attribution to policymakers' decision variables. We illustrate this for the case of housing and water, which differ in the extent of influence of geography.

\subsection{Housing Supply Energy Requirements}

For housing, climatic conditions and building materials (accounting for local availability) are key sources of uncertainty and are analysed in this section (see Table 1 for all sources of uncertainty). We include country-specific options of traditional mud-based construction in India, and wooden homes in Brazil. For this paper, we use a single-storey building archetype for an average family in each country. Due to different average household sizes, the sample house is $30 \mathrm{~m}^{2}$ in Brazil and $40 \mathrm{~m}^{2}$ in India. Figure 3 shows the results, where the bars in the 
Fig. 3 Life-cycle energy requirements of housing with different construction technologies and materials. Whiskers reflect the range of climatic conditions

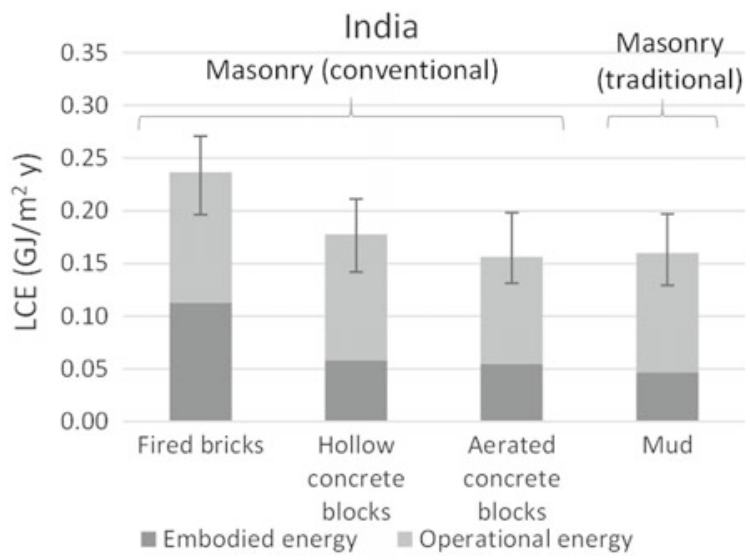

(a) India - Reference climatic zone is 'composite climate' (location: Allahabad).

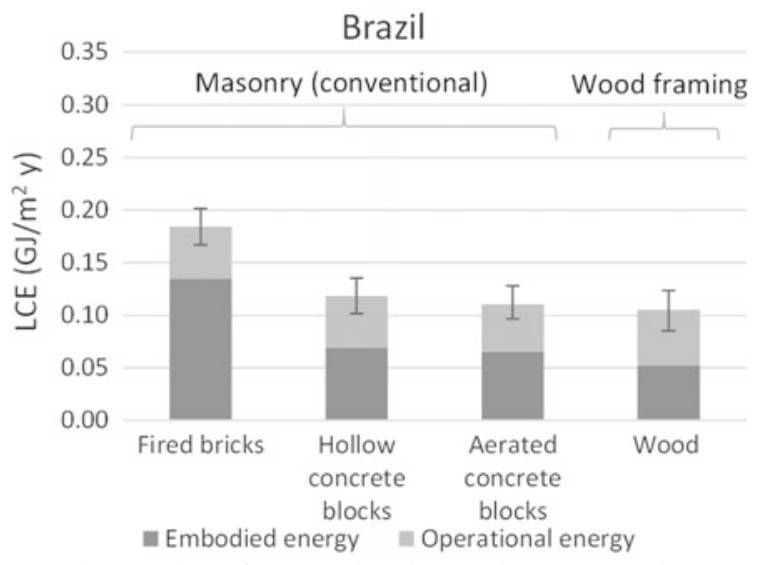

(b) Brazil - reference climatic zone is warm-humid (location: Belem).

histogram indicate energy requirements for different building technologies, and the whiskers show the range of variation in operating energy covering all climate conditions in both countries.

An important takeaway regarding the use of traditional mud housing in India is that despite moderately higher operating energy (due to poorer thermal insulation of the building), the savings in construction energy lead to a net energy reduction in comparison to fired bricks, the most common choice of construction today. Furthermore, the most efficient (aerated concrete blocks) has very marginally lower overall energy requirements than the least efficient (traditional mud) technology. However, aerated concrete is likely to be far more expensive than traditional mud. 
On an energy cost basis, therefore, this preliminary analysis would suggest that the traditional mud option may be the most favourable option compared to the present technology of choice, fired bricks. Other inconveniences, such as water penetration and termite attack, may, however, limit its application. Another insight is that the difference in total energy requirements is driven almost entirely by construction energy differences. The variation in operating energy is much smaller. One implication of this finding is that the favourable choice of construction materials is unlikely to vary much across India due to varying climate conditions. However, if one were to consider a broader range of energy savings measures (e.g. thermal insulation, high-performance glazing, shading devices, etc.) operational energy reductions may have a more influential role.

In Brazil, building operating energy is even less important than in India, due to more moderate climatic conditions. The variation in energy from the heterogeneity of climatic conditions in the country is correspondingly lower as well. Notably, the building materials show similar relative merits with respect to total energy requirements, with the exception that the traditional (in this case, wood) housing is even more favourable, having the least total energy requirement.

\subsection{Water Supply Energy Requirements}

Key sources of uncertainty for water supply include the geography and normative standard for water demand. We compare two different systems with different geographies - one with low water conveyance (in South Africa [7]), and another with high conveyance requirements (in Florida [8]). We also vary the normative standard for water demand, between a normative minimum of 501 per cap per day [9], and a reference of 100 1/cap/day (representing approximately the average demand in India). Notably, nothing in this analysis is actually dependent on local conditions in India or Brazil, so we show only one set of results.

In contrast to housing, water energy requirements are almost entirely composed of operating energy, which comprises pumping water through the distribution system and treating water. In this sector, construction materials matter far less than pumping technology and location. The range of variation for the two systems is higher than the mean. There may well be other locations in these countries that entail even higher conveyance costs (Fig. 4). 
Fig. 4 Water supply in India and Brazil. The bars show different normative requirements; the whiskers show the impact of source water distance

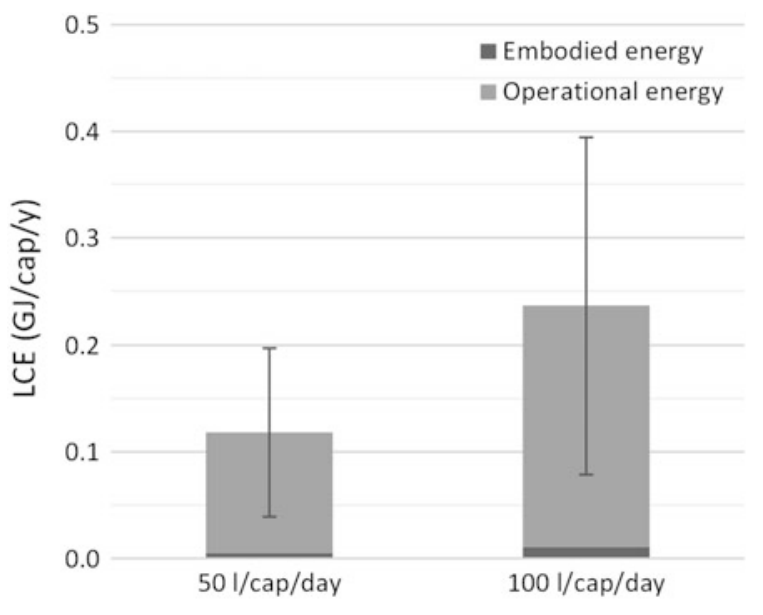

\section{Conclusions}

This paper illustrated the use of LCA methods to link human wellbeing to resource consumption. This linkage enables sectoral LCA analyses to be integrated and compared for use in holistic development policy planning. This provides policymakers with insights on trade-offs, and helps assign priorities among sectors. We illustrate the relative contribution of technological uncertainty in different sectors, as well the trade-offs between upfront and long-term operating energy costs. We also show how these trade-offs differ in different geographic conditions, illustrated with India and Brazil.

We did not aim for this study to provide comprehensive empirical findings on development energy needs-for instance, we do not include here the energy associated with building out infrastructure to meet development gaps - rather, we focused on annual energy outflows and their drivers. We illustrate, nevertheless, several empirical insights. For instance, the transport sector dominates energy needs to meet a basic living standard in Brazil, but not in India. In both country we show that traditional building materials in the long term may offer a win-win for both reducing energy use and costs of housing.

\section{References}

1. Finnveden G, et al., Recent developments in Life Cycle Assessment, Journal of Environmental Management, Vol. 91, 2009, pp. 1-21.

2. http://economictimes.indiatimes.com/news/economy/infrastructure/india-needs-1-5-trillion-forinfrastructure-arun-jaitley/printarticle/52922015.cms, (Accessed 15.06.2017).

3. Tiwari P, Rao J, Day J, Development Paradigms for Urban Housing in BRICS Countries. 2016. 
4. Rao N.D, Min J, Decent Living Standards: material prerequisites for human wellbeing, Soc Indic Res, 2017.

5. Buyle M, Braet J, Audenaert A, Life cycle assessment in the construction sector: A review, Renew Sust Energ Rev, Vol. 26, 2013, pp. 379-388.

6. Mastrucci A, Rao N.D, Decent housing in the developing world: Reducing life-cycle energy requirements. Energ. Buildings, Vol. 152, 2017, pp. 629-642.

7. Friedrich E, Life-cycle assessment as an environmental management tool in the production of potable water, Water Sci Technol, Vol. 46, No. 9, 2002, pp. 29-36.

8. Mo A, et al., Embodied energy comparison of surface water and ground-water supply options, Water Res, vol. 45, no. 17, 2011, pp. 5577-5586.

9. Gleick P, The human right to water. Water Policy, 1, 1998, pp. 487-503.

Open Access This chapter is licensed under the terms of the Creative Commons Attribution 4.0 International License (http://creativecommons.org/licenses/by/4.0/), which permits use, sharing, adaptation, distribution and reproduction in any medium or format, as long as you give appropriate credit to the original author(s) and the source, provide a link to the Creative Commons license and indicate if changes were made.

The images or other third party material in this chapter are included in the chapter's Creative Commons license, unless indicated otherwise in a credit line to the material. If material is not included in the chapter's Creative Commons license and your intended use is not permitted by statutory regulation or exceeds the permitted use, you will need to obtain permission directly from the copyright holder. 


\title{
Integrating the Concept of Planetary Boundaries into Decision Making Processes
}

\author{
Marcial Vargas-Gonzalez
}

\begin{abstract}
What is absolute sustainability? This question has been the centre of discussion for many years now. During this session, several stakeholders tried to bring their own answer to the question, discussing issues like the current Science-Based Targets approach, SDGs, resource criticality and the development of targets based on the earth's regenerative capacity. And while different topics were addressed and the need to develop more robust approaches was apparent, all presenters were aligned on the need to help companies incorporate the notion of planetary boundaries in their business practices in order to address as quickly as possible the environmental issues of the 21 st century.
\end{abstract}

\section{Introduction}

What is sustainability? This question has been the centre of discussion for many years now. From the environmental point of view, sustainability was considered up until now as improving the environmental performance of your company or product. This was therefore about being more sustainable than someone else's company or product but not about becoming sustainable in absolute terms.

The Planetary Boundaries concept provides insights on what environmental sustainability means at the global level, i.e. what is the planet's resilience threshold within which we can keep developing. This being said, a major question arises: how can we translate such concepts at a corporate level to enable better decision-making processes?

Two levers are being currently investigated:

- Using Planetary Boundaries as a new normalization system in LCA approaches to assess the product's sustainability and the material challenges it faces $[1,2]$.

M. Vargas-Gonzalez ( $\bowtie)$

Quantis International, Paris, France

e-mail: Marcial.vargas-gonzalez@quantis-intl.com

(C) The Author(s) 2018

E. Benetto et al. (eds.), Designing Sustainable Technologies,

Products and Policies, https://doi.org/10.1007/978-3-319-66981-6_45 
- Using Planetary Boundaries to define science-based corporate targets to provide a way to define environmental strategies strengthened by a recognized scientific approach. A method that has gained considerable momentum thanks to the Science-Based Targets Initiative, which focuses on the climate change issue [3].

This session was the opportunity for companies and academia to discuss the concept of Planetary Boundaries, its integration at the corporate level (the approaches and methods being developed), its operationalization but also the limits of such an approach.

\section{Presentation Summary}

Trough 5 presentations, this session helped understand how the planetary boundaries concept was first applied to carbon emissions by the existing sectorial decarbonisation approach, paving the way for new methodologies and the assessment of different aspects and sectors including: the carbon emissions of the agri-food sector, the development of targets beyond carbon, resource criticality and the alignment of SDGs and Planetary Boundaries.

\subsection{Sectoral Pathways to Low Carbon Economy Shall Drive Transition Planning and Companies' Decision Processes: The Key Learning's from Assessing Low Carbon Transition Initiative's Pilot Phase}

Recently, the main environmental concern for companies and states alike has been to drastically reduce carbon emissions in order to remain below the $2{ }^{\circ} \mathrm{C}$ target set during COP21. And while climate leaders have assimilated existing tools like carbon reporting and the Sectoral Decarbonisation Approach (SDA), carbon emissions continue to rise.

During this presentation, it was discussed how the ACT approach developed by CDP and ADEME can help companies ensure that their approach is aligned with the level of decarbonisation needed. It provides sectorial assessment methods with a holistic view helping companies in the electric utilities, automobile and retail sectors improve their climate strategy, business mode, investments, operations and GHG emissions and GHG emission management.

While ADEME's work shows that companies are ready for the transition, several hurdles remain like the inclusion of new sectors in the existing methodologies, the inclusion of SMEs in the carbon discussions and the extension of the approach to include other environmental indicators. 


\subsection{Defining Science-Based Targets for an Agri-food Company: A Case Study}

In line with the observation made by ADEME, today, more than 300 companies have committed to the Science Based Targets initiative showing the way to a $2{ }^{\circ} \mathrm{C}$ temperature rise scenario. However, as previously mentioned, in order to make sure those targets are reached, it is key to define manageable targets in line with the company's structure which can be difficult when considering that several sectors are not described in the SDA approach.

This is the main purpose of Quantis \& Ecofys' work, defining science-based targets for a couple of agri-food companies using the SDA approach. This showcases not only the needed methodological developments, including the development of reduction pathways for several crops, but also the need to have a common structure when building a "SBT strategy". In order to meet their goals, companies need to understand the calculations and commitments that have been validated before companies starts strategizing and acting.

The key message is that even though developments are still ongoing, it is possible for companies to start acting immediately with a pragmatic framework and approach.

\subsection{One Planet Thinking: Towards Companies That Perform Within the Earth's Regenerative Capacity}

While the main focus has been carbon issues, the fact that we used the regenerative capacity of 1.6 planets in 2012 at present consumption levels demonstrates the urgent need to act within the earth's regenerative capacity beyond carbon and to start addressing all environmental issues. As previously proved, this can be managed with a pragmatic and operational approach in line with industrial needs.

Inspired by the concept of planetary boundaries, which was introduced by Rockström et al. in 2009 [4], Ecofys developed One Planet Thinking (OPT) together with Eneco, an energy utilities company in the Netherlands, with the aim to link corporate activities to global, regional and local boundaries to set targets for climate change, particulate matter, fossil and mineral resources.

The approach has its limitations but the overall framework shows that the use of pragmatic approach can ensure the development of sustainable business practices in a company. Moreover, this presentation shows that including other environmental issues in the "science-based targets" approach is possible, even though different issues (spatial focus, time variability) must be understood and included before we consider the methodology robust and streamlined. The issue of criticality exemplifies this issue. 


\subsection{Criticality Methodology for Resources: How to Apply to Construction in the Future?}

Criticality as a concept refers both to the potential impact of shortage of a resource and to the probability of such a shortage, as such it is an essential issue when discussing the absolute sustainability of resource use.

Understanding criticality is therefore a first step in the understanding of resource use mechanisms and how setting a target for resource use might be attained in the future. And even though the presented approach did not focus on the target setting aspect of criticality, it detailed what should be assessed when evaluating the sustainability of resource use including rarity and spatial level of focus. This last aspect itself is quite innovative, as resource scarcity has been classically considered as a global issue while this methodology suggests it might not be the case for all resources.

Overall, this work seems necessary in order to better understand the implications of resource scarcity and the elements to be considered before defining reduction targets for resource use.

\subsection{Operationalization of Sustainable Development Goals Using a Planetary Boundaries-Based Life-Cycle Assessment Framework}

In 2015, the international community formulated the Sustainable Development Goals (SDGs) [5], this set of goals is supposed to help achieve sustainable development. This implies that existing SDGs should lead companies and countries to develop within the earth's regenerative capacity.

However, until recently, no framework had been developed on how to integrate the idea of Planetary Boundaries with the SDGs. The work presented showed how planetary boundaries could be used to compare countries' current activities with overall SDGs, assessing if economic sectors are aligned with the international targets.

This offers a new vision of how companies and countries can simplify their approaches and use the Planetary Boundaries approach as a key to identify material issues and be aligned with SDGs, the overall goal being to reduce the number of KPIs needed to assess their activities. This underlines once more how sustainability must be operationalized if we want international targets to be met by the industrial sectors. 


\section{Discussion}

Through these five presentations several common issues transpired, showing that companies and governmental are both struggling with absolute sustainability and its implications. Most issues arise from the lack of robust methodologies and data that could be applied for different environmental aspects and for varied economic sectors. Leading to the question: are science-based and context-based approaches scientific enough?

Presenters emphasized on the need to further detail, improve and complete existing methodologies in order to consolidate the efforts and build an approach that is both scientific and operational. However, it seems unreasonable to expect stakeholders to wait before they start acting and setting targets, as environmental issues must be addressed with urgency. It therefore seems that while current approaches are only the first building blocks in the development of absolute sustainability targets, they should be integrated into decision-making quickly and improved on a regular basis.

Moreover, the work presented during these presentations showed that there is a real academic and industrial demand for science-based and context-based approaches. This leads presenters to believe that these new methodologies will develop quickly and will be aligned with current scientific practices and evolutions in the near future, with developments focusing on: the inclusion of new sectors in existing methodologies, the development of a general framework for planetary boundaries-based targets and the operationalization of existing approaches at the corporate and national level.

The main hurdle remaining is the definition of an approach that is scientific and robust but that can also be easily implemented at the corporate level and included in the decision-making process.

\section{Conclusions}

With the definition of an international target during COP21, the attention turned away from conventional target-setting ways and companies stated their need to define their own science-based targets. The approach has quickly caught on despite the different difficulties companies have identified. It seems however necessary to address these issues in order to develop more robust approaches, including the development of approaches for issues beyond carbon, that remain operational and easy to implement and track.

And while most of the presentations focused on the methodological evolutions that will lead to a more transparent approach to target setting, all presenters were aligned on the need to help companies incorporate the notion of planetary boundaries in their business practices in order to address as quickly as possible the environmental issues of the 21 st century. 


\section{References}

1. Bjørn A, Hauschild M.Z, Introducing carrying capacity-based normalisation in LCA: framework and development of references at midpoint level, The International Journal of Life Cycle Assessment, 20(7), 1005-1018, 2015, http://doi.org/10.1007/s11367-015-0899-2.

2. Bjørn A, Margni M, Roy P.O, Bulle C, Hauschild, M.Z, A proposal to measure absolute environmental sustainability in life cycle assessment. Ecological Indicators, 63, 1-13, 2016. http://doi.org/10.1016/j.ecolind.2015.11.046.

3. Sandin G, Peters G.M, Svanström M, Using the planetary boundaries framework for setting impact-reduction targets in LCA contexts. The International Journal of Life Cycle Assessment, 20(12), 1684-1700, 2015, http://doi.org/10.1007/s11367-015-0984-6.

4. Rockström J, Steffen W, Noone K, Persson Å, Chapin III F. S, Lambin E, Lenton T.M, Scheffer M, Folke C, Schellnhuber H, Nykvist B, De Wit C.A, Hughes T, van der Leeuw S, Rodhe H, Sörlin S, Snyder P.K, Costanza R, Svedin U, Falkenmark M, Karlberg L, Corell R. W, Fabry V.J, Hansen J, Walker B, Liverman D, Richardson K, Crutzen P, Foley J, Planetary boundaries: exploring the safe operating space for humanity. Ecology and Society 14(2): 32, 2009, [online] URL: http://www.ecologyandsociety.org/vol14/iss 2/art32/.

5. World leaders adopt Sustainable Development Goals. United Nations Development Programme. Retrieved 25 September 2015.

Open Access This chapter is licensed under the terms of the Creative Commons Attribution 4.0 International License (http://creativecommons.org/licenses/by/4.0/), which permits use, sharing, adaptation, distribution and reproduction in any medium or format, as long as you give appropriate credit to the original author(s) and the source, provide a link to the Creative Commons license and indicate if changes were made.

The images or other third party material in this chapter are included in the chapter's Creative Commons license, unless indicated otherwise in a credit line to the material. If material is not included in the chapter's Creative Commons license and your intended use is not permitted by statutory regulation or exceeds the permitted use, you will need to obtain permission directly from the copyright holder.

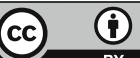




\title{
Exploring the Linkages Between the Environmental Sustainable Development Goals and Planetary Boundaries Using the DPSIR Impact Pathway Framework
}

\author{
Chanjief Chandrakumar and Sarah J. McLaren
}

\begin{abstract}
Most of the conventional environmental sustainability assessment methods, such as Life Cycle Assessment and environmental footprints, evaluate economic goods and services in terms of the nature or the function of the studied systems. As such, these methods overlook the variations in the overall magnitude of production and consumption patterns for the examined systems. As a result, the progress achieved in mitigating global environmental problems is likely to be slow and may be insignificant. Hence this study explores the interlinkages between the Sustainable Development Goals (SDGs) and Planetary Boundaries (PBs) using an DPSIR (Drivers-Pressures-State of the Environment-Impacts-Responses) impact pathway framework - in support of developing an absolute sustainability assessment method (ASAM). The study demonstrates that there is a substantial overlap between the SDGs and PBs. The science-based thresholds listed in the PBs can therefore be adopted as a complementary set of environmental boundaries for the SDG indicators. Overall, the study lays the foundation for advancing an ASAM that can guide policy- and decision-makers to operationalize the SDGs effectively.
\end{abstract}

\section{Introduction}

The Planetary Boundaries (PBs) concept was introduced by Rockström and his associates [1], and was updated by Steffen et al. [2]. Rockström et al. proposed nine critical Earth system processes and associated control variables and thresholds, claiming that transgressing any of the thresholds would potentially be devastating

C. Chandrakumar $(\square)$

New Zealand Life Cycle Management Centre, School of Agriculture and Environment, Massey University, Palmerston North 4442, New Zealand

e-mail: C.Chandrakumar@massey.ac.nz

S. J. McLaren

School of Engineering and Advanced Technology, Massey University,

Palmerston North 4442, New Zealand

(C) The Author(s) 2018

E. Benetto et al. (eds.), Designing Sustainable Technologies,

Products and Policies, https://doi.org/10.1007/978-3-319-66981-6_46 
for human societies [1]. Based on the nine PBs, a safe operating space for humanity was determined $[1,2]$. Here, the safe operating space refers to a relatively stable state called the Holocene epoch, in which human societies can continue to develop and thrive [2]. Today, both the scientific and political communities have agreed upon the notion that there are global limits for the Earth system and they should be respected. Consequently, studies adopting the PBs have started proliferating, and they can be classified into works that (i) define or refine the control variables and the associated thresholds [e.g. 1, 2], (ii) downscale the global PBs to sub-global levels [e.g. 3, 4], (iii) set impact reduction targets [e.g. 3, 4], and (iv) devise policies and strategies [e.g. 3, 5].

While the PBs concept sets global limits for environmental impacts to benchmark a system's performance globally, environmental sustainability assessment methods (ESAMs) such as Life Cycle Assessment (LCA) and environmental footprints evaluate the environmental performance of a so-called product system (which is usually defined in terms of supplying a specified quantity of an economic product or service). Generally, the outcomes of an LCA or environmental footprint study guide decision makers to improve the eco-efficiency of the chosen product system through identifying the environmental hotspots along its life cycle [6]. As a result, use of LCA and other related life cycle thinking approaches to support decision-making has become common within the business and academic communities [6]. However, although the outcomes of these studies guide eco-efficiency improvements, the overall progress achieved in mitigating environmental problems still remains slow and insignificant [7-9]. One contributing factor is that conventional ESAMs like LCA do not benchmark the environmental sustainability performance of a system against a set of environmental boundaries (or standards). Instead, they rank a particular system in relative terms, by comparing it with a reference system that is relevant to the nature or the function of the system under investigation, and thus, the variations in the consumption and production patterns of the examined products and services are overlooked [7-9]. For example, Product A may be superior (or more sustainable) than Product B in terms of eco-efficiency, but neither could be sustainable on an absolute scale due to the predicted growth in global production and consumption volumes of the product [7, p. 325].

Therefore, recently, the scientific community began to focus on the so-called concept of absolute sustainability. Absolute sustainability is focused on how human societies can operate within the carrying capacity of the Earth system [8, 9]. Here, the term "carrying capacity" refers to "the maximum sustained environmental interference a particular system can withstand without experiencing negative changes in structure or functioning that are difficult or impossible to revert" [6, p. 1007]. As a result of growing interest in absolute sustainability, scientists have started developing absolute sustainability assessment methods (ASAMs) by supplementing the existing ESAMs with the Earth's carrying capacity [6, 8, 9], for instance, supplementing the ecological footprint with the Earth's biocapacity (available bio-productive area) [e.g. 10], LCA with PBs [e.g. 6, 8] and environmental footprints with PBs [e.g. 5]. 


\section{Operationalisation of Sustainable Development Goals}

The United Nations agreed on a set of Sustainable Development Goals (SDGs) in 2015 comprising 17 goals, 169 targets and 232 indicators [11, 12]. The SDGs aim to cover a wide range of sustainable development problems [12-14]. Overall, the SDGs are intended to be universal with a shared common vision of progressing towards a safe, just and sustainable operating space for human societies $[12,16]$. However, the SDGs have been criticised as being difficult to implement due to having too many goals and targets, lacking clarity, and having overlapping objectives [16]. Additionally, the SDG proposed for safeguarding the Earth system have been criticised as being neither sufficiently comprehensive nor ambitious [13, 14]. For instance, many of the SDG indicators have been proposed without a relevant environmental boundary. Researchers therefore have begun exploring how to operationalize the SDGs within the Earth's carrying capacity, and specifically how to link them to the PBs and then to LCA [13, 15]. Dong and Hauschild classified the indicators proposed in the SDGs, PBs and LCA using an DPSIR (Drivers-Pressures-State of the Environment-Impacts-Responses) impact pathway framework (see [20] for DPSIR impact pathway framework) and showed that all three approaches overlap in terms of seven impact categories (climate change, acidification, ozone depletion, eutrophication, chemical pollution, freshwater use and change in biosphere integrity) [15]. However, the study used the older version of the SDGs listed in [11] and until recently, no studies have explored the interlinkages between the latest SDGs listed in [12], the PBs and LCA. Additionally, the potential for operationalizing the SDGs using an ASAM had not been explored yet. Therefore, this study identifies the SDG indicators that evaluate environmental problems, and then systematically explores the interlinkages with the PBs.

To that end, the rest of the paper is organised as follows: Sect. 3 outlines the ASAM framework presented in [17], Sect. 4 establishes the interlinkages between the environmental SDGs and PBs, and Sect. 5 summarises how this work underpins the development of the proposed ASAM.

\section{Outline of the Proposed Approach}

The aim of the proposed ASAM is to operationalize the SDGs at sub-global levels (e.g. country, region, organisation, product) by estimating environmental boundaries at these different levels [17]. These boundaries can then be used to calculate distance-to-target measurements through benchmarking the system's (e.g. country, region, organisation, product) performance against the estimated boundaries. This involves, firstly, identifying the SDG indicators concerned with the conventional areas of protection (AoPs) used in LCA i.e. human health, ecosystem quality, resource scarcity and man-made environment [18]. 
According to $[6,19]$, many of the PB control variables differ from the indicators of the conventional ESAMs (including LCA), particularly with respect to the point of impact assessment although these indicators evaluate similar kinds of environmental impacts to those reported in the PBs. Hence, the chosen SDG indicators and the $\mathrm{PB}$ control variables are further classified into driver, pressure, state, impact and response indicators using an DPSIR impact pathway framework. Having classified them, the interlinkages between the SDGs and PBs are explored. This enables subsequent development of a complementary set of global boundaries for the SDG indicators using, where appropriate, the thresholds proposed for the control variables in the PBs. Afterwards, the global boundaries can be allocated to lower economic levels using a top-down approach. The method is operationalised by developing distance-to-target measurements based on the calculated environmental boundaries compared with the current environmental performance of the systems under analysis (calculated using conventional ESAMs like LCA and environmental footprint studies). These distance-to-target measurements could be positive or negative depending on whether the system is performing in line with the goals and targets reported under the SDGs.

\section{Linkages Between the SDGs and PBs}

This section details how the PBs can be employed as a complementary set of global boundaries for the SDGs by providing a systematic comparison between the SDG indicators and the PB control variables. Firstly, the SDG indicators concerned with the AoPs of human health, ecosystem quality, resource scarcity and man-made environment were chosen (a total of 73 indicators). This set of SDG indicators comprised all the SDG indicators under the SDGs for clean water and sanitation (SDG 6), responsible consumption and production (SDG 12), climate action (SDG 13), life below water (SDG 14) and life on land (SDG 15), plus $\mathrm{CO}_{2}$ emission per unit of value added (SDG Indicator 9.4.1), economic loss due to natural disasters (SDG Indicator 1.5.2), levels of fine particulate matter in cities (SDG Indicator 11.6.2), and proportion of land for sustainable agriculture (SDG Indicator 2.4.1). Then, as outlined in Sect. 3, the chosen SDG indicators were mapped onto a network of cause-effect chains (developed based on the environmental problems addressed in the SDGs and PBs) along with the PB control variables and linked together wherever relevant (see Fig. 1). This mapping step further classified the SDG indicators into driver (0 SDG indicators), pressure (2 SDG indicators), state (19 SDG indicators), impact (14 SDG indicators) and response (38 SDG indicators) indicator categories.

As emphasised in [1, 2], human societies should be operating within the thresholds reported in the PBs. Therefore, this section focuses on the PBs and discusses how each PB (shown in bold text) is related to different SDGs. Steffen et al. introduced a PB called "freshwater use" and two control variables to evaluate the challenges resulting from absolute water withdrawals [2]. The proposed control 


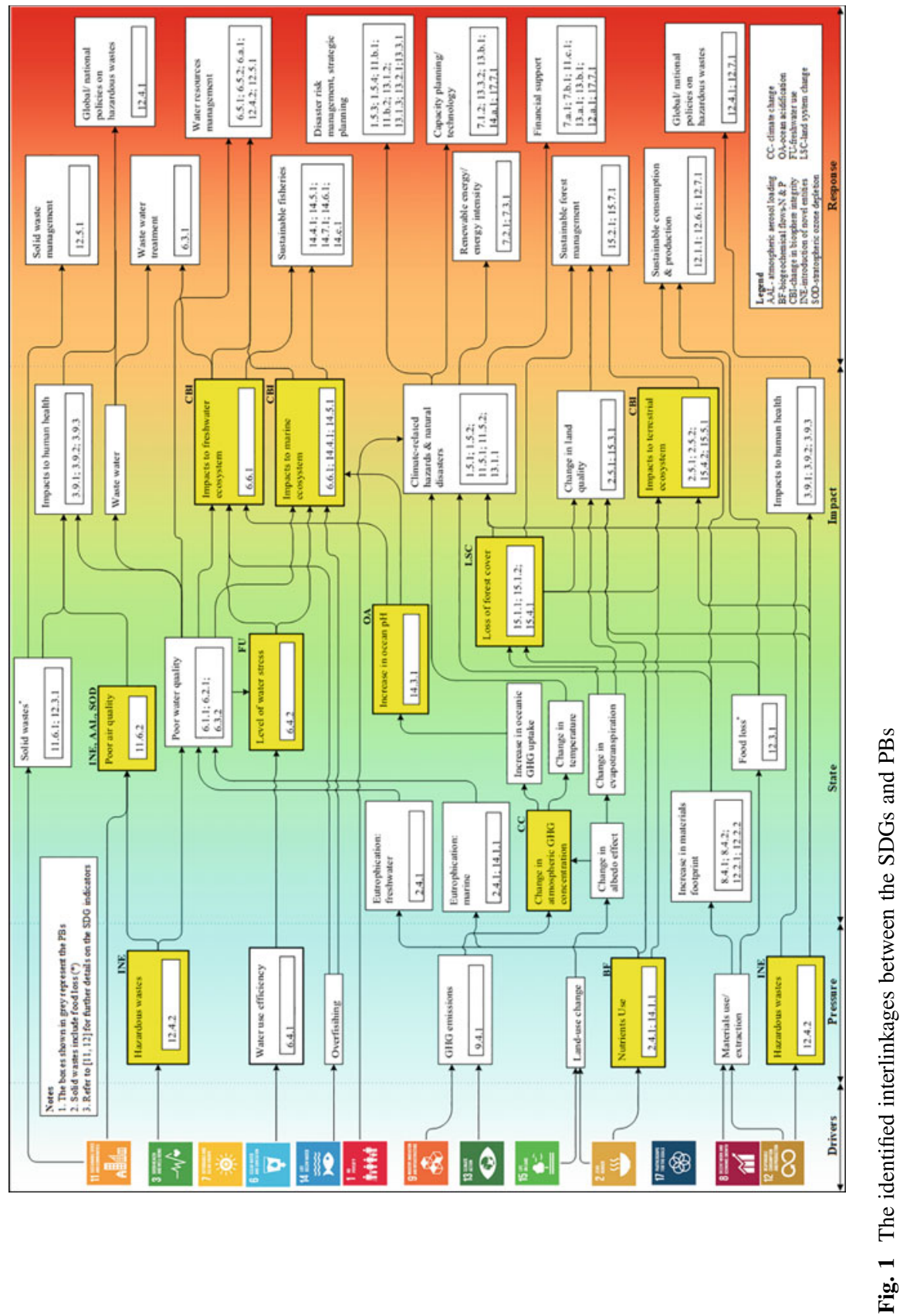


variables estimate the associated impacts at the global as well as the basin levels [2], and inform the impacts at the pressure point of the DPSIR impact pathway (see Fig. 1). Likewise, SDG Indicator 6.4.1 evaluates the same problem at the pressure point in the impact pathway (in terms of water use efficiency). Consequently, at the state point, SDG Indicator 6.4.2 accounts for the effects of excessive water withdrawals (i.e. the level of water stress), which is similar to the PB control variable [12]. However, the proposed SDG indicators do not include any absolute limits. We, therefore, recommend deploying the thresholds proposed for the freshwater use PB because the control variables and the SDG indicators largely overlap; and both inform the impacts at the pressure or state point in the impact pathway.

Increasing atmospheric $\mathrm{CO}_{2}$ concentration and the associated $\mathrm{CO}_{2}$ uptake by the oceans have resulted in ocean acidification problems [2]. As a consequence, a PB called "ocean acidification" was introduced with a control variable (state point) and a threshold for carbonate ion concentration in terms of aragonite [2]. Meanwhile, the SDGs advanced an indicator (SDG Indicator 14.3.1) that estimates the $\mathrm{pH}$ level of the oceans [12]. Although the control variable and the SDG Indicator apply different units to track the ocean acidification effects, the objective and the point of assessment in the DPSIR framework are the same. Moreover, both the SDG indicator and the control variable evaluate the impacts on an absolute scale. We, therefore, comprehend that the ocean acidification impacts can be measured in terms of either aragonite or $\mathrm{pH}$ level of the oceans.

The "changes in biosphere integrity" $\mathrm{PB}$ adopts two control variables to assess the two components of the biosphere: genetic and functional diversity $[1,2]$. The first component evaluates the extinction of species due to human pressures, whereas the second estimates the loss of biodiversity at different ecosystem levels. According to Fig. 1, the impacts pertaining to the both components are expressed at the impact point of the DPSIR impact pathway. In this regard, the SDGs also propose a set of indicators for protecting terrestrial, marine and freshwater ecosystems [12]. SDG Indicators 14.4.1 and 14.5.1 estimate the proportion of fish stocks existing within the biologically sustainable levels and the coverage of protected marine areas, respectively. Moreover, SDG Indicator 6.6.1 tracks the changes occurring in both marine and freshwater ecosystems due to water quality degradation. Although these SDG indicators implicitly underpin the significance of operating within the Earth's carrying capacity, no relevant boundaries have been reported. However, given that the objectives of these control variables overlap with the SDG indicators, it makes sense to supplement the SDG indicators with the thresholds proposed for the "changes in biosphere integrity" PB to inform the environmental impacts in terms of genetic and functional diversities on an absolute scale.

Considering the intensive use of nutrients and the associated eutrophication effects in major ecosystems, Steffen et al. proposed the so-called "biogeochemical flows" PB and two associated control variables [2]. These control variables evaluate the eutrophication effects in oceanic, freshwater and terrestrial ecosystems. Given that the major eutrophication problems arise from $\mathrm{N}$ and $\mathrm{P}$ fertiliser use and the control variables evaluate the impacts at the pressure point (as shown in Fig. 1), 
thresholds have been set for $\mathrm{N}$ and $\mathrm{P}$ fertiliser application [2]. Likewise, SDG Indicator 14.1.1 evaluates the problem of marine eutrophication resulting from land-based activities, including nutrient pollution [12]. Since both SDG Indicator 14.1.1 and the relevant control variables refer to the same problem of eutrophication, and particularly at the same point of the impact pathway (i.e. pressure), the PB thresholds can be used as they are complementary to SDG Indicator 14.1.1.

Changes in land use have significant effects on several biological and ecological systems, including climate and water. For example, changes in the area of boreal forests particularly affect the albedo of the land surface, and changes in the area of tropical forests specifically affect global evapotranspiration rates [2]. For this purpose, a PB called "land-system change" is suggested, which estimates the loss of forest cover at the state point of the impact pathway (see Fig. 1). Similarly, the SDGs report a set of indicators that evaluate the environmental problems resulting from forest cover loss as well as loss of other biomes [12]. For instance, SDG Indicator 15.1.1 estimates the ratio between forest and total land area, SDG Indicator 15.1.2 measures the proportion of protected areas for terrestrial, freshwater and mountain biodiversity, and, SDG Indicator 15.4.1 estimates the land coverage allocated for mountain biodiversity. Nonetheless, none of these SDG indicators assesses the environmental degradation on an absolute scale. Rather, they merely report the proportions of the protected and degraded lands.

We, hence, recommend using SDG Indicator 15.1.1 along with the threshold proposed for the land-system change PB for estimating the loss of forest cover on an absolute scale. However, since the PB focuses solely on the loss of forest cover, there remains a research gap in identifying relevant thresholds for other biomes addressed in SDG Indicators 15.1.2 and 15.4.1.

The "climate change" $\mathrm{PB}$ and its associated control variables emphasise that the atmospheric $\mathrm{CO}_{2}$ concentration and the radiative forcing from greenhouse gases (GHGs) should be reduced to $350 \mathrm{ppm} \mathrm{CO}_{2}(350-450 \mathrm{ppm})$ and to $1 \mathrm{Wm}^{-2}$ respectively (at the state point in the DPSIR impact pathway) [2]. But limiting the atmospheric concentration to $350 \mathrm{ppm} \mathrm{CO}_{2}$ is unlikely as the current values of the control variables are $399 \mathrm{ppm} \mathrm{CO}_{2}$ and $2.3 \mathrm{Wm}^{-2}$ [2], and the world population and economy are still growing [21]. The IPCC therefore suggests that achieving a concentration of $450 \mathrm{ppm} \mathrm{CO}$ is more likely [21, p. 12]. On the other hand, the SDGs present a set of SDG indicators concerned with mitigation of climate change problems [12]. At the response point, SDG Indicator 13.1.1 estimates the fatalities and injuries due to climate change impacts, whereas SDG Indicators 13.1.2, 13.1.3 and 13.2.1 focus on adopting policies and strategies to avert climate change impacts. SDG Indicators 13.3.1, 13.3.2, 13.a.1 and 13.b.1 aim to strengthening institutional, systemic and individual capacity-building to implement adaptation, mitigation and technology transfer and development actions. In addition, SDG Indicator 9.4.1 quantifies the carbon intensity of industries at the pressure point. In general, except SDG Indicator 9.4.1, others focus only on averting the climate change impacts (as seen in Fig. 1), and none of them evaluates the climate change impacts on an absolute scale. Therefore, to our understanding, the PB thresholds 
(and the corresponding global carbon budget) can be used as a set of global boundaries for SDG Indicator 9.4.1.

The "atmospheric aerosol loading" PB evaluates the impact resulting from the emissions of black and organic carbon from sources like cooking and heating with biofuels and diesel transportation, whereas the "introduction of novel entities" PB refers to the persistence, mobility and impacts of chemicals and other types of engineered materials or organisms produced by human activities [2]. Similarly, the "stratospheric ozone depletion" PB concentrates on the ozone concentration variations resulting due to synthetic chemicals release [2]. Interestingly, the associated control variables of these three PBs express the impacts at the state point of the DPSIR impact pathway. As far as we understand, some of the SDG indicators evaluate similar environmental impacts, but not explicitly. SDG Indicator 11.6.2 monitors the levels of fine particulate matter in cities at the state point (with a focus on human health), whereas SDG Indicators 11.6.1 and 12.4.2 quantify the solid and hazardous waste generated (pressure point). SDG Indicator 12.4.2 also quantifies the amount of hazardous waste treated (response point) and SDG Indicators 12.4.1 and 12.7.1 focus on the global initiatives taken to develop multinational agreements and policies on hazardous waste and other chemicals (response point). Although there are some overlaps between the above-listed three PBs and the SDG indicators, it would not be advisable to use them in a complementary way for the following reasons: (i) existence of an enormous number of hazardous substances (including chemicals); (ii) no SDG indicators directly assess the effects of ozone depletion; (iii) no control variables and thresholds have been proposed for the "introduction of novel entities" PB; (iv) the effects of some substances are still unknown, and some effects are not readily reversible; and (v) the PBs are located closer to the original activities that cause the environmental impacts, whereas the SDGs focus on waste management, and are generally located at the response point [2]. Further research is therefore needed to understand better the complementarities between these PBs and the SDGs.

In sum, the environmental SDG indicators mostly address the environmental problems reported in the $\mathrm{PBs}$, which are primarily associated with the ecosystem quality AoP. However, in contrast to the PBs, the SDGs additionally concentrate on the AoPs human health, resource scarcity and man-made environment through addressing the global challenges of unsustainable food and agriculture, soil quality degradation, impacts of ecosystem degradation on human health, direct human impacts on ecosystem (wildlife trafficking and poaching, and overfishing) and lack of infrastructure for water quality and resources management by communities [12]. Regarding unsustainable food and agriculture, SDG Indicator 2.5.1 estimates the number of plant and genetic resources secured for sustainable food and agriculture, whereas SDG Indicator 2.5.2 estimates the local breeds under risk of extinction. Likewise, SDG Indicator 2.4.1 evaluates the soil quality degradation. Nevertheless, these SDG indicators are suboptimal because they use a relative scale, and lack clarity; for instance, SDG Target 2.4 (which includes SDG Indicator 2.4.1) focuses on multiple environmental problems, including climate change and soil quality degradation $[12,14]$. 
On the other hand, a set of SDG indicators has been reported to evaluate the impacts of degradation of ecosystems on human health. SDG Indicators 3.9.1, 3.9.2, 6.3.1 and 6.3.2 evaluate the human health problems resulting due to ambient air pollution, unsafe water, sanitation and hygiene, whereas SDG Indicator 3.9.3 assesses the health problems resulting from unintentional poisoning [12]. Additionally, SDG Indicators 2.4.1, 2.5.1 and 2.5.2 explicitly refer to the impacts on human health as a consequence of unsustainable food production and agricultural practices. Likewise, SDG Indicators 15.7.1 and 15.c.1 estimate the impacts of wildlife trafficking and poaching, whereas SDG Indicators 14.4.1 and 14.5.1 addresses the impacts associated with overfishing. Regarding lack of infrastructure for water quality and resources management by communities, SDG Indicators 6.1.1, 6.2.1 and 6.a.1 concentrate on developing infrastructure for effective water resources management [e.g. 12, 16].

\section{Conclusions}

The study underpins the development of an ASAM framework by systematically exploring the interlinkages between the SDGs and PBs using an DPSIR impact pathway framework. With to the analysis presented in Sect. 4, the two approaches demonstrate notable overlaps with regard to their indicators and control variables. Each of the PBs is linked to one (or more) SDG indicator(s), as shown in Fig. 1. In particular, the "freshwater use", "ocean acidification", "biogeochemical flows", "land system change" and "change in biosphere integrity" PBs exhibit sound linkages with the SDG indicators. Interestingly, some of the control variables of these five PBs are located at the same point of the DPSIR impact pathway as the SDG indicators. But, in contrast, the "climate change" PB control variable is located at the state point of the impact pathway, whereas the SDG indicators associated with climate change are mostly located at the response point, except SDG Indicator 9.4.1, which estimates the carbon intensity of industries at the pressure point. Moreover, no SDG indicators report an absolute limit for GHG emissions. The "introduction of novel entities" and "atmospheric aerosol loading" PBs show some overlaps with SDG Indicators 11.6.1, 11.6.2 and 12.4.2, whereas no explicit linkages are found between the "stratospheric ozone depletion" PB and the SDG Indicators. Furthermore, as discussed in Sect. 4, the SDGs additionally shed light on some other global challenges not explicitly addressed in the PBs such as unsustainable food and agriculture, soil quality degradation, impacts of ecosystem degradation on human health, direct human impacts on ecosystem (wildlife trafficking and poaching, and overfishing), and lack of infrastructure for water quality and resources management by communities.

Overall, according to this study, it seems potentially feasible to adopt the science-based thresholds reported in the PBs as a complementary set of global boundaries for the SDG indicators. Moreover, advancing appropriate environmental boundaries for the additional challenges addressed in the SDGs, and using them 
alongside the PB thresholds, will provide a platform to benchmark the environmental sustainability performance of a system at a global level. However, further research is necessary to benchmark similar environmental impacts on a sub-global level, given that most of the impacts are, in fact, a result of the accumulated effects of discrete regional and local problems [2, 4]. Hence, some suggest allocating the global boundaries to sub-global levels using a variety of allocation principles [e.g. 3 , 4], while others propose developing appropriate independent boundaries at sub-global levels [e.g. 2, 6, 8].

Finally, in order to develop an ASAM framework, future studies should focus on linking the SDGs and PBs with the Impact Assessment phase of LCA. Such an ASAM has potential to inform whether the chosen system is aligned (or not) with the environmental goals and targets listed in the SDGs as well as whether they are operating within the carrying capacity of the Earth system.

\section{References}

1. Rockström J, Steffen W, et al., Planetary boundaries: exploring the safe operating space for humanity, Ecology and Society, vol. 14, 2009.

2. Steffen W, Richardson K, et al., Sustainability. Planetary boundaries: guiding human development on a changing planet, Science, vol. 347, p. 1259855, 2015.

3. Roos S, Zamani B, et al., A life cycle assessment (LCA)-based approach to guiding an industry sector towards sustainability: the case of the Swedish apparel sector, Journal of Cleaner Production, vol. 133, pp. 691-700, 2016.

4. Sandin G, Peters G.M, Svanström M, Using the planetary boundaries framework for setting impact-reduction targets in LCA contexts, The International Journal of Life Cycle Assessment, vol. 20, pp. 1684-1700, 2015.

5. Nykvist B, Persson A, et al., National Environmental Performance on Planetary Boundaries, A Study for the Swedish Environmental Protection Agency, Naturvårdsverket: Stockholm, Sweden2013.

6. Bjørn A, Hauschild M.Z, Introducing carrying capacity-based normalisation in LCA: framework and development of references at midpoint level, The International Journal of Life Cycle Assessment, vol. 20, pp. 1005-1018, 2015.

7. Bjørn A, Hauschild M.Z, Absolute versus Relative Environmental Sustainability, Journal of Industrial Ecology, vol. 17, pp. 321-332, 2013.

8. Bjørn A, Margni M, et al., A proposal to measure absolute environmental sustainability in life cycle assessment, Ecological Indicators, vol. 63, pp. 1-13, 2016.

9. Hauschild M.Z, Better-But is it Good Enough? On the Need to Consider Both Eco-efficiency and Eco-effectiveness to Gauge Industrial Sustainability, Procedia CIRP, vol. 29, pp. 1-7, 2015.

10. Borucke M, Moore D, et al., Accounting for demand and supply of the biosphere's regenerative capacity: The National Footprint Accounts' underlying methodology and framework, Ecological Indicators, vol. 24, pp. 518-533, 2013.

11. United Nations, Transforming Our World: The 2030 Agenda for Sustainable Development, 2015.

12. United Nations, Revised list of global Sustainable Development Goal Indicators <https:// unstats.un.org/sdgs/indicators/indicators-list/>, (Accessed 23.04.2017).

13. Brandi C, Safeguarding the earth system as a priority for sustainable development and global ethics: the need for an earth system SDG, Journal of Global Ethics, vol. 11, pp. 32-36, 2015. 
14. Wackernagel M, Hanscom L, and Lin D, Making the Sustainable Development Goals Consistent with Sustainability, Frontiers in Energy Research, vol. 5, 2017-July-11 2017.

15. Dong Y, Hauschild M.Z, Indicators for Environmental Sustainability, Procedia CIRP, vol. 61, pp. 697-702, 2017.

16. Maier S, Beck T, et al., Methodological Approach for the Sustainability Assessment of Development Cooperation Projects for Built Innovations Based on the SDGs and Life Cycle Thinking, Sustainability, vol. 8, p. 1006, 2016.

17. Chandrakumar C, McLaren S.J, Jayamaha N, Childerhouse P. Implementation of Sustainable Development Goals Based On a Planetary Boundaries-Based Life Cycle Assessment. Proceedings of Student Seminar on Life Cycle Management and Industrial Ecology, 30 March 2017, Massey University, Auckland, New Zealand. <http://lcm.org.nz/implementationsustainable-development-goals-using-planetary-boundaries-based-life-cycle-assessment>, (Accessed 03.07.2017).

18. Huijbregts M.A.J, Steinmann Z.J.N, et al., ReCiPe2016: a harmonised life cycle impact assessment method at midpoint and endpoint level, The International Journal of Life Cycle Assessment, vol. 22, pp. 138-147, 2016.

19. Ryberg M.W, Owsianiak M, Richardson K, Hauschild M.Z, Challenges in implementing a Planetary Boundaries based Life-Cycle Impact Assessment methodology, Journal of Cleaner Production, vol. 139, pp. 450-459, 2016.

20. Song X, Frostell B, The DPSIR Framework and a Pressure-Oriented Water Quality Monitoring Approach to Ecological River Restoration, Water, vol. 4, pp. 670-682, 2012.

21. IPCC, Summary for Policymakers. In Climate Change 2014: Mitigation of Climate Change. Contribution of Work-ing Group III to the Fifth Assessment Report of the Intergovernmental Panel on Climate Change. Cambridge University Press, Cambridge, United Kingdom and New York, NY, USA.

Open Access This chapter is licensed under the terms of the Creative Commons Attribution 4.0 International License (http://creativecommons.org/licenses/by/4.0/), which permits use, sharing, adaptation, distribution and reproduction in any medium or format, as long as you give appropriate credit to the original author(s) and the source, provide a link to the Creative Commons license and indicate if changes were made.

The images or other third party material in this chapter are included in the chapter's Creative Commons license, unless indicated otherwise in a credit line to the material. If material is not included in the chapter's Creative Commons license and your intended use is not permitted by statutory regulation or exceeds the permitted use, you will need to obtain permission directly from the copyright holder. 


\section{Part IV \\ How to Develop and Sustain LCM-Based Innovations}




\title{
Financing Innovation and Circular Economy
}

\author{
L. Goovaerts, C. Schempp, L. Busato, A. Smits, L. Žutelija \\ and R. Piechocki
}

\begin{abstract}
In the seminar on "Financing Innovation and the Circular Economy (CE)", organized by the European Investment Bank (EIB) in the context of the Life Cycle Management Conference 2017, experts of the EIB and EIB partner institutions (European Commission, Rabobank) spoke about the specific challenges involved in the transition to a $\mathrm{CE}$ and the role played by their institutions in accelerating this transition by means of appropriate funding, financing and advisory instruments. In short presentations, the speakers first explained the broader role of the EIB and the European Commission (EC) in supporting the transition to CE, as well as the general types of $\mathrm{CE}$ projects and the specific questions that project promoters can expect in project appraisal. EC, EIB and Rabobank representatives also presented the concrete support and products offered to innovative circular economy project promoters.
\end{abstract}

\section{Introduction}

In view of the world's limited resources and the predicted demographic developments, the currently prevailing linear economic model appears to be unsustainable from both economic and environmental points of view. The answer lies in transition to the CE business model, which requires nothing less than an economic paradigm shift and a radical change in the mind-set of policy-makers, businesses, consumers and financiers towards more life-cycle thinking. CE, as defined by the EIB and the EC, "attempts to encompass all economic systems where the resources used for a product or a service are maximally reduced and/or recycled, while either main-

L. Goovaerts · C. Schempp $(\bowtie) \cdot$ L. Busato · A. Smits

European Investment Bank, Luxembourg City, Luxembourg

e-mail: c.schempp@eib.org

L. Žutelija

European Commission, Brussels, Belgium

R. Piechocki

Rabobank, Utrecht, The Netherlands

(C) The Author(s) 2018

E. Benetto et al. (eds.), Designing Sustainable Technologies,

Products and Policies, https://doi.org/10.1007/978-3-319-66981-6_47 
taining to the best extent possible their economic value at all times and/or ensuring that they are biologically degraded. CE-related projects focus on re-thinking and redesigning products, processes, value chains, business and service models in order to achieve the above-specified purpose" [1].

The circular economy can be seen as a green giant on the rise, on account of its enormous potential to unleash sustainable growth and job creation as well as the increasing traction it is gaining in the business world. Many businesses in different sectors have already started to tap into this potential, displaying a large variety of innovative technologies and business models. However, the transition to a $\mathrm{CE}$ is no certain success and needs to tackle a number of hurdles. Ensuring access to finance for innovative circular businesses, throughout their different growth phases, is one of them.

\section{Challenges and Opportunities in Financing CE Projects}

Besides its environmental benefits, the circular economy offers tremendous economic potential, which businesses in different sectors have started tapping into, displaying a large variety of innovative technologies and business models. However, CE projects present a number of different challenges to access return-based finance. On the one hand, the new technologies and business models proposed are often unproven and complex, rely on uncertain supply chains and operate in uncertain markets. Furthermore, CE projects usually involve small sub-investment grade promoters, with limited collateral or few physical assets. Because of the increased market and credit risks involved, the CE promoters often have limited access-to-finance or face increased cost of capital. A study published in 2015 by the Innovation Finance Advisory unit of the EIB on access-to-finance conditions of projects supporting CE concluded that while market forces (commodity price fluctuations) alone could create a circular economy there is a risk of a slow transition and high opportunity costs [1]. Hence, there is space (and need) for policy intervention and support in form of innovative funding and financing instruments.

\section{Financing of Innovation and Circular Economy in Practice}

\subsection{The EIB in the Circular Economy}

Mr. Schempp explained the crucial role of the EIB, as the EU Bank, in bridging financing gaps and catalysing strategic investments in key EU policy areas such as $\mathrm{CE}$, not only through tailored financial instruments but also through specialized 
advisory services. With the EC's support, the EIB has reinforced the technical and financial advisory services available to projects through the European Investment Advisory Hub (EIAH) and the Innovation Finance Advisory unit. As further explained by Ms. Busato, given the unique combination of risk features, the EIB provides advice to $\mathrm{CE}$ project promoters on structuring and financing to improve the bankability of their projects.

CE projects are generally eligible for EIB financing as these are well aligned with one or more of the bank's central policy objectives including the support of environmental protection and resource efficiency, research and innovation and SME financing. In the last 5 years, the EIB lent around 2.4 billion to $\mathrm{CE}$ projects in diverse sectors including typical infrastructure sectors such as water and waste management, as well as in the agribusiness and bio-economy and industry and services sectors.

\subsection{The European Commission's Perspective: From Funding to Financing}

The representative of the European Commission provided an overview of EU's general policy and available funding sources from the Horizon 2020 and LIFE programmes as well as from the European Structural and Investment Funds (ESIF). These programmes are designed to attract funding from other public or private investors in key EU priority areas including CE. Non-reimbursable EU funding in the form of grants will continue to play a role in reducing the overall cost and risk, and help boost the credit rating of innovative projects that otherwise would not have seen the light of day. Simultaneously, the EC is increasingly promoting the integration of available EU funds with financial instruments and products such as loans, guarantees, equity and other risk-bearing mechanisms, as a means of enhancing the scope and leveraging the impact of the EU budget.

In this regard the EC representative mentioned the combined efforts of the EC and the EIB Group in setting up such financial instruments and risk-bearing mechanisms.

\subsection{Joint Initiatives of the EIB and the European Commission in Support of CE}

With the backing of EU guarantees granted to the European Fund for Strategic Investment (EFSI) and the "EU Finance for Innovators" (InnovFin) Programme, the EIB is being enabled to take on more risk and help more CE projects with medium to high risk profiles get off the ground. 
EFSI is one of the three pillars of the Investment Plan for Europe introduced in 2015 and aims to help to finance strategic investments in key areas with relevance for the $\mathrm{CE}$ such as infrastructure, research and innovation, renewable energy and energy efficiency as well as risk finance for small and medium-sized enterprises (SMEs) [2].

InnovFin is available since 2014 and consists of a range of tailored productsfrom guarantees for intermediaries that lend to SMEs to direct loans to enterprises -helping support the smallest to the largest R\&I projects in the EU and countries associated to Horizon 2020 [3]. Following the recommendation of the EIB study on access-to-finance conditions for projects in CE, the EC and EIB amended the InnovFin Programme to include business model innovation (not only technology innovation) as an eligibility criterion [4]. Further, InnovFin also has dedicated financing instruments in key areas relevant to CE. This is the case of the Energy Demonstration Projects facility and the Circular Bio-economy investment platform.

A third example of a successful EIB-EC collaboration with relevance for the $\mathrm{CE}$ is the programme for the Competitiveness of Enterprises and Small and Medium-sized Enterprises (COSME), managed by the European Investment Fund (EIF). The programme aims to improve access to finance for SMEs through two financial instruments that have been available since August 2014: Loan Guarantee Facility and Equity Facility for Growth [5].

Finally, the European Commission has partnered with the EIB to launch the Circular Economy Finance Support Platform. The platform aims to "enhance the link between existing instruments, such as the European Fund for Strategic Investments and the InnovFin-EU Finance for Innovators initiative backed by Horizon 2020, and potentially develop new financial instruments for circular economy projects." The platform is based on three pillars: the first pillar will ensure coordination and awareness raising by providing support to the Circular Economy Financing Expert Group. The second pillar will focus on advisory services. The third pillar will assess the possibility and the need for new dedicated financial instruments for circular economy projects [6].

\subsection{Practical Issues for Financing CE Projects}

As explained by Mrs. Goovaerts and Mr. Piechocki, CE specialists of EIB and Rabobank, their institutions only finance sound and sustainable CE projects. A sound circular project optimises the use and life of assets and products, closes material loops to maintain or recover their economic value at end-of-life, and uses sustainably sourced secondary raw materials, renewable or regenerative resources. Secondly, a sound circular project is expected to be a part of the long term strategy of the company and integrated in the business operation and its environment, including a well-established cooperation with reliable partners within the value chain. Moreover, a sound circular business model shows clearly identifiable demand for circular products, materials or services resulting in reliable and 
sufficient cash-flows to support the repayment of the loan. At last, a sound circular project is run by a competent, experienced promoter able to successfully implement a challenging CE project.

As explained by Mr. Smits, EIB loan officer, the EIB can either lend directly to individual borrowers/projects or, in the case of smaller borrowers/projects, through financial intermediaries. Direct financing is typically made available in the form of senior or subordinated loans for larger projects. The EIB can provide direct financing where the project investment cost is of a minimum of EUR $15 \mathrm{~m}$. In such cases, the EIB carries its own due diligence of the borrower and the project. In all cases the EIB finances a maximum 50\% of the project investment cost.

In the case of smaller projects or promoters (SMEs and MidCaps), the EIB provides intermediated financing via local banks, investment platforms and funds in the form of loans or guarantees. For instance, the EIB has provided several credit lines to its Dutch partner bank Rabobank, which the bank on-lends to 'eligible' SMEs and Mid-Caps at a reduced interest rate. A recent example is the Rabobank Impact Loan for SMEs and MidCaps III with a total credit line of EUR $200 \mathrm{~m}$. The impact loan includes circular businesses as 'eligible' borrowers. In case of intermediated financing, the EIB establishes the eligibility criteria for projects and promoters to be financed, while the due diligence of the final borrower/project is the responsibility of the intermediary financing institution.

\section{Q\&A and Future Perspectives}

The workshop concluded with a Q\&A session. The opening question enquired to what extent the banking sector considers companies' environmental risk profile. As explained by Ms. Goovaerts, EIB specialist on innovative industries, environmental and social impact is always taken into consideration, and compliance with relevant EU legislation is verified. An adjustment of risk assessment methodologies to reflect the unique character of CE projects is something the EIB is currently looking into. In a similar vein, Mr. Piechocki, sustainable business specialist at Rabobank, confirmed that supply chain policies and sustainability risk are always assessed. The bank also assists the clients in finding solutions, in case a risk has been identified.

The next question was directed towards the European Commission representative. Ms. Žutelija, CE policy officer, was asked about the future perspective to develop a set of environmental indicators harmonised at EU level to provide environmental profile of a company including its supply chain. Ms. Žutelija reconfirmed that a harmonised approach at EU level is needed and pointed out that the EC continues working to set up a harmonised system. In this context, the EC is currently finalising the pilot phase of the Product and Organisation Environmental Footprint life-cycle based methodology (PEF and OEF), and is considering future policy options related to measuring the environmental performance of products and organisations throughout their lifecycle. 
Another participant enquired which gaps in CE financing should be addressed in the future. Mr. Schempp, EIB specialist on CE, replied that in the past the EIB has built up a long track record in financing traditional recycling and resource efficiency projects. In the last few years, the bank has increased its risk taking capacity through the establishment of the EFSI and InnovFin programmes and is thus in a better position to provide financing to more innovative circular economy projects and business models. While the financing risk may be increased, such projects are expected to deliver higher economic and environmental benefits. Ms. Busato, EIB Innovation Finance Advisor, pointed out that as long as providers of return based finance and credit rating assessment institutions fail to account for the additional risk associated with the linear economy projects, there will be a gap in methodologies for assessment. The next step is establishing standard methodologies calculating economic return based on a balanced approach to linear and circular risks.

\section{References}

1. European Investment Bank Advisory Services, Access-to-finance conditions for Projects supporting Circular Economy, Report, Luxembourg 2015

2. <http://ec.europa.eu/growth/industry/innovation/funding/efsi_en> (Accessed 12.10.2017)

3. <https://ec.europa.eu/programmes/horizon2020/en/news/innovfin-\%E2\%80\%93-eu-financeinnovators-new-financial-instruments-help-innovative-firms-access-finance> (Accessed 12.10. 2017)

4. <http://www.eib.org/infocentre/press/releases/all/2015/2015-299-eu-opens-up-eur24bn-ofexisting-finance-to-circular-economy-businesses-in-support-of-eu-climate-goals.htm> (Accessed 12.10.2017)

5. <http://ec.europa.eu/growth/access-to-finance/cosme-financial-instruments_en> (Accessed 12. 10.2017)

6. <http://europa.eu/rapid/press-release_IP-17-104_en.htm> (Accessed 12.10.2017)

Open Access This chapter is licensed under the terms of the Creative Commons Attribution 4.0 International License (http://creativecommons.org/licenses/by/4.0/), which permits use, sharing, adaptation, distribution and reproduction in any medium or format, as long as you give appropriate credit to the original author(s) and the source, provide a link to the Creative Commons license and indicate if changes were made.

The images or other third party material in this chapter are included in the chapter's Creative Commons license, unless indicated otherwise in a credit line to the material. If material is not included in the chapter's Creative Commons license and your intended use is not permitted by statutory regulation or exceeds the permitted use, you will need to obtain permission directly from the copyright holder. 


\title{
Life Cycle Approaches to Sustainable Regional Development
}

\author{
Fritz Balkau and Timothy Grant
}

\begin{abstract}
Regional development that is truly sustainable depends on the adoption of systematic and long-term criteria for decision-making at different levels, taking into account the full life cycle of raw materials and products, as well as of infrastructure projects. This session invited the presentation of life cycle management applications from both a scientific and a practitioner point of view, highlighting examples and case studies at regional level. The applications are relevant to private actors from different economic sectors as well as for public representatives responsible for regional planning and administrative procedures.
\end{abstract}

\section{Introduction and Session Content}

Regional development that is truly sustainable depends on the adoption of systematic and long-term criteria for decision-making at different levels, taking into account the full life cycle of raw materials and products, as well as of infrastructure projects. This session invited the presentation of life cycle management applications from both a scientific and a practitioner point of view, highlighting examples and case studies at regional level. The applications are relevant to private actors from different economic sectors as well as for public representatives responsible for regional planning and administrative procedures.

Life cycle tools presented in this session included LCA, Materials Flow Analysis (MFA), supply-chain management, eco-design, environmental product declarations (EPD), and sustainable public purchasing (SPP). These were applied to a variety of industry sectors such as building and construction, chemicals production, agriculture and food, wood production. All the above life cycle tools are applicable at

\author{
F. Balkau ( $\square)$ \\ Sustainable Solutions, Paris, France \\ e-mail: fbalkau@gmail.com \\ T. Grant \\ Lifecycles Australia, Melbourne, Australia \\ (C) The Author(s) 2018 \\ E. Benetto et al. (eds.), Designing Sustainable Technologies, \\ Products and Policies, https://doi.org/10.1007/978-3-319-66981-6_48
}


regional level, with broad-scale applications into initiatives aimed at circular economy, bio-economy and fostering local industries.

The presenters reiterated that the systematic use of the above tools depends on an extensive programme of awareness-raising, education and training of both private and public sector actors, as well as the creation of collaborative partnerships that reach along the life chain of materials and programmes. All these need to be underpinned by adequate life cycle based databases and suitable metrics that allow assessments to be reliably performed. It is also useful to undertake pilot studies and compile case studies of successful initiatives.

\section{Appreciation of the Presentations}

The five presentations in this session described a variety of life cycle tools and methodologies. While each presentation was on a different subject, they nevertheless formed a complementary set of approaches that can be of use to regions in their pursuit of more sustainable forms of development. The case studies were drawn from five different regions around the world-France (2), Germany, Australia, South America (Peru), indicating that the use of life cycle tools for regional development has universal relevance.

Benoit Ribon gave an interesting presentation of territorial metabolism (or materials flows) in a north-eastern region in France (Alsace). Flows are calculated according to a set of internal nodes that are connected by materials and energy movements. This allows an estimation of internal consumption as well as of import and export volumes. Such an analysis provides information towards the design of a circular economy, and at a different level, the needs and options for local waste management.

The presentation described a model to calculate regional MFA, revealing the dynamics of resources in transit, waste management and internal (regional) production and consumption. All these have important sustainability implications. Concerning the management considerations, through an MFA both the energy sources as well as points of wastage can be more clearly identified, potentially allowing efficient intervention to improve the path towards regional sustainability.

The study also highlighted some constraints, in particular that imposed by the nomenclature of freight statistics. Freight is one of the few data sources at regional level relating to materials importation and exportation, however the structure of the data does not reflect well the life cycle of products. This inevitably leads to many assumptions in data processing and modelling. A direct conclusion is thus that regions themselves may need to ensure sufficient statistical data if they are to better understand their circular economy dynamics.

Timothy Grant described a recent study in the State of South Australia aimed at enhancing the circularity of materials flows to increase local employment, reduce energy use and improve materials efficiency while diminishing waste generation. The ensuing scenario development looked also at other benefits such as energy 
independence, entrepreneurship, and generally encouraging the evolution to more sustainable forms of business that favour use of local materials and labour. Greater reliance on renewable energy is an important centrepiece of the South Australian scenarios, compensating for the closure of fossil fuelled power stations there, and present use of fossil fuel for heating.

The use of MFA as illustrated in this presentation covered not only energy but also various materials flows that have implications for sustainability. The use of MFA gives greater reliability and certainty to scenario development, giving public policy makers more confidence in the various future visions that circular economy scenarios attempt to provide.

The implications of the materials flow calculations of the scenarios are now being evaluated by the Government with an expectation of early adoption and application.

Vanessa Pasquet described the life cycle management framework put in place by the French region 'Hauts de France' where various industry sectors become more deeply linked with their supply chains by using life cycle management procedures. These include methods such as environmental design, eco-labels, environmental product declarations (EPD) and advanced building standards. Similar tools can also be applied to other industry sectors such fisheries, agriculture, textiles among others.

Life cycle assessment provides much of the essential information on which the management tools are based. In view of the breadth of the sustainability agenda, a variety of LCA procedures may be necessary to capture all the key issues of regional concern. It is also important that all stakeholders along the value chains are identified during the LCA process to ensure adequate co-operation in implementation.

The life cycle management framework described by Pasquet is strongly oriented towards collective action rather than authoritarian regulation, although the latter is eventually needed to ensure that all players have the same targets. The building of stakeholder alliances depends on credible information (from the LCA process), identification of common objectives for all the stakeholders, effective metrics to measure progress and of remaining hotspots, communication procedures adapted to the needs of supply-chain stakeholders, and transparency in monitoring and reporting the outcomes. In many cases further education and training of supply-chain partners may be needed.

Sinéad O'Keefe described an initiative by the by the Helmholtz-Centre for Environmental Research GmbH-UFZ to model the industrial infrastructure and stakeholder collaborations necessary for greater use of locally sourced organic material (mainly wood products) in the eastern regions of Germany. The region already has an extensive chemicals industry based on imported raw materials (mainly gas) but also has extensive forest lands and agricultural outputs. The perspective of establishing a regional bio-economy as part of an industrial transformation process would have many advantages.

The study considers the sustainability and social impacts of a number of product mixes that could be based on a regional bio-economy rather than on imported materials as at present. Various life cycle assessment tools underpin this initiative; materials flow analysis, life cycle assessment, social impact assessment, and 
eco-footprint being the principal ones. Climate footprint was the main sustainability factor considered, however a full impact suite was taken into account to avoid possible burden shifting and spill-over effects.

It is likely that a fully-fledged move to transform the resource base of a regional economy will eventually require further tools as a supplement. Monitoring and evaluating the development of a regional bio-economy needs to take into account technical, social and environmental factors, as well as paying regard to employment conditions and local amenity objectives. Tools for some of these were developed as part of the initiative, and were applied to several scenarios of biomass sources, product mixes and social structures.

O'Keefe describes an integrated approach - the SUMINISTRO frameworkthat considers the above factors using methodologies based on both traditional and social LCA, evaluating the environmental and socio-economic impacts from different product mixes of the bio-economy(s) being modelled. The study concludes that an integrative assessment framework and knowledge base can indeed facilitate industrial transformation towards regional bio-economies based on local resources. The establishment of participative stakeholder networks helps to identify key opportunities and risk in such a transformation. A number of actual local case studies of biomass-derived products confirms the feasibility of this approach.

Ian Vázquez-Rowe. Consequential life cycle assessment methods can be used to evaluate the various environmental, social and economic consequences of public policy decisions for further development of regional agriculture. A particular preoccupation for agriculture these days is water use and climate change, not only in terms of adaptation but also for GHG generation from agriculture itself. Vázquez-Rowe presented an evaluation of potential agricultural developments in Peru, in particular for the growing of grapes for production of pisco (an alcoholic beverage made from grapes) for export. The market for this is expected to increase, making a sustainability (GHG and water) assessment very pertinent. Potential impacts have to be seen in the light of crop replacement and possible use of fallow land that do not require additional new lands to be brought into production. The study had to take into account questions of land-use, property markets, and the optimum way to allocate production under different economic and market scenarios. Nevertheless, not all parameters influencing farmers' decision could be included in the model. In the end it was found that there need not be an increase in net GHG emissions if appropriate farming practices were employed, but water use was dependent on the area farmed.

\section{Overview}

Individually, the presentations describe some interesting research and regional case studies. Several broke new ground in the application of life cycle tools at regional level, both in assessment and in LCM, as for example in social LCA related to bio-economy transformation in Germany. Several others described the relevance of 
standard LC tools to other regions that had not so far been highlighted in regional LCM work (e.g. MFA in Alsace, and LCA in Peru, respectively). Two presentations relating to South Australia and Hauts de France respectively, describe the application of life cycle management and circular economy principles by regional authorities to move their development policies forward.

This Session discussed extended LCA for new industrial situations, as well as the application of standard LCA and MFA to additional regions. Both are valuable in promoting the further use of life cycle methodologies in regions, echoing the conclusion of a recent book [1] that what is needed is both a further evolution of LC tools, and raising the profile of LCM among regional managers and policy makers. The utility of life cycle approaches to regional development is relevant to all continents as we saw, and it is encouraging that LCA societies are nowadays found in many countries. It remains to encourage these societies to take a broader view than simply refining the present assessment methodologies - they also need an eye on new client groups who have different needs.

\section{Future Perspectives}

Neither the development agenda nor the LCA environment is standing still. Regions and local governments are under increasing stakeholder and trade pressure, and are looking for ways to meet upcoming challenges, whether climate change, biodiversity loss, land preservation or social and employment issues. While certain life cycle techniques are occasionally used by regions, few base their future development on a systematic life cycle approach. This is particularly unfortunate at a time when many are experimenting with, for example, circular economy, industrial ecology, long-term infrastructure planning or 'sustainable' forms of manufacture and business. Now is the right formative moment for the life cycle community to open its doors to regional government as an important stakeholder and client group, to speak to them in their language, and to discuss their needs. The incorporation of a municipality in the recent road test of Organisational Life Cycle Assessment (O-LCA) [2] is a step (but only a small one) in the right direction. To borrow a phrase from other area, a dose of positive discrimination in the direction of the public sector would be a helpful move in enlarging the scope of LCA/LCM application.

At the same time the life cycle community could usefully accelerate the effort to further evolve life cycle methodologies that are of interest to regions, such as landscape and land-use assessment techniques, biodiversity assessment, natural resource accounting, eco-footprints and so on. There are embryonic moves in these areas - they could usefully be moved further along to mainstream level in conferences, teaching and research. 


\section{References}

1. Life cycle approaches to sustainable regional development, Balkau F, Massari S, Sonnemann G, eds., Taylor and Francis 2017.

2. Road testing Organizational Life Cycle Assessment around the world: Applications, experiences and lessons learned", http://www.lifecycleinitiative.org/download/6060.

Open Access This chapter is licensed under the terms of the Creative Commons Attribution 4.0 International License (http://creativecommons.org/licenses/by/4.0/), which permits use, sharing, adaptation, distribution and reproduction in any medium or format, as long as you give appropriate credit to the original author(s) and the source, provide a link to the Creative Commons license and indicate if changes were made.

The images or other third party material in this chapter are included in the chapter's Creative Commons license, unless indicated otherwise in a credit line to the material. If material is not included in the chapter's Creative Commons license and your intended use is not permitted by statutory regulation or exceeds the permitted use, you will need to obtain permission directly from the copyright holder.

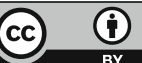




\title{
Turning the Lens Around: LCA Success Stories Outside-In
}

\author{
Eric Mieras and Alain Wathelet
}

\begin{abstract}
Life Cycle Assessment experts are working on environmental assessments with both feet on the ground and faced every day with challenges around data quality, allocation and other challenging topics. But what's the perspective of the people that are the audience of the results: "How do they look at LCA?" The objective of this session was to show the value LCA has for people outside the LCA Community that are not as familiar with the topic as the Experts. Sanjeevan Bajaj (FICCI), Namy Espinoza-Orias (Nestlé), Pawin Boonyaporn (Advanced Biochemical), Jens-Christian Holst (Siemens) and Aubin Roy (AvniR) shared their experiences.
\end{abstract}

\section{Introduction}

Life Cycle Assessment (LCA) studies are often prepared by high level scientists and are focused on specific subjects for the interest of a small community. Nevertheless, the development of Life Cycle Thinking in a business organization leads to the use of a large panel of tools to demonstrate the sustainability of their business solutions (products and/or services), and an LCA approach perfectly fits this goal. During LCM, the benefits of this LCA approach are presented not only by LCA practitioners but also from the different users of Life Cycle approaches.

The "outside-in" session is conducted from the LCA perspective with a look from the perspective of users outside of the LCA community, including strategic decision making, marketing team, R\&I team and product development team.

E. Mieras $(\bowtie)$

PRé Sustainability, Stationsplein 121, 3818LE Amersfoort, The Netherlands e-mail: mieras@pre-sustainability.com

A. Wathelet

Solvay SA, 1120 Brussels, Belgium

(C) The Author(s) 2018

E. Benetto et al. (eds.), Designing Sustainable Technologies,

Products and Policies, https://doi.org/10.1007/978-3-319-66981-6_49 


\section{Internalizing Life Cycle Thinking in Business Strategy Through Amalgamation of Life Cycle Knowledge with Strategy Development Tools [1]}

Human societies today need business models that allow wealth generation along with regeneration of natural and social capital, which means going beyond the usual financial performance focus to manage environmental, social, developmental impacts of doing business. On top of this, Life Cycle approaches call for an assessment of these impacts not just from production and delivery, but across product/service life cycles from material extraction to end-of-life disposal. Given the dominant business paradigm of profit-maximization, why would business leaders succumb to hard sell by LCA practitioners and make life more complex for themselves?

To be fair, Life Cycle Thinking is not new to business management. The Value Chain concept is integral to business strategy. Based on a process view of organizations, the idea of seeing a business as a system, made up of subsystems with inputs, transformation processes and outputs is already internalized in strategy literature. How value chain activities are carried out determines costs and impacts profits. While converting inputs to outputs, business organizations engage in hundreds of activities which can be categorized as primary or support activities, and their depiction through the Value Chain diagram is pervasive in business management literature. Thus product development requires businesses to research where the raw materials might come from, which manufacturing processes may be needed, who will use the product, what additional costs will be incurred during use, what maintenance support will be needed, what types of waste will be created before/ during/after use, and where the product remnants will go when discarded. In the LCA practitioner's lingo, this translates to 'designers conduct life cycle studies and measure the potential impacts of various options, requesting information from suppliers, calculating full life cycle cost of goods consumers purchase including point-of-purchase price as well as costs of transporting, storing, installing, cleaning, operating, repairing, and eventually discarding those goods'.

Despite common ground across strategy development and Life Cycle approaches, there is little uptake of Life Cycle approaches at a truly strategic level. Most success stories about business value from Life Cycle studies talk about cost reductions through materials/energy saving, or customer loyalty through sustainability posturing. Cost reductions accompany operational improvements, while sustainability posturing builds image. Business leaders aren't fundamentally rethinking their business to increase aggregated well-being of the planet and human society. At best, they are trying to do less harm while going about their business.

For Life Cycle approaches to qualify as tools enabling paradigm shift, like Strategy tools, they must provide a structure to generate and evaluate strategic options of domain selection (what business to be in) and domain navigation (how to compete in this business). Business strategy deals with competition, business 
leaders choose the most attractive industries and/or influence industry structures to make them attractive for themselves.

The field of strategy is constantly evolving at a fast pace. LCA can be used to support strategic decision making for instance on which technology to invest in. However, most companies $(95 \%)$ do not include sustainability yet, so there's still a lot of companies to win.

\section{Integrated Product Development at Nestlé [2]}

At Nestlé, the world's largest nutrition, health and wellness company, the life cycle approach is applied to multi-criteria product innovation and renovation, which involves Nestlé's partners and stakeholders from farm to consumer and beyond.

In order to embed environmental sustainability into their products, the Sustainability by Design program led by the Research \& Development unit assesses and optimizes the environmental performance across the entire value chain at the earliest stages of product development. The identification of environmental hotspots where relevant, and the definition of actions to address them is carried out using the internal eco-design tool EcodEX. Furthermore, a new tool aimed at identifying opportunities for projects to deliver on Nestlé in Society commitments is being launched in 2017 across the R\&D organization.

On the other hand, successful product renovation and innovation requires building sufficient and necessary consumer, technical and product mastership knowledge. This consumer-centric approach entails the close cooperation of marketing, operations and R\&D units and also spans over the entire product life cycle.

This holistic and comprehensive methodology, as applied by Nestlé, delivers competitive advantage, leads to the fulfillment of public commitments at company level and drives the company contribution as member of the food industry to the achievement of the Sustainable Development Goals. Informed decision making takes place at the various steps of project execution, risks and opportunities are identified, and early warning is brought up at the appropriate time.

However, given the breadth of technical areas concerned and what is at stake, the interpretation of data and insights resulting from this way of working can be complex and raise some challenges. The deployment of support tools can only be justified as long as they are properly and widely used. Deciding which function will take up the identified actions for improvement, either R\&D, operations or both in a concerted way, requires alignment of objectives and priorities as well as clear allocation of resources. For a company as large as Nestlé, it is fundamental to progress from starting the conversation and raising awareness on multiple criteria for product development to consistently accomplishing it.

Being proactive and understanding that what matters to consumers entails all aspects of the product - going beyond quality and including sustainabilityenriches the product development process and provides consumers with a product they prefer, knowing that Nestlé has taken care of what matters to them. 
At Nestlé, LCA is one of the assessments used in a customer focused innovation approach. Nestlé created an overall dashboard for innovation decisions that is available for everyone involved. Within this multi-criteria assessment there's a clear role for LCA in combination with other impacts.

\section{Innovative Partnership to Promote Credible and Sustainable Bio-based Materials Through the Value Chain [3]}

Bio-based materials derived from plant residues open up exciting opportunities for environmentally-responsible products. Trendsetters such as bio-based epichlorohydrin $(\mathrm{ECH})$ can provide more sustainable alternatives for traditional raw materials. To this end, AkzoNobel, Advanced Biochemical (Thailand) Co., Ltd. (ABT) and EY (Ernst \& Young) have joined forces to develop a monitoring system which could track and quantify the use of renewable raw materials in paints, coatings and further applications. The partnership built on a previous agreement, whereby AkzoNobel would progressively increase the use of epoxy resins derived from Epicerol ${ }^{\circledR}$, a bio-based ECH, in its coatings products.

Throughout 2017, the partnership will roll out an online system which will support a comprehensive bio-based certification. ABT, AkzoNobel and EY have developed a 'chain of custody' methodology to ensure that even in situations where no physical segregation of petro and bio-based materials is practiced, volumes may still be assigned and reported.

Rather than buying ECH directly, AkzoNobel is sourcing epoxy resins from a number of intermediate producers. The company then uses epoxy resins as ingredients in various coatings. By creating market pull through e-certification, the partnership promotes bio-based materials through the value chain.

Epicerol $^{\circledR}$ is an ECH based on $100 \%$ renewable glycerine, a by-product from the transformation of vegetable oils. Manufactured by ABT using an innovative and patented process, the drop-in was developed and commercialised because of demand for a truly sustainable ECH.

A comparative Life Cycle Assessment (LCA) benchmarked Epicerol ${ }^{\circledR}$ against state-of-the-art propylene-based processes. It showed that incorporating 1 MT of Epicerol $^{\circledR}$ can reduce a product's carbon footprint (defined as the global warming potential (GWP) from cradle-to-gate, including biogenic $\mathrm{CO}_{2}$ ) by $2.56 \mathrm{MT} \mathrm{CO}_{2}$ equivalent.

The technology also reduces the volume of chlorinated by-products from the process by over $80 \%$, while another distinctive technology enables brine recycling and drastically reduces liquid effluents.

A wider rollout is targeted before end-2017. By promoting sustainable alternatives through pro-active procurement of bio-based feedstock and a transparent transfer of e-certificates across the value chain, this innovative project will demonstrate a credible approach to sourcing better materials. 


\section{The City Performance Tool-How Cities Use LCM Based Decision Support [4]}

Modern cities have an increasingly vital role to play in finding new ways to protect the environment. Understanding the environmental and economic impact of infrastructure choice is crucial for urban decision makers especially as linked to long term strategies and investment have to be taken. Siemens deep technology expertise can support this understanding however technology performance indicators have to be transformed to the solution embedded into a city specific context and in combination with other infrastructure choices across city domains.

Understanding these cities requirements and perspective of the decision makers Siemens developed the City Performance Tool to explore the environmental and economic impact of more than 70 technologies from Building, Transport and Energy Technologies - at different time periods and implementation rates.

It is designed to reduce the environmental impacts of everyday activities in cities. It covers greenhouse gas emissions from buildings and transport, as well as air pollutants such as particulate matter $(\mathrm{PM})$ and nitrogen oxides $\left(\mathrm{NO}_{\mathrm{x}}\right)$. It also looks at the creation of new local jobs to install, operate and maintain city solutions.

The common model determines the variety of activities in process blocks to build any city in a common LCA tool (SimaPro):

- $20+$ electricity and heat generation

- $10+$ building types its electricity, heat and cooling demand

- 10 freight modes; 35 passenger transport modes; 15 road and rail infrastructure elements

The CyPT provides environmental results relative to the amount invested. It enables city managers to prioritise projects based upon their likely environmental and economic impacts. It can offer city managers a roadmap towards meeting carbon emission targets, clean air standards and local job growth. The work presents the methodology, details about the underlying LCA models and its implementation into the tool. In transport, for example, CyPT assesses how a technology would reduce demand (reduce parking search traffic), shift the mode (public transport instead of cars) or improve efficiency (automated trains). The model is based on LCA methodology and builds upon Siemens' technology expertise and global databases of deep vertical process knowledge.

Now urban decision makers can use the City Performance Tool (CyPT) by Siemens to select urban infrastructure technologies that offer their own cities maximum environmental and economic benefits. The CyPT studies are performed in more than 25 cities worldwide and experiences gained will be shared in this presentation. 


\section{Implementing LCM All Along the Supply Chain: From Compliance to Collaborative Value Creation [5]}

In a complex society sustainable production and consumption patterns can only be achieved through the participation of entire supply chains, including designers, suppliers, manufacturers, etc. The involvement of all these actors and their collaboration is critical to mainstream LCM in a large scale and to create shared value. In this context, the aim of this contribution is to present the [avniR] "customer/ supplier" working group. In parallel, a set of success stories will be presented together with an assessment of challenges and opportunities to integrate LCM all along the supply chain.

Since 2012, a new governance based on different working groups was launched to strengthen collaboration within the [avniR] platform in North of France. A working group was initiated in this context to mainstream LCM all along the supply chain called "customer/supplier working group". Different actions were conducted in the context of the working group including awareness rising, workshops and collaborative working groups based on identified needs from the stakeholders.

One of the leading ongoing actions launched in collaboration with Renault, is the customer/supplier, Collaborative Life Cycle Activities (CoLCA). The first series of workshops was organized in 2016 with a large set of businesses both big and small ones. Seven businesses joined the working group (ALSTOM, FAVI, HAPPY CHIC, HELIOPAC, LE RELAIS-MÉTISSE, POLYNT COMPOSITES and SNCF).

As the first step, the "Collaborative Life Cycle Activities" approach was presented and discussed with participants. Then participant businesses conducted their own assessment internally involving various department (production, R\&D, top management, marketing, communication, etc.). A second meeting of the working group permit to present the self-maturity assessment results of each company and to discuss their plan to go to next level and begin interactions with their clients/ suppliers. In 2017 the working group aims to share and discuss the strategic plan and the learning from businesses involved, also to initiate the same dynamic with other businesses from variety of sectors.

As over $80 \%$ of a company's impact occur in the value chain, it's crucial to involve suppliers. Often these suppliers are SMEs that are less mature when it comes to environmental impact. Therefore, it's essential to involve them in the process. 


\section{References}

1. Bajaj S, Internalizing Life Cycle Thinking in Business Strategy through Amalgamation of Life Cycle Knowledge with Strategy Development Tools, LCM 2017 conference, Luxembourg, 2017.

2. Espinoza-Orias N, Cooper K, Integrated product development at Nestlé, LCM 2017 conference, Luxembourg, 2017.

3. Holst J.C, Heidinger K, Müller K, Jaeger F, The City Performance Tool- How cities use LCM based decision support, LCM 2017 conference, Luxembourg, 2017.

4. Boonyaporn P, Innovative Partnership to Promote Credible and Sustainable Bio-based Materials Through the Value Chain, LCM 2017 conference, Luxembourg, 2017.

5. Roy A, Adibi N, Pasquet V, Morel S, Implementing LCM all along the supply chain: from compliance to collaborative value creation, LCM 2017 conference, Luxembourg, 2017.

Open Access This chapter is licensed under the terms of the Creative Commons Attribution 4.0 International License (http://creativecommons.org/licenses/by/4.0/), which permits use, sharing, adaptation, distribution and reproduction in any medium or format, as long as you give appropriate credit to the original author(s) and the source, provide a link to the Creative Commons license and indicate if changes were made.

The images or other third party material in this chapter are included in the chapter's Creative Commons license, unless indicated otherwise in a credit line to the material. If material is not included in the chapter's Creative Commons license and your intended use is not permitted by statutory regulation or exceeds the permitted use, you will need to obtain permission directly from the copyright holder.

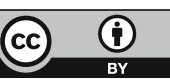




\title{
Integrated Product Development at Nestlé
}

\author{
Namy Espinoza-Orias, Karen Cooper and Sofiane Lariani
}

\begin{abstract}
Nestlé's purpose is to enhance the quality of life and contribute to a healthier future. In practice, it is translated into product development through a life cycle, multi-criteria, and integrated approach engaging internal and external stakeholders. An overview of the company's values is presented as well as its creating shared value business principles, followed by an explanation of the Sustainability by Design program and how it is embedded into product innovation and renovation. The integrated development of a breakfast solution for children who skip breakfast exemplifies this approach. Being proactive and understanding that what matters to consumers entails all aspects of the product-going beyond quality and including sustainability - enriches the product development process, informs decision-making timely, provides consumers with a product they prefer, delivers competitive advantage, and supports the fulfilment of Nestlé's public commitments.
\end{abstract}

\section{Introduction}

In 2016, Nestlé celebrated its 150th anniversary and is currently the largest food and beverage company worldwide. From the beginning, when Henri Nestlé invented the Farine Lactée to save the life of an infant, Nestlé's purpose has been to enhance the quality of life and contribute to a healthier future. What drives our value creation, both for business and society, is a nutrition, health and wellness strategy. With more than 2000 brands, whose products are sold across seven product categories in 191 countries, understanding and anticipating consumer needs in an ever evolving and competitive environment is imperative for Nestlé's long-term business success. This is precisely the starting point of our integrated product development. It follows a life cycle, multi-criteria process, with the engagement of

\footnotetext{
N. Espinoza-Orias $(\bowtie) \cdot$ K. Cooper $\cdot$ S. Lariani

Nestlé Research Center, Rue Du Jorat 57, 1000 Lausanne, Switzerland

e-mail: NamyDaniela.EspinozaOrias@rdls.nestle.com

(C) The Author(s) 2018

E. Benetto et al. (eds.), Designing Sustainable Technologies,

Products and Policies, https://doi.org/10.1007/978-3-319-66981-6_50
} 
internal and external stakeholders and the firm inclusion of Nestlé's values, business principles and public commitments against which our company and products are held accountable.

The purpose of this paper is to explain how key elements bring in practice product innovation and renovation with a competitive advantage. Our values framework, business model, Sustainability by Design program and integrated product development approach are reviewed. A breakfast solution for schoolchildren who skip breakfast is presented as an example of consumer-centric product development.

\section{Creating Shared Value, the Nestlé Way}

Guided by values rooted in respect, Nestlé works alongside partners and stakeholders to create shared value (CSV) across all the activities of the company, which contribute to society while ensuring the long-term success of our business.

Three interconnected focus areas are identified wherein our purpose is realized:

(1) Individuals and families: to whom Nestlé offers products and services that enable them to lead healthier and happier lives.

(2) Our communities: helping those we live and work with in developing into thriving and resilient communities, enhancing rural livelihoods, and respecting and promoting human rights.

(3) Our planet: stewarding resources for the future, caring for water, acting on climate change and shaping sustainable consumption.

Our CSV priorities and efforts are supported by 42 specific, public commitments with clear short-term timelines (2020) as well as three overarching medium term (2030) ambitions for our focus areas. In particular, Nestlé aims to help 50 million children lead healthier and happier lives, help improve the livelihoods of 30 million in communities directly connected to our business activities and strive for zero environmental impact in our business operations [1].

The recently updated and reframed commitments as well as newly introduced ones [1] go further and deeper than the first set of them launched back in 2012. The commitments were defined taking into account stakeholder recommendations and issues material to our consumers, stakeholders and the company. The materiality assessment is carried out every two years in collaboration with SustainAbility - an independent consultancy specialized in corporate sustainability - using a formal process entailing extensive consultations of stakeholders, investors and key opinion leaders. Issues of concern in the environmental, social and governance areas are identified and assessed in order to determine their business impact in terms of risks and opportunities along with the level of interest stated by stakeholders. The commitments were also aligned in scope and timeline with the Sustainable Development Goals (SDGs) [2]. 
In particular, those SDGs where Nestlé has specific responsibilities as a member of the private sector and can have the most positive and meaningful impact are emphasized. This is achieved by mapping our material issues to the SDGs; as a result, it is possible to find where our activities contribute, directly or indirectly, to the realization of the SDGs.

\section{Sustainability by Design}

In order to embed sustainability into our products, the Sustainability by Design program led by the Nestle Research organization assesses the sustainability aspects at the earliest phase of the product development cycle. The aim is to optimize the impacts across the entire value chain through an iterative approach that spans the product development process. The various elements taken into account are schematised in Fig. 1.

From the early stages of a product development, projects are systematically evaluated with the following steps:

(1) Identification - as early as possible - of the opportunities or hotspots, either environmental or social. A tool has been developed that guides projects through the process of discussing its potential impact on individuals and families, our communities and the planet. Project managers collaborate with sustainability

\begin{tabular}{|c|c|c|}
\hline \multicolumn{2}{|c|}{ WHEN TO ASSESS? } & WHERE IN THE VALUE CHAIN? \\
\hline \multicolumn{2}{|c|}{$\begin{array}{l}\text { At the various stages of the } \\
\text { Produa Development Process }\end{array}$} & $\begin{array}{l}\text { At the various } \\
\text { life cycle stages of the product }\end{array}$ \\
\hline \multicolumn{2}{|l|}{ 1. Ideation } & 1. Agricultural production of ingredients \\
\hline \multicolumn{2}{|c|}{ 2. Conceptualization } & 2. Manufacture of packaging materials \\
\hline \multirow{2}{*}{\multicolumn{2}{|c|}{ 3. Development }} & 3. Manufacture of food products \\
\hline & & 4. Distribution \\
\hline \multicolumn{2}{|c|}{ 5. Launch } & 5. Retail \\
\hline \multirow{2}{*}{\multicolumn{2}{|c|}{ 6. Post-Launch }} & 6. Consumption \\
\hline & & 7. End of life \\
\hline \multicolumn{2}{|c|}{ WHO IS IMPACTED? } & WHAT IS ADDRESSED? \\
\hline \multicolumn{2}{|c|}{ Intemal and external stakeholders } & Contribution through our products to: \\
\hline 1. Farmers & 5. Pets & 1. Nesté Public Commitments \\
\hline 2. Suppliers & 6. Families & 2. Materiality issues \\
\hline 3. Employees & 7. Communities & 3. Ambitions for our focus areas \\
\hline 4. Consumers & 8. The planet & 4. Sustainable Development Goals \\
\hline
\end{tabular}

Fig. 1 Elements considered in the sustainability assessment of products at Nestlé 
champions to identify which societal commitments their project may affect, positively or negatively. This is revisited at key moments in the project, i.e. when passing a stage gate or a major change occurs.

(2) Comparison to the Nestlé materiality matrix (Fig. 2), which summarizes the issues of concern to the company and its stakeholders. As the 42 commitments are not exhaustive to all potential issues, this enables project managers to understand if their project may assist in other areas of concern.

(3) An action plan is developed to either mitigate or leverage the potential impacts and is then recorded as part of the project process. The actions can consist of carrying out further in-depth assessments as the project progresses and more information becomes available.

(4) The tool covers every step in the value chain from ingredients, processing in our factories, packaging, consumer use right through to end of life. It allows a deep dive into specific aspects; for example, into ingredients and responsible sourcing, linking into the Procurement side of the company.

(5) At this point, a project is also assigned a specific rating for potential environmental sustainability impact, which is a key KPI tracked at a strategic level across the project portfolio. This tracking allows the calculation of time spent on projects of different ratings, an important indicator as to whether the portfolio will deliver against our environmental commitments. This rating can be reviewed or ratified at various points in the project process.

(6) The assessment itself ends at this point, having taken approximately an hour but delivering key information on how the project will deliver against sustainability and an action plan for improvement.

Following up on the agreed actions usually involves the use of EcodEX, a simplified environmental life cycle assessment tool developed with an outside IT company, Selerant [3]. This tool allows the comparison of multiple scenarios, for instance a current product in the market versus an innovation. Early use allows detailed quantitative information on potential environmental issues and benefits to be available for decision making at a point where there is more design freedom and less cost to make changes.

Future potential work to develop this tool may include a stronger social focus and Nestlé is evaluating the latest methodologies in this area.

\section{Integrated Product Development}

\subsection{PrIME}

Consumer centric product development, renovation or innovation must also take into account the different elements influencing the decision to buy food and beverage products. These are taste, price, healthiness, convenience and sustainability [4]. 


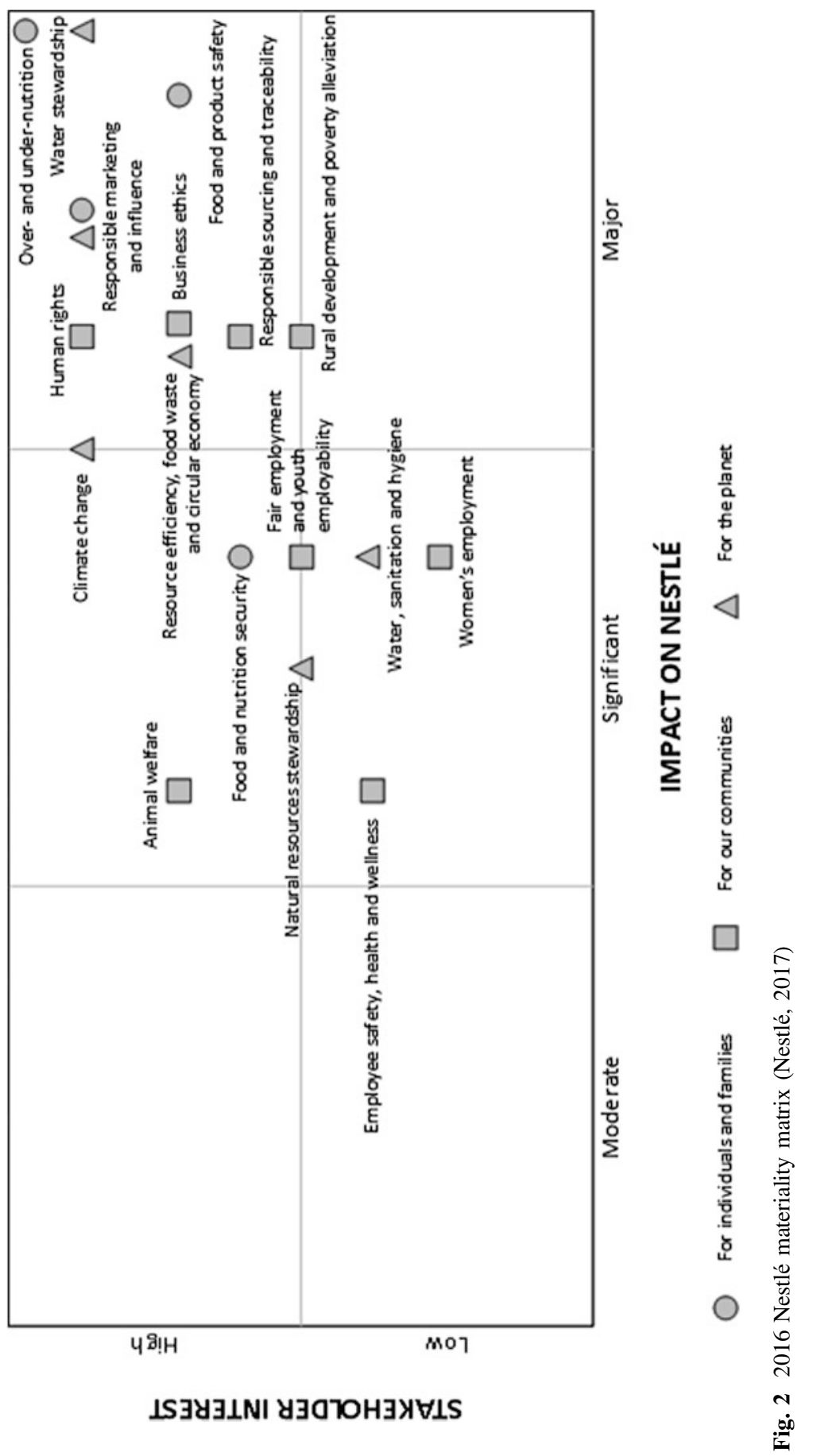


The incremental approaches optimizing one element at the time in a complex environment where Nestlé has to come up with heathy, sustainable products that the consumer wants and can afford, have quickly shown their limitations.

Therefore, Nestlé Research put in place a holistic approach, called PrIME, optimizing at the same time consumer healthiness, preference, sustainability and affordability. Integrating these four elements into product development through deep understanding of consumer needs and preferences has helped identifying optimal recipes by maximizing these different elements simultaneously.

Nestlé is applying the PrIME approach to product design and packaging to optimize the whole product experience by:

(1) Mastering healthiness and sensorial product features. For example, delivering product sweetness with less sugar through optimal product design.

(2) Improving the packaging functionality to ensure:

1. Inclusions of all individuals (for instance the elderly), and

2. Frustration free packaging experience.

Nestlé Research has developed this consumer centric product development approach in all key global brands for the past years and we are now deploying it in the main markets to support our local brands.

This consumer-centric approach is built around a process that uses a collection of standardized methodologies and tools to connect people understanding and product knowledge. The management of our knowledge and data is the foundation of this process and the way to accelerate it, iteration after iteration. Therefore, several activities are in place to optimize the management and sharing of consumer and product knowledge and data. PrIME is using data from different sources and big data techniques to ensure we constantly satisfy the evolution of needs of individuals and families.

PrIME consists of the following steps:

(1) Identification of consumer value drivers: what is important for the individuals and families? How do they like consuming their products? What are their needs? What are their dissatisfactions? This first step is important to dive into the heart of the consumer preferences but not sufficient to decode individuals and family's needs.

(2) Understanding people needs and behaviours is at the heart of this approach. We use state of the art consumer research to translate multi-dimensional needs into objective product features and attributes.

(3) Consumer centric product and technological mastership is ultimately the way to:

a. Fast track product development. b. Identify our product knowledge gaps and develop innovative, differentiating and sustainable technologies to better satisfy the consumer on the elements cited above: healthiness, preference, sustainability and affordability. c. Control our manufacturing settings and provide day after day high and sustainable product quality to individuals and families. 
The PrIME approach has shown its success developing efficiently and effectively the products the consumer wants first time right and contributes to push us to constantly innovate in technologies relevant for consumers, communities and the planet.

\subsection{Nutritional Considerations for Product Development at Nestlé}

Nestlé Research developed in 2004 the Nestlé Nutritional Profiling System (NNPS) that guides the nutritional dimension of formulation and reformulation of Nestlé products nutritional product development [5]. The NNPS translates nutritional recommendations from the World Health Organization and other international and national organizations - taking also technical feasibility into account - into tangible product targets, e.g. the amounts of nutrients to limit and nutrients to encourage in a product. If all targets defined by the NNPS are met, then the product achieves the Nutritional Foundation (NF). The nutritional assessment is facilitated through a tool, globally rolled out in Nestlé and used by more than 1000 product developers and nutritionists. This assessment is a fundamental step within the development process and guides further improvements in the products.

\section{Case Study: Nestlé Nido GoldenStart $\subset$}

Although breakfast is considered the most important meal of the day, it is common that children skip breakfast frequently, thus missing the opportunity to consume a meal rich in nutrients recommended for a wholesome diet. Existing studies and reviews on breakfast consumption habits in different regions and countries [6-8] report that between 10 and $30 \%$ of schoolchildren skip breakfast.

The reasons leading to skipping breakfast are varied, and include socio-demographic factors, limited nutritional knowledge, lack of time and planning for breakfast (preparation and/or consumption), absence of hunger in the morning, limited or no availability of breakfast foods that are affordable and convenient, as well as concerns about body weight [9-11].

A review was made to summarize recommendations for a complete, healthful breakfast in order to offer guidance on its ideal composition and energy intake [12, 13]. A breakfast should provide between 20 and $25 \%$ of the overall daily energy intake, a range that already takes into account the number of eating occasions in a day (breakfast, lunch, dinner and snacks). A complete breakfast meal should consist of solid and liquid foods, with the purpose of maximizing nutrient intake and reducing or closing nutrient gaps. Foods containing protein, whole grains, fruits and vegetables are specifically recommended. 
Following the holistic approaches described earlier (Sustainability by Design, PrIME and nutritional assessment through the NNPS), Nestlé developed a breakfast solution targeting the segment of schoolchildren aged 4-8 who are breakfast skippers. This product, marketed under the brand Nido GoldenStart $\mathrm{C}$, provides the right combination of essential nutrients required for breakfast and $12 \%$ of the daily energy intake for this age group. Its ingredients are milk, cereals and fruits, delivered in a convenient ready to drink format (beverage carton, $200 \mathrm{ml}$ ). A first generation of the product (powder beverage) was launched in Central America in 2015; a second generation ready to drink version was launched in Ecuador in September 2017.

The various steps followed in the integrated product development process can be recapped as follows:

(1) Consolidation of consumer data: The drivers of preference, expressed in terms of sensory attributes (taste, texture, aroma, colour, etc.) were identified by mining internal consumer insight data.

(2) Definition of the right nutritional targets through the NNPS and a project to define specific nutritional targets for complete breakfasts and "breakfast-on-the-go".

(3) Product and process mastership: Product experts at Research and Development units as well as factories represented the key unit operations required to screen the potential ingredients, design the recipe, define the process parameters and finally manufacture the product.

(4) A large number of possible recipes was reduced to an optimal number. These recipes were produced for consumer trials considering factors such as consumer and sensory data, recipe cost and technical constraints.

(5) Preference mapping: The recipes most liked by parents and children were selected and the reasons for liking or disliking them were explored. In parallel, a monadic sensory profiling was performed with an expert panel to understand the consumers' feedback. A satiation test was also conducted to assess the effect of the product texture on satiation and thus validate the portion size.

(6) Validation test: The superiority of the final recipe was validated using a number of internal methods, among which the Nutritional Foundation (NF) and 60/40+ taste preference criteria can be highlighted. 60/40 is a Nestlé method whereby consumers are asked to state their preference in a blind test between an own product and a competitor or benchmark. A Nestlé product passes the test when more than $60 \%$ of the consumers would prefer it to the competitor product or benchmark. The "+" equals achieving the Nutritional Foundation, meaning all nutritional targets defined by the NNPS are met.

(7) Environmental sustainability evaluation: The eco-design of the product was carried out using the EcodEX tool. On a basis of 100 kilocalories delivered, Nido GoldenStart $(\mathrm{C}$ showed improved environmental performance on all five environmental indicators assessed (climate change, freshwater consumption scarcity, abiotic depletion, ecosystems quality and land use impact on biodiversity) when compared to a reference product. 
Moreover, the integrated development of Nido GoldenStart $\subset$ satisfies a clear consumer need while contributing to the achievement of Nestlé commitments on:

(1) Inspiring people to lead healthier lives: Empower parents to foster healthy behaviours in children (not skipping breakfast).

(2) Offering tastier and healthier choices: Launching more nutritious foods and beverages especially for children. Increase the fibre and grains content in our foods and beverages.

(3) Safeguarding the environment: Assess and optimize the environmental impact of our products.

\section{Conclusions}

This holistic and comprehensive approach, as applied by Nestlé, delivers competitive advantage, leads to the fulfilment of public commitments at company level and drives our contribution as member of the food industry to the attainment of the Sustainable Development Goals. Informed decision-making takes place at the various steps of project execution, risks and opportunities are identified, with early warning brought up in a timely manner.

However, given the breadth of technical areas concerned and what is at stake, the interpretation of data and insights resulting from this way of working can be complex and raise some challenges. The deployment of support tools can only be justified as long as they are used properly and extensively. Deciding which business function will take up the identified actions for improvement, either Research and Development, Operations, Markets or a combination requires alignment of objectives and priorities as well as clear allocation of resources. For a company as large as Nestlé, it is fundamental to progress from starting the conversation and raising awareness on integrated product development, to consistently accomplishing it.

Being proactive and understanding that what matters to consumers entails all aspects of the product-going beyond quality and including sustainability - enriches the product development process and provides consumers with a product they prefer.

\section{References}

1. https://www.nestle.com/asset-library/documents/library/documents/corporate_social_responsibility/ nestle-csv-full-report-2017-en.pdf. (Accessed 04.04.2018).

2. http://www.un.org/ga/search/view_doc.asp?symbol=A/RES/70/1\&Lang=E. (Accessed 30.05. 2017).

3. http://www.selerant.com/eco-design/. (Accessed 30.05.2017).

4. http://www.foodinsight.org/sites/default/files/2016-Food-and-Health-Survey-Report_FINAL1. pdf. (Accessed 30.05.2017). 
5. Vlassopoulos A, Masset G, Rheiner Charles V, Hoover C, Chesneau-Guillemont C, Leroy F, Lehmann U, Spieldenner J, Tee E-S, Gibney M, Drewnowski A, A nutrient profiling system for the (re)formulation of a global food and beverage portfolio, European Journal of Nutrition, Vol. 56, 2016, pp. 1-18.

6. Rampersaud G, Pereira M, Girard B, Adams J, Metzl J, Breakfast habits, nutritional status, body weight, and academic performance in children and adolescents, Journal of the American Dietetic Association, Vol. 105, No. 5, 2005, pp. 743-760.

7. Deshmukh-Taskar P, Nicklas T, O'Neil C, Keast D, Radcliffe J, Cho S, The relationship of breakfast skipping and type of breakfast consumption with nutrient intake and weight status in children and adolescents: The National Health and Nutrition Examination Survey 1999-2006, Journal of the American Dietetic Association, Vol. 110, No. 6, 2010, pp. 869-878.

8. Utter J, Scragg R, Mhurchu C.N, Schaaf D, At-home breakfast consumption among New Zealand children: Associations with body mass index and related nutrition behaviors, Journal of the American Dietetic Association, Vol. 107, No. 4, 2007, pp. 570-576.

9. Sweeney N, Horishita N, The breakfast-eating habits of inner city high school students, Journal of School Nursing, Vol. 21, No. 2, 2005, pp. 100-105.

10. Malinauskas B, Raedeke T, Aeby V, Smith J, Dallas M, Dieting practices, weight perceptions, and body composition: a comparison of normal weight, overweight, and obese college females, Nutrition Journal, Vol. 5, 2006, p. 11.

11. Reddan J, Wahlstrom K, Reicks M, Children's perceived benefits and barriers in relation to eating breakfast in schools with or without Universal School Breakfast, Journal of Nutrition Education and Behaviour, Vol. 34, No. 1, 2002, pp. 47-52.

12. O'Neil C, Byrd-Bredbenner C, Hayes D, Jana L, Klinger S, Stephenson-Martin S, The role of breakfast in health: Definition and criteria for a quality breakfast, Journal of the Academy of Nutrition and Dietetics, Vol. 114, No. 12, Suppl. 3, 2014, pp. S8-S26.

13. Vlassopoulos A, Nutrition criteria to be considered for breakfast drink innovation, Nestlé Research Center, 2017 (Internal note).

Open Access This chapter is licensed under the terms of the Creative Commons Attribution 4.0 International License (http://creativecommons.org/licenses/by/4.0/), which permits use, sharing, adaptation, distribution and reproduction in any medium or format, as long as you give appropriate credit to the original author(s) and the source, provide a link to the Creative Commons license and indicate if changes were made.

The images or other third party material in this chapter are included in the chapter's Creative Commons license, unless indicated otherwise in a credit line to the material. If material is not included in the chapter's Creative Commons license and your intended use is not permitted by statutory regulation or exceeds the permitted use, you will need to obtain permission directly from the copyright holder. 


\title{
The City Performance Tool-How Cities Use LCM Based Decision Support
}

\author{
Jens-Christian Holst, Katrin Müller, Florian-Ansgar Jäger \\ and Klaus Heidinger
}

\begin{abstract}
This paper presents the City Performance Tool (CyPT) approach, a LCA-based methodology for sustainable infrastructure decision support. The work presents an outside-in view about the methodology, details about the underlying LCA models and its implementation into a tool. The results for three different cities will be presented and a general conclusion for the use of LCM-based decision support from a business perspective will be given.
\end{abstract}

\section{Introduction}

All over the world, cities are shaped by profound forces: their population, their technologies and their infrastructures. Even today, these forces collide and urbanization and climate change will spur dramatic changes in metropolitan areas. Cities need to pave the way for constant evolution: digital technologies are becoming increasingly important and urban infrastructures and buildings require a more efficient and sustainable setup.

These changing environments set free a swarm of urban challenges. Developed cities for instance need to focus on cutting carbon emissions, improving efficiency in infrastructure and buildings, stimulating a market shift towards cleaner vehicles and more efficient and environmentally friendly public transportation. At the same time, infrastructure quality in many advanced economies is deteriorating. Looking forward to 2030 , more than $\$ 50$ trillion will need to be invested in infrastructure globally to keep up with GDP and population growth [1].

Cities in emerging markets on the other hand face issues such as power outages and inadequate public transport and roads, which brake on growth and development. Infrastructures cannot be built fast enough to keep pace with economic and

J.-C. Holst $(\bowtie) \cdot$ K. Müller · F.-A. Jäger

Siemens AG, Corporate Technology, 13629 Berlin, Germany

e-mail: jens-christian.holst@siemens.com

K. Heidinger

Siemens AG Austria, Siemensstrasse 90, 1210 Vienna, Austria

(C) The Author(s) 2018

E. Benetto et al. (eds.), Designing Sustainable Technologies,

Products and Policies, https://doi.org/10.1007/978-3-319-66981-6_51 

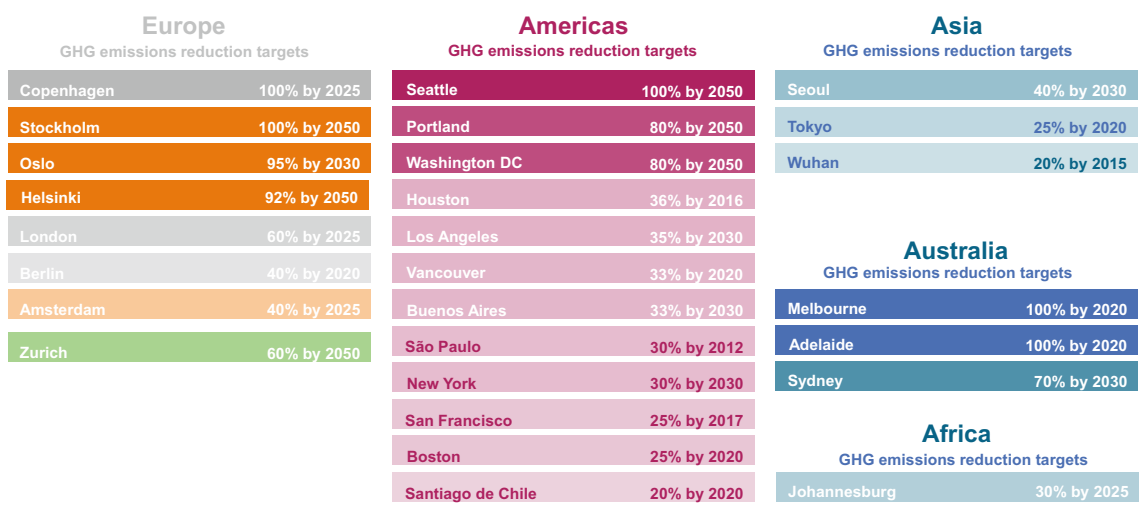

Fig. 1 Environmental reduction targets of different cities worldwide

urban development. In times of constrained budgets city leaders carefully need to identify their infrastructure investments ensuring that their investments address their environmental and economic priorities. Technologies need to be adapted to serve local needs to ensure that the right technologies are applied in the right environments, tailored to the specific characteristics of the individual city (Fig. 1).

To tackle these questions, Siemens has developed an interactive and comprehensive tool-the City Performance Tool (CyPT) [2]. It gives guidance to a city on how to achieve their environmental targets while providing an indication on how each infrastructure-related decision will influence job creation and the infrastructure sector growth. The results of the CyPT approach for a city is shown in Fig. 2. The main categories depicted are $\mathrm{GhG}$ emission reductions in $\mathrm{MtCO}_{2}$ equivalents per year, air quality from $\mathrm{kt} \mathrm{NO}_{\mathrm{x}}$ and kt PM10 per year for time horizons from 2020 through 2050. The darker line represents the city target and the lighter line the reduction results calculated from the parameterized LCA model.

\section{The City Performance Tool-Methodology and Process}

The City Performance Tool is a dynamic simulation tool which studies a series of more than 70 technologies from Building, Transport and Energy Technologies - at different time periods and implementation rates. It covers greenhouse gas emissions from buildings and transport, as well as air pollutants such as particulate matter (PM) and nitrogen oxides $\left(\mathrm{NO}_{\mathrm{x}}\right)$. It also looks at the creation of new local jobs to install, operate and maintain city solutions. In transport, for example, CyPT assesses how a technology would reduce demand (reduce parking search traffic), shift the mode (public transport instead of cars) or improve efficiency (automated trains). The model is based on Life Cycle Assessment (LCA) methodology and builds upon Siemens' technology expertise and global databases of deep vertical 


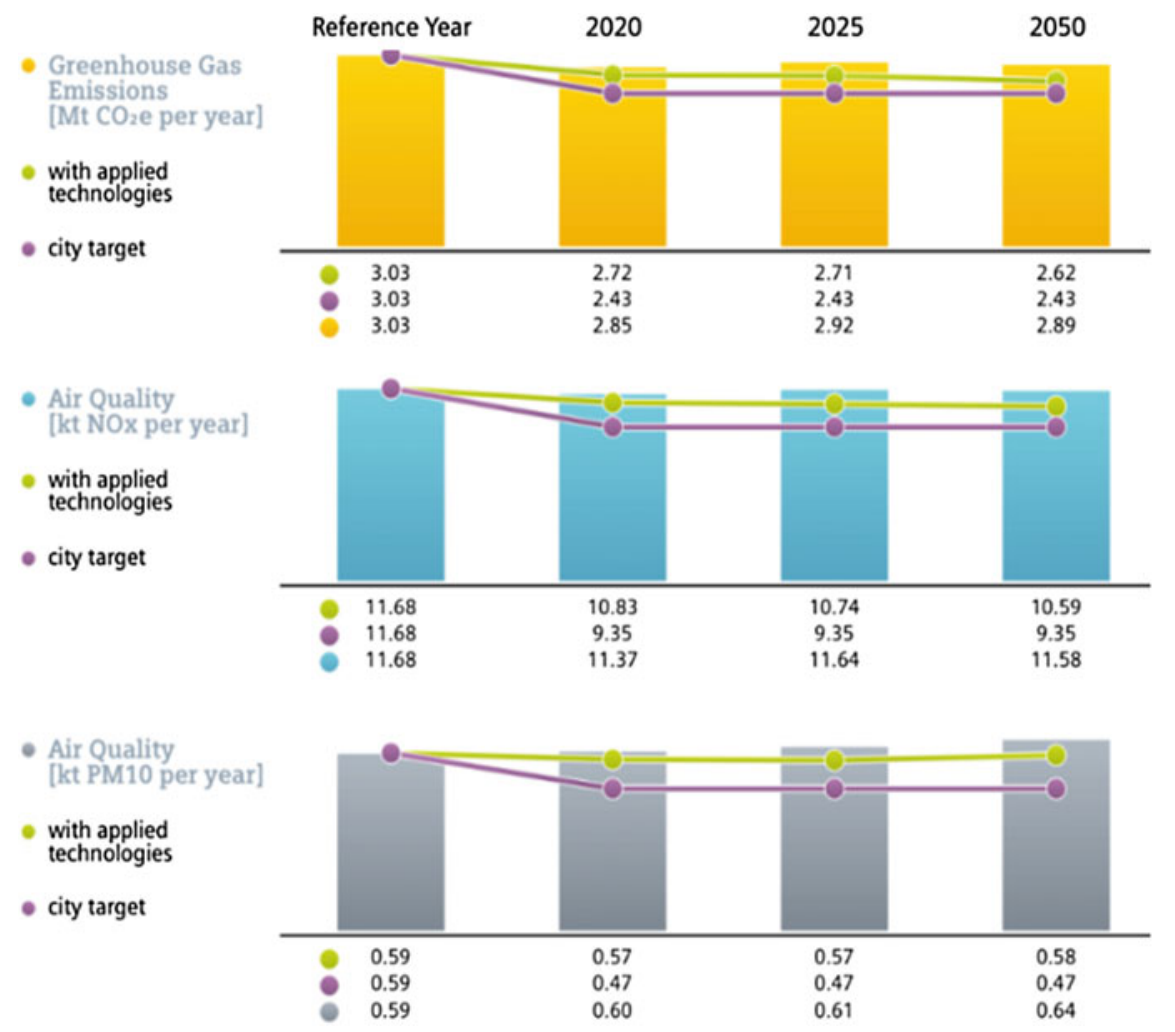

Fig. 2 Environmental reduction using CyPT methodology: results of applying a combination of different technology levers (light) are compared to City targets for the categories GhG-emissions and air quality

process knowledge. It can determine the implementation rate needed for your city to meet its future environmental targets. It can also measure the impacts of a city's strategic plans, and compare traditional methods with state-of-the-art technologies for their benefits and value for money.

The methods and tools are a combination of conventional LCA, consequential LCA, greenhouse gas accounting for cities and futurology. Even though the software and modelling concept such as the functional units are common LCA-based, the model is aligned with the GPC-Protocol [3]. Forecasting elements are included from HBEFA [4] for market and technology development.

In total 70 technology levers from energy system, buildings and transport are included in the LCA parameter model. Thus are 20 electricity generation and 20 heat generation technologies, 10+ building types with its electricity, heat and cooling demand, 10 freight modes; 35 passenger transport modes; 15 road and rail infrastructure elements. To establish a baseline, the model requires data to determine the level of maturity of the infrastructure system and its individual 
Table 1 Lever description of the 70 technology levers in buildings, transportation and energy

\begin{tabular}{|c|c|c|}
\hline Buildings & Transportation & Energy \\
\hline Wall insulation & Tram & Photovoltaic \\
\hline Glazing & Metro & $\begin{array}{l}\text { Wind power generation, } \\
\text { combined cycle gas } \\
\text { turbine }\end{array}$ \\
\hline Building envelop & $\begin{array}{l}\text { Regional train: new vehicles, new line, } \\
\text { reduced headway }\end{array}$ & Combined heat and power \\
\hline Efficient lighting & ATO Automated Train Operation & $\begin{array}{l}\text { Network optimization, } \\
\text { smart grid for monitoring } \\
\text { and automation }\end{array}$ \\
\hline $\begin{array}{l}\text { Demand oriented } \\
\text { lighting }\end{array}$ & Regenerative braking & $\begin{array}{l}\text { Power system automation } \\
\& \text { optimized network }\end{array}$ \\
\hline $\begin{array}{l}\text { Demand controlled } \\
\text { ventilation }\end{array}$ & Bus & Smart metering \\
\hline $\begin{array}{l}\text { Building efficiency } \\
\text { monitoring }\end{array}$ & $\begin{array}{l}\text { Car: CNG, hybrid, plug-in, electric, } \\
\text { hydrogen }\end{array}$ & $\begin{array}{l}\text { On shore power supply in } \\
\text { harbors }\end{array}$ \\
\hline $\begin{array}{l}\text { Efficient motors \& } \\
\text { drives }\end{array}$ & LED street lighting & \\
\hline Room automation & $\begin{array}{l}\text { Smart street lighting, intelligent traffic } \\
\text { light management }\end{array}$ & \\
\hline $\begin{array}{l}\text { HVAC \& } \\
\text { lighting + blinds }\end{array}$ & Intermodal traffic management & \\
\hline $\begin{array}{l}\text { Building automation, } \\
\text { BACS A,B,C, remote } \\
\text { monitoring }\end{array}$ & Low emission zone (truck) & \\
\hline Heat recovery & $\begin{array}{l}\text { Eco driving training, urban bike } \\
\text { sharing, cycling highway, occupancy } \\
\text { dependent } \mathrm{T} \text {, tolling, city tolling }\end{array}$ & \\
\hline \multirow{6}{*}{$\begin{array}{l}\text { Building performance } \\
\text { optimization }\end{array}$} & E-Ticketing & \\
\hline & E-Car sharing & \\
\hline & E-Highway & \\
\hline & e-BRT & \\
\hline & Freight ram & \\
\hline & Freight rail electrification & \\
\hline
\end{tabular}

components. This is necessary to later calculate the impact of levers. Levers are new technology or measures that are introduced to the system. The list of levers is depicted in Table 1.

The common CYPT model determines the variety of process blocks to build any city in a common LCA tool (SimaPro) [5]: in Fig. 3 the underlying LCA model is depicted for the building sector.

The baseline model is using city data publicly available mostly from statistics, studies, and transport or household surveys. Around 300 city-specific data points are used for three main sectors: electricity, heating, and cooling demand for 


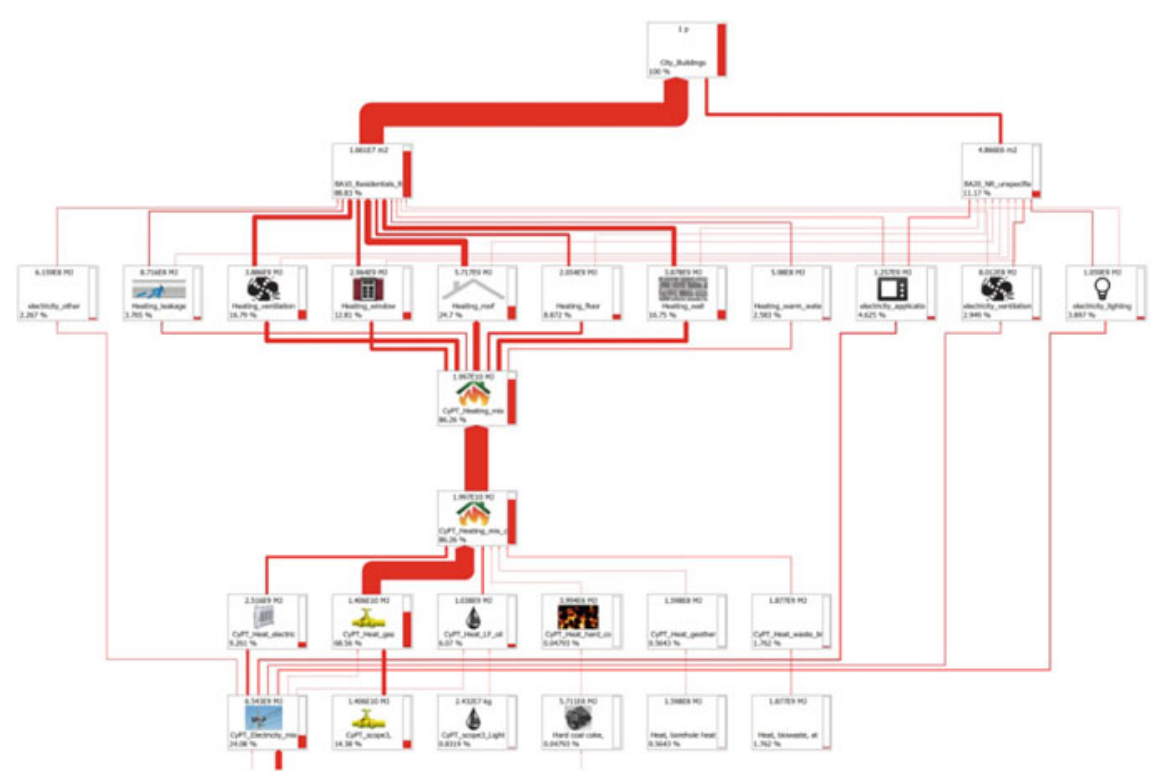

Fig. 3 Subset of lever model: each technology is parameterized by input emissions factors from the SimaPro model

buildings; passenger and freight transport demand; and the transport network. The model baseline of the of a City's lever network includes more than 2000 single processes, and more than 1000 customized parameters. This baseline has three KPIs - PM10 and $\mathrm{NO}_{\mathrm{x}}$ (air quality) and $\mathrm{CO}_{2}$ eq. emissions - which are also projected into the future based on a business-as-usual scenario and your own city's demand projection (if available). This calculation delivers a basic understanding of the root causes of emissions in your city's buildings and transport. A range of impacts can be studied based on a single lever and its implementation rates, comparing options, or combining several levers in one or more sectors [6].

Then mitigation strategies are applied in terms of what-if scenarios, by answering the following questions: Which and how much energy is required to serve the city's demand in transport and buildings? Which infrastructure technologies are available, what is the efficiency of, how can the demand shifted or reduced?

The calculation of the KPI's starts with the determination of demand which is than split into the contributing technologies. This is displayed in Fig. 3. Emissions related to buildings are a sum of heating, electricity etc. and its contributing technologies i.e. heat from gas. The CyPT model is multi-process LCA model, the change of one parameter defines a scenario, describing the transformation of the given infrastructure over time (Fig. 4).

Due to the scenario-based approach of the CyPT a process was developed to ensure the full stakeholder involvement of city representatives. At the beginning of 


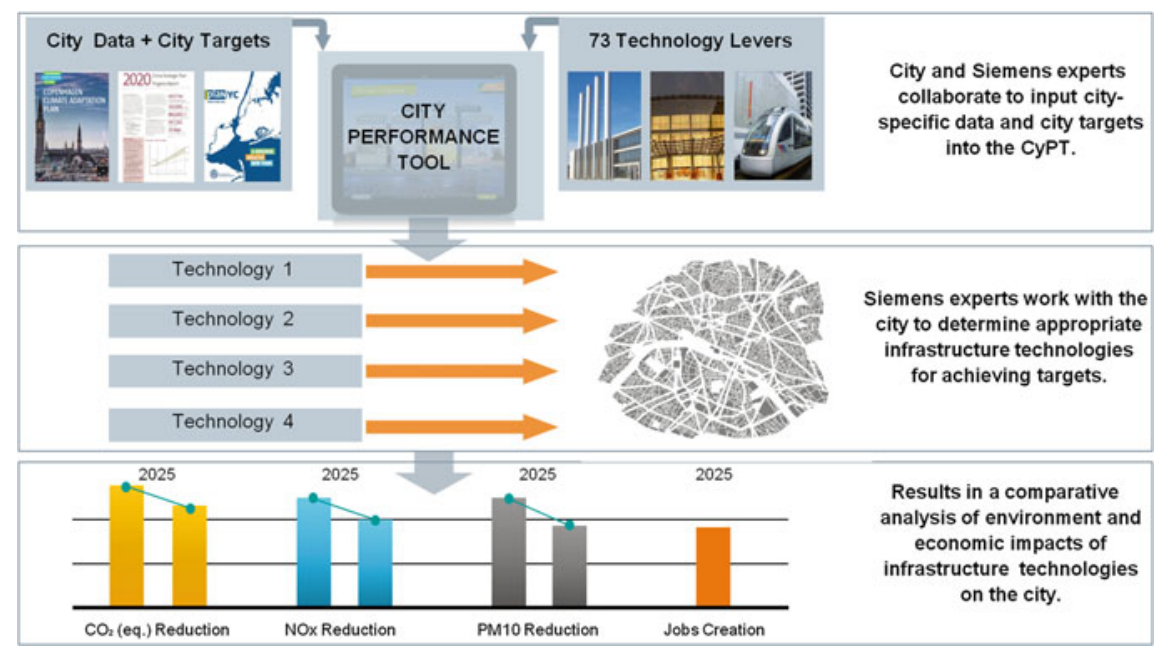

Fig. 4 Process of CyPT application in cities: collaboration starts in data collection using the LCAmodel and the impacts of technologies for KPI's like $\mathrm{CO}_{2}$ emission or air quality reduction

the process, data is collected on your city's energy, buildings and transport infrastructure to customize the CyPT model. Once all the data is collected, the CyPT Manager calculates the environmental baseline for your city based on activities in the three sectors. Comparing the results with your own targets it identifies future needs and improvement areas. The CyPT Manager also calculates the city-specific impacts of individual technologies. Levers can be pre-selected based on results, and city specific plans and policy can also be considered valuable mix of solutions.

A specially developed CyPT app visualizes the effects of individual levers on a city, its buildings and its transport system [7].

\section{Inside-Out View-Results from Vienna, Copenhagen and Minneapolis}

To understand the outside-in view we will present and discuss results of the CyPT projects and the resulting infrastructure reports from three different cities: Vienna [8], Copenhagen [9] and Minneapolis [10].

Vienna has set 2025 as the ambitious target year for the city to implement major infrastructure technologies in order to meet its environmental and economic targets. This study quantifies the benefits of over thirty building, energy and transport technologies and makes recommendations of which infrastructure solutions the city should be prioritizing in the run up to 2025, see Fig. 5. This study tested some of the technologies in Vienna's climate action programs named KLiP I and II. It was 


\begin{tabular}{|c|}
\hline $\begin{array}{l}\text { Vienna First } \\
\text { Technologies implemented }\end{array}$ \\
\hline Increased CHP contribution \\
\hline Residential wall insulation \\
\hline Residential doublentriple glazing \\
\hline Commercial wall insulation \\
\hline Commercial doubleftriple glazing \\
\hline LED street lighting \\
\hline Metro ATO \\
\hline CNG cars \\
\hline Hybrid electric vehicles \\
\hline Intermodal trafic management \\
\hline
\end{tabular}

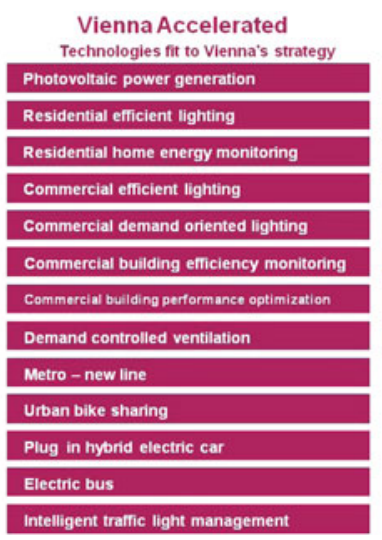

Vienna Experimental

Additional technologies to be implemented Residential home automation

Commercial heat recovery

Hybrid electric bus

Train - ATO

Electric cars

Electric taxis

Demand oriented street lighting

Electric car sharing

Fig. 5 Lever mix used in the Vienna CyPT project from already implemented to experimental technologies

found that solutions are cost effective in reducing $\mathrm{CO}_{2}$ eq. levels and improving air quality, the city will need to implement more of these intensive carbon reduction technologies if it is to meet its 2030 targets.

An investment of $€ 8$ bn over the next decade is needed to implement a set of technologies that can reduce $\mathrm{CO}_{2}$ eq. emissions in Vienna by $9 \mathrm{Mt}$ and add 85,000 full time equivalent jobs to the local economy. In this model, the city is currently achieving its reductions predominantly through building and energy technologies that are more expensive and do not improve air quality as effectively. Vienna has some untapped cost-effective technology opportunities to meet its $\mathrm{CO}_{2}$ mitigation targets. In the energy sector, the city should continue its implementation of combined heat and power (Fig. 6).

In the transport sector, the city should invest in new cutting-edge technologies such as intermodal traffic management applications, electric car and electric taxi solutions. These are win-win cost effective solutions that tackle $\mathrm{CO}_{2}$ emissions, improve air quality and increase local jobs.

Minneapolis and its development organization Xcel has set a "80 by 50" target, meaning $80 \%$ reduction of GhG emissions by 2050 [8]. The outcome of CyPT study shows that its utilities need to use $65 \%$ clean energy sources as a base of their energy mix (Fig. 7).

But even with this step, Xcel will have to continue greening its electricity by implementing 40 buildings and transportation technologies and adopted at their highest implementation rates by 2050 to ensure that targets are met. Our results show that, if the electricity mix gets significantly cleaner, the top performing technologies for reducing carbon emissions include (1) electrifying both passenger and freight road transport and (2) improving energy efficiency in buildings, particularly in commercial and government buildings. The technology mix and its resulting improvements in air quality are depicted in Fig. 8. Additional benefits could be realized from public transit, if more people could be attracted to use it. 

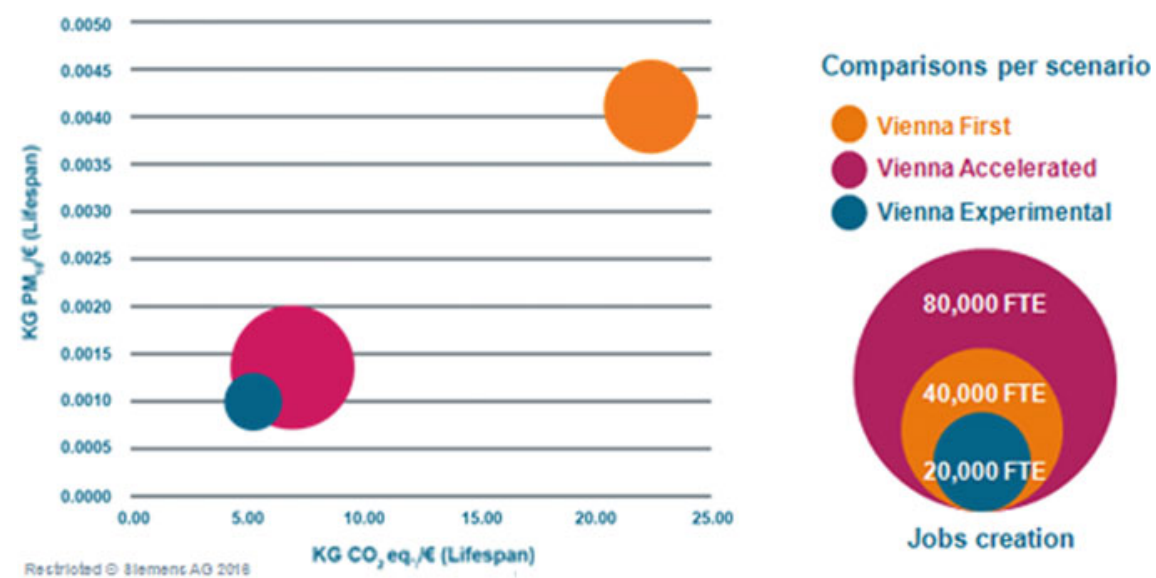

Fig. 6 Cost effectiveness comparison: the larger the bubble the more jobs the technology will induce. Bubbles on the top right of the chart, reduce the larger amount of greenhouse gases and PM10 levels per unit of investment Technologies such as plug-in hybrid electric cars that improve air quality and reduce $\mathrm{CO}_{2}$ most effectively

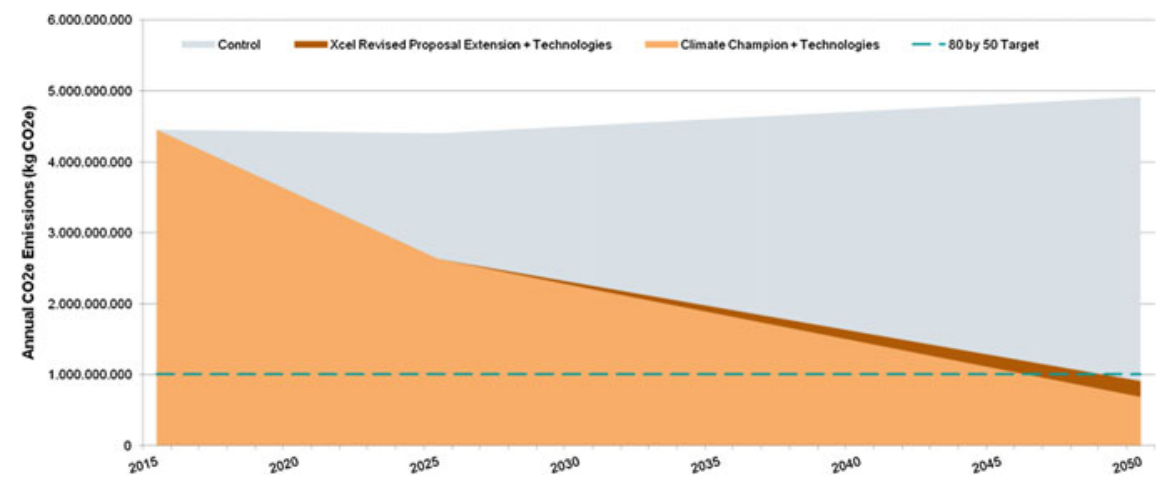

Fig. 7 Resulting emission reduction $\left(\mathrm{CO}_{2}\right.$ eq.) applying a $65 \%$ clean energy mix

Copenhagen has been and continues to lead cities on climate policy globally. It ranked top of Siemens' European Green City Index in 2009 [11], and it is making great strides meeting its 2025 target for carbon neutrality. Siemens has used the CyPT and found that the City of Copenhagen is reducing emissions from nearly all of the sources where it has significant control in energy and transport.

Copenhagen is decoupling its $\mathrm{CO}_{2}$ eq. emissions so that despite a growing population and increasing building floor-space, carbon emissions are expected to decrease over the next 10 years. This is predominantly derived from greener national electricity and local heating mixes. Using our accounting standard that captures direct and indirect emissions, our model quantifies these savings as high as 


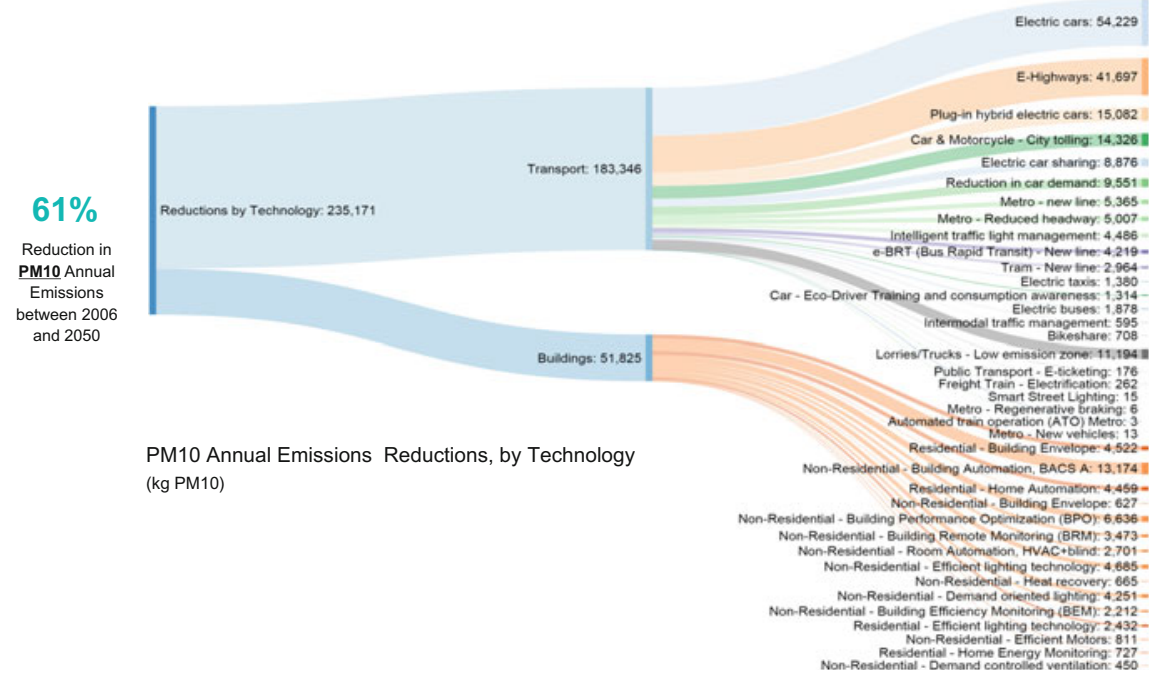

Fig. 8 The CyPT for Minneapolis shows which technology has the greatest air quality improvement potential and its reduction values

$12 \%$ of current annual emissions. These are savings achieved from the expansion of wind power and biomass for combined heat and power in the 2025 Climate Plan. Maintaining its global leadership position will require delivering further building upgrades within its own public stock and incentivizing private building owners to take action to improve the efficiency of their buildings. The city will also have to stimulate a market shift towards cleaner vehicles and a modal shift towards low carbon public transportation. The report indicates that intelligent administrative inner-city infrastructure investments in energy or mass transit must be accompanied by effective incentives for the private sector, as well as a good national framework to promote alternative mobility and cost-effective renewable energy (Fig. 9).

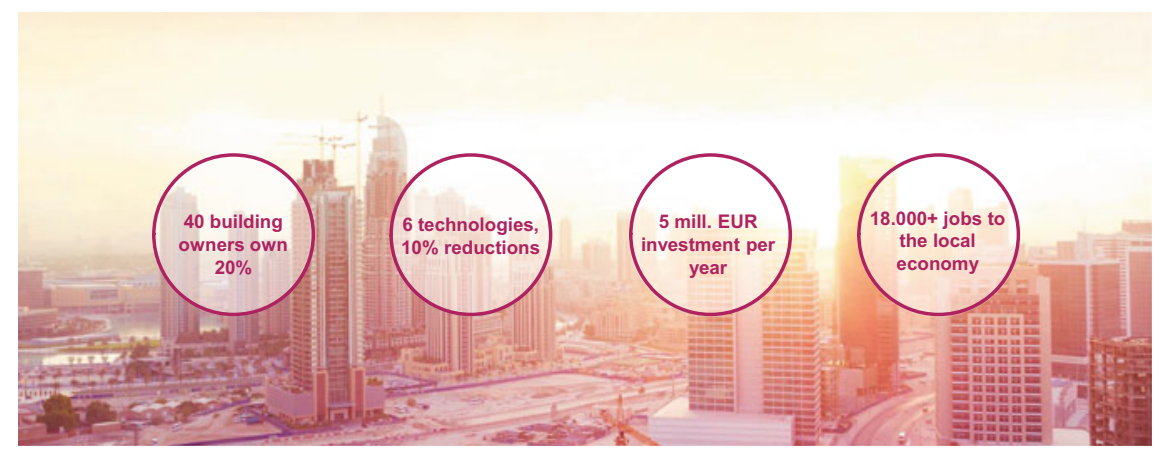

Fig. 9 Major CyPT results for Copenhagen 


\begin{tabular}{|l|l|l|l|}
\hline Lever & \% Reductions CO.e & $\mathrm{kg}$ CO, eq savings / CAPEX & Total jobs \\
\hline Bullding Effiliclency Monitoring (BEM) & $0.7 \%$ & 1.3 & 900 \\
\hline Bullding Performance Optimization (BPO) & $2 \%$ & 5 & 400 \\
\hline Heat recovery & $1.3 \%$ & 0.1 & 2,600 \\
\hline Bullding Automation, BACS A & $4 \%$ & 0.1 & 5,400 \\
\hline Room Automation, HVAC+bllind & $1 \%$ & 0 & 8,000 \\
\hline Bullding Remote Monitoring (BRM) & $1 \%$ & 2 & 1,400 \\
\hline
\end{tabular}

Fig. 10 Shortlisted smart technologies providing the largest savings

For example, buildings are responsible for around $80 \%$ of Copenhagen's greenhouse emissions - and almost a third of the commercial building stock is managed by just 40 owners. If these owners have an incentive to invest in six cutting-edge building technologies selected by the CyPT, the city could save $10 \%$ of its emissions and create more 18,000 jobs over the next 10 years.

As buildings become more sustainable, transportation begins to have a bigger contribution to emissions. The report shows that, by 2050, transportation will account for $27 \%$ of Copenhagen's emissions, as opposed to $20 \%$ today. The big surprise in the Siemens "Copenhagen's Big Incentive" report is that the most effective technology investments are outside of the city's budgeting program.

It is about "national investments in wind, private investments in building retrofitting, and household and business investment in alternative cars." In figures: $95 \%$ of the estimated three billion euros needed to save an additional $26 \%$ of city-wide emissions over the next 10 years are outside of the city budget. This indicates the investment strategy of Copenhagen must be aligned to the state or governmental plans (Fig. 10).

\section{Conclusions}

Modern cities constantly need to balance long-term targets such as reducing greenhouse gas emissions with shorter term aims such as improving air quality and creating jobs. All too often, lack of public support means long-term objectives are sacrificed for more immediate needs. The cities in the world have different focuses on environmental, economic or social aspects/KPIs. These differ by region, in scope (Carbon Accounting Scope 1-3), in time (in terms of target setting, short term for air pollutants, long term for GHG-emissions) and at last by infrastructure vertical. The LCA based decision support approach CyPT is presented in this paper. A parameterized LCA model is used to calculate the $\mathrm{GhG}$ and air quality emissions from 70 technologies applied in cities. CyPT takes on the challenges of this balancing act by helping decision makers to recognise and select technologies that offer win-win solutions. This is shown in the example of Minneapolis and the air 
quality improvement potentials and the underlying technology mix. CyPT highlights the benefits from key performance indicators to enable city leaders to deliver long-term advantages strongly linked to short-term gains. Cities can maximise CyPT results by identifying the most cost-effective solutions for improving air quality and reducing greenhouse gas emissions. By studying capital and operational investments across the lifespan of each technology, CyPT can report on kilograms of $\mathrm{CO}_{2}$ eq. as well as PM10 and $\mathrm{NO}_{\mathrm{x}}$ levels for every euro spent. This was shown in the example of Vienna, where untapped cost-effective technology opportunities show how to meet its $\mathrm{CO}_{2}$ mitigation targets. The CyPT helps to optimize investment strategies for your city's long-term needs. This was shown at the example of Copenhagen, where almost all of the technology investments must come from sources outside of the city budget.

\section{References}

1. Economist.com (2014),. Investing in infrastructure: The trillion dollar ga. Available at: http:// www.economist.com/news/leaders/21599358-how-get-more-wolrds-savings-pay-new-roadsairports-and-electricity.

2. https://www.siemens.com/global/en/home/company/topic-areas/intelligent-infrastructure/ cities-on-the-move.html??stc=wwcg 106121 .

3. Fong W, Sotos M, Doust M, et al., The Global Protocol for Community-Scale Greenhouse Gas Emission Inventories (GPC), 1st Ed., Greensouse Gas Protocol, 2014. http://ghgprotocol. org/greenhouse-gas-protocol-accounting-reporting-standard-cities, (Accessed 18.04.2017).

4. Keller M, Wuethrich P, Ickert L, Schmied M, Stutzer B, et al., Handbook Emission Factors for Road Transport, 3.2 Ed., INFRAS AG, 25.7.2014.

5. https://simapro.com/licences/\#/business.

6. https://www.siemens.com/de/de/home/unternehmen/themenfelder/intelligente-infrastruktur/ city-performance-tool.html.

7. http://www.dgterritorio.pt/2015-4-23-10-20-42-78__SavvasVerdisUrbanAnalyticsLisbon 2015. pdf.

8. https://www.siemens.com/content/dam/internet/siemens-com/global/company/topic-areas/ intelligent-infrastructuree/resilience/report/summary-vienna-report-oct-2014.pdf.

9. https://www.siemens.com/content/dam/internet/siemens-com/global/company/topic-areas/ intelligent-infrastructuree/resilience/report/summary-copenhagen-cypt-report-oct-2015.pdf.

10. https://www.siemens.com/content/dam/internet/siemens-com/global/company/topic-areas/ intelligent-infrastructuree/resilience/report/summary-minneapolis-cypt-report-dec-2015.pdf.

11. http://www.siemens.com/entry/cc/features/greencityindex_international/all/de/pdf/gci_report_ summary.pdf. 
Open Access This chapter is licensed under the terms of the Creative Commons Attribution 4.0 International License (http://creativecommons.org/licenses/by/4.0/), which permits use, sharing, adaptation, distribution and reproduction in any medium or format, as long as you give appropriate credit to the original author(s) and the source, provide a link to the Creative Commons license and indicate if changes were made.

The images or other third party material in this chapter are included in the chapter's Creative Commons license, unless indicated otherwise in a credit line to the material. If material is not included in the chapter's Creative Commons license and your intended use is not permitted by statutory regulation or exceeds the permitted use, you will need to obtain permission directly from the copyright holder.

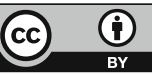




\title{
Sustainability of Bio-based Products: Linking Life Cycle Thinking with Standards, Certification and Labelling Schemes
}

\author{
Mauro Cordella and Renata Kaps
}

\begin{abstract}
This session gathered contributions showing how Life Cycle Thinking can feed in the development of sustainability criteria, the certification and, ultimately, the policy making for bio-based products and processes. The session was composed of five presentations related to methodological approaches, case studies and real life examples, which were followed by a panel discussion. The addressed subjects covered the sustainability assessment of bio-based plastics, marine biotechnology processes, bio-based lubricants and forestry sector, and the assessment of the water scarcity. Key issues which emerged from the discussion include: the methodological difficulties in comparing bio-based and conventional products; the availability of data; the need to keep research focused on practical applications and increase the interest of industry.
\end{abstract}

\section{Introduction}

During the LCM 2017 conference, the Circular Economy \& Industrial Leadership unit of the Joint Research Centre chaired a session on 'Sustainability of bio-based products: linking life cycle thinking with standards, certification and labelling schemes'.

This session aimed at gathering contributions showing how Life Cycle Thinking can be used to feed into the development of sustainability criteria, the certification and ultimately the policy making for bio-based products and processes, as for instance lubricants, cosmetics, plastics, construction materials.

Products and processes using bio-based materials play an important role in the economy and, consequently, in the European policy. Bio-based materials may offer potential benefits in terms of reducing fossil fuel depletion and emission of greenhouse gases and creating employment opportunities in rural areas, among others.

M. Cordella $(\bowtie) \cdot$ R. Kaps

Circular Economy and Industrial Leadership Unit, European Commission,

Joint Research Centre, Seville, Spain

e-mail: mauro.cordella@ec.europa.eu

(C) The Author(s) 2018

E. Benetto et al. (eds.), Designing Sustainable Technologies,

Products and Policies, https://doi.org/10.1007/978-3-319-66981-6_52 
However, their sustainability depends on the geographical and technical characteristics of each biomass-to-product value chain, which may result in trade-offs between different aspects (e.g. land use, water consumption, deforestation).

Efforts to develop multi-disciplinary approaches to assess the sustainability of bio-based products have yielded a broad range of case studies. Nevertheless, no commonly agreed approaches exist and the information available in the literature seems to have only limited practical application.

\section{Session Wrap-up}

The session was composed of five contributions and a closing panel discussion concerning the session's theme. Above 70 participants attended this session. Results and discussion are summarised below.

\section{$2.1 \quad$ Integrating Life Cycle Assessment (LCA) and Eco-design Strategies for a Sustainable Production of Bio-based Plastics}

This presentation was given by Mr. Venkateshwaran Venkatachalam from the Institute for Bioplastics and Biocomposites of University of Applied Sciences and Arts of Hannover (Germany).

The authors described an approach for addressing the development of sustainable bio-plastic products since their early design phase [1]. The approach aims to integrate LCA considerations in the definition of eco-design strategies of a product, with the ambition to cover technical, economic, ergonomic, market, and environmental aspects along the entire production chain of a product system.

A personal computer's mouse was taken as a case study to show how the approach could work in practice. Production and supply of materials were identified as the life cycle hot spots. Criteria required for the selection of bio-polymers were proposed based on technical and environmental aspects. Finally, further development needs and challenges of this approach were presented. These in particular refer to the comparison of bio- versus conventional plastic products and the integration of consequential LCA to better understand market mechanisms.

The presenter was asked about the future perspectives of their research and which eco-design requirements should be set to ensure the production and supply of sustainable materials, being these the main hot-spots from a life cycle point of view. It was answered that future perspectives may embrace biodegradability and composting of bio-plastics and that the identification of eco-design strategies has so far focused on processes and not on the applications. 


\subsection{Sustainability Assessment of Blue Biotechnology Processes: Addressing Environmental, Social and Economic Dimensions}

This presentation was given by Ms. Maria Teresa Moreira from the Department of Chemical Engineering of the University of Santiago de Compostela (Spain).

The authors showed the results of their research on environmental, social and economic assessment of a marine biotechnology process for the production of the red carotenoid astaxanthin by the green microalga Haematococcus pluvialis [2].

This is an interesting and novel case study, since only few research projects have dealt so far with the assessment of bioactive compounds and pharmaceutical ingredients, mainly due to the lack of information from commercial-scale facilities. In this study, process data for the compilation of the life cycle inventory was collected from real facilities at lab, semi-pilot and pilot scale.

Consumption of electricity during the algal cultivation was spotted as the largest contribution to the environmental impacts. However, a significant improvement was observed in the scale-up of the process. A socio-economic evaluation completed the environmental assessment, identifying the main strengths of the process from a holistic perspective.

The presenter was asked how the social assessment was carried-out and also if a comparison with the conventional chemical process was conducted. It was explained that the social assessment was based on the reply of two SMEs to a survey sent in the course of the study. The assessed biotechnology process was compared with a conventional one and was found to have lower environmental impacts.

\subsection{Addressing the Sustainability of Lubricants from an LCA Perspective}

This presentation was given by Ms. Candela Vidal-Abarca Garrido from the Circular Economy and Industrial Leadership Unit of the Joint Research Centre.

The authors described their work about the revision of the EU Ecolabel for lubricants, a voluntary label targeting the best lubricants available on the market in terms of their environmental performance throughout the life cycle [3].

The existing award criteria focus on renewability of raw materials, aquatic toxicity, biodegradability and bio-accumulation of the ingredients. The label is currently suitable only for bio-based lubricants and considered the main reference to assess their sustainability.

A review of twelve LCA studies on lubricants was carried-out to identify hot-spots and improvement areas. Although impacts during use and disposal stages were found critical, most of the reviewed LCAs are cradle-to-gate studies, which calls for a more comprehensive assessment of the full life cycle. Outcomes of the 
LCA review did not support the environmental preference of bio-based lubricants. Based on this, the approach suggested for this EU Ecolabel revision is to focus on 'loss lubricants' (i.e. released during use phase) independently from their sourcing. Toxicity and biodegradability are considered the main aspects to be addressed.

Some clarifications were asked about how the LCA review was conducted and which differences were found between various types of lubricants. Trade-offs were identified between conventional and bio-based lubricants. For the latter ones, environmental concerns are significantly associated with the agricultural stage, impacts of which could be mitigated through third-party certified sourcing of sustainable bio-based materials. Additionally, the life cycle performance was found to depend on the application. For instance, conventional and/or synthetic lubricants tend to have longer lifetime, which can be advantageous in some cases. However, some synthetic (e.g. PAGs, PAOs, esters) and bio-based lubricants could have better performance with respect to toxicity and biodegradability than the conventional mineral ones.

\subsection{Recommended Water Scarcity Footprint Method AWARE: Learning from 11 Case Studies}

This presentation was given by Ms. Anne-Marie Boulay representing LIRIDE (University of Sherbrooke, Canada) and CIRAIG (Polytechnique of Montreal, Canada).

The authors described the testing phase of AWARE, a consensus-based indicator developed by the WULCA working group of the UNEP-SETAC Life Cycle Initiative in order to assess impacts from water consumption at the midpoint level. This method can be an important milestone for harmonising the assessment of products with respect to water scarcity [4].

The methodology, candidate for inclusion in the European Commission's Environmental Footprint (EF) guide, has been applied to eleven case studies to gain insights on method, associated results and reference values. The selected case studies were: an Italian beer, a biofuel, a bio-based plastic, cement, sweet beverages, a flow-regulator, an average basket of food products, a burger, a Volkswagen car, a bowl of rice, the EU total consumption.

No major problems were found although some challenges were encountered for interpreting country level data and testing the sensitivity of modelling choices (e.g. cut-offs and water demand for ecosystems). All in all, the results are considered to build trust in this and contributing to its wider acceptance.

The presenter was asked to provide further arguments supporting the choice of AWARE since other methods can also be used to yield similar outcomes. It was clarified that AWARE is the result of a consensus process aiming to develop a widely agreed method. 


\subsection{Sustainability Assessment of the Portuguese Forest Sector}

This poster spotlight was provided by Ms. Ana Dias from the Centre for Environmental and Marine Studies (CESAM) and the Department of Environment and Planning of University of Aveiro (Portugal).

Authors of this contribution introduced the audience to Sustain For, a project aiming at assessing the possible effects of a transition to a bio-economy in Portugal [5].

The project will select environmental, economic and social indicators to evaluate the sustainability of the forest sector and apply them to evaluate current impacts of two Portuguese forest sectors (eucalypt and maritime pine); and will also identify opportunities for improving the sustainability of these sectors.

The full value chain of a representative basket of products (paper, solid wood products, fuels and bio-based chemical products) will be assessed. Trade-offs between sustainability pillars, sectors, subsectors and impacts will be identified.

The authors wisely point out that a transition to a bio-economy, despite its opportunities and advantages, could reduce the availability of wood resources for other applications. This is already a reality in some countries as Portugal. Different management and policy strategies for using wood resources more efficiently should be adopted.

\subsection{Panel Discussion}

A panel discussion was opened after the five presentations. The first aspects discussed related to the comparison of bio-based and conventional products. It is clear that trade-offs exist. Raw materials and land use were pointed out by several presenters as hot-spots, which need to be carefully evaluated in the case of bio-based products. A consistent assessment should moreover include the end of life stage, which is quite often neglected.

A second point of discussion was related to the decision making process and what should be done to achieve practical effects. The main barrier seems to be the lack of LCA data. Improving data sharing could have positive effects. Another driver could be increasing the interest of industry. A presenter reported that environmental assessments conducted by industry are often driven by external factors rather than by real company interests. This calls for enhancing the interaction between research and industrial sectors, also to make clearer which are the data needs and the added value of outcomes. Another presenter raised the attention also to the importance of consumer behaviour and perception. 


\section{Conclusions}

The session confirmed that there are still data and methodological gaps which need to be filled to get a comprehensive understanding of the life cycle impacts of bio-based materials. Challenging aspects include in particular the multiple assessment of alternative sources and uses of biomass feedstock, also in comparison with conventional materials, and the comprehensive understanding of the consequences at macro-level. Moreover, it seems necessary to incentivise the transition from theoretical assessments to more practical applications.

Although further developments are still needed, the contributions presented, which ranged from methodological approaches to case studies and real life examples, can hopefully contribute to make progresses in the assessment of bio-based products and to support the related decision making processes.

\section{References}

1. Venkatachala V, Spierling S, Endres H-J, Integrating Life Cycle Assessment (LCA) and Eco-design strategies for a sustainable production of bio-based plastics, Proceedings of the LCM 2017 conference, Luxembourg, 2017.

2. Pérez-López P, Feijoo G, Moreira M-T, Sustainability assessment of blue biotechnology processes: addressing environmental, social and economic dimensions, Proceedings of the LCM 2017 conference, Luxembourg, 2017.

3. Vidal-Abarca Garrido C, Kaps R, Cordella M, Wolf O, Addressing the sustainability of lubricants from an LCA perspective, Proceedings of the LCM 2017 conference, Luxembourg, 2017.

4. Boulay A-M, Bayart J-B, Beley J-J, Berger M, Caldeira C, Frischknecht R, Kabe Y-H, Manzardo A, Tirado P, Pfister S, Puerto M, Sala S, Schenker U, Recommended water scarcity footprint method AWARE: learning from 11 case studies, Proceedings of the LCM 2017 conference, Luxembourg, 2017.

5. Dias A, Quinteiro P, Costa T, Arroja L, Castanheira E, Garcia R, Marques P, Malça J, Freire F, Sustainability assessment of the Portuguese forest sector, Proceedings of the LCM 2017 conference, Luxembourg, 2017.

Open Access This chapter is licensed under the terms of the Creative Commons Attribution 4.0 International License (http://creativecommons.org/licenses/by/4.0/), which permits use, sharing, adaptation, distribution and reproduction in any medium or format, as long as you give appropriate credit to the original author(s) and the source, provide a link to the Creative Commons license and indicate if changes were made.

The images or other third party material in this chapter are included in the chapter's Creative Commons license, unless indicated otherwise in a credit line to the material. If material is not included in the chapter's Creative Commons license and your intended use is not permitted by statutory regulation or exceeds the permitted use, you will need to obtain permission directly from the copyright holder.

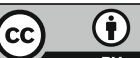




\title{
Sustainability Assessment of Blue Biotechnology Processes: Addressing Environmental, Social and Economic Dimensions
}

\author{
Paula Pérez-López, Gumersindo Feijoo and María Teresa Moreira
}

\begin{abstract}
The biotechnological development has traditionally focused on the compliance with regulatory demands rather than optimising the processes or analysing their sustainability. This work proposes the combination of available tools for the comprehensive sustainability assessment of a blue biotechnology process based on the cultivation of the microalgae Haematococcus pluvialis. The work aims to include environmental, economic and social dimensions to measure the sustainability of the production of a carotenoid with potential applications in food, nutraceutical, cosmetics and eventually pharmaceutical industries. Electricity for cultivation was identified as the major contributor to the environmental impacts, which depended significantly on the production scale. Social benefits were mainly related to workers and consumers, while the economic assessment suggested a profitable process with a relatively short period to recover the initial investment.
\end{abstract}

\section{Introduction}

Biotechnology is a wide industrial sector that ranges from high value, low volume products such as pharmaceuticals to low value commodities such as biofuels. The main effort to date has focused on implementing processes effectively to meet the regulatory demands more than optimising the operations or analysing the sustainability, especially in the case of fine chemicals [1]. Nevertheless, there have been several attempts to develop methodologies for the measurement of bioprocess sustainability in the last decade [2, 3].

\footnotetext{
P. Pérez-López $(\bowtie) \cdot$ G. Feijoo · M. T. Moreira

Department of Chemical Engineering, Institute of Technology,

University of Santiago de Compostela, 15782 Santiago de Compostela, Spain

e-mail: paula.perez_lopez@mines-paristech.fr

P. Pérez-López

Centre Observation, Impacts, Energie (O.I.E.), MINES ParisTech,

PSL Research University, 1 Rue Claude Daunesse CS 10207, 06904

Sophia Antipolis Cedex, France

(C) The Author(s) 2018

E. Benetto et al. (eds.), Designing Sustainable Technologies,

Products and Policies, https://doi.org/10.1007/978-3-319-66981-6_53
} 
Life Cycle Assessment (LCA) is one of the available methodologies to measure this sustainability holistically, although few LCAs applied to bioactive compounds and pharmaceutical ingredients are found in literature [2-4]. In the case of blue (i.e. marine) biotechnology, which involves the exploration and exploitation of new products from marine organisms, the LCA studies conducted to date mainly analysed the cultivation and extraction of fractions from microalgae and seaweed. They focus on relatively low value commodities, namely biofuels [5]. Most of the work dealt with the production of biodiesel by transesterification, although other bioenergy sources (bioethanol, biogas ...) have also been evaluated. Few examples of LCA studies addressing the production of high-value chemicals and bioactive compounds from marine sources are available, due to the lack of information from commercial-scale facilities $[4,5]$. The studies generally rely on extrapolations and simulation models rather than field data from operating systems [5].

According to the principles of sustainable development, measuring sustainability for supply chain decision-making requires the integration of social and economic dimensions together with environmental aspects [6]. To this end, an integrated framework for Life Cycle Sustainability Assessment (LCSA) is proposed. It combines conventional LCA with social LCA (SLCA) and Life Cycle Costing (LCC) [7], based on UNEP/SETAC guidelines [8] for SLCA and [9, 10] for LCC.

In this work, the sustainability of a blue biotechnology process, namely the production of the red carotenoid astaxanthin by the green microalga Haematococcus pluvialis, is evaluated. Astaxanthin is a high-value red carotenoid with numerous applications in food and feed industries, nutraceuticals and cosmetics [11]. Given its antioxidant and anti-inflammatory properties, astaxanthin is suggested to play a beneficial role in human health for the protection of skin cells against UV-light photo-oxidation, slowing of age-related diseases and even control of carcinogenic processes $[11,12]$. Astaxanthin can be obtained from microalgae in a two-stage process: in the first stage the biomass is cultivated in a photo-bioreactor (PBR) under favourable growth conditions whereas in the second stage, adverse conditions are promoted to induce the accumulation of astaxanthin within the biomass [13].

A comprehensive sustainability assessment is here presented, based on process data from real facilities for algae cultivation and astaxanthin extraction at lab, semi-pilot and pilot scale. An environmental LCA jointly with a socio-economic assessment was conducted following SLCA existing guidelines and a Cost-Benefit Analysis (CBA) approach to complete the evaluation by taking the three dimensions of sustainability into account.

\section{Methodology}

The three dimensions of sustainability (environmental, social and economic) are evaluated in this work according to a cradle-to-gate perspective based on the Life Cycle Thinking principles. The environmental LCA followed ISO14040 
standards [14]. A socio-economic assessment was conducted following UNEP/ SETAC SLCA guidelines and CBA approach to complete the assessment [7-9].

\subsection{Environmental LCA}

The environmental performance of microalgal astaxanthin production was evaluated by conducting an attributional LCA with a cradle-to-gate approach. The study included the environmental burdens associated with the production of the different inputs to the system, the microalgae cultivation, harvesting and final carotenoid extraction. This process competes with two alternative routes that consist in: (i) the synthetic production from petrochemical feedstock and (ii) the fermentation process by the yeast Phaffia rhodozyma [15]. Although synthetic astaxanthin dominates the current market and has a more competitive price, it is not approved for human consumption as a nutraceutical or pharmaceutical. Recent studies suggest that the antioxidant activity of natural astaxanthin is significantly higher [11]. Thus, both the microalgae- and the yeast-based routes are suitable for its production, although microalgae process tends to have a higher yield.

The goal of the LCA was the identification of the main stages contributing to the environmental impacts of a nutraceutical oleoresin containing $10 \%$ astaxanthin while taking the influence of scale-up into account. Three real facilities were analysed, including a lab-scale system (15 L tubular airlift PBR), a semi-pilot system (80 L annular PBR) and a pilot system (1000 L sequential airlift PBRs). The production system was divided into 5 stages depicted in Fig. 1: (i) cleaning and sterilisation, (ii) preparation of the inoculum and culture medium, (iii) cultivation, (iv) harvesting and (v) extraction. The functional unit (FU) was defined as $1 \mathrm{~kg}$ astaxanthin, considered as a realistic production level for the pilot-scale process. Although this FU was not a realistic value for the lab process (which produces approximately $1 \mathrm{~g}$ per batch), the results are referred to the same unit as the pilot systems to facilitate the comparison of the environmental profiles.

The Life Cycle Inventory (LCI) data for the foreground system consisted of average data obtained by on-site measurements. Water emissions were calculated assuming that the remaining nutrients in the culture medium after algae growth were directly discharged to water. Data for the background inventory were taken from Ecoinvent v2.2 [16]. A system expansion approach was considered to include the potential use of residual algal biomass as fertiliser. The biomass content of nitrogen and phosphorous were calculated according to [11].

The Life Cycle Impact Assessment included the classification and characterisation stages, which were conducted according to CML 2 baseline 2001 V2.05 impact categories [17]. Environmental indicators of ten impact categories were evaluated: abiotic depletion potential (ADP), acidification potential (AP), eutrophication potential (EP), global warming potential over a 100-year timeframe (GWP), ozone layer depletion potential (ODP), photochemical oxidants formation potential (POFP) and toxicity related impact categories: human toxicity (HTP), 


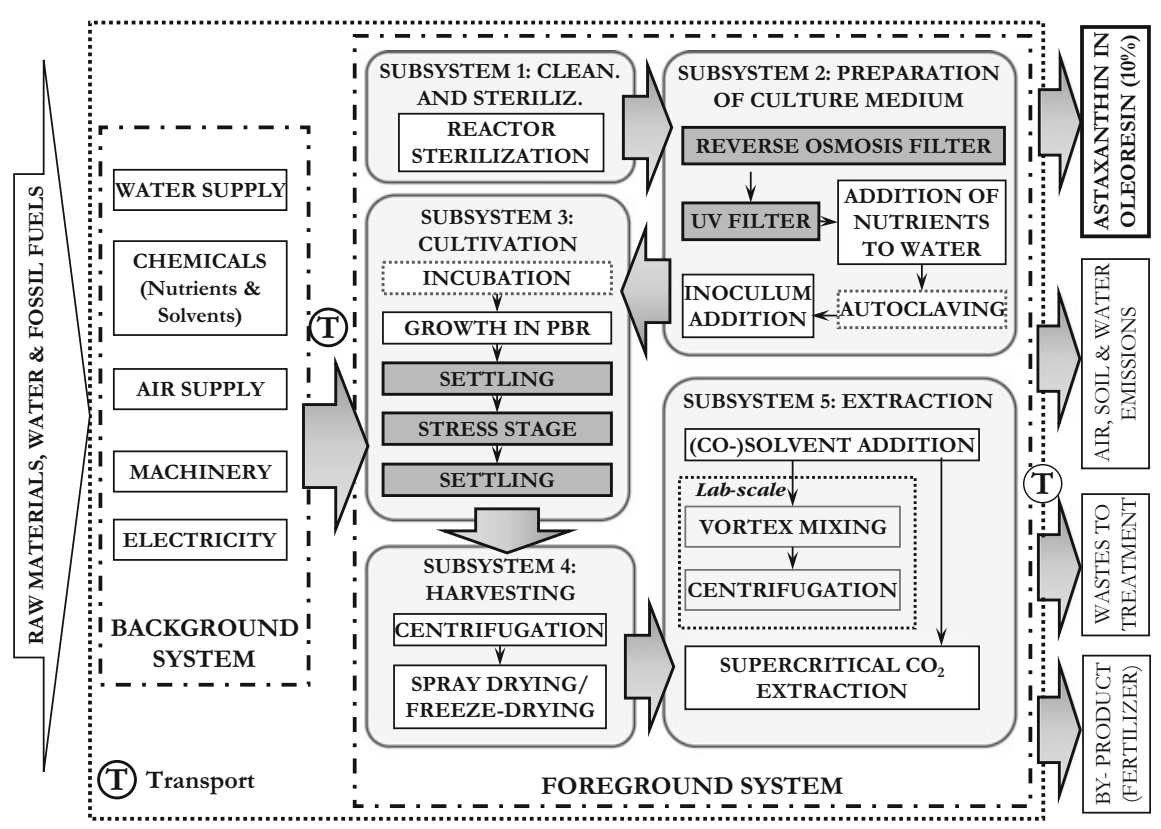

Fig. 1 Process chain and system boundaries for the production of $H$. pluvialis astaxanthin (blocks in dark grey correspond to steps that are specific to the pilot-scale production, blocks with dotted lines correspond to steps that are specific of lab- and semi-pilot production)

freshwater aquatic eco-toxicity (FEP), marine aquatic eco-toxicity (MEP) and terrestrial eco-toxicity (TEP). SimaPro 8.0.2 was used for the implementation [18].

\subsection{Social Assessment}

The social dimension is frequently considered as the weakest pillar of sustainable development, as reflected by the limited analytical and theoretical tools for its evaluation [19]. In order to develop a standardized methodology, UNEP-SETAC published the Guidelines for Social Life Cycle Assessment of Products, together with the methodological sheets for subcategories in Social Life Cycle Assessment (SLCA) $[8,20]$. The methodological sheets contain all the necessary information to collect data for 31 defined impact sub-categories, which correspond to relevant characterised social issues. The sub-categories representing social impacts are classified into five stakeholder categories: workers, consumers, local community, society and value chain actors. The given information includes a definition of each sub-category and an explanation of issues associated with it, as well as examples of inventory indicators, units of measurement and data sources [20]. 
In this case, the methodological sheets were taken as a basis to perform the social assessment of the $H$. pluvialis astaxanthin production. Thus, a specific questionnaire was developed, dealing with key issues and possible indicators related to the sub-categories that were relevant for the scope of the assessment. The questionnaire was fulfilled by two small and medium enterprises (SMEs) located in France and Ireland. For confidentiality reasons, the companies are referred to as SME 1 and SME 2 in the results section.

Three stakeholder groups were considered as the most representative in the specific context of the study: workers, consumers and society. For each of them, the selected indicators were grouped into sub-categories and measured in quantitative or semi-quantitative terms. Indicators for workers included annual salary, women-to-man ratio considering the total number of employees of each SME, women-to-man salary difference for the country and working hours per week. For consumers, impacts and benefits of the product were measured in terms of tests and safety information provided, customer service and information on formulation and use, value added of the product, natural origin, etc. Indicators for the society included the relative importance of blue biotechnology in the country, potential market share for the studied companies, compliance with certifications, existence of signed codes of conduct on sustainability, etc.

Following the approach of previous social LCA works [21], each indicator was expressed according to a numeric index based on the risk level in order to better understand the social impact information. As shown in Fig. 2, the index for each indicator ranged from 1 to 4 , being 1 the corresponding value for the worst scenario (highest risk) and 4 the index for the ideal scenario (no risk at all). In the case of stakeholder "workers", all the selected indicators were quantitative. The index for each indicator was calculated with respect to minimum and maximum risk levels in the world according to the values reported by OECD [22], Statista [23] and the World Economic Forum [24]. For each subcategory, the index was then obtained as the average index of the set of indicators assigned to this subcategory. For the stakeholders "consumers" and "society", most indicators had a Yes/No format; therefore, all the impacts related to these subcategories were converted into semi-quantitative terms through a scoring system. For Yes/No indicators, a value of 1 was assigned to negative response and a value of 4 was considered for affirmative response. In the case of some specific indicators, intermediate values were assigned according to expertise knowledge.

\begin{tabular}{|c|c|c|c|c|}
\hline & $\begin{array}{l}\text { Quantification of } \\
\text { indicators }\end{array}$ & & $\begin{array}{c}\text { Normalization } \\
\text { (index between 1-4) }\end{array}$ & $\begin{array}{c}\text { Classification into } \\
\text { stakeholders and subcat. }\end{array}$ \\
\hline$\pi$ 인 & $\begin{array}{l}\text { Quantitative (real value } \\
\text { of the company) }\end{array}$ & $\rightarrow$ & $\begin{array}{l}\text { Minimum and maximum in the } \\
\text { world represent extreme values }\end{array}$ & $\begin{array}{l}\text { WORKERS Gender } \\
\text { equality }\end{array}$ \\
\hline 욤음 & $\begin{array}{l}\text { Semi-quantitative } \\
\text { (Yes/No or Low/ } \\
\text { Intermediate/High) }\end{array}$ & $\rightarrow$ & $\begin{array}{c}\text { Yes/No => extreme values } \\
\text { Low/High }=>\text { rated by expert }\end{array}$ & $\begin{array}{c}\text { SOCIETY } \\
\text { Contribution to econ. } \\
\text { development }\end{array}$ \\
\hline
\end{tabular}

Fig. 2 Scheme of the method applied for the social impact assessment 


\subsection{Economic Analysis}

As economic aspects cannot be neglected in life cycle based sustainability assessment, a CBA approach is here proposed to evaluate this dimension. CBA is a basic decision-making tool included by Huppes et al. [9] among the methods to address the economic dimension of sustainability. It allows the comparison between different proposals according to the net profit of each option. As the integration of both CBA and LCA is now being widespread for the combined assessment of economic and environmental aspects $[10,25]$, this methodology has been selected for the evaluation of astaxanthin production.

CBA aims to compare the economic feasibility of a project or process by taking into account the costs and benefits over its life time $[10,25]$. The starting point of the tool is the premise that a project should only be developed if all the benefits exceed the aggregated costs. This premise is checked out by considering the net profit of a process as the difference between benefits and costs:

$$
N P=\sum B_{i}+\sum C_{i}
$$

Where NP is the net profit, $\mathrm{Bi}>0$ is the value of the benefit item $\mathrm{i}$ and $\mathrm{Ci}<0$ is the value of the cost item $i$. Thus, if the result of the calculation is NP $>0$, then the project is economically viable, whereas if $\mathrm{NP}<0$ the project is not viable in economic terms. The implementation of CBA requires that all benefits and costs are expressed in the same units. In projects related to environmental issues (e.g. operation of wastewater treatment plants), this restriction may require a complex homogenisation method for the quantification in monetary terms. However, in the case of the addressed process, the only benefit corresponded to the production of a high value molecule with biological properties, so the benefits could be measured in the same units as for costs (monetary units) and no method of homogenisation was needed.

Firstly, the assessment followed the CBA approach proposed by [10] to determine the NP by only considering the benefit of astaxanthin production and the variable operating costs in terms of energy consumption, chemicals, staff and other raw materials. In a second stage, the economic feasibility of the process was evaluated by considering two additional parameters: the net present value (NPV) and the pay-back period. Four different types of internal costs were included for the study of economic feasibility throughout the whole life time of the project, whereas externalities were excluded to avoid double counting of issues already covered by the environmental LCA:

- Investment costs, including the equipment required for the operation of the plant.

- Overhead costs, related to renting, insurances, travel costs, taxes and interests.

- Variable operating costs (already considered in the first stage, in which NP was determined according to the approach of [10]) associated with the consumption 
Fig. 3 Algorithm for the calculation of cash flows in the determination of the net present value of a project

\begin{tabular}{l}
\hline (+) Revenue \\
$(-)$ Variable operating costs \\
$(-)$ Overhead costs \\
$(-)$ Amortization \\
\hline BENEFITS BEFORE TAXES \\
$(-)$ Taxes \\
\hline BENEFITS BEFORE INTERESTS \\
(-) Interests \\
\hline NET BENEFIT \\
(+) Amortization \\
(-) Investment \\
\hline NET CASH FLOW RATE
\end{tabular}

of water, chemicals and other raw materials (material costs), as well as energy, operating labor costs, and also disposal costs.

- Research and development costs (calculated in relation to revenue).

With this information, the NPV was calculated according to Eq. 2:

$$
\text { Total } N P V \text { of the project }=\sum_{n=1}^{n=t} \frac{\text { Cash flow in year } \mathrm{t} \cdot(1+\mathrm{i})^{n}}{(1+\mathrm{r})^{n}}
$$

Where " $n$ " is the number of years of analysis, " $i$ " is the inflation rate and " $r$ " the nominal discount rate. The determination of cash flows was carried out according to the algorithm shown in Fig. 3.

The pay-back period of a project is defined as the period of time during which a facility must operate to recover the initial investment, according to the total capital costs and the estimated annual profits. It was determined by accumulating the annual profits until an equal value to the capital sum invested was obtained.

\section{Results and Discussion}

\subsection{Environmental Performance of $\mathrm{H}$. pluvialis Astaxanthin}

The results of the environmental LCA for the astaxanthin production process are depicted in Fig. 4. According to the results, there is a strong dependence of the environmental impacts with respect to the production scale. Thus, the total contributions were found to be from $10 \%$ up to four times higher for the lab-scale process than for the semi-pilot system. Regarding the pilot two-stage process, the semi-pilot system was found to have impacts between 10 and 100 times higher, whereas the lab process showed contributions between 25 and 122 times above those of the pilot process. Differences between production scales are mainly linked to low yields and oversized equipment in the smaller-scale processes. These differences are expected to decrease as the production scale increases, until an 


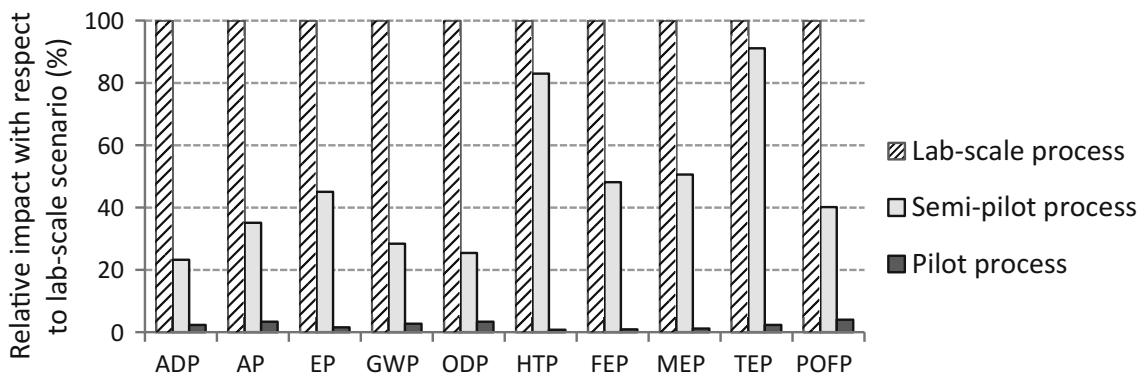

Fig. 4 Environmental LCA results associated with the production of $1 \mathrm{~kg}$ astaxanthin by H. pluvialis at lab, semi-pilot and pilot scale

asymptotic minimum level is achieved. Regardless of the environmental indicator and the production scale, most of the impact was linked to cultivation stage (S3). In particular, the production of electricity (mainly associated with artificial illumination and air supply) was the main hot-spot responsible for these environmental burdens, as further discussed in [11].

\subsection{Social Hotspots of $\mathbf{H}$. pluvialis Astaxanthin}

The indexes for the selected social indicators of each stakeholder category were aggregated by sub-category and depicted in a spider chart (Fig. 5) to obtain a visual representation and identify the hot spots or main social concerns of the process. As shown in Fig. 5, the results of the social impact assessment show the profiles for both SMEs, with most indexes near the maximum possible value. However, the outcome differs depending on the strategic management of the company and on the

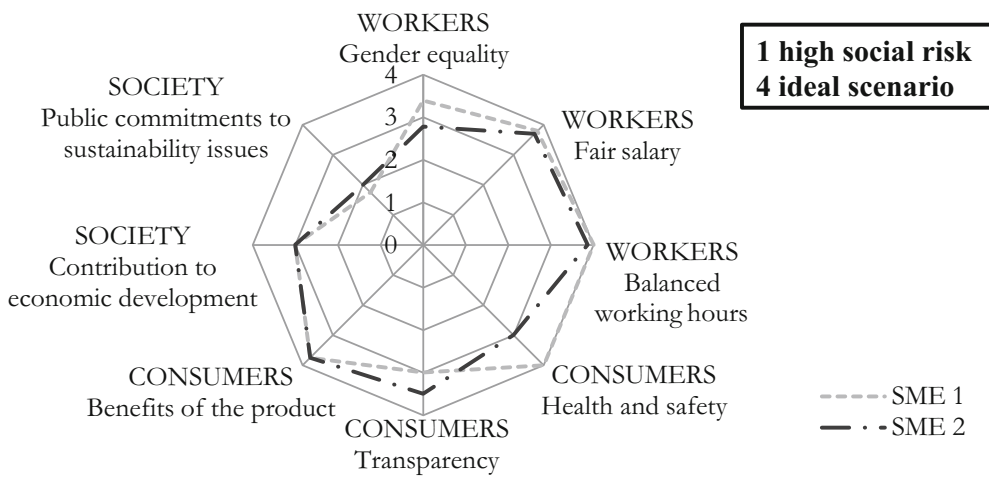

Fig. 5 Radar chart representing prominent social issues of the corporate strategy of two SMEs involved in the production of $H$. pluvialis astaxanthin 
considered stakeholder category. Thus, while the performance related to workers and consumers show quite different profiles for the two companies, the sub-categories related to society present a similar behaviour. Nevertheless, the score of both companies in terms of benefits to workers and consumers revealed a relatively favourable performance. Despite the importance of the respective countries in a national scale, the performance in terms of benefits to society was, in the specific case of the evaluated SMEs, limited by the small size of the two companies, which resulted in a low potential market share, compared to the total market volume of blue biotechnology in the corresponding countries.

\subsection{Economic Evaluation of $\mathbf{H}$. pluvialis Astaxanthin}

The NPV was obtained considering a production of $120 \mathrm{~kg}$ astaxanthin/year, a $12.5 \%$ nominal discount rate (according to typical values of $10-15 \%$ for biomass products) and a $1.7 \%$ inflation rate (average inflation rate in the country for the year 2012). The calculated value was 2,068,203 €, which means that the assessed process would be economically feasible, since NPV $>0$. Among the different groups of costs, the variable production costs were responsible for up to $75 \%$ of the total cost. The highest fraction of these variable operating costs corresponded to the staff, which represented more than three fourths of the production costs. Among the other items, energy consumption would be the most relevant cost, with $20 \%$ of the total. Regarding the pay-back period, Fig. 6 shows that one year and four months of operation of the facility, would be a sufficient period of time to recover the total initial investment according to the estimated costs and revenues.

The results obtained for the two indicators (net present value and pay-back period) indicate that microalgal astaxanthin could allow significant economic benefits. Although the performed economic assessment is subject to a considerable level of uncertainty, related to the inaccurate estimation of the different costs and final revenue for a sector that is still immature, the high profitability and low payback time suggest that the process would still be viable in a wide range of conditions (including less favourable contexts). Additional co-products may be obtained from the residual algal paste in the future, increasing the potential revenues of the process.

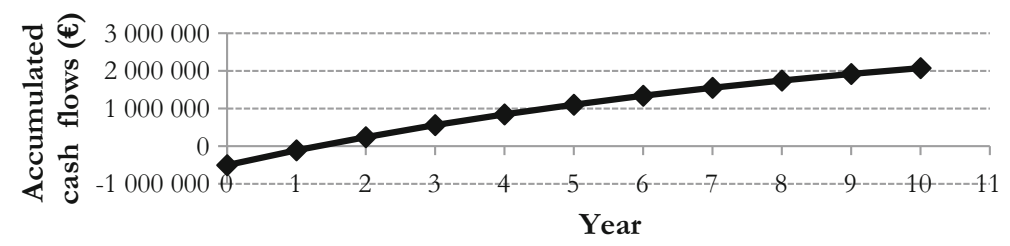

Fig. 6 Pay-back period for astaxanthin production at pilot scale 


\section{Conclusions}

In this study, the existing tools to estimate the environmental, social and economic performances were applied to a novel production process in the sector of blue biotechnology. The results allowed evaluating the high influence of upscaling in the environmental profile of microalgal production process, as well as identifying the main contributors to the environmental impacts (electricity related to cultivation stage). The results also indicate that the process may have remarkable societal benefits (especially for workers and consumers) and the economic indicators suggest that the process could be feasible if operated under similar conditions as in the case of the studied SMEs. The combined outcome of the environmental, social and economic assessment of microalgal astaxanthin production constitutes a valuable basis for the successful incorporation of sustainability criteria in the design of blue biotechnology processes. The results may help to orient actions towards a more eco-efficient microalgae-related industry as well as to feed the debate for the development of appropriate environmental and socio-economic policies in the sector.

Acknowledgements This research was financially supported by BAMMBO Project (FP7 KBBE-2010-4-265896). The authors belong to GRC 2013-032, co-funded by FEDER. P. Pérez-López thanks the Spanish Ministry of Education for the grant AP2012-1605.

\section{References}

1. Woodley J.M, New opportunities for process systems engineering in industrial biotechnology, Computer Aided Chemical Engineering, Vol. 27, 2009, pp 157-162.

2. Kim S, Jiménez-González C, Dale B, Enzymes for pharmaceutical applications-a cradle-to-gate life cycle assessment, The International Journal of Life Cycle Assessment, Vol. 14, No. 5, 2009, pp 392-400.

3. Jiménez-González C, Woodley J.M, Bioprocesses: Modeling needs for process evaluation and sustainability assessment, Computers \& Chemical Engineering, Vol. 34, No. 7, 2010, pp 1009-1017.

4. Pérez-López P, Ternon E, González-García S, Genta-Jouve G, Feijoo G, Thomas OP, Moreira MT, Environmental solutions for the sustainable production of bioactive natural products from the marine sponge Crambe crambe, Science of the Total Environment, Vol. 475, 2014, pp 71-82.

5. Collet P, Hélias A, Lardon L, Steyer J.P, Bernard O, Recommendations for Life Cycle Assessment of algal fuels, Applied Energy, Vol. 154, 2015, pp 1089-1102.

6. Hutchins M.J, Sutherland J.W, An exploration of measures of social sustainability and their application to supply chain decisions, Journal of Cleaner Production, Vol. 16, No. 15, 2008, pp 1688-1698.

7. Sala S, Farioli F, Zamagni A, Life cycle sustainability assessment in the context of sustainability science progress (part 2), The International Journal of Life Cycle Assessment, Vol. 18, No. 9, 2013, pp 1686-1697. 
8. UNEP-SETAC, Guidelines for social life cycle assessment of products, United Nations Environment Programme (UNEP), Paris, France, 2009, <http://www.unep.org/publications/>, (Accessed 31.05.2017).

9. Huppes G, van Rooijen M, Kleijn R, Heijungs R, de Koning A, van Oers L, Life cycle costing and the environment, National Institute for Public Health and the Environment, Bilthoven, The Netherlands, 2004.

10. Molinos-Senante M, Hernández-Sancho F, Sala-Garrido R, Cost-benefit analysis of water-reuse projects for environmental purposes: A case study for Spanish wastewater treatment plants, Journal of Environmental Management, Vol. 92, No. 12, 2011, pp 30913097.

11. Pérez-López P, González-García S, Jeffryes C, Agathos S.N, McHugh E, Walsh D, Murray P, Moane S, Feijoo G, Moreira M.T, Life cycle assessment of the production of the red anti-oxidant carotenoid astaxanthin by microalgae: From lab to pilot scale, Journal of Cleaner Production, Vol. 64, 2014, pp 332-344.

12. Barros M.P, Poppe S.C, Bondan E.F, Neuroprotective properties of the marine carotenoid astaxanthin and omega-3 fatty acids, and perspectives for the natural combination of both in krill oil, Nutrients, Vol. 6, No. 3 2014, pp 1293-1317.

13. Aflalo C, Meshulam Y, Zarka A, Boussiba S, On the relative efficiency of two- vs. one-stage production of astaxanthin by the green alga Haematococcus pluvialis, Biotechnology and Bioengineering, Vol. 98, No. 1, 2007, pp 300-305.

14. ISO 14040, Environmental management-Life Cycle Assessment-Principles and framework, Geneva, Switzerland, 2006.

15. Baker R, Saling P, Comparing natural with chemical additive production, Feed mix $2003 ; 11$ (1):12-14.

16. Frischknecht R, Jungbluth N, Althaus H.J, Doka G, Heck T, Hellweg S, Hischier R, Nemecek T, Rebitzer G, Spielmann M, Wernet G, Overview and methodology, Ecoinvent report No. 1 v2.0. Swiss Center for Life Cycle Inventories, Dübendorf, Switzerland, 2007.

17. Guinée J.B, Gorrée M, Heijungs R, et al., Handbook on life cycle assessment: Operational guide to the ISO standards, Kluwer Academic Publishers, Dordrecht, the Netherlands, 2002.

18. Goedkoop M, Oele M, Leijting J, Ponsioen T, Meijer E, SimaPro 8. Introduction to LCA with SimaPro, Pré Consultants, 2013.

19. Lehtonen M, The environmental-social interface of sustainable development capabilities, social capital, institutions, Ecological Economics, Vol. 49, No. 2, 2004, pp 199-214.

20. UNEP-SETAC, The methodological sheets for subcategories in Social Life Cycle Assessment (S-LCA), United Nations Environment Programme (UNEP), Paris, France, 2013, <http:// www.lifecycleinitiative.org/>, (Accessed 31.05.2017).

21. Benoît Norris C, Cavan D.A, Norris G, Identifying social impacts in product supply chains: overview and application of the Social Hotspot Database, Sustainability, Vol. 4, No. 9, 2012, pp 1946-1965.

22. <http://stats.oecd.org/>, (Accessed 31.05.2017).

23. <https://www.statista.com/statistics/226956/average-world-wages-in-purchasing-powerparity-dollars/>, (Accessed 31.05.2017).

24. World Economic Forum, The Global Gender Gap Report 2013, Colgny/Geneva, Switzerland, 2013, <http://www.weforum.org/reports/global-gender-gap-report-2013>, (Accessed 31.05. 2017).

25. Birol E, Koundouri P, Kountouris Y, Assessing the economic viability of alternative water resources in water-scarce regions: Combining economic valuation, cost-benefit analysis and discounting, Ecological Economics, Vol. 69, No. 4, 2010, pp 839-847. 
Open Access This chapter is licensed under the terms of the Creative Commons Attribution 4.0 International License (http://creativecommons.org/licenses/by/4.0/), which permits use, sharing, adaptation, distribution and reproduction in any medium or format, as long as you give appropriate credit to the original author(s) and the source, provide a link to the Creative Commons license and indicate if changes were made.

The images or other third party material in this chapter are included in the chapter's Creative Commons license, unless indicated otherwise in a credit line to the material. If material is not included in the chapter's Creative Commons license and your intended use is not permitted by statutory regulation or exceeds the permitted use, you will need to obtain permission directly from the copyright holder.

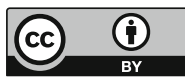




\title{
Integrating Life Cycle Assessment and Eco-design Strategies for a Sustainable Production of Bio-based Plastics
}

\author{
Venkateshwaran Venkatachalam, Sebastian Spierling, \\ Hans-Josef Endres and Andrea Siebert-Raths
}

\begin{abstract}
In order to design and develop a sustainable bio-based plastic product right from its development phase, it is necessary to analyse and interpret the direct and indirect environmental impacts along the life cycle of the product system through life cycle thinking. Eco-design is a well-established approach in designing products with the consideration and integration of the environmental impacts of the product system, and Life Cycle Assessment (LCA) is a standardized methodology to quantify and analyse the environmental impacts of the product system along the production chain. This paper identifies the practical needs for integrating LCA and eco-design strategies into the production of bio-based plastic products, taking a bio-based computer mouse body made of Poly Lactic Acid (PLA) as a case study.
\end{abstract}

\section{Introduction}

Plastics have an important role in our daily life. From packaging to automotive areas, the use of plastics has steadily increased during the last 100 years and is predicted to keep growing in the future [1]. The major part of the current plastics is based on fossil resources like crude oil. However, in the past few decades, bio-based plastics have become a focus in plastic research [2], due to the finiteness of fossil-based raw materials, renewability of the feedstock used in the bio-based plastics and the current challenges of climate change. Bio-based plastics are defined as ready to use blends, which consist of biopolymers and additives [2]. With increasing bio-based plastic production, the question of whether the bio-based plastics a sustainable alternative to conventional plastics, have been raised repeatedly. Many LCA studies have been conducted for biopolymers [3] but most of these LCA studies are used as a communication tool to show its environmental performance rather than integrated to the design of products manufactured from these

\footnotetext{
V. Venkatachalam $(\bowtie) \cdot$ S. Spierling $\cdot$ H.-J. Endres $\cdot$ A. Siebert-Raths Faculty II-Mechanical Engineering and Bio Process Engineering, Institute for Bioplastics and Biocomposites, University of Applied Sciences and Arts, Hannover, Germany e-mail: venkateshwaran.venkatachalam@hs-hannover.de

(C) The Author(s) 2018

E. Benetto et al. (eds.), Designing Sustainable Technologies,

Products and Policies, https://doi.org/10.1007/978-3-319-66981-6_54
} 
biopolymers. Even though various eco-design studies have been done for the selection of biopolymers [4, 5], very few exist for bio-based plastics. The main objective of this paper is to identify the eco-design strategies for the production of a bio-based plastic material based on its material properties and the results of LCA. To perform this study, an eco-design team was setup, composed of material and process engineers, sustainability experts and marketing representatives. This team analysed the cost, sustainability aspects, chemical and mechanical properties of the different biopolymers and additives before the manufacture of a bio-based plastic product. The cradle to gate LCA has been performed for the product, after the most suitable polymers were chosen. The results of this LCA and its influence on the proposed eco-design strategies will be discussed in this paper.

\section{Description of the Eco-design Process Stages, as Applied to the Case-Study}

\subsection{Motivation Factors}

Benefits and motivation factors [6] for performing product or process eco-design have been analysed as a very first stage, even before LCA and product properties analysis, so that the eco-design team can analyse and identify the strategies within the framework of the product development. The motivation factors not only arise from the manufacturing company but also from external factors such as policies and markets. Some of them are provided here as example:

- Increase the marketability of the bio-based plastic products through product innovation, thereby increasing its demand and reducing costs of raw materials

- Improve the products mechanical and environmental performances by reducing energy and material consumption, having circular economy as a global target,

- Clear communication to the customers and consumers with regards to the products mechanical and environmental performances.

\subsection{Methodology of Eco-design for Bio-based Plastics}

As eco-design process requires to consider a vast array of inter-related aspects such as product economic, technical, ergonomic, market and environmental properties, it requires a multi-disciplinary approach. Based on literature review of eco-design applied to bio-based products [7,8], the different stages considered for the present case study are shown in Fig. 1. It can be seen that the identification and implementation of eco-design strategies is an iterative approach supported by LCA implementation. 
Fig. 1 Methodological stages of eco-design applied to bio-based plastics

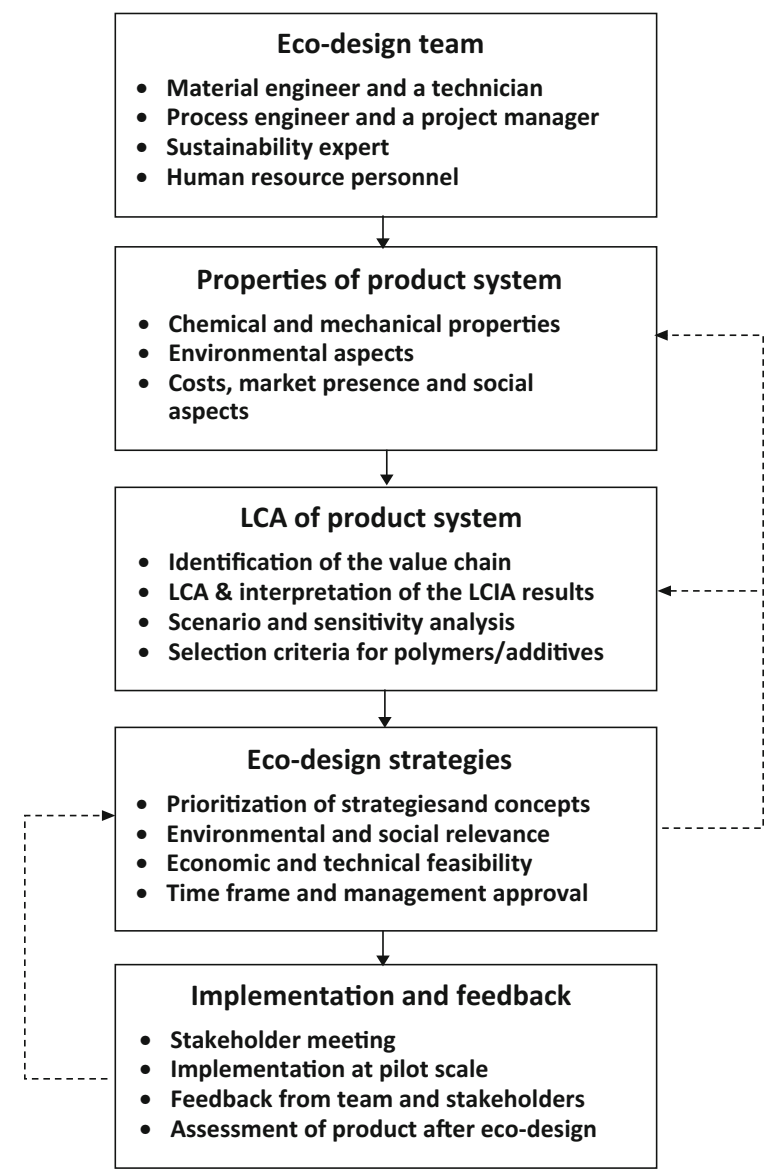

\section{LCA Application}

\subsection{Goal and Scope Definition}

The main goal of this LCA study is to analyse the environmental performance of the product made out of bio-based plastics and then identify opportunities to optimize this product. The results of this LCA study will be helpful to analyse the individual contribution of different processes towards the total environmental impacts. The Life Cycle Impact Assessment (LCIA) results, along with the hot spot and scenario analyses will then be used to develop eco-design strategies for the product. 


\subsubsection{Functional Unit}

The functional unit of the LCA study is the production of $1 \mathrm{~kg}$ of bio-based plastic blend, made primarily of biopolymer PLA, to be used to manufacture 25 pieces of computer mouse bodies, which include right mouse button, left mouse button, palm rest and the base [9].

\subsubsection{System Boundaries}

The system boundaries are set from cradle to gate, which includes raw material acquisition phase, production of the bio-based plastics blends and finally the processing of the blends to manufacture the computer mouse pieces. The use and End of Life (EoL) phases of the product system are not considered for this study due to the limited availability of data on the consumer behaviour and the strategies to use and dispose the products after its use phase. However, some factors influencing these two phases are included as a part of the eco-design strategy definition, which will be explained in Sect. 4. This LCA study is done in accordance to ISO 14040 [10] and ISO 14044 [11] and supported by the software GaBi 6 [12]. From Fig. 2, it can be seen that the raw material acquisition phase includes the manufacture, processing and the transport of the polymers, additives and other chemicals from their original sites to Germany, where they will be extruded, granulated, crystallized and packed as bio-based plastics blends. The main components of the blend are the biopolymers Poly-L-Lactic Acid (PLLA) and Poly-D-Lactic Acid (PDLA), which are produced from sugarcane and sugar beets respectively. Both PLLA and PDLA come under the biopolymer type PLA and is one of the widely used biopolymers with suitable mechanical properties. The processing phase involves in the pre-processing of these bio-based plastic blends before bringing it to a specific form (here, body of the computer mouse) by injection moulding. The finished product is then sent to an assembly site (electronic parts of mouse are not included in the system boundaries) and finally distributed to the consumers. The utilities such as process steam, process water, compressed air and output flows such as emissions and wastes are considered within each process even though they are not explicitly shown in Fig. 2.

\subsubsection{Life Cycle Inventory Data Sources and Data Quality Requirements}

The bio-based plastic blend, which is used in the manufacture of the body of a computer mouse, is made of biopolymers and additives. The production of the bio-based plastic blend takes place in Germany. Inventory data for the manufacture of PLLA, PDLA and other additives in the raw material acquisition phase were picked up from different literature sources [13, 14]. The background Life Cycle Inventory (LCI) datasets were considered for different utilities like process steam, 


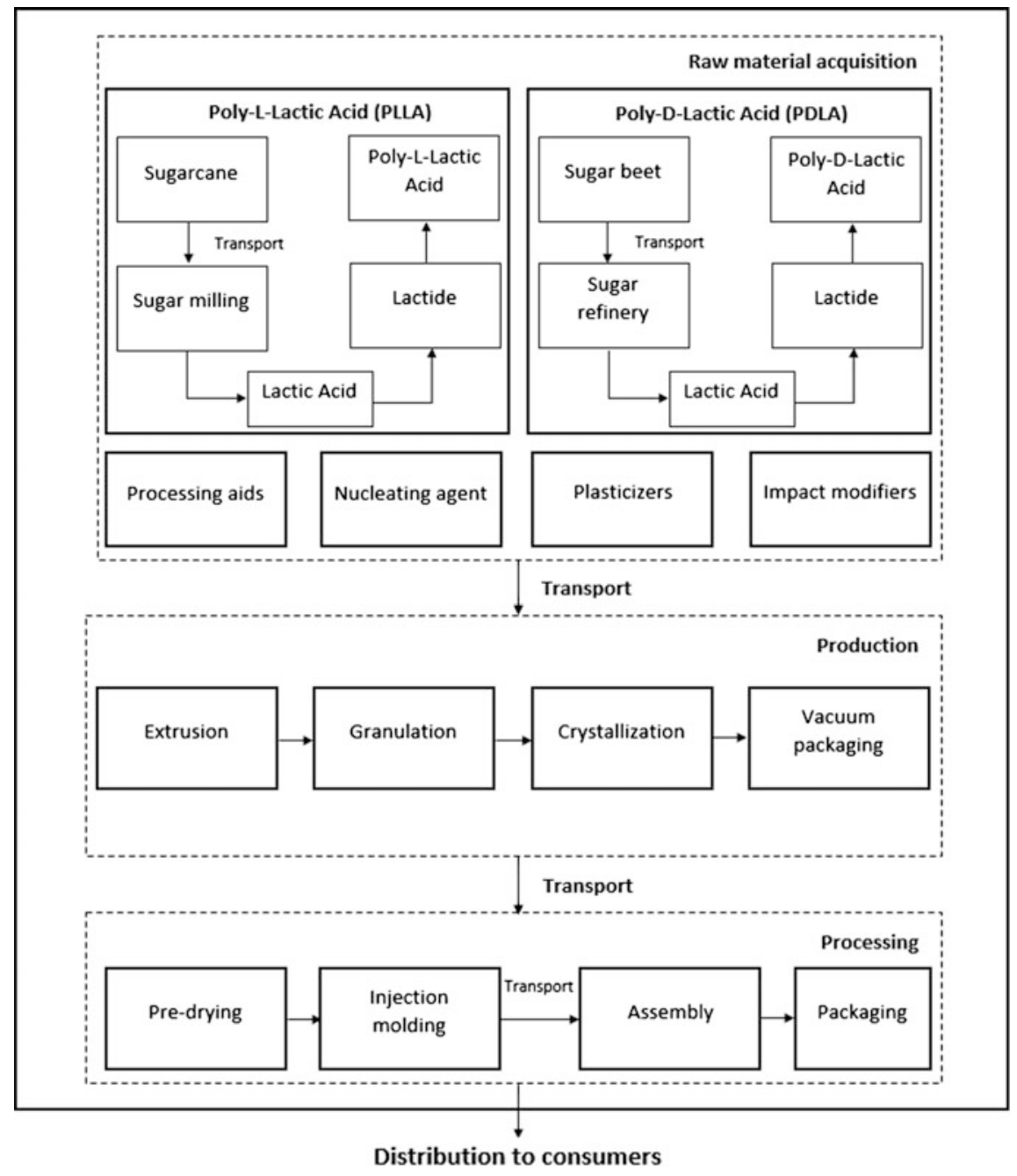

Fig. 2 System boundaries of the product system - computer mouse

natural gas and water, which are involved in the manufacturing of biopolymers and these were selected from GaBi LCI databases. PLLA used in the blend, originally comes from Thailand, whereas PDLA comes from Spain even though both of the raw materials are produced and supplied by the same company. The two polymers along with the additives (from different companies) are then transported to the supplier site and finally to Germany, where they are subjected to extrusion, granulation and crystallization. Primary inventory data were obtained on-site for the production and processing phases, in co-operation with the industrial partners. 
In the case of PLLA and PDLA, we could not obtain the primary inventory data from the polymer manufacturers due to confidentiality issues. However, regional specific secondary datasets are used in the modelling of product system wherever possible, in order to close the data gaps related to the geographical settings. Transoceanic, intra-continental and the local transportation distance of raw materials from the production plant (PLLA and PDLA from Thailand and Spain) to the supplier transformation site (Our institute in Germany where the blends are manufactured with the help of PLLA and PDLA) have been calculated with the help of an online tool EcoTransIT [15], which calculates the distance in accordance to realistic transportation scenarios. The availability and clarity of inventory data is one of the main challenges for this study and is identified as an eco-design strategy in Sect. 4.

\subsection{Impact Assessment and Interpretation}

Different impact assessment methods are available to perform the Life Cycle Impact Assessment (LCIA). The recommendations from the International Reference Life Cycle Data System (ILCD)/Product Environmental Footprint (PEF) were considered for the characterisation of each midpoint impact, and the LCIA results were obtained from the method 'Impacts ILCD/PEF recommendation v1.09' [16] in the $\mathrm{GaBi}$ software. The LCIA results of the study, for the selected impact categories are presented in Table 1.

Global Warming Potential (GWP) presented in Table 1 includes biogenic carbon, as the feedstock comes from renewable resources and biogenic carbon storage during plant growth should be accounted for, in any cradle to gate assessment of

Table 1 Impact assessment results of $1 \mathrm{~kg}$ of bio-based plastic blend, used in the manufacture of computer mouse body

\begin{tabular}{l|l|l}
\hline Impact category (midpoint) & Unit & $\begin{array}{l}\text { Value per functional } \\
\text { unit }\end{array}$ \\
\hline Acidification potential (AP) & Mole $\mathrm{H}^{+}$-eq. & 0.030 \\
\hline Global warming potential (GWP) & $\mathrm{kg} \mathrm{CO}_{2}$-eq. & 1.462 \\
\hline Eutrophication Potential (EP) & Mole N-eq. & 0.099 \\
\hline $\begin{array}{l}\text { Photochemical ozone creation potential } \\
\text { (POCP) }\end{array}$ & $\mathrm{kg} \mathrm{NMVOC}$ & 0.020 \\
\hline Ozone depletion potential (ODP) & kg CFC-11-eq. & $2.933 \mathrm{E}-11$ \\
\hline Abiotic depletion potential (ADP) & kg Sb-eq. & $1.267 \mathrm{E}-04$ \\
\hline Land use (LU) & $\mathrm{kg} \mathrm{C} \mathrm{deficit} \mathrm{eq.}$ & 31.084 \\
\hline Ecotoxicity (ET) & $\mathrm{CTUe}$ & 0.564 \\
\hline Human toxicity (cancer effects) [HT (CE)] & $\mathrm{CTUh}$ & $2.196 \mathrm{E}-08$ \\
\hline Ionizing radiation (IR) & $\mathrm{kBq} \mathrm{U235} \mathrm{eq.}$ & 0.160 \\
\hline Particulate matter (PM) & $\mathrm{kg} \mathrm{PM} \mathrm{2.5-eq.}$ & 0.003 \\
\hline
\end{tabular}




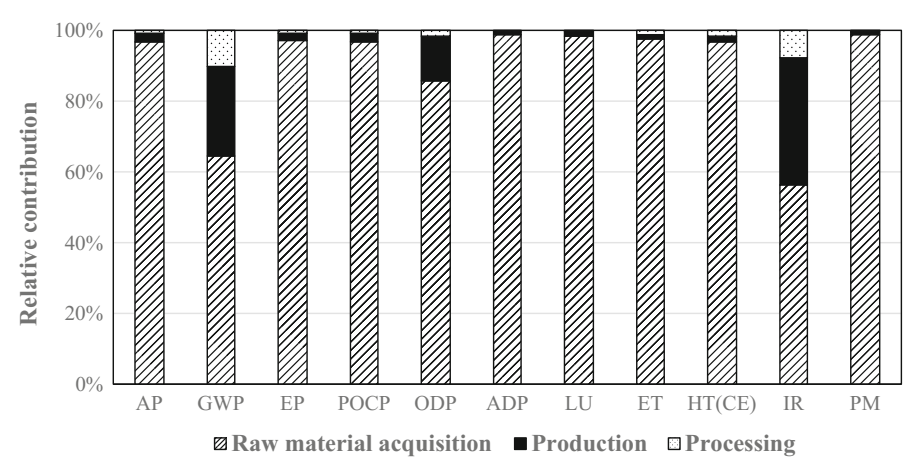

Fig. 3 Relative contribution of life cycle phases (cradle to gate) towards each impact category indicator

bio-based materials [17]. Since use and EoL phases are not considered in this study, it is important to mention that the benefits of accounting for biogenic carbon storage during plant growing is generally counter-balanced with the release of biogenic carbon during use and/or EoL phases. In the results presented here, biogenic carbon storage was accounted for but its release during use and/or EoL not. The relative contribution of each life cycle phase to the different impact categories is shown in Fig. 3 .

It can be found out that the raw material acquisition phase is the most important phase in terms of contribution to all impact categories, contributing to more than $95 \%$ in 8 out of 11 environmental impact categories. For all impact categories, contribution from raw material acquisition phase is mainly induced by the production and transport of PLLA and by the additives required for the production of bio-based plastic blends. Considering GWP impact category, impact from raw material acquisition is mainly due to process steam and power consumption from the production of PLLA. Regarding the production phase, the power consumption and compressed air used for different production processes contribute mainly to the impact on GWP (25.5\%). Finally, contribution from processing phase to the GWP $(10 \%)$ comes from the power consumption for the injection moulding of the plastic blends and the transportation of the finished products to the final assembly. Even though the contribution of the production phase to the impact on ODP (12.6\%) and the processing phase (1.4\%) mainly comes from the power consumption, in the case of raw material acquisition phase (86\%), major contribution comes from the impact modifiers (fossil-based acrylic additives used to improve the product impact strength, one of its mechanical properties) and the polymer (PLLA). IR follows the same tendency as that of ODP, with the contribution in raw material acquisition $(56.3 \%)$ coming from the polymer (PLLA) manufacture, for the production (36.1\%) and processing $(7.5 \%)$, contribution coming from the power consumption. Some of the eco-design strategies are identified based on the interpretation of the LCIA results. 


\subsection{Scenario Analysis}

Scenario analysis is a method, where boundary conditions (i.e. type of process and associated quantity) of some of the key process parameters are modified in the inventory, in order to analyse the variation induced on LCIA results. Scenario analysis can be a powerful method to identify different potential strategies supporting the optimisation of product's environmental performance.

The LCIA results highlighted that a significant amount of environmental impacts comes from the transoceanic transportation of the polymer PLLA i.e. the transportation of polymer from Thailand, where it was originally manufactured, to Germany, where it will be used in the manufacture of bio-based plastic product. In the scenario analysis, the trans-oceanic cargo transportation of PLLA is kept as a base scenario, compared to a scenario of cargo transportation of PLLA from Spain, and another scenario of truck transportation of PLLA from Spain. These scenarios appeared as feasible since the same company supply PLLA from Thailand and PDLA from Spain. Although there was no significant change in most of the impact categories, the change in the most significant impact categories AP, GWP, EP and POCP for the two scenarios is shown alongside the base scenario in Fig. 4. At least $21 \%$ reduction is observed in the total impact on AP when the PLLA is produced and transported by truck from Spain to Netherlands when compared to the base scenario.

Additives are also used in the manufacture of bio-based plastic product in order to increase and optimize the mechanical properties of the product. One such additive is the impact modifiers, which are used for increasing flexibility and impact strength to meet physical property requirements of rigid parts [18]. These modifiers are mostly fossil-based acrylic compounds and production of these modifiers contributes to a significant amount of environmental impacts. A new variant of bio-based impact modifiers has been developed and is already available on the market [19]; however, the scale of its production is much smaller compared to the already established conventional impact modifiers. These bio-based impact modifiers are Polyhydroxyalkanoate (PHA) based, used in the polymer blends to improve the mechanical properties of PLA [19]. A scenario analysis was performed

Fig. 4 Scenario analysis for the transportation of PLLA from Thailand to Europe

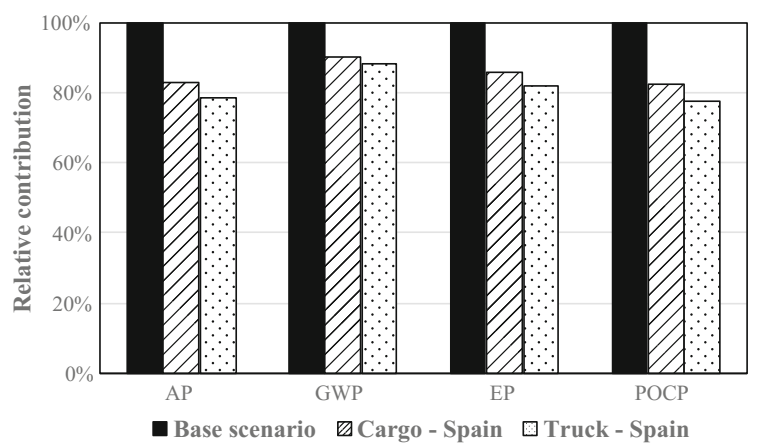


with the replacement of the fossil-based impact modifiers by PHA based additives. Eco-design strategies were identified based on these scenario analyses.

\section{Identification of Eco-design Strategies}

From the LCIA results and scenario analysis, considered together with costs and materials properties, eco-design strategies have been proposed and some of the important strategies are listed in Table 2, taking into account the different aspects involved in the product development. Environmental impacts, economic and technical feasibility are scored arbitrarily on a scale going from 1 to 5 defined by the eco-design team and stakeholders, with 1 being least feasible (for economic and technical feasibility) or least relevant (for environmental improvement) and 5 being highly feasible and highly relevant. A 'Time frame', ranging from Short Term (ST), Medium Term (MT) and Long Term (LT) is also set, for the likelihood of implementing these strategies, based on the previously existing manuals on eco-design such as 'Practical Manual of Ecodesign. Implementation in 7 steps' [6], 'Design for Sustainability - A practical approach for developing economies' [20] and 'Product Design and Life Cycle Assessment' [21]. The first two strategies presented in Table 2 are based on the results of scenario analysis and the related environmental performances. Therefore, it is required that inventory data considered for polymers and additives must be transparent and representative, allowing to perform a comprehensive LCA and to communicate the LCIA results based on a consistent model, which will also affect the material development. Improving the mechanical, ergonomic, recycling and biodegradable properties of the product not only have a high environmental relevance but also helpful to position bio-based plastic products as a sustainable option in a long term. The implemented strategies must be reviewed among the team and stakeholders continuously, as there is always a potential for improvement.

Table 2 Proposed eco-design strategies after analysing different aspects of the bio-based computer mouse body

\begin{tabular}{l|l|l|l|l}
\hline Strategies & $\begin{array}{l}\text { Environmental } \\
\text { improvement }\end{array}$ & $\begin{array}{l}\text { Economic } \\
\text { feasibility }\end{array}$ & $\begin{array}{l}\text { Technical } \\
\text { feasibility }\end{array}$ & $\begin{array}{l}\text { Time } \\
\text { frame }\end{array}$ \\
\hline $\begin{array}{l}\text { Sourcing of polymers } \\
\text { from Europe }\end{array}$ & 4 & 2 & 5 & LT \\
\hline Bio-based additives & 3 & 2 & 3 & MT \\
\hline Clarity in inventory data & 2 & 5 & 4 & ST \\
\hline $\begin{array}{l}\text { Mechanical properties of } \\
\text { the polymers }\end{array}$ & 3 & 2 & 2 & MT \\
\hline $\begin{array}{l}\text { Ergonomics of the } \\
\text { product }\end{array}$ & 4 & 2 & 2 & MT \\
\hline Recyclability & 5 & 1 & 4 & MT \\
\hline Biodegradability & 3 & 3 & 2 & MT \\
\hline
\end{tabular}




\section{Conclusion}

LCA of a bio-based computer mouse body was conducted on a cradle to gate basis. It was found out that the raw material acquisition phase constitutes the major share in all of the environmental impacts. Several strategies for an eco-design of a bio-based product were identified based on the LCIA results, scenario analyses and consideration of some alternative aspects of the product. Some of the methodological and practical requirements for implementing eco-design strategies at the level of bio-based plastics manufacturing were also shown. Once implemented these strategies should pave the way for a sustainable production of bio-based plastics.

Acknowledgements The authors would like to thank the German Federal Ministry of Education and Research as well as the German Federal Ministry of Food and Agriculture for the funding and support to the research project in which this study has been conducted.

\section{References}

1. http://www.plasticseurope.org/documents/document/20161014113313-plastics_the_facts_ 2016_final_version.pdf, (Accessed 10.06.2017).

2. Endres H-J, Siebert-Raths A, Engineering Biopolymers Markets, Manufacturing, Properties and Applications, Carl Hanser Verlag GmbH \& Co. KG, 2011.

3. Yates M, Barlow C.Y, Life Cycle Assessments of biodegradable, commercial biopolymers A critical review, Resources Conservation and Recycling, Vol. 78, 2013, pp. 54-66.

4. Olwill J.A., Wright E.I, Rahimifard S, A holistic approach to design support for bio-polymer based packaging. Journal of Polymers and the Environment, 20, 2012, pp. 1112-1123.

5. Ribeiro I, Peças P, Henriques E, A life cycle framework to support materials selection for Ecodesign: A case study on biodegradable polymers, Materials \& Design, Vol. 51, 2013, pp. 300-308.

6. IHOBE S.A, Manual Práctico de Ecodiseño-Operativa de implantación en 7 pasos, Sociedad pública de gestión ambiental, 2001.

7. González-García S, Silva F.J, Moreira M.T, Castilla Pascual R, García Lozano R, Gabarrell X, Rieradevall Pons J, Feijoo G, Combined application of LCA and eco-design for the sustainable production of wood boxes for wine bottles storage, International Journal of Life Cycle Assessment, Vol. 16, 2011, pp. 224-237.

8. Muñoz I, Gazulla C, Bala A, Puig R, Fullana P, LCA and ecodesign in the toy industry: case study of a teddy bear incorporating electric and electronic components, International Journal of Life Cycle Assessment, Vol. 14, 2009, pp. 64-72.

9. https://www.nager-it.de/maus, (Accessed 05.03.2017).

10. ISO 14040:2006 Environmental management-life cycle assessment-principles and framework.

11. ISO 14044:2006 Environmental management-life cycle assessment-requirements and guidelines.

12. GaBi, Thinkstep AG; LBP-GaBi, University of Stuttgart: GaBi Software System, Leinfelden-Echterdingen/Germany, 2013.

13. Groot W.J, Boren T, Life cycle assessment of the manufacture of lactide and PLA biopolymers from sugarcane in Thailand, International Journal of Life Cycle Assessment, Vol. 15, No. 9, 2010, pp. 970-984. 
14. Harbec A, Lactic acid production from agribusiness waste starch fermentation with Lactobacillus Amylophilus and its cradle-to-gate life cycle assessment as a precursor to Poly-L-Lactide, 2010.

15. http://www.ecotransit.org/index.de.html, (Accessed 12.03.2017).

16. http://eplca.jrc.ec.europa.eu/uploads/ILCD-Recommendation-of-methods-for-LCIA-def.pdf, (Accessed 15.04.2017).

17. Pawelzik P, Carus M, Hotchkiss J, Narayan R, Selke S, Wellisch M, Weiss M, Wicke B, Patel M, Critical aspects in the LCA of bio-based materials-Reviewing methodologies and deriving recommendations, Resources, Conservation and Recycling, Vol. 73, 2013, pp. 211-228.

18. Impact modifiers: How to make your compound tougher https://www.pharosproject.net/ uploads/files/sources/1828/1359960957.pdf, (Accessed 09.01.2018).

19. http://www.yield10bio.com/sites/default/files/ANTEC-PLA_PVC.pdf, (Accessed 16.05.2017).

20. Design for Sustainability -A practical approach for developing economies http://www.d4sde.org/manual/d4stotalmanual.pdf, (Accessed 12.05.2017).

21. Zbicinski I, Stavenuiter J, Kozlowska B, van de Coevering H.P.M., Product Design and Life Cycle Assessment, The Baltic University Press, 2006.

Open Access This chapter is licensed under the terms of the Creative Commons Attribution 4.0 International License (http://creativecommons.org/licenses/by/4.0/), which permits use, sharing, adaptation, distribution and reproduction in any medium or format, as long as you give appropriate credit to the original author(s) and the source, provide a link to the Creative Commons license and indicate if changes were made.

The images or other third party material in this chapter are included in the chapter's Creative Commons license, unless indicated otherwise in a credit line to the material. If material is not included in the chapter's Creative Commons license and your intended use is not permitted by statutory regulation or exceeds the permitted use, you will need to obtain permission directly from the copyright holder.

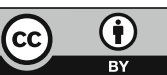




\title{
Using LCA and EPD in Public Procurement Within the Construction Sector
}

\author{
Kristian Jelse and Kristof Peerens
}

\begin{abstract}
Life cycle assessments (LCAs) and Environmental Product Declarations (EPDs) have long been pointed to as tools to be used in green public procurement (GPP), but doing so in practice is challenging. How can such information be requested without breaking some of the general principles for public procurement, for example non-discrimination and transparency? This session was meant to give insights from different stakeholders through five presentations providing different perspectives on this issue. The individual presentations were followed up on by a panel discussion.
\end{abstract}

\section{Introduction}

With the European Union being responsible for more than a third of global resource consumption, $40 \%$ of global energy consumption, $36 \%$ of global carbon dioxide emissions, and one third of global waste streams [1], there is an increasing need for this region to take a leading position when it comes to reducing the overall environmental impact.

Specifically for the construction sector, energy efficient housing is one of the key elements in being able to reduce Europe's overall environmental impact, with studies showing that the embodied energy in construction represents the amount of energy equivalent to more than 75 years of heating [1]. The construction sector overall is more than just housing, and each of the different components have a significant impact on the environment. A rough estimate by the Norwegian authorities has shown that the yearly carbon footprint of the public road system (including construction and maintenance)

K. Jelse $(\bowtie)$

EPD International AB, Stockholm, Sweden

e-mail: kristian@environdec.com

K. Peerens

3 M Belgium bvba/sprl, Diegem, Belgium

(C) The Author(s) 2018

E. Benetto et al. (eds.), Designing Sustainable Technologies,

Products and Policies, https://doi.org/10.1007/978-3-319-66981-6_55 
equates to $\sim 2.3$ million tons of carbon dioxide equivalents, excluding direct traffic emissions (which add 20-25\%) [2].

\section{Challenges in Using LCAs and EPDs as a Standard Assessment Method in Green Public Procurement}

Even though LCA and EPD data is commonly used to quantify the potential environmental impact of construction elements or construction works, reality shows that in GPP, despite environmental considerations being implemented in a variety of European Member States, this data is not used in order to come to conclusions and make decisions related to product or supplier selection [3]. The presentations at the session showed different challenges to different types of stakeholder groups.

\subsection{Challenges for Industry}

LCA is a time and resource intensive undertaking for industry, which requires significant effort in gathering data, creating life cycle models in dedicated software packages, appropriately interpreting the results, and reporting the information in an understandable format. This means that for small and medium sized enterprises, cost is often seen as a barrier to starting such studies [1]. As a result, those companies that have limited resources available cannot always justify the cost, and as a result will not have LCA or EPD data available.

In addition, there are a number of challenges related to communicating LCA and EPD information: some of the information must be specific to the product application and use, other data is relevant for different types of audiences (general public versus architects or project developers). Unfortunately, even when only considering the European Union, means of communication, as well as the different LCA and EPD elements differ [1]. When publishing LCA and EPD data for a number of different countries, there may be a need to generate multiple LCA reports or EPDs, have them verified multiple times (in line with the countries' requirements), and often have them registered locally, each of which comes at a cost. Harmonisation of results can therefore be seen as an absolute must when it comes to promoting the consistent use of LCAs and EPDs [1].

\subsection{Challenges for the Public Sector}

No matter how it is approached, LCA is a complex topic that requires a certain skill set to be able to interpret information generated by the study. So even if LCA and EPD data was to be available for all products participating in a public tender, there 
would need to be quite some knowledge required from the procurement departments in order to identify the best possible product for a certain application [3].

Environmental benchmark data is not readily available, which means that this adds a layer of complexity for public authorities for product differentiation and selection. With the risk of duplicating efforts and lack of consistency, public authorities often use national/regional expertise in order to determine GPP criteria, which can result in different regulatory frameworks, and a lack of harmonisation.

For public authorities, sustainability criteria must go beyond environmental impacts, and must include social, humanitarian and economic aspects as well. Weighting and characterisation of environmental criteria based on the significance of the differences in environmental impacts are therefore a must [4].

\section{Tool and Platform Development to Assist in Interpretation of LCA and EPD Data}

A number of industry sectors, depending on their maturity in the area of sustainability, have built tool sets that help in assessing the overall sustainability impact of their sector's products, technologies and solutions. As a sector, it has therefore been possible to meet the growing demands related to the availability of data. The schemes as developed and deployed in e.g. the steel industry have therefore resulted in collaboration and engagement, increasing transparency and improvements in environmental performance, thus triggering innovation and continuous improvement [4].

Similarly, regional and federal governments (e.g. Germany) as well as building certification schemes are developing databases and software packages that allow for generic and product-specific data to be made available to different stakeholders to allow them to assess the impact of new construction developments or maintenance [5]. Not only have these tools reduced the cost of an LCA, but it ensures that LCA is considered during the construction design and planning [5].

\section{Discussion on Further Efforts to Integrate Data from LCAs and EPDs in GPP}

When considering the different options for integrating LCA and EPD in GPP criteria setting and purchasing decision consistently within any given sector on European level, the key element that must be considered is harmonisation:

- It is a must that schemes operated in different European countries work in the same way and avoid setting national special features in addition to the standards to allow companies to consistently report LCA and EPD data; 
- Benchmarks must be made available in order to ensure that products that are regarded as true differentiators are considered as such regardless of the country they are sold into;

- GPP award criteria must be harmonised, in order to ensure there is a consistent message from the public sector on what the focus areas are supposed to be, and these should be driven by the European Commission;

- For those organisations that are not in a position to generate LCA data themselves, financial support or publicly available data repositories should be made available, driven by the public sector.

Truly implementing science-based product or service selection criteria for purchasing materials or services is the single most important element that will drive a true reduction of the environmental impact of the European construction sector. Based on today's assessment methodologies and data availability, LCAs and EPDs are a critical element in this approach, and should therefore be much more visible in public and private efforts to drive down the environmental impact.

\section{References}

1. Adibi N, Pasquet V, Darul M, Roy A, Salamon A, Challenges to mainstream the use of EPDs in construction Public Procurement in European context, LCM 2017 conference, Luxembourg, 2017.

2. Fredriksen K-S, O'Born R, Implementing climate emission demands in road construction contracts: Insights from the KRAKK project and the Norwegian Public Roads Administration, LCM 2017 conference, Luxembourg, 2017.

3. Ganassali S, Lavagna M, Campioli A, Saporetti S, Green Public Procurement and Construction Sector: EPD and LCA based benchmarks of the whole-building, LCM 2017 conference, Luxembourg, 2017.

4. Brankley L, Tugrul A, Anderson J, Knight D, A Manufacturing Sector's Experience of integrating EPD alongside product, management system and responsible sourcing certification for public procurement, LCM 2017 conference, Luxembourg, 2017.

5. Kusche O, Brockmann T, Figl H, How LCA and EPD are used for implementing Green Public Procurement in the construction sector in Germany, LCM 2017 conference, Luxembourg, 2017.

Open Access This chapter is licensed under the terms of the Creative Commons Attribution 4.0 International License (http://creativecommons.org/licenses/by/4.0/), which permits use, sharing, adaptation, distribution and reproduction in any medium or format, as long as you give appropriate credit to the original author(s) and the source, provide a link to the Creative Commons license and indicate if changes were made.

The images or other third party material in this chapter are included in the chapter's Creative Commons license, unless indicated otherwise in a credit line to the material. If material is not included in the chapter's Creative Commons license and your intended use is not permitted by statutory regulation or exceeds the permitted use, you will need to obtain permission directly from the copyright holder. 


\title{
Green Public Procurement and Construction Sector: EPD and LCA Based Benchmarks of the Whole-Building
}

\author{
Sara Ganassali, Monica Lavagna, Andrea Campioli \\ and Sergio Saporetti
}

\begin{abstract}
The paper discusses the possible use of Life Cycle Assessment (LCA) and Environmental Product Declarations (EPDs) in European and Italian Green Public Procurement (GPP) for the building sector, in order to define targets based on objective and reliable building environmental impacts information. The research objective is to define how to set LCA benchmarks towards the improvement of GPP requirements. The study analyses the GPP criteria based on LCA in Europe with a focus on Italy; it proposes LCA benchmarks for selected groups of construction materials and scenarios for the implementation of LCA benchmarks related to buildings elements and whole-buildings. It also illustrates how public administrations can verify the GPP criteria achievement using EPDs.
\end{abstract}

\section{Introduction}

Life Cycle Assessment (LCA) practice is increasing in the construction sector to evaluate the environmental sustainability of buildings. Generally, the definition of the better environmental profile between buildings providing a similar function is performed through the simple comparison of the LCA impacts results. This approach should be completed by objective LCA-based environmental benchmarks (reference values) to be set, which can be used for setting sustainable construction and production targets. Environmental LCA-based benchmark values are used in several Green Building Rating Systems (GBRSs) and in buildings energy certifications. In these certifications (such as LEED, BREEAM, Verde, Green Star,

\footnotetext{
S. Ganassali $(\bowtie) \cdot$ M. Lavagna · A. Campioli

Department of Architecture, Built Environment and Construction

Engineering, Politecnico di Milano, 20133 Milan, Italy

e-mail: sara.ganassali@polimi.it

S. Saporetti

Ministero dell'ambiente e della tutela del territorio e del mare,

Direzione clima ed energia, Divisione II certificazione ambientale ed acquisti pubblici verdi, 00147 Rome, Italy

(C) The Author(s) 2018

E. Benetto et al. (eds.), Designing Sustainable Technologies,

Products and Policies, https://doi.org/10.1007/978-3-319-66981-6_56
} 
Minergie, etc.) the LCA benchmarks are not performed on a common basis, inducing the consideration of different repositories for each, making the comparison of different LCA-based benchmarks impossible for the building sector. The benchmarks used in these certifications are often established from the statistical analysis of LCA data obtained from a reference buildings sample with specific environmental performances [1, 2], or from a reference building modelled according to national standards and prescriptions [3-5].

Considering the lack of a common framework for setting environmental sustainable targets for the construction sector, the present study aims at presenting a new benchmarking approach, fixing LCA-based benchmark "code of practice" for construction materials, building elements and whole-building. LCA benchmark values can improve European and Italian Green Public Procurement (GPP) criteria required for the construction sector, and it can also support stakeholders with sustainable construction strategies and improvement of buildings environmental performances. It could also support the GPP verification process made by public administrations.

The study is divided into four sections. First, the use of LCA in the GPP for buildings is examined, illustrating which EU Members have already included LCA approach and consideration of Environmental Product Declarations (EPDs) in the national GPP. Second, the benchmarking methodology and the environmental benchmarks related to building materials are illustrated, as well as the proposed EPD-based verification process for the contracting authorities. Finally, the approach used to set LCA-based environmental benchmarks for building elements and whole-buildings is explained.

\section{LCA Data, EPDs and GPP in Europe}

This section investigate the possibility to introduce LCA in GPP criteria for the construction sector, through the analysis of GPP requirements from the EU Commission. The EU Nations are encouraged to draw up National Action Plans (NAPs) for reducing environmental impacts from public tenders managed by contracting authorities and the associated procurements of goods, services and works. GPP criteria for buildings are often developed in NAPs, including environmental design and planning strategies in the national political framework. GPP is a voluntary instrument, aiming at stimulate the market demand for more sustainable goods and services. LCA-based criteria are required in the optional EU-GPP for "Office Building Design, Construction and Management" [6], including the environmental evaluation of impacts during all the stages of the buildings life cycle (production of materials and elements, construction, use stage and end of life), promoting the application of a cradle-to-grave LCA. In GPP requirements for buildings, the promotion of energy efficiency and the use of products with a specific percentage of recycled content material are also integrated. There is only one LCA-based indicator required in GPP for new office buildings 
which is the total amount of Global Warming Potential (GWP) for the building whole-life. It can be partly calculated by practitioners through the aggregation of EPDs results for the main building elements (foundations and substructures, structural frame, external walls, floors and ceilings, internal walls, windows and roofs), and/or based on a complete cradle-to-grave LCA. The LCA and use of EPDs provide a quantitative assessment of the building environmental performances, however, the comparability between two options remains sensitive even if the EPDs are compliant with the related existing standards and Product Category Rule (PCR).

The use of GPP for buildings in European Nations has been investigated (Table 1), analysing the use of the LCA approach in GPP criteria and the related use of EPDs for the LCA in order to understand how the environmental products labels are used by contracting authorities to verify the GPP criteria achievement.

For each EU member State, Table 1 shows the presence of National Action Plans (NAP), the adoption of GPP for buildings, the use of the Life Cycle Assessment (LCA) and the use of Environmental Product Declarations (EPDs).

Table 1 shows a restricted view of the use of LCA and EPDs in GPP for buildings, however the information found is useful in order to understand how some EU Nations insert environmental criteria in public procurement. Belgium inserts LCA in GPP-Office Buildings criteria using the GBRSs (i.e. LEED and BREEAM) which just involve the life cycle approach in their criteria; Denmark suggests the use of LCA and EPDs for an environmental products comparison; Lithuania introduces the LCA and the Life Cycle Costing as mandatory analysis; Luxembourg introduces voluntary LCA criteria; The Netherlands requires mandatory LCA analysis for groundworks, roads and hydraulic engineering constructions.

The study focused on the Italian GPP requirements for building products, in order to develop propositions for improving environmental sustainability criteria. The new Italian procurement code (Codice degli Appalti) requires compulsory environmental criteria (Criteri Ambientali Minimi-CAMs) to enhance the sustainability process of construction products, of new public and refurbished buildings and of public construction sites management [7]. CAMs criteria are set on three design reference steps: the sustainable site analysis, in which the considered building is located; the buildings technical specifications, which include dynamic energetic simulations, the use of new energy supplies, indoor air quality and comfort and the materials technical specifications, which set the recycled content value for specific material categories.

The CAMs criteria related to the environmental sustainability of building products, do not include LCA. CAMs set mandatory recycled content percentage value for different material categories (i.e. the concrete must contain at least $5 \%$ of recycled content on the product's weight, as well as bricks and gypsum plasterboards). The contracting authorities can check the latter criteria achievement with the use of materials environmental performances issued from environmental/energy building certifications (i.e. LEED, BREEAM, etc.) and/or construction products environmental labels (labels type I, II and III). However, the use of EPDs in CAMs is underestimated, despite for the first time the use of EPDs is incentivised. 
Table 1 Analysis of GPP-LCA criteria application for buildings in European Nations

\begin{tabular}{|c|c|c|c|c|}
\hline Nations & $\begin{array}{l}\text { National } \\
\text { Action Plans } \\
\text { Provided by "National GPP } \\
\text { Action Plans (policies and } \\
\text { guidelines)", EU Commission, } \\
\text { DG Environment (updated to } \\
\text { May 2017) }\end{array}$ & \begin{tabular}{|l} 
GPP \\
Buildings \\
Collected \\
through the \\
national GPP \\
website
\end{tabular} & $\begin{array}{l}\text { Life Cycle } \\
\text { Assessment } \\
\text { Collected } \\
\text { through the } \\
\text { national GPP } \\
\text { website }\end{array}$ & EPDs \\
\hline Austria & $\bullet$ & $\bullet$ & 0 & 0 \\
\hline Belgium & $\bullet$ & $\bullet$ & $\bullet$ & $\bullet$ \\
\hline Bulgaria & $\bullet$ & $\circ$ & n.a. & n.a. \\
\hline Croatia & $\bullet$ & $\bullet$ & $\circ$ & $\circ$ \\
\hline Cyprus & $\bullet$ & n.a. & n.a. & n.a. \\
\hline $\begin{array}{l}\text { Czech } \\
\text { Republic }\end{array}$ & $\bullet$ & n.a. & n.a. & n.a. \\
\hline Denmark & $\bullet$ & $\bullet$ & - & $\bullet$ \\
\hline Estonia & $\circ$ & $\circ$ & n.a. & n.a. \\
\hline Finland & $\bullet$ & $\bullet$ & $\circ$ & $\bullet$ \\
\hline France & $\bullet$ & $\bullet$ & n.a. & n.a. \\
\hline Germany & $\bullet$ & $\bullet$ & $\circ$ & $\circ$ \\
\hline Greece & $\circ$ & $\circ$ & n.a. & n.a. \\
\hline Hungary & 0 & $\circ$ & n.a. & n.a. \\
\hline Ireland & $\bullet$ & u.d. & n.a. & n.a. \\
\hline Italy & $\bullet$ & $\bullet$ & $\circ$ & $\bullet$ \\
\hline Latvia & $\bullet$ & n.a. & n.a. & n.a. \\
\hline Lithuania & $\bullet$ & $\bullet$ & $\bullet$ & $\circ$ \\
\hline Luxembourg & 0 & $\bullet$ & $\bullet$ & $\circ$ \\
\hline Malta & $\bullet$ & $\bullet$ & n.a. & n.a. \\
\hline $\begin{array}{l}\text { The } \\
\text { Netherlands }\end{array}$ & $\bullet$ & $\bullet$ & $\bullet$ & $\circ$ \\
\hline Poland & $\bullet$ & $\circ$ & n.a. & n.a. \\
\hline Portugal & $\bullet$ & u.d. & n.a. & n.a. \\
\hline Romania & 0 & $\circ$ & n.a. & n.a. \\
\hline Slovakia & $\bullet$ & u.d. & n.a. & n.a. \\
\hline Slovenia & $\bullet$ & $\bullet$ & n.a. & n.a. \\
\hline Spain & $\bullet$ & $\bullet$ & n.a. & n.a. \\
\hline Sweden & $\bullet$ & $\bullet$ & n.a. & n.a. \\
\hline Norway & $\bullet$ & $\bullet$ & $\circ$ & 0 \\
\hline Swiss & $\bullet$ & n.a. & n.a. & n.a. \\
\hline UK & $\bullet$ & $\bullet$ & n.a. & n.a. \\
\hline
\end{tabular}

\section{Legend}

Black circle: present topic

White circle: not present topic n.a. information not available u.d. topic under development 
Therefore it induced the promotion of EPDs on the market: the manufacturers are encouraged to certify their products in order to compete in public tenders, while the practitioners are stimulated toward environmental strategies choosing certified products. Increasing the use of EPDs in the construction sector could lead to a growth of available transparent LCA data on market and to a possible introduction of LCA benchmarks in CAMs criteria. Moreover, with the LCA benchmarks insertion in CAMs, the EPDs could become simple verification instruments for public administrations.

\section{LCA Benchmarks for Construction Products}

This section aims at demonstrating how LCA-based benchmark values for construction products can be defined, and how could EPDs be integrated into CAMs criteria in order to facilitate the verification process by the public administrations in charge of analysing different options.

The benchmark values for construction materials developed in this study are fixed for different LCA impact categories and they could be integrated in GPP criteria, in order to set environmental sustainable levels for building products. The benchmarking methodology has been developed after a detailed literature review of five recent studies in which existing environmental benchmarks practices in the construction sector have been applied. The benchmarking methods were all based on the statistical analysis and interpretation of LCA data. In the analysed case-studies, LCA-based benchmarks were related to healthcare buildings in Portugal [8], for school buildings in South Korea [9] and for residential buildings in Italy [12], France [11] and Germany. In order to set LCA-based benchmarks for construction products, a reference sample composed by eight construction product categories was processed, categories are also contained in CAMs criteria (cement, bricks, wood-based materials, steel, gypsum plasterboards, glass wool panels, stone wool panels and ceramic tiles). The sample was made by building materials manufactured and sold in Europe in recent times (after the year of 2010) and it was considered representative of the European production/construction practices. The related LCA data were collected through EPDs, reporting transparent environmental impacts results for each product life cycle phase. The LCA system boundaries considered in this study are the one related to the product stage (LCA modules A1, A2 A3). Thirty-two EPDs were analysed for cement, eight for bricks, eleven for wooden-based materials, forty-five for steel, thirty-one for gypsum plasterboard, fifty for glass-wool panels, fifteen for stone-wool panels and twenty-five for ceramic tiles.

The EPDs collected were taken from European EPD Program Operators: BAU EPD (Austria), IBU (Germany), International EPD ${ }^{\circledR}$ System (Sweden), GlobalEPD (Spain), EPD Denmark (Denmark), DAPHabitat System (Portugal), FDES INIES (France), EPD Italy (Italy) and EPDNorge (Norway). In order to guarantee the comparability and the homogeneity between LCA data from different 
national repositories, it was checked that the EPDs were compliant with the European standard EN 15804:2012 "Sustainability of construction works", and that they have mutual recognition between the Product Category Rules (PCRs).

Ten environmental impacts categories were considered: GWP, Ozone Depletion Potential (ODP), Acidification Potential (AP), Eutrophication Potential (EP), Photochemical Ozone Creation (POCP), Abiotic Depletion Potential for Non-fossil resources (ADNP), Abiotic Depletion Potential for fossil resources (ADP0), Total use of Renewable Primary Energy resources (PERT), Total use of Non-Renewable Primary Energy resources (PENRT), and the consumption of Fresh Water (FW). The method applied to set LCA-based benchmarks was a statistical analysis and interpretation of LCA data collected. It allows verification of wrong data (the outliers) and the assessment of the reliability of final results. For each material category and for each environmental impact category, three benchmark values have been set up: the limit value, the reference value and target value. The reference value represents the median value, which is not sensitive to the outliers in a sample composed of a small number of data. The target and the limit value are set using the quartile division of LCA data: the target value is fixed by the $1^{\circ}$ quartile, which indicates a value of $25 \%$ lower than the median value (it represents the best manufacturing practice); the limit value is fixed by the $3^{\circ}$ quartile, which represents a value of $25 \%$ higher than the median value (the lowest acceptable value in the evaluation scale).

In Table 2, the benchmarks fix the limit, the reference and the target values of GWP, AP, PENRT and FW.

In Table 2, the negative GWP value observed for wooden products is due to the $\mathrm{CO}_{2}$ stored in the products, which is released into the atmosphere at the end of use stage. The LCA benchmarks presented in the study are European benchmark values, the LCA benchmarks could be divided and analysed per country, extrapolating single threshold values for each Nation (Fig. 1).

The three LCA-based benchmarks (limit, reference and target values) fix a sustainable range of values for each material group, so the public administration could decide which is the better sustainable level to apply in the public tender requirements. They can then check the GPP criteria achievement, verifying if the LCA data of specific building products (certified with EPD labels) fall into the LCA-based benchmark range.

The LCA benchmarks are based on a limited number of data due to the lack of EPDs and LCA-based data available on the market, despite the number of information published is increasing. 
Table 2 LCA-based benchmarks related to $1 \mathrm{~kg}$ of eight construction materials

\begin{tabular}{|c|c|c|c|}
\hline Materials & Limit value & Reference value & Target value \\
\hline \multicolumn{4}{|l|}{$G W P\left(\mathrm{~kg} \mathrm{CO}_{2} \mathrm{eq}\right)$} \\
\hline Brick & $2.63 \mathrm{E}-01$ & $2.56 \mathrm{E}-01$ & $2.18 \mathrm{E}-01$ \\
\hline Cement & $8.49 \mathrm{E}-01$ & $7.52 \mathrm{E}-01$ & $6.48 \mathrm{E}-01$ \\
\hline Ceramic & $4.89 \mathrm{E}-01$ & $3.56 \mathrm{E}-01$ & $3.25 \mathrm{E}-01$ \\
\hline Plasterboard & $2.59 \mathrm{E}-01$ & $2.22 \mathrm{E}-01$ & $2.12 \mathrm{E}-01$ \\
\hline Glass wool & $1.12 \mathrm{E}+00$ & $1.08 \mathrm{E}+00$ & $1.06 \mathrm{E}+00$ \\
\hline Stone wool & $1.22 \mathrm{E}+00$ & $1.19 \mathrm{E}+00$ & $8.86 \mathrm{E}-01$ \\
\hline Steel & $1.24 \mathrm{E}+00$ & $5.46 \mathrm{E}-01$ & $5.11 \mathrm{E}-01$ \\
\hline Wood fibreboard & $-9.81 \mathrm{E}-01$ & $-1.20 \mathrm{E}+00$ & $-1.22 \mathrm{E}+00$ \\
\hline \multicolumn{4}{|l|}{$A P\left(\mathrm{~kg} \mathrm{SO}_{2} \mathrm{eq}\right)$} \\
\hline Brick & $7.32 \mathrm{E}-04$ & $6.41 \mathrm{E}-04$ & $5.51 \mathrm{E}-04$ \\
\hline Ceramic & $1.81 \mathrm{E}-03$ & $1.23 \mathrm{E}-03$ & $7.85 \mathrm{E}-04$ \\
\hline Cement & $1.37 \mathrm{E}-03$ & $1.30 \mathrm{E}-03$ & $9.98 \mathrm{E}-04$ \\
\hline Plasterboard & $6.53 \mathrm{E}-04$ & $5.03 \mathrm{E}-04$ & $3.40 \mathrm{E}-04$ \\
\hline Glass wool & $8.41 \mathrm{E}-03$ & $6.31 \mathrm{E}-03$ & $6.20 \mathrm{E}-03$ \\
\hline Stone wool & $1.11 \mathrm{E}-02$ & $6.73 \mathrm{E}-03$ & $5.35 \mathrm{E}-03$ \\
\hline Steel & $3.18 \mathrm{E}-03$ & $2.59 \mathrm{E}-03$ & $1.10 \mathrm{E}-03$ \\
\hline Wood fibreboard & $2.10 \mathrm{E}-03$ & $1.70 \mathrm{E}-03$ & $1.64 \mathrm{E}-03$ \\
\hline \multicolumn{4}{|l|}{ PENRT (MJ) } \\
\hline Brick & $3.53 \mathrm{E}+00$ & $3.39 \mathrm{E}+00$ & $2.88 \mathrm{E}+00$ \\
\hline Ceramic & $4.96 \mathrm{E}+00$ & $3.40 \mathrm{E}+00$ & $2.52 \mathrm{E}+00$ \\
\hline Cement & $9.08 \mathrm{E}+00$ & $7.32 \mathrm{E}+00$ & $6.77 \mathrm{E}+00$ \\
\hline Plasterboard & $4.72 \mathrm{E}+00$ & $4.09 \mathrm{E}+00$ & $3.23 \mathrm{E}+00$ \\
\hline Glass wool & $3.22 \mathrm{E}+01$ & $3.13 \mathrm{E}+01$ & $3.02 \mathrm{E}+01$ \\
\hline Stone wool & $1.85 \mathrm{E}+01$ & $1.71 \mathrm{E}+01$ & $1.24 \mathrm{E}+01$ \\
\hline Steel & $1.50 \mathrm{E}+01$ & $8.75 \mathrm{E}+00$ & $8.71 \mathrm{E}+00$ \\
\hline Wood fibreboard & $1.09 \mathrm{E}+01$ & $7.87 \mathrm{E}+00$ & $6.73 \mathrm{E}+00$ \\
\hline \multicolumn{4}{|l|}{$F W\left(\mathrm{~m}^{3}\right)$} \\
\hline Brick & $8.52 \mathrm{E}-04$ & $6.59 \mathrm{E}-04$ & $3.66 \mathrm{E}-04$ \\
\hline Ceramic & $1.80 \mathrm{E}-01$ & $1.68 \mathrm{E}-03$ & $1.06 \mathrm{E}-03$ \\
\hline Cement & $3.47 \mathrm{E}-03$ & $3.16 \mathrm{E}-03$ & $2.28 \mathrm{E}-03$ \\
\hline Plasterboard & $1.33 \mathrm{E}-03$ & $1.00 \mathrm{E}-03$ & $8.55 \mathrm{E}-04$ \\
\hline Glass wool & $1.82 \mathrm{E}-02$ & $1.79 \mathrm{E}-02$ & $1.35 \mathrm{E}-02$ \\
\hline Stone wool & $4.42 \mathrm{E}-03$ & $4.16 \mathrm{E}-03$ & $3.48 \mathrm{E}-03$ \\
\hline Steel & $4.00 \mathrm{E}-02$ & $3.21 \mathrm{E}-03$ & $2.62 \mathrm{E}-03$ \\
\hline Wood fibreboard & $1.13 \mathrm{E}-02$ & $3.83 \mathrm{E}-03$ & $2.32 \mathrm{E}-03$ \\
\hline
\end{tabular}




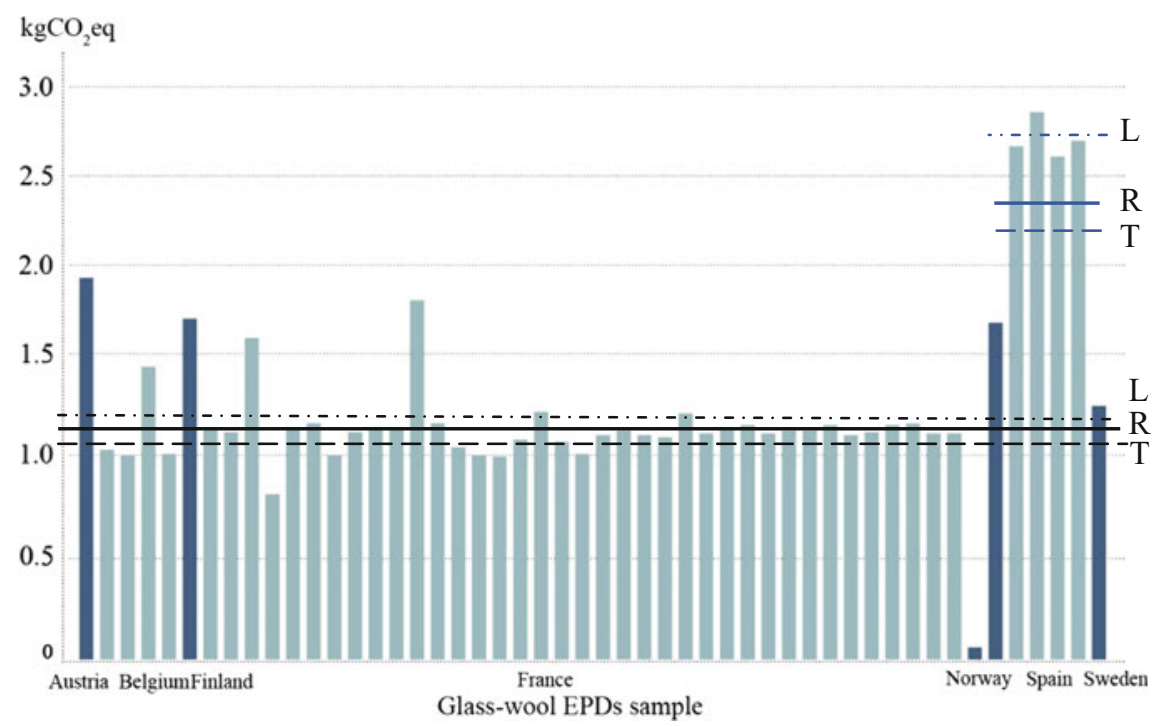

Fig. 1 European GWP benchmarks (black lines) and Spain GWP benchmarks (grey bars) related to $1 \mathrm{~kg}$ of glass-wool panel (LCA phases A1-3). Legend limit value (L), reference value (R), target value $(\mathrm{T})$

\section{LCA Benchmarks for Building Elements and Whole-Building}

The LCA-based benchmarks could also be applied to the main building elements and the whole-buildings in the GPP for buildings and in the CAMs criteria.

LCA benchmark values for the main building elements are already used in the construction sector [13]. According to the main studies analysed from the literature review [8-12], this study tried to define a benchmarking methodology to set environmental LCA-based benchmarks for building elements, explaining the possible methodological processes. The building elements categories to be considered should refer to construction technologies and practices commonly used, in this work four categories were considered: structure frame, floors, roof, external walls and internal walls. The related LCA-benchmarks should be set at least for five different generic building types, which are based on the classification often used in the main GBRSs: commercial buildings, educational buildings, healthcare buildings, residential buildings and retail. The elements analysed should be related to a specific context, recent (after 2010), in order to analyse the construction practices employed in average in the country of concern. According to the national energy parameters, prescriptions and construction practices, a range of similar constructing solutions it is modelled for each building element identified. The data collection for the building elements could be carried out using national databases, where buildings can be selected based on energy and environmental certifications [9, 10], 
or through the screening of the calls for tenders [11]. As for materials the LCA data related to each building element should be processed through statistical analysis and interpretation, in order to establish the reference value [10]. The reference study period should be based on 100 years and the LCA system boundaries should be cradle-to-grave. The environmental impacts should be normalised to $1 \mathrm{~m}^{2}$ of the building element, in order to allow comparison of building elements.

The benchmarking methods could be applied to set LCA benchmarks for whole-buildings. LCA-based benchmarks for the whole-buildings could be fixed through the statistical analysis of the LCA data related to a specific built environment $[1,2,5]$ or modelling a reference building starting from national construction standards and prescriptions. The buildings sample should be composed by buildings realised after the year of 2010 and they should belong to a specific construction context, according to the national energy standards and the current construction practices. The buildings analysed should refer to the main five different generic buildings types: commercial buildings, educational buildings, healthcare buildings, residential buildings and retail. The LCA benchmarks should refer to the single building function, avoiding issues linked to building operational energy use and occupations (i.e. the educational buildings have different energy consumption than an office building related to the worktime and equipment use) [14]. The building typologies related to the functions can be divided into building archetypes, according to the specific built environment characteristics (i.e. the residential building could be divided into single-family house, terraced house, multi-family house and apartment block). A reference building should be modelled for each building archetype and each building function, fixing the reference service life (equal to 100 years) and the functional unit ( $1 \mathrm{~m}^{2}$ of gross floor area), in order to compare the building environmental performances. The LCA system boundaries for the whole-building benchmark should be cradle-to-grave. Based on a statistical analysis, the median value should be set as the reference LCA benchmark for the whole-buildings [8-12]. As an example the Swedish developer and construction company Folkhem have certified a wooden residential house with the Swedish Program Operator "The International EPD ${ }^{\circledR}$ System": the EPD assesses the life cycle of a Folkhem's concept building, providing objective and reliable information on the environmental impacts of the whole-building. The use of EPDs, as well as the Folkhem's EPD, could be an easy tool for contracting authorities, in order to verify the GPP criteria achievement and to permit an appropriate check of the procurement contract executions.

\section{Conclusions}

The study proposes a simple benchmarking to set LCA-based benchmarks, incentivising the use of environmental performances values in GPP criteria, in order to facilitate the verification activities of public administrations. In this study, LCA benchmarks for construction products were fixed and an approach to fix building 
elements and whole-building benchmarks is articulated. The inclusion of LCA benchmark values in GPP criteria for buildings can stimulate the use and the production of EPDs for the construction market, making available transparent LCA data and facilitating the integration of LCA in the GPP criteria. The EPDs could also be an instrument to verify the GPP criteria achievement by contracting authorities.

The use of LCA-based benchmarks could also be a way to stimulate the building market to reach new environmental sustainability targets.

\section{References}

1. Garnett N, Pickrell S, Benchmarking for construction: theory and practice, Construction Management and Economics, 2000, Vol 18, pp. 55-63.

2. Haapio A, Viitaniemi P, A critical review of building environmental assessment tools, Environmental Impact Assessment Review, Vol. 28, 2008, pp. 469-482.

3. Eberl S, DGNB vs. LEED: A comparative analysis, Conference on Central Europe towards Sustainable Building, 2010.

4. Collinge W.O, Thiel C.L, Campion N.A, Al-Ghamdi S.G, Woloschin C.L, Soratana K, Bilec M.M, Integrating Life Cycle Assessment with Green Building and Product Rating Systems: North American Perspective, Procedia Engineering, Vol. 118, 2015, pp. 662-669.

5. Ganassali S, Lavagna M, Campioli A, LCA benchmarks in buildings environmental certification systems, 41st IAHS World Congress "Sustainability and Innovation for the Future", Portugal, 2016.

6. Dodd N, Garbarino E, Gama Caldas M, Green Public Procurement Criteria for Office Building Design, Construction and Management. Procurement practice guidance document, EUR 28006 EN, 2016.

7. Allegato 1 del G.U. n.16 del 21 Gennaio 2016, Criteri ambientali minimi per l'affidamento di servizi di progettazione e lavori per la nuova costruzione, ristrutturazione e manutenzione di edifici pubblici, Ministero dell'Ambiente, del Territorio e del Mare, 2016.

8. Castro M.d.F, Mateus R, Serodio F, Braganca L, Development of benchmarks for operating ad resources consumption to be used in healthcare building sustainability assessment methods, Sustainability, Vol. 7, 2015, pp. 13222-13248.

9. Ji C, Hong T, Jeong J, Kim J, Lee M, Jeong K., Establishing environmental benchmarks to determine the environmental performance of elementary school buildings using LCA. Energy and Buildings, Vol. 127, 2016, pp. 818-829.

10. Köning H, De Cristofaro M.L, Benchmarks for Life Cycle Costs and Life Cycle Assessment benchmarks of residential buildings. Building Research \& Information, Vol. 40, No. 5, 2012, pp. 558-580.

11. Lasvaux S, Lebert A, Achim F, Grannec F, Hoxha E, Nibel S, Chevalier J, Towards guidance values for the environmental performance of buildings: application to the statistical analysis of 40 low-energy single family houses' LCA in France, The International Journal of Life Cycle Assessment, Vol. 22, No. 5, 2016, pp. 657-674.

12. Moschetti R, Mazzarella L, Nord N, An overall methodology to define reference values for building sustainability parameters, Energy and Buildings, Vol. 88, 2015, pp. 413-427.

13. Anderson J, Shiers D, Green guide to specification, John Wiley \& Sons, 2016.

14. Hong S, Paterson G, Burman E, Steadman P, Mumovic D, A comparative study of benchmarking approaches for non-domestic buildings: Part 1-top-down approach, International Journal of Sustainable Built Environment, Vol. 2, 2013, pp. 119-130. 
Open Access This chapter is licensed under the terms of the Creative Commons Attribution 4.0 International License (http://creativecommons.org/licenses/by/4.0/), which permits use, sharing, adaptation, distribution and reproduction in any medium or format, as long as you give appropriate credit to the original author(s) and the source, provide a link to the Creative Commons license and indicate if changes were made.

The images or other third party material in this chapter are included in the chapter's Creative Commons license, unless indicated otherwise in a credit line to the material. If material is not included in the chapter's Creative Commons license and your intended use is not permitted by statutory regulation or exceeds the permitted use, you will need to obtain permission directly from the copyright holder.

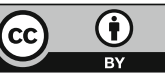




\title{
Special Session on Product Environmental Footprint
}

\author{
Mélanie Guiton and Enrico Benetto
}

\begin{abstract}
The Product Environmental Footprint (PEF) Life Cycle Assessment (LCA) method has been adopted by the European Commission in 2013 as part of the Communication "Building the Single Market for Green Products" [1]. Since then a pilot phase involving about 300 companies, industry associations, NGOs and governments has been launched to develop product category rules for more than 20 different product groups. In this session it was discussed the status of play of the work done till now and its future perspective.
\end{abstract}

\section{Introduction}

In 2010 a coalition of big companies from different sectors asked to the at the time European Commissioner for Environment Janez Potočnik to intervene to solve a problem that was undermining their competitiveness in the European market. The problem was the proliferation of environmental labels and certification schemes that was taking place in different European Member States. From the industry viewpoint this situation constrained them in particular to produce different studies and calculations reporting the environmental performance of the same product in case that product was going to be sold in different countries. This situation was created by the fact that different countries (e.g. France, Italy, United Kingdom, Switzerland) were developing product-related environmental information policies based on similar-but-different methodological approaches (being them ISO 14040-44, ISO 14025, WRI GHG protocol, PAS 2050, BP X30, and many more). The same year the European Council put forward the same request to the Commission, that is to develop a harmonised method to calculate the environmental performance of products along their entire supply chain. Based on this double request the Commission developed the Product Environmental

M. Guiton $(\bowtie) \cdot$ E. Benetto

Department ERIN-Environmental Research and Innovation,

Luxembourg Institute of Science and Technology (LIST),

Life Cycle Sustainability and Risk Assessment, 4422 Belvaux, Luxembourg

e-mail: melanie.guiton@list.lu

(C) The Author(s) 2018

E. Benetto et al. (eds.), Designing Sustainable Technologies,

Products and Policies, https://doi.org/10.1007/978-3-319-66981-6_57 
Footprint (PEF) and Organisation Environmental Footprint (OEF) methods. They were adopted by the Commission in April 2013 [2] and published in May 2013 on the Official Journal of the European Union.

The intention was to provide industry, member states and NGOs with a solid science-based LCA calculation tool that would lead to results more reproducible, reliable and verifiable. The achievement of these ambitious objectives, however, requires the development of product category rules (organisation sectoral rules for the implementation of $\mathrm{OEF}$ ) and a number of additional features like clear rules on verification, communication, data quality, etc. In order to develop and test all these new features the Commission decided to launch, in tight collaboration with all stakeholders, a pilot phase (2013-2017).

During the special session on PEF at LCM 2017 conference the work done during the pilot phase has been analysed from different viewpoints and some preliminary insight about possible future application of PEF/OEF has been drawn.

\section{Summary of the Presentations Delivered}

The first presentation was delivered by An De Schryver from the European Commission. Ms. De Schryver presented the main outcomes of the Environmental Footprint (EF) pilot phase. The EF pilot phase has gathered about 300 stakeholders from worldwide, actively developing Product Environmental Footprint Category Rules (PEFCRs) and Organisation Environmental Footprint Sectoral Rules (OEFSRs). These organisations (companies, industry associations, governments, NGOs) have invested important resources to develop a work that they consider key for their future respective interests. On top of the 300 stakeholders "doing" the work, there have been more than 2000 stakeholders who have been monitoring the developments and participating to the several online consultations run during these years. This has been the first case of such a wide exercise run by the European Commission and surely shows the high political and technical relevance of the dossier.

Ms. De Schryver highlighted the main results achieved during the pilot phase. In particular she stressed the following ones:

- Implementation of the materiality principle. It is well known to LCA practitioners that even for the most complicated products there are a limited number of processes that drives the majority of the impacts. The hotspot analysis procedure developed for PEF allows to identify the most relevant impact categories, life cycle stages, processes, and direct elementary flows, i.e. those contributing to at least $80 \%$ of the environmental impacts. The driving processes will be those for which company and/or site specific data will be requested.

- Implementation of the Data Needs Matrix (DNM). Once the most relevant processes are identified, clear instruction on which data shall be collected and 
what kind of secondary datasets can be used are listed in the DNM, based on the level of operational control the company performing the study has.

- Reduction of costs. One of the barriers towards a wider implementation of LCA, especially in Small and Medium Enterprises (SMEs) has been the cost of implementing such studies, due to a number of factors, including the amount of data to be collected/found, the time requested to do calculations, the costs of verification, the cost to access to secondary LCI database. The implementation of PEF will bring down many of the costs of performing a PEF/LCA study, up to $80 \%$, depending on the specific situations. This result will be achieved thank to the availability of common rules (PEFCRs/OEFSRs), high quality secondary LCI datasets available for free to any user around the world, free availability of the models used, the availability of user-friendly software, of e-learning packages developed in several languages, and clear verification rules.

- Harmonisation of secondary data sources. By having clear modelling requirements for secondary dataset tendered, the PEF initiative is steering the harmonisation of existing LCI commercial databases. The Commission will make available more than 8000 secondary LCI datasets coming from 6 different data providers. They will all have the same modelling requirements for the foreground data, the same energy and transport datasets for the background. Moreover, they will all use the same format and nomenclature, therefore making them directly usable in most of the LCA software available on the market.

The second presentation was delivered by Mr Erwin M. Schau from the European Commission Joint Research Centre. He focused his presentation on the concept of classes of performance, based on the example of the PEFCR on olive oil. One of the novelty introduced by the PEF method is the concept of benchmark, corresponding to the PEF profile of the average product sold in Europe. Each PEFCR includes one or more representative products. Each of them is modelled based on the different production techniques and their respective "weight" in terms of consumption based European market. The representative product therefore represents the weighted average for that group and its PEF profile, calculated and reported in the PEFCR, it is the benchmark for that product group. Therefore, by having this reference publicly available, each producer will be able to know if their own product score better or worse than the benchmark. Once the benchmark is available, the next logical step is to consider the possibility of developing classes of environmental performance. During the pilot phase the stakeholders leading the development of PEFCRs were invited to test this concept, in particular trying to develop 5 classes of performance, where the benchmark is always class $\mathrm{C}$. This is an optional requirement. The approach taken to define the classes of performance was to identify the most relevant parameters (in terms of activity data and emissions) and then identify the theoretical minimum and maximum values possible for such parameters. Once these were identified the calculations were run to identify the range of performance that could be achieved with different combinations of the various parameters. Mr. Schau highlighted the pros and cons of this approach, clearly stating that the ideal solution would be to base the 
definition of the classes of performance on a statistically representative sample of PEF profiles calculated for real products.

The third presentation was delivered by Mr. Quentin De Hults, speaking on behalf of PlasticsEurope. PlasticsEurope has been committed in LCA for decades by managing a dataset program for plastics, and by contributing to LCA developments e.g. through the UNEP SETAC Life Cycle Initiative. PlasticsEurope also believes in LCA to be completed by other tools like risk assessment. With this constructive state of mind, PlasticsEurope is participating to the European Product Environmental Footprint, officially in 3 pilots and in many other working groups and workshops. According to Mr. De Hults the EF pilot phase contributed to improve the best practice in the LCA domain thanks to the transparency of the approach and the high quality of the technical discussions that take place at the Technical Advisory Board. He also mentioned a number of issues to be further improved, referring e.g. to the improvement of the toxicity impact categories, with the development of more reliable characterisation factors. He mentioned that the approach taken in PEF should be complemented with other approaches like Proscale (a specific presentation of Proscale took place during the same conference). Another area of improvement referred to the development of secondary datasets when there are only few companies running certain processes, taking into account the associated industrial confidentiality issues to be addressed. According to PlasticsEurope, there is room for the use of PEF into existing or new product policies provided that it remains a voluntary approach.

The fourth presentation was delivered by Mr. Pau Huguet Ferran from Ecomatters. Mr. Ferran spoke on behalf also of PRé Consultants. The two companies supported the European Commission during the EF pilot phase by reviewing a number of PEF studies carried out by companies on real products to test the feasibility of implementation of the draft PEFCR developed. The shortcomings identified when reviewing these studies have been very important to improve the quality of the PEFCRs and to correct some flaws in the methodological requirements developed during the pilot phase. Some of the most relevant problems highlighted by the reviewers were that (i) the PEFCRs were not strict enough in terms of modelling requirements, (ii) the DNM was not systematically implemented, (iii) the Circular Footprint Formula, that is the single formula to calculate credits and burden related to the end of use of a product, was too complicated and wrongly implemented by some companies, (iv) the way how the representative product has been modelled by the stakeholders leading the PEFCR development is not necessarily supported the companies implementing it.

Based on the outcome of these reviews a number of changes have been implemented in the Guidance documents supporting the pilot phase.

The fifth and last presentation was delivered by Ms. Mihaela Thuring from VITO, institute actively involved in two pilots and some supporting contracts. According to Ms. Thuring environmental information on organisations and products are important tools that companies can use for achieving environmental goals towards a sustainable society. Both ISO 14001 (Environmental management systems) and PEF are aimed at providing such information to market players. 
The PEF method explores a new way of communicating the environmental impacts of products through the application of the PEF formula(s), as part of the efforts towards a Circular Economy. This new way expresses no longer the actual potential environmental impact, but includes also the potential benefits of the recycling/ recoverability process, as well as the energy recovery potential of the materials. While understandable for professionals in the field, this new approach poses multiple questions in determining how this complex message gets across to consumers and how it will contribute to fulfilling the original goal of streamlining the communication of environmental information. The wide participation in the PEF/OEF pilot of actors across most industries and beyond European borders showed that the relevance of LCA methods in taking decisions and communicating on environmental related aspects is more actual than ever, as well as the interest in the unifying approach which the PEF/OEF method is bringing.

VITO stated that the identification of information flows between PEF and ISO 14001 determines how an organisation can integrate both the PEF and ISO 14001 in a coherent and efficient manner.

\section{Conclusions}

The discussion during this special session highlighted the great amount of work done by the European Commission and all stakeholders in the context of the Environmental Footprint pilot phase. It is widely acknowledged that major improvements from the methodological point of view have been achieved, leading to a new generation of LCA/PEF studies that should deliver results that are more reliable, more reproducible and more verifiable. The pilot phase highlighted issues to be solved in the area of characterisation factors in existing impact assessment methods, the need to develop more and better defined impact categories (e.g. in the area of biotic and abiotic resources). There are important quality issues found in all commercial databases that would require paying more attention and, in particular, to the review process of these databases. There is work to be done to better identify the benchmarks, and to develop classes of performance. There is an important "educational" work to be done within practitioners, policy makers, and NGOs when it comes to the development and use of weighting factors. However, notwithstanding all these possible improvements, the LCA community is aware that their credibility depends highly on the success of PEF. There are market needs to be addressed, there are policies that could highly benefit from a consistent use of LCA principle. There are problems related to misleading green claims and the way we implement green public procurement that could be addressed and maybe solved by embedding PEF into a number of existing or new policies. After the pilot phase the European Commission will engage with all interested stakeholders in an open conversation about the future role of PEF/OEF and LCA into European policies. 
Acknowledgements The session on Product Environmental Footprint has been chaired by Mr. Michele Galatola, Product Team Leader at European Commission-DG ENV, and Mr. Alberto Frausin, CEO at Carlsberg Italy. The authors would like to greatly acknowledge Mr. Michele Galatola for having provided the complete content of this paper. As agreed between the declared authors of the paper and Mr. Galatola, he remains the corresponding author Michele.GALATOLA@ec.europa.eu.

\section{References}

1. European Commission, Communication from the Commission to the European Parliament and the Council: Building the Single Market for Green Products-Facilitating better information on the environmental performance of products and organisations, April 2013, COM/2013/0196 final.

2. European Commission, Recommendation of 9 April 2013 on the use of common methods to measure and communicate the life cycle environmental performance of products and organisations, 2013/179/EU.

Open Access This chapter is licensed under the terms of the Creative Commons Attribution 4.0 International License (http://creativecommons.org/licenses/by/4.0/), which permits use, sharing, adaptation, distribution and reproduction in any medium or format, as long as you give appropriate credit to the original author(s) and the source, provide a link to the Creative Commons license and indicate if changes were made.

The images or other third party material in this chapter are included in the chapter's Creative Commons license, unless indicated otherwise in a credit line to the material. If material is not included in the chapter's Creative Commons license and your intended use is not permitted by statutory regulation or exceeds the permitted use, you will need to obtain permission directly from the copyright holder. 